Florida International University FIU Digital Commons

$3-7-2014$

\title{
Physics-Based Modeling of Power System Components for the Evaluation of Low-Frequency Radiated Electromagnetic Fields
}

Mohammadreza Barzegaran

Florida International University, barzegaran@gmail.com

DOI: $10.25148 /$ etd.FI14040832

Follow this and additional works at: https://digitalcommons.fiu.edu/etd

Part of the Power and Energy Commons

\section{Recommended Citation}

Barzegaran, Mohammadreza, "Physics-Based Modeling of Power System Components for the Evaluation of Low-Frequency Radiated Electromagnetic Fields" (2014). FIU Electronic Theses and Dissertations. 1239.

https://digitalcommons.fiu.edu/etd/1239 


\section{FLORIDA INTERNATIONAL UNIVERSITY}

Miami, Florida

PHYSICS-BASED MODELING OF POWER SYSTEM COMPONENTS FOR THE EVALUATION OF LOW-FREQUENCY RADIATED ELECTROMAGNETIC FIELDS

A dissertation submitted in partial fulfillment of the requirement for the degree of

DOCTOR OF PHILOSOPHY

in

ELECTRICAL ENGINEERING

By

Mohammadreza Barzegaranbaboli

2014 
To: Dean Amir Mirmiran

College of Engineering and Computing

This dissertation, written by Mohammadreza Barzegaranbaboli, and entitled PhysicsBased Modeling of Power System Components for The Evaluation of Low-Frequency Radiated Electromagnetic Fields, having been approved in respect to style and intellectual contents, is referred to you for judgment.

We have read this dissertation and recommend that it be approved.

Ibrahim Tansel

Jean Andrian

Sakhrat Khizroev

Nezih Pala

Osama A. Mohammed, Major Professor

Date of Defense: March 07, 2014

The dissertation of Mohammadreza Barzegaranbaboli is approved.

Dean Amir Mirmiran

College of Engineering and Computing

Dean Lakshmi N. Reddi

University Graduate School

Florida International University, 2014 
C Copyright 2014 by Mohammadreza Barzegaranbaboli

All rights reserved. 


\section{DEDICATION}

This dissertation is dedicated to my compassionate, supportive, intelligent, and patient wife, Parvin Rafiee, and to my always ever faithful parents, Masoumeh Mirbozorgi and Alireza Barzegaran, who brought me up with their love and encouraged me to pursue advanced degrees. 


\section{ACKNOWLEDGMENTS}

I owe several years of my research life to my advisor Professor Osama A. Mohammed for his willingness to accept me in his laboratory and for providing me with a great research motivations, and research facilities. Hence, I wish to express in words my deepest gratitude to my adviser, Professor Osama Mohammed, for his guidance, encouragement, and support during my graduate study. His creative thinking, knowledge and expertise on computational electromagnetic were indeed the fuse of my propulsion motor in conducting this research.

I would like to thank my dissertation committee members Professor Sakhrat Khirzroev, Professor Ibrahim Tansel, Professor Jean Andrian, and Dr. Nezih Pala for their insightful comments and constructive suggestions in the review of my research proposal and dissertation.

I would like to acknowledge the partial research support provided from the office of Naval Research and the US department of Energy throughout my years of research in Energy Systems Laboratory. I also acknowledge the doctoral DEA fellowship from FIU graduate school during the summer semester of 2013 of my studies at Florida International University.

Although this doctoral dissertation is considered to be my personal accomplishment; I would like to acknowledge my colleagues in Energy Systems Research Laboratory for their creative support, encouragement, and collaborations during my graduate studies.

I also appreciate the trust and support of the ECE Department staff; Ms. Pat Brammer, Ms. Ana Saenz, and Mr. Oscar Silveria during my studies in department of electrical and computer engineering. 


\author{
ABSTRACT OF THE DISSERTATION \\ PHYSICS-BASED MODELING OF POWER SYSTEM COMPONENTS FOR THE \\ EVALUATION OF LOW-FREQUENCY RADIATED ELECTROMAGNETIC FIELDS \\ by \\ Mohammadreza Barzegaranbaboli \\ Florida International University, 2014 \\ Miami, Florida, USA
}

Professor Osama A. Mohammed, Major Professor

The low-frequency electromagnetic compatibility (EMC) is an increasingly important aspect in the design of practical systems to ensure the functional safety and reliability of complex products. The opportunities for using numerical techniques to predict and analyze system's EMC are therefore of considerable interest in many industries.

As the first phase of study, a proper model, including all the details of the component, was required. Therefore, the advances in EMC modeling were studied with classifying analytical and numerical models. The selected model was finite element (FE) modeling, coupled with the distributed network method, to generate the model of the converter's components and obtain the frequency behavioral model of the converter. The method has the ability to reveal the behavior of parasitic elements and higher resonances, which have critical impacts in studying EMI problems.

For the EMC and signature studies of the machine drives, the equivalent source modeling was studied. Considering the details of the multi-machine environment, including actual models, some innovation in equivalent source modeling was performed 
to decrease the simulation time dramatically. Several models were designed in this study and the voltage current cube model and wire model have the best result. The GA-based PSO method is used as the optimization process. Superposition and suppression of the fields in coupling the components were also studied and verified. The simulation time of the equivalent model is $80-100$ times lower than the detailed model. All tests were verified experimentally.

For the EMC study of the switching activities, 3DFE modeling of a typical power electronic drive was implemented with an innovation in defining switching activities. The measurement was also applied for verification of the numerical results, and for investigating the stray fields under different operating conditions.

As the application of EMC and signature study, the fault diagnosis and condition monitoring of an induction motor drive was developed using radiated fields. In addition to experimental tests, the $3 \mathrm{DFE}$ analysis was coupled with circuit-based software to implement the incipient fault cases. The identification was implemented using ANN for seventy various faulty cases. The simulation results were verified experimentally. Finally, the identification of the types of power components were implemented. More than 170 circumstances of the combinations of the typical power components were tested experimentally and the identification is explained. The results show that it is possible to identify the type of components, as well as the faulty components, by comparing the amplitudes of their stray field harmonics. The identification using the stray fields is nondestructive and can be used for the setups that cannot go offline and be dismantled. 


\section{TABLE OF CONTENTS}

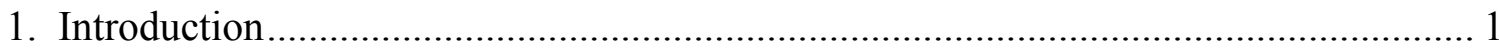

1.1 INTRODUCTION TO ELECTROMAGNETIC COMPATIBILITY..................................... 2

1.2 THE SOURCES OF ELECTROMAGNETIC INTERFERENCE …........................................5

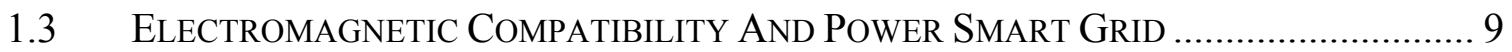

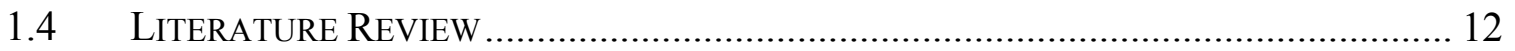

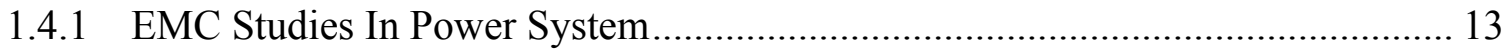

1.4.2 Electromagnetic Computational Modeling Studies …………………………...... 25

1.4.3 Electromagnetic Signature Studies ………………......................................... 26

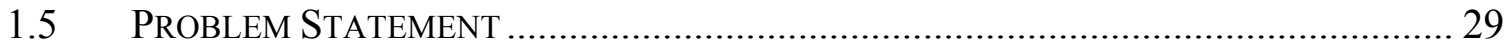

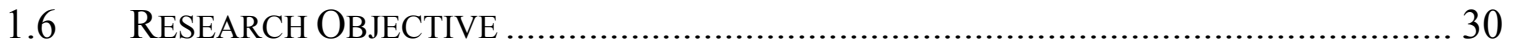

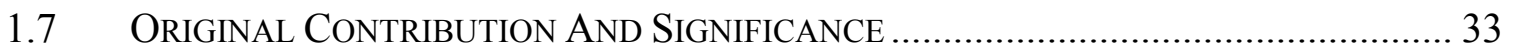

1.8 ORganization Of THE DisSERTATION............................................................. 36

2. Advanced Modeling in Computational Electromagnetic Compatibility ................... 38

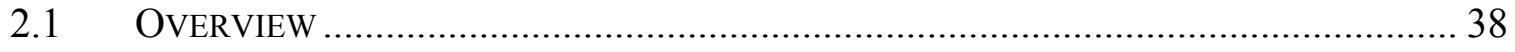

2.2 EMC COMPUTATIONAL ModELS AND SOLUTION METHODS …………………...... 41

2.3 CLASSIFICATION OF EMC MODELS ................................................................. 43

2.4 TIME DOMAIN VS. FREQUENCY DOMAIN MODELING.............................................. 46

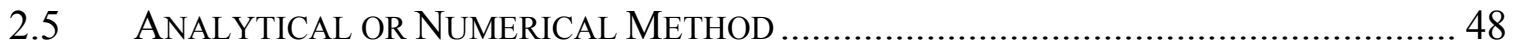

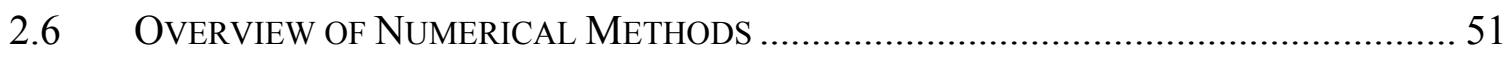

2.6.1 Modeling of Problems via the BEM and MoM ................................................. 52

2.6.2 Modeling of problems via the domain methods: FDM and FEM......................... 52

3. 3-D Finite Element Method Coupled with Transmission-Line Modeling in Emc Study 55

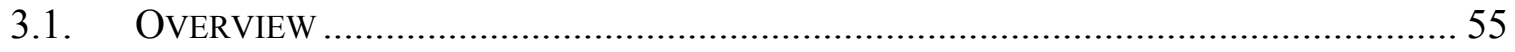

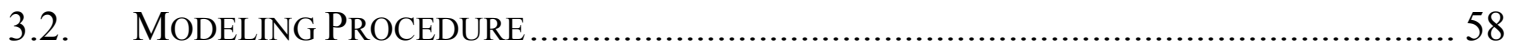

3.2.1 Physics based modeling using 3-D FEM ....................................................... 59

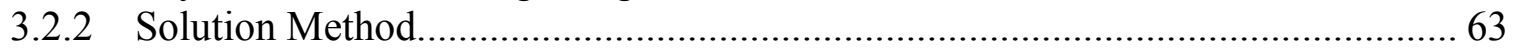

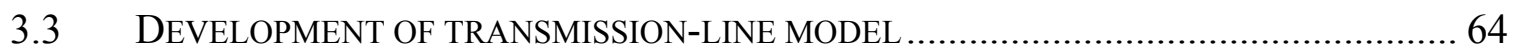

3.4 RESONANT CIRCUIT DESIGN OPTIMIZATION ………………................. 71

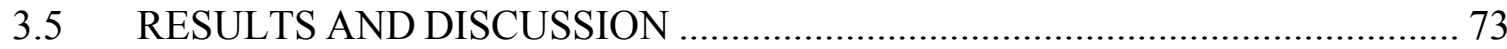

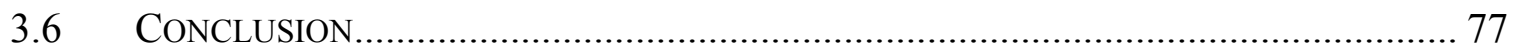

4. Equivalent Source Modeling and 3-D Full Finite Element Modeling for the

Studying Radiated Electromagnetic Field ............................................................... 79

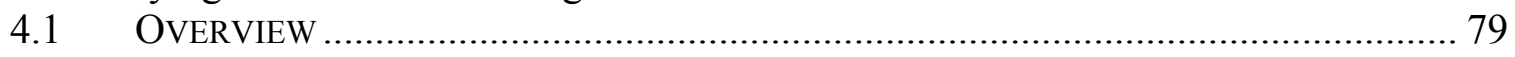

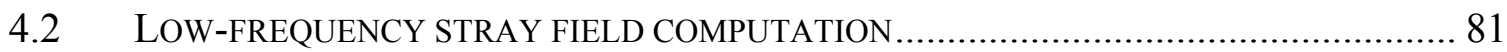

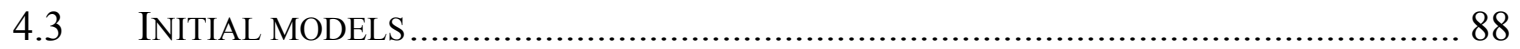

4.3.1 Single current loop model............................................................................. 90

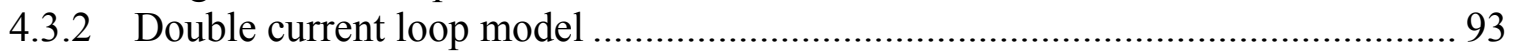

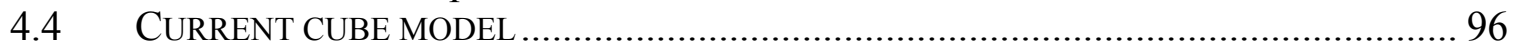

4.4.1 Magnetic field as the objective function ............................................................ 99 


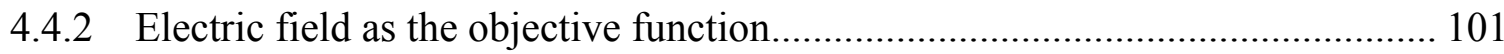

4.5 VOLTAGE-CURRENT RECTANGULAR PRISM MODEL …...................................... 104

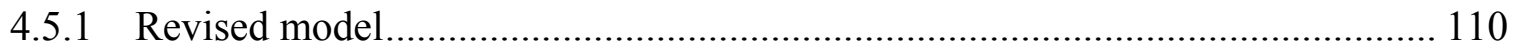

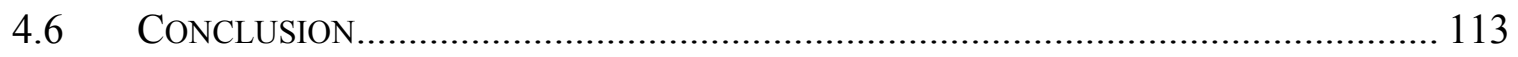

5. Instruction for the Low Frequency Electromagnetic Signature Experimental

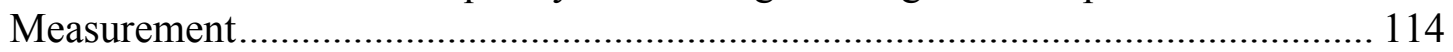

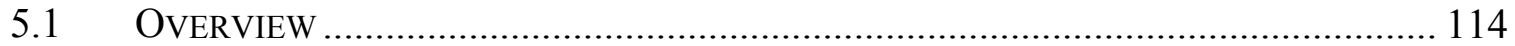

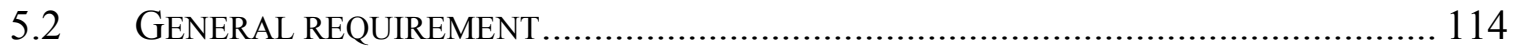

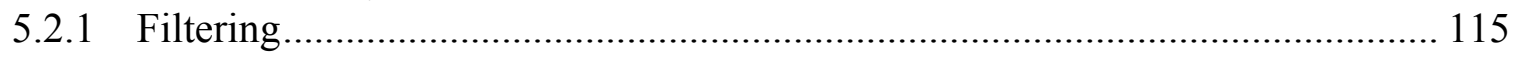

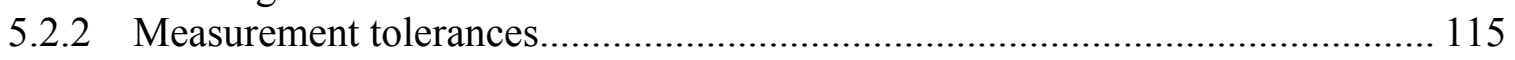

5.2.3 Ambient electromagnetic level ................................................................. 116

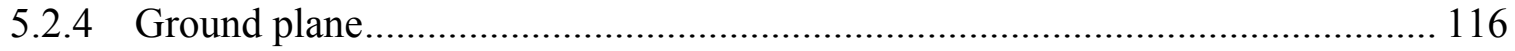

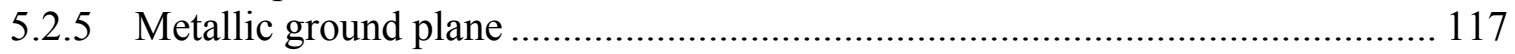

5.2.6 Power source impedance........................................................................... 120

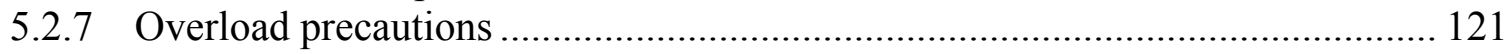

5.2.8 Construction and arrangement of Interconnecting leads and cables.................... 121

5.2.9 Construction and arrangement of Input power leads ........................................ 122

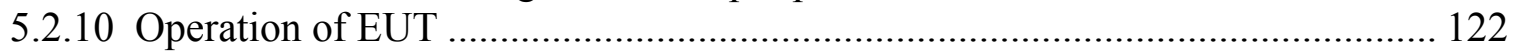

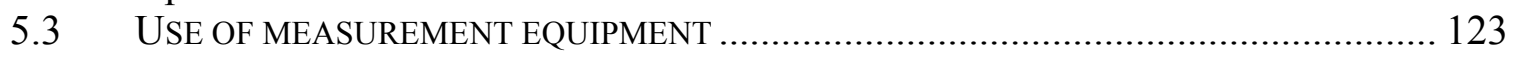

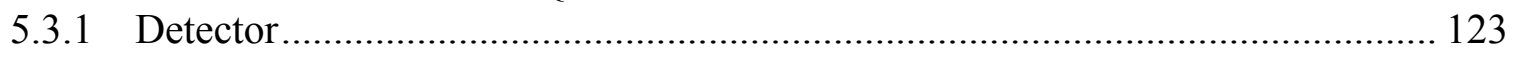

5.3.2 Computer-controlled receivers..................................................................... 123

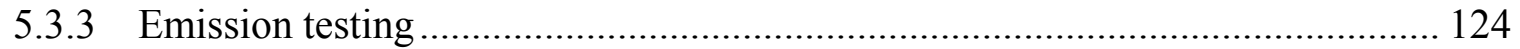

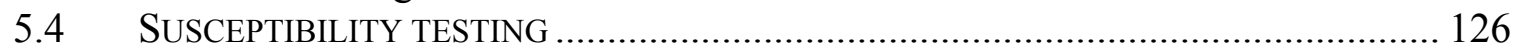

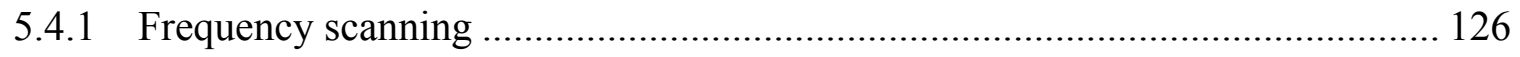

5.4.2 Thresholds of susceptibility .................................................................... 126

5.4.3 Calibration of measuring equipment............................................................ 127

5.5 DETAILED REQUIREMENTS AND PROCEDURE OF THE EXPERIMENTS …………...... 127

5.5.1 Conducted emissions, power leads, $30 \mathrm{~Hz}$ to $10 \mathrm{kHz}$...................................... 128

5.5.2 Radiated Emissions, magnetic field $30 \mathrm{~Hz}-100 \mathrm{kHz} \ldots \ldots \ldots \ldots \ldots \ldots \ldots \ldots \ldots \ldots \ldots \ldots \ldots \ldots . . . . . . . . . . . . . . . . .133$

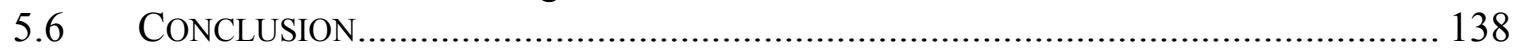

6. Wire Modeling and Experimental Verification ………........................................ 139

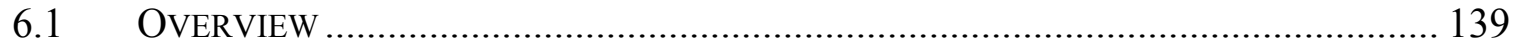

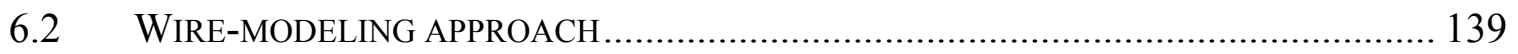

6.3 MODELS OF THE COMPONENTS WITH SIMULATION AND EXPERIMENTAL RESULTS 141

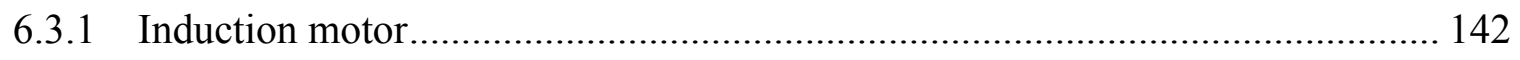

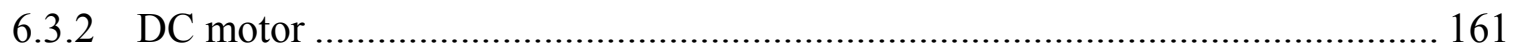

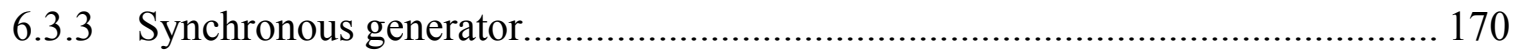

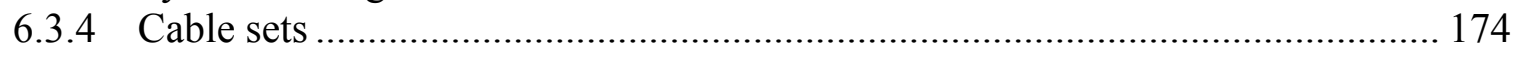

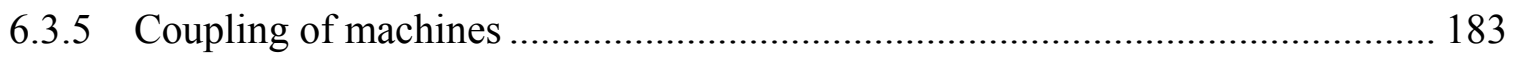

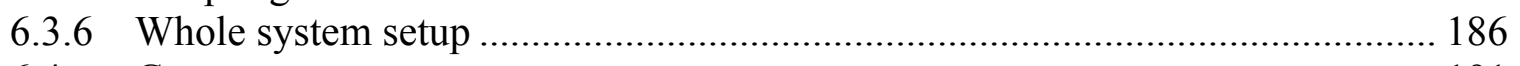

6.4 GENERALIZATION OF THE WIRE MODEL.......................................................... 191

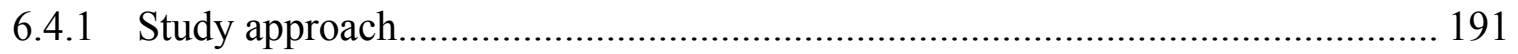

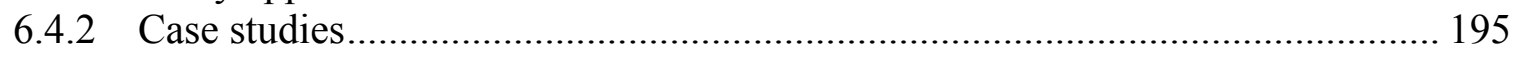




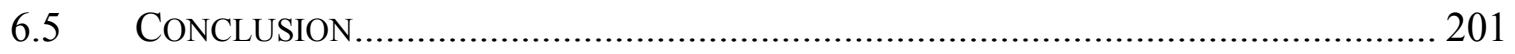

7. Modified 3d Finite Element Modeling of Converters for System Monitoring.......... 203

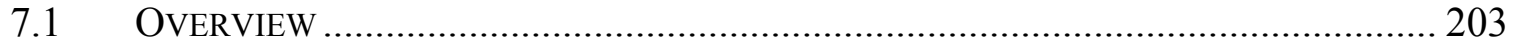

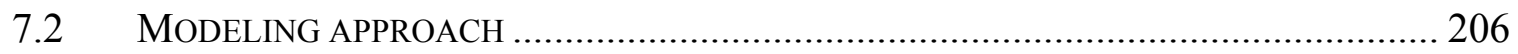

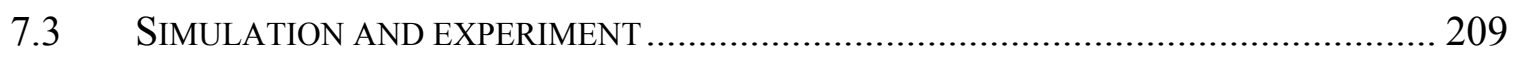

7.3.1 Case 1: converter connected to the load ......................................................... 209

7.3.2 Case 2: converter connected to the motor......................................................... 213

7.4 APPLICATIONS OF THE FREQUENCY RESPONSE ANALYSIS OF THE STRAY FIELD .... 218

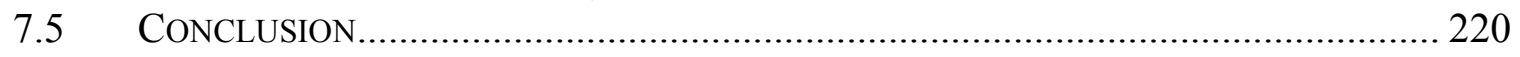

8. Fault Type Diagnosis of Induction Motor Using Magnetic Stray Field .................... 222

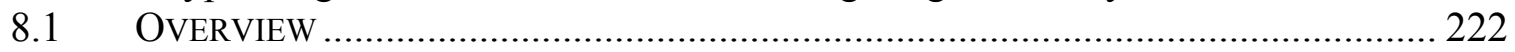

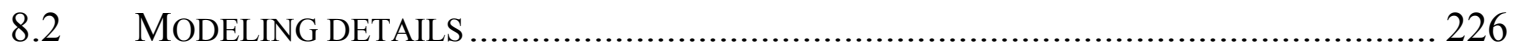

8.3 UNBALANCED CURRENT STUDY, EXPERIMENT AND SIMULATION ……………...... 229

8.4 IDENTIFICATION OF UNBALANCED CURRENT PHASE THROUGH ARTIFICIAL

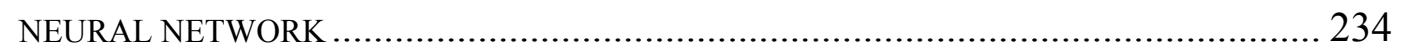

8.5 SHORT-CIRCUIT STUDY, EXPERIMENT AND SIMULATION ……………………..... 235

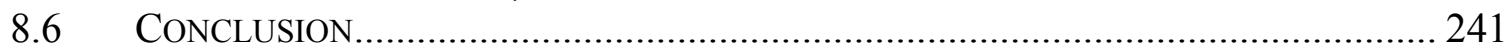

9. Identification of Short-Circuit Location in the Stator Windings of Induction Motors

Using Radiated Electromagnetic Field Signatures .................................................. 242

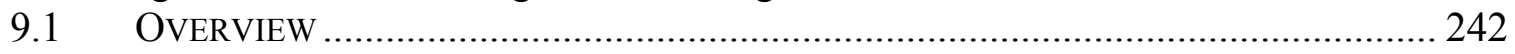

9.2 MAGNETIC STRAY FIELD MONITORING OF THE SHORT-CIRCUIT IN THE WINDING.. 247

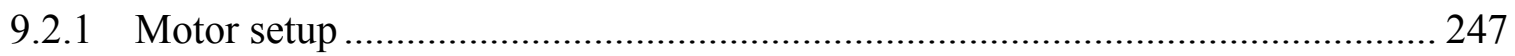

9.2.2 Monitoring the radiated magnetic field............................................................ 248

9.3 INTERCOIL SHORT-CIRCUIT STUDY, EXPERIMENTAL AND SIMULATION ………....... 250

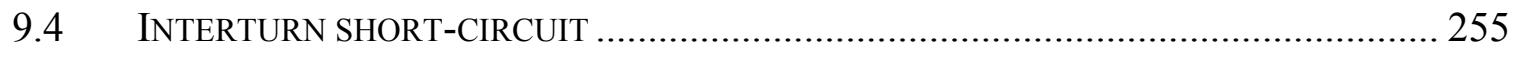

9.4.1 Singular-optimum location of antenna.......................................................... 258

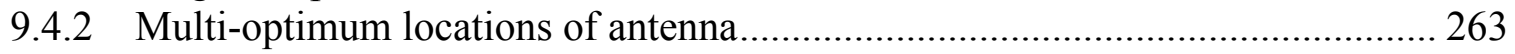

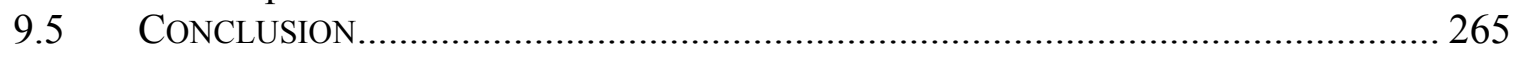

10. Power Source Condition Monitoring Based on the Electromagnetic Signatures .... 267

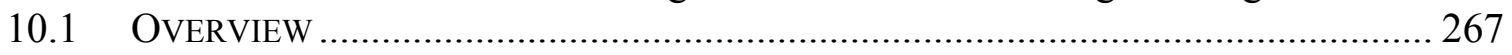

10.2 POWER COMPONENT MONITORING PROCEDURE.................................................. 270

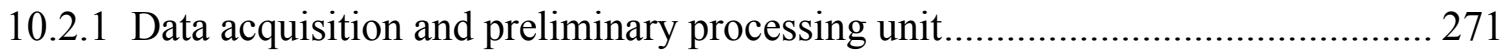

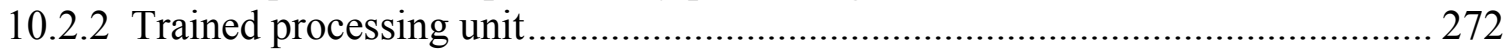

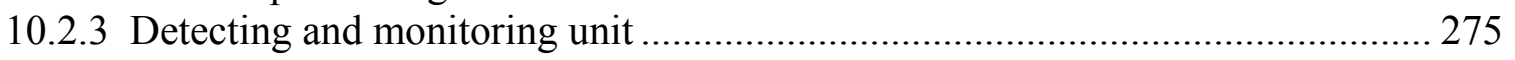

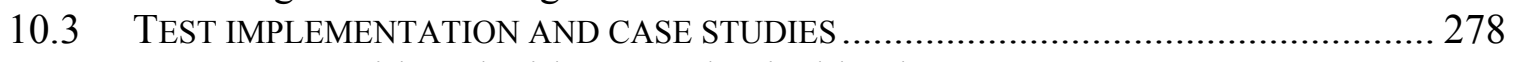

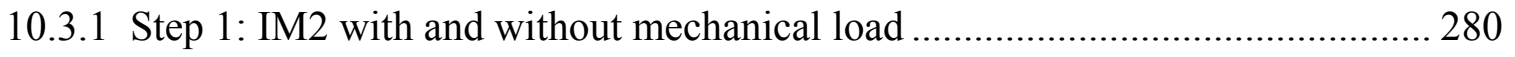

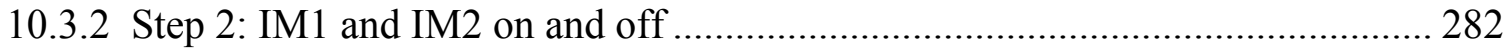

10.3.3 Step 3: IM1 faulty: short-circuited) and IM2 (healthy) ………...................... 284

10.3.4 Step 4: $\mathrm{IM}_{1}$ ( unbalanced current, healthy ) and $\mathrm{IM}_{2}$ ( unbalanced current,

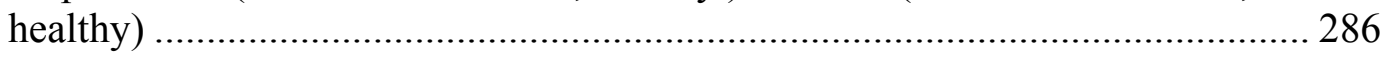

10.3.5 Step 5: Converter connected to the $\mathrm{IM}_{1}$ at different switching frequencies with

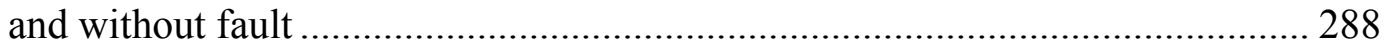


10.3.6 Step 6: Synchronous Generator connected to the DC motor and $\mathrm{IM}_{3}$ (3-HP

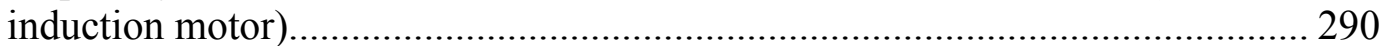

10.4 FAULT DIAGNOSIS AND MONITORING OF POWER CONVERTER................................ 292

10.4.1 Harmonic and Inter-Harmonic Study of the Radiated Fields ............................. 293

10.4.2 Switching Patterns (SPWM, SVPWM) ………….......................................... 296

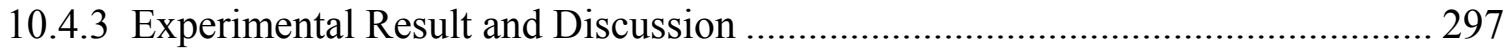

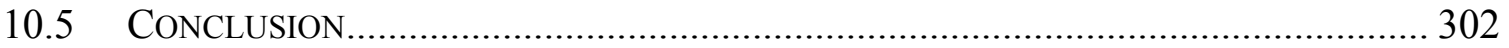

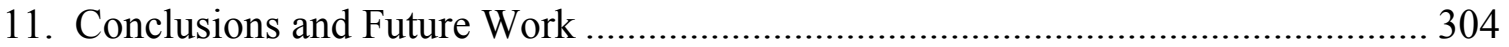

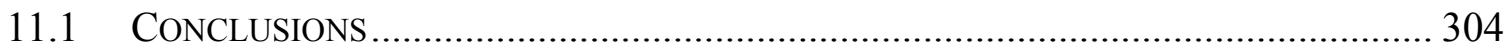

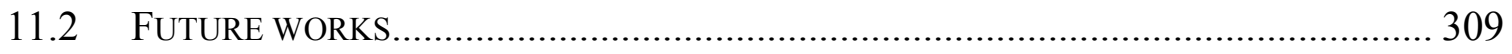

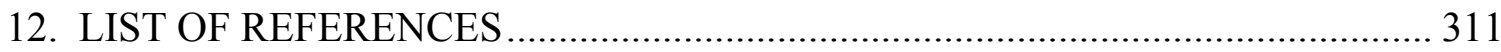




\section{LIST OF TABLES}

Table 3.1 Optimal values for designed inductor resulted from GA.............................. 73

Table 4.1 The calculated currents for the cube branches ......................................... 99

Table 4.2 The calculated current for cube legs .................................................. 102

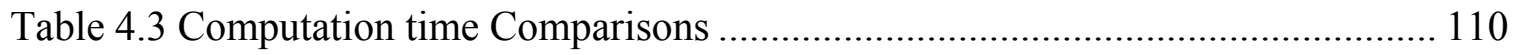

Table 5-1 Bandwidth and measurement time ....................................................... 124

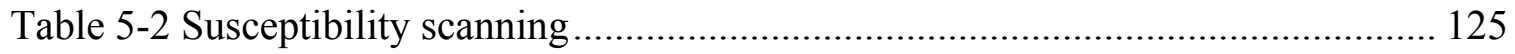

Table 6.1 Computation time Comparisons ........................................................ 150

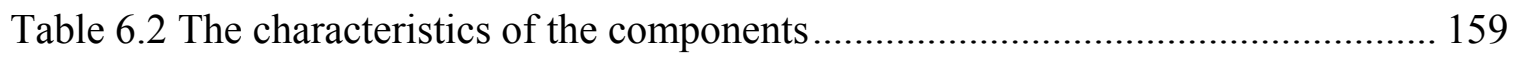

Table 6.3 The details of the components in the tested setup .................................... 186

Table 6.4 Some patterns of size variation of induction motor and synchronous (syn)

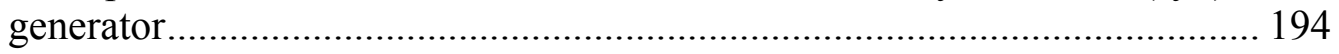

Table 6.5 Some patterns of terminal voltage variation of induction motor and synchronous (syn) generator .......................................................... 194

Table 6.6 Simulation characteristics comparison ................................................ 200

Table 7-1 The details of the components in the tested setup .................................... 207

Table 8.1 The unbalanced states applied to the system ........................................ 230

Table 8.2 The short-circuit states applied to the system......................................... 237

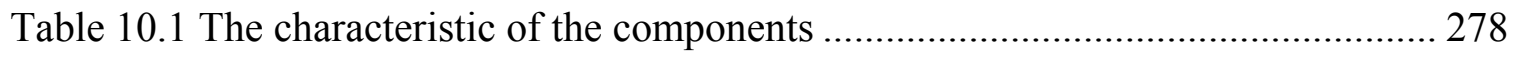

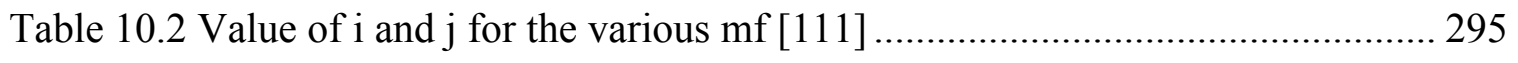




\section{TABLE OF FIGURES}

Fig. 1.1 The main origins of the electromagnetic interference in low and high frequency 4

Fig. 1.2 Low order odd harmonic superimposed by the electromagnetic interference....... 7

Fig. 1.3 High frequency harmonics produced by the electromagnetic interference ........... 7

Fig. 1.4 Power system made-up of equipment................................................................. 11

Fig. 1.5 EMI filter. (a) Overall filter. (b) CM equivalent filter.

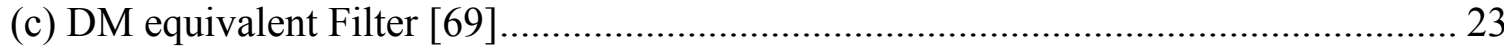

Fig. 1.6 Feedback AEFs. (a) Voltage-sense voltage compensation. (b) Current-sense voltage compensation. (c) Voltage-sense current compensation. (d) Current-sense current compensation [69] ................................................................................... 23

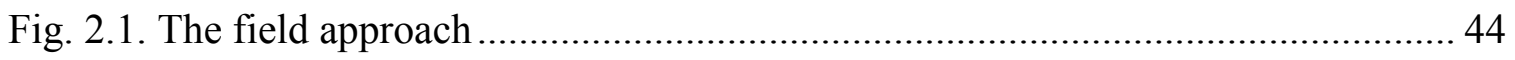

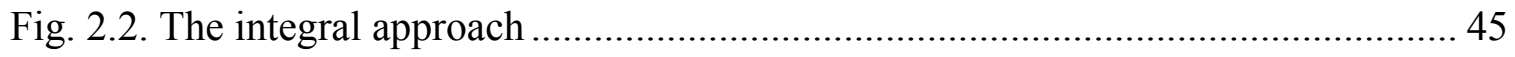

Fig. 3.1. Half wave zero current switching buck converter's circuit model.................... 57

Fig. 3.2 Field spectrum from the finite element analysis for the calculation of high frequency model of this component. (a) magnetic field intensity of spiral planar inductor (b) capacitor (c) electric field in small part of the capacitor (d) mesh in capacitor.

Fig. 3.3 Energy tolerance versus degrees of freedom ........................................................ 64

Fig. 3.4 Infinitesimal section of a two-layer winding of planar inductor. ........................ 67

Fig. 3.5 Frequency response analysis of the ZCS buck converter. (a) simple model (b) distributed-parameter frequency model, (c) optimized distributed-parameter

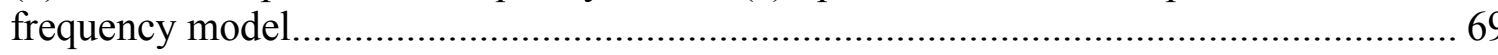

Fig. 3.6 Circuit model for high-frequency representation of the resonant circuit ........... 70

Fig. 3.7 The optimization process ................................................................................ 72

Fig. 3.8 Iteration accomplished by GA to minimize the objective function..................... 73

Fig. 3.9. Schematic of the converter's experimental setup ................................................ 74

Fig. 3.10. Voltage and current of the switches in ZCS-Buck converter,

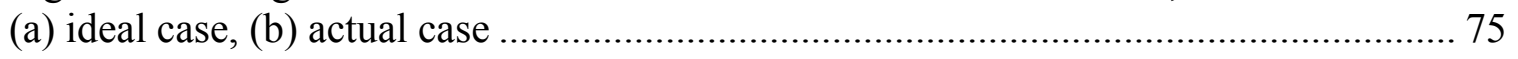

Fig. 3.11. FFT spectrum of the resonant circuit. a)Initial design, b) Modified design..... 75 
Fig. 3.12. Measured voltage and current of the switches in ZCS-Buck converter,

(a) primary designed circuit, (b) optimized circuit 77

Fig. 4.1 The model's environment for calculating field in far distance......................... 83

Fig. 4.2 convergence of the problem using GMRES solver ........................................ 88

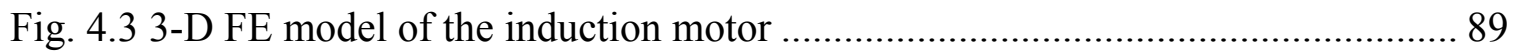

Fig. 4.4 the stray electric and magnetic spatial fields in meter in $\mathrm{x}$ axis

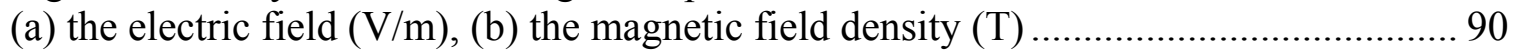

Fig. 4.5 Prototype of the proposed machine (SCIM) in Finite Element Analysis

(a) actual model, (b) Initial equivalent current loop model for EMI and signature studies

Fig. 4.6 Magnetic Flux Density (normal) spatial in (a) single phase of actual machine and (b) single loop model

Fig. 4.8 Magnetic Flux Density spectrum in Y component in (a) single phase of actual machine and (b) single loop model

Fig. 4.9 Magnetic Flux Density spectrum in $\mathrm{Z}$ component in (a) single phase of actual machine and (b) single loop model

Fig. 4.10 The FE model of the double current loop model. 93

Fig. 4.11 Magnetic Flux Density (norm) spatial fields in (a) actual machine and (b) double current loop model.

Fig. 4.12 Magnetic Flux Density spatial fields in X component in (a) actual machine and (b) double current loop model

Fig. 4.13 Magnetic Flux Density spatial fields in Y component in (a) actual machine and (b) double current loop model

Fig. 4.15 Prototype of the proposed machine (SCIM) in Finite Element Analysis (a) actual model, (b) An equivalent current loop model for EMI and signature studies

Fig. 4.16 (a) the lines and coordinates on which the field is measured (b) Comparison between magnetic flux density in real and cubic model for one motor (simulation time of full $3 \mathrm{D}$ is 1 hour whereas the simulation time of cube model is 1.5 minutes),.. 100

Fig. 4.17 (a) Two motor case of study for validation of the model (b) Comparison between electric field in real and cubic model for two motor 101 
Fig. 4.18 (a) The lines and coordinates on which the field is measured

(b) Comparison of electric field between actual and cubic model for one motor 103

Fig. 4.19 (a) Two motor case of study for validation of the model (b) Comparison between electric field in real and cubic model for two motor 104

Fig. 4.20 (a) Prototype of the detailed geometrical model (b) and the equivalent rectangular prism model 106

Fig. 4.21 Comparison between magnetic flux density calculated by 3DFE analysis for equivalent cubic model and real detail machine model, the one motor case of study....

Fig. 4.22 Normal Magnetic flux density in the X-Y plane (a) for the equivalent source model, (b) for the detailed model, Normal electric field in the X-Y plane (c) for the equivalent source model, (d) for the detailed model 108

Fig. 4.23 (a) Two machine case of study for validation of the model (b) Comparison between normal magnetic flux density computed by 3DFE analysis for the equivalent cubic model and the real detail machine model, the two motor case of study.

Fig. 4.24 Comparison between Electric fields computed by 3DFE for the equivalent cubic model and the real detail machine model, the two motor case of study.....

Fig. 4.25 Revised version of the cube model (a), Normal Magnetic flux density throughout X-Y plane for the equivalent source model (b), for the detail model (c), Normal electric field throughout XY plane for the equivalent source model (d), for the detail model (e), all for the one motor case of study

Fig. 4.26 Comparison between magnetic flux density calculated by 3DFE analysis for revised equivalent source model and detail machine model, (a) the one machine (b) and two machine case of studies

Fig. 4.27 Comparison of the near fields, 0.25 meter from machines, Normal Magnetic flux density in the X-Y plane for (a) the revised model, (b) for the detailed geometrical model.

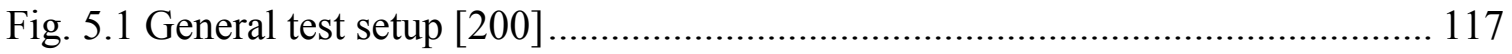

Fig. 5.2 Test setup for non-conductive surface mounted EUT ................................. 118

Fig. 5.3 Test setup for free standing EUT in shielded enclosure ............................... 119

Fig. 5.4 Test setup for free standing EUT [192], [200] ........................................... 119 
Fig. 5.5 LISN Schematic and impedance characteristics....................................... 120

Fig. 5.6 Limit for Conducted emission - submarine application [200] ........................ 129

Fig. 5.7 Limit for Conducted emission - surface ship application $(60 \mathrm{~Hz})$ [200] .......... 129

Fig. 5.8 Limit for Conducted emission - surface ship and submarine application $(400 \mathrm{~Hz})[200]$

Fig. 5.9 Measurement System check

Fig. 5.10 Measurement setup .............................................................................. 131

Fig. 5.11 Radiation emission limit for all Army applications..................................... 134

Fig. 5.12 Radiation emission limit for all Navy applications ..................................... 134

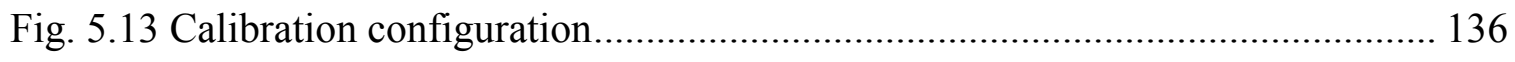

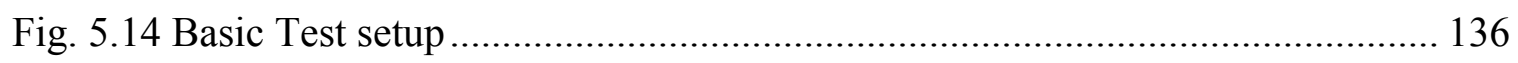

Fig. 6.1 Prototype of the proposed machine (SCIM) in Finite Element Analysis

(a) actual model, (b) An equivalent line-shape model for EMI and signature studies. . 140

Fig. 6.2 Prototype of the proposed cube model for replicating electric field of

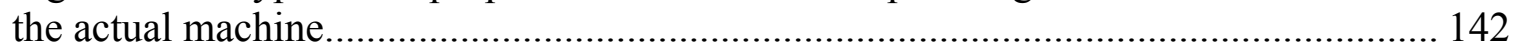

Fig. 6.4 Mesh of the equivalent source model ...................................................... 144

Fig. 6.5 (a) Reference lines from which propagated electric and magnetic field are measured, (b) Propagated magnetic field from actual and proposed model in all three axes

Fig. 6.6 Propagated electric field from actual and proposed model in all three axes ..... 145

Fig. 6.7 Radiated magnetic field from actual and proposed model in $\mathrm{X}$ axis at 1 meter distance to the models 146

Fig. 6.8 Magnetic and electric field spectrum throughout XY plane propagated from actual machine and model.

Fig. 6.9 Propagated magnetic field from actual and proposed model for two motor in all three axes 149

Fig. 6.10 Propagated electric field from actual and proposed model for two motor in all three axes

Fig. 6.11 Arrow plot of magnetic field density (T) of (a) actual machine 
(b) equivalent source model in xy-plane

Fig. 6.12 Stream-line of H-Field of (a) actual machine model xy-plane (A/m)

(b) equivalent source model in xy-plane $(\mathrm{A} / \mathrm{m})$

Fig. 6.13 Decomposition and reconstruction by MRA at two levels (a), bandwidth of sub-signals (b) [203].

Fig. 6.14 Normal magnetic (left) and electric field (right) comparisons at different frequency band, equivalent (solid blue) real machine (dash line), one machine case of study

Fig. 6.15 Normal magnetic (left) and electric field (right) comparisons at different frequency bands, equivalent (solid ) real machine ( dash line), two machine case of study..... 154

Fig. 6.16 Voltage amplitude of the terminal of the model during one time cycle 155

Fig. 6.17 Magnetic field density (B) of equivalent source model in four different moments of time at near distance. 156

Fig. 6.18 Magnetic field density (B) of equivalent source model at the four different intendances of time at far distance.

Fig. 6.19 Deviation of magnetic field density (B) of the equivalent source model due to the rotation of the whole machine around z-axis 158

Fig. 6.20 The studied experimental setup including the machine and measurement tools

Fig. 6.21 (a) The magnetic field intensity at $55 \mathrm{~cm}$ away from the setup in $\mathrm{Y}$ axis while all components except IM were off at $60 \mathrm{~Hz}(\mathrm{~dB} \mu \mathrm{A} / \mathrm{m})$, (b) the region of the model (the model is in center, and the measured line is shown in read) .................. 160

Fig. 6.22 Typical Schematic of the equivalent source model (optimization aspect) ...... 162

Fig. 6.23 Equivalent source models of (a) armature winding (b) commutation winding (c) compensation winding (d) field winding in equivalent dc machine 162

Fig. 6.24 Final Equivalent source model of the Propulsion DC Machine 163

Fig. 6.25 Schematic of (a) the detailed model of the dc machine and (b) the mesh in FE domain. 163

Fig. 6.26 (A). Radiated magnetic field density in (case a): XZ plane when $\mathrm{X}$ varies between -20 to 20 (case b): $X Y$ plane when $X$ varies between -20 to 20 (case c): $Y Z$ plane when $\mathrm{Z}$ varies between -20 to 20, (B) geometry of the three cases.. 165 
Fig. 6.27 Radiated electric field of in (case b): XY plane when $X$ varies between -20 to 20 (case c): YZ plane when $\mathrm{Z}$ varies between -20 to 20 165

Fig. 6.28 Radiated magnetic field density in (case a): XZ plane when X varies between -20 to 20 166

Fig. 6.29 Magnetic field density of (a) the actual machine (b) the equivalent source model $(\mathrm{T})$

Fig. 6.30 Electric field of (a) the actual machine (b) the equivalent source model $(\mathrm{mV} / \mathrm{m})$. 167

Fig. 6.31 (A). Radiated magnetic field density of two machine case in (case a): XZ plane when X varies between -20 to 20 (case b): XY plane when $X$ varies between -20 to 20 (case c): YZ plane when $Z$ varies between -20 to 20, (B) geometry of the three cases 168

Fig. 6.32 Radiated electric field of two machine case in (case b): XY plane when X varies between -20 to 20 (case c): YZ plane when $\mathrm{Z}$ varies between -20 to 20 169

Fig. 6.33 Magnetic field density of (a) the actual machine (b) the equivalent source model 170

Fig. 6.34 Electric field of (a) the actual machine (b) the equivalent source model ........ 170

Fig. 6.35 prototype of synchronous machine (a) actual machine (b) equivalent source model (c) terminal voltage of the equivalent source model

Fig. 6.36 Equivalent source model of individual windings (a) armature winding (b) field winding 172

Fig. 6.37 Magnetic field density propagated with and without the armature winding along (a) $\mathrm{X}$ axis in $\mathrm{XZ}$ plane (b) $\mathrm{X}$ axis in $\mathrm{XY}$ plane

Fig. 6.38 Electromagnetic field comparisons in 3 planes: (a) Magnetic field density of actual machine (T), (b) Magnetic field density of equivalent source model (T), (c) Electric field of actual machine $(\mathrm{V} / \mathrm{m}),(\mathrm{d})$ Electric field of equivalent source model $(\mathrm{V} / \mathrm{m})$.

Fig. 6.39 models of the proposed cable in finite element design (a) typical model (b) orignial FE model (c) deformed FE model 175

Fig. 6.40 Prototype of the multi-dipole models of the studied cable 176

Fig. 6.41 Radiated magnetic field density of (a) actual model (b) equivalent source model in tesla $(\mathrm{T})$. Note that the cable is very small compared to the region 177 
Fig. 6.42 Radiated electric field of unit length of the cable (a) actual model

(b) equivalent source model $(\mathrm{mV} / \mathrm{m})$ 177

Fig. 6.43 Radiated magnetic field density of perpendicular cables case

(a) actual model (b) equivalent source model in tesla (T) 178

Fig. 6.44 Radiated electric field of perpendicular cables case (a) actual model

(b) equivalent source model $(\mathrm{mV} / \mathrm{m})$.

Fig. 6.45 A sample of multi-directional discontinuous cables.

Fig. 6.46 Radiated magnetic field of multi-directional cables case (a) actual model

(b) equivalent source model (T)

Fig. 6.47 Radiated electric field of multi-directional cables case (a) actual model

(b) equivalent source model $(\mathrm{mV} / \mathrm{m})$....

Fig. 6.48 Radiated electric field of the cables in multi permittivity area

(a) actual model (b) equivalent source model (V/m)

Fig. 6.49 Schematic of the synchronous machine connected to the cable

(a) the detailed model (b) the equivalent source model.

Fig. 6.50 Radiated Magnetic field density along X axis in XY plane

Fig. 6.51 Radiated Magnetic field density along X axis in XY plane

Fig. 6.52 Schematic of the power setup (a) the full FE model (b) the equivalent source model

Fig. 6.53 Radiated electric field of (a) actual model (b) equivalent source model in tesla (T) while the synchronous generator is turned $\mathrm{ON}$ and other components are off.

Fig. 6.54 Radiated magnetic field density of (a) actual model (b) equivalent source model in tesla (T) while the synchronous generator is turned ON and other components are off...

Fig. 6.55 Radiated electric field of (a) actual model (b) equivalent source model in tesla $(\mathrm{T})$ while the coupling of machines (generator-motor) is turned $\mathrm{ON}$ and others are off. 185

Fig. 6.56 magnetic stray field density of (a) actual model (b) equivalent source model in tesla $(\mathrm{T})$ while the coupling of machines (generator-motor) is turned $\mathrm{ON}$ and others are off.. 186

Fig. 6.57 Schematic of the power setup (a) full FE model (b) equivalent source model 187 
Fig. 6.58 Radiated magnetic field of the induction machine on the cable, while only the induction motor is turned on 188

Fig. 6.59 Radiated magnetic flux density of (a) actual model (b) wire model in tesla $(\mathrm{T})$.

Fig. 6.60 Radiated Magnetic flux density of the optimized wire model (T)

Fig. 6.61 The studied setup including machines, measurement stuff and control drive (for switching) 190

Fig. 6.62 The measured magnetic field intensity at $55 \mathrm{~cm}$ far from the setup in $\mathrm{Y}$ axis while all components were turned on at $60 \mathrm{~Hz}(\mathrm{~dB} \mu \mathrm{A} / \mathrm{m})$...

Fig. $6.63 \mathrm{~K}_{\mathrm{B}}$ due to size variation of synchronous generator and induction motor 195

Fig. $6.64 \mathrm{~K}_{\mathrm{E}}$ due to change of size of synchronous generator and induction motor.

Fig. 6.66 Field spectrum of induction motor while the terminal voltage decreased $40 \%$ (a) B of actual model (b) B of equivalent source model (c) E of actual model (d) E of equivalent source model 196

Fig. 6.67 Field spectrum of induction motor while the terminal voltage decreased $40 \%$ and geometric size increased $20 \%$ (a) B of actual model (b) B of equivalent source model (c) E of actual model (d) E of equivalent source model.

Fig. 6.68 Field spectrum of synchronous generator while the terminal voltage decreased $20 \%$ and geometric size increased $70 \%$ (a) B of the actual model (b) B of equivalent source model (c) E of actual model (d) E of equivalent source model.... 198

Fig. 6.69 Calculation diagram of optimization factors for the two AC machines. SYN G and IM stand for synchronous generator and induction motor....

Fig. 6.70 Radiated field spectrums from both synchronous generator and induction motor (a) B of actual model (b) B of equivalent source model (c) E of actual model (d) E of equivalent source model 200

Fig. 7.1 The prototype of the inverter, load and the connection cable 206

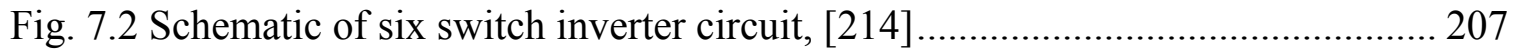

Fig. 7.3 Line Current and Voltage in the case of SVPWM ..................................... 208

Fig. 7.4 Physical model of the inverter switches ..................................................... 209

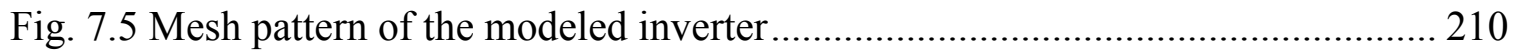


Fig. 7.6 Stray magnetic field density of the system: (a) IGBT switched on (b) IGBT switched off $(\mu \mathrm{T})$......

Fig. 7.7 Stray electric field of the system: (a) IGBT switched on (b) IGBT switched off $(\mu \mathrm{V} / \mathrm{m})$.

Fig. 7.8 Stray magnetic field density of the system ( $\mu \mathrm{T})$ : (a) only the cable is switched on, (b) only the inverter is switched on, (c) only the load is switched on, (d) the whole system is switched on 212

Fig. 7.9 The scheme of the setup of case 2 (a) FE simulation (b) measurement 214

Fig. 7.10 commutation curve of some material used in the simulation 215

Fig. 7.11 Stray magnetic field intensity of the setup case 2 at $5 \mathrm{kHz}$ simulated in FE $(\mu \mathrm{A} / \mathrm{m})$

Fig. 7.12 Measured frequency response of the stray magnetic field intensity of the setup case 2 from $\mathrm{DC}$ to $20 \mathrm{kHz}(\mathrm{dB} \mu \mathrm{A} / \mathrm{m})$

Fig. 7.13 Stray magnetic field intensity of the setup case 2 from DC to $7.5 \mathrm{kHz}$ $(\mathrm{dB} \mu \mathrm{A} / \mathrm{m})$ at $5 \mathrm{~cm}$ away from the inverter with and without shield by means of simulation and measurement.

Fig. 7.14 Stray magnetic field intensity of the setup at case 2 from DC to $20 \mathrm{kHz}$ $(\mathrm{dB} \mu \mathrm{A} / \mathrm{m})$ at $5 \mathrm{~cm}$ away from the inverter (a) without shield and (b) with shield 220

Fig. 8.1 Finite element model of the studied induction motor 226

Fig. 8.2 Schematic of the circuit connected to the model for the unbalanced current study. 227

Fig. 8.3 Schematic of the circuit connected to the model for the inter-turn short-circuit study

Fig. 8.4 Location of the selected turn for inter-turn and turn-terminal short-circuits (TB: terminal of phase B, S1 and S2: selected turns of phase B) 228

Fig. 8.5 Finite element (FE) model of the studied induction motor with the radiated magnetic field density at $30 \mathrm{~cm}(\mathrm{~T})$. The arrows show the propagated field.... 229

Fig. 8.6 Experimental test setup. 230

Fig. 8.7 The radiated magnetic field intensity at the given distance captured by experimental and simulation tests. (a) The normal case along a line in $\mathrm{Y}$ axis (b) frequency response of the case phase A 
Fig. 8.8 Harmonic orders of the magnetic field radiated from the motor in the various unbalanced states. (a) first harmonic, (b) third harmonic (c) sixth harmonic, (d) eighth harmonic, (e) ninth harmonic, (f) eleventh harmonic 233

Fig. 8.9 Harmonic orders of the magnetic field radiated from the motor in the various unbalanced states. (a) first harmonic, (b) third harmonic (c) sixth harmonic, (d) eighth harmonic, (e) ninth harmonic, (f) eleventh harmonic 234

Fig. 8.10 The diagram of the neural network. 235

Fig. 8.11 The details of the training process of ANN (a) performance (b) training state 236

Fig. 8.12 Radiated $\mathrm{H}$ field of the motor, while there is a complete short-circuit between terminal $\mathrm{T}_{\mathrm{B}}$ and the turn $\mathrm{S} 1\left(\mathrm{SH}_{100 \mathrm{~V}}\right)$ (a) measurement (frequency response with the span of $1 \mathrm{kHz}$ ), (b) simulation with the plot at $\mathrm{x}=30 \mathrm{~cm}$ along the $\mathrm{Y}$ axis ..... 238

Fig. 8.13 Several harmonic orders of the magnetic field radiated from the motor in the various short-circuit states. (a) first harmonic, (b) third harmonic (c) fifth harmonic

Fig. 9.1 The schematic of the test setup.

Fig. 9.2 The studied setup including machines, measurement stuff.....

Fig. 9.3 Location of the selected turn for inter-turn and turn-terminal shortcircuits (TB: terminal of phase B, S1 and S2: selected turns of phase B).... 251

Fig. 9.4 Radiated H-field of the motor, while there is a complete short-circuit between terminal $\mathrm{T}_{\mathrm{B}}$ and the turn $\mathrm{S}_{1}$. 252

Fig. 9.5 Radiated H-field of the motor, while there is a short-circuit between terminal TB and the turn $\mathrm{S} 1$ while the rheostat is set to $0.4 \Omega$ 252

Fig. 9.6 Finite element model of the studied induction motor. 253

Fig. 9.7 Schematic of the circuit connected to the model for the short-circuit study ..... 254

Fig. 9.8 Radiated H-field in various cases of the significant short-circuits in the induction motor's stator winding $(\mathrm{dB} \mu \mathrm{A} / \mathrm{m})$. Note that the terminalturn short-circuit is between $\mathrm{T}_{\mathrm{B}}-\mathrm{S}_{1}$ of fig. 9.3 and the intercoil short-circuit is between $S_{1}-S_{2}$. The $R$ is the re resistance of the rheostat. 254

Fig. 9.9 The coil of the winding and the necessity of the appropriate location of antenna.... 256 
Fig. 9.10 The selected six points in the winding of phase A for the interturn short-circuit. Note that the displayed terms such as S25-1 means the turn number 1 in the 25 th slot

Fig. 9.11 The rotation angle of the magnetic antenna around the machine. 260

Fig. 9.12 Radiated H-field of the interturn short-circuit between S28-1 - S28-2 and normal case at $\alpha=90^{\circ}(\mathrm{dB} \mu \mathrm{A} / \mathrm{m})$ 260

Fig. 9.13 Difference of the Radiated H-field of the 3 interturn short-circuit cases and normal case at $\alpha=90^{\circ}(\mathrm{dB} \mu \mathrm{A} / \mathrm{m})$, (a): interturn short-circuit between S28-1-S28-2, (b): interturn short-circuit between S27-1-S27-2, (c): interturn short-circuit between S25-1-S

Fig. 9.14 Comparison of the Radiated H-field of the 3 interturn short-circuit cases at $\alpha=60^{\circ}(\mathrm{dB} \mu \mathrm{A} / \mathrm{m}),(\mathrm{a})$ : interturn short-circuit between S28-1-S28-2, (b): interturn short-circuit between S27-1-S27-2, (c): interturn short-circuit between S25-1-S25-2... 261

Fig. 9.15 The schematic of the stator winding. 262

Fig. 9.16 Comparison of the Radiated $\mathrm{H}$-field of the 3 interturn short-circuit cases $(\mathrm{dB} \mu \mathrm{A} / \mathrm{m}),(\mathrm{a})$ : interturn short-circuit between S28-1-S28-2 at $\alpha=45^{\circ}$ (b): interturn short-circuit between S27-1-S27-2 at $\alpha=40^{\circ}$ (c): interturn short-circuit between S25-1-S25-2 at $\alpha$ 264

Fig. 10.1 Scheme of the system and identification procedure 271

Fig. 10.2 Flowchart of the component identification............................................. 276

Fig. 10.3 Flowchart of the component identification............................................. 278

Fig. 10.4 Electric stray field of the step 1 in $\mathrm{dB} \mu \mathrm{V} / \mathrm{m}$ (main harmonic orders) .............. 280

Fig. 10.5 Electric stray field of the step 1 in $\mathrm{dB} \mu \mathrm{V} / \mathrm{m}$ (inter-harmonic orders)............. 281

Fig. 10.6 The locations of the antennas and motor in the tests of step2, $3,4 \ldots \ldots \ldots \ldots \ldots . . .283$

Fig. 10.7 Electric stray field of the step2 in $\mathrm{dB} \mu \mathrm{V} / \mathrm{m}$ (the antenna is located at point A) 284

Fig. 10.8 Location of the selected turn for inter-coil and turn-terminal shortcircuits $\left(T_{B}\right.$ : terminal of phase $B, S_{1}$ and $S_{2}$ : selected turns of phase $B$ ) 285

Fig. 10.9 Electric stray field of the step 3 in $\mathrm{dB} \mu \mathrm{V} / \mathrm{m}$ (the antenna is located at point $\mathrm{B}$ and both machines are switched on) 286 
Fig. 10.10 Electric stray field of the step4 in $\mathrm{dB} \mu \mathrm{V} / \mathrm{m}$ (the antenna is located at point $\mathrm{B}$, both machines are switched on while there is an unbalance current in phase A)

Fig. 10.11 The locations of the antennas and components in the tests of step5 288

Fig. 10.12 Electric stray field of the step5 in $\mathrm{dB} \mu \mathrm{V} / \mathrm{m}$ (the antenna is located at point B)

Fig. 10.13 Electric stray field of the step 5 in $\mathrm{dB} \mu \mathrm{V} / \mathrm{m}$ while the $\mathrm{IM}_{1}$ is faulty (the antenna is located at point B). 290

Fig. 10.14 The locations of the antennas and components in the tests of step6 290

Fig. 10.15 Electric stray field of the step6 in $\mathrm{dB} \mu \mathrm{V} / \mathrm{m}$ (the antenna is located at point $A$ )

Fig. 10.16 Electric stray field of the step6 in $\mathrm{dB} \mu \mathrm{V} / \mathrm{m}$ (the antenna is located at point $\mathrm{B}$ )

Fig. 10.17 Schematic of the test setup (PWM VSI drive) 294

Fig. 10.18 The drive system schematic diagram: (a) the inverter circuit; (b) A vector representation of the SVPWM technique and the margins of SPWM versus SVPWM.

Fig. 10.19 The radiated electric field from the setup with SPWM pattern in two different switching frequencies (fs), (a) fs: $1380 \mathrm{~Hz}$, (b) fs: $1740 \mathrm{~Hz}$

Fig. 10.20 The radiated electric field from the setup with SVPWM pattern in two different switching frequencies (fs), (a) fs: $1380 \mathrm{~Hz}$, (b) fs: $1740 \mathrm{~Hz}$. 299

Fig. 10.21 The radiated (a) E-field and (b) H-field from the setup with SPWM strategy with $\mathrm{fs}=1380 \mathrm{~Hz}$ in healthy and faulty states

Fig. 10.22 The radiated E-field from the setup with SPWM strategy with $\mathrm{fs}=1380 \mathrm{~Hz}$ in healthy and unbalanced voltage faulty states 302 


\section{LIST OF ABBREVIATIONS AND ACRONYMS}

3DFE

2DFE

ANN

ASD

BEM

$\mathrm{CM}$

DWT

E-field

EMC

EMI

EMP

ESD

EUT

FDM

FDTD

GA

GMRES

H-field

HF

MOM

MRA
Three dimensional finite element

Two dimensional finite element

Artificial neural network

Adjustable speed drive

Boundary Element method

Common mode

Discrete wavelet transform

Electric field

Electromagnetic compatibility

Electromagnetic interference

Electromagnetic pulse

Electrostatic discharge

Equipment under test

Finite differential method

Finite differential time domain method

Genetic algorithm

Generalized minimum residual

Magnetic field intensity

High frequency

Method of moment

Multi resolution analysis 
PFC

PSO

PWM

RFI

SOR

SVM

VVVF
Power factor correction

Particle swarm optimization

Pulse width modulation

Radio frequency interference

Successive over relaxation

Space vector modulation

Variable voltage variable frequency 


\section{Introduction}

Compliance with electromagnetic compatibility (EMC) standards is an increasingly important aspect in the design of practical engineering systems. Consideration of EMC issues at the design stage is necessary to ensure functional safety and reliability of complex modern products. These products are increasingly reliant on electronic subsystems to provide powering, communication, control and monitoring function that are needed to provide enhanced levels of functionality of systems. Typical examples include; transportation vehicles (road, rail, sea and air), manufacturing plants, power generation and distribution as well as communication systems.

Opportunities for using numerical simulation techniques to predict and analyze the system EMC and related issues (e.g. human electromagnetic field exposure and installed antenna performance) are therefore of considerable interest in many industries.

For efficient control and use of electric energy, electronics and power electronics are increasingly used within electrical systems. Examples of such technologies are solar and wind power conversion systems, electric vehicles, variable speed drives and energyefficient lighting systems. These technologies are also used in evolving Smart Grid applications. A basic performance of such modern electrical systems is related to the EMC in the area of low frequency disturbances. Based on the above background, the importance of low frequency EMC study is considerably increasing.

On the other hand, the power electronic technologies are also used in evolving machine-drive equipment such as vessels and aircrafts. The electromagnetic signature is 
observable at low frequency in the local magnetic field, however, several threats are present in military applications: detection and classification, and subsequent detonation of sea mines, detection and localization of submarines out of the air. Due to the improvement in the sensitivity of electromagnetic field sensors and smart signal processing, electromagnetic signature reduction is vital. Thus, the first goal is to decrease the detection range by complying with strict electromagnetic signature requirements.

The other electromagnetic signature study aspect of the radiated fields in lowfrequency is condition monitoring of the components. Faults in the winding of the machines, as well as switch's failure and many other issues, can be detected without the need of system dismantling. This is critically beneficial for sensitive applications, which may not be easily possible to reach the components for online testing. On the other hand, offline testing of the component may be costly.

\subsection{Introduction to electromagnetic compatibility}

The presence of unwanted voltages or currents in electrical equipment is stated as interference in electrical systems, which can damage the equipment or degrade its insulation and performance. Electromagnetic interference (EMI) is a fairly comprehensive term that covers a wide range of undesirable electrical voltages and currents with a frequency spectrum from the $\mathrm{DC}$ frequency level up to the $\mathrm{GHz}$ range. EMI may be introduced into an electric circuit through the following paths:

- Conducted over the power cables or signal cables. 
- Radiated as magnetic or electric field from components, which is the source of the interference, and then coupled into another electric component, which is the victim.

There are two main sources of EMI:

- Natural events, such as snow storms, electrical storms, rain particles, and solar radiation, as well as lightning, electrostatic discharges (ESD) and cosmic discharges. This type of interference is commonly called static or atmospheric noise.

- Inherent interference, which is noise within a piece of electronic equipment, caused by thermal agitation of electrons flowing through circuit resistance. (This noise is usually noticed as the background noise heard in a radio receiver when it is tuned to a frequency between stations).

- Man-made interference, which is principally generated by electrical equipment used for industrial and domestic power supply, communications and control applications [1].

This study concentrates on the man-made sources of EMI and mainly those present in the industrial environment. Every electrical circuit should be considered to be a potential source of electrical interference, particularly those where switching of inductive or capacitive circuits takes place. Fortunately, most electrical interference is of such a sufficiently low level that it has no noticeable effect on other items of electrical equipment. 
EMC refers to the ability of equipment to function satisfactorily without producing emissions that degrade the performance of other equipment, and also are not affected by emissions from other equipment.

Electromagnetic interference (EMI) covers various main groups as shown in figure 1.1.

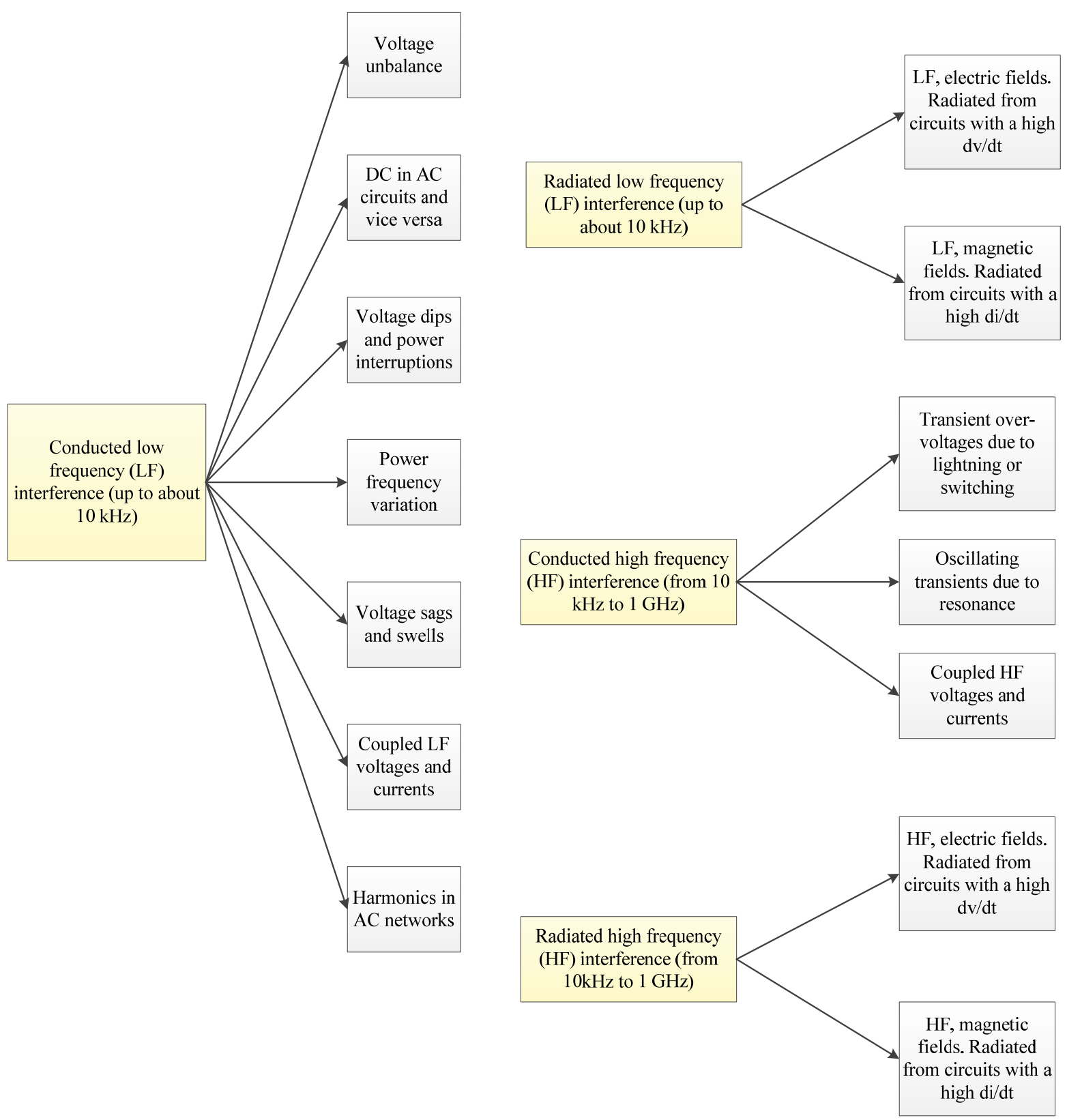

Fig. 1.1 The main origins of the electromagnetic interference in low and high frequency 
The rapid increase in the use of non-linear power electronics devices, such as AC and DC variable speed drives, has increased the overall importance of EMI in industry. To compound the problem, there has been a rapid increase in the number of electronic control and communications devices, which operate at low voltages and high speeds, and are susceptible to this high level of interference.

A simple but effective way to understand interference problems is to remember that there are always three elements to every interference issue:

1. There must be a source of interference energy.

2. There must be a receptor or victim that is upset by the interference energy.

3. There must be a coupling path between the source and the receptor.

The management of EMI and EMC in industrial environments falls into two categories:

- The establishment of standards for the containment of EMI by setting maximum limits on EMI emissions from electrical equipment.

- The establishment of standards for the susceptibility (or immunity) of electronic devices through good design and shielding of electronic equipment, which will enable them to operate within certain levels of interference.

\subsection{The sources of electromagnetic interference}

It is not practical to completely eliminate the electrical interference. The main objective is to minimize its effect on neighboring electronic equipment [1].

The main sources of EMI in the industrial environment are:

- Any circuit which produces arcs. 
- Circuits which generate non-sinusoidal voltages, produce electric fields.

- Circuits which generate non-sinusoidal currents, produce magnetic fields.

The $\mathrm{AC}$ variable speed drives use power electronic techniques to convert $\mathrm{AC}$ to $\mathrm{DC}$ (rectifier) and then to convert DC to AC (inverter) to provide a variable voltage variable frequency (VVVF) output. The overall efficiency and performance of the electric motor depends on the quality of the current to the motor. Over the past decade, a smooth sinusoidal current waveform has been achieved through the use of pulse width modulation (PWM) and high frequency switching (10 kHz to $20 \mathrm{kHz})$. Unfortunately, the AC converter has become a major source of both conducted and radiated electromagnetic interference (EMI).

The two main areas of EMI generation are:

- Supply side (mains)

The switching frequency of a 6-pulse diode bridge is $360 \mathrm{~Hz}$ on a $60 \mathrm{~Hz}$ power supply system. The harmonics generated by the rectifier fall into the frequency spectrum up to about $3 \mathrm{kHz}$ and are conducted back into the power system. The radiated EMI from the rectifier is of relatively low frequency (low $\mathrm{di} / \mathrm{dt})$.

- Machine side

Due to the high inverter switching frequencies (typically between $2 \mathrm{kHz}$ to $20 \mathrm{kHz}$ ), high frequency harmonics up to $10 \mathrm{MHz}$ (RFI) are generated by the inverter and conducted along the cable to the motor. The EMI radiated from this cable is therefore of relatively high frequency, often with high $\mathrm{dv} / \mathrm{dt}$ [1]. 
Supply-side harmonic interference is a continuous distortion (up to $3 \mathrm{kHz}$ ) of the normal sinusoidal current waveform. The distortion frequencies are multiples of the fundamental $60 \mathrm{~Hz}$ frequency.

Harmonic interference relates mainly to low-order odd harmonics, similar to the image shown in fig. 1.2.

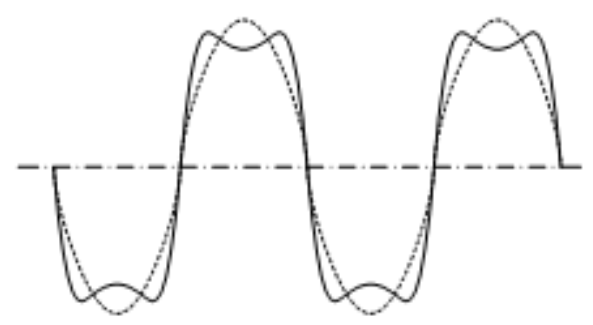

Fig. 1.2 Low order odd harmonic superimposed by the electromagnetic interference

Motor-side interference is a continuous high frequency distortion superimposed on top of the normal sinusoidal waveform.

The high frequency (RFI) superimposed on a sinusoidal waveform is shown in fig. 1.3. These orders of harmonics generates while the harmonic orders of the machine couple with the harmonic orders of the inverter (switching frequencies).

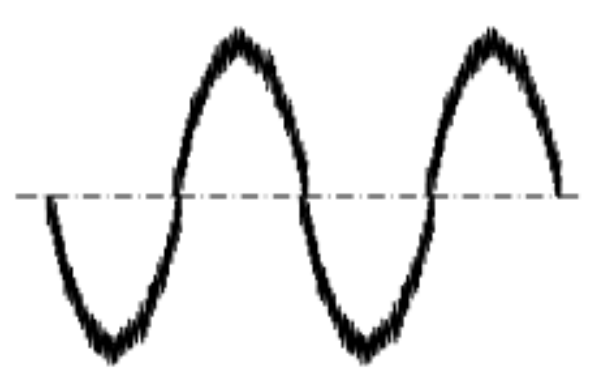

Fig. 1.3 High frequency harmonics produced by the electromagnetic interference

AC converters do not themselves radiate a high level of EMI energy. The electromagnetic fields in the immediate area $(<100 \mathrm{~mm})$ of the converter can be quite 
high, but these weaken quite quickly according to the inverse square law and are insignificant at a distance of about $300 \mathrm{~mm}$. When AC converters are mounted in metal enclosures, the electromagnetic radiation is largely eliminated. The main mechanism of propagation of the EMI is through the supply cables, the cables to the motor and most important through the earth connections. The supply cable is the most important route for the transfer of EMI. Conduction along the control and communication cables is fairly rare because these cables are usually well shielded and their source impedance is high.

In addition to the inverter and machine, railroad and mass transit systems have some unique types of EMI source problems. These include:

- Propulsion system's high voltage and high current operational mode emissions.

- Train signaling systems and their associated computer operating codes.

- Third rail shoes arcing broadband emissions.

- High voltage contact switching arcing broadband emissions.

- Train control system's emissions.

- Track train control circuits.

- Right away emission sources.

Moreover, medical equipment utilized in medical facilities has numerous EMI sources. Some of the more prominent of these are listed below:

- Life support equipment, such as ventilators, cardiac defibrillators, infusion pumps, etc.

- Patient telemetry and assistance equipment, which includes electrocardiographs and motorized wheelchairs.

- Electrical surgical units and their associated support equipment. 
- Magnetic Resonance Imagine (MRIs) systems.

- X-ray units, both therapeutic and diagnostic.

- Gamma Beam Electron Accelerators and Therapeutic equipment.

\subsection{Electromagnetic compatibility and power smart grid}

After explaining the various sources of EMI and their reason, the EMI in the power smart grid is discussed in this section. The smart grid is a very remarkable issue nowadays. The term is widely used by many, especially politicians. The International Electro-technical Commission (IEC) has defined the concept of smart grid [2]. The definition states that the smart grid is an electrical energy system that uses information technology. The smart grid is thus not only related to electrical networks, but to the entire power system. With smart grid technologies, as well as power technologies for renewables and improved energy efficiency, there is an increased use of electronics. We see growth in the use of power electronics, as well as electronics for information technologies.

One example of smart grid application is the possibility of charging electric car batteries during the hours with a surplus of low cost renewable energy. When the

electricity price is high, electric cars may feed energy back to the electrical network. This can be achieved using a continuous transfer of electricity price information with automatic control of the power flow to and from the electric cars. The term smart grid is thus enabling a "smart" electrical system where the entire power system, with networks as well as connected equipment, is converting between electrical energy and other forms of useful energy. 
The physical characteristic of smart grid technologies, with an increased incorporation of potentially sensitive electronics, naturally has implications with respect to electromagnetic compatibility (EMC). The satisfactory function of electrical and electronic equipment with respect to the electromagnetic disturbances is the aim of EMC. The IEC defines [3], [4] EMC as "the ability of an equipment or system to function satisfactorily in its electromagnetic environment without introducing intolerable electromagnetic disturbances to anything in that environment". In the European Union EMC Directive the "equipment or system" of IEC corresponds to the EU term "equipment", where equipment in turn is subdivided into apparatus and fixed installation.

Electromagnetic disturbances may be radiated or conducted, and electrical/electronic equipment is potentially sensitive to any or to both of these types of disturbances. Disturbances are in turn subdivided into a number of low and high frequency phenomena, where IEC defines low-frequency up to and including 9 kilohertz.

Examples of lack of EMC in relation to evolving smart grid technologies have been reported in Sweden. Kilo-watt hour meters in households sending data signals through power lines have caused interference with, for example, dimmer controlled lamps and electrical appliances. There are also cases reported where electrical apparatuses in households have interfered with electronic kilowatt-hour meters with adverse errors in registration of energy. Power electronics in wind power plants have emitted disturbances interfering with transfer of kilowatt-hour meter readings as signals on power lines. Power electronic-based photovoltaic solar and wind energy equipment may emit disturbances causing variations, such as voltage fluctuations and unbalance [4]. However, with a 
proper design such equipment may well improve voltage quality, for instance by reducing depth of voltage dips [5]. Voltage quality can be seen as an umbrella name for deviations from ideal voltage conditions at a site in a typical network, shown in fig.1.4 [6].

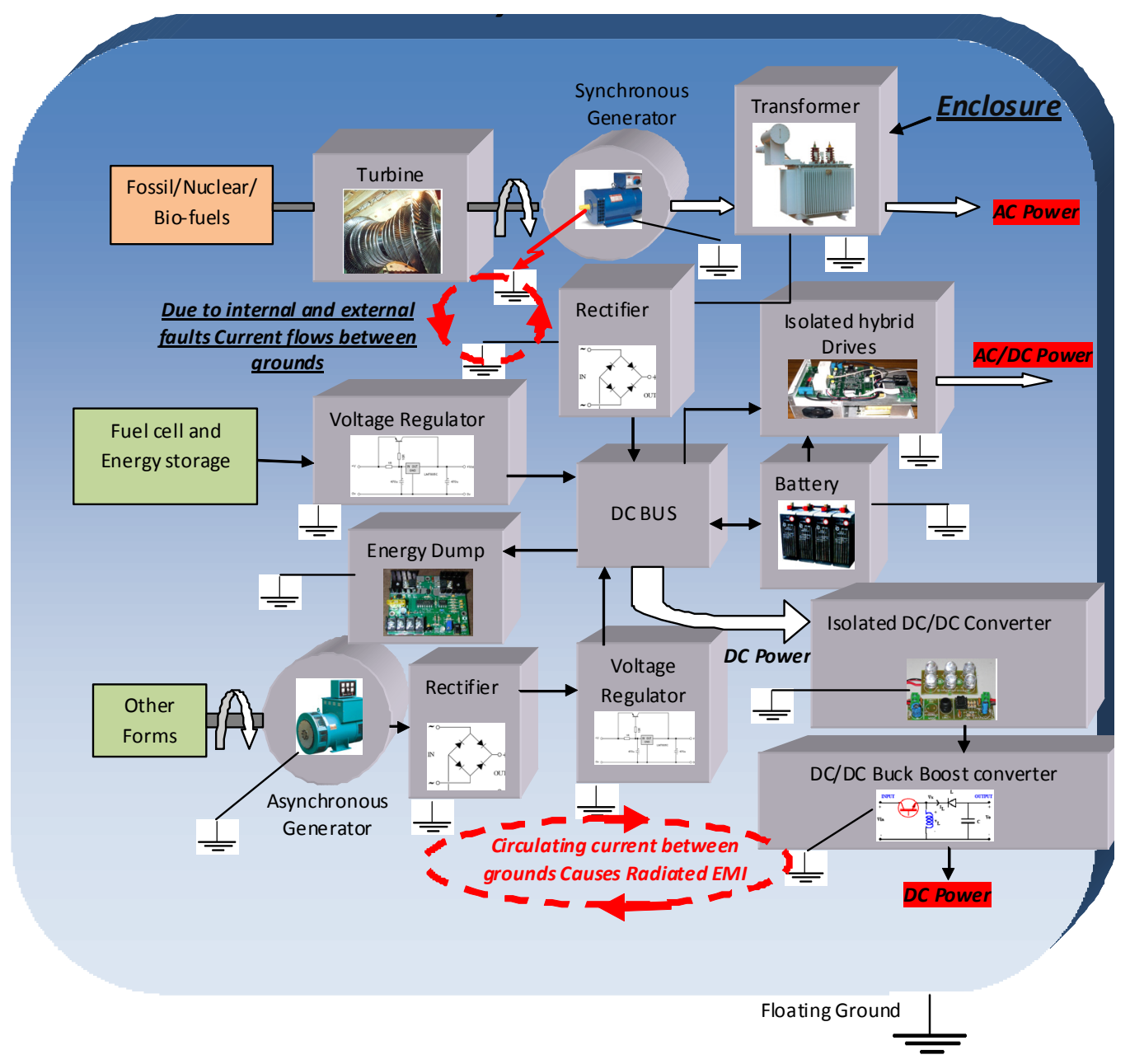

Fig. 1.4 Power system made-up of equipment

This is equivalent to electromagnetic disturbances of the voltage at the site. With no disturbances the voltage quality is perfect, otherwise, it is not. Electromagnetic disturbances are defined as electromagnetic phenomena that may degrade the performance of equipment [7]. Adequate voltage quality contributes to the satisfactory function of electrical and electronic equipment in terms of EMC. Electromagnetic 
disturbances as imperfect voltage quality at a site in a network can be regarded as electromagnetic emission from the network [8].

Over the years, focus has shifted between various electromagnetic phenomena. During the 1980s, low-order harmonics were important to the program due to the introduction of thyristor and diode based current stiff line commutated power electronics. Similarly, voltage fluctuations causing flickering lights were of great concern where arc furnaces were the main source of disturbances. In the 1990s, the use of variable speed drives for induction motors was introduced on a large scale. Voltage dips were causing interruptions in industrial processes due to inadequate immunity for those drive systems [9].

Presently, there is an increased awareness of electromagnetic disturbances in the frequency range up to a few kilohertz. This is due to switched converter technologies used over time in an increasing number of apparatuses, from energy-efficient luminaries to charging units for electrical vehicles. Due to connection of wind and solar power at the end of weak feeders, the occurrence of temporary over-voltages is a disturbance of increased concern. Regardless of the phenomena, it is clear that an appropriate division of responsibilities for networks and connected equipment is dominant.

\subsection{Literature review}

The previous works of the investigation of the radiated fields in the power system can be categorized into the following five sections:

- EMC studies in power system.

- Electromagnetic computational modeling studies. 
- Electromagnetic signature studies.

- System monitoring studies.

- Fault and failure diagnosis.

\subsubsection{EMC studies in power system}

The EMC studies in the power system, which can be categorized as the low frequency EMC studies, include those on the source of EMC in the power system, such as lightning pre-stroke phenomena, the lightning return-stroke phase and related electromagnetic scenario lightning electromagnetic pulse (LEMP), geomagnetic-storm-originated electromagnetic disturbance on power transmission lines, and evaluation of the $\mathrm{AC}$ interferences between transmission lines and metallic underground structures. Moreover, the degradation mechanisms affecting the shielding properties of a generic conducting enclosure under critical exposure conditions were studied. The critical situation can be namely when such a thin-walled structure is introduced into a quasi static magnetic field. Finally, the EMC studies regarding the power converter are reviewed. As mentioned before, one of the main source of the EMI is the presence of power electronic elements; therefore, a vast part of the literature review belongs to this issue.

\subsubsection{The lightning pre-stroke phase}

Through [10-21], the lightning pre-stroke phase is investigated and its essential role in determining the reliability of structural protection systems is acknowledged. Even the efficiency of the supplementary protection system, to which the task of mitigating induced effects on inner victims is assigned, is critically dependent on the striking performances. Hence, special attention is paid to the so far overlooked electrodynamic 
mechanism involved in the attachment process, since a pure electrostatics-based method, traditionally applied to air termination placement, is verified to be only partially satisfactory. The problem of mitigating the interference of a LEMP with the sensitive circuitry placed inside a building is an important problem, which is studied. However, such a victim can be equipped with largely available lightning protection devices. Applying reliable guidelines to the primary protection system still turns out to be the requirement for a successful EMC design of the secondary protection system. Several subjects are methodically treated and stimulating arguments, for ultimately exploring more reliable estimation methods applied to the interception efficiency of air terminals, are provided. The thundercloud electrostatic modeling, along with the study of the prestroke electrostatic and electrodynamics, is investigated in the mentioned literature.

In lightning physics, the stepped leader attachment process and recognizing of location of the lightning point where a flash will strike are subjects of paramount importance for safety/security and EMC problems. By perceiving that the striking points exactly positioned on the inception and launch location of an attaching leader in response to an advancing stepped leader, the connection between the attachment process and the striking point is explained. However, when the attachment phenomenon is limited to the late moments of the pre-stroke phase, the striking point is unpredictable. This is most probable when the physical and geometrical properties of the striking object and its surroundings are expected to play an influencing role. In general, the struck structure can be a natural or man-made global object, or an aircraft. Regarding the former class of victims, a considerable number of models and experimental methods have been adopted for evaluating the induced effects on detecting the loops placed inside a building. In these 
investigations, the lightning flash is assumed to strike a metallic part of a protected building or outer points, for instance lying on the earth plane [11]. In both case studies, a lightning electromagnetic pulse (LEMP) prediction is utilized for progressing the theoretical evaluation of the induced mechanism, which of course carefully takes into account the shielding properties of the protection system. Specifically, the supposed primary protection system is configured as a pair of air (rods, catenary wires or meshed conductors) and earth terminations connected to the opposite ends of an interposed set of down conductors. Such an all-metal structural protection system can also be effective against inner LEMPs. The effectiveness of this protection system against fire, explosion and injury to occupants has long been verified. However, a secondary protection, generally involving surge protective devices, is often additionally needed [1].

\subsubsection{Electromagnetic scenario lightning electromagnetic pulse related to the lightning return-stroke}

The corresponding to the above material are the subject of the research which analyzes the lightning return-stroke phase and related electromagnetic scenario lightning electromagnetic pulse (LEMP) [11],[22]-[38].

The induced effects on the canonical victim represented by an overhead horizontal line positioned near a flash are investigated. Although the LEMP quasi-static component is assumed to be near to the return-stroke channel, the more surprising coupling feature emerged here is that the LEMP quasistatic component is not involved significantly. The significant reasons explaining such an unexplored behavior are to be enquired into the 
combinative electromagnetic performances of the wet earth plane, channel and upper cloud, all together envisioned to form an interconnected discharging system.

The interest in the lightning phenomenon and the related effects have increased recently due to a the variety of reasons. Although the physics of lightning has never changed, a large debate on the progressive tropicalization of the climate in some regions is currently underway, (for example, this seems to occur in the European nations facing the Mediterranean basin), a phenomenon which is showing significant changes in the currently available thunderstorm maps and lightning statistics from earlier studies. Moreover, the growing number of exposed structures and systems and their non-uniform territorial distribution is making the application of security criteria an increasingly pressing and diversified problem. It is an increasing social necessity for a careful charting of the notions of risk awareness and safety with special reference to household and office appliances connected to distribution and communication lines. Also, as mentioned before, the reduction of electronic components' size and optimization of the insulating compartment of power systems coincide in significantly increasing the sensitivity and vulnerability to the lightning generated electromagnetic impact.

The large amount of latest experimental data and models (see, for example, the review books or selected collections of articles ([11], [25], [27], [38]) allows an insight into such an attractive and important subject. On the other hand, the required collection of evidence prior to standardization (especially addressed here to protection/mitigation criteria and devices), seems to be still in progress (see, for example, [17], [35]). Lightning can be substantially considered as an unstable product of little understood electrification processes, which develop inside a thunder-cloud or banks of thunderclouds. Therefore, 
the currently available models are questionable because the incidental, changeable and irreproducible nature of such a phenomenon makes the interpretation more hypothetical. Typically, available referential data are sets of recorded oscillograms arranged in the form of excitation-field components, in which the detection at a distance from the discharging channel is approximately known [1]. This representation of a LEMP, which is the usual demonstration of it, unfortunately, can no longer be considered as a proper benchmark to substantiate the reliability of electromagnetic models of lightning. Consequently, the impressive reproductions of experimental oscillograms can be derived from different, and sometimes conflicting, models owing to the large number of variables and assumptions invariably introduced and combined successfully.

\subsubsection{Effects of geometrical storms on long distance AC transmission system}

The EMC effects of geometrical storms on long distance AC transmission system are also discussed in the literature [39-47]. Solar Induced Currents (SICs) are the cause of many detrimental effects on power systems. It is worth noting that the scientific interest for this topic is cyclical and follows the relevant periods of the solar activity. The solar flares cause the ejection of particles with particular trajectories that could interact with the earth's magnetic field. After the incidence of the solar flare (about twenty to forty hours), these particles can cause relatively rapid transient variations in the earth's magnetic field, well-known as geomagnetic disturbances or geomagnetic storms. This magnetic interaction can cause problems on electric power system and on the communications system. This is primarily due to the fact that the geomagnetic disturbances produce an induced Earth-Surface Potential (ESP). The induced potential 
and the size of the system increase compatibly. Subsequently, the induced ESP gives rise to spurious currents in long transmission lines and equipment that are in some way grounded at points geographically remote from each other. Therefore, the strategy is to build the longer transmission lines, which are able to cover large geographical areas. For this reason, some of the problems caused by geomagnetic storms can become more pronounced. Several studies on the occurrence of relevant ejection of particles by solar flares have been conducted in the past and it has been observed that this phenomenon has a cyclic characteristic with an estimated period of about eleven years.

As mentioned above, the spurious currents due to the geomagnetic disturbances are called Solar Induced Currents (SICs) in power systems. Some measurements of SICs on actual power systems are reported in the literature ([39-41]). The effects on equipment and irregular operations of power systems are the main concern and it was shown in the literature that they all can nearly be traced to the presence of the SIC in the windings of transformers ([42-46]). The SIC can be considered quasi-DC and their main effect is the simultaneous AC and DC excitation of energized transformers, since the SICs have a fundamental period of several minutes (from 6 to 15 minutes). The SICs have been measured in excess of 100A in transformer neutral leads in extreme cases [47].

\subsubsection{Interferences between the AC transmission lines and metallic underground structures}

The evaluation of the interferences between the AC currents transmission lines and metallic underground structures and pipelines are investigated [48-50]. The requisite to develop mathematical tools and demonstrations for the computation of electromagnetic 
interference with underground metallic assemblies has originated from the general trend in the last few decades, of concentrating most utility structures (overhead AC transmission lines, traction lines, telecommunication lines, pipelines for fluid transportation, etc.) in the same corridors. The interest towards the influence of $\mathrm{AC}$ on metallic structures is related to the possible resulting hazards regarding the safety of people coming into contact with the pipeline, the risks of damage to the pipeline coating and metal and the risks of damage to the equipment connected to the pipeline (cathodic protection systems [48]).

\subsubsection{Metallic enclosures exposed to quasi-static fields}

One of the most appealing EMC problems consists of predicting the shielding degradation of discontinued metallic enclosures exposed to quasi-static fields [51-56]. The two main origins should be considered. First, irrespective of the presence of apertures, regular non-magnetic conducting panels often organized in a way to form an enclosure, perpetually make the interior vulnerable system less effectively impermeable to the magnetic than to the electric field. The negligible reflective and absorptive behaviors of the enclosure wall at low frequencies causes the unfavorable performance against magnetic fields. Similarly, the large conductivity of the adopted materials causes the favorable performance against electric fields, which leads to the displacement current collected by the hollow body to company the low-resistance wall, where it flows in the form of a conduction current. Moreover, the coupling to an interior wire crossing an aperture represents a paradigmatic case study with reference to the concentrated field penetration. Therefore, the fields of interest often reduce to the components of the net 
electric field tangential to them, and the simultaneous magnetic field linking the exposed segment of the wire. Due to the structures assumed by the above fields passing through the hole and actually coupled to the inside conductor, it is confirmed that the former field often approaches zero, while the latter is important.

The above observations especially apply to high-current source cases, namely when the application is for example constructed of the "bulk current injection" (BCI) for laboratory tests [51]. Classically, such simulations have long been adopted to explore the induced effects of a lightning discharge directly striking an aircraft, in which the current spreads up over the conducting portions of the enclosure and takes the path of least impedance. Specifically, replicating the development of the residual field in the shielding volume is the requirement for a careful knowledge of induced effects on the complex wiring laid onboard aircraft and running nearby the apertures. In this case, a large amount of the produced magnetic field may be assumed to be of quasi-static nature due to the limited spectral components of the return-stroke current and the short geometrical amounts often involved (hole dimensions and spectator's distance, both small in comparison to the free-space wavelength).

\subsubsection{Power quality}

Power quality (PQ) monitoring is a very multipart task, and recently it is assuming a growing significance because of the large diffusion of non-linear and time-varying loads and their interactions with the power supply system. Consequently, the distributor/ customer responsibility question is currently challenging the EMC community. The brief description of the PQ scenario and an overview on the disturbances affecting electrical 
power systems and related international standards are described in the literature [1], [5766]. The capability of evaluating the PQ at the point of common coupling in terms of harmonic distortion is described in [1]. The value of the procedure is sustained even by a comparison with other harmonic assessment procedures. The main causes of the PQ disturbances, as well as the detrimental effects they have on the electrical systems, are described. Among these issues, harmonics are investigated more accurately.

In terms of Electromagnetic Compatibility, the sufficient voltage quality contributes to the satisfactory function of electrical and electronic equipment. Electromagnetic disturbances as imperfect voltage quality at a site in a network can be regarded as electromagnetic emission from the network [61]. According to the EMC Directive network in equipment, this is in line with the original name of IEC Technical Committee (TC) 77 which was "EMC between electrical equipment including networks"; now simply EMC [61]. The electromagnetic energy transfer with adequate voltage quality at its sites is the technical function of an electrical network. Similarly, immunity of an electrical network can be seen as the ability to absorb disturbing emissions, such as distorted current with adequate voltage quality while transferring energy, i.e. with satisfactory function. For instance, low order harmonics and voltage fluctuations, and network strength are relevant for network immunity [62], [63]. In terms of geomagnetic, the induced current caused by space weather is another example of electromagnetic immunity relevant to an electric grid [64] to keep its function satisfactory. 


\subsubsection{Power converters}

One of the main practical aspects of studying EMC is the power converters and their contribution in the EMC problems of the power network. There are numerous publications about these articles. They include modeling, prediction and reduction of the EMI regarding the power electronic components and power converters in the system [6791].The appearance of EMI noise in different types of power converters used in SMPS units has also been explained in several articles. While the references [70] and [71] deal with buck converters, the generation of conducted noise in boost-power-factor-correction (PFC) converters is discussed through [72]-[74], and [75]-[77] deal with forward converters, and [78] and [79] deal with offline fly-back converters. The popular boost acdc PFC converter is utilized next as an instance to provide some background regarding the generation and measurement of EMI noise in SMPS units. In details, many researchers developed the EMI filters for mitigating the conducted EMI. Both passive and active EMI filters (AEFs) have been used along with the input power supply lines to mitigate the noise. The design of passive filters for use in power electronics is particularly challenging, since the filters are terminated with varying noise source (SMPS) and load (power line) impedances [70], [80], [81].

A commonly used filter topology is shown in fig. 1.5(a) [80], [82]-[84]. The equivalent $\mathrm{CM}$ and DM filter circuits of this filter are shown in fig. 1.5(b) and (c). In practice, often the leakage inductance of the CM inductor, CM leakage alone, is used as the DM inductor. 

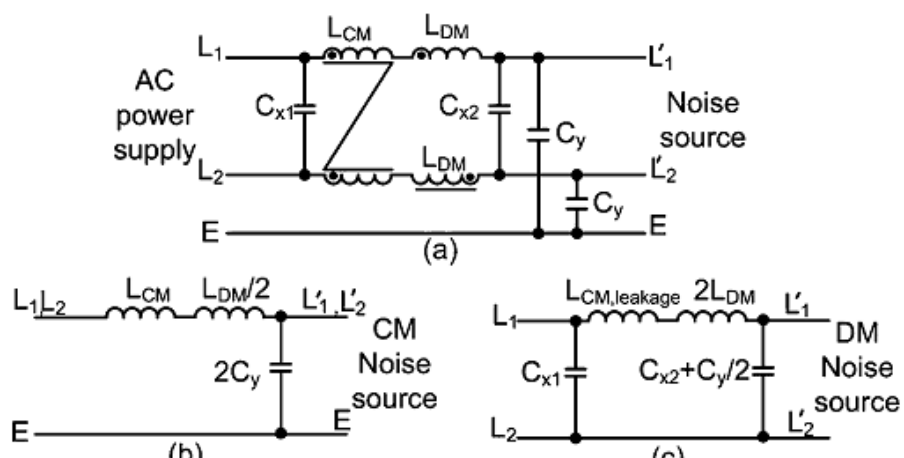

Fig. 1.5 EMI filter. (a) Overall filter. (b) CM equivalent filter. (c) DM Equivalent Filter [69]

The detailed surveys of the EMI passive filters are mentioned in [69].AEFs use active electronic circuits to suppress the unwanted signals and are possible alternatives to immense passive EMI filters. Based on the method used, an AEF can be of feed forward or feedback type, or a combination of both types. A feed forward AEF injects an equal amount of noise of opposite polarity to reduce the noises. A feedback AEF, on the other hand, uses a high-gain feedback-control loop to reduce the noise level.

Fig. 1.6 shows four possible topologies for feedback AEF. The inductor or the voltage across the AEF can be sensed or the compensation can be through injection of a series voltage or a shunt current [85], [86].

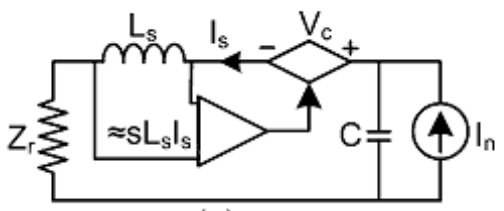

(a)

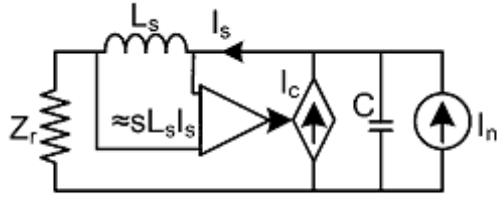

(c)

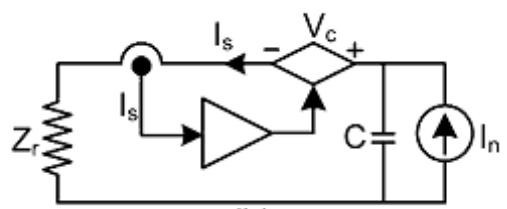

(b)

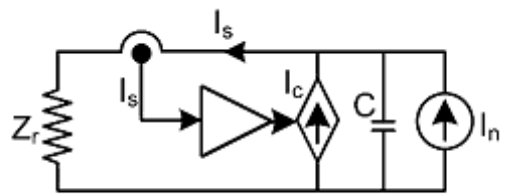

(d)

Fig. 1.6 Feedback AEFs. (a) Voltage-sense voltage compensation. (b) Current-sense voltage compensation. (c) Voltage-sense current compensation. (d) Current-sense current compensation [69]. 
Series-voltage compensation shown in fig. 1.6(a) and (b) increases the effective impedance of the series path, whereas the shunt-current compensation [see fig. 1.6(c) and (d)] reduces the effective impedance of the shunt path. In general, all the feedback configurations of AEF can be applied for reduction of both $\mathrm{CM}$ and DM noises. Although the main function of switch control is to regulate the power flow, the control can also be changed to influence the radiated and conducted EMI generated by the power converter.

The first and one of the simplest techniques to reduce the EMI is the selection of low switching frequency. That is to say, the switching frequency plays an important role in minimizing the EMI filter requirements with a reduced switching frequency, resulting in lower EMI noise at the expense of large-sized passive components [87]. In [88], a very low $100 \mathrm{~Hz}$ switching frequency, corresponding to one switching per half of an ac cycle, has been utilized in the boost PFC converter to help in meeting the EMC standards. Conducted EMI needs have been encountered with just a simple first-order filter. However, the passive elements used in the converter are expected to be larger as a result of the very low switching frequency.

The other technique, which is used for the reduction of noises, is utilizing different kinds of switching patterns of pulse width modulation (PWM). The investigation of the frequency-modulation spread range approach has been done in [76] and [89], which associate the modulation parameters with the resulting spread in the spectrum. It has been suggested in [89] that the modulation frequency must be close to or greater than the measurement resolution bandwidth (RBW) set by the standards. Rossetto et al [87] used a 600-W boost PFC converter for modulation at the switching frequency, $100 \mathrm{~Hz}$, in synchronism with the rectified ac-voltage waveform. The switching frequency is tried to 
be modulated in a way that it is at its minimum value when the ac current is at its maximum. In addition to spreading the EMI noise, this would also reduce the switching losses. Mihalic and Kos [90] adopted a random PWM (RPWM) scheme to spread the line noise spectrum. In RPWM, the pulse width is randomly changed at a constant switching frequency, while the average pulse width value is reserved matching to the required duty cycle. Using simulations, [91] has compared the EMI performance of different control schemes as follows: 1) synchronous PWM; 2) asynchronous PWM; 3) hysteresis with varying band; 4) hysteresis with fixed band; and 5) constant on-time methods and resolved that hysteresis control with fixed band performs better than the other methods.

\subsubsection{Electromagnetic computational Modeling studies}

The modeling process in the field of electromagnetic compatibility means the establishment of a connection between the source of interference or any other cause and its effect, which can be the response of the component as the part of the system. This relationship can be established in several ways, depending on the type of problem, its complexity, and the degree of approximations with respect to an exact formulation. The possible methods involve:

- Using circuit theory for designating the conducted disturbance, such as voltage dips, over-voltages, voltage stoppages, harmonics, and common ground coupling [72], [92]-[94].

- Using an equivalent source model (usually circuit) with either distributed or lumped parameters, such as in low-frequency electromagnetic field coupling expressed in terms of mutual inductances and stray capacitances, field-to-line 
coupling using the transmission line approximation, and cable crosstalk [95][97].

- Formulating the problem in terms of formal solutions to Maxwell's equation and making analytical models based on that [98-101].

- Physics based modeling using numerical methods, such as finite element method, finite differential method, method of moment, and so forth [102][110].

Generally, the methods used in EMC modeling are not only to visualize electromagnetic phenomena but also to predict and suppress interferences, which can be regarded as either theoretical or experimental.

More details about the EMC modeling will be discussed in the second chapter.

\subsubsection{Electromagnetic signature studies}

The definition of the electromagnetic signature study is opposite to the EMC/EMI application, which is the reduction of the radiated fields. It is tried in this study to utilize the radiated field that is mainly in low frequencies from a few $\mathrm{Hz}$ to several $\mathrm{kHz}$. The radiated fields can be processes through numerical and data acquisition techniques. The application of this process is monitoring the behavior of the components through the electromagnetic signatures, as well as diagnosing the faults and failures. For this purpose, various modeling techniques, as well as experimental performances are used, which are summarized in the following. 


\subsubsection{System monitoring studies}

Many researches have been implemented in characterization and detection of the source of harmonics and inter-harmonics [111]-[118]. In [111], the principle of the harmonic and inter-harmonic is discussed and the proposed model is suggested. Then, the inter-harmonic assessment is considered with particular attention to the problem of the frequency resolution and of the computational burden associated with the analysis of periodic steady-state waveforms. Finally, modeling of different kinds of inter-harmonic sources and the extension of the classical models developed for power system harmonic analysis to include inter-harmonics, are discussed. The modeling for the assessment of the sources is discussed more in [112]. A new algorithm termed hybrid blind source separation is used for the localization. Although, the application of this localization is for high frequency components, identifying and locating the sources on the basis of predicted measures, which are used in this research, can be inferred and used for the low frequency components. Some literature such as [113], [114] focused on the improving the measurement and extraction techniques to enhance the investigation of harmonics and inter-harmonics. The enhancements are in about higher degree of accuracy, structural/performance robustness, and frequency adaptivity. In [115], simulation and experimental study of the identification of the inter-harmonic source location in power systems are implemented. The method is based on the inter-harmonic impedances measured at the metering points and comparing them with the harmonics of the utility system. The main idea behind this method is that the inter-harmonic impedance of the system is much smaller than that of an inter-harmonic-generating load. 
Besides inter-harmonic, some other methods are used for source identification. In [116], an approximate technique was proposed for the reconstruction of magnetic field distribution in the proximity of unknown sources based on two nested optimization algorithm. Moreover, in [117], the cascade correlation network was used for the harmonic source detection. The Current-injection-based harmonic power flow was used to calculate bus voltages and total harmonic distortion. D. Srinivasan et al [118] proposes a neural-network (NN)-based approach to nonintrusive harmonic source identification. In this approach, NNs were trained to extract important features from the input current waveform to uniquely identify various types of devices using their distinct harmonic "electromagnetic signatures". The identification was particularly implemented based on the measurement of current at the incoming supply point and comparing the magnitude of different components from home appliances to computer apparatuses and power components.

\subsubsection{Fault and failure diagnosis}

In early research, the fault detection in machines involved simple techniques, such as overcurrent or overvoltage detection. Following the detection, it was required to bring the machine offline to clear the fault. In safety-critical applications; however, a shutdown of the motor may not be an option. This demands better fault detection approaches. The first step in dealing with a fault in the drive is the ability to detect it quickly and determine its location and severity accurately. Through the analysis of electromagnetic signature, the problems of off-line analysis of the failures has been resolved. 
Between the online methods, the air gap flux electromagnetic signature technique measures the rotating field of the machine by utilizing the sensor installed in the machine to detect faults. Valuable research efforts were presented regarding this technique [119][128]. The broken bar and stator winding short-circuit are studied in [119] and [120] through data acquisition methods and experiments in low and medium frequencies. Similarly, Chadebec et al [121] showed the trustworthiness of fault detection on electrical machines by analysis of the low frequency magnetic field signature. The other advantage of using stray field in the fault diagnosis, such as the fault in lamination and broken bars, were explained in several publications such as [122]- [126].

The air-gap magnetic flux can give good results in detecting interturn short-circuit; however, installing and maintaining the sensor is the drawback of this method. Hence, in this research, we will measure the radiated magnetic field outside the motor using the magnetic antenna and use the frequency response analysis for interturn fault detection. Since it's possible to locate the field antenna close to the machine, the magnetic behavior of only the faulty machine would be evaluated. This is also useful in cases where there are other machines around the faulty one.

\subsection{Problem statement}

As mentioned in the previous parts and the literature reviews, power electronic devices are increasingly used within electrical systems for efficient control and use of electric energy. Solar and wind power, electric vehicles, variable speed drives and smart grid applications are examples of such technologies. Electromagnetic interference occurs

due to the presence of these electronic devices in these systems. It can be conducted or 
radiated to neighboring equipment. This radiated or conducted EMI may cause malfunction or de-rating at low power frequency and high frequency applications (such as radar). The de-rating of systems may cause them to work under their normal rates. Based on these issues, the importance of low frequency field signature analysis is considerably increasing.

Although in low frequencies the reduction of radiated or conducted interferences are proposed, they can also be used for investigating the behavior of the components. The system monitoring and fault/failure diagnosis of the power components can be achieved through studying the radiated and conducted electromagnetic signatures.

One of the most important aspects of electromagnetic signature study in power system is the modeling of components, including electrical machines, cable runs, power converters, and their enclosures. Since testing various techniques in actual machines, drives and other components are cost prohibitive, one needs to study and develop techniques that reduce the radiated fields and evaluate their levels with a high level of precision. Some researchers are involved in the modeling of these devices for the mitigation of radiated fields [129]-[132]. Most of the works reported in the literature were related to high frequencies. As discussed above, low-frequency devices also radiate electromagnetic fields that disturb other devices in their vicinity, and therefore the detection and mitigation of electromagnetic fields are increasingly important.

\subsection{Research objective}

The objectives of this research are based on the issues that are stated above. In order to achieve the optimum performance of a component in the power system, many studies 
should be implemented. Moreover, a well-designed algorithm for the purpose of fault and failure diagnosis requires numerous tests. However, testing various techniques on the power components experimentally costs a huge amount of money and time; in addition, it may cause destructive damages. Therefore, primitive tests for finding optimum performances are fulfilled by using physics-based modeling. The full three-dimensional (3-D) finite element method is used as the physics-based modeling. The detailed modeling of typical components in a power system setup are implemented for the purpose of studying the radiated field at given distances. Hence, the radiated electric and magnetic fields of the sources at the area around the components are obtained and investigated. However, the full 3-D finite element modeling for the purpose of studying the multi components cases becomes difficult, which is because of the significant increase of the number of meshes and the presence of very tiny elements in the components and the proportion of them to the large area of the simulation. Simulating in the large area is because of the need to observe the electromagnetic signatures at the far distances. Therefore, equivalent source modeling is used. The particle swarm optimization method is utilized to implement the equivalent source model physically. The other equivalent source models in the process of embedding the equivalent source models, such as dipole models and quadra-pole models are utilized.

Finally, the particle swarm optimized based on genetic algorithm is used. This model decreases the simulation times more than 100 times, while it keeps the accuracy up to 95 percent. The drawback of this modeling method is that the model should be revised and the optimization process should be implemented for each different state of the components. Therefore, the wire modeling is utilized. This new model uses the magnetic 
and electric field densities and the current density around the wire and improvises the wire model with the modified value of current and voltages. Consequently, the equivalent source model would be created. This model has the ability to be used for various situations and state of the components while it keeps the accuracy up to more than 98 percent.

As the EMC applications point of view, the equivalent source models, as well as the full finite element models, can be used for testing different kinds of shielding. Different electrical and chemical features of the materials are utilized to have the optimum shielding of the components. Moreover, the proper locations of the shields are tested. The other application is improving the performance of the components, such as optimizing different types of switching techniques of the converters. The optimum switching patterns can be considered for the purpose of less EMI, as well as the fault diagnosis of the switches.

As the electromagnetic signature study applications' point of view, the full finite element modeling should be used; however, there is a limitation of the number of meshes for the multi-components modeling. For this issue, the multi-symmetry and multi-linear modeling are used, which resolve this limitation and decrease the simulation time. This is explained more in the next section. Then, the system monitoring of the system is implemented. The source identification is fulfilled for the purpose of detecting the component, which is not operating properly. Various couples of the components, such as generator-motor, converter-fed motors, and controlled drives are tested to define the proper algorithm for this purpose. In detail, the fault detections of the machines, as well as the converter switches, are employed using the electromagnetic signatures. Moreover, 
the type of short-circuit in the induction motors, as well as the identification of the location of short-circuit in the winding of the induction motors, are investigated all through the radiated electromagnetic signatures.

\subsection{Original contribution and significance}

In order to implement the full finite element for the electromagnetic signature study applications and EMC applications, several modifications in the solver are implemented. We used the fast generalized minimal residual technique, GMRES, as the iterative solver with the krylov as the pre-conditioner. The fast GMRES is a variant of the GMRES method with flexible preconditioning that enables the use of a different pre-conditioner at each step of the Arnoldi process. In particular, a few steps of GMRES can be used as a pre-conditioner for fast GMRES. The flexibility of this solution method is beneficial for the problem with nonlinear material characteristics, such as the core of the motor. Consequently, the multi-symmetric problems will generate that can be solved much faster than the problem with the regular settings.

Since the proposed setup consists of the electric machines, the nonlinearity of the features of ferromagnetic materials makes the solution of finite element problems complex. In this case, since there are several materials with nonlinear characteristics, the linear solver cannot be used. On the other hand, using nonlinear material rises the simulation time dramatically. Hence, a modification in choosing the solver and the iterative solver is employed. Instead of having a linear or curved B-H curve, the ramp of the curve in several zones is calculated $\left(\mu_{\mathrm{r} 1}, \mu_{\mathrm{r} 2} \ldots\right)$ and used instead of the B-H curve. The benefit of this modification for this study is that the magnetic flux density of a 
component alters in a very small period due to the steady state condition of the proposed system. For higher frequencies, it goes down to under 1T. Therefore, in this case, a specific zone of the permeability can be chosen for this component. Similarly, the permeability of the other components of the system can be chosen based on the working frequency of them. Therefore, having the idle parts of the B-H curves of the elements would be avoided, and the simulation time decreases. This algorithm can be defined in the material properties part of the FE simulation.

For the equivalent source modeling, the optimizations in the values of the model parameters of component, such as voltage and current, are employed. Moreover, the model is a cube model; therefore, the optimum dimensions should be obtained to have similar electromagnetic signatures as the actual results. All of these are obtained by using particle swarm optimization based on the genetic algorithm. Having numerous objective functions for the optimization procedure increases the process time; hence, the objective functions were considered either E-field or H-field in addition to the dimensions of the cube. Both situations satisfy the requirements and the equivalent source model radiates similar fields as the actual model.

In terms of the types of results, the frequency response analysis in addition to time domain analysis through equivalent source models and full models are achieved. The application of time-domain analysis is studying the effect of rotation, which is considered.

The optimized models have one drawback, which is the application of the models for a defined situation. In other words, if any change in the voltage, power or variation in the construction of the component is required to be implemented, the objective functions 
should be solved again, which takes time (sometime more than the simulation time of the full finite element models). Hence, the wire model based on the structure of the actual model of the proposed component is designed. The parameters of the wire model are voltage and current, which are obtained based on the electric and magnetic field density and current density of the actual components. In order to have a comprehensive model for different levels of voltages and currents, as well as sizes of the components, the appropriate factors are considered that enable the model to be used as a generalized model for designed components. The accuracy of the wire model is so close to the actual results.

Modeling the power electronic-based components, such as the power converter, has the problem of simulating the switching activities, while the low frequency model is proposed. In order to achieve this, the modification in the material of the insulator attached to the switch is done, which makes the current loop based on the switching frequency. The effect of this switching frequency in the low frequency response is demonstrated and discussed.

The application of the electromagnetic signature study is monitoring the behavior of the components and diagnosing the faults that occurred in the components. This is achieved though the harmonic and inter-harmonic study of the radiated electric and magnetic fields. To do this, the harmonic orders of each component should be addressed to their physical operation. In consequence, the types of fault, as well as the location of them, can be identified by analyzing the frequency response of the electromagnetic signatures. This study is implemented experimentally and by simulating the full finite element of the components. The equivalent source modeling cannot be used, because the 
diagnosis process can be accomplished by studying the harmonic orders, which are affected from the material features of the cores, as well as the wires, whereas the wire equivalent source models consist of lines and nodes. In addition to the fault diagnosis and system monitoring, the optimization in the performance of the components are achieved by studying the electromagnetic signatures. For instance, different types of switching are implemented and their radiated fields are observed. Based on the fields, the optimum switching technique for the purpose of fault diagnosis, as well as having less EMI, is obtained.

\subsection{Organization of the dissertation}

The dissertation is organized as follows:

In chapter 2, the advances in computational modeling of the electromagnetic compatibility were discussed. The models are classified. The utilized method in the research was described in details. Then, the full 3-D finite element modeling of the components were described and the related novelties were mentioned in chapter 3. The 3D FEM was coupled with the transmission line modeling and utilized in EMC modeling of the printed circuit board. Based on this, the optimization process in the modeling was performed and explained.

The equivalent source modeling methods from the simple dipole model to the PSOGA based cube model are explained in chapter 4. Before discussing the experimental implementation of the low-frequency EMC analysis, as well as electromagnetic signature analysis, the requirements for low-frequency EMC experiments are reflected in chapter 5. The wire model, which is based on the physics of the components as the most developed 
equivalent source model, is explained in chapter 6 . Then, the modified 3-D finite element modeling for the purpose of fault diagnosis is demonstrated in chapter 7. The first application of this modeling was fault type diagnosis, which is presented in chapter 8 . The different types of unbalanced currents applied to an induction machine and the electromagnetic signature is studied with the trained neural network are present in chapter 9. Finding the location of the short-circuit as the main fault in the electrical machines are discussed along with the study of finding the optimum location of antenna. In chapter 10, the various combinations of components are studied experimentally for the purpose of source identification with and without faults. Moreover, the improvements of their performance of them were employed. Finally, the conclusion and recommendation for the future work are presented in chapter 11, respectively. 


\section{Advanced Modeling in Computational Electromagnetic Compatibility}

\subsection{Overview}

Before starting the modeling of the components, the advances in modeling the computational electromagnetic compatibility along with the types of modeling in this field are discussed and compared.

James Clerk Maxwell derived his celebrated four equations and established electromagnetics as a rigorous theory and published this work in the famous treatise in $1865[133]$

Relating the behavior of electromagnetic fields and sources, several other physical relationships are necessary for their solution, in addition to Maxwell's equations themselves. The most important are Ohm's law, the equation of continuity, and the constitutive relations of the medium and the imposed boundary conditions of the physical problem of interest. Before Maxwell, the science of electromagnetism had existed mostly as an experimental discipline for several centuries through the works of scientists, such as Benjamin Franklin, Charles Augustin de Coulomb, Andre' Marie Ampere, Hans Christian Oersted, and Michael Faraday. The early doubt about Maxwell's theory vanished in 1888 when Heinrich Hertz transmitted and received radio waves, thus having demonstrated the validity of the Maxwell theory.

The early works on analytical solution methods in electromagnetics, based on Maxwell's equations, were mainly focused on the area of radio science. Some of such applications of the electromagnetic theory started to appear not long after Maxwell's treatise had established. Among the analyzed simple geometries were the fields radiated 
from the Hertzian dipole, an infinitely long straight circular wire and two coaxial cones [134]. In most of the cases, the equations were solved as boundary value problems having yielded to the solution in terms of infinite series expansions.

By the incidence of computational electromagnetics, analytical methods in electromagnetics enabled handling the practical engineering problems. This was brought on by the advent of the digital computer in the 1960s. While much effort of the early research based on analytical methods was focused on the study of antennas in radio science, the emergence of computational electromagnetics opened up a number of new areas of applications. At the beginning, the emphasis was on high frequency radar systems related to defense. This was mainly due to the concentration of available research funds for such a work during that time. Indeed, in what is reported as one of the earliest cases of solving electromagnetic problems on a digital computer in the 1950s, the machine was built due to the need for calculating artillery ballistic tables [135].

Since that time, a continuing advancement in computational methods has been prompted by the rapid progress in computer hardware. Frequency domain integral equation techniques having simpler mathematical framework than their time domain counterpart started to appear in the mid-1960s $[136,137]$. One of the first digital computer solutions of the Pocklington's equation was reported in 1965 [138]. This was followed by one of the first implementations of the finite difference method (FDM) to the solution of partial differential equations in 1966 [139] and time domain integral equation formulations in 1968 [140] and 1973 [141,142]. Through the 1970s, the finite element method (FEM) became widely used in almost all areas of applied electromagnetism encompassing power engineering and electronics applications, microwaves, antennas and 
propagation, and electromagnetic compatibility. Good review of various types of FEM along with many important FEM applications in electrical engineering and electronics till date has been presented in Ref. [143]. The boundary element method (BEM) developed in the late seventies for the purposes of civil and mechanical engineering [144] started to be used in electromagnetics in the 1980s. Nevertheless, there have been many applications of BEM in electromagnetics; the primer of BEM for electrical engineers appeared quite recently [145].

Up to a dozen or more computational methods are nowadays commonly in use for electromagnetic modeling purposes, there is no particular method, which can claim superiority over the whole range of applications [146]. The physical and mathematical base on which a specific method has been built often gives it some advantage when dealing with a particular class of problem. Nevertheless, the integral equation formulation handled via the method of moments (MoM) with its wide application and versatility is accepted by many researchers to be a sort of "workhorse" in computational electromagnetics [147]. An excellent review of the numerical methods used in computational electromagnetics has been given in literature in 1998 [148]. Among many others, a rather comprehensive textbook on numerical methods in electromagnetics is the one by Sadiku [149], whereas a relevant review of theoretical models and computational methods used in electromagnetic compatibility is available in Ref. [150].

Analysis of wire antennas and scatterers and related EMC applications by using the integral equation approach has been presented in Ref. [151]. Direct time domain techniques for the solution of certain classes of EMC problems have been documented in Refs. [151,152]. 


\subsection{EMC Computational Models and Solution Methods}

Generally, the methods used in EMC are not only to visualize electromagnetic phenomena but also to predict and suppress interferences which can be regarded as either theoretical or experimental. This research is oriented to the consideration of theoretical approach and related computational models in the analysis of EMC problems. The experimental approach is also used for verification of the theoretical approach, which is explained in the next chapters.

It is the rapid progress in the development of digital computers that has provided advances in EMC computational models in the last few decades.

Electromagnetic modeling provides the simulation of an electrical system to investigate the electromagnetic behavior for a rather wide variety of parameters including different initial and boundary conditions, excitation types, and different configuration of the system itself. The important fact is that the modeling is undertaken within a significant shorter time than it would be necessary for building and testing the appropriate prototype via experimental procedures. The basic purpose of an EMC computational model or electromagnetic signature model is to predict a victim response to the external excitation generated by a certain EMI source.

A basic electromagnetic signature model includes the sources that could be either low frequency electrical power components or high frequency radio transmitters or any kind of undesired electromagnetic pulse (EMP). If the study is in the case of EMC, then the EMI victim is also important that can be any electrical equipment, medical electronic equipment or even the human body itself. 
In principle, all EMC models arise from the challenging electromagnetic theory concepts and foundations are based on Maxwell equations. The governing equations of a particular problem in differential, integral, or integro-differential form can be readily derived from the four Maxwell equations.

EMC models are analyzed using either analytical or numerical methods. Though both approaches can be used in the design of the electrical systems, analytical models are not useful for accurate simulation of electric systems or their use is restricted to the solution of rather simplified geometries with a high degree of symmetry (canonical problems).

On the contrary, a more accurate simulation of various practical engineering problems is possible by the use of numerical methods. In this case, errors are primarily related to the limitations of the mathematical model itself and the applied numerical method of solution, respectively.

Commonly, analytical and numerical techniques are used for a wide variety of purposes such as:

- Predicting system-level response to EMI sources

- Evaluating the behavior of EMC protection measures

- Processing measures system test data

The development of analytical and numerical modeling techniques has had a marked impact on the area of EMC. These techniques are used in the design, construction, test, and evaluation phases.

- Defense electric systems

- Communications and data transmission systems

- Power utilities 
- Consumer electronics

EMC computational models can be validated via experimental measurements or theoretical study, comparing the results to already well-established numerical models.

\subsection{Classification of EMC Models}

There are many possible classifications of EMC computational models used in research and practical purposes [150-154].

Based on the mentioned literatures, EMC models can be classified as:

- Circuit theory models featuring the concentrated electrical parameters

- Transmission line models using distributed parameters in which low frequency electromagnetic field coupling are taken into account

- Models based on full-wave approach taking into account radiation effects for the treatment of electromagnetic wave propagation problems

It is worth emphasizing that, for the purpose of modeling using full wave, it's mostly based on the thin-wire scattering theory.

Almost all problems in science and engineering can be formulated in terms of differential, integral, and variational equations. Generally, these are two basic approaches to solve problems in electromagnetics:

- Differential or the field approach

- Integral or the source approach.

The field approach deals with a solution of a corresponding differential equation with associated boundary conditions, specified at a boundary of a computational domain. Historically, this approach has been derived by Gilbert, Faraday, and Maxwell. The field 
approach is very useful for handling the interior (inner, closed, or bounded) field problems.

The electromagnetic field sources' concept, or the integral approach, is based on the solution of a corresponding integral equation. Historically, this approach was promoted by Franklin, Cavendish, and Ampere, among others. It is worth noting that the source approach is convenient for the treatment of the exterior (open, outer, or bounded) field problems.

Mostly, the methods for solving partial differential equations or integral equations can be classified as analytical or numerical ones.

The principal drawback of the analytical methods is the inability to handle problems involving complex geometries and inhomogeneous domains. This difficulty can be overcome by applying numerical methods.

The main problems arising in the application of numerical methods is related to the spurious solutions, convergence rate, and accuracy.

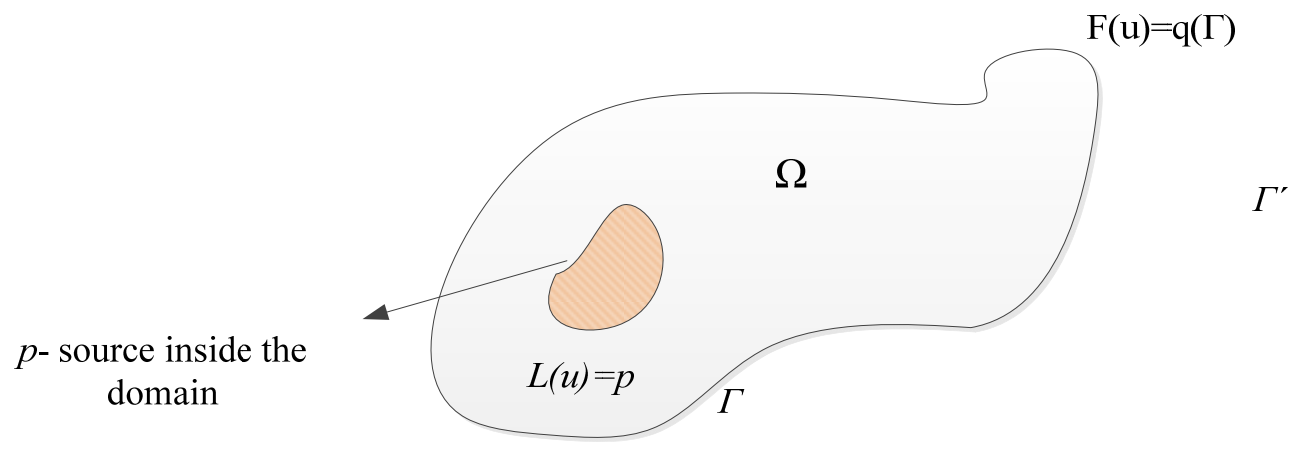

Fig. 2.1. The field approach

A classical boundary-value problem can be formulated in terms of the operator equation: 
On the domain $\Omega$ with conditions $\mathrm{F}(\mathrm{u})=\mathrm{q}(\Gamma)$ prescribed on the boundary $\Gamma$ (fig. 2.1 ), where $\mathrm{L}$ is the linear differential operator, $\mathrm{u}$ is the solution of the problem, and $\mathrm{p}$ is the excitation function representing the known sources inside the domain. Methods for the solution of the interior field problem are generally referred to as differential methods or field methods.

On the contrary, if $\mathrm{L}$ represents an integral operator, unknowns are related to field sources, that is, charge densities or current densities distributed along the boundary $\Gamma^{\prime}$ (fig. 2.2). Once the sources are determined, the field at an arbitrary point, inside or outside the domain, can be obtained by integrating the sources.

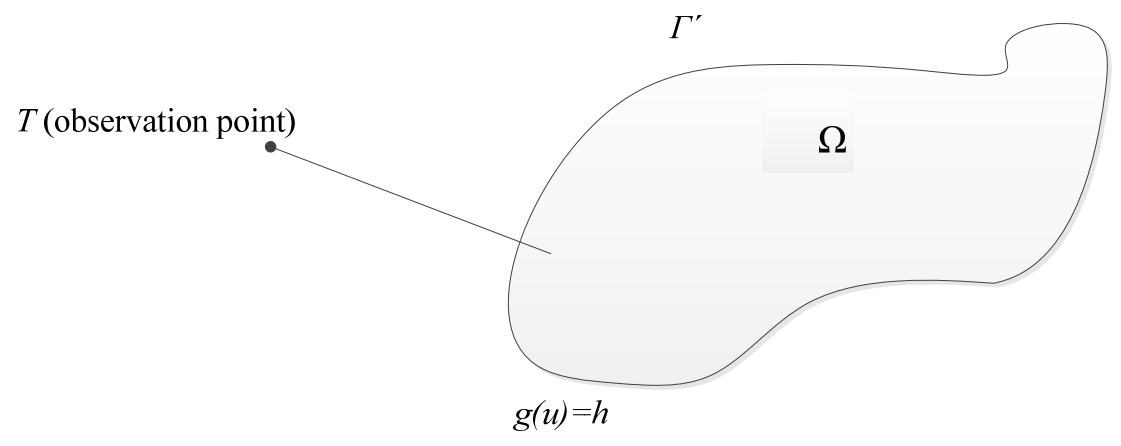

Fig. 2.2. The integral approach

Methods of solutions for the exterior field problems are referred to as the integral methods, or method of sources. The boundary conditions used in electromagnetic field problems are usually of the Dirichlet (forced) type, the Neumann (natural) type, or their combination (mixed boundary conditions). These boundary conditions can be either homogeneous or inhomogeneous. 


\subsection{Time domain vs. Frequency Domain Modeling}

The problems being analyzed can be regarded as steady state or transient, and the solution methods are usually classified as frequency or time domain. The frequency and time domain techniques for solving transient electromagnetic phenomena have been fully documented in [149].

A frequency domain solution is commonly applied for many sources but as one single frequency whereas with the time domain, it is for a single source but many frequencies [150]. While this is true for the continuous integral Fourier transform, it is often not possible in practice to obtain the equivalent solution from another domain for highly resonant structures, when the discrete Fourier transform is used. Equivalence of the results in the two respective domains is true only under a precise set of conditions, which are hard to meet in practice.

One important difference between the two approaches is that the time domain solution obtained is specific to the temporal variation of the excitation source. The transient response of a structure when subjected to different excitations, for example, a stepfunction or a Gaussian voltage source will require the computation process to be repeated for the respective solutions. Whereas, in the frequency domain approach, the solution from one set of computations can be applied to obtain transient results from different sources if the geometry of the structure is unchanged. This difference is a significant factor when considering the relative merits of the two approaches.

Comparison based on computational efficiency between the two approaches yields the general conclusion that the computer time required for a solution using both the integral equation frequency domain and the implicit time domain approaches is proportional to 
$(L / \Delta L)^{3(\mathrm{D}-1)}$, where $L$ is a characteristic dimension of the structure, $\Delta L$ the frequency dependent space discretization, and $D$ the dimensionality, which for a wire is 2 . For the explicit time domain approach, the time is proportional to $(L / \Delta L)^{2(\mathrm{D}-1)+1+r}$, where $0 \leq \mathrm{r} \leq 1$. Although in many situations, the time domain approach has better advantages, it can be said that the computational efficiency of each approach is dependent on the structure being analyzed and the form of the result being sought.

Other statements arising from the concession between the frequency and the time domain approach are as follows [149]:

- Better physical insight when using the time domain approach. However, an understanding of the resonant characteristic can only be obtained from the frequency spectrum.

- Nonlinearities are more conveniently handled in the time domain.

- Interactions (e.g., pulse reflection) may be isolated in the time domain using timerange gating.

- It is possible to obtain singularity expansion method poles more directly and efficiently in the time domain.

- Frequency domain formulation is relatively simpler and easier to use, thus allowing more complex structures to be analyzed more conveniently.

- For complicated geometry, larger computing effort may be required for the more complex formulation of the time domain approach.

One factor of increasingly importance in favor of the frequency domain approach is in analyzing electromagnetic compatibility (EMC) properties. Accurate frequency domain information is required for such work as regulatory standards are specified in this form. 
Due to its simpler formulation, versatile general purpose frequency domain codes are widely available and are used by professionals and amateurs alike. An efficient frequency domain transient analysis methodology is likely to find applications in many fields by users of such codes.

The problem of excessive computer time becomes critical in transient electromagnetics. In the frequency domain approach to a transient problem, the computation must be repeated over the bandwidth for hundreds or even thousands of times at a frequency sampling interval. For a highly resonant structure, this straightforward unabridged approach will simply be beyond the practical limit in terms of the computer time required to solve a problem.

Much effort in computational electromagnetics research has been directed toward reducing the computation operation count for solving problems. This is achieved through several ways in the analytical formulation, using specialized Green's function approximations applied particularly to integration and matrix solving techniques. Very often improvements in computation efficiency are achieved at the expense of sacrificing accuracy and the utility of a formulation that is, narrowing the range of applications for a particular formulation. Several techniques for reducing the computation operation count in frequency sampling, when analyzing the transient behavior of resonant structures have been discussed in [155].

\subsection{Analytical or Numerical Method}

A general mutual concession between analytical and numerical methods can be done, as follows: 
Analytical solution methods yield exact solutions but are limited to a narrow range of applications, mostly to canonical problems. There are not many practical engineering problems that can be worked out using these techniques. On the other hand, the numerical techniques are applicable to almost all scientific engineering problems, but the main drawbacks are related to the approximation limit in the model itself, space and time discretization. Moreover, the criteria for accuracy, stability, and convergence are not always straightforward and clear to researchers.

The most commonly used analytical methods in electromagnetics are as follows [149]:

- Separation of variables

- Series expansion

- Conformal mapping

- Integral solutions such as Laplace and Fourier transform

- Perturbation methods

While the numerical methods used in electromagnetics, among others, are as follows:

- Finite difference method (FDM)

- Finite element method (FEM)

- Boundary element method (BEM)

- Method of moment (MOM)

- Transmission-Line modeling

- Monte Carlo method

- Method of lines 
Practicably, the most powerful analytical method is the separation of variables; it is the method that will be summarized in the following.

The method of separation of variables (sometimes called the method of Fourier) is a convenient method for solving a partial differential equation (PDE). Basically, it entails seeking a solution which breaks up into a product of functions, each of which involves only one of the variables. For example, if we are seeking a solution $\Theta(x, y, z, t)$ to some PDE, we require that it has the product form

$$
\Theta(x, y, z, t)=X(x) Y(y) Z(z) T(t)
$$

A solution of the form in Eq. (2.2) is said to be separable in $x, y, z$, and $t$. For example, consider the following functions: (a) $x \cos (t) t^{3} y$, (b) $x y^{2}+\frac{2}{t}$, (c) $(2 x+\sqrt{y}) z \cos 10 t$. (a) is completely separable, (b) is not separable, while (c) is separable only in $z$ and $t$.

To determine whether the method of independent separation of variables can be applied to a given physical problem, we must consider the PDE describing the problem, the shape of the solution region, and the boundary conditions - the three elements that uniquely define a problem. For example, to apply the method to a problem involving two variables $x$ and $y$ (or $\rho$ and $\varphi$, etc.), three things must be considered:

The differential operator $L$ must be separable, i.e., it must be a function of $\Theta(\mathrm{x}, \mathrm{y})$ such that

$$
\frac{L\{X(x) Y(y)\}}{\Theta(x, y) X(x) Y(y)}
$$

is a sum of a function of $x$ only and a function of $y$ only. All initial and boundary conditions must be on constant-coordinate surfaces, i.e., $x=$ constant, $y=$ constant. 
The linear operators defining the boundary conditions at $\mathrm{x}=$ constant (or $\mathrm{y}=$ constant) must involve no partial derivatives of $\Theta$ with respect to $\mathrm{y}$ (or $\mathrm{x}$ ), and their coefficient must be independent of $\mathrm{y}$ ( or $\mathrm{x}$ ).

With this preliminary discussion, we will now apply the method of separation of variables to PDEs in rectangular, circular, cylindrical and spherical coordinate systems. In each of these applications, we shall always take these three major steps:

1) Separate the (independent) variables

2) Find particular solutions of the separated equations, which satisfy some of the boundary conditions

3) Combine these solutions to satisfy the remaining boundary conditions

On the other hand, the applications of numerical methods are in electromagnetics and many other industrial uses, such as continuum problems that includes: hydrodynamics, thermodynamics, or acoustics, which are based on the advantage to model a particular problem without a requirement of high level mathematics and physical knowledge, respectively. Because of the complexity of the proposed models, numerical method is highly recommended.

\subsection{Overview of Numerical Methods}

It is important to clarify some principles and ideas of how to describe field problems via partial differential or integral equations. Namely, there are some basic differences between domain methods (e.g. finite element method), boundary methods (e.g. boundary element method), and source simulation methods (charge or current simulation method). This section deals with fundamentals of the FDM, FEM, and direct BEM. 


\subsubsection{Modeling of Problems via the BEM and MoM}

Integral formulations of partial differential equations along the boundary are carried out using the Green integral theorem. The resulting equations are modeled discretizing only the boundary and by integrating any known $\Omega_{\mathrm{s}}$ within a given subdomain. Modeling of partial differential equations through their boundary integral formulations results in less unknowns but dense matrices. The formulation of the boundary element method using field and potential quantities rather than field sources is usually called the direct BEM formulation. Integral equation over unknown sources $\Omega$ 's (sources outside the boundary) can also be derived from the green integral theorem, and the solution method can be referred to as a special variant of the boundary element method - indirect boundary element method.

The well-known method of moment $(\mathrm{MoM})$ is equivalent to BEM when using sub sectional bases and Dirac delta function as weighting functions. BEM, on the contrary, offers a more general relationship in which shape functions corresponding to sub sectional bases are taking systematically as in the finite element method (FEM). Thus, BEM can be regarded as a combination of the classical boundary integral equation method and the discretization concept originated from FEM.

\subsubsection{Modeling of problems via the domain methods: FDM and FEM}

FDM is generally one of the simplest numerical methods. Modeling via FDM is undertaken discretizing the entire domain and converting the differential operator into difference equations. 
FEM modeling of partial differential equations is performed by discretizing the entire calculation domain $\Omega$, and integrating any known source $\Omega$ s within the domain. Dirichlet and Neumann boundary conditions can be prescribed along the boundary $\Gamma$ for both FDM and FEM. Modeling of differential equations with FEM results in an algebraic equation system, which provides a sparse matrix. This matrix is usually banded and in many cases symmetric depending on the solution of the problem.

The finite difference method is a highly versatile method and has been used to analyze objects with an extremely wide range of size and complexity.

Historically, the finite difference method was developed in the 1920s for some applications in hydrodynamics. The finite difference method is based on the approximation of the function derivatives using finite differences. That is, a differential equation is replaced by a finite difference equation.

FDM is one of the simplest numerical methods but at the same time suffers from relatively poor convergence rate. This drawback can be overcome by increasing the number of unknowns in the corresponding matrix systems. More details about the FDM are explained in [149].

The finite element method is one of the most commonly used numerical methods in science and engineering. The method is highly automatized and very convenient for computer implementation based on step by step algorithms. The special features of FEM are related to efficient modeling of complex shape geometries and inhomogeneous domain, and also to the relatively simple mathematical formulation, providing a highly banded and symmetric matrix, which is similar for accuracy refinement by higher order approximation and automatic inclusion of natural (Neumann) boundary conditions. This 
method generally gives better results than the FDTD in modeling complicated boundaries and is particularly suited for problems with closed boundaries. In this method, the entire domain space to be analyzed is discretized into a gird of elements of finite size.

Particularly for the purpose of low frequency EMC study, FEM is used, since the analysis is in a huge region and there are numerous types of materials and boundaries. For high frequency, MoM is more appropriate [143]. 


\section{3-D Finite Element Method Coupled with Transmission-Line Modeling in EMC Study}

\subsection{Overview}

After explaining the details of the computational electromagnetic model in EMC study, the 3-D finite element method as the well-known and reliable method for the low frequency EMC study is selected as the physics based modeling and coupled with the transmission line modeling method for evaluating the behavior of the proposed component which is converted in this chapter. The other components are studied in the next chapter along with the concept of the equivalent source modeling.

In order to evaluate the actual behavior of the converter at the design stage, a numerical model of the inductor was created and implemented using the finite element (FE) analysis. Using the numerical model, the physics based circuit model was obtained for the converter's resonant circuit. The acquired physics based circuit model was used to approximate the electrical behavior of the resonant circuit. The operating condition of the half-wave ZCS buck converter was verified both numerically and experimentally. It was shown that, by using the proposed model, it is possible to evaluate realistic waveforms of voltages and currents including the effects of parasitic elements. This is essential step for studying electromagnetic field emissions in power converters for the evaluation of their EMI interactions for EMC compliant designs.

Soft-switching converters are becoming more popular as they have lower loss and noise characteristics, as compared to pulse width modulation (PWM) converters. In these 
types of converters, the resonance circuit is a major contributor to the creation of EMI. It can cause unexpected current noise flow in the common mode path [156].

Generally, in order to control the conducted emissions (CE) noise in the softswitching converters, the issue of parasitic elements must be considered during the design stage. These parasitics are circuit elements (resistance, inductance or capacitance) that are possessed by electrical components, but are not desirable for them to have it.

So far, various algorithms were introduced to eliminate or minimize the effects of these unwanted elements [157-159]. Previously, methods based on the parallel-plate waveguide and FEM analysis were introduced to solve the PCB unwanted coupling problems and reduce the EMI effects of the converters [160-163].

All components and interconnections contain unintentional (parasitic) circuit elements which often a combination of them can make a change in the operating condition of the whole power converter. This makes the EMI issues more complicated. Furthermore, the switching action causes various parasitic elements in the converter and results in conducted and radiated energy at unpredictable frequencies. In practice, these parasitic oscillation frequencies are most difficult to filter out. They often cause the most interference with signal processing circuitry.

Therefore, an understanding of the magnitude of these parasitic elements and the characteristics of the components over a range of frequencies will ensure the correct choice of their application. Moreover, it is important for investigation of the effects of switching-frequency control on EMI generation.

The operating principle of the Quasi-resonant converters is described in [164]. These converters are obtained by adding the resonant elements $L_{r}$ and $c_{r}$ to the PWM switching 
converters. Fig. 3.1 shows the simplified circuit model of the ZCS-Buck converter. The parasitic elements in the configuration of the resonant components can affect the operating condition of the whole converter. In order to have a more precise study on the converter behavior, a distributed model should be obtained for each of the elements.

A planar inductor based resonant circuit is designed for the converter's resonant inductor (shown by $\mathrm{L}_{\mathrm{r}}$ in fig. 3.1). This design can meet the packaging constraints in power electronic converters in addition to the required EMI compliance levels. The design and characteristic optimization of the planar inductor is studied in references [165168]. The electromagnetic radiation of a PCB planar inductor is discussed in [169], [170]. As an example, based on the procedure in reference [164], the inductance value is selected to be $L_{r}=1 \mu \mathrm{H}$, and the resonant capacitor is selected to be $C_{r}=400 n f$. With this selection, the resonant frequency will be $f_{0}=\frac{1}{2 \pi \sqrt{L_{r} C_{r}}}=251.65 \mathrm{kHz}$.

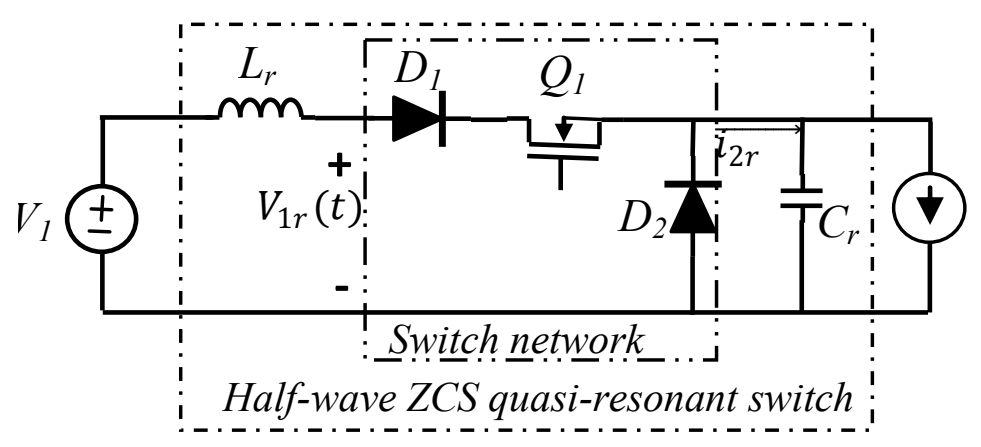

Fig. 3.1. Half wave zero current switching buck converter's circuit model.

Subsequently, in order to find the parameters of the converter, a finite element analysis is performed on the resonant part of the converter. After obtaining the high frequency physics based model of the resonant circuit, the resonant behavior of the proposed planar inductor based resonant circuit is compared with the ideal resonant 
circuit. The FE analysis is used to study the electromagnetic behavior of the proposed resonant circuits in section 3 of this chapter. As a result, a high frequency model is obtained for the resonant circuit under a wide range of switching frequencies. Finally, the resonant circuit design was optimized in order to compensate the destructive effects of parasitic elements in the operation of the converter. The results were verified through the simulation and experimentation.

\subsection{Modeling Procedure}

Under PWM operating conditions, the inductor's resistance, inductance and capacitance behave differently from the low frequency operation. The windings have skin and proximity effects, which cause the resistance to be much higher than the low frequency value. The inductance value decreases with the increase in the operating frequency, while the small capacitance effect comes in the picture at high frequencies. Under such PWM operation, the inductor winding's capacitance is distributed between several parts of the winding (turn-to-turn and turn-to-ground). Hence, to obtain accurate values of the resistances, inductances (self and mutual), and capacitances (self and mutual), a detailed numerical model for the inductor should be used. Also, this strategy is used for the capacitor of the converter since, in higher frequencies, the self and mutual inductances, as well as the self and mutual capacitances, have effects in the frequency response analysis.

In reference [171], a method based on the vector fitting algorithm was proposed for modeling the physics-based representation of transformers. This is also applicable to the inductor design. The method used in this study is based on the lumped-parameter model 
presented in [172], [173]. This method is used to obtain the s-domain model of a spiral winding planar inductor, which can be used to find the frequency response of the inductor. The proposed model includes the windings' resistances, self-inductances, ground capacitances, the inter-turn capacitances within each winding and the mutual inductive and capacitive couplings between the two windings.

\subsubsection{Physics based modeling using 3-D FEM}

A three-dimensional finite element study was performed on the resonant circuit components, in order to calculate the parasitic elements of these components, which can affect the operating condition of the converter.

The electromagnetic field inside the inductor and capacitor is governed by the following set of nonlinear partial differential equations [171]:

$\nabla \times((v) \nabla \times \mathbf{A})=\mathbf{J}, \nabla \cdot \sigma((\partial \mathbf{A}) / \partial t+\nabla V)=0$

where $\mathbf{A}$ is the magnetic vector potential, $\mathbf{J}$ is the total current density, $v$ is the magnetic reluctivity, $\mathrm{V}$ is the electric scalar potential, and $\sigma$ is the electric conductivity. By solving these equations, the resistance and inductance of the inductor are calculated as a function of frequency.

Also the electrostatic problem is solved for the calculation of capacitances matrix as capacitances are a function of geometry rather than frequency. An electrostatic analysis is assumed to be a linear analysis, which means that the electric field is proportional to the applied voltage. The analysis determines the electric scalar potential distribution caused by the applied voltage. The following Maxwell equation is solved during electrostatic analysis. 
$\nabla \cdot(\varepsilon \nabla V)=-\rho$

where $\rho$ is surface charge density, $\varepsilon$ is permittivity, $V$ is electric scalar potential. By coupling electric and magnetic analyses, electromagnetic analysis for frequency response analysis based on (3-3) are applied in this simulation.

Typically, the quasi-static electromagnetic analysis is used as the analysis approach in these cases, but this method has one problem. The changing electric displacement field over time is considered as zero $(\partial \mathrm{D} / \partial \mathrm{t}=0)$. This assumption can be used for low frequency analysis. However, for higher frequency analysis, this assumption affects the result [174], [175]. Therefore, a new approach was used in this research in which $\partial \mathrm{D} / \partial \mathrm{t}$ is considered.

To derive the time harmonic equation, this physics interface solves magnetic and electric interface. The analysis is started with Maxwell-Ampere's law including displacement current. This does not involve any extra computational cost in the frequency domain. Firstly a time-harmonic field is assumed as (3-3).

$$
\nabla \times \mathbf{H}=\mathbf{J}=\sigma(\mathbf{E}+v \times \mathbf{B})+j \omega \mathbf{D}+\mathbf{J}^{e}
$$

Using the definitions of the fields $\mathbf{B}=\nabla \times \mathbf{A}$ and $\mathbf{E}=-\nabla V-j \omega \mathbf{A}$ and combining them with the constitutive relationships, $\mathbf{B}=\mu_{0}(\mathbf{H}+\mathbf{M})$ and $\boldsymbol{D}=\varepsilon_{0} \mathbf{E}$, the Ampere's law can be rewritten as (3-4).

$$
\left(j \omega \sigma-\omega^{2} \varepsilon_{0}\right) \mathbf{A}+\nabla \times\left(\mu_{0}^{-1} \nabla \times \mathbf{A}-\mathbf{M}\right)-\sigma v \times(\nabla \times \mathbf{A})+\left(\sigma+j \omega \varepsilon_{0}\right) \nabla V=\mathbf{J}^{e}
$$

The equation of continuity is again obtained by taking the divergence of Ampere's law. It is the equation solved for the electric potential. Thus the following equations for $\mathrm{V}$ and $\mathbf{A}$ are achieved: 
$-\nabla \cdot\left(\left(j \omega \sigma-\omega^{2} \varepsilon_{0}\right) \mathbf{A}-\sigma \boldsymbol{v} \times(\nabla \times \mathbf{A})+\left(\sigma+j \omega \varepsilon_{0}\right) \nabla V-\left(\mathbf{J}^{e}+j \omega \mathbf{P}\right)\right)=0$

A particular gauge can be obtained with reducing the system of equation by choosing $\Psi=-j V / \omega$ in the gauge transformation. Therefore, the modified magnetic vector potential is obtained.

$$
\tilde{\mathbf{A}}=\mathbf{A}-\frac{j}{\omega} \nabla V
$$

Working with $\widetilde{\mathbf{A}}$ is often the best option when it is possible to specify all source currents as external currents $\mathbf{J}^{e}$ or as surface currents on boundaries.

$$
\left(j \omega \sigma-\omega^{2} \varepsilon_{0}\right) \tilde{\mathbf{A}}+\nabla \times\left(\mu_{0}^{-1} \nabla \times \tilde{\mathbf{A}}-\mathbf{M}\right)-\sigma v \times(\nabla \times \tilde{\mathbf{A}})+\left(\sigma+j \omega \varepsilon_{0}\right) \nabla V=\mathbf{J}^{e}+j \omega \mathbf{P}
$$

where $\mathbf{A}$ is magnetic potential, $\mathbf{J}^{e}$ is external current density, $\mathbf{M}$ is magnetization and $\boldsymbol{v}$ is the motion speed which here, it is equal to zero.

The equation (3-7) is modified version of a classic quasi-static equation (3-4), which is implemented in FE softwares [176]. Further modification in this study is applied by linking MATLAB software with FE software. This can be done by defining a variable in MATLAB codes as $\mathbf{D}$ and making a link to the FE software, then considering this $\mathbf{D}$ (electric displacement field) instead of the default D. This new defined electric displacement field is based on the electric field obtained from the solution of software. The other element $(\widetilde{\mathbf{A}})$ is defined in the same way.

Another necessity of this type of analysis is the need of simultaneous estimation of capacitance and inductance that the former one can be calculated by the electrostatic analysis and the latter one by the magnetostatic analysis. This type of interface can be used for 3D, 2D in-plane, and 2D axisymmetric models. Note that the magnetic and 
electric currents physics interfaces support the stationary and frequency domain study types better than the transient domain study.

Fig. 3.2 shows the planar spiral inductor and capacitor models used in the power converter.
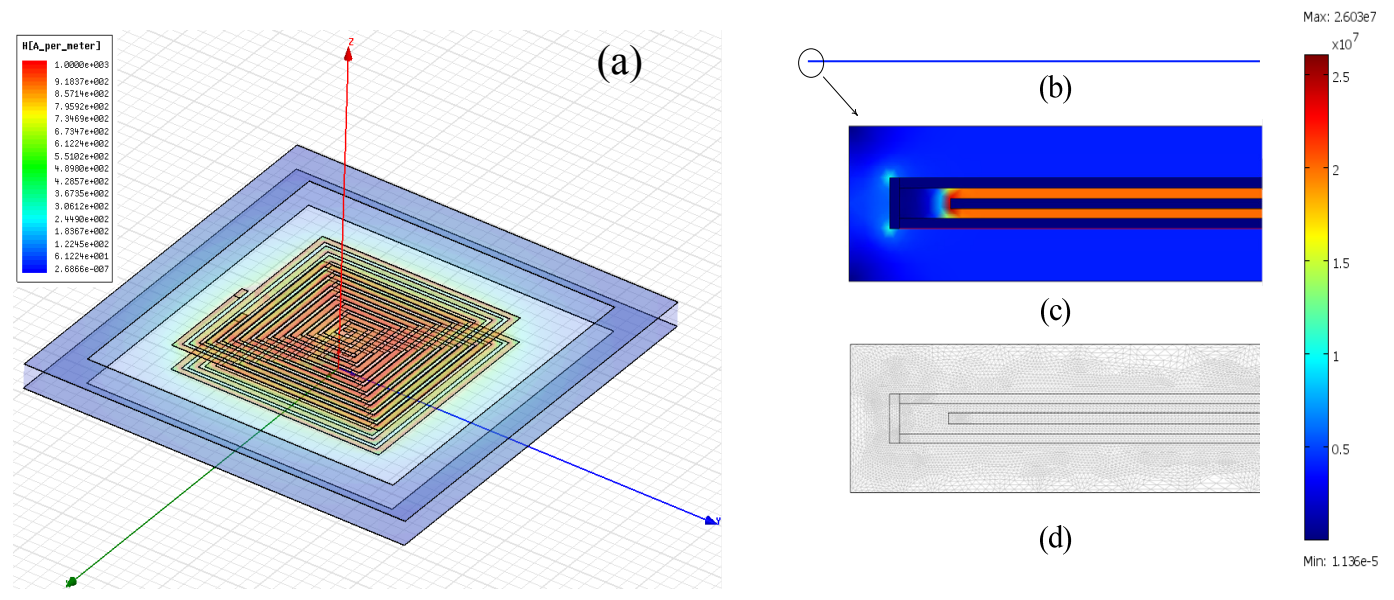

Fig. 3.2 Field spectrum from the finite element analysis for the calculation of high frequency model of this component. (a) Magnetic field intensity of spiral planar inductor (b) Capacitor (c) Electric field in small part of the capacitor (d) Mesh in capacitor.

To find a numerical solution for this problem, the coupling of the FE and a circuit based analysis is utilized. The amount of energy, magnetic and electric field are estimated from the FE model. The results are then imported to the circuit-based software (Spice), in order to evaluate the performance of the whole circuit.

After solving the FE model, the inductance, capacitance and resistive matrices were obtained via calculation of the energy matrix in the FE model. The magnetic energy is calculated by:

$W=\frac{1}{2} \iiint_{V} \mu \cdot \operatorname{Re}\left(\mathbf{H}_{1} \cdot \mathbf{H}_{1}^{*}\right) d v+\frac{1}{2} \iiint_{V} \mu \cdot \operatorname{Re}\left(\mathbf{H}_{2} \cdot \mathbf{H}_{2}^{*}\right) d v+\frac{1}{2} \iiint_{V} \mu \cdot \operatorname{Re}\left(\mathbf{H}_{1} \cdot \mathbf{H}_{2}^{*}\right) d v$ 
where $\mathrm{H}_{\mathrm{i}}$ is the magnetic field intensity inside the model and $\mu$ is the permeability of the model.

Following the calculation of the magnetic energy in the FE model, equation (3-8), all self and mutual inductances were estimated based on the magnetic energy value, [177], [178]. Also the explanation about deriving capacitances and resistances is mentioned in the same references.

\subsubsection{Solution Method}

As it was discussed, a numerical technique based on adaptive MEI-FEM (magneticelectric interface finite element method) is used. This method has much more accurate result, as compared to quasi-static electromagnetic finite element method, electrostatic finite element method, and magnetostatic finite element method. All experiments are performed on a x5677 dual core $3.47 \mathrm{GHz}$ CPU and 192GB RAM. The iteration process is terminated when the normalized backward error (tolerance) is reduced by $10^{-3}$.

To implement the MEI-FEM, the adaptive grouping method is used to reduce the memory consumption and captures the fine details of the structure. The simulation time for the analysis of the inductor with five million degrees of freedom and $10^{-3}$ percent tolerance of energy was 3 hours. Also, the simulation time for the analysis of the capacitor with three million degrees of freedom, fig. 3-2(d), with $1 \mathrm{e}^{-7}$ percent tolerance of energy was about 2 hours. Fig. 3-3 shows the energy tolerance versus the number of degrees of freedom.

The analysis method, which is used in this analysis is generalized minimal residual method (usually abbreviated GMRES) with successive over-relaxation (SOR) as pre and 
post smothers. The GMRES is an iterative method for the numerical solution of a system of linear equations [179].

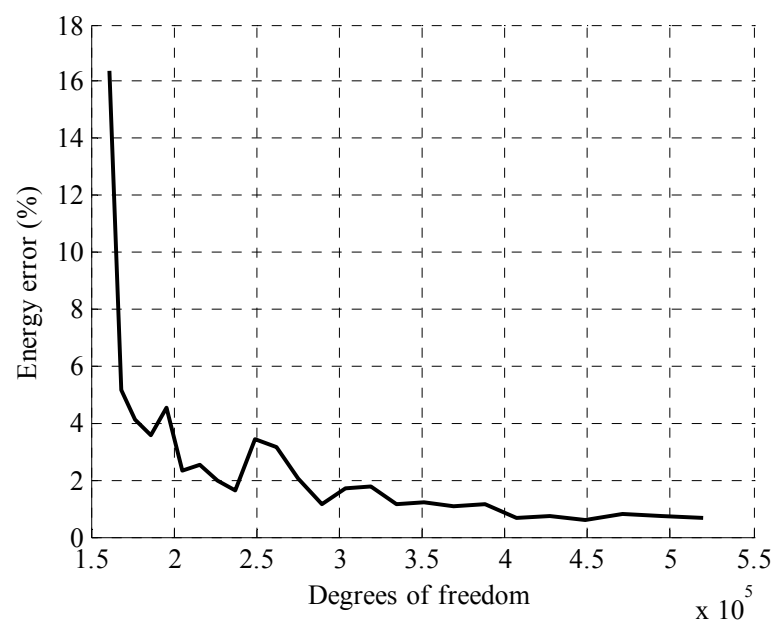

Fig. 3.3 Energy tolerance versus degrees of freedom

In numerical linear algebra, the method of SOR is a variant of the Gauss-Seidel method for solving a linear system of equations, resulting in faster convergence. A similar method can be used for any slowly converging iterative process. The SOR method uses a more accurate approximation of the matrix, which leads to fewer iterations but slightly more work per iteration than in the Jacobi method.

The geometry of the elements are non-uniform (The length of some elements are much bigger than the width of them). Therefore, the geometric multi-grid method is chosen as the pre-conditioner and F-cycle as multi-grid cycle. In addition, the parallel sparse direct linear solver (PARDISO) method with $1 \mathrm{e}^{-8}$ pivoting perturbation is chosen as the coarse solver.

\subsection{Development of transmission-line model}

After obtaining the parameters of the component, the transmission line model is developed. 
The influence of the capacitive reactance is increased by increasing the switching frequency. Therefore, very small capacitive reactances can affect the input transfer function in higher frequencies. In addition, mutual inductances between elements at far distances are more significant in the transfer function in high frequencies. Therefore, a model, which considers all these reactances is essential. The transmission-line or distributed-section model considers almost all tiny capacitive reactances and mutual inductances, so it is eligible for frequency response analysis.

Following the calculation of all required parameters for the distributed model, these parameters are located in the model, then the model evaluation procedure starts. As in the lumped parameter model the evaluation procedure from the distributed parameter nature of the problem at hand, we start at a location $x$ and moving an infinitesimal distance $\Delta x$ toward the lower end of the winding, the potential difference $\Delta V$ is calculated as:

$\left[\begin{array}{l}\Delta V_{1}(x, s) \\ \Delta V_{2}(x, s)\end{array}\right]=\left[\begin{array}{ll}Z_{1}(s) \Delta x & Z_{m}(s) \Delta x \\ Z_{m}(s) \Delta x & Z_{2}(s) \Delta x\end{array}\right]\left[\begin{array}{l}\hat{I}_{1}(x, s) \\ \hat{I}_{2}(x, s)\end{array}\right]$

where $\hat{I}_{1}(x, s)$ and $\hat{I}_{2}(x, s)$ are the current following in $Z_{1}$ and $Z_{2}$, respectively. The indices 1 and 2, indicates the first and the second layers of the inductor's and capacitor's models. A detailed description of this method is presented in reference [172]. By transferring the modal domain into a phase domain, the voltages of the top terminal in two layers are calculated as:

$$
\left[\begin{array}{l}
V_{s 1}(s) \\
V_{s 2}(s)
\end{array}\right]=\left[\begin{array}{ll}
M_{11}(s) & M_{12}(s) \\
M_{21}(s) & M_{22}(s)
\end{array}\right]\left[\begin{array}{c}
I_{s 1}(s) \\
I_{s 2}(s)
\end{array}\right]
$$

where $M_{i j}(s)$ are elements of impedance matrix in s-domain. 
To determine the frequency characteristics of the inductor, we set $s=j \omega$ in the related equations. The input impedance is given by:

$Z_{i n}(j \omega)=\frac{V_{s 1}(j \omega)}{I_{s 1}(j \omega)}$

Using the above equations, input impedance on the component is calculated as the following:

$Z_{\text {in }}(j \omega)=M_{11}(j \omega)$

The rational function given by (3-12) can be represented by an equivalent electrical network as shown in fig. 3.4. Note that, the circuit model in fig. 3.4 is not unique and can be shown in various configurations. This network reflects the frequency dependence of the inductor resistance, inductance and capacitance. Using $Z_{\text {in }}(j \omega)$, the natural frequencies are determined. 


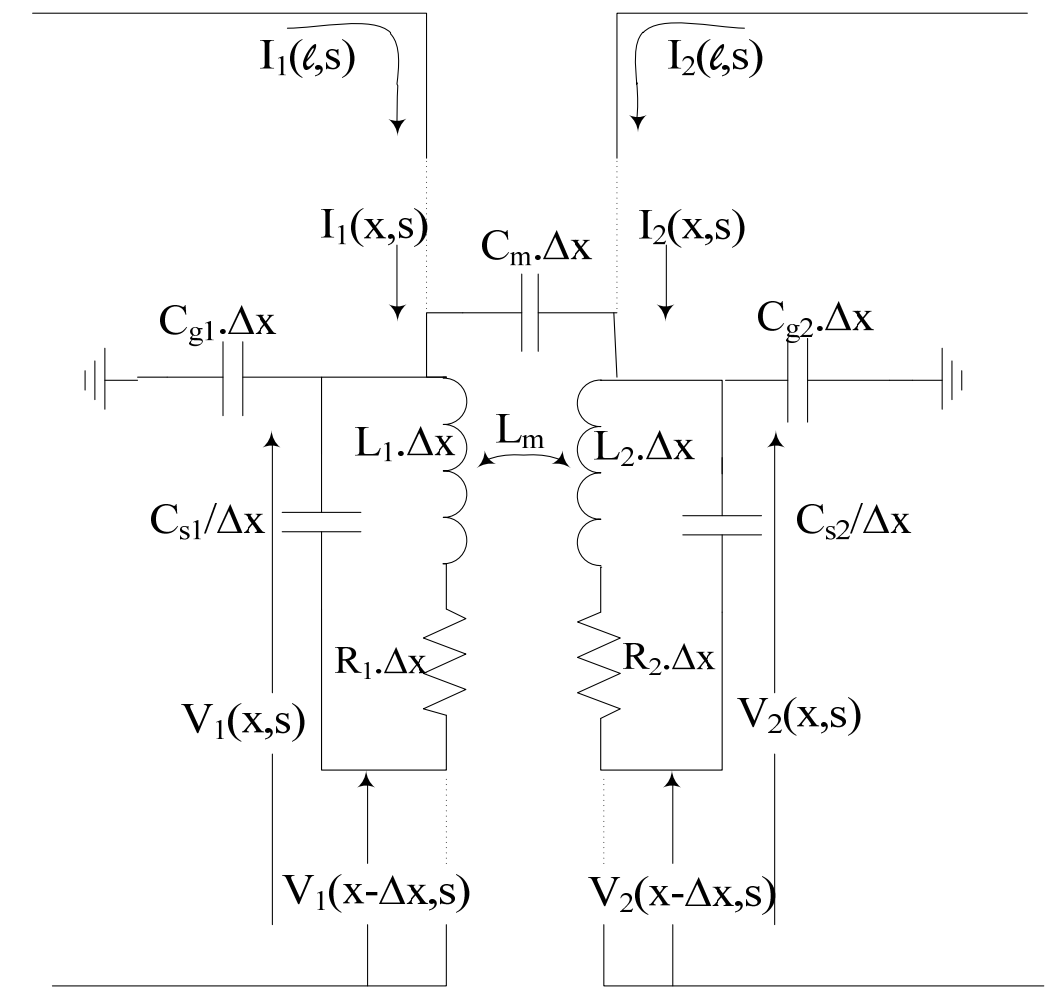

Fig. 3.4 Infinitesimal section of a two-layer winding of planar inductor.

The distributed model parameters of the designed inductor, from the finite element analysis were; $R_{l}=0.2713 \Omega, R_{2}=0.2713 \Omega, L_{l}=0.557 \mu \mathrm{H}, L_{2}=0.557 \mu \mathrm{H}, L_{m}=0.307 \mu \mathrm{H}$, $C_{s l}=8 \mathrm{pF}, C_{s 2}=8 \mathrm{pF}, C_{g 1}=0.33 \mathrm{pF}, C_{g 2}=0.33 \mathrm{pF}, C_{m}=6.7 \mathrm{pF}$. Also, the distributed model parameters of the capacitor were; $R_{c l, 2,3}=0.006725 \Omega, R_{c m}=10 \mathrm{k} \Omega, L_{c 1,2,3}=5 \mathrm{pH}, C_{s 1,2,3}=$ $0.49 \mathrm{pF}, C_{g l, 2,3}=4.42 \mathrm{pF}, C_{m}=177 \mathrm{nF}$. As mentioned earlier, the indices 1 and 2 of the inductor parameters stand for the first and the second layers of the inductor, as shown in fig. 3.2 (b). Note that the neutral-end terminal point of the first layer is connected to the line-end terminal of the second layer.

An advantage of the transmission-line model is to reveal the frequency response behavior of the model. Therefore, using only the simplified modeling of the resonant 
stage of HW ZCS-Buck converter doesn't show all the resonances and only shows the basic resonance as depicted in fig. 3.5 (a).

The embedded distributed model, which is shown in fig. 3.4 should have at least 5 sections to have reasonable result. We considered 10 sections in this study to have better and more accurate results. The schematic of this model is shown in fig. 3.6. As mentioned in [172], the result will be more accurate with more number of sections but from specific number of section like 10 the results will not change significantly.

Utilizing the distributed parameter model developed in this paper, as shown in fig. 3.5 (b), a realistic picture emerges. The number of resonances becomes more practical and representative of the real device than the one given in fig. 3.5 (a). Accounting for parasitic resonances in higher frequencies will enable a realistic inclusion of EMC issues in the design stage.

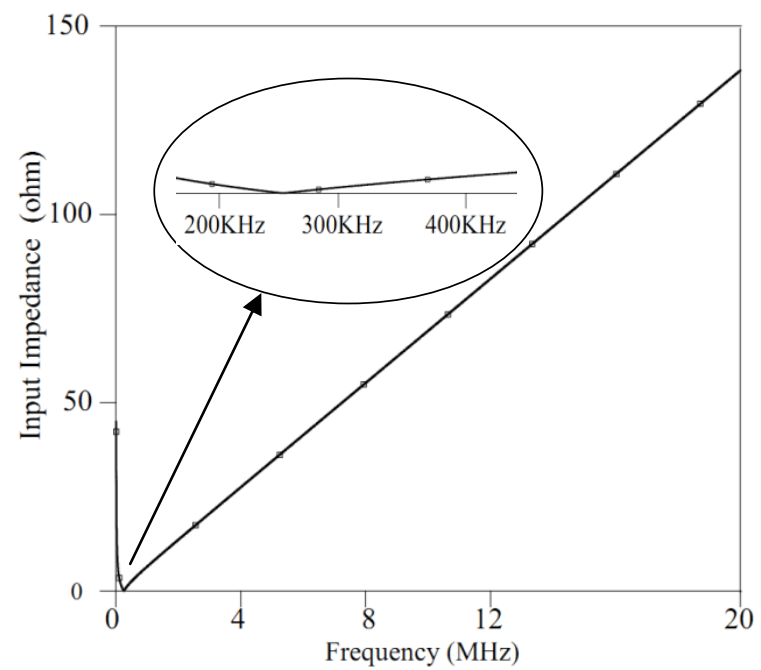

(a)

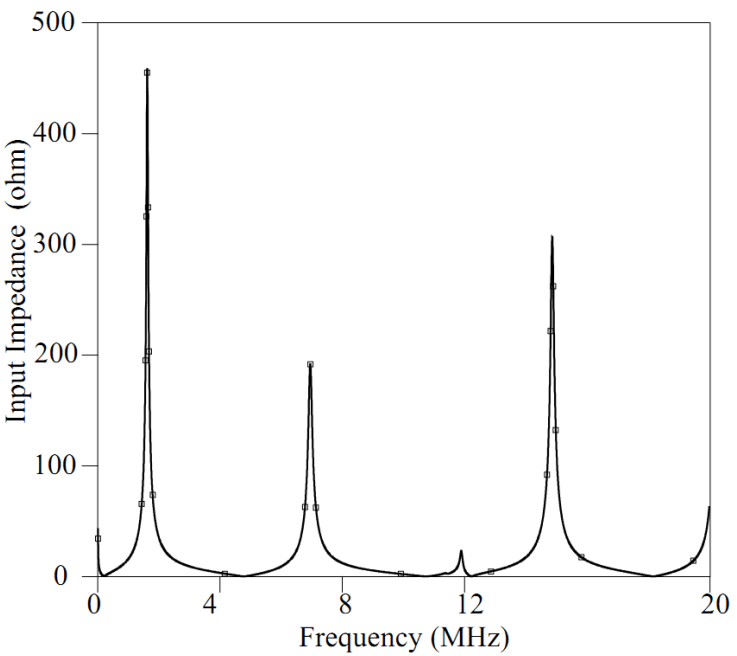

(b) 


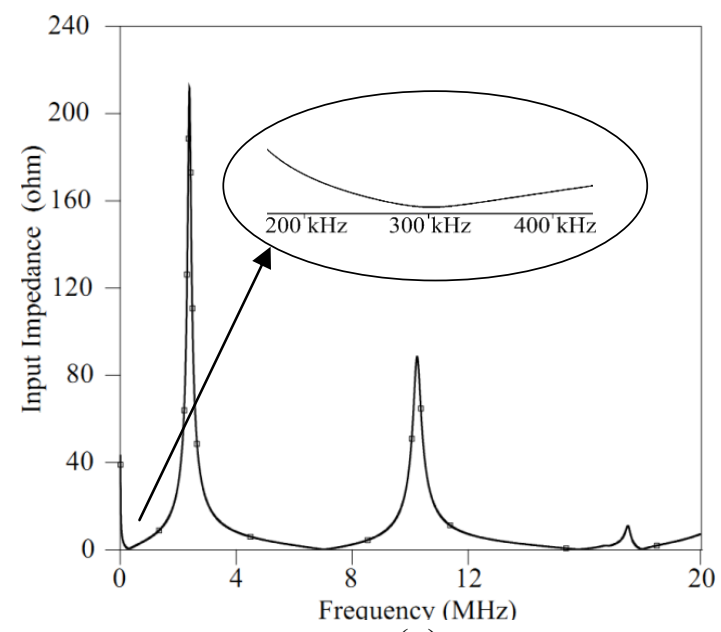

(c)

Fig. 3.5 Frequency response analysis of the ZCS buck converter. (a) simple model (b) distributed-parameter frequency model, (c) optimized distributed-parameter frequency model.

Through the optimization procedure [180], the size and geometry of the spiral planar inductor are changed in a way to minimize the effects of the parasitic parameters on the operating condition of the converter. The proposed algorithm is summarized as follows:

- Step 1: Design the planar inductor in the FE software.

- Step 2: Calculate the magnetic and electric fields based on equations (3-9)-(3-12).

- Step 3: Calculate resistances, inductances and capacitances based on equation (312).

- Step 4: Substitute the obtained parameters into the circuit shown in fig. 3-4. 


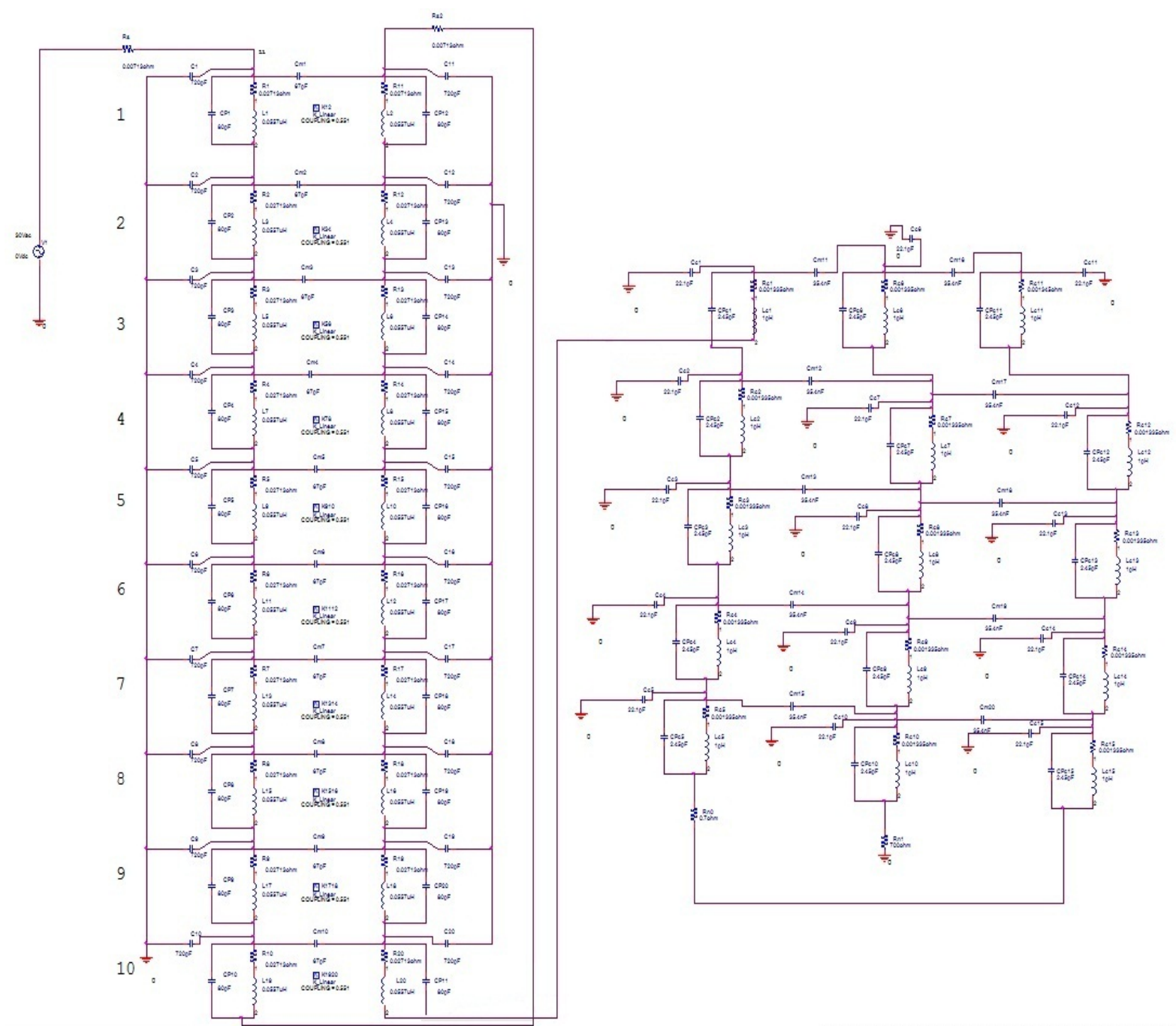

Fig. 3.6 Circuit model for high-frequency representation of the resonant circuit

The circuit shown on the left hand side of fig. 3.6 depicts the high-frequency model for inductor used in the resonant circuit and the circuit on the right hand side is the highfrequency model of the capacitor. Configuration of this circuit is based on the proposed transmission-line model shown in fig. 3.4. The numbers of transmission lines are chosen as ten for inductor and 5 for capacitor for better accuracy [172], [173]. Models for the inductor and capacitor are connected to build the resonant circuit used in the topology of the converter. 


\subsection{RESONANT CIRCUIT DESIGN OPTIMIZATION}

As shown in fig. 3.5, the first resonance frequency in fig. 3.5(b) is much lower than the expected resonance frequency (the first resonance frequency shown in fig. 3.5(a)). This phenomenon may deteriorate the operation of the converter. Because the resonance part of the converter is designed to resonate in $250 \mathrm{kHz}$ (in this example), but in practice, it's resonating at lower frequencies.

In order to solve this problem, an optimization procedure is utilized in the design of the planar inductor. The main objective in the procedure of the optimization is to set the resonance frequency to desired value (i.e $250 \mathrm{KHz}$ ), while reducing the amplitude of higher order harmonics. To achieve this goal, through the optimization process the planar inductor trace thickness and voltage clearance is changed, as well as the number of turns. Therefore, a multi-objective function is formed as a combination of each of these single objectives. The first part of this multi-objective function is calculated from a transient non-linear FE analysis to calculate the value of the inductor. Moreover, the second part of this multi-objective function is calculated from the circuit simulation of the resonant circuit, which is implemented in the Pspice environment. A classic genetic algorithm multi-objective optimization scheme is utilized to do the optimization task automatically. The variable of the genetic algorithm are indeed the inductance value of the planar inductor and magnitude of the harmonics in the resonant circuit output.

Fig. 3-7 depicts the flowchart of the parameter optimizing procedure using GA. GA is a population based global search procedure, which is inspired by natural selection and genetics law [181]. Parameters for optimization are number of turns in each layer $(\mathrm{N})$ and inductor's trace dimensions. The GA evolves the given population of individuals. 


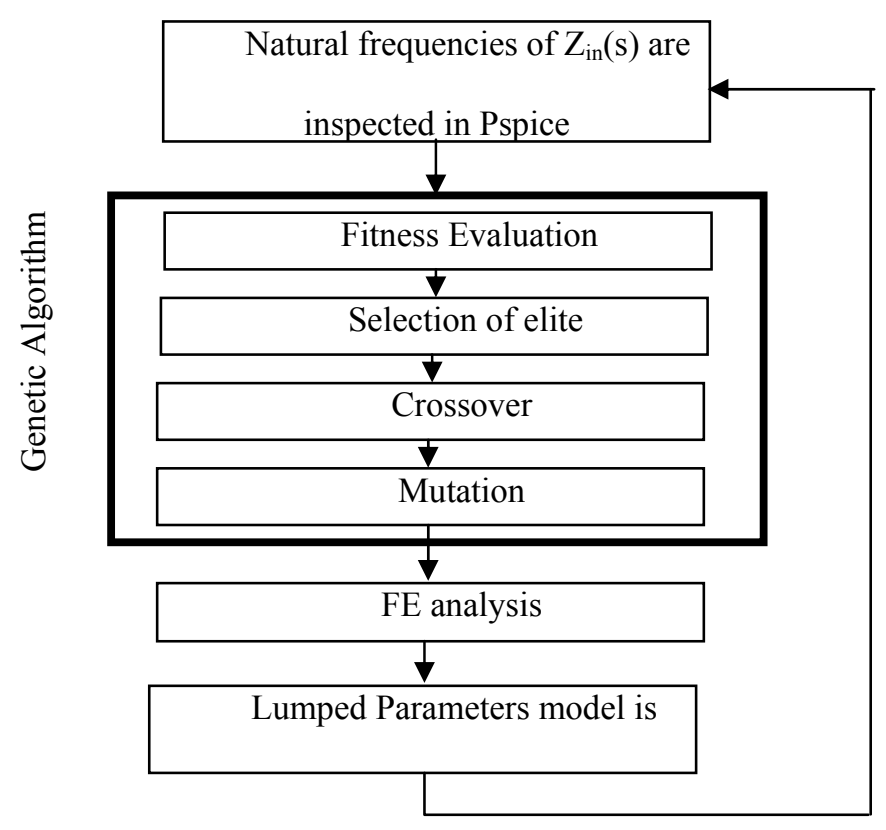

Fig. 3.7 The optimization process

The objective function is as follows: Objectiove function $=\alpha_{1} \cdot|\Delta f|^{2}+\alpha_{2} \cdot\left|Z_{\text {in }}(s)\right|^{2}$ where, $\alpha_{1,2}=$ Weights signifying the importance of the objective function (taken as 1 in our case), $\Delta f$ : frequency of the main resonance (resonant circuit first natural frequency) and $Z_{i n}(s)$ : input impedance of the resonant circuit. The main purpose of GA is to find the minimum for objective function.

The fitness function computes the error of the simulated and specified reference signal at each time point. The errors are then squared and added together to give a single scalar objective value.

The fitness values shown in fig. 3.8, illustrate that after about 10 number of iteration the error reaches to an acceptable value. Table 3.1 shows the results of the GA-algorithm and are compared to the conventional inductors. Note that the optimization procedure is mainly applied on the design of the planar inductor and the resonance capacitor is not considered in the design optimization. 


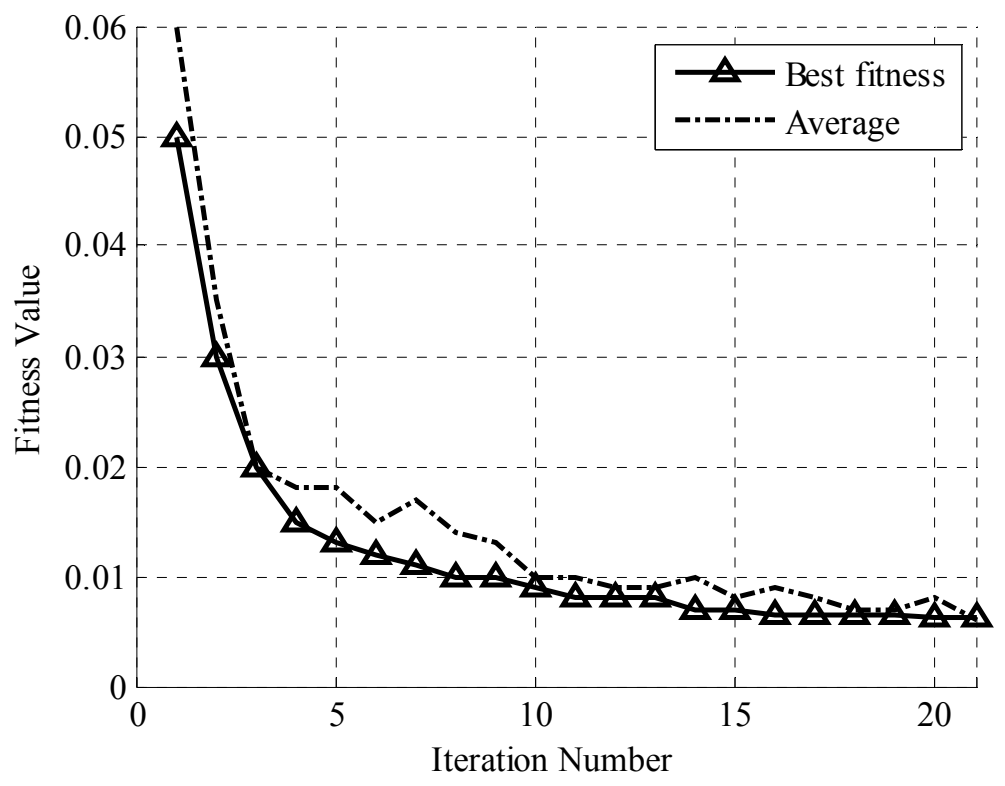

Fig. 3.8 Iteration accomplished by GA to minimize the objective function Table 3.1: Optimal values for designed inductor resulted from GA

\begin{tabular}{c|c|c|c}
\hline Planar inductor design & $\mathrm{L} \mu \mathrm{H})$ & Trace width (mil) & Number of turns \\
\hline Un-optimized & 1 & 40 & 8 \\
\hline Optimized & 0.76 & 29.75 & 6 \\
\hline
\end{tabular}

Fig. 3.5 (c) shows the frequency spectrum of the optimized resonant circuit. In fig. 3.5 (c), compared to fig. 3.5 (b), the unwanted higher frequency resonances are reduced. Also, the main resonance frequency is set to its desired value. (i.e. $250 \mathrm{KHz}$ )

\subsection{RESULTS AND DISCUSSION}

Fig. 3.9, shows the experimental setup used to test and verify the results. In this setup, to inspect and record the results, a $600 \mathrm{MHz}, 10 \mathrm{G}$ sample/s oscilloscope was used. The bandwidth for both the voltage and current probes were $100 \mathrm{MHz}$. The converter should operate in zero-current switching condition. Therefore, the switching frequency and duty cycle are set by the digital function generator. 


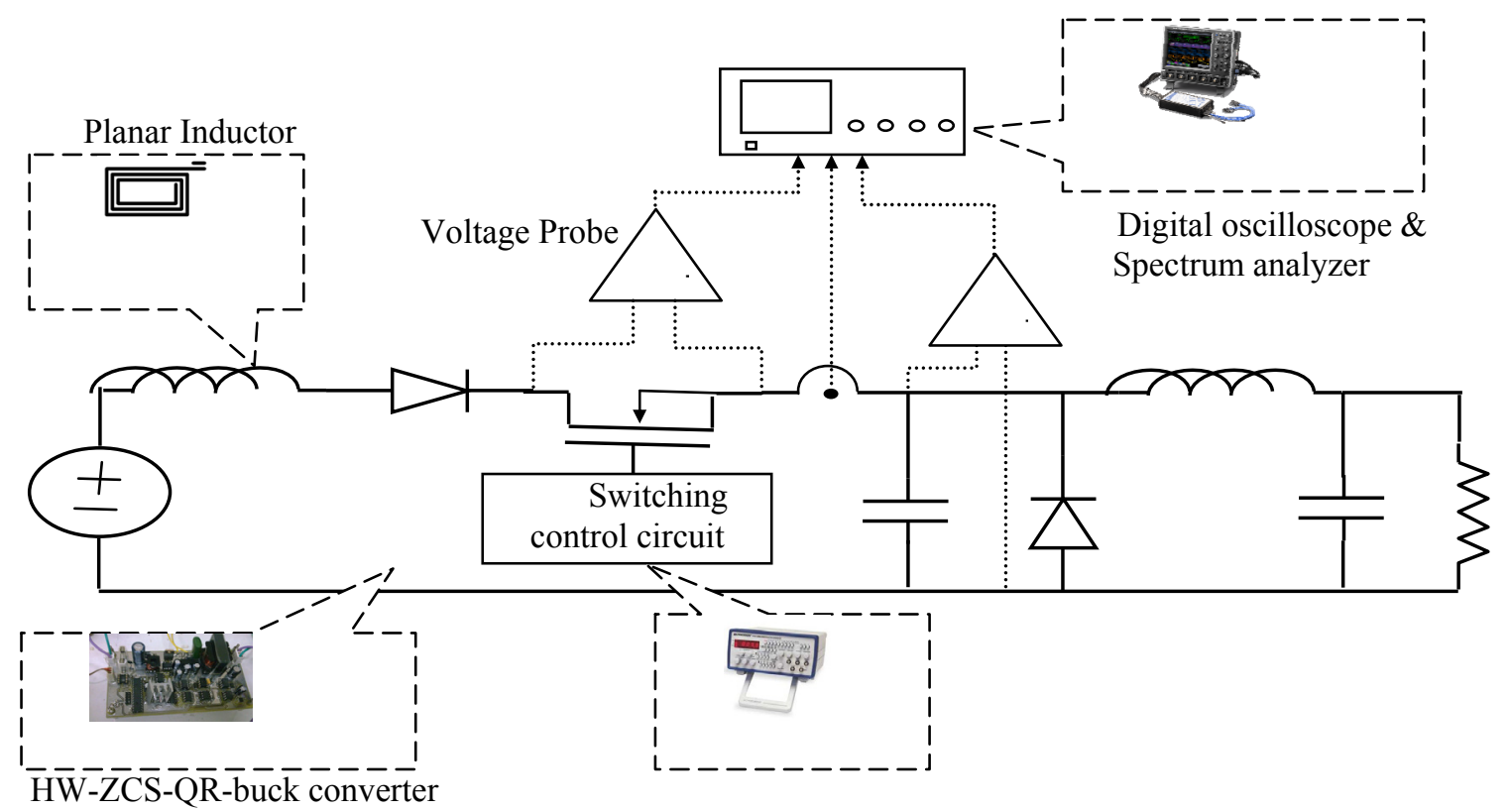

Fig. 3.9. Schematic of the converter's experimental setup

Fig. 3.10 shows the effects of high-frequency operating conditions on the zero current switching (ZCS) of the MOSFET in the converter circuit. In this configuration, it was expected to increase the output voltage of the converter by increasing the operating frequency of the MOSFET, while keeping the ZCS sequence as shown in the ideal case (without considering all parasitic elements) in fig. 3.10 (a). The simulation results show that the ZCS behavior of the converter is completely lost as a result of using the highfrequency circuit model for the resonance circuit as shown in fig. 3.10 (b). The parameters in these circuits were evaluated from the FE implementation described in the previous section. 


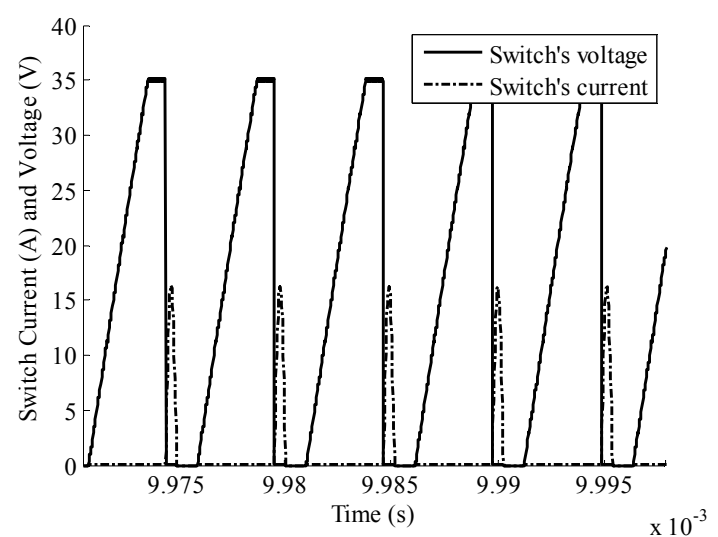

(a)

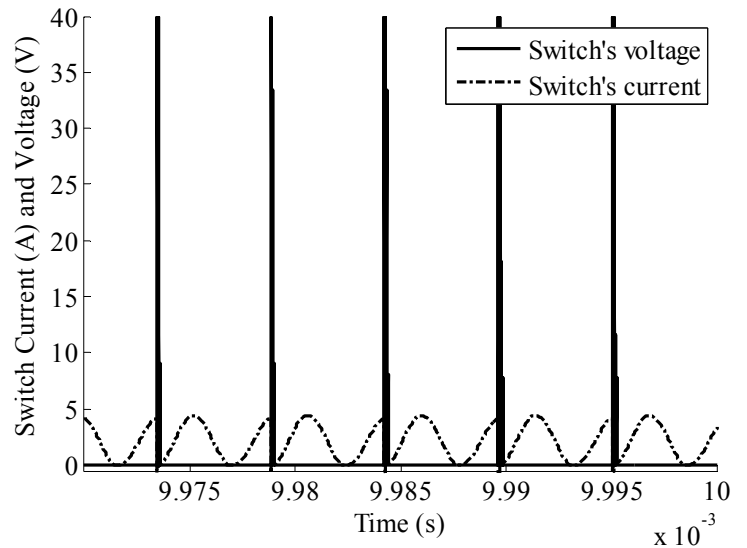

(b)

Fig. 3.10. Voltage and current of the switches in ZCS-Buck converter, (a) ideal case, (b) actual case

To keep the desired performance of the converter, the switching frequency of the converter should be kept below the value shown in fig. 3.10 (b). In an actual case (distributed-parameter frequency model), it can be observed from fig. 3.10 (b) that the next resonant frequencies happen at the frequencies starting from $4.5 \mathrm{MHz}$ and above. The fig 3.11 shows a comparison between two different designs of the resonant circuit. The fig. 3.11 (a) shows the FFT analysis of the primary design of the resonant circuit and fig. 3.11 (b) shows the FFT of the optimized resonant circuit output signal. It is noticed that, the number and amplitude of harmonics is reduced by design optimization.

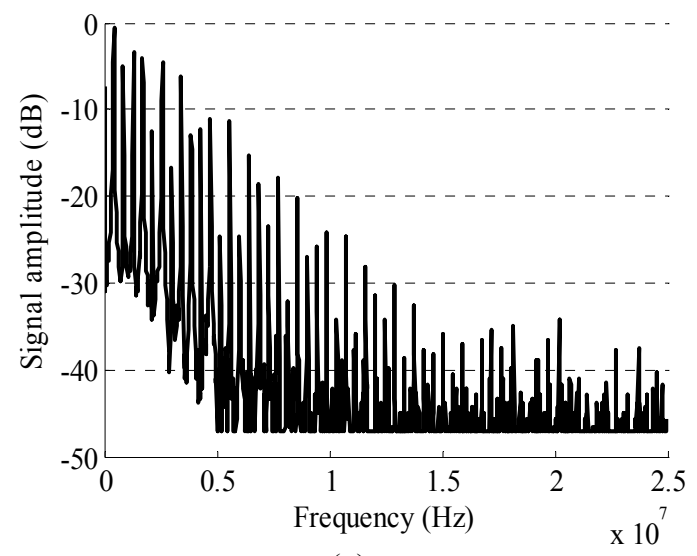

(a)

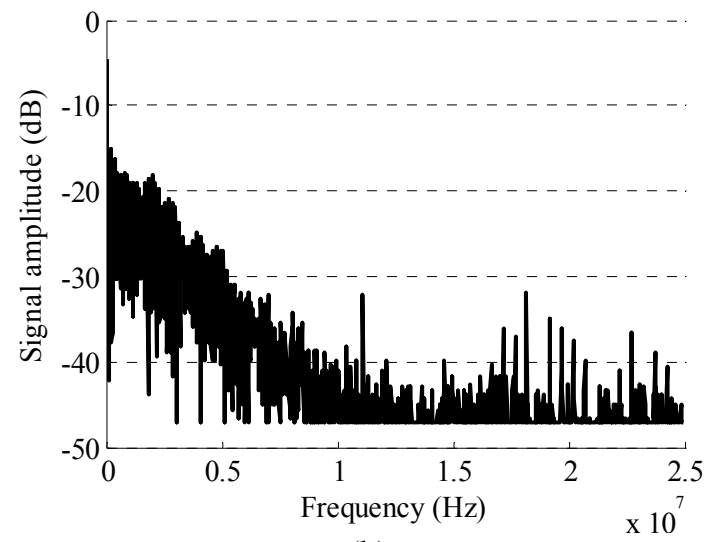

(b)

Fig. 3.11. FFT spectrum of the resonant circuit. a)Initial design, b) Modified design 
To verify the simulation results in fig. 3.10, an experimental test was carried out. The fig. 3.12 shows the measured voltage and current of the MOSFET, which is located in series with the resonance inductor (see fig. 3.1). As it is illustrated in fig. 3.12(a), in the primary design (Un-optimized) of the resonance circuit, by increasing the switching frequency above a certain value the unwanted resonances will appear. These resonances can harm the proper zero-current switching operation of the converter.

The fig. 3.12 (b) shows the switch's voltage and current, in the circuit with the optimized resonance circuit. As it is demonstrated in this figure, the converter is operating in zero-current switching condition, as it was expected.

In the fig. 3.12 (a), some distortions are observed in the switch's voltage waveform. These distortions are mainly because of the parasitic elements of the circuit and poor layout of the PCB, which are not considered in this study.

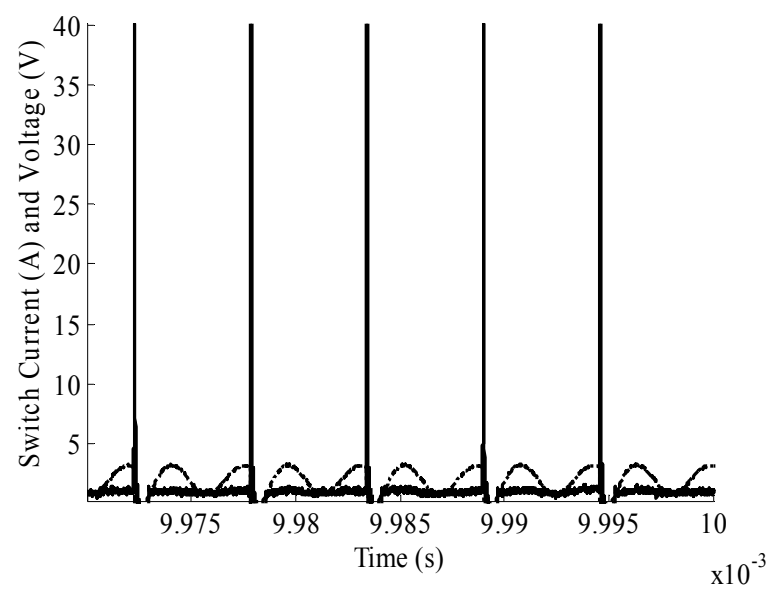


(a)

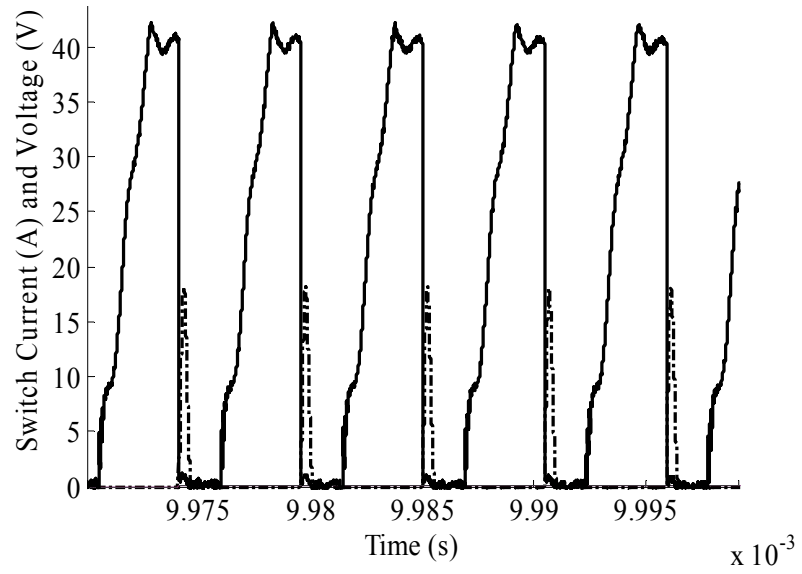

(b)

Fig. 3.12. Measured voltage and current of the switches in ZCS-Buck converter, (a) primary designed circuit, (b) optimized circuit

\subsection{Conclusion}

In this chapter, a method for the frequency response model of the converter's components is presented using coupled FE-transmission line method computations. FE analysis was performed to obtain the frequency behavioral model of the converter. The natural frequencies, computed from the proposed s-domain model, was used to analyze the circuit's electrical behavior and operation. The results show that the s-domain model of the converter has the ability to reveal the behavior of parasitic elements, as well as higher resonances, which has critical impact in studying EMI problems. This model can also be implemented for other types of converters making it practical for the evaluation on EMI/EMC issues in the design and development stages. The power converter discussed in this chapter is not a representative of all power converter designs but can show the value of considering the effects of parasitic components on operation of the 
power converters, especially when their switching frequencies are increased. By considering the effects of parasitic components, one can modify the converter's circuit design in a way to reduce the effects of parasitic components and set the operating condition, so that satisfies the converter's behavioral characteristics. 


\section{Equivalent Source Modeling and 3-D Full Finite Element Modeling for the Studying Radiated Electromagnetic Field}

\subsection{Overview}

In recent years, there has been an increased interest in the expansion of multi-level numerical simulation methods for investigation of EMI issues, in the early stages of the design of electrical apparatus at low frequency. Systems such as electric drives and power converter circuits are in this category of systems being studied. EMI studies of a complete motor drive system were performed by a number of researchers [108], [182][189]. In [182, 183], the $\mathrm{kHz}$ range frequency models of the various components of a complete motor drive were developed. The efforts have been made to measure the EMI emissions from $\mathrm{kHz}$ up to the $\mathrm{GHz}$ range in the case of adjustable speed drives (ASD). The EMI modeling and simulation for inverters were also performed. For EMI caused by the ground current in [182], the coupled FE-circuit high-frequency electric machine model for simulating electromagnetic interference in a motor drive was presented. The model can predict the EMI caused by the ground current and motor terminal overvoltage. The proposed model in [183] can be used as a computational prototyping tool for evaluating the high-frequency operating conditions of electric machines numerically. Likewise, studies in lower frequency are performed. For electromagnetic signature studies outside the machine, Le Coat et al evaluated electromagnetic signatures of induction machines [108]. Two types of these machines were studied experimentally and theoretically using $2 \mathrm{D}$ and $3 \mathrm{D}$ methods. The $3 \mathrm{D}$ model consists of several frames as conductors and specific frame with suitable permeability as stators. The results show that 
this model has good accuracy and is suitable for single machine case studies. More similar works, in this area, were conducted by others [185-189].

In order to study the electric and magnetic behavior of power components in multi component study, an accurate physics-based model of each component is considered in this paper. For instance, all windings, types of connections, in addition to the geometrical features slot shapes, rotor and stator structures, as well as material properties, are considered in developing the actual machine model.

In addition to electric machines, other components, such as power converters, cables and transformers, have very tiny elements. Considering all of these in the FEA model causes an increase in the computational complexity. In addition to keeping in mind the essential elements of each parameter for the EMI study, there is a need to observe the fields at far distances. Therefore, a very large region must be considered in such a model. Consequently, the number of degrees of freedom in meshing increases dramatically, and subsequently the speed of analysis will decrease. Accordingly, logical simplification in designing components should be implemented.

In this chapter, it is proposed to develop the complete geometrical model of the machine to a simple rectangular prism, but it should produce similar electromagnetic behaviors for studying the EMI issues. The basic concept for EMI signature evaluation for this case is explained in the following section. Then, the study approach is investigated for firstly primitive models and then the embedded model for the purpose of simplifying the model of the electrical machine. The optimization process and other theoretical aspects are explained in this part. The magnetic and electric field are chosen as the objective function, which is the most important parameter in the optimization. The 
various aspects of the model, including time analysis, are also studied. Since it is proposed to use this model as a typical model for an induction machine, other operating conditions of this machine are investigated. For verification purposes, in all parts of this study, the results of the model are compared with the result of the actual model.

\subsection{Low-frequency stray field computation}

The interest is primarily in the radiation pattern of the electric component (in this case electrical machines) at a distance from the source. Hence, the electromagnetic fields are usually measured at a considerably far distance from the components. The electric and magnetic fields specifically at an infinite distance can be the most effective index in investigating the EMI [175]. Since the wave length in power frequency, $60 \mathrm{~Hz}$, is so large, the far distance means thousands of kilometers or more. Therefore, the region and proposed measurement points, which are intended to be far, should be considered at a very far distance. However, computationally utilizing such a large region could be impossible. Hence, we need to obtain the fields virtually. The process of obtaining the fields, in this case, is investigated.

Time-varying electric and magnetic fields generate mmfs and emfs that can sustain the fields that compromise the flux. Also, obviously a consequence of the interaction of electric and magnetic fields is that they tend to propagate as waves. Therefore, it can be concluded that a time-varying current will radiate electromagnetic waves. On a more fundamental level, since the current is moving charges, the time-varying current is accelerated charge and it can be declared that a charge that undergoes accelerated motion 
will radiate an electromagnetic wave. Based on the fact that currents and fields in the DC machine are time-varying, the same rule can be applied for the direct current machine.

Fig. 4.1 shows the geometry for calculating the radiated field from a typical power component at a far distance.

To determine the electromagnetic field, the machine is considered to be situated at the origin and oriented along the z-axis. We shall use an approach based on the magnetic vector potential and obtain electric and magnetic fields consistent with Maxwell's equations, while fulfilling certain other pertinent requirements. The magnetic potential for the static case at a point $\mathrm{P}(r, \theta, \phi)$ will be as follows: $A=\frac{\mu I d l}{4 \pi r} \mathbf{a}_{z}$

where I is the current of the machine and $\mu$ is the permeability. If the current of the element is assumed to be time-varying in the manner $I=I_{0} \cos \omega t$, we expect the corresponding magnetic vector potential to be that in (4-2), with $I$ replaced by $I=I_{0} \cos \omega t$. Proceeding in this manner would however lead to fields inconsistent with Maxwell's equations. The reason is that the time-varying electric and magnetic fields give rise to wave propagation [188].

$A=\frac{\mu I_{0} d l}{4 \pi r} \cos (\omega t-\beta r) \mathbf{a}_{z}$

where $\beta$ is the phase constant $\left(\omega / v_{p}\right)$ and $v_{p}$ is the velocity of propagation of the wave. 


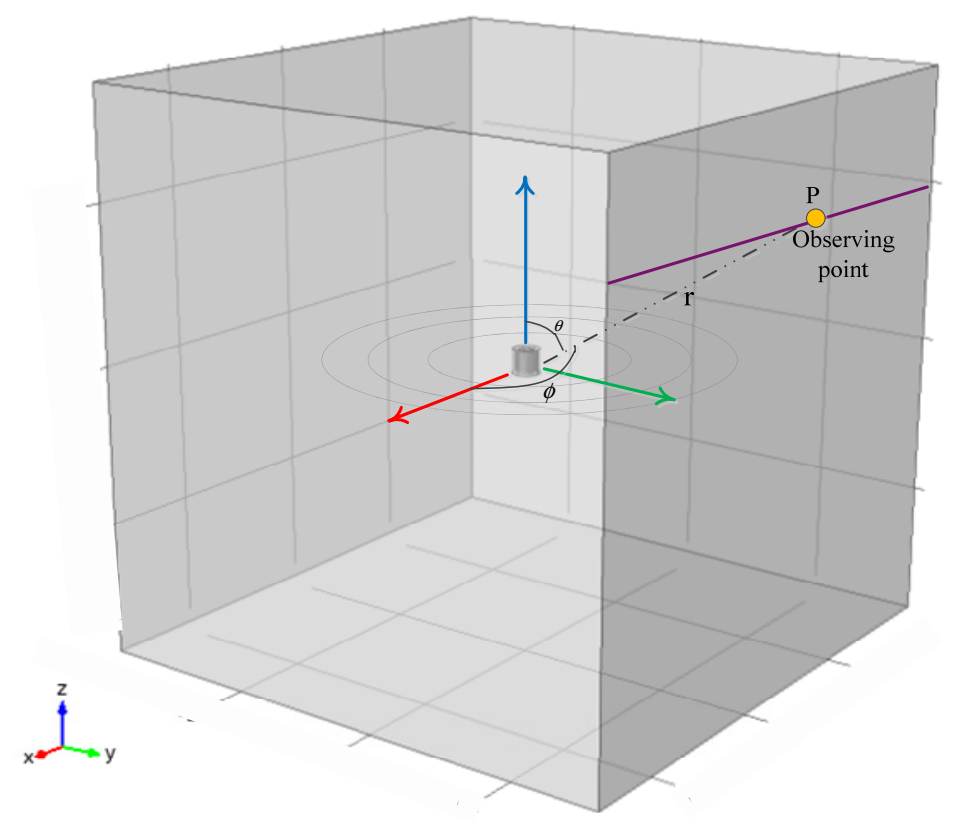

Fig. 4.1 The model's environment for calculating field in far distance

The Magnetic potential in terms of its components in spherical coordinates is expressed in (4-3). Also, the magnetic field according to Maxwell's equations is obtained $(4-4)$.

$$
\begin{aligned}
& A=\frac{\mu I_{0} d l \cos (\omega t-\beta r)}{4 \pi r}\left(\cos \theta \mathbf{a}_{r}-\sin \theta \mathbf{a}_{\theta}\right) \\
& H=\frac{I_{0} d l \sin \theta}{4 \pi r}\left[\frac{\cos (\omega t-\beta r)}{r^{2}}-\frac{\beta \sin (\omega t-\beta r)}{r}\right] \mathbf{a}_{\theta}
\end{aligned}
$$

Using Maxwell's curl equation for $\mathrm{H}$ we will have the electric field.

$$
\begin{aligned}
& E=\frac{2 I_{0} d l \cos \theta}{4 \pi \varepsilon \omega}\left[\frac{\sin (\omega t-\beta r)}{r^{3}}+\frac{\beta \cos (\omega t-\beta r)}{r^{2}}\right] \mathbf{a}_{r} \\
& +\frac{I_{0} d l \sin \theta}{4 \pi \varepsilon \omega}\left[\frac{\sin (\omega t-\beta r)}{r^{3}}+\frac{\beta \cos (\omega t-\beta r)}{r^{2}}-\frac{\beta^{2} \sin (\omega t-\beta r)}{r}\right] \mathbf{a}_{\theta}
\end{aligned}
$$

The expression for the complete electromagnetic field, equations (4-4) and (4-5) appears to be complicated. Fortunately, it is seldom necessary to work with the complete 
field expression. This is because one is often interested in the field far from the electric machine that is governed predominantly by the terms involving $1 / r$. Thus, from (4-4) and (4-5), we find that for the machine of length $d l$ oriented along the z-axis and carrying current $I=I_{0} \cos \omega t$, the electric and magnetic fields at values of $\mathrm{r}$ far from the dipole are given by:

$$
E=\frac{\beta^{2} I_{0} d l \sin \theta}{4 \pi \varepsilon \omega r} \sin (\omega t-\beta r) \mathbf{a}_{\theta}=-\frac{\eta \beta I_{0} d l \sin \theta}{4 \pi r} \sin (\omega t-\beta r) \mathbf{a}_{\theta}
$$

$H=-\frac{\beta I_{0} d l \sin \theta}{4 \pi r} \sin (\omega t-\beta r) \mathbf{a}_{\theta}$

These fields in equations (4-6) and (4-7) are known as the radiation fields, since they are the components of total fields that contribute to the time-varying radiated power away from the component.

The present concept is based up on the Stratton-Chu [184] magnetic field intensity solution to the time-harmonic form of Maxwell's equations. The mathematical form used here, given by Silver [185],[186], yields the magnetic flux density as a volume integral of the electric and magnetic current densities, $\mathrm{J}$ and $\mathrm{M}$, respectively:

$\bar{B}(P)=\frac{-j}{4 \pi \omega} \int_{V}\left[(\mathbf{M} \cdot \bar{\nabla}) \bar{\nabla}+k^{2} \mathbf{M}+j \omega \mu \mathbf{J} \times \bar{\nabla}\right] \cdot \frac{e^{-j k R}}{R} d V$

In the above equation, $\mathrm{P}$ denotes the observation point, $V$ is the volume occupied by $\mathbf{J}$ and $\mathbf{M}$. $\mathbf{R}$ is the vector from the current density elements, $\mathbf{J}$ and $\mathbf{M}$, to the observation point P. $\omega$ is equal to $2 \pi f$, where $f$ is the frequency of the currents, $k$ is the wave number given by $\mathrm{k}=\omega(\mu \varepsilon)^{\frac{1}{2}}$, and $\varepsilon$ and $\mu$ are the permittivity and 
permeability of the medium, respectively. Also, the time-harmonic variation $\mathrm{e}^{\mathrm{j} \omega \mathrm{t}} \mathrm{has}$ been suppressed.

It is assumed that the volumetric current density elements, $\mathrm{JdV}$, can be represented as linear current elements I dl, located at infinity and aligned with the current flow directions. Recalling that $\mathrm{M}=0$, and completing the indicated vector operations, (4-9) becomes:

$$
\bar{B}(P)=\frac{\mu}{4 \pi} \int_{l_{1}}^{l_{2}}\left[\left(j k+\frac{1}{R}\right) \frac{e^{-j k R}}{R}\right] \cdot I(l)\left(\overline{d l} \times \overline{u_{R}}\right)
$$

Where $u_{R}$ is the unit vector pointing in the direction of $R$. The bracketed term in (4-9) describes the time-harmonic retardation effects for the current element I dl, and suggests a criterion for using a quasi-static electromagnetic formulation. (The geometry is illustrated in fig. 4.1) when examining (4-9), it is seen that whenever

$$
\mathrm{R}<<\frac{\mathrm{c}}{2 \pi \mathrm{f}}
$$

(where $\mathrm{c}$ is the homogenous medium light speed), the bracketed term may be approximated by $1 / \mathrm{R} 2$ and (4-2) reduces to the electromagnetic quasi-static result

$$
\left.\bar{B}(P) \cong \frac{\mu}{4 \pi} \sum_{i=1}^{2} \int_{l_{1}}^{l_{2}}\left[\frac{1}{\boldsymbol{R}_{i}^{3}} I_{i}(l)\right] \cdot\left(\overline{d l}_{i} \times \bar{R}_{i}\right)\right]
$$

In (4-11), the summation is over the two points at the far distance in the region with $\mathrm{I}$ is the currents in the motor wires, $\mathrm{dl}$ is the current elements along the motor wires, and $\mathrm{R}$ is the vector from the current elements $\mathrm{dl}$, to the observation point $\mathrm{P}$ ( $\mathrm{i}=$ 1 and 2 for the corresponding points). Additionally, to facilitate computer programming, the vector $R$, is used in the cross product of (4-11), in contrast to the 
unit vector $\mathrm{u}_{\mathrm{R}}$ which was used in (4-12), resulting in $\mathrm{R}_{\mathrm{i}}{ }^{3}$ in the integrand denominator of (4-13).

Returning to (4-11), the distances $R_{i}$ between the differential line elements $d l_{i}$ and the observation point $P$ are:

$$
\begin{aligned}
& R_{1}=\left[\rho^{2}+a^{2}-2 \rho a \cos (\varphi-2 \pi z)+(Z-z)^{2}\right]^{1 / 2} \\
& R_{2}=\left[\rho^{2}+a^{2}+2 \rho a \cos (\varphi-2 \pi z)+(Z-z)^{2}\right]^{1 / 2}
\end{aligned}
$$

where $\rho, \varphi, z$ are the cylindrical coordinates of the observation point P. Assuming that the currents $I_{i}$, are oppositely directed, identical, and uniform over the length of the winding in motor, substitution of the differential current elements dli and the distances $R_{i}$ into (11) yields the Cartesian components of the magnetic flux density:

$$
\begin{aligned}
& B_{x}=\frac{\mu I}{4 \pi} \int_{z_{1}}^{z_{2}}\left\{\boldsymbol{R}_{1}^{-3}[2 \pi a(Z-z) \cos (2 \pi z)+a \sin (2 \pi z)-\rho \sin (\varphi)]+\right. \\
& \left.R_{2}^{-3}[2 \pi a(Z-z) \cos (2 \pi z)+a \sin (2 \pi z)+\rho \sin (\varphi)]\right\} d z \\
& B_{y}=\frac{\mu I}{4 \pi} \int_{z_{1}}^{z_{2}}\left\{R_{1}^{-3}[2 \pi a(Z-z) \sin (2 \pi z)-a \cos (2 \pi z)+\rho \cos (\varphi)]+\right. \\
& \left.R_{2}^{-3}[2 \pi a(Z-z) \sin (2 \pi z)-a \cos (2 \pi z)-\rho \cos (\varphi)]\right\} d z \\
& B_{z}=\frac{\mu I}{4 \pi} \int_{z_{1}}^{z_{2}} 2 \pi a\left\{R_{1}^{-3}[a-\rho \cos (\varphi-2 \pi z)]\right. \\
& \left.-R_{2}^{-3}[a+\rho \cos (\varphi-2 \pi z)]\right\} d z
\end{aligned}
$$

In the above equations, $I$ is the total current in each wire of the motor in phase A, $Z_{1}$ and $Z_{2}$ are axial coordinates of the electrical machine end points, and $R_{1}$ and $R_{2}$ are given by (4-12) and (4-13). According to standards, when $\rho=0,(4-14)-(4-16)$ reduce 
to well-known expression for the on-axis magnetic flux densities of motors in the region [187], [188].

In order to deduce the electric and magnetic fields, with the spherical coordinate components of B known, the next step is straightforward. The free space relationship (417) is applied to (4-18):

$H=\mu_{0}^{-1} B$

$E=\frac{1}{j \omega \varepsilon_{0}} \nabla \times H$

The analysis method, which is used in this analysis, is the generalized minimal residual method (usually abbreviated GMRES) with successive over-relaxation (SOR) pre and post smoothers. The GMRES is an iterative method for the numerical solution of the system of linear equations [179]. In numerical linear algebra, the SOR is a variant of the Gauss-Seidel method for solving a linear system of equations, resulting in faster convergence. The convergence is shown in fig. 4.2. A similar method can be used for any slowly converging iterative process. The SOR method uses a more accurate approximation of the matrix, which leads to fewer iterations but slightly more work is required per iteration than in the Jacobi method. It should be noted that the problem consists of nonlinear equations due to the presence of the B-H curve of the core of the motor. The iterative solver solves the problem by dividing the nonlinear curves to the sets of linear one, as $\mu_{\mathrm{r}}$ ramp and solving each of them individually, [179]. 


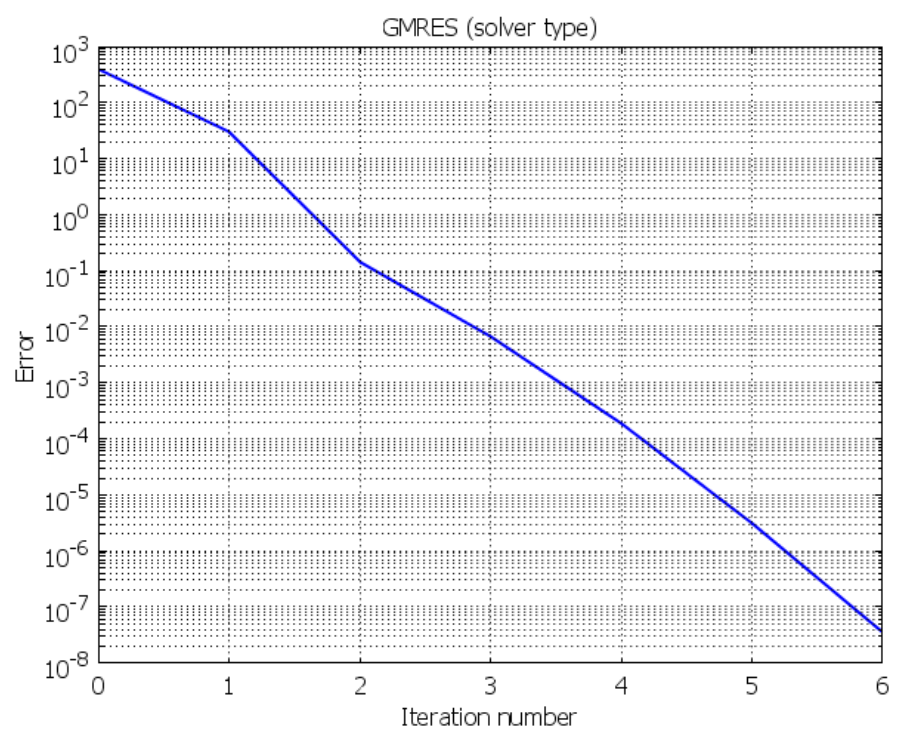

Fig. 4.2 convergence of the problem using GMRES solver

\subsection{Initial models}

The electromagnetic signature study of the electrical machine in fig. 4.1 can be estimated based on equations (4-14)-(4-18). Since the electric and magnetic fields are interconnected to each other through (4-17) and (4-18), studying each of them shows the behavior of the electric machine at a far distance. However, as mentioned earlier, estimating the parameters of electrical machines at far distance requires significant time, especially for multi-component studies using physics-based simulations. Therefore, a logical simplification is used here throughout the optimization. The details of the modified quasi-static method used for solving the 3D modeling were explained fully in chapter 3 .

For simulation purposes, a 3-phase, 380-V, 5-A, 120-turn/phase induction machine with stack length of $15(\mathrm{~cm})$ and outer diameter of $17.5(\mathrm{~cm})$ is simulated in the 3D electromagnetic FE domain for one instant of time at the frequency of $60 \mathrm{~Hz}$. 
The mesh of this full 3D model is applied from volumes to nodes to have a great accuracy (see fig. 4.3). The mesh of critical situations, such as gaps between stator and rotor, the teeth, curves of windings, is selected finely. On the other hand, the mesh of the air in the environment is considered coarsely. Nevertheless, the number of degrees of freedom is about 2.26 million, which took about 6 hours to solve the model for a single instant of time at the frequency of $60 \mathrm{~Hz}$. The radiated electric and magnetic fields of this model are shown in fig. 4.4. The equivalent source modeling tries to keep the accuracy in comparison with this model and decrease the simulation time to be able to have the low frequency radiated field analysis in a sequence of time or as frequency response analysis.
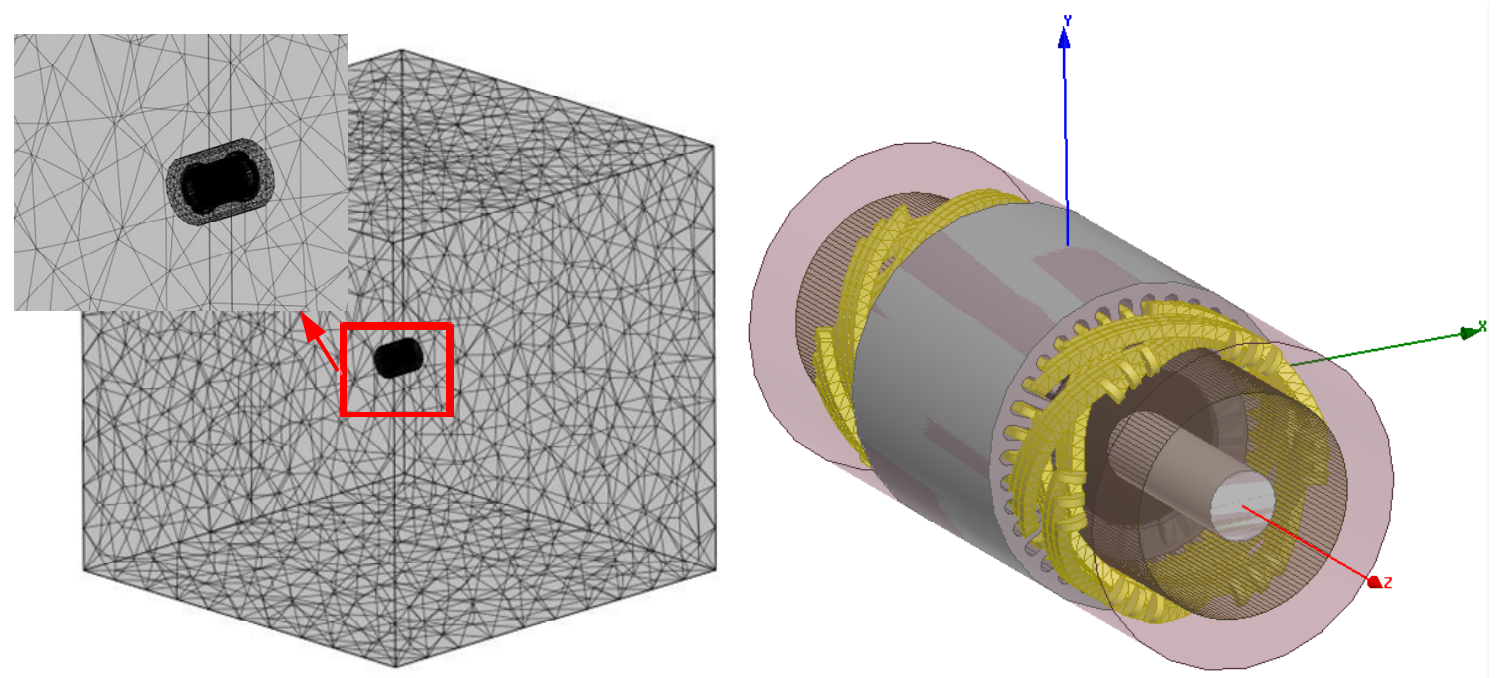

Fig. 4.3 3-D FE model of the induction motor

The spatial view of the stray magnetic and electric fields from the motor is shown in fig. 4.4. It is proposed to design a model to have the similar stray field. 


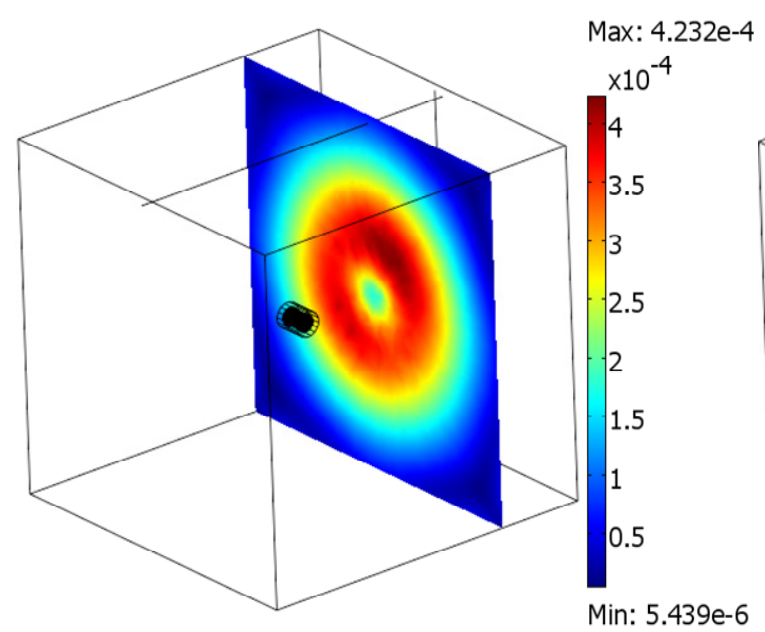

(a)

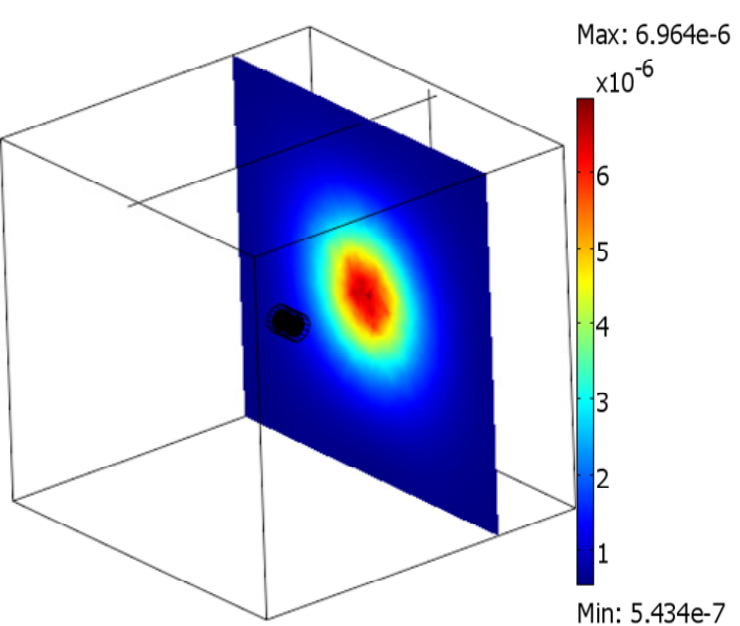

(b)

Fig. 4.4 the stray electric and magnetic spatial fields in meter in $\mathrm{x}$ axis (a) the electric field $(\mathrm{V} / \mathrm{m}),(\mathrm{b})$ the magnetic field density $(\mathrm{T})$

\subsubsection{Single current loop model}

In order to find a comprehensive and accurate model for the electric machine, a simple current loop instead of an accurate model of the electric machine is first analyzed in this part. The reason for using the single current loop is that the stray field at far distance will be dipoles. Therefore, a current loop may be able to resemble this dipole.

The current loop is located in the stator, which is shown in fig. 4.5. In order to analyze the accuracy of this model, all components of electric and magnetic fields are investigated at far distances and compared with the stray fields of the single phase of the actual machine. As it is shown in fig. 4.5 to fig. 4.9, the magnetic field density of $x$ and $y$ components $\left(\mathrm{B}_{\mathrm{x}}\right.$ and $\left.\mathrm{B}_{\mathrm{y}}\right)$ of these two models are almost the same but the magnetic field density of the $\mathrm{z}$ component is different. Consequently, this model has a problem in one component. 


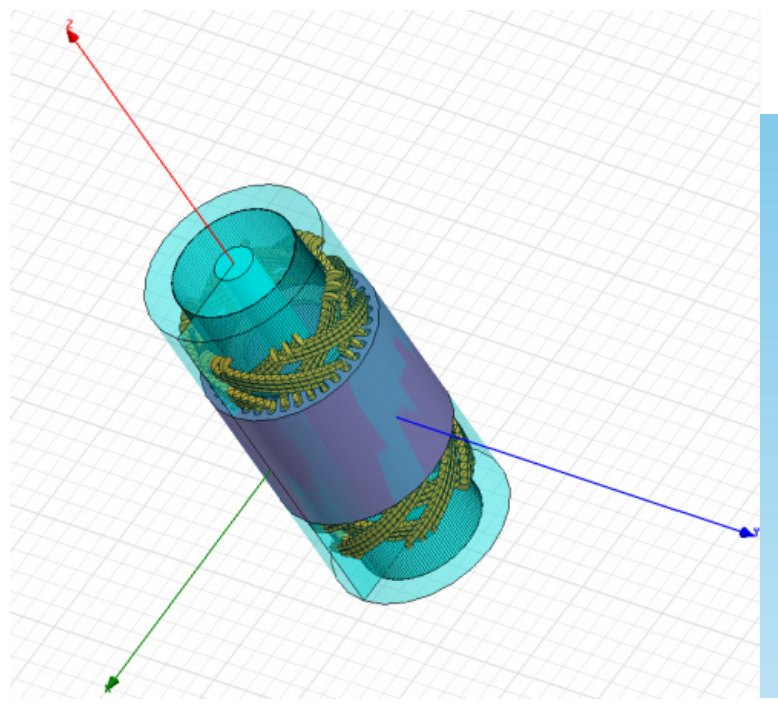

(a)

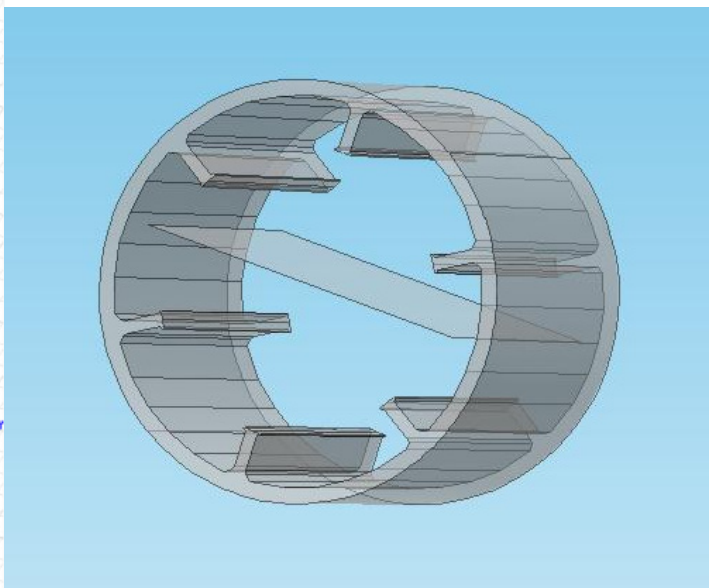

(b)

Fig. 4.5 Prototype of the proposed machine (SCIM) in Finite Element Analysis (a) actual model, (b) Initial equivalent current loop model for EMI and electromagnetic signature studies

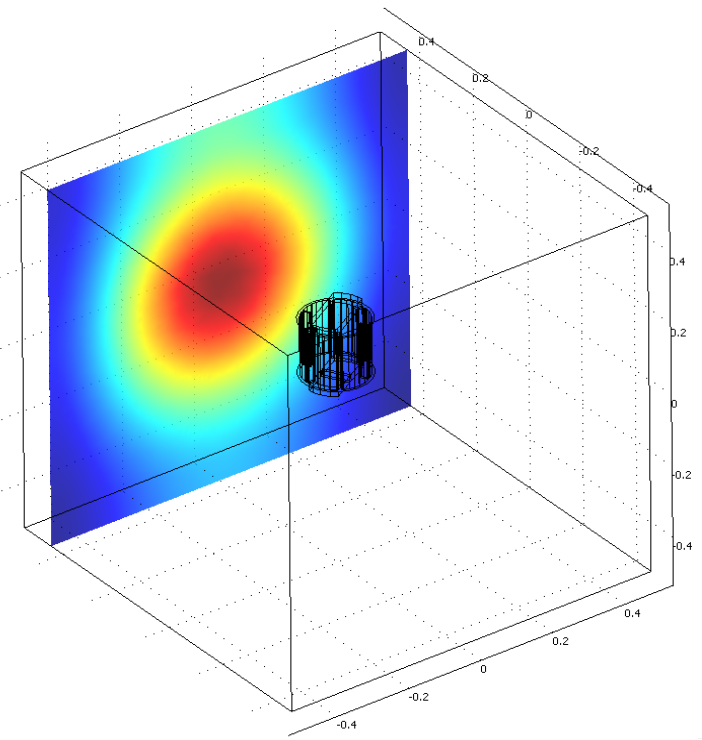

(a)

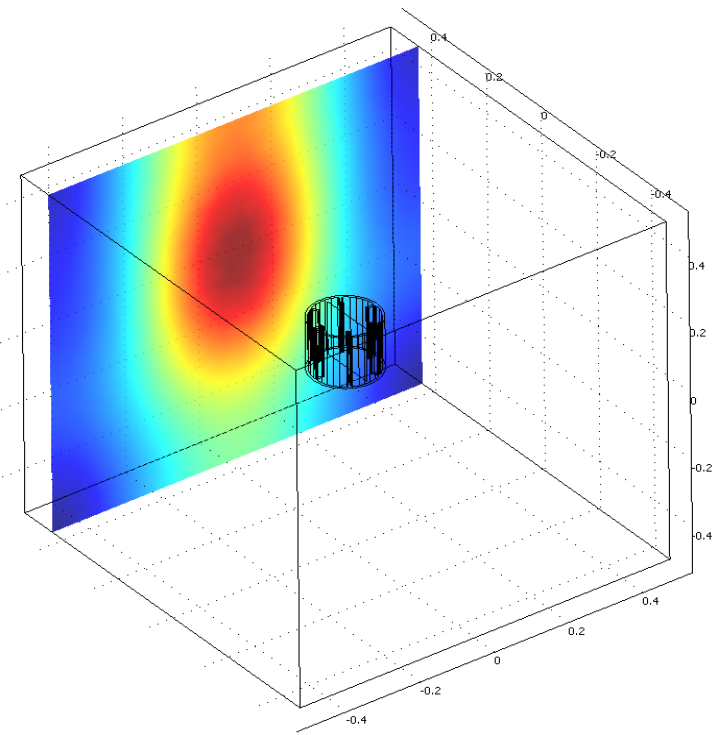

(b)

Fig. 4.6 Magnetic Flux Density (normal) spatial in (a) single phase of actual machine and (b) single loop model 


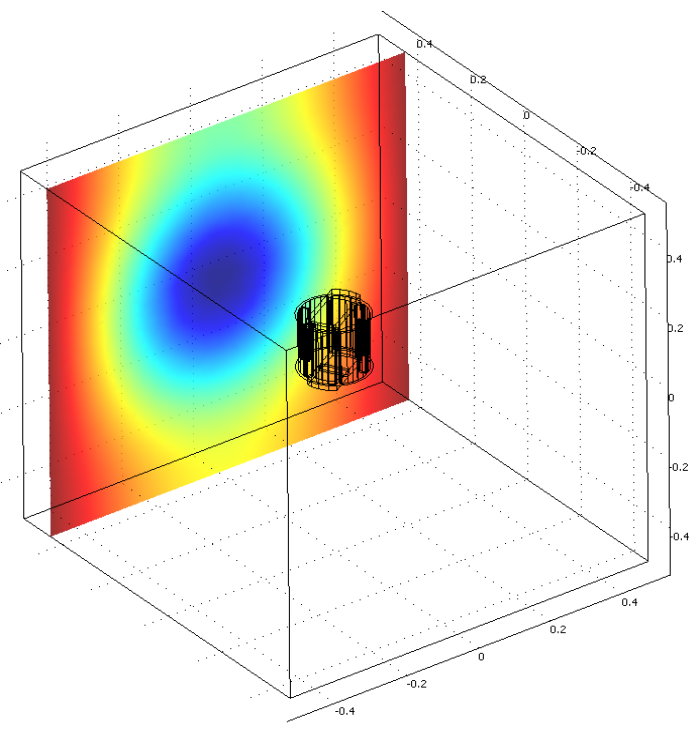

(a)

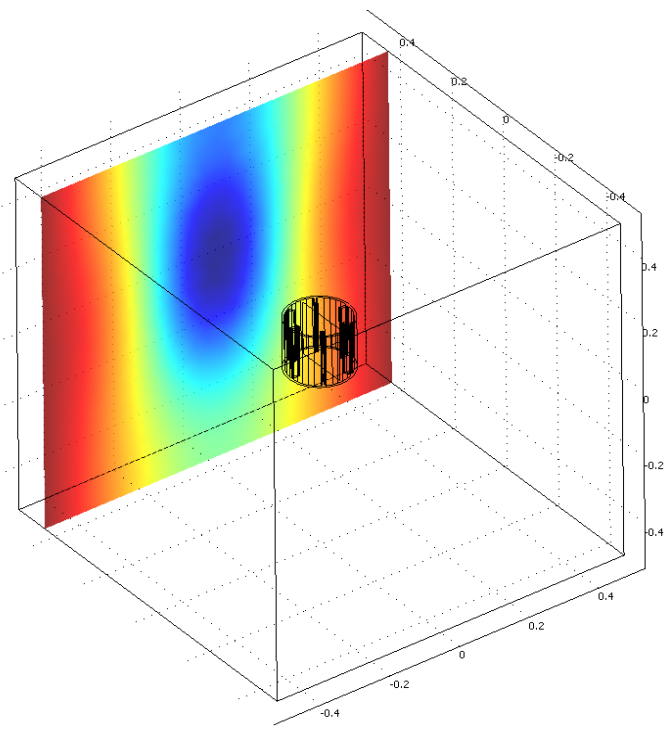

(b)

Fig. 4.7 Magnetic Flux Density spatial, X component in (a) single phase of actual machine and (b) single loop model

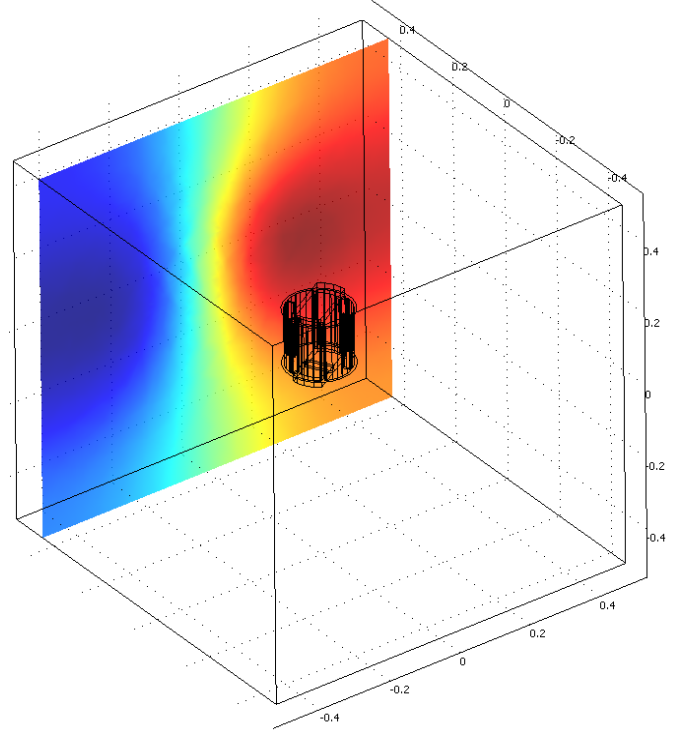

(a)

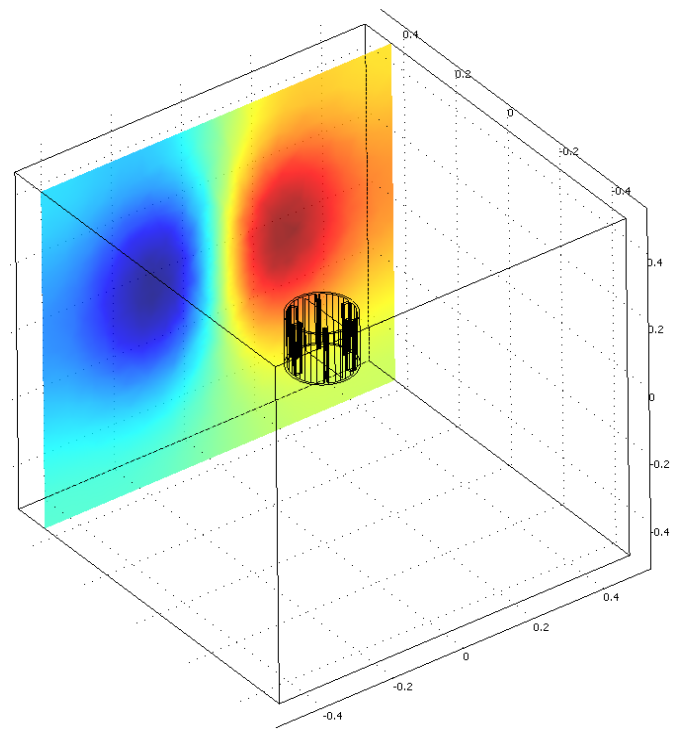

(b)

Fig. 4.8 Magnetic Flux Density spectrum in Y component in (a) single phase of actual machine and (b) single loop model 


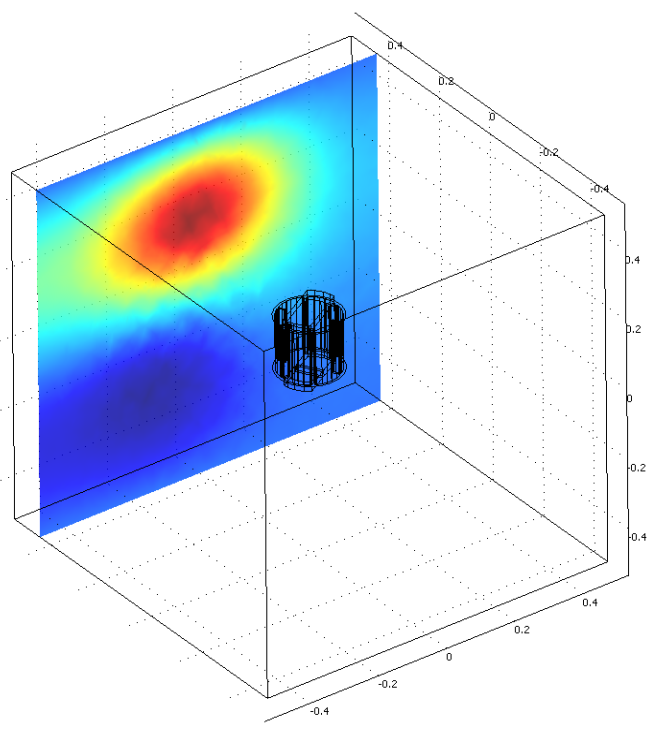

(a)

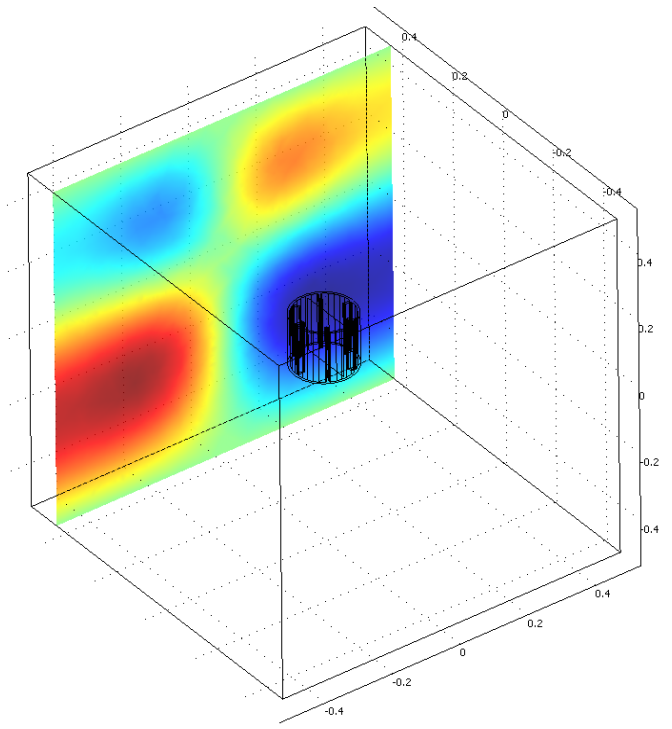

(b)

Fig. 4.9 Magnetic Flux Density spectrum in $\mathrm{Z}$ component in (a) single phase of actual machine and (b) single loop model

\subsubsection{Double current loop model}

As it is obtained from the last part, the single current loop model cannot be used instead of the model of even a single phase of the actual machine for electromagnetic studies at far distances. Therefore, the double current loop model with variable angles in between loops is used instead. The FE model of the double current loop is shown in fig. 4.10.

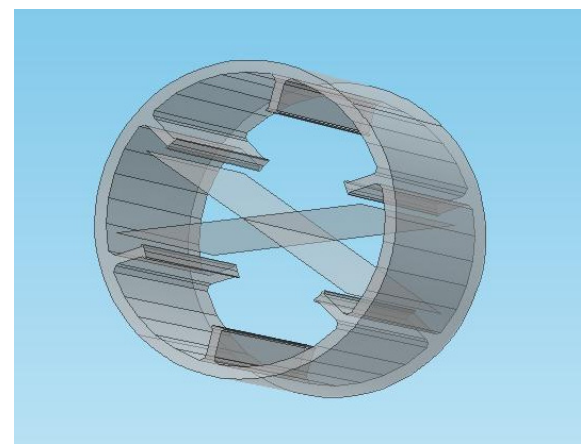

Fig. 4.10 The FE model of the double current loop model 
The current of the lines of the loops can be adjusted to have similar amplitude of the actual machine, but the main issue is the spatial fields, which should be the same in all three $\mathrm{X}, \mathrm{Y}$ and $\mathrm{Z}$ components. Therefore, the spatial stray fields from the double current loop are captured in one meter and shown in the following, from fig. 4.11 to fig. 4.14.

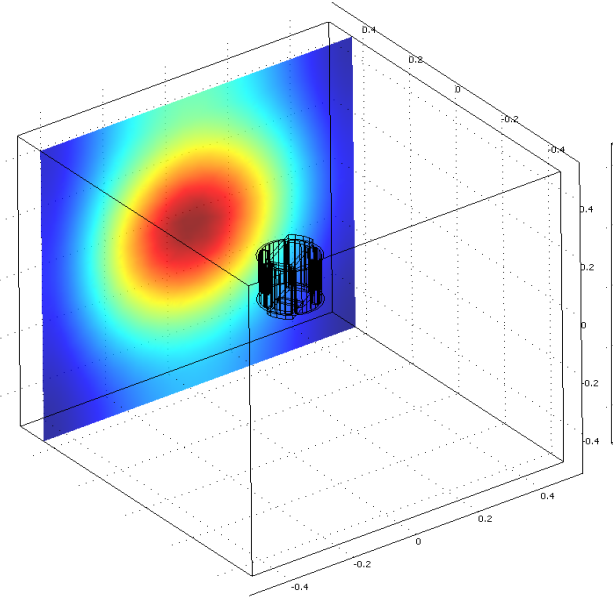

(a)

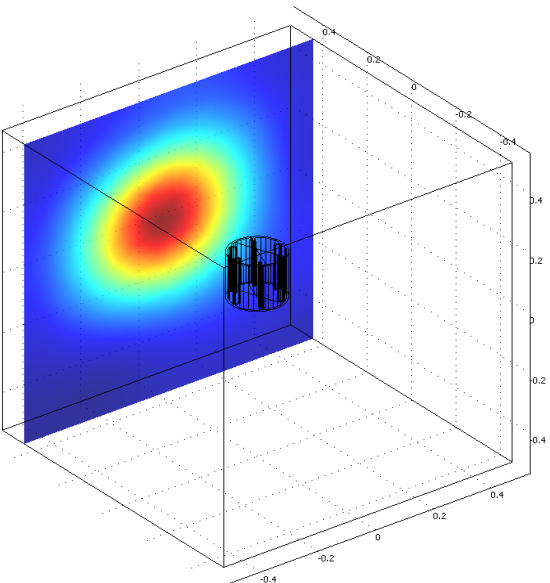

(b)

Fig. 4.11 Magnetic Flux Density (norm) spatial fields in (a) actual machine and (b) double current loop model

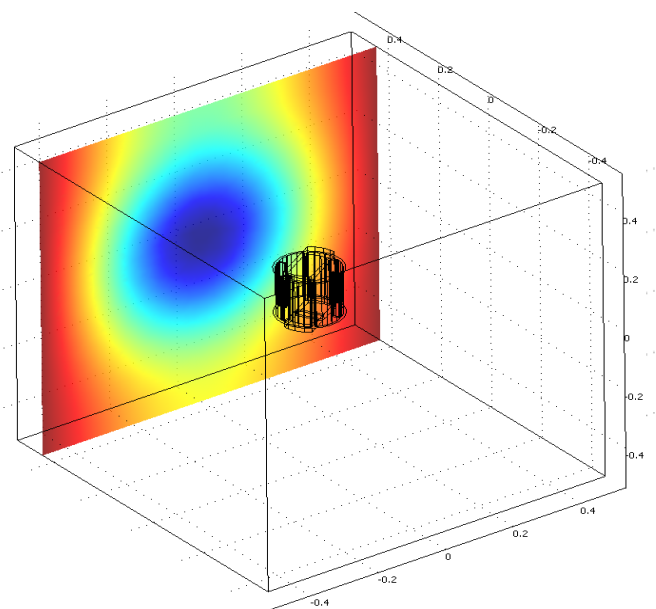

(a)

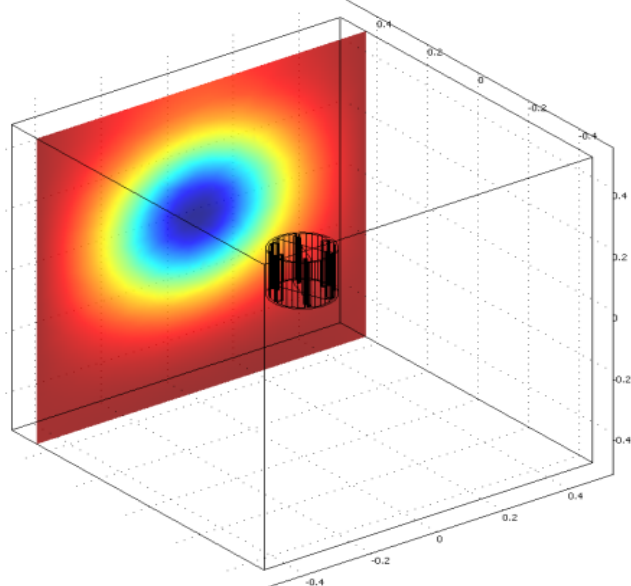

(b)

Fig. 4.12 Magnetic Flux Density spatial fields in X component in (a) actual machine and (b) double current loop model 


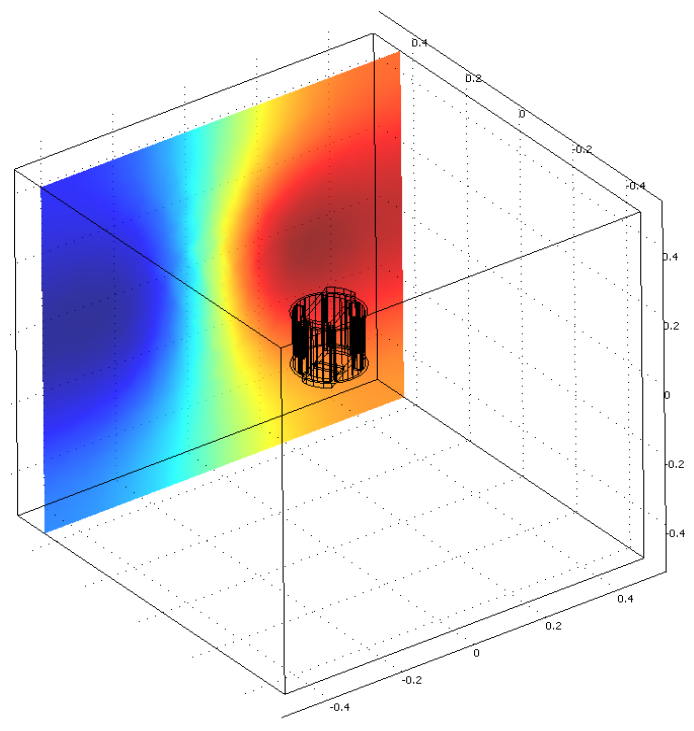

(a)

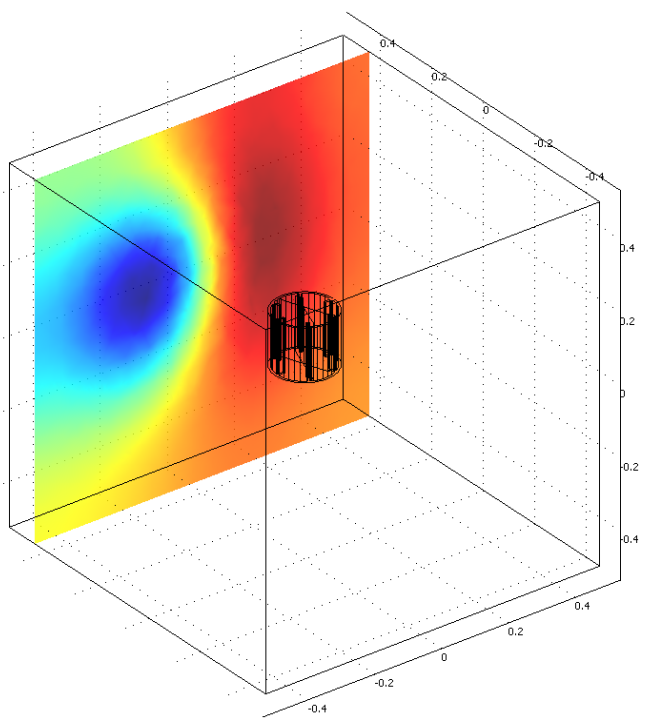

(b)

Fig. 4.13 Magnetic Flux Density spatial fields in Y component in (a) actual machine and (b) double current loop model

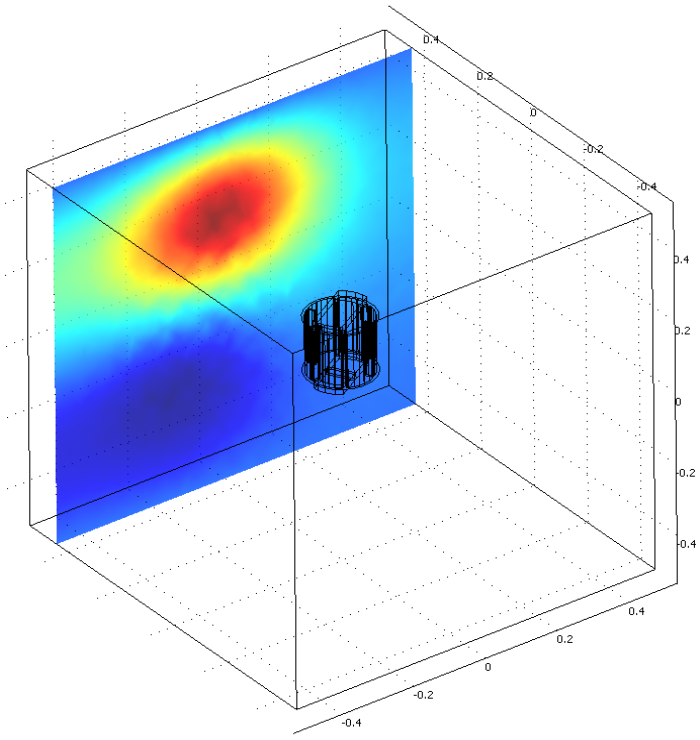

(a)

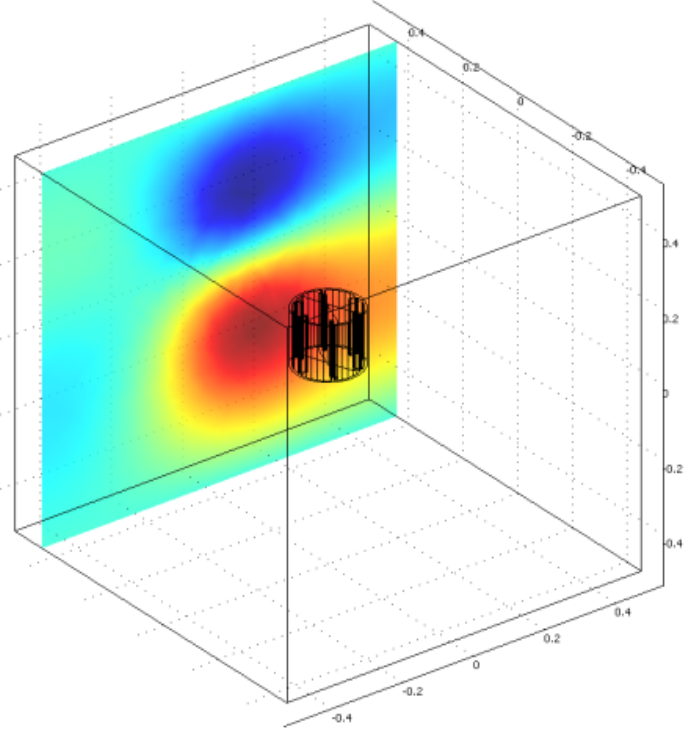

(b)

Fig. 4.14 Magnetic Flux Density spatial fields in Z component in (a) actual machine and (b) double current loop model 
All components of the magnetic field are demonstrated in fig. 4.11 to fig. 4.14. As shown in the figures, the numbers of poles are the same in both cases, but there are still some difference between the actual and the double current loop model case in the position of the maximum magnetic field density. For example, the concentration of the stray fields from the equivalent source model in fig. 4.11 and fig. 4.12 are not similar to the actual model's one. Hence, equivalent source modeling endeavors to increase the parameters of the model to have more control of all parameters, including concentration, amplitude and the number of poles. Therefore, the cube model is implemented.

\subsection{Current cube model}

After investigating single and double current loop models, investigating the cube model is proposed in this part. Since there is more variables in the cube model, there is more possibility to reach better results. The cube model has twelve legs, as well as all other cubes, whereas each lag has a current, which can be in each direction of the leg. The prototype of the model is shown in fig. 4.15.

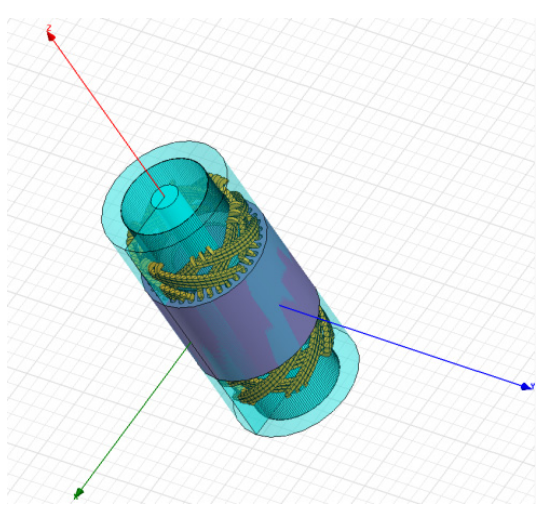

(a)

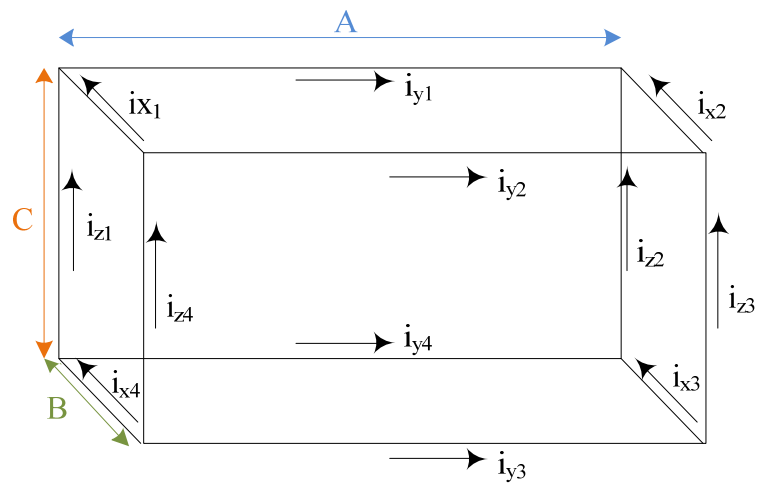

(b)

Fig. 4.15 Prototype of the proposed machine (SCIM) in Finite Element Analysis (a) actual model, (b) An equivalent current loop model for EMI and electromagnetic signature studies 
As shown in fig. 4.15, the model has current in all of its branches, which are evaluated based upon an intelligent optimization process. To enhance the possibility to get similar results, legs of the plane also defined as variables. Then, the cube branches' lengths $(A, B, C)$ and the corresponding currents to each leg $\left(i_{x 1} i_{x 2} i_{x 3} i_{x 4}, i_{y 1} i_{y 2} i_{y 3} i_{y 4}\right.$, and $\left.\begin{array}{lllll}i_{z 1} & i_{z 2} & i_{z 3} & i_{z 4}\end{array}\right)$ are calculated. Therefore, the model may change to a prism, when it's optimized. The proposed optimization process (GA-based PSO) is as follows:

PSO is a population-based algorithm that exploits a population of individuals to probe the promising region of the search space. In this context, the population is called swarm and the individuals are called particles. Each particle moves with an adaptable velocity within the search space and retains in its memory the best position it ever encountered. The global variant of PSO, which is the best position ever attained by all individuals of the swarm, is communicated to all the particles. The general principles for the PSO algorithm are stated in [190]:

The Particle swarm optimization is launched by initializing an N set of K-dimensional flock of birds randomly over the K-dimensional search space. In other word, of a Kdimensional search space, the $\mathrm{i}^{\text {th }}$ particle, can be represented by an K-dimensional vector, $\mathrm{X}_{\mathrm{i}}=\left[\mathrm{x}_{\mathrm{i} 1}, \mathrm{X}_{\mathrm{i} 2}, \ldots, \mathrm{X}_{\mathrm{ik}}\right]^{\mathrm{T}}$, and the velocity $\mathrm{U}_{\mathrm{i}}=\left[\mathrm{u}_{\mathrm{i} 1}, \mathrm{u}_{\mathrm{i} 2}, \ldots, \mathrm{u}_{\mathrm{ik}}\right]^{\mathrm{T}}$. Therefore, the total size of each population is a $\mathrm{k} \times \mathrm{N}$ matrix. In $\mathrm{PSO}$, the best position that the particle " $\mathrm{i}$ " visited so far is referred to as $\mathrm{P}_{\mathrm{i}}=\left[\mathrm{p}_{\mathrm{i} 1}, \ldots, \mathrm{p}_{\mathrm{is}}, \ldots\right]^{\mathrm{T}}$, and the best position of the best particle in the swarm is referred to as $\mathrm{G}=\left[\mathrm{g}_{1}, \ldots, \mathrm{g}_{\mathrm{s}}, \ldots\right]^{\mathrm{T}}$. Therefore, each particle "i" adjusts its position in the next iteration s+1 with respect to equations (4-19) and (4-20) [190]:

$v_{i k}(s+1)=\omega^{0}(s) v_{i k}(s)+C_{1} r_{1}\left(P_{i}(s)-x_{i k}(s)\right)+C_{2} r_{2}\left(G(s)-x_{i k}(s)\right)$ 
$x_{i k}(s+1)=x_{i k}(s)+\chi \cdot u_{i k}(s+1)$

where $\omega^{\circ}(t)$ is the inertia coefficient, which is employed to control the effect of the previous history of velocities on the current velocity. Here, $\chi$ is a constriction factor, which is used to limit the velocity. Also, $\mathrm{C}_{1}$ and $\mathrm{C}_{2}$ denote the cognitive and social parameters, while $r_{1}$ and $r_{2}$ are random real numbers drawn from the uniformly distributed interval $[0,1]$. The $\omega^{\circ}(t)$ was initialized as a large value (here we used 1 ) . Parameters $\mathrm{C}_{1}$ and $\mathrm{C}_{2}$ adjust dynamically [191].

In order to prevent premature convergence to suboptimal solutions, Raymond R. Tan augmented a binary PSO with a GA-based mutation operator and achieved significant improvement in the rate of successful convergence [191]. Therefore, in the current study, we incorporated mutation operator into our continuous-space PSO algorithm. This mutation operator reinitiates the value of each continuous variable into its feasible range by a predefined probability (e.g. 5\%).

In this study, the number of the population is set to 7 , and for preventing explosion of swarm, the maximum allowable velocity along each dimension is set to half of its feasible range. The results show that, in this application, for different number of switching angles, the algorithm converges within 100 to 150 iterations. Hence, in a conservative manner, the number of iterations is set to 30. Also, for enhancing the PSO's ability in escaping from local minima, a mutation operator is incorporated into the algorithm. The results indicate that, it is better to utilize this operator in discrete iteration intervals, with different probability. In this study, the mutation probabilities for iteration 
intervals of $\{5,7\}$ and $\{7,30\}$ are $1 \%$ and $3 \%$, respectively. Other iteration intervals are not influenced by mutation.

The objective function of the optimization process can be defined into two categories of electric fields or magnetic fields.

\subsubsection{Magnetic field as the objective function}

First, the magnetic field densities of the three components are considered as the objective function:

obj = mean $\left(\left|B x_{\text {motor }}\right|-\left|B x_{\text {cube }}\right|+\left|B y_{\text {motor }}\right|-\left|B y_{\text {cube }}\right|+\left|B z_{\text {motor }}\right|-\left|B z_{\text {cube }}\right|\right)$

where $B x, B y$, and $B z$ represent the normal magnetic field density along a line in $x, y$, and $z$ directions. The indices, motor and cube stands for the actual 3D motor model, and its corresponding cube current loop model (see fig. 4.15 (a) and (b)).

For simulation purposes, the same motor is used with the same setup to compare with the initial equivalent source modeling methods. The magnetic field along three lines in the $\mathrm{x}, \mathrm{y}, \mathrm{z}$ directions are calculated, and then the optimization process is implemented.

Table 4.1 The calculated currents for the cube branches

\begin{tabular}{cccc}
\hline $\boldsymbol{i}_{\boldsymbol{x} 1}$ & $\boldsymbol{i}_{\boldsymbol{x} 2}$ & $\boldsymbol{i}_{\boldsymbol{x} 3}$ & $\boldsymbol{i}_{\boldsymbol{x} 4}$ \\
\hline 67.95 & -30.4 & -24.2 & 14.4 \\
$\boldsymbol{i}_{\boldsymbol{y} 1}$ & $\boldsymbol{i}_{\boldsymbol{y} 2}$ & $\boldsymbol{i}_{\boldsymbol{y} 3}$ & $\boldsymbol{i}_{\boldsymbol{y} 4}$ \\
82.25 & -32.23 & 44.42 & -80.4 \\
$\boldsymbol{i}_{z 1}$ & $\boldsymbol{i}_{z 2}$ & $\boldsymbol{i}_{z 3}$ & $\boldsymbol{i}_{z 4}$ \\
62.9 & -73.5 & -1.26 & -64.5 \\
\hline
\end{tabular}

Within the optimization process, the cube length were calculated as $(A=0.1009(\mathrm{~m})$, $\mathrm{B}=0.125(\mathrm{~m}), \mathrm{C}=0.1282(\mathrm{~m}))$. Table 4.1 shows the calculated current for the cube branches after the optimization process. The comparison between the normal magnetic 
field of the actual 3D model and the cube model for one motor case is illustrated in fig. 4.16.

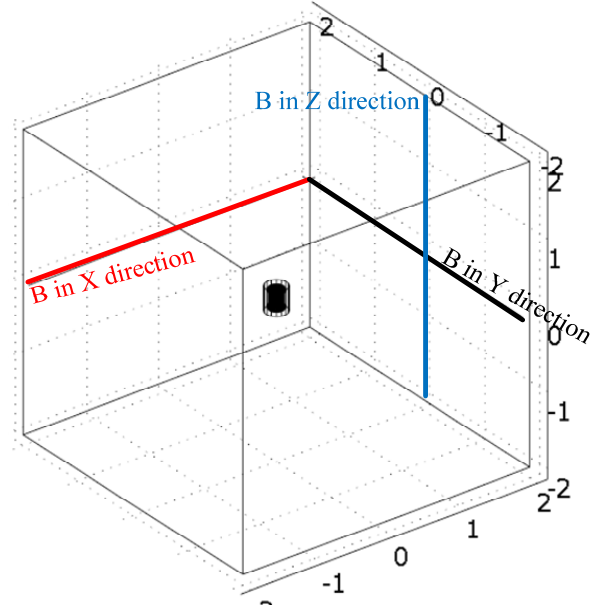

(a)

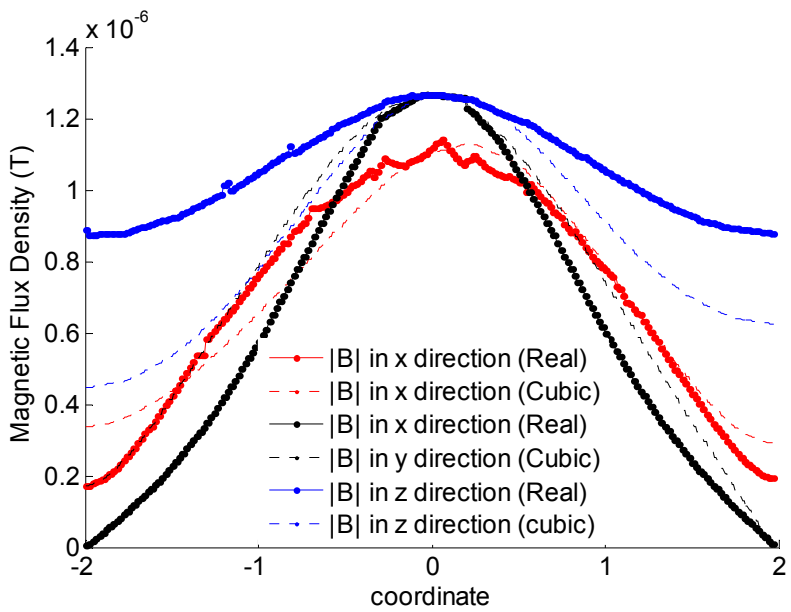

(b)

Fig. 4.16 (a) the lines and coordinates on which the field is measured (b) Comparison between magnetic flux density in real and cubic model for one motor (simulation time of full $3 \mathrm{D}$ is 1 hour whereas the simulation time of cube model is 1.5 minutes),

As can be seen from fig. 4.16(b), the equivalent source model shows good accuracy at the center of the lines. However, the accuracy in the two ends of the lines is not as good. simulation time of the full 3D is 6 hours, whereas the simulation time of the cube model is 1.5 minutes.

For validation of the calculated cube model from one motor case, the model is compared with the two motors, fig. 4-17 (a), while the current in the branches of the cube remains the same as the first cube. The centers of the coordinate of the two cubes are exactly the same as the 3D motor's model. Fig. 4-17 (b) shows the comparison between the magnetic field in the actual and cube model for the two motor cases. As can be seen, the magnetic fields again follow the same patterns with an acceptable accuracy. The 
comparison between the simulation time shows that, this approach makes the simulation time at least 100 times faster than full 3D model. Moreover, it was observed that in case of evaluation of the field in just two directions, more accurate results can be calculated.

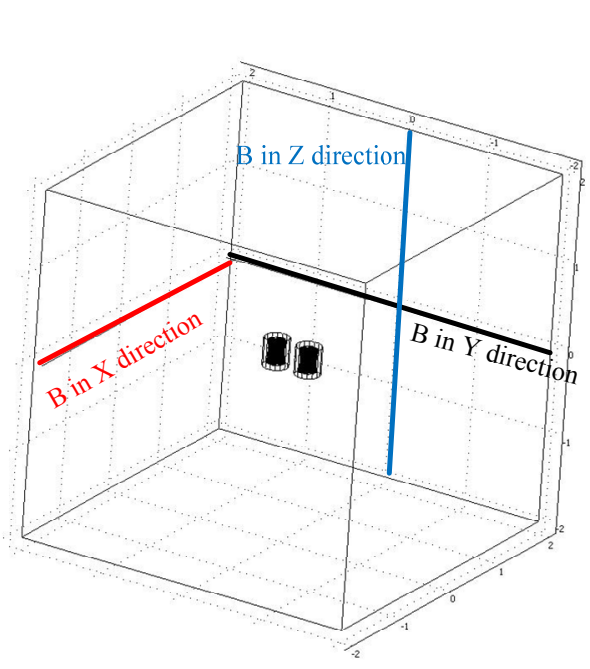

(a)

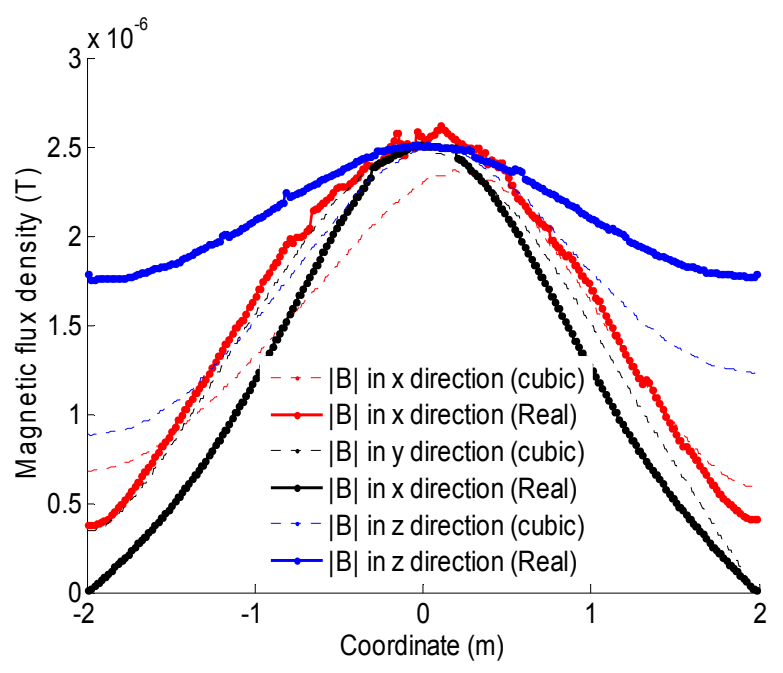

(b)

Fig. 4.17 (a) Two motor case of study for validation of the model (b) Comparison between electric field in real and cubic model for two motor

However, there is still a problem with the currents of legs mentioned above in Table 4.1 only the magnetic field of the cubic model has a similar result compared with the actual model of the electric machine, but the electric field doesn't have similar results at all. Therefore, the electric field as the objective function is also studied.

\subsubsection{Electric field as the objective function}

To find good results for the electric field, another optimization is performed based on the electric field objective function. The objective function of the optimization process for this case is the main index for evaluating the values of the model. This is because in the initial and the later one in the process, the modified values are just compared by 
means of the objective function. The objective function of the optimization process is defined as:

$\operatorname{obj}=\operatorname{mean}\left(\left|E x_{\text {motor }}\right|-\left|E x_{\text {cube }}\right|+\left|E y_{\text {motor }}\right|-\left|E y_{\text {cube }}\right|+\left|E z_{\text {motor }}\right|-\left|E z_{\text {cube }}\right|\right)$

where $E x, E y$, and $E z$ represent the normal magnetic field density along a line in $x, y$, and $z$ directions.

Following the optimization process, the cube or actually the rectangular prism length are calculated as $(A=0.1009(\mathrm{~m}), \mathrm{B}=0.125(\mathrm{~m}), \mathrm{C}=0.1282(\mathrm{~m}))$. Table 4.1 shows the calculated current for the rectangular prism branches following the optimization process in one case. The optimized currents of the cube based on the equation (4-22) as the objective function are achieved and shown in Table 4.2.

The comparison between the normal electric field of the actual 3D model and the cube model for one motor case is illustrated in various circumstances. For brevity, only some indices, including field spectrum, arrow-line and stream-line, are selected for the study. For instance, electric field spectrum radiated from the actual machine and the equivalent source models are compared, as shown in fig. 4.18.

Table 4.2 The calculated current for cube legs

\begin{tabular}{cccc}
\hline $\boldsymbol{i}_{x 1}$ & $\boldsymbol{i}_{\boldsymbol{x} 2}$ & $\boldsymbol{i}_{\boldsymbol{x} 3}$ & $\boldsymbol{i}_{\boldsymbol{x} 4}$ \\
\hline-61.18 & 528.12 & -267.44 & -107.82 \\
$\boldsymbol{i}_{\boldsymbol{y} 1}$ & $\boldsymbol{i}_{\boldsymbol{y} 2}$ & $\boldsymbol{i}_{\boldsymbol{y} 3}$ & $\boldsymbol{i}_{\boldsymbol{y} 4}$ \\
-115.25 & -251.54 & 36.107 & 424.1 \\
$\boldsymbol{i}_{z 1}$ & $\boldsymbol{i}_{z 2}$ & $\boldsymbol{i}_{z 3}$ & $\boldsymbol{i}_{z 4}$ \\
-150.03 & -99.84 & 4.46 & -135.6 \\
\hline
\end{tabular}




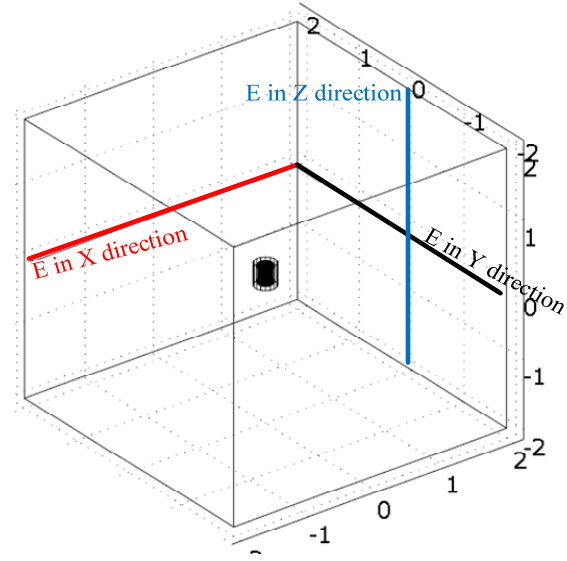

(a)

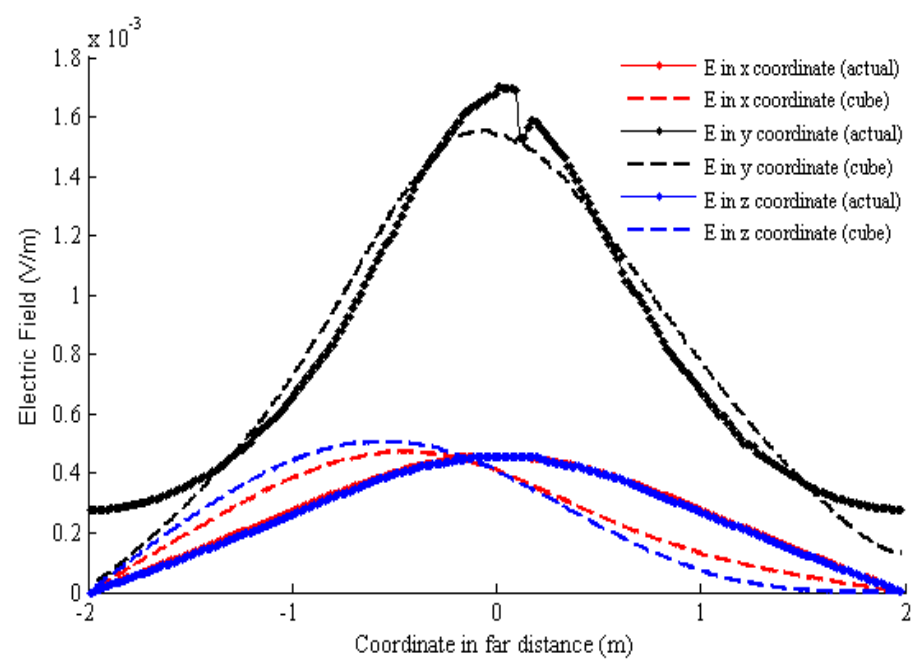

(b)

Fig. 4.18 (a) The lines and coordinates on which the field is measured (b) Comparison of electric field between actual and cubic model for one motor

Considering fig. 4.18, the accuracy of the E-fields in all coordinates are better than the case in which the magnetic field was the objective function. The E-field in the $\mathrm{Y}$ direction has great accuracy, but in the other two legs, the peaks are shifted to right.

The main goal of this modeling is to study the electromagnetic signature of a multicomponent system. For validation of the calculated equivalent source model (rectangular prism) from one motor case, the model is compared in a case containing two motors, as shown in fig. 4.19(a), while the current in the branches of the rectangular prism model remains the same as the first case. The centers of the coordinates of the two rectangular prisms are exactly the same as the $3 \mathrm{D}$ motor model. The figure shows a comparison between the electric field spectrum in the actual and cube model for two motor cases. As can be seen from fig. 4.19(b), the accuracy is the same as the one motor case. 


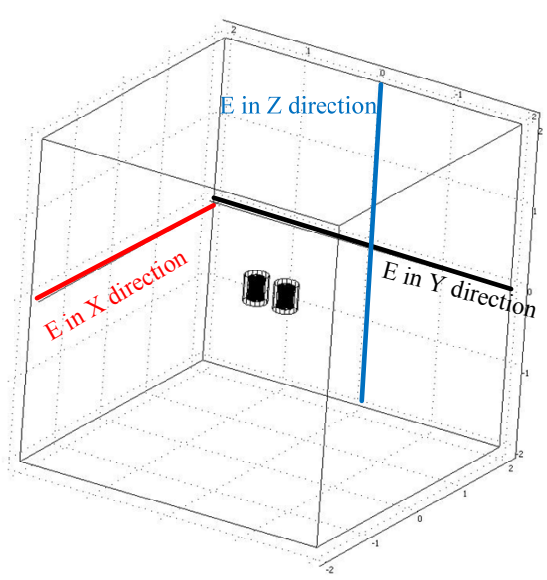

(a)

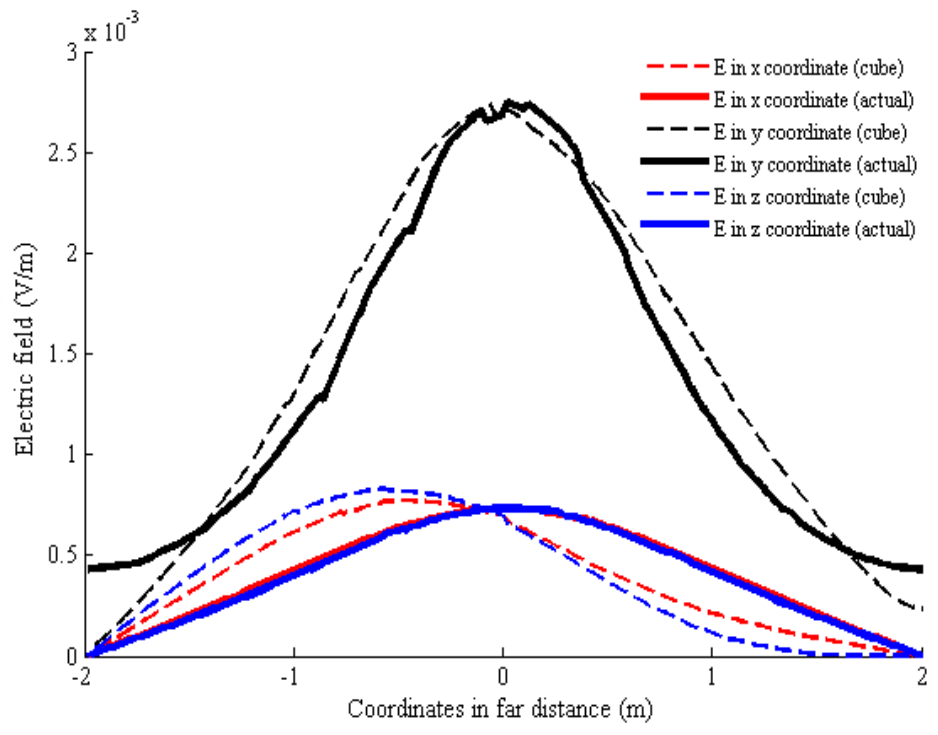

(b)

Fig. 4.19 (a) Two motor case of study for validation of the model (b) Comparison between electric field in real and cubic model for two motor

The problem with this model is that the currents in Table 2.2 is totally different from the current in table 4.1; therefore, simultaneous obtaining of electric and magnetic fields from the cube model similar to the actual model is not possible by using the current cube model. Consequently, the new model is designed.

\subsection{Voltage-current rectangular prism model}

As mentioned before, in the static magnetic field domain, the far field definition defines the fact that the normal magnetic flux density of a magnetic dipole is always coincident with the definition of the far field at any distance. Where a magnetic dipole can be simply created from a simple current loop, for simulation of the far field of multiconductor components, such as electrical machines, fig 4.3 shows that the whole multiconductive component can be replaced by a simple current loop. This simple idea allows 
the simplification of the complex machine structure to one or several loops, resulting in a more numerically simple model for radiated fields in all directions. Although substitution of the complex machine structure with several current loops evaluate the normal magnetic flux density close to the results of the detailed model of the machine, the electric field cannot be accurately predicted at the same time. This was the problem of the current cube model. Similar ideas for the magnetic field evaluation can be used for the recreation of far electric fields emitted by the equivalent loop model.

In the static electric field domain, an electric dipole with a finite length is what helps to revise the electric field. The electric dipole can be created by two points with different potentials along a finite line. Similar to magnetic field, several electric dipoles can be used for the recreation of the electric field in all directions.

In several simulation studies, it was observed that the path and direction of current and the value of current density of the electric machine and its winding potential have direct influence on establishing magnetic and electric stray fields at a far distances. A large number trial and error process may be needed to guess the optimal shape of the equivalent source model from the complicated structure of the machine. Nevertheless, the symmetry of the far field, calculated by the detailed geometrical model, along the axial direction of the machine in any $\mathrm{X}=\mathrm{a}, \mathrm{Y}=\mathrm{b}$ planes, and the symmetry of the far field, calculated by the detailed geometrical model in the radial direction in any $\mathrm{Z}=\mathrm{c}$ plane, are the main factors of the choice of the equivalent cuboid (rectangular prism) shape, illustrated in fig. 4.20. The variables of this equivalent rectangular prism model are:

- The currents in its branches: $\left(i_{x} 0 \ldots i_{x 3}, i_{y 0} \ldots i_{y 3}, i_{z 0} \ldots i_{z 3}\right)$

- The voltages at its nodes: $\left(\mathrm{V}_{1}, \mathrm{~V}_{2}\right)$, 
- The size of the rectangular prism: (A, B, C)

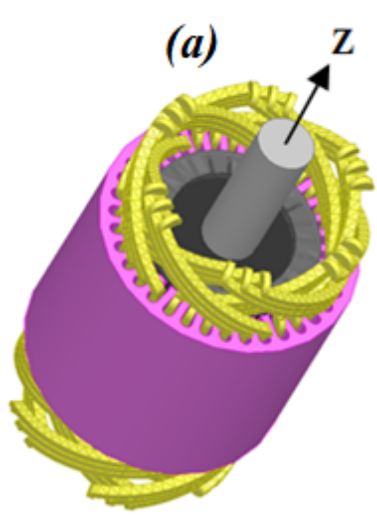

(a)

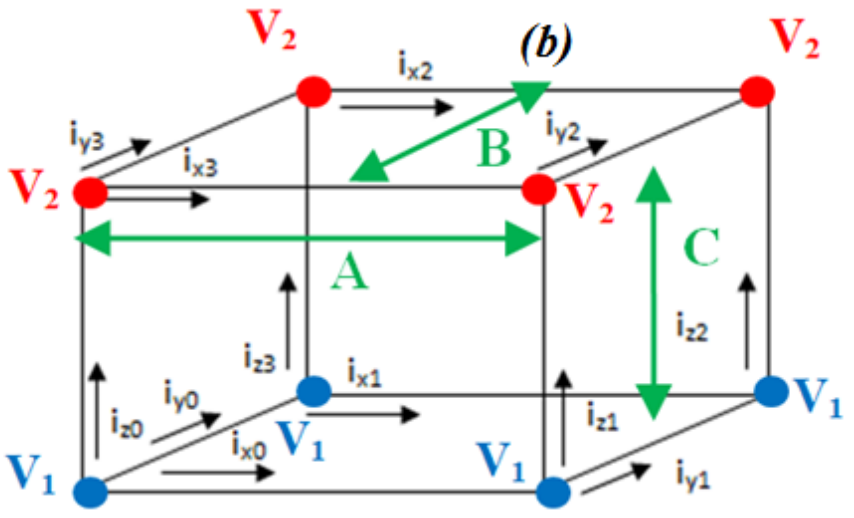

(b)

Fig. 4.20 (a) Prototype of the detailed geometrical model (b) and the equivalent rectangular prism model

In order to evaluate these variables properly, an optimization problem was defined. The multi-objective function, equation (4-23), was defined as the difference between the far magnetic plus electric fields of the equivalent source model and the detailed machine 3D FE model. In this model, the voltage on nodes, as well as the current of the legs are used as variables. Therefore, as shown in fig. 4.20 , there are 12 current variables, 8 voltage variables. There are 3 variables as dimensions of the prism. Using this model, the variables for the optimization are more than previous model, therefore the possibility of obtaining accurate results is better. Also, the voltage parameter which has a great impact on the electric field is available. The optimization process sets the objective function to a minimum by changing the variables of the equivalent source model. This process was performed utilizing an evolutionary-based optimization algorithm.

$$
\operatorname{obj}=\text { mean }\left(\sum_{\mathrm{i}=1}^{3}\left|\mathbf{B}_{\mathrm{i}}(\mathrm{X}, \mathrm{Y}, \mathrm{Z})_{3 \mathrm{DFE}}-\mathbf{B}_{\mathrm{i}}(\mathrm{X}, \mathrm{Y}, \mathrm{Z})_{\mathrm{Eq}, \text { model }}\right|+\sum_{\mathrm{i}=1}^{3}\left|\mathbf{E}_{\mathrm{i}}(\mathrm{X}, \mathrm{Y}, \mathrm{Z})_{3 \mathrm{BFE}}-\mathbf{E}_{\mathrm{i}}(\mathrm{X}, \mathrm{Y}, \mathrm{Z})_{\mathrm{Eq}, \text { model }}\right|\right)
$$


where $\mathbf{B}_{\mathrm{i}}(\mathrm{X}, \mathrm{Y}, \mathrm{Z})_{3 \mathrm{DFE}}$, and $\mathbf{E}_{\mathrm{i}}(\mathrm{X}, \mathrm{Y}, \mathrm{Z})_{3 \mathrm{DFE}}$, are normal magnetic flux densities and normal electric fields calculated along three finite lines for the detailed geometrical models, fig. 4.18 (a). Here, $\mathbf{B}_{\mathrm{i}}(\mathrm{X}, \mathrm{Y}, \mathrm{Z})_{\text {Eq.model, }}$ and electric field, $\mathbf{E}_{\mathrm{i}}(\mathrm{X}, \mathrm{Y}, \mathrm{Z})_{\text {Eq.model }}$ are the corresponding measurements from the equivalent cube model. The B and $\mathrm{E}$ are each $3 \times \mathrm{N}$ matrix, with $\mathrm{N}$ standing for the number of samples.

A snapshot of the normal magnetic flux densities, $\mathrm{B}_{\mathrm{i}}(\mathrm{X}, \mathrm{Y}, \mathrm{Z})_{3 \mathrm{DFE}}$, and electric fields, $\mathrm{E}_{\mathrm{i}}(\mathrm{X}, \mathrm{Y}, \mathrm{Z})_{3 \mathrm{DFE}}$, along three straight lines in the $\mathrm{X}, \mathrm{Y}$, and $\mathrm{Z}$ directions, were calculated and stored. The optimization process runs iteratively to minimize the objective function. Following the optimization process, the cube side lengths were computed as $(\mathrm{A}=0.279$ $(\mathrm{m}), \mathrm{B}=0.2(\mathrm{~m}), \mathrm{C}=0.31(\mathrm{~m}))$. The comparison between the normal magnetic flux densities of the full 3DFE model and the proposed cube model with one motor case is illustrated in fig. 4.21 and 4.22 .

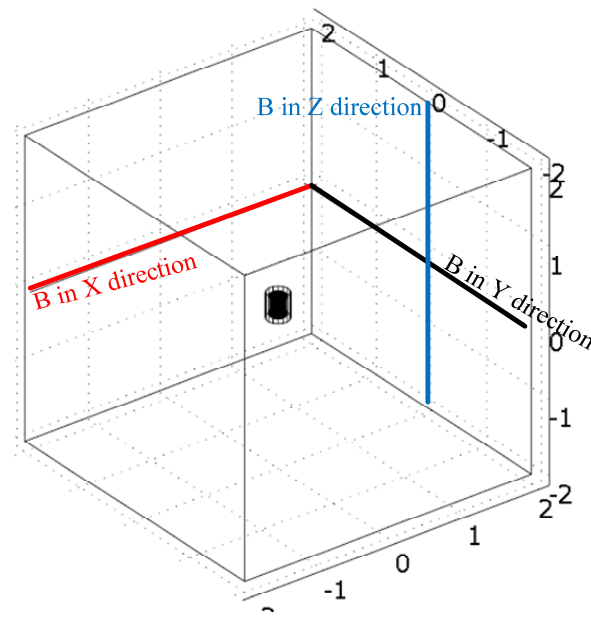

(a)

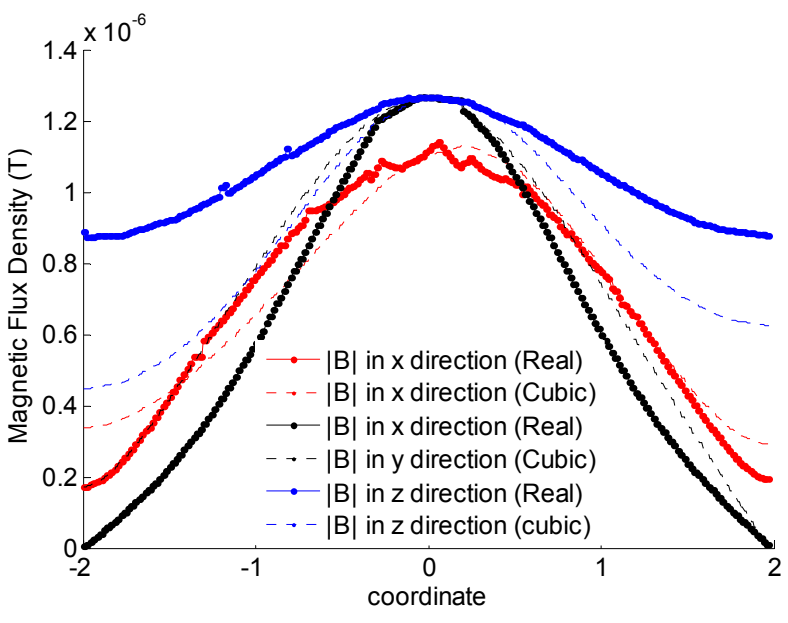

(b)

Fig. 4.21 Comparison between magnetic flux density calculated by 3DFE analysis for equivalent cubic model and real detail machine model, the one motor case of study 


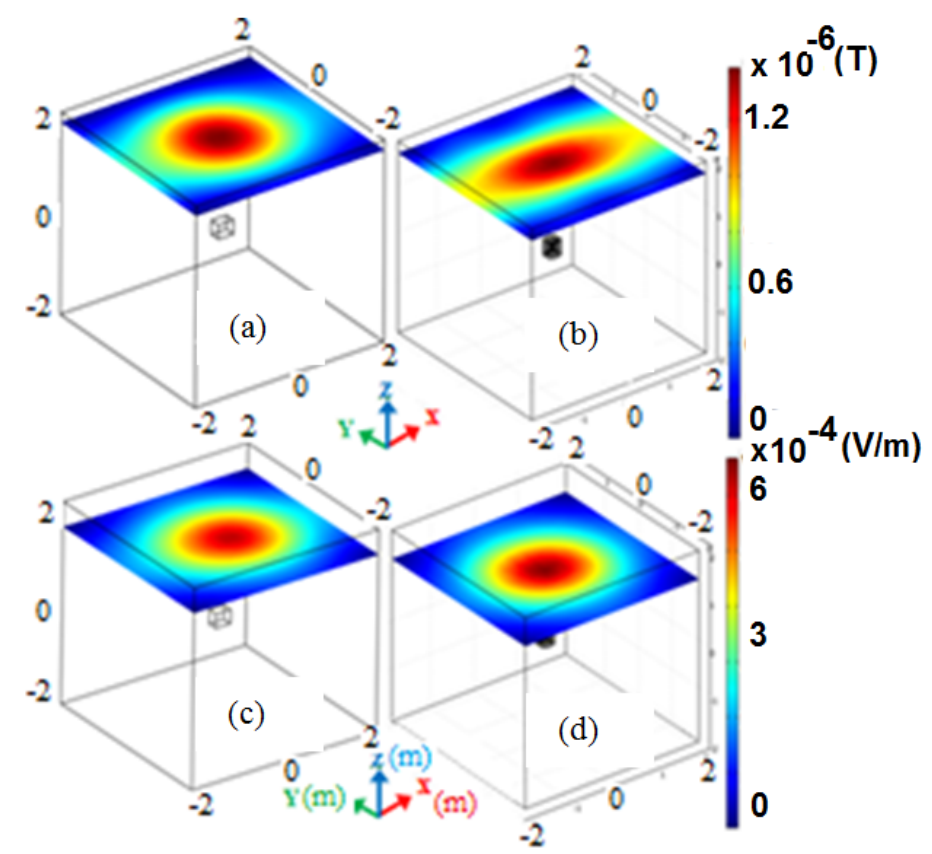

Fig. 4.22 Normal Magnetic flux density in the X-Y plane (a) for the equivalent source model, (b) for the detailed model, Normal electric field in the X-Y plane (c) for the equivalent source model, (d) for the detailed model

As long as the environment is assumed to have linear properties, the superposition of the far fields of a set of cube models will be equal to the superposition of the far fields of detailed geometrical models. This is the case when the observation point and the centers of machines and cubes are the same. In order to validate this, another case study including two motors, shown in fig. 4.23(a), was investigated. Figures 4.23(b) and 4.24, respectively show a comparison between the normal magnetic flux density and the electric field of the detailed geometrical model and the equivalent cube model for the two machine environment. The magnetic flux densities and electric fields again follow similar patterns with an acceptable accuracy. 


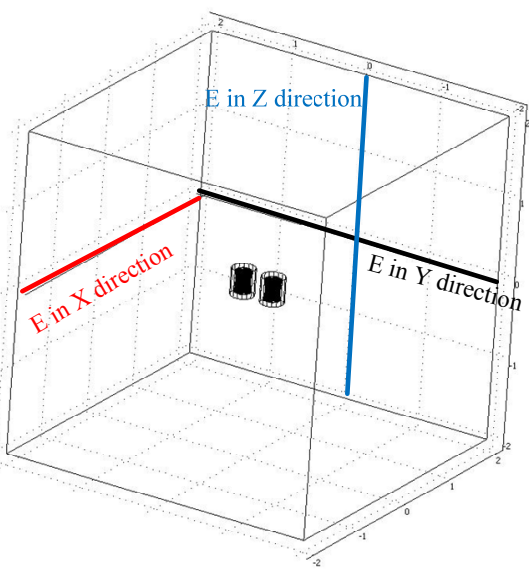

(a)

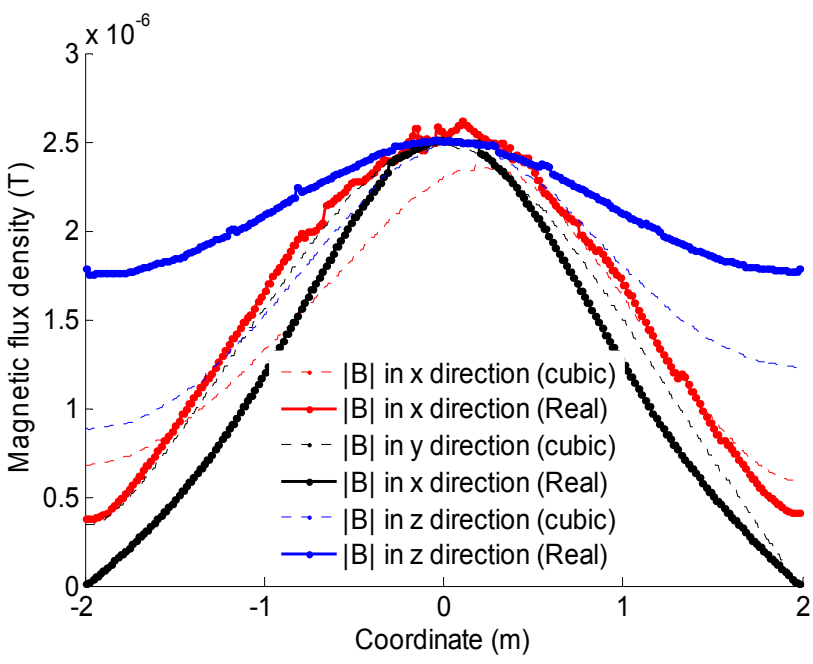

(b)

Fig. 4.23 (a) Two machine case of study for validation of the model (b) Comparison between normal magnetic flux density computed by 3DFE analysis for the equivalent cubic model and the real detail machine model, the two motor case of study

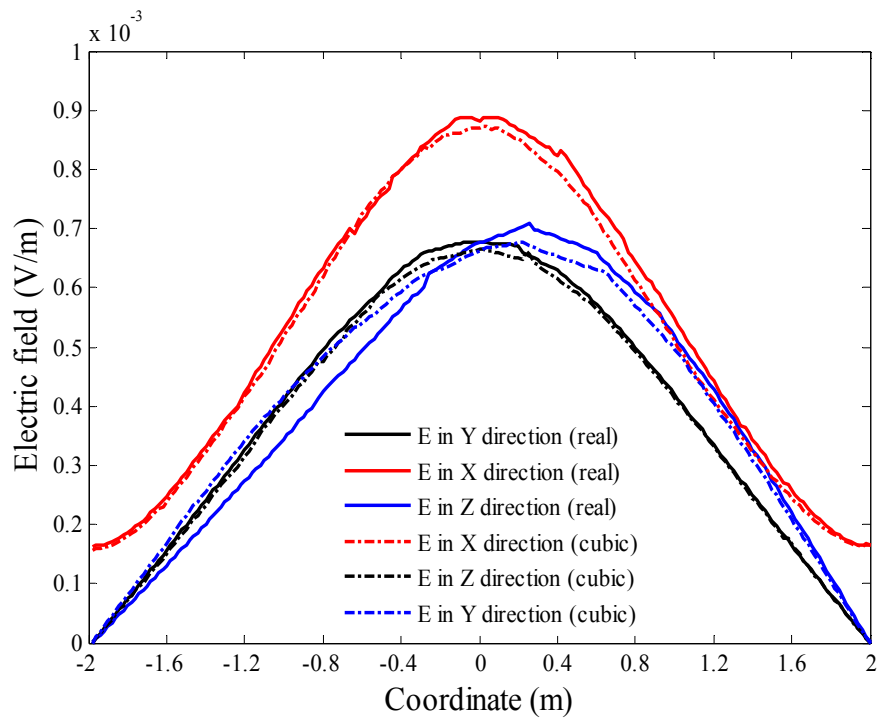

Fig. 4.24 Comparison between Electric fields computed by 3DFE for the equivalent cubic model and the real detail machine model, the two motor case of study

The comparison between the average of various simulation times shows that using the proposed approach makes the simulation time at least 216 times faster than using the full 
3DFE model with an acceptable minor difference. The comparison between the simulations time in cases of one motor, two motor, as well as the optimization time, are shown in Table 4.3. The computation time of the optimization process is four times that of the computation time elapsed of the detail geometrical model. However, it is only done one time for one motor case study.

Table 4.3 Computation time Comparisons

\begin{tabular}{|c|c|c|}
\hline Case of Studies & One Machine case & Two Machines case \\
\hline 3DFE model & 6(Hours) & 11(Hours) \\
\hline Equivalent Cube model & 100(Sec.) & 160(Sec.) \\
\hline Revised model & 250 (Sec.) & 400(Sec.) \\
\hline Optimization Process & 23(Hours) & ---------- \\
\hline
\end{tabular}

\subsubsection{Revised model}

The comparison of fig. 4.21 and 4.22 reveals the special location and the magnitude of the maximum field. This is predicted accurately by the equivalent source model. However, the magnetic field pattern typically in the $\mathrm{X}-\mathrm{Y}$ plane does not match precisely with that of the detailed geometrical model. Although, the equivalent source model seems to be the minimalist model for this kind of geometry simplifications, the studies have shown that without revision of the model by adding additional current loops or curving the current flowing in each side, the improvement of the accuracy of the magnetic field in all directions is impossible. This is because based on the presented theory in the previous section, for each direction, an independent current loop is needed, while the current loops 
of the cube model are dependent. Fig. 4.25 (a) demonstrates a revised version of the cube model. The magnetic and electric fields of this model, figs. 4.25 (b) and 4.25 (d), are very close to that of the 3DFE model in figs. 4.25 (c) and 4.25 (e).

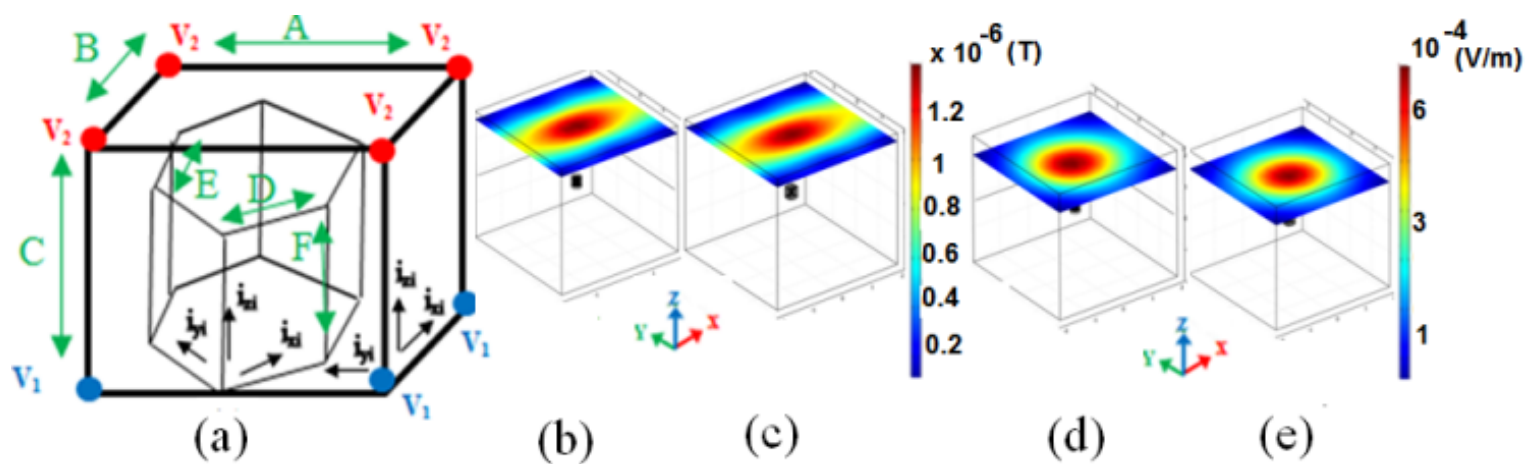

Fig. 4.25 Revised version of the cube model (a), Normal Magnetic flux density throughout X-Y plane for the equivalent source model (b), for the detail model (c), Normal electric field throughout XY plane for the equivalent source model (d), for the detail model (e), all for the one motor case of study

Fig. 4.26 shows the comparison between the far magnetic field of the revised model and detailed geometrical model for one and two motor case of study. It is observed that this kind of model revision may lead to more accurate results. However, it increases the number of model variable to twice the cube model and the computation time by 2.5 times of that of the cube model. Indeed, the cube model is a tradeoff between several factors, including accuracy, simulation times, simplicity, and generality. Fig. 4.27 shows the comparison of the near magnetic fields of the revised and the detailed model of the machines. Interestingly, the revised model could also predict a very similar pattern to that of the detailed model, thought it was not a direct objective. 

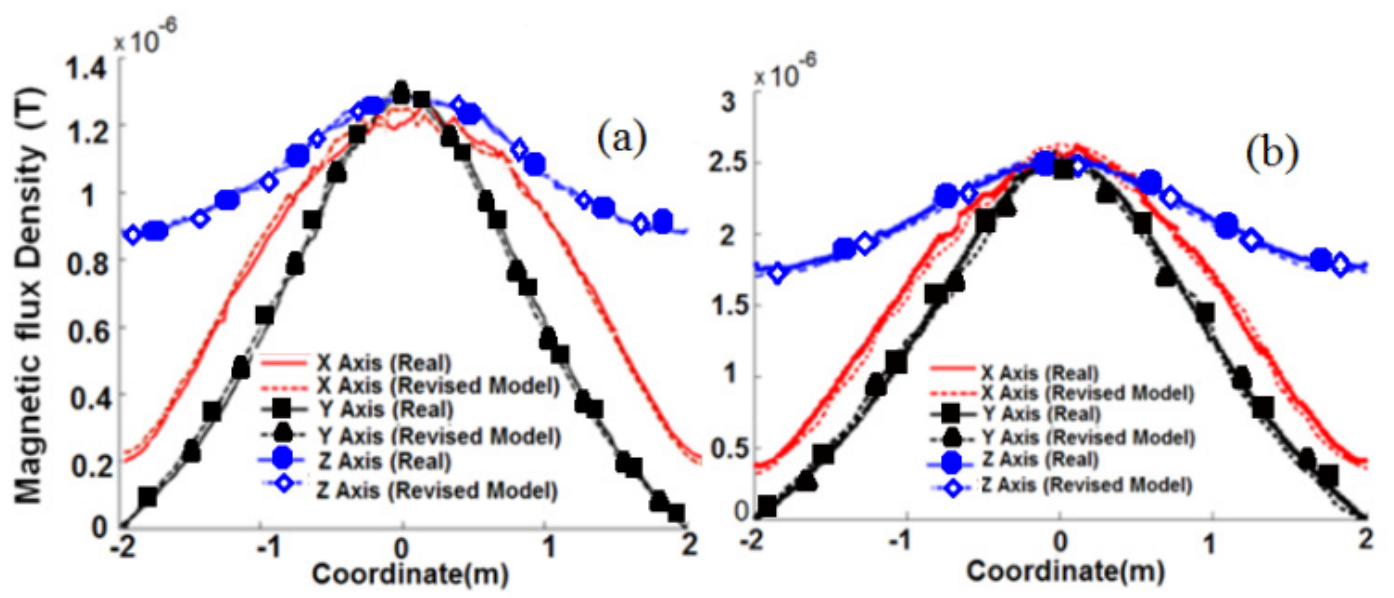

Fig. 4.26 Comparison between magnetic flux density calculated by 3DFE analysis for revised equivalent source model and detail machine model, (a) the one machine (b) and two machine case of studies

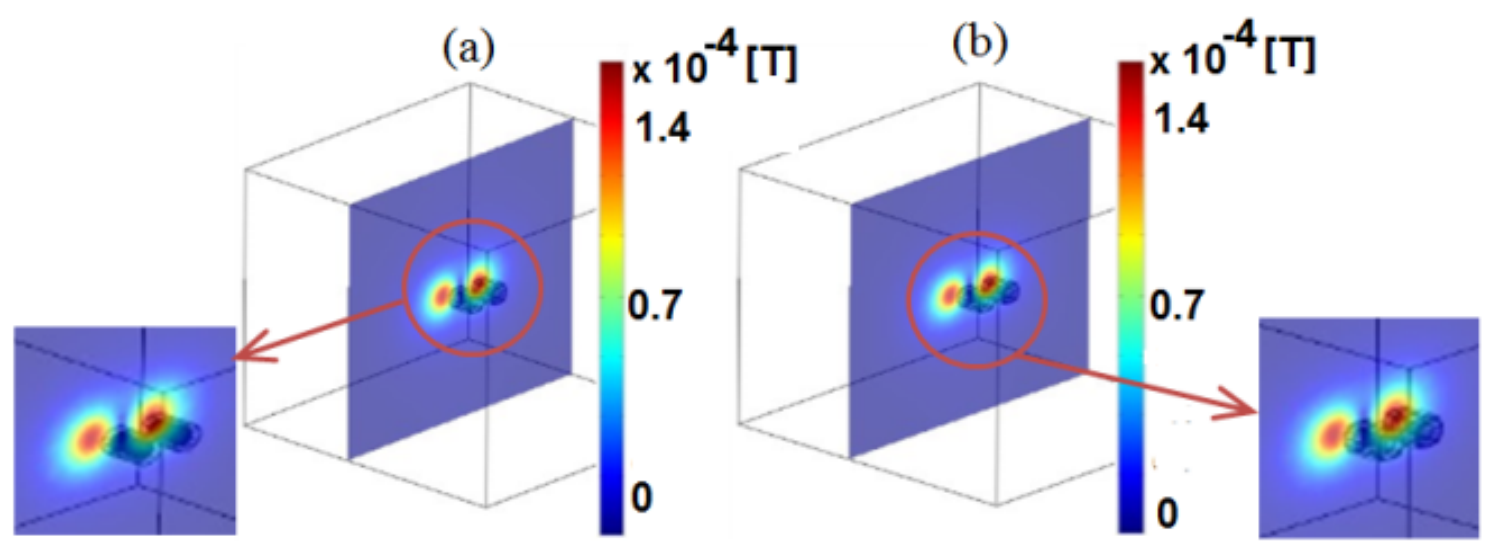

Fig. 4.27 Comparison of the near fields, 0.25 meter from machines, Normal Magnetic flux density in the X-Y plane for (a) the revised model, (b) for the detailed geometrical model

Since the assumption of quasi static fields in this problem is valid, the iron core saturation is ignored, there is a linear relationship among the magnetic field and the actual phase current of the machine, and the current in sides of the equivalent source model. There is also a linear relationship among the electric field and the terminal voltage 
of the machine, and the voltage nodes $\left(\mathrm{V}_{1}\right.$ and $\left.\mathrm{V}_{2}\right)$ of the equivalent source model. Therefore, the equivalent source models can be simply recreated when the phase current, terminal voltage or the frequency of the machine changes over the time.

\subsection{Conclusion}

Since there is a considerable necessity for analyzing the radiated electromagnetic field around electrical machines and also considering that simulating multi-machine environment with actual models requires significant time and computing resources, designing an equivalent source model was studied in this chapter. Several models are investigated in this study and the voltage current cube model has the best result between these proposed models. The GA-based PSO method is used for evaluating currents of cube branches, as well as voltages of nodes. The finite element analysis was used for the simulation. The results show that it is possible to replace actual model of the electrical machines with the equivalent rectangular cube model. The simulation time of the cube model is approximately 100 times less than the actual model, enabling the numerical simulation of multiple cases. The model was validated based upon the simulation of two similar induction machine with two cube models with the same center of coordinates.

However, the final model has great accuracy in resembling the stray fields; it can only be used in one particular occasion. In other words, by changing the voltage, power or construction the machine, such as the number of poles and size, the model should be reoptimized. Therefore, a new type of modeling is designed, which is discussed with the experimental verification in chapter 6 . 


\section{Instruction for the Low Frequency Electromagnetic Signature Experimental}

\section{Measurement}

\subsection{Overview}

Based on the organization of the dissertation, the study from chapter 7 consists of many experimental tests. Therefore, the instruction for the low-frequency electromagnetic experimental measurement is collected from several standards (MIL, IEEE, ANSI and ASTM standards) [192-200].

This instruction establishes the interface and associated verification requirements for the control of the electromagnetic interference (emission and susceptibility) characteristics of electric power equipment and subsystems in design and implementation. Such equipment and subsystems may be used independently or as an integral part of other subsystems or systems. In the following, first the general requirements are mentioned, then detail requirements for the control for the conducted and radiated emission in low frequencies are explained.

\subsection{General requirement}

Electronic, electrical, and electromechanical equipment and subsystems should comply with the applicable general interface requirements in section 5.2.1. General requirements for verification should be in accordance with section 5.2.2. These general requirements are in addition to the applicable detailed emission and susceptibility requirements, and associated test procedures defined in 5.3. 


\subsubsection{Filtering}

The use of line-to-ground filters for the EMI control should be minimized. Such filters establish low impedance paths for structure (common-mode) currents through the ground plane and can be a major cause of interference in systems, platforms, or installations because the currents can couple into other equipment using the same ground plane. If such a filter must be employed, the line-to-ground capacitance for each line should not exceed 0.1 microfarads $(\mu \mathrm{F})$ for $60 \mathrm{Hertz}(\mathrm{Hz})$ equipment or $0.02 \mu \mathrm{F}$ for 400 $\mathrm{Hz}$ equipment. For submarine DC-powered equipment and aircraft DC-powered equipment, the filter capacitance from each line-to-ground at the user interface should not exceed $0.075 \mu \mathrm{F} / \mathrm{kW}$ of connected load. For DC loads less than $0.5 \mathrm{~kW}$, the filter capacitance should not exceed $0.03 \mu \mathrm{F}$. The filtering employed should be fully described in the equipment or subsystem technical manual and the Electromagnetic Interference Control Procedures (EMICP).

\subsubsection{Measurement tolerances}

Unless otherwise stated for a particular measurement, the tolerance should be as follows:

a. Distance: $\pm 5 \%$

b. Frequency: $\pm 2 \%$

c. Amplitude, measurement receiver: $\pm 2 \mathrm{~dB}$

d. Amplitude, measurement system (includes measurement receivers, transducers, cables, and so forth): $\pm 3 \mathrm{~dB}$

e. Time (waveforms): $\pm 5 \%$ 
f. Resistors: $\pm 5 \%$

e. Capacitors: $\pm 20 \%$

\subsubsection{Ambient electromagnetic level}

The ambient electromagnetic level measured with the EUT de-energized and all auxiliary equipment turned on during tests, must be at least $6 \mathrm{~dB}$ below the permissible specified limits when the tests are performed in a shielded enclosure. Ambient conducted levels on power leads should be measured with the leads disconnected from the EUT and connected to a resistive load, which pulls the same rated current as the EUT. At what time tests are performed in a shielded enclosure and the EUT is in compliance with required limits, the ambient profile doesn't require to be recorded in the EMITR. When measurements are made outside a shielded enclosure, the tests should be performed during times and conditions when the ambient is at its lowest level. The ambient should be recorded in the EMITR and should not compromise the test results [192].

\subsubsection{Ground plane}

The EUT should be installed on a ground plane that simulates the actual installation. If the actual installation is unidentified or multiple installations are expected, then a metallic ground plane should be used. Unless otherwise specified below, ground planes should be 2.25 square meters or larger in space with the smaller side no less than 76 centimeters. When a ground plane is not present in the EUT installation, the EUT should be placed on a non-conductive table. 
width. Metallic ground planes used outside a shielded enclosure should extend at least 1.5 meters beyond the test setup boundary in each direction.

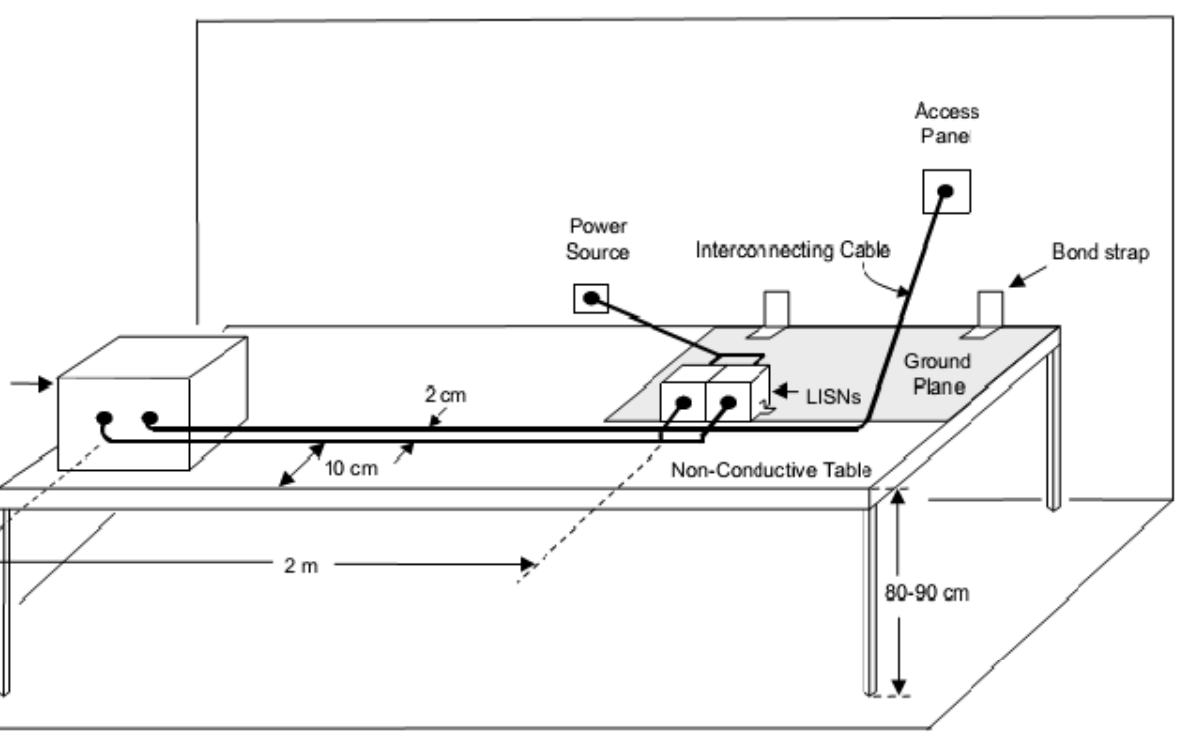

Fig. 5.2 Test setup for non-conductive surface mounted EUT 


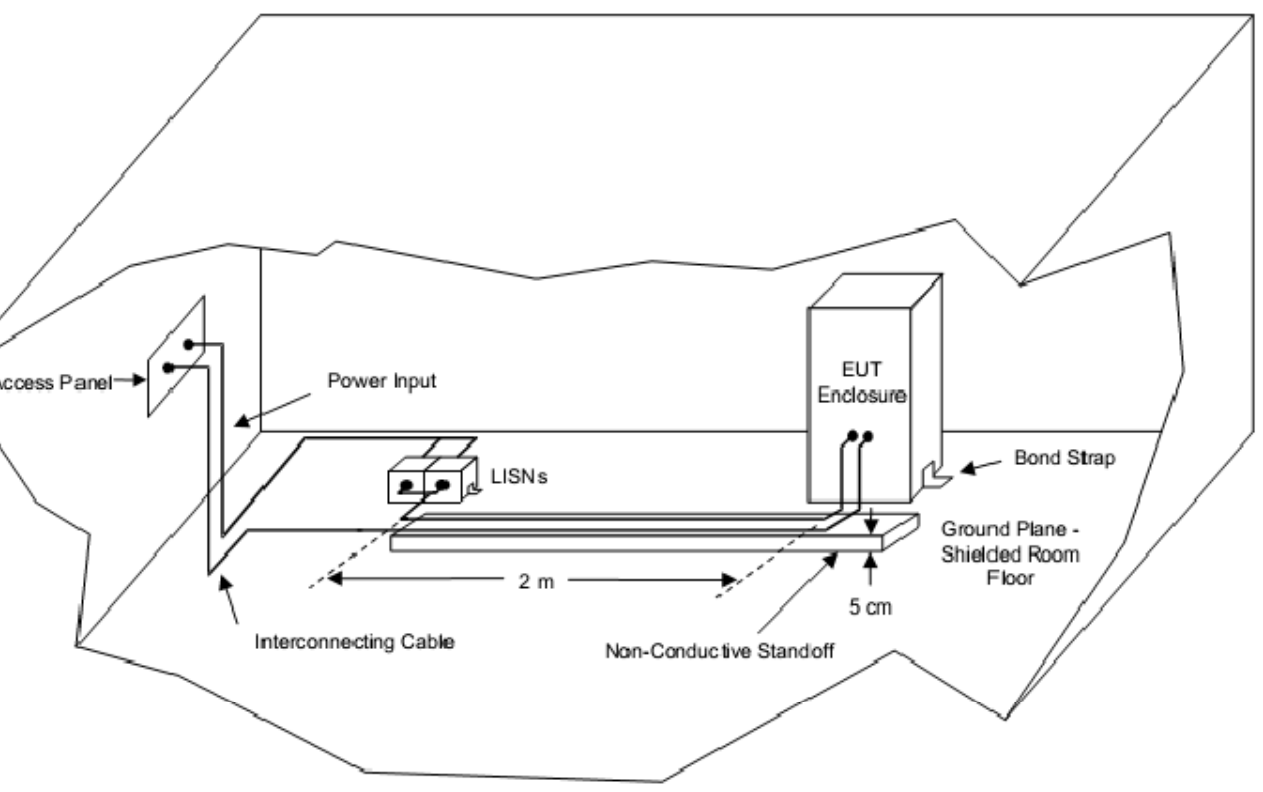

Fig. 5.3 Test setup for free standing EUT in shielded enclosure

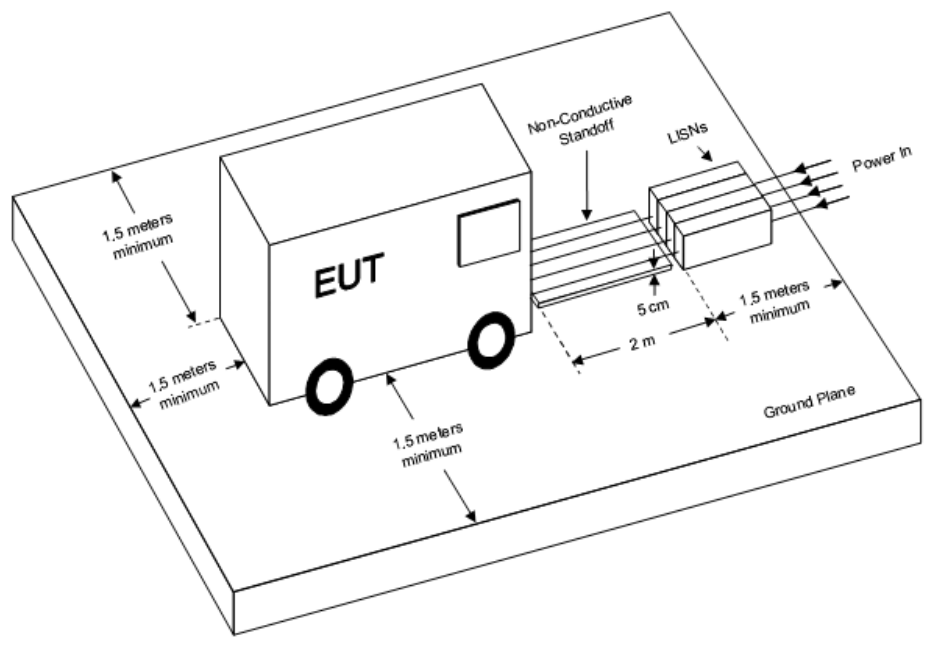

Fig. 5.4 Test setup for free standing EUT [192], [200] 


\subsubsection{Power source impedance}

The impedance of power sources providing input power to the EUT should be controlled by Line Impedance Stabilization Networks (LISNs) for all measurement procedures of this document unless otherwise stated in a particular test procedure. LISNs should not be used on output power leads. The LISNs should be located at the power source end of the exposed length of power leads specified in paragraph 4.3.8.6.2 of [200]. The LISN circuit should be in accordance with the schematic shown in fig. 5.5. The LISN impedance characteristics should be in accordance with fig. 5.5. The LISN impedance should be measured at least annually under the following conditions:

a. The impedance should be measured between the power output lead on the load side of the LISN and the metal enclosure of the LISN.

b. The signal output port of the LISN should be terminated in fifty ohms.

c. The power input terminal on the power source side of the LISN should be unterminated.

The impedance measurement results should be provided in the EMITR.
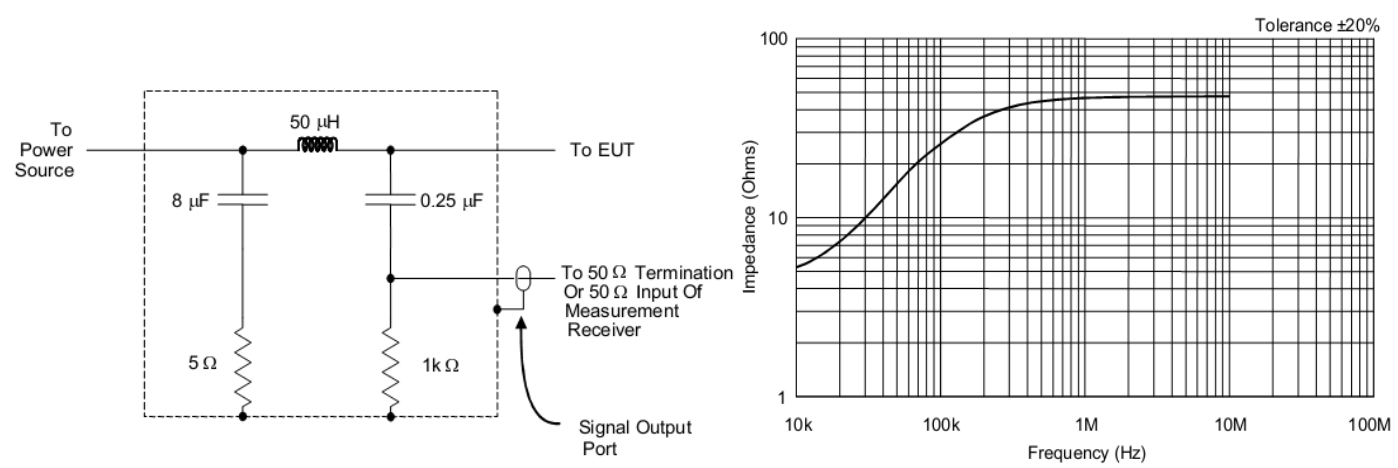

Fig. 5.5 LISN Schematic and impedance characteristics 


\subsubsection{Overload precautions}

Measurement receivers and transducers are subject to overload, especially receivers without pre-selectors and active transducers. Periodic checks should be performed to assure that an overload condition does not exist. Instrumentation changes should be implemented to correct any overload condition.

\subsubsection{Construction and arrangement of Interconnecting leads and cables}

Individual leads should be grouped into cables in the same manner as in the actual installation. Total interconnecting cable lengths in the setup should be the same as in the actual platform installation. If a cable is longer than 10 meters, at least 10 meters should be included. When cable lengths are not specified for the installation, cables should be sufficiently long to satisfy the conditions specified below. At least the first 2 meters (except for cables which are shorter in the actual installation) of each interconnecting cable associated with each enclosure of the EUT should be run parallel to the front boundary of the setup. Remaining cable lengths should be routed to the back of the setup and should be placed in a zig-zagged arrangement. When the setup includes more than one cable, individual cables should be separated by 2 centimeters measured from their outer circumference. For bench top setups using ground planes, the cable closest to the front boundary should be placed 10 centimeters from the front edge of the ground plane. All cables should be supported 5 centimeters above the ground plane. 


\subsubsection{Construction and arrangement of Input power leads}

Two meters of input power leads (including neutrals and returns) should be routed parallel to the front edge of the setup in the same manner as the interconnecting leads. Each input power lead, including neutrals and returns, should be connected to a LISN (see fig. 5.5). Power leads that are bundled as part of an interconnecting cable in the actual installation should be configured in the same fashion for the 2 meter exposed length and then should be separated from the bundle and routed to the LISNs. After the 2 meter exposed length, the power leads should be terminated at the LISNs in as short a distance as possible. The total length of power lead from the EUT electrical connector to the LISNs should not exceed 2.5 meters. All power leads should be supported 5 centimeters above the ground plane. If the power leads are twisted in the actual installation, they should be twisted up to the LISNs.

\subsubsection{Operation of EUT}

During emission measurements, the EUT should be placed in an operating mode which produces maximum emissions. During susceptibility testing, the EUT should be placed in its most susceptible operating mode. For EUTs with several available modes

(including software controlled operational modes), a sufficient number of modes should be tested for emissions and susceptibility such that all circuitry is evaluated. The rationale for modes selected should be included in the EMITP. 


\subsection{Use of measurement equipment}

Any frequency selective measurement receiver may be used for performing the testing described in this standard provided that the receiver characteristics (that is, sensitivity, selection of bandwidths, detector functions, dynamic range, and frequency of operation) meet the constraints specified in this standard and are sufficient to demonstrate compliance with the applicable limits. Typical instrumentation characteristics may be found in ANSI C63.2.

\subsubsection{Detector}

A peak detector should be used for all frequency domain emission and susceptibility measurements. This device detects the peak value of the modulation envelope in the receiver bandpass. Measurement receivers are calibrated in terms of an equivalent Root Mean Square (RMS) value of a sine wave that produces the same peak value. When other measurement devices, such as oscilloscopes, non-selective voltmeters, or broadband field strength sensors are used for susceptibility testing, correction factors should be applied for test signals to adjust the reading to equivalent RMS values under the peak of the modulation envelope.

\subsubsection{Computer-controlled receivers}

A description of the operations being directed by software for computer-controlled receivers should be included in the EMITP. Verification techniques used to demonstrate proper performance of the software should also be included. 


\subsubsection{Emission testing}

\subsubsection{Bandwidth}

The measurement receiver bandwidths listed in Table 5.1 should be used for emission testing. These bandwidths are specified at the $6 \mathrm{~dB}$ down points for the overall selectivity curve of the receivers. The video filtering should not be used to bandwidth limit the receiver response. If a controlled video bandwidth is available on the measurement receiver, it should be set to its greatest value. Larger receiver bandwidths may be used; however, they may result in higher measured emission levels. No bandwidth correction factors should be applied to test data due to the use of larger bandwidths.

Table 5-1 Bandwidth and measurement time

\begin{tabular}{cccc}
\hline Frequency Range & $6 \mathrm{~dB}$ Bandwidth & Dwell time & $\begin{array}{c}\text { Minimum } \\
\text { Measurement Time }\end{array}$ \\
\hline $30 \mathrm{~Hz}-1 \mathrm{kHz}$ & $10 \mathrm{~Hz}$ & $0.15 \mathrm{sec}$ & $0.015 \mathrm{sec} / \mathrm{Hz}$ \\
$1 \mathrm{kHz}-10 \mathrm{kHz}$ & $100 \mathrm{~Hz}$ & $0.015 \mathrm{sec}$ & $0.15 \mathrm{sec} / \mathrm{kHz}$ \\
$10 \mathrm{kHz}-150 \mathrm{kHz}$ & $1 \mathrm{kHz}$ & $0.015 \mathrm{sec}$ & $0.015 \mathrm{sec} / \mathrm{kHz}$ \\
$150 \mathrm{kHz}-30 \mathrm{MHz}$ & $10 \mathrm{kHz}$ & $0.015 \mathrm{sec}$ & $1.5 \mathrm{sec} / \mathrm{MHz}$ \\
$30 \mathrm{MHz}-1 \mathrm{GHz}$ & $100 \mathrm{kHz}$ & $0.015 \mathrm{sec}$ & $0.15 \mathrm{sec} / \mathrm{MHz}$ \\
Above $1 \mathrm{GHz}$ & $1 \mathrm{MHz}$ & $0.015 \mathrm{sec}$ & $15 \mathrm{sec} / \mathrm{GHz}$ \\
\hline
\end{tabular}

\subsubsection{Emission identification}

All emissions regardless of characteristics should be measured with the measurement receiver bandwidths specified in Table 5.1 and compared against the applicable limits. 
Identification of emissions with regard to a narrowband or broadband categorization is not applicable.

\subsubsection{Frequency scanning}

For emission measurements, the entire frequency range for each applicable test should be scanned. Minimum measurement time for analog measurement receivers during emission testing should be as specified in Table 5.2. Synthesized measurement receivers should step in one-half bandwidth increments or less, and the measurement dwell time should be as specified in Table 5.2. For equipment that operates such that potential emissions are produced at only infrequent intervals, times for frequency scanning should be increased as necessary to capture any emissions.

Table 5-2 Susceptibility scanning

\begin{tabular}{ccc}
\hline Frequency Range & $6 \mathrm{~dB}$ Bandwidth & Dwell time \\
\hline $30 \mathrm{~Hz}-1 \mathrm{MHz}$ & $0.0333 \mathrm{f}_{0} / \mathrm{sec}$ & $0.05 \mathrm{f}_{0}$ \\
$1 \mathrm{MHz}-30 \mathrm{MHz}$ & $0.00667 \mathrm{f}_{0} / \mathrm{sec}$ & $0.01 \mathrm{f}_{0}$ \\
$30 \mathrm{MHz}-1 \mathrm{GHz}$ & $0.00333 \mathrm{f}_{0} / \mathrm{sec}$ & $0.005 \mathrm{f}_{0}$ \\
$1 \mathrm{GHz}-40 \mathrm{GHz}$ & $0.00167 \mathrm{f}_{0} / \mathrm{sec}$ & $0.0025 \mathrm{f}_{0}$ \\
\hline
\end{tabular}

\subsubsection{Emission data presentation}

Amplitude versus frequency profiles of emission data should be automatically generated and displayed at the time of test and should be continuous. The displayed information should account for all applicable correction factors (transducers, attenuators, cable loss, and the like) and should include the applicable limit. Manually gathered data is not acceptable except for verification of the validity of the output. Plots of the displayed data should provide a minimum frequency resolution of $1 \%$ or twice the measurement receiver bandwidth, whichever is less stringent, and minimum amplitude 
resolution of $1 \mathrm{~dB}$. The above resolution requirements should be maintained in the reported results of the EMITR.

\subsection{Susceptibility testing}

\subsubsection{Frequency scanning}

For susceptibility measurements, the entire frequency range for each applicable test should be scanned. For swept frequency susceptibility testing, frequency scan rates and frequency step sizes of signal sources should not exceed the values listed in Table 5.2. The rates and step sizes are specified in terms of a multiplier of the tuned frequency $\left(f_{0}\right)$ of the signal source. Analog scans refer to signal sources, which are continuously tuned. Stepped scans refer to signal sources, which are sequentially tuned to discrete frequencies. Stepped scans should dwell at each tuned frequency for the greater of 3 seconds or the EUT response time. Scan rates and step sizes should be decreased when necessary to permit observation of a response.

\subsubsection{Thresholds of susceptibility}

Susceptibilities and anomalies that are not in conformance with contractual requirements are not acceptable. However, all susceptibilities and anomalies observed during conduct of the test should be documented. When susceptibility indications are noted in EUT operation, a threshold level should be determined, where the susceptible condition is no longer present. Thresholds of susceptibility should be determined as follows and described in the EMITR:

a. When a susceptibility condition is detected, the interference signal should be reduce until the EUT recovers. 
b. The interference signal should be reduce by an additional $6 \mathrm{~dB}$.

c. The interference signal should gradually increase until the susceptibility condition reoccurs. The resulting level is the threshold of susceptibility.

d. The level, frequency range of occurrence, frequency and level of greatest susceptibility, and other test parameters, as applicable must be record.

\subsubsection{Calibration of measuring equipment}

Test equipment and accessories required for measurement in accordance with this standard should be calibrated in accordance with ANSI/NCSL Z540-1 or ISO 10012 or under an approved calibration program traceable to the National Institute for Standards and Technology. In particular, measurement antennas, current probes, field sensors, LISNs (see fig. 5.5 for required impedance), and other devices used in the measurement loop should be calibrated at least every 2 years unless otherwise specified by the procuring activity, or when damage is apparent.

\subsection{Detailed requirements and procedure of the experiments}

This section specifies detailed emissions requirements and the associated test procedures. General test procedures are included in this section. Specific test procedures are implemented by the Government approved EMITP. All results of tests performed to demonstrate compliance with the requirements are to be documented in the EMITR and forwarded to the Command or agency concerned for evaluation prior to acceptance of the

equipment or subsystem. Design procedures and techniques for the control of EMI should be described in the EMICP. Approval of design procedures and techniques described in 
the EMICP does not relieve the supplier of the responsibility of meeting the contractual emission, susceptibility, and design requirements.

\subsubsection{Conducted emissions, power leads, $30 \mathrm{~Hz}$ to $10 \mathrm{kHz}$}

\subsubsection{Applicability}

This requirement is applicable for power leads, including returns that obtain power from other sources not part of the EUT for surface ships, submarines, Army aircraft (including flight line) and Navy aircraft

* For equipment intended to be installed on Navy aircraft, this requirement is applicable only for aircraft with Anti-Submarine Warfare (ASW) capability.

* For AC applications, this requirement is applicable starting at the second harmonic of the EUT power frequency.

\subsubsection{Limits}

Conducted emissions on power leads should not exceed the applicable values shown on fig. 5.6 through 5.8, as appropriate, for low frequency applications.

\subsubsection{Test procedure}

\subsection{Setup and requirements}

This test procedure is used to verify that electromagnetic emissions from the EUT and do not exceed the specified requirements for power input leads including returns. 


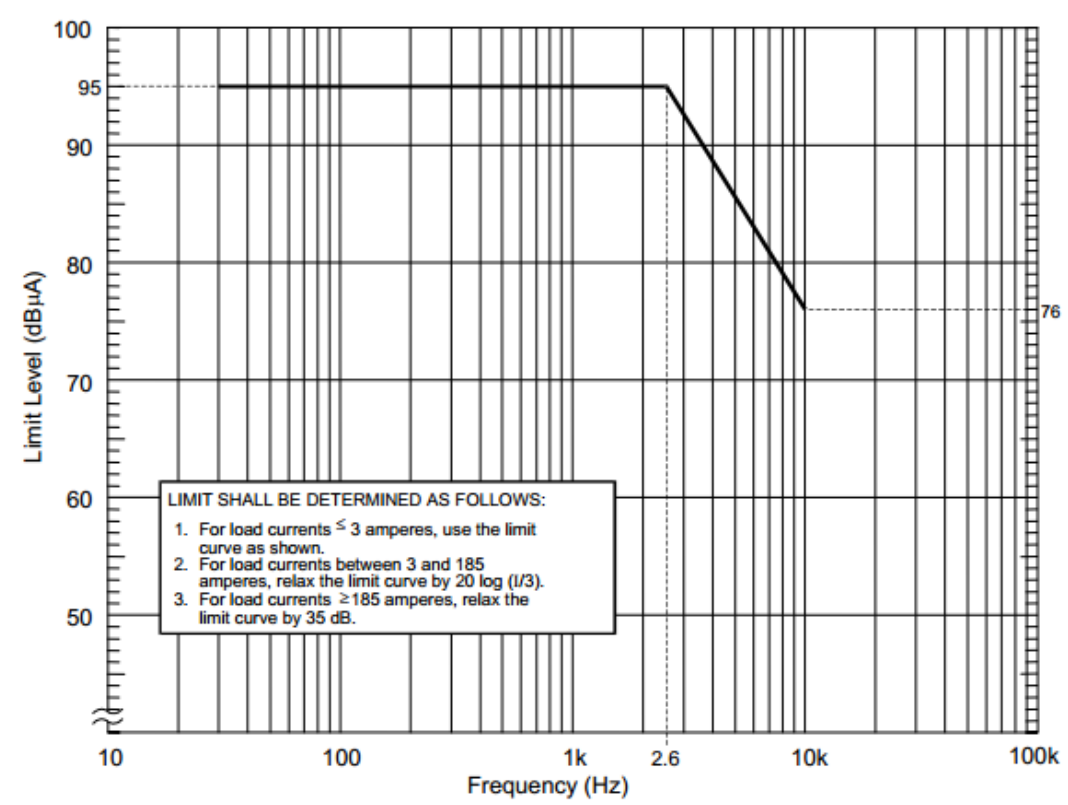

Fig. 5.6 Limit for Conducted emission - submarine application [200]

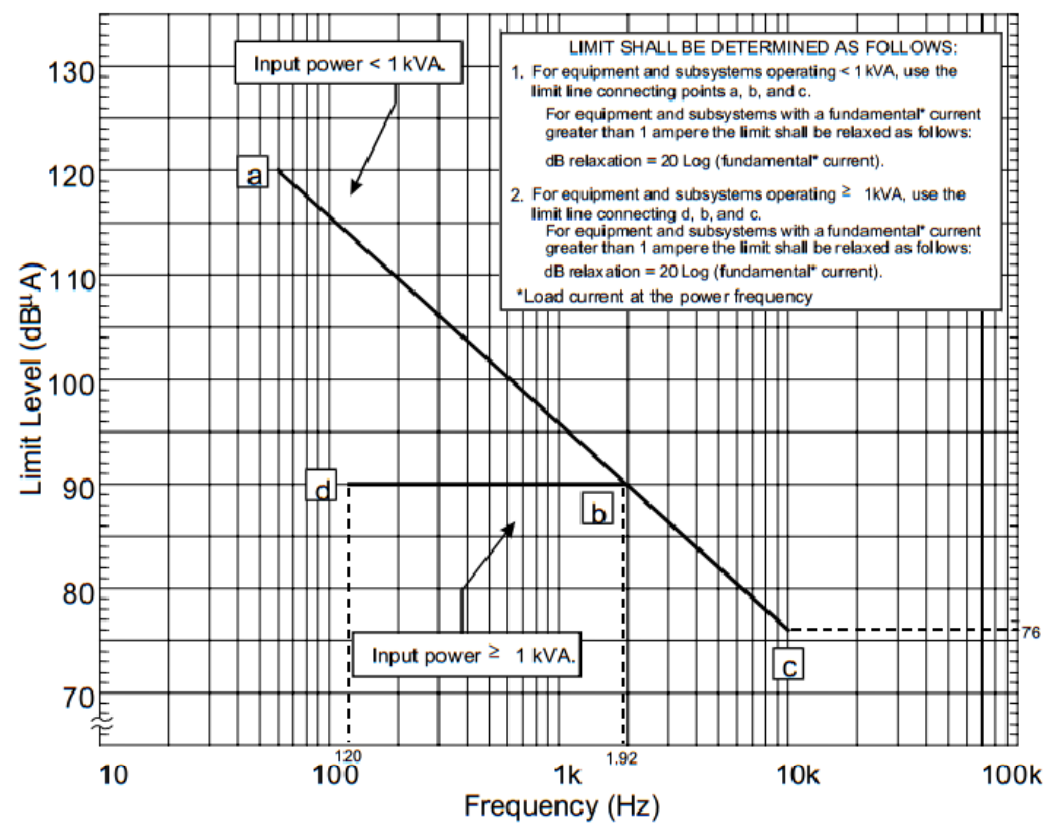

Fig. 5.7 Limit for Conducted emission - surface ship application (60Hz) [200] 


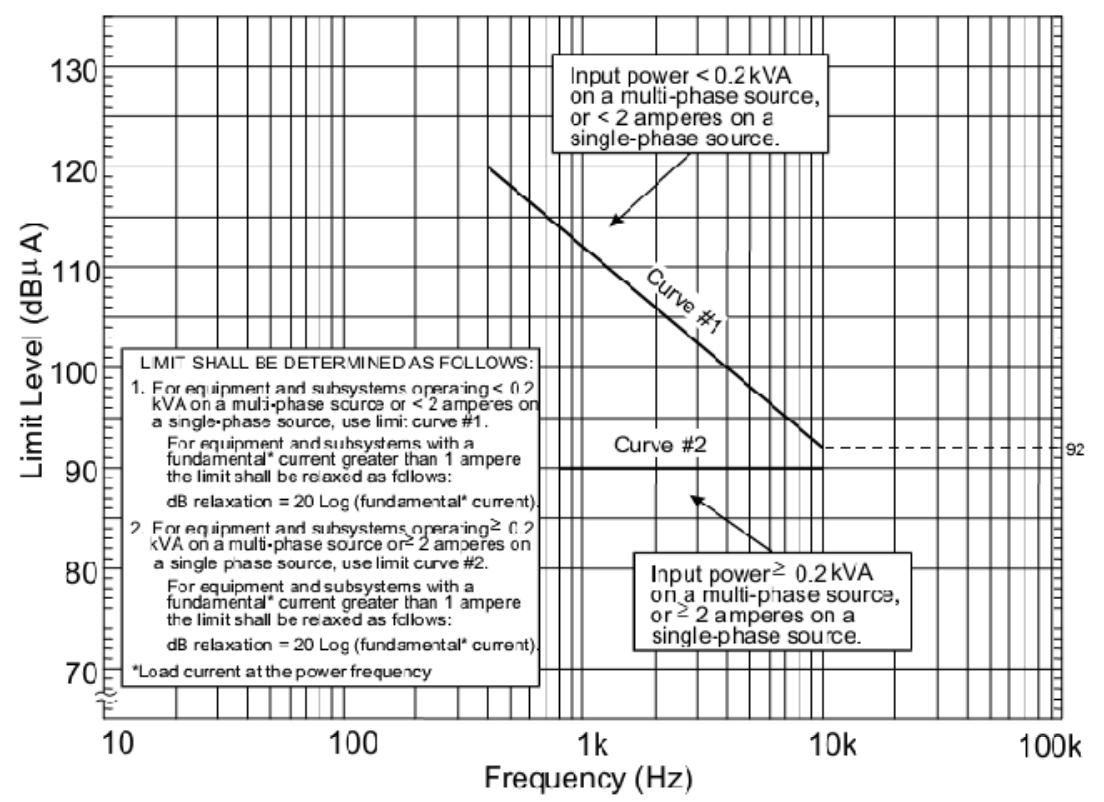

Fig. 5.8 Limit for Conducted emission - surface ship and submarine application $(400 \mathrm{~Hz})$ [200]

The test equipment should be as follows:
a. Measurement receivers
b. Current probes
c. Signal generator
d. Data recording device
e. Oscilloscope
f. Resistor (R)

\section{g. LISNs}

The test setup should be as follows: 
a. Maintain a basic test setup for the EUT as shown and described in fig. 5.1 through 5.4. The LISN may be removed or replaced with an alternative stabilization device when approved by the procuring activity.

b. Calibration. Configure the test setup for the measurement system check as shown in fig. 5.9.

c. EUT testing.

(1) Configure the test setup for compliance testing of the EUT as shown in fig. 5.10.

(2) Position the current probe $5 \mathrm{~cm}$ from the LISN

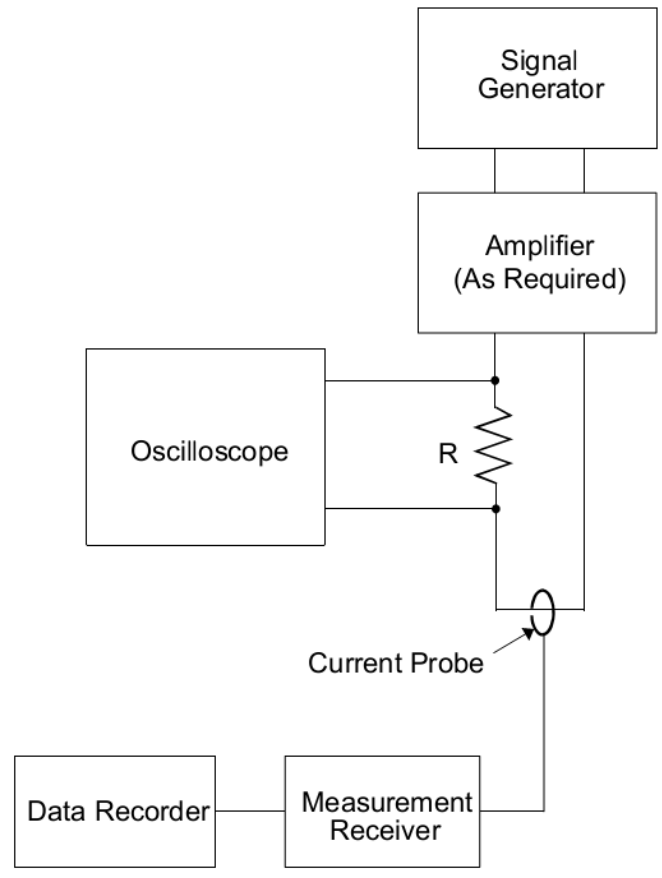

Fig. 5.9 Measurement System check

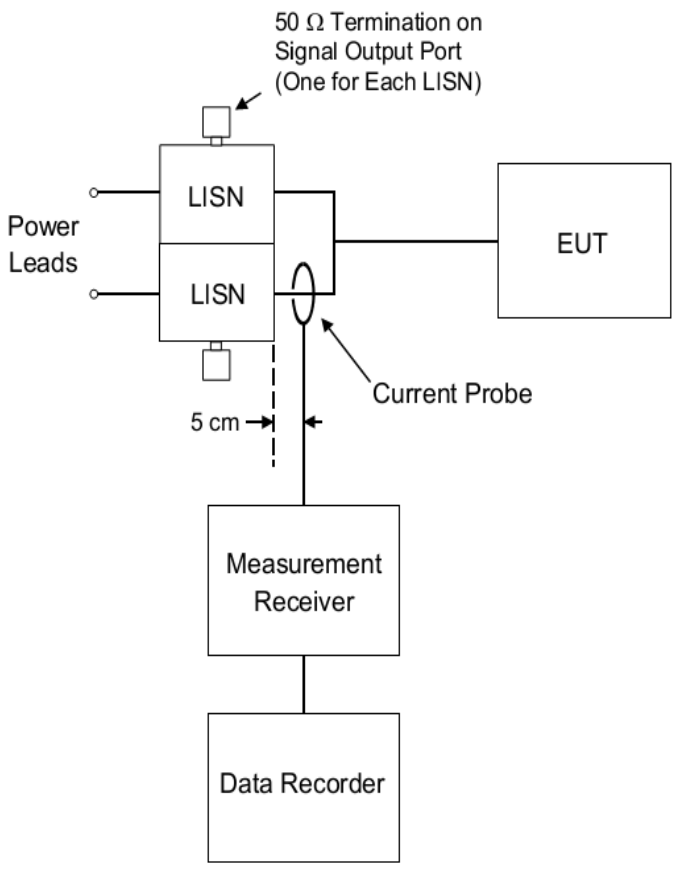

Fig. 5.10 Measurement setup

\subsection{Procedure}

The test procedures should be as follows:

a. Turn on the measurement equipment and allow a sufficient time for stabilization. 
b. Calibration. Evaluate the overall measurement system from the current probe to the data output device.

(1) Apply a calibrated signal level, which is at least $6 \mathrm{~dB}$ below the applicable limit at $1 \mathrm{kHz}, 3 \mathrm{kHz}$, and $10 \mathrm{kHz}$, to the current probe.

(2) Verify the current level, using the oscilloscope and load resistor; also, verify that the current waveform is sinusoidal.

(3) Scan the measurement receiver for each frequency in the same manner as a normal data scan. Verify that the data recording device indicates a level within $\pm 3 \mathrm{~dB}$ of the injected level.

(4) If readings are obtained which deviate by more than $\pm 3 \mathrm{~dB}$, locate the source of the error and correct the deficiency prior to proceeding with the testing.

c. EUT testing. Determine the conducted emissions from the EUT input power leads, including returns.

(1) Turn on the EUT and allow sufficient time for stabilization.

(2) Select an appropriate lead for testing and clamp the current probe into position.

(3) Scan the measurement receiver over the applicable frequency range, using the bandwidths and minimum measurement times specified in Table 5.1.

(4) Repeat 3.2.3.4c (3) for each power lead.

\subsection{Data presentation}

Data presentation should be as follows:

a. Continuously and automatically plot amplitude versus frequency profiles on $\mathrm{X}-\mathrm{Y}$ axis outputs. Manually gathered data is not acceptable except for plot verification. 
b. Display the applicable limit on each plot.

c. Provide a minimum frequency resolution of $1 \%$ or twice the measurement receiver bandwidth, whichever is less stringent, and a minimum amplitude resolution of $1 \mathrm{~dB}$ for each plot.

d. Provide plots for both the measurement and system check portions of the procedure.

\subsubsection{Radiated Emissions, magnetic field $30 \mathrm{~Hz}-100 \mathrm{kHz}$}

\subsubsection{Applicability}

This requirement is applicable for radiated emissions from equipment and subsystem enclosures, including electrical cable interfaces. The requirement does not apply to radiation from antennas.

\subsubsection{Limits}

Magnetic field emissions should not be radiated in excess of the levels shown in figures 5.11 and 5.12 at a distance of $7 \mathrm{~cm}$. 


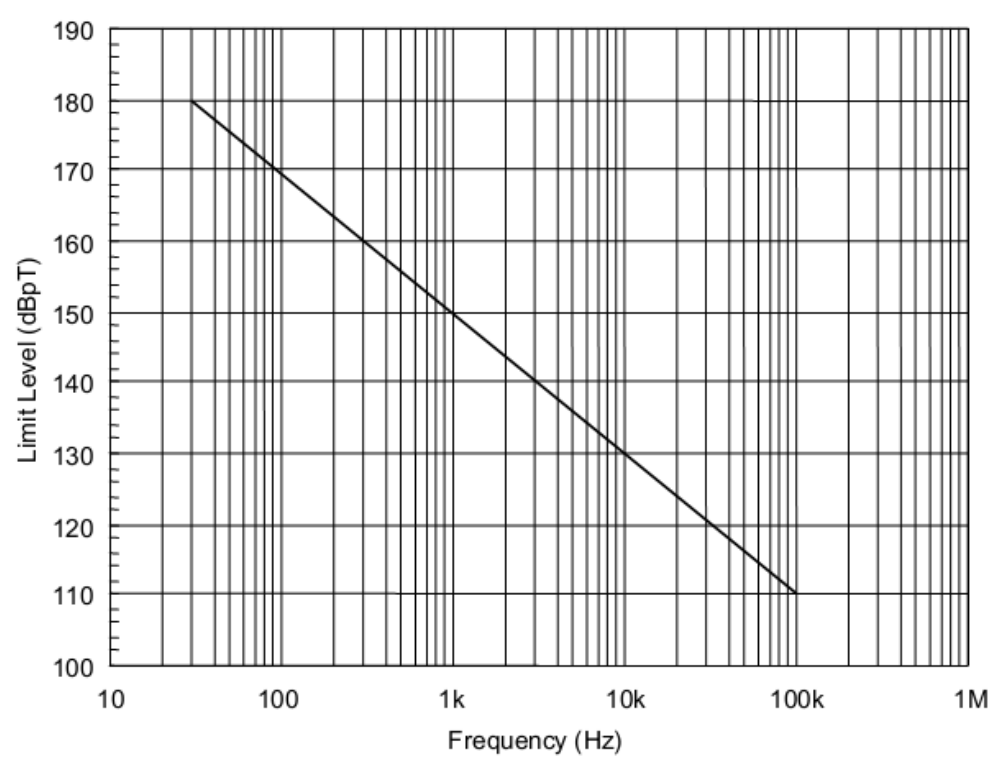

Fig. 5.11 Radiation emission limit for all Army applications

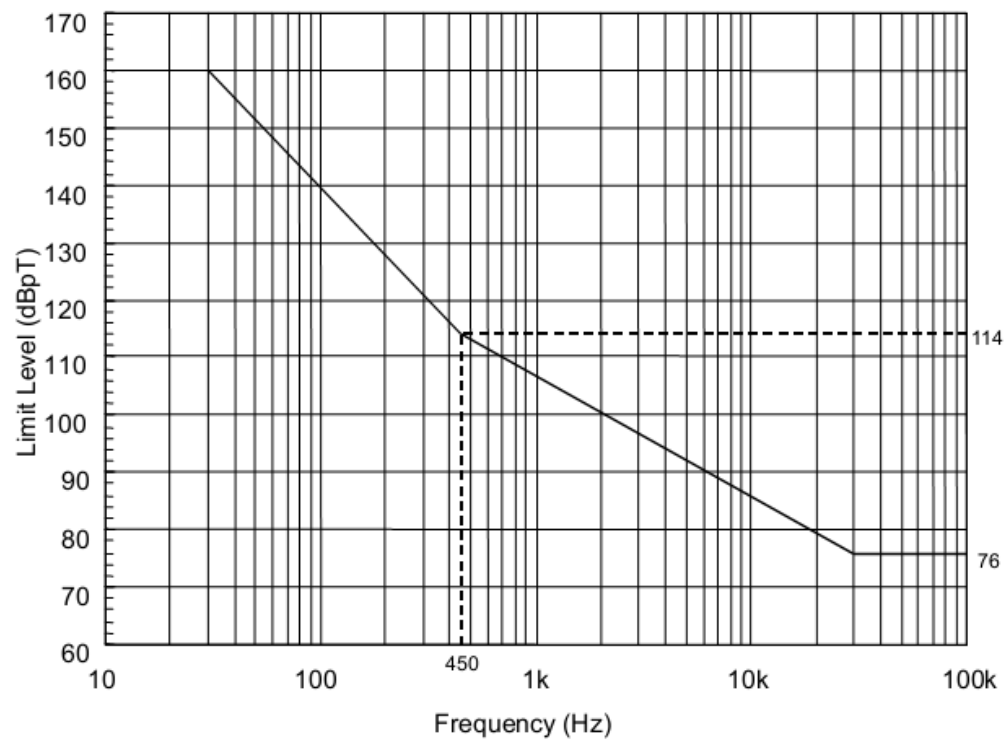

Fig. 5.12 Radiation emission limit for all Navy applications

\subsubsection{Test procedures}

This test procedure is used to verify that the magnetic field emissions from the EUT and its associated electrical interfaces do not exceed specified requirements. 


\subsection{Test equipment and setup}

The test equipment should be as follows:

a. Measurement receivers

b. Data recording device

c. Loop sensor having the following specifications:

(1) Diameter: $13.3 \mathrm{~cm}$

(2) Number of turns: 36

(3) Wire: DC resistance between 5 and 10 ohms (7-41 litz (7strand, No. 41 AWG))

(4) Shielding: Electrostatic

(5) Correction factor: To convert measurement receiver readings expressed in decibels above one microvolts $(\mathrm{dBuV})$ to decibels above one picotesla $(\mathrm{dBpT})$, add the factor shown in fig. 5.11.

d. LISNs

e. Ohmmeter

f. Signal generator

The test setup should be as follows:

a. Maintain a basic test setup for the EUT as shown and described in figures 5.1 through 5.4.

b. Calibration. Configure the measurement setup as shown in fig. 5.13.

c. EUT Testing. Configure the measurement receiving loop and EUT as shown in fig. 5.14 . 


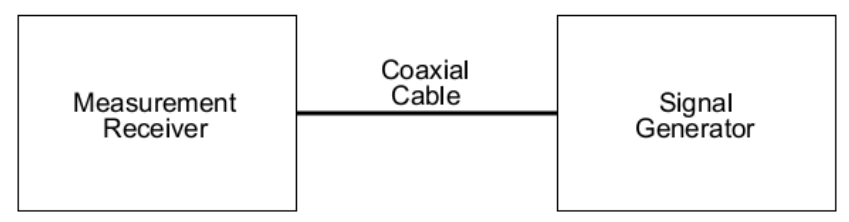

Fig. 5.13 Calibration configuration

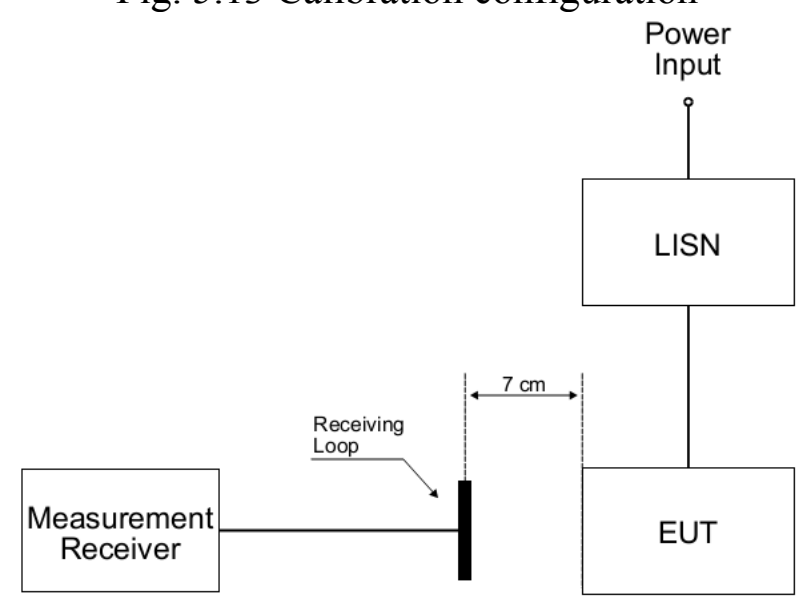

Fig. 5.14 Basic Test setup

\subsection{Procedures}

The test procedures should be as follows:

a. Turn on the measurement equipment and allow sufficient time for stabilization.

b. Calibration.

(1) Apply a calibrated signal level, which is at least $6 \mathrm{~dB}$ below the limit (limit minus the loop sensor correction factor), at a frequency of $50 \mathrm{kHz}$. Tune the measurement receiver to a center frequency of $50 \mathrm{kHz}$. Record the measured level.

(2) Verify that the measurement receiver indicates a level within $\pm 3 \mathrm{~dB}$ of the injected signal level.

(3) If readings are obtained which deviate by more than $\pm 3 \mathrm{~dB}$, locate the source of the error and correct the deficiency prior to proceeding with the testing. 
(4) Using an ohmmeter, verify that the resistance of the loop sensor winding is approximately 10 ohms.

c. EUT Testing.

(1) Turn on the EUT and allow sufficient time for stabilization.

(2) Locate the loop sensor $7 \mathrm{~cm}$ from the EUT face or electrical interface connector being probed. Orient the plane of the loop sensor parallel to the EUT faces and parallel to the axis of connectors.

(3) Scan the measurement receiver over the applicable frequency range to locate the frequencies of maximum radiation, using the bandwidths and minimum measurement times of Table 5.2.

(4) Tune the measurement receiver to one of the frequencies or band of frequencies identified in c of (3) above.

(5) Monitor the output of the measurement receiver while moving the loop sensor (maintaining the $7 \mathrm{~cm}$ spacing) over the face of the EUT or around the connector. Note the point of maximum radiation for each frequency identified in step c (4).

(6) At $7 \mathrm{~cm}$ from the point of maximum radiation, orient the plane of the loop sensor to give a maximum reading on the measurement receiver and record the reading. If the measured emission exceeds the limit at the $7 \mathrm{~cm}$ distance, increase the measurement distance until the emission falls within the specified limit. Record the emissions and the measurement distance for assessment by the procuring activity. NOTE: The EUT should comply with the applicable radiated emission $(30 \mathrm{~Hz}-10 \mathrm{kHz})$ limit at $7 \mathrm{~cm}$. 
(7) Repeat step c (4) through step c (6) for at least two frequencies of maximum radiation per octave of frequencies below $200 \mathrm{~Hz}$ and for at least three frequencies of maximum radiation per octave above $200 \mathrm{~Hz}$.

(8) Repeat step c (2) through step c (7) for each face of the EUT and for each EUT electrical connector.

\subsection{Data presentation}

Data presentation should be as follows:

Provide graphs of scans and tabular listings of each measurement frequency, mode of operation, measured magnetic field, and magnetic field limit level.

\subsection{Conclusion}

In this chapter, the requirement and procedure of electromagnetic compatibility testing were explained. The procedure for the measurement of radiated emission, as well as conducted emission, was described in details; however, the conducted emission is not the purposed of this dissertation.

All the details were related to the low frequency analysis. Note that the requirements for high frequency analysis are different for both emission and susceptibility studies. 


\section{Wire Modeling and Experimental Verification}

\subsection{Overview}

The 3D full finite element modeling was described in chapter 3 and 4. Also, the equivalent source modeling using initial dipole models and cube models were explained in chapter 4 . The final model, which was voltage-current rectangular prism model, has a good accuracy for the magnetic and electric stray fields. However, there is an issue that this model is designed based on optimization processes, which means that the model is applicable for one particular voltage or specific amount of power. If sizes of the component change, the model should be optimized again to find new parameters of branches and new dimensions. Therefore, the new model is proposed based on the structure and operation of the components to be applicable for all parameter sets without any need to be modeled or simulated again.

Consequently, the principle of the model is explained in the next section. Then, modeling strategies of each component with the simulation results are demonstrated. Afterward, the whole setup is modeled and verified experimentally. Finally, the procedure of generalization of the model is explained in detail along with the simulation results.

\subsection{Wire-modeling approach}

As explained in chapter 4, the electromagnetic signature study of an electric machine can be estimated at a far distance based on (4-14) through (4-18). 
The path and direction of currents, as well as the value of the current density, of the electric machine winding have a very important role in establishing magnetic stray fields at far distances. The magnetic field's waveform at a far distance is under the influence of direction of wires in winding arrangements. Therefore, a line-shape model is proposed instead of the actual model and the related current based on the current density of the actual machine is applied to the lines. The model of the proposed actual machine (Squirrel Cage Induction Motor, SCIM) and the proposed model are shown in fig. 6.1.

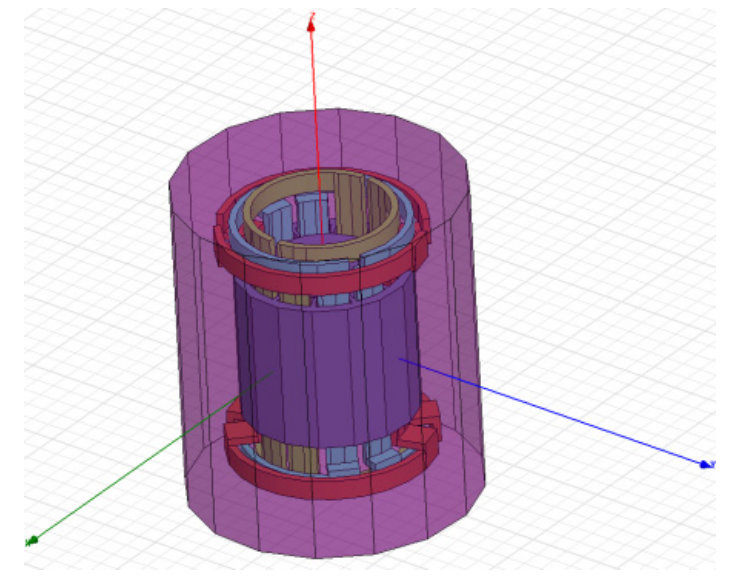

(a)

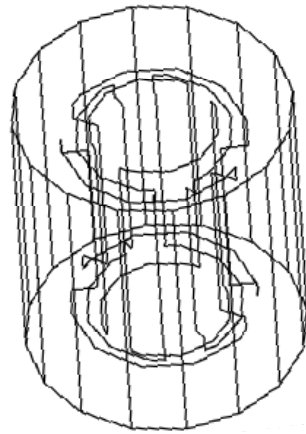

(b)

Fig. 6.1 Prototype of the proposed machine (SCIM) in Finite Element Analysis (a) actual model, (b) An equivalent line-shape model for EMI and electromagnetic signature studies.

The wire model of the system is designed and created based on the current directions. The path of the winding arrangement for the machine and other components, including the position of voltage terminals, should be identified. As shown in this figure, the wire model consists of numerous lines with specific currents flowing and voltages established at nodes of these wires. Currents of the wire model are calculated based on equalizing the 
magnetic field densities. Using the Biot-Savart law, the radiated magnetic field density of a line at an $\mathrm{R}$ distance away from the line is as follows:

$B_{l}=\frac{\mu_{0}}{4 \pi} \int \frac{I_{l} d l \times \hat{a}_{R}}{R^{2}}$

Where the $l$ is the length of the line and $\mathrm{I}_{l}$ is the carrying current of the line and $\hat{a}_{R}$ is the distance vector between $d l$ and the observation point. Similarly, for a volume current, the radiated magnetic field density at an $\mathrm{R}$ distance is as follows:

$B_{v}=\frac{\mu_{0}}{4 \pi} \int \frac{J d v \times \hat{a}_{R}}{R^{2}}$

The idea of this model is to have the same field, while the model is a line and doesn't have a cross-section. Hence, by equalizing two above equations and considering $\mathrm{J}, \mathrm{R}, d s$ and $d l$ as known parameters, then, the $\mathrm{I}_{l}$, the current amplitude of the line, can be calculated. The voltage of nodes are similarly calculated by equalizing the electric field due to the charge distribution of the line and volume. Each component has some parameters which should be considered about this modeling and explained in their section. More details about basics of the model are mentioned in [201].

\subsection{Models of the components with simulation and experimental results}

A typical power setup consists of electrical generators, such as synchronous generator, electrical motors, such as induction and DC motors, and connection cables and power converters. All of these components except the converter are modeled using the wire model and some of them are verified experimentally. The converter has some consideration and its modeling for stray field analysis is explained in the next chapter. In 
addition to the study of each component, their coupling is also studied. Finally, the whole setup is investigated.

\subsubsection{Induction motor}

\subsubsection{The modeling concept}

Before investigating complete wire model, the combination of wire model for magnetic field and cube model for the electric field are investigated and introduced in this part. The procedure for designing the wire model is explained in 6.2. Since the modeling is based on the electric current, it gives good results just for the magnetic field, so the model doesn't show logical results for the electric field. In order to have the electric field of the actual machine at a far distance, a cube model is proposed, which is shown in fig 6.2. The details of making this cube are explained in chapter 4 .

As shown in fig. 6.2, eight voltages are applied to the nodes at the corners of the cube. The value of these voltages are based on the maximum electric potential difference and also the electric displacement field in the winding.

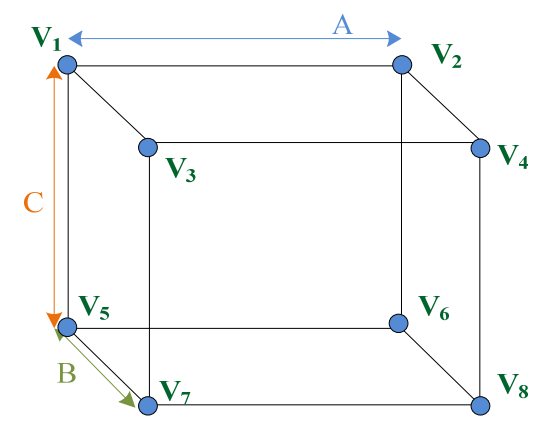

Fig. 6.2 Prototype of the proposed cube model for replicating electric field of the actual machine 
Consequently, to have both the magnetic and electric field of the model simultaneously, these two models (fig. 6.1(b) and fig. 6.2) are combined together. The combined model propagates similar electric and magnetic fields at far distances. This model is shown in fig. 6.3.

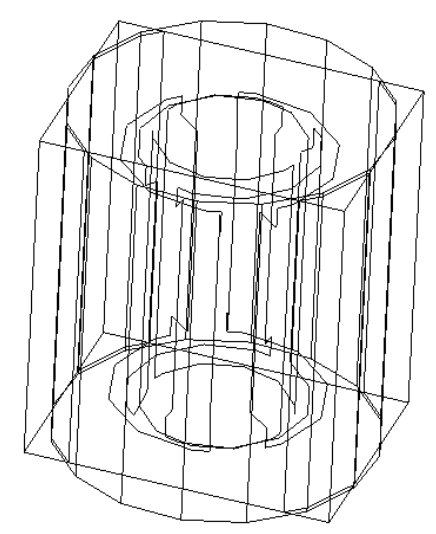

Fig. 6.3 The equivalent cylinder-cube model for reproducing radiated electric and magnetic fields of the actual machine

For simulation purposes, a 3-phase, $380 \mathrm{~V}, 5 \mathrm{~A}, 120$ turn/phase induction machine with a stack length of $0.15 \mathrm{~m}$ and outer diameter of $0.175 \mathrm{~m}$ is simulated in the $3 \mathrm{D}$ electromagnetic finite element domain for a specific time. The meshed final model is shown in fig. 6.4. The number of degrees of freedom of the source model is considered as large as possible, in order to have accurate results of the propagation in measured areas. In addition, appropriate element growth rate is applied to the model and the tolerance of analysis is considered at $1 \mathrm{e}-6$.

Using the optimization process, the cube lengths were calculated as $(A=0.1009 \mathrm{~m}$, $\mathrm{B}=0.125 \mathrm{~m}$ and $\mathrm{C}=0.1282 \mathrm{~m}$ ). Moreover, as mentioned earlier, the size of the cylinder is based on the size of the actual machine. Although the analysis is implemented for a 
typical machine, this equivalent source model can be used for similar types of machines (induction machine) with slight modification, for example, the size of the model. This modification in size can be based on the ratio of the size of any actual machine to a basic actual machine model. This basic model can be the machine studied in this section. Other parameters, such as the voltage and current values can be considered as well. Although the optimization is used for the design of the cube model for having the correct electric field, the new model, which eliminates the cube and optimization process, is employed and explained in 6.3.3.

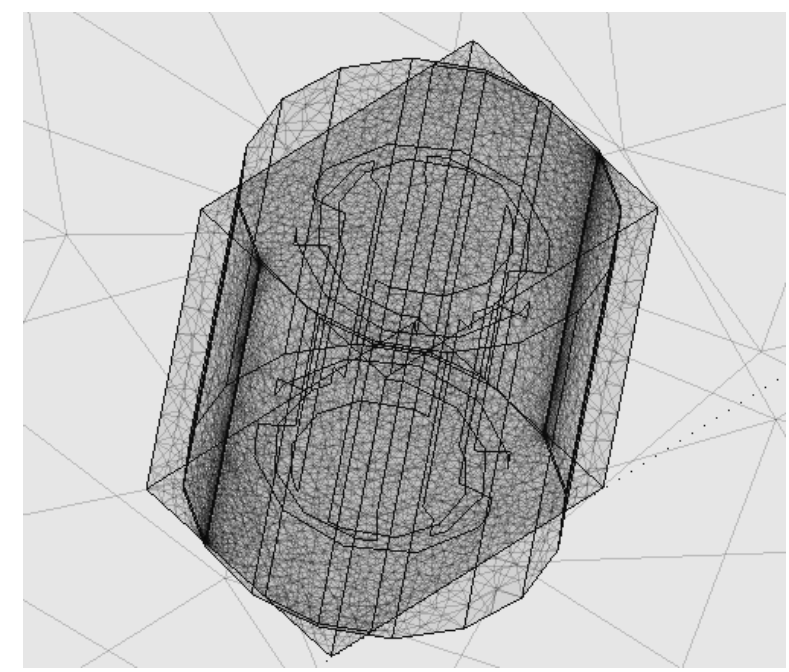

Fig. 6.4 Mesh of the equivalent source model

\subsubsection{Simulation results}

In order to verify the accuracy of the model, propagated electric and magnetic fields from the proposed model and the actual machine along three lines in the $\mathrm{x}, \mathrm{y}$, and $\mathrm{z}$ directions at a far distance, fig. 6.5(a), were calculated and compared. This comparison is shown in fig. 6.5(b). The position of the reference lines from which propagated electric and magnetic fields are measured is also shown in fig. 6.5(a). As shown in fig. 6.5(b) and 6.6, the model propagates similar electric and magnetic fields in comparison with actual 
model at far distances. Because of the adjacency of the radiated electric fields in the $\mathrm{x}$ and y lines, a magnified view depicting details is shown in fig. 6.6.

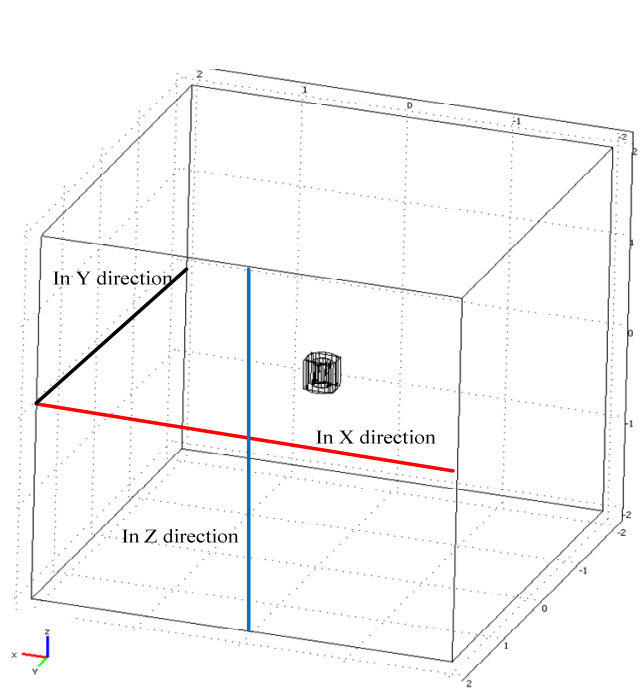

(a)

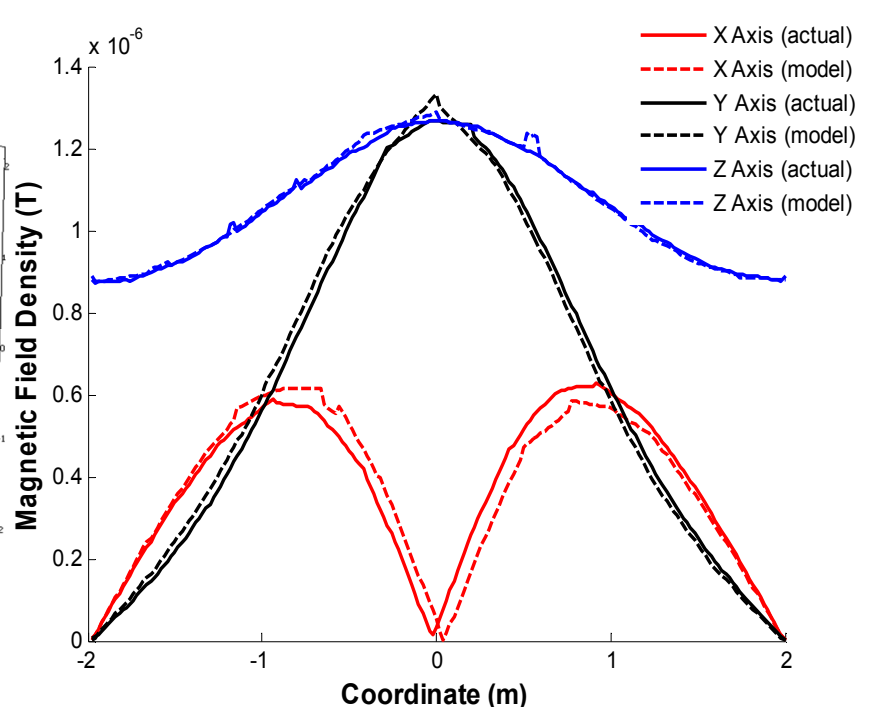

(b)

Fig. 6.5 (a) Reference lines from which propagated electric and magnetic field are measured, (b) Propagated magnetic field from actual and proposed model in all three axes

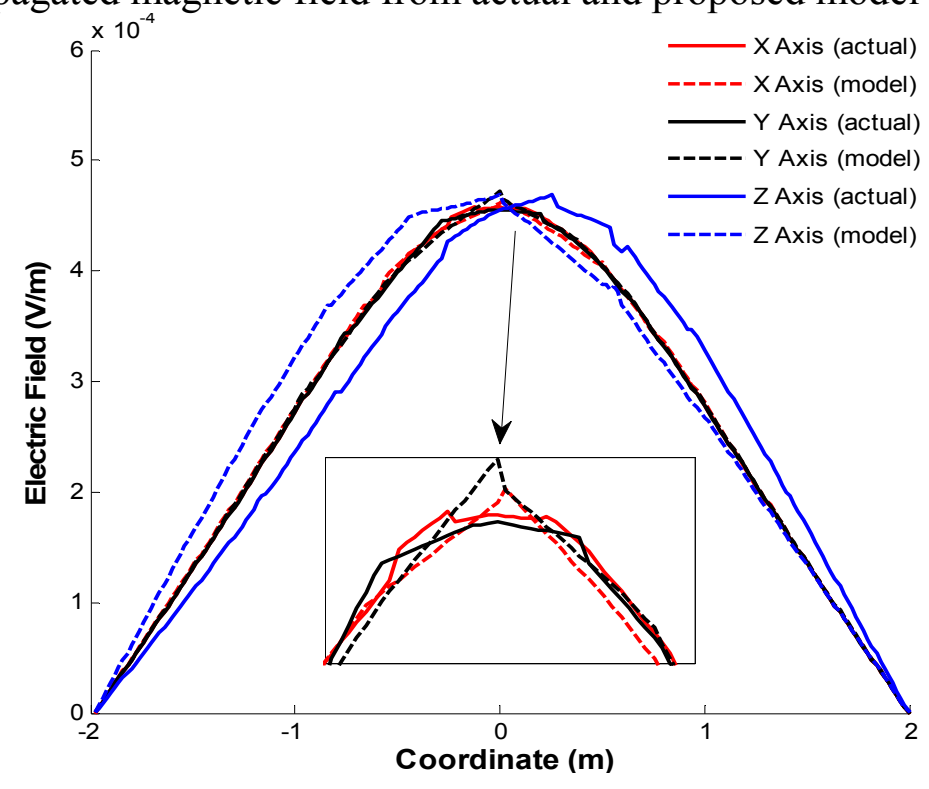

Fig. 6.6 Propagated electric field from actual and proposed model in all three axes

As shown in fig. 6.5(a), radiated magnetic flux densities along different axes are different in various planes and even in the same plane. For example, the measured 
magnetic flux density along the $\mathrm{Z}$ axis shows the lower amplitude compared with the one along the $\mathrm{Y}$ axis. Since the structure of winding in which the shaft of the motor and windings are along the $\mathrm{Z}$ axis, the current flows mostly along the same axis and subsequently doesn't change significantly. Consequently, the field doesn't change considerably along the axis.

Following the investigation of far fields, the checking of fields at closer distances to the model was conducted. The propagated magnetic field along the $\mathrm{X}$ axis is obtained and shown in fig. 6.7. This figure shows the propagated magnetic field density at one meter closer to the model. The difference between the propagated magnetic field density in the $\mathrm{X}$ axis direction, shown in figs. 6.5 and 6.7 , is because of the butterfly effect of magnetic field density in farther distances [108]. Furthermore, fig. 6.7 shows that there are some ripples in the fields. These ripples are related to the high frequency response. Therefore, since the result of the equivalent source model in rippled parts follows the actual one, it can be inferred that the model can also be used in the high frequency analysis.

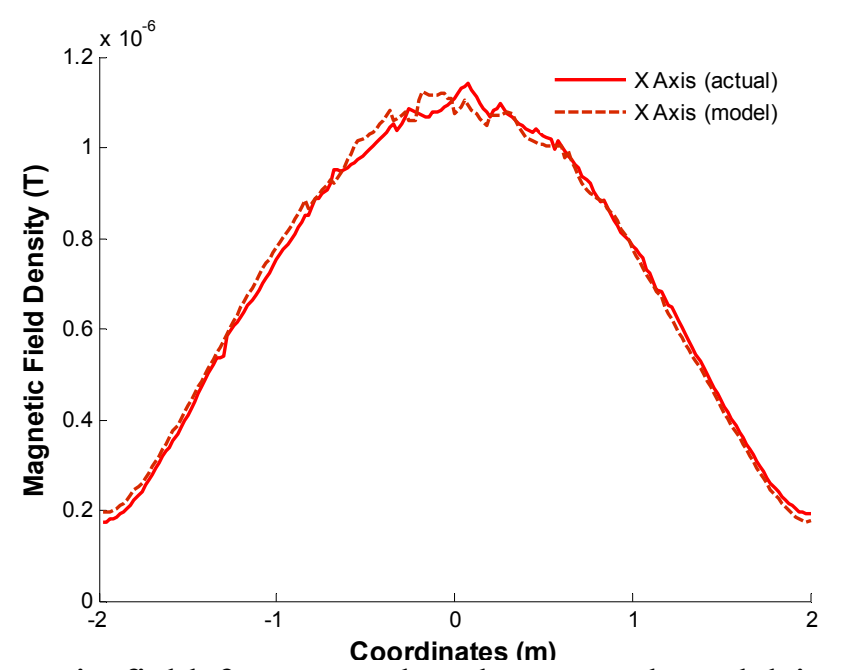

Fig. 6.7 Radiated magnetic field from actual and proposed model in $\mathrm{X}$ axis at1 meter distance to the models 
Figures $6.5(\mathrm{~b})$ to 6.7 show the fields at one line in the planes, which are shown in fig. 6.5(a). This analysis is essential but not sufficient for investigating the accuracy, because one may think that the fields may resemble each other just in the center line due to symmetry. For further investigation and to assure that the fields propagated from the actual machine and equivalent source models resemble each other at all positions in the study area, the propagated magnetic and electric fields spectrum throughout the whole XY plane were obtained and shown in fig. 6.8.

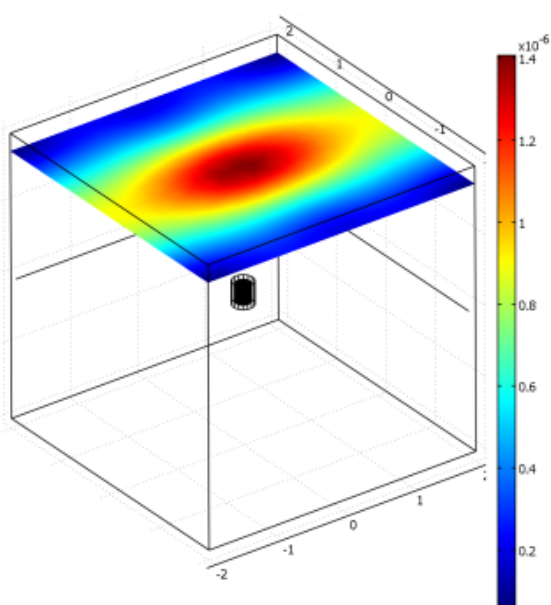

(a)

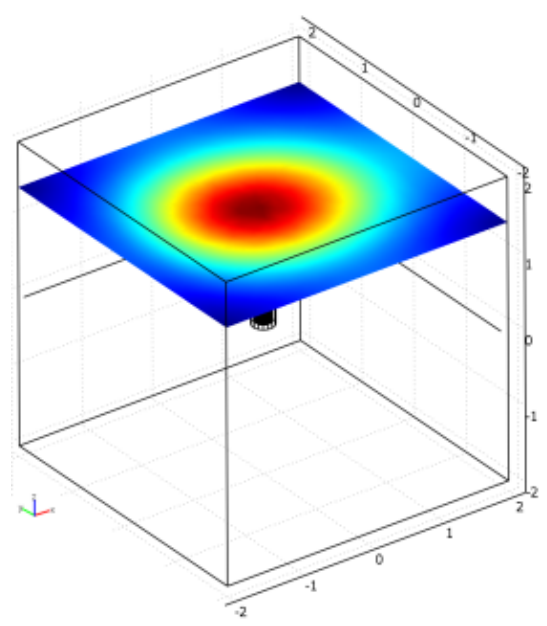

(c)

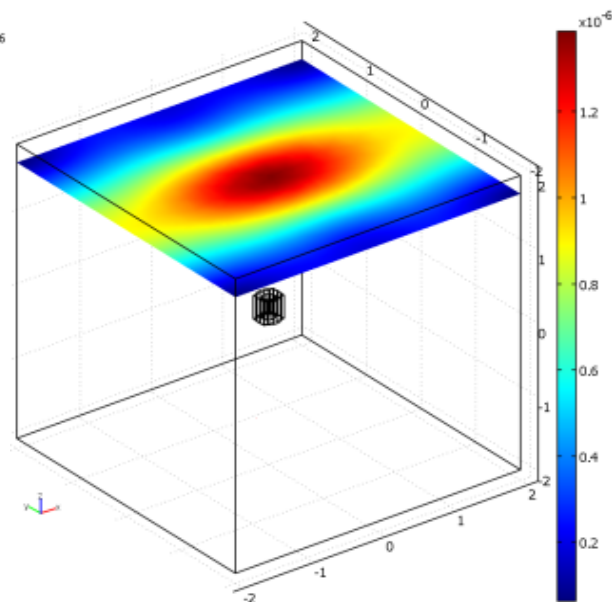

(b)
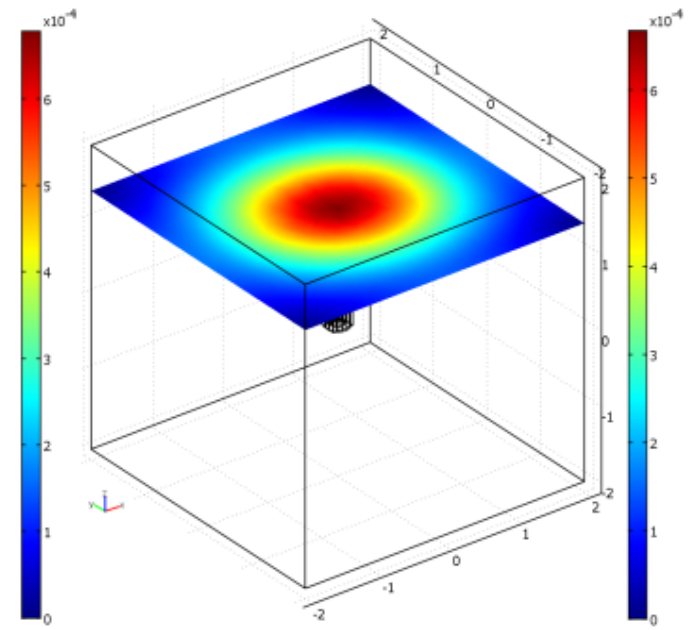

(d)

Fig. 6.8 Magnetic and electric field spectrum throughout XY plane propagated from actual machine and model 
Comparing figs 6.8 (a) and (b), it can be seen that, not only so the wave-shapes of the magnetic field density of the two models match, but also their amplitude is almost the same in all points of the plane. Also, the electric fields of both models, which are shown in figs. 6.8 (c) and (d), are the same at almost all points. This is also valid for all other planes around the model. In conclusion, it is verified that the equivalent source model can replace the actual model for the electromagnetic signature study analysis of one case machine.

Since the final goal of this research is to use this model in multi-component systems, the model is studied for the two-motor case. This can also be considered as validation of the obtained cylinder-cube model from one motor case, and inserted into the model to investigate a multi-machine drive, while the currents in the branches of the cylinder and voltages at the nodes of the cube remain the same as in the first case (single case model). The centers of the coordinates of the two cubes and cylinders are exactly the same as the actual machine model. Figures 6.9 and 6.10 show the comparison between magnetic and electric fields propagated from the actual and proposed models for the two-motor case. Note that the proposed planes and lines for measuring the fields are the same as the single machine case (fig. 6.5(a)). As can be seen, magnetic and electric fields, like the single machine case, follow the same patterns with good accuracy.

The shift in the electric field signatures measured along the $Z$ axis (fig. 6.10) is because of the size of the equivalent source model. As discussed before, an optimization method can be used for fitting the size of the model. If parameters of the optimization varies for example mutation factor are modified to bigger values, this shift would be decreased. This becomes true for the magnetic field as well. 


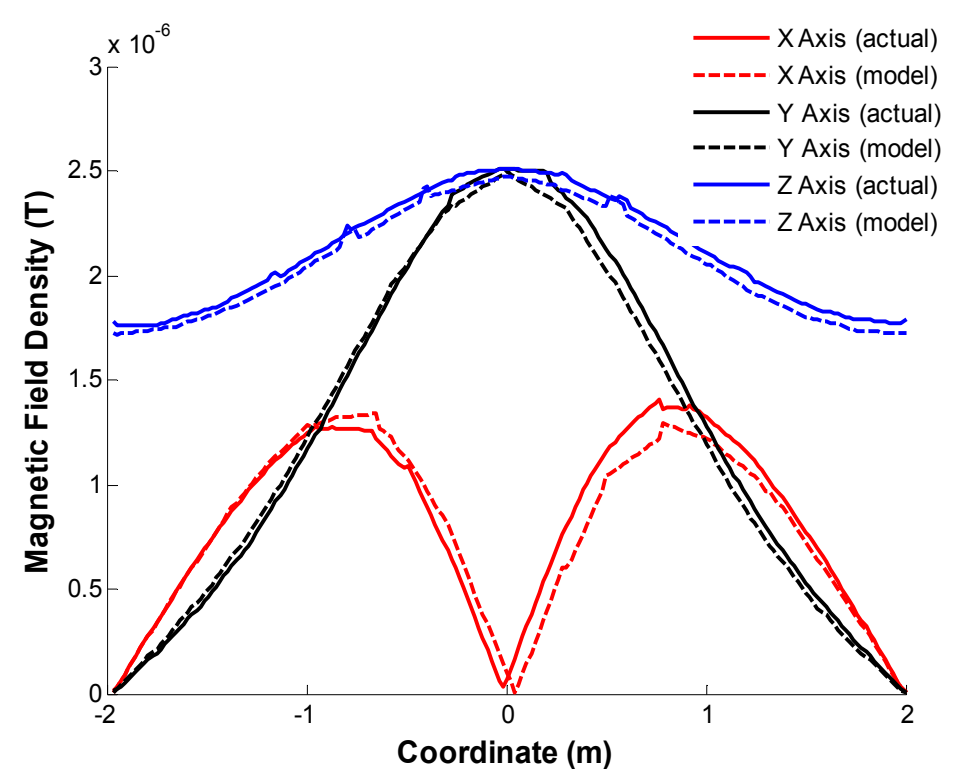

Fig. 6.9 Propagated magnetic field from actual and proposed model for two motor in all three axes

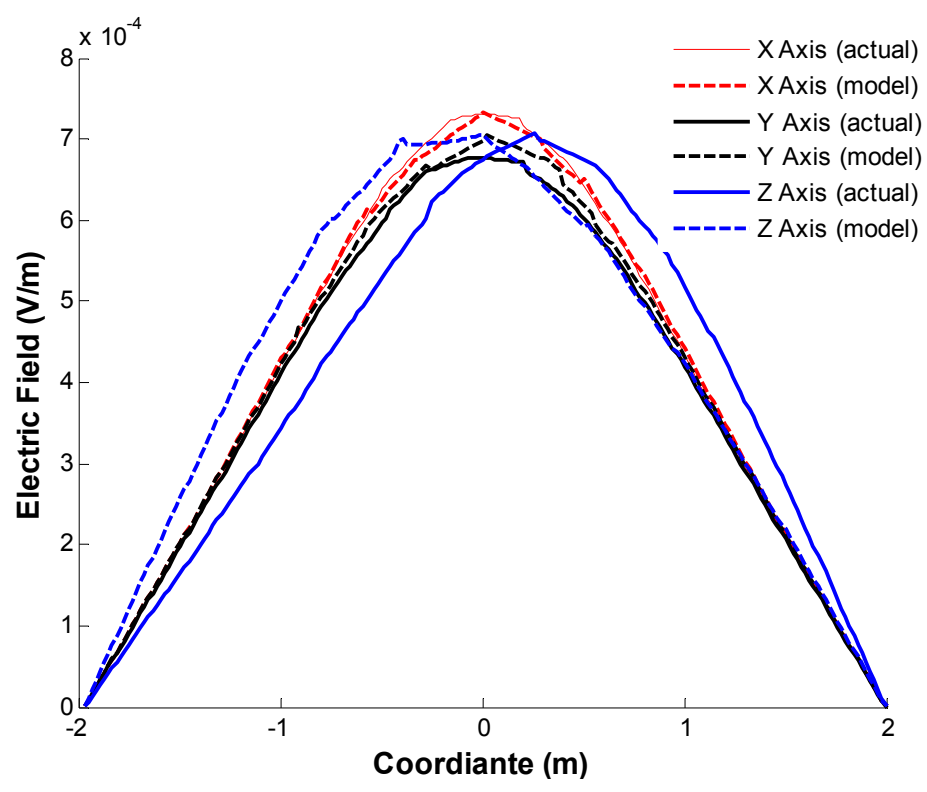

Fig. 6.10 Propagated electric field from actual and proposed model for two motor in all three axes

One of the achievements of this research is the simulation time reduction. The comparison between the simulation time shows that, this approach makes the simulation 
time of the model at least 100 times faster than a full 3D model. More details of the comparison are illustrated in Table 6.1.

Table 6.1 computation time Comparisons

\begin{tabular}{ccc}
\hline Case of Studies & One Machine case & Two Machines case \\
\hline 3DFE model & 6 (Hours) & 11 (Hours) \\
Equivalent model & $100($ Sec. $)$ & $160($ Sec. $)$ \\
\hline
\end{tabular}

In addition to the importance of the accuracy of the field spectrums, the direction of the flowing fields is significant because the field spatial figures, fig. 6.8(a)-(d), don't show the direction of the fields. Hence, the arrow line of the magnetic field density of the actual and equivalent machines are compared and shown in fig. 6.11. As displayed in this figure, the magnetic field in the actual case around the motor is denser in comparison to the equivalent source model case. However, the radiated magnetic field at further distance is almost the same in these two models. This is more important because the model is designed for far distances. While the arrow plot in fig. 6.11 shows the magnetic field density, this can be classified as discrete streamline of this field. A continuous stream-line of the H-Field (magnetic field intensity) of the two models (actual and equivalent source models) is obtained as shown in fig. 6.12. The H-Field streamline also shows that the equivalent source model has very similar results to the actual model. It also shows that the dipoles establish around the equivalent source model also in near distance. It should be noted that the purpose of this model was to obtain resembling fields at far distances. 


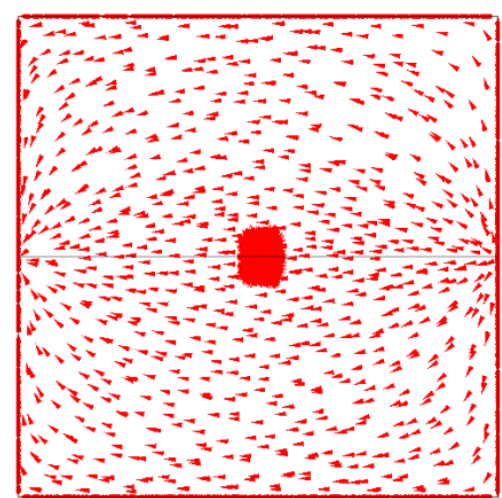

(a)

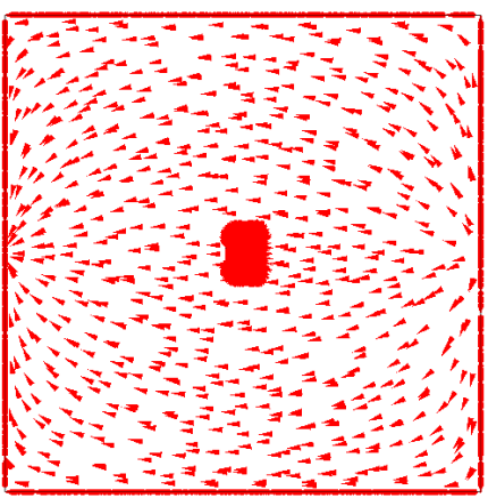

(b)

Fig. 6.11 Arrow plot of magnetic field density (T) of (a) actual machine (b) equivalent source model in xy-plane

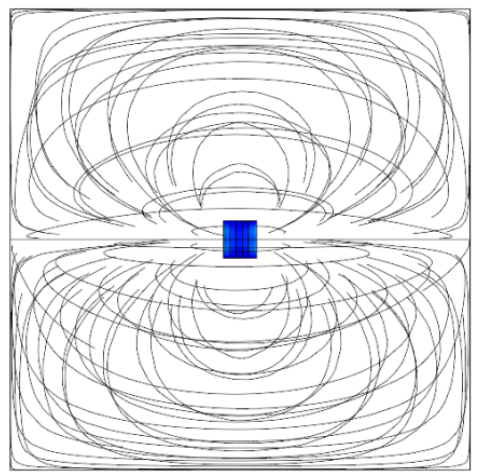

(a)

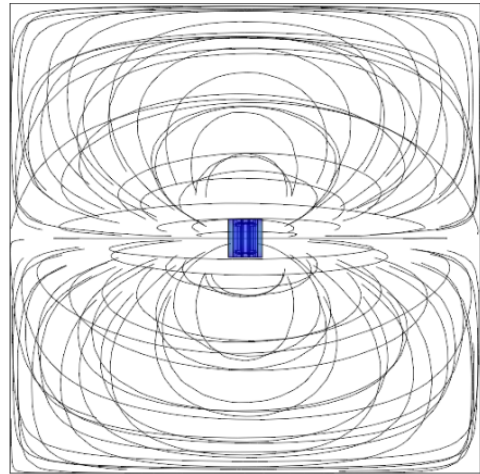

(b)

Fig. 6.12 Stream-line of H-Field of (a) actual machine model xy-plane (A/m) (b) equivalent source model in xy-plane $(\mathrm{A} / \mathrm{m})$

\subsubsection{Frequency response analysis - Multi-Resolution Analysis (MRA)}

Frequency analysis helps to extract the waveform's information that is not readily available in time domain. On the other hand, it determines how the field's waveforms, from real and equivalent source models, are comparable at all of the various frequencies within a certain bandwidth. Multi-Resolution Analysis (MRA) offers a way for analysis of non-stationary waveforms bounded in both frequency and time durations. 
This method breaks up the signal into hierarchical levels of different resolutions, which are matched to different frequency bands as shown in fig. 6.13(b) [202]. The MRA consists of two general successive processes of decomposition and reconstruction. In the orthogonal wavelet decomposition procedure, the decomposition is started with the original signal and it is repeated by consecutive approximations down into coefficient vectors with lower resolution as shown schematically in fig. 6.13 (a). The employed mathematical manipulation for the decomposition step is called discrete WT (DWT). As shown in fig. 6.13(a), along with the decomposition process, low and high pass decomposition filters ( $\mathrm{L}$ and $\mathrm{H})$ and down sampling of the PD signals are applied at each level. The wavelet coefficient vectors may be modified before the reconstruction procedure is commenced. Various types of modifications of the wavelet coefficient vectors with many known applications were employed; de-noising and compression are more drastic and well known among them. All of the detail coefficient vectors and only the final approximations coefficient vector at level $G$ are applied to reconstruct the original signal, but after some modifications, such as de-noising and compression, if required. This reconstruction is performed by up-sampling and another low and high pass reconstruction filters shown in fig. 6.13(a) by L' and H', respectively. The reconstruction procedure is originated from the inverse discrete Wavelet Transform (IDWT) concept. The reversed coefficient vectors, which are calculated by the equal number of reconstruction and decomposition levels, are termed as reconstructed details and approximation $[202,203]$. Here, The Nyquist frequency is defined as the half of the sampling frequency. The sampling frequency is calculated by the division of the displacement by the unit speed of $1(\mathrm{~m} / \mathrm{s})$. 


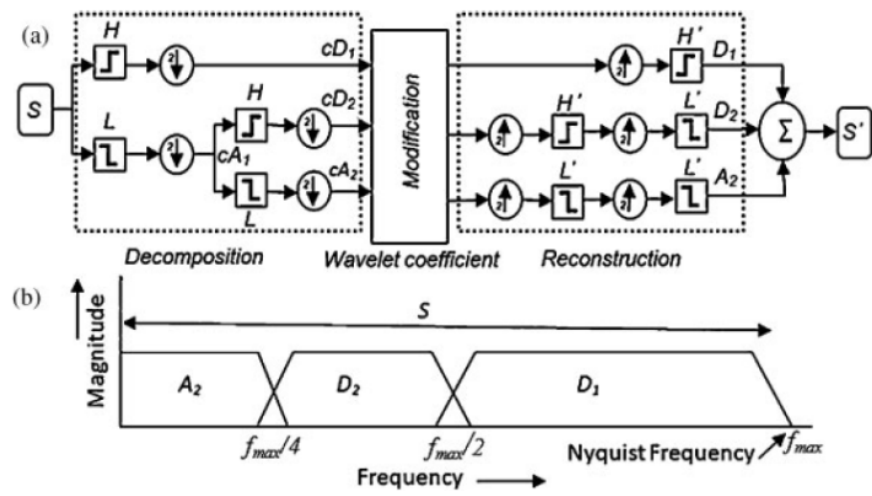

Fig. 6.13 Decomposition and reconstruction by MRA at two levels (a), bandwidth of subsignals (b) [203]

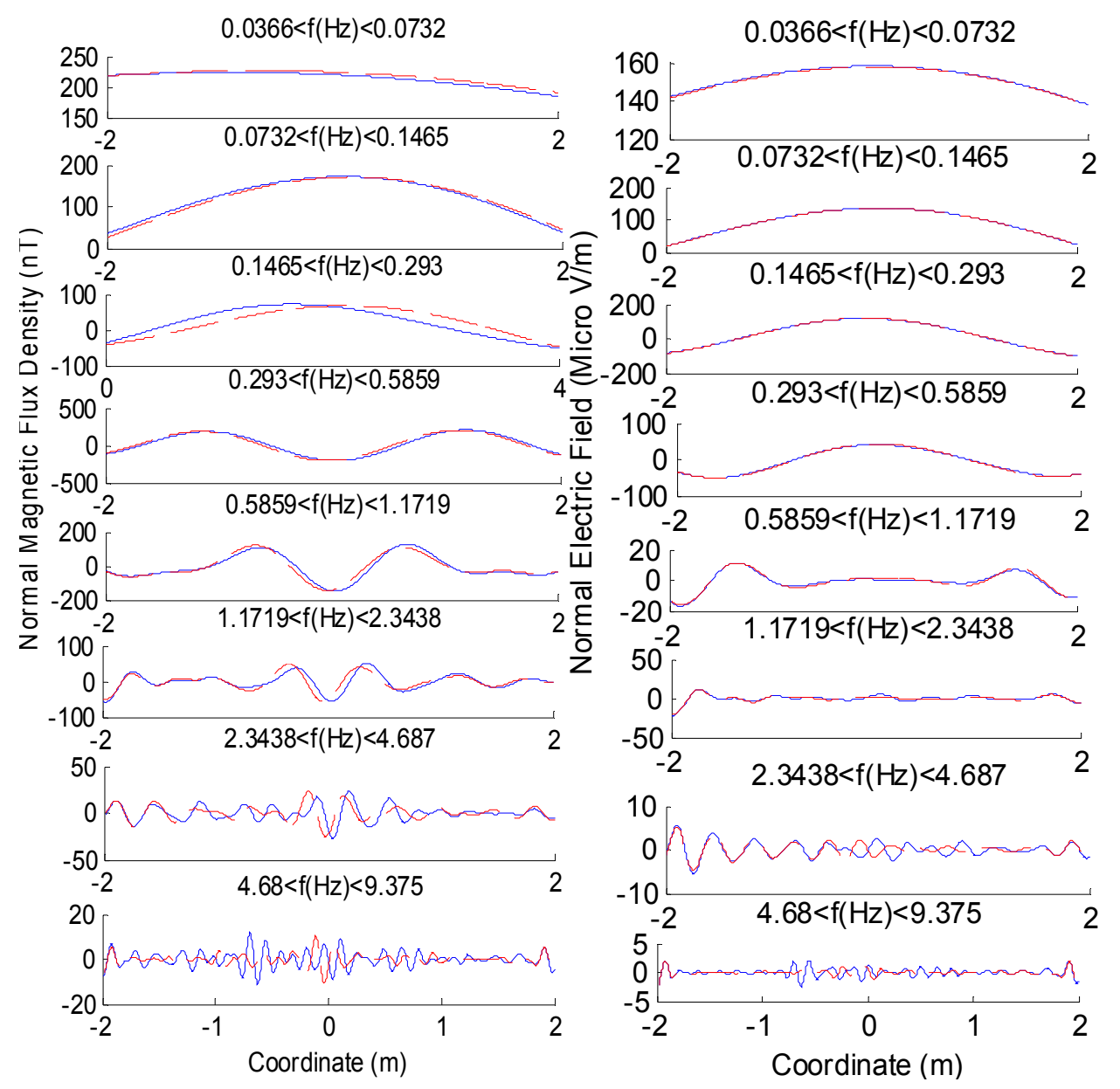

Fig. 6.14 Normal magnetic (left) and electric field (right) comparisons at different frequency band, equivalent (solid blue) real machine (dash line), one machine case of study 
Fig. 6.14 show the reconstructed magnetic and electric fields in the $\mathrm{Y}$ direction at different frequency bands for one machine, respectively. It can be observed that an acceptable matching at different frequency bands exists between the equivalent and real machines almost in the entire frequency band.
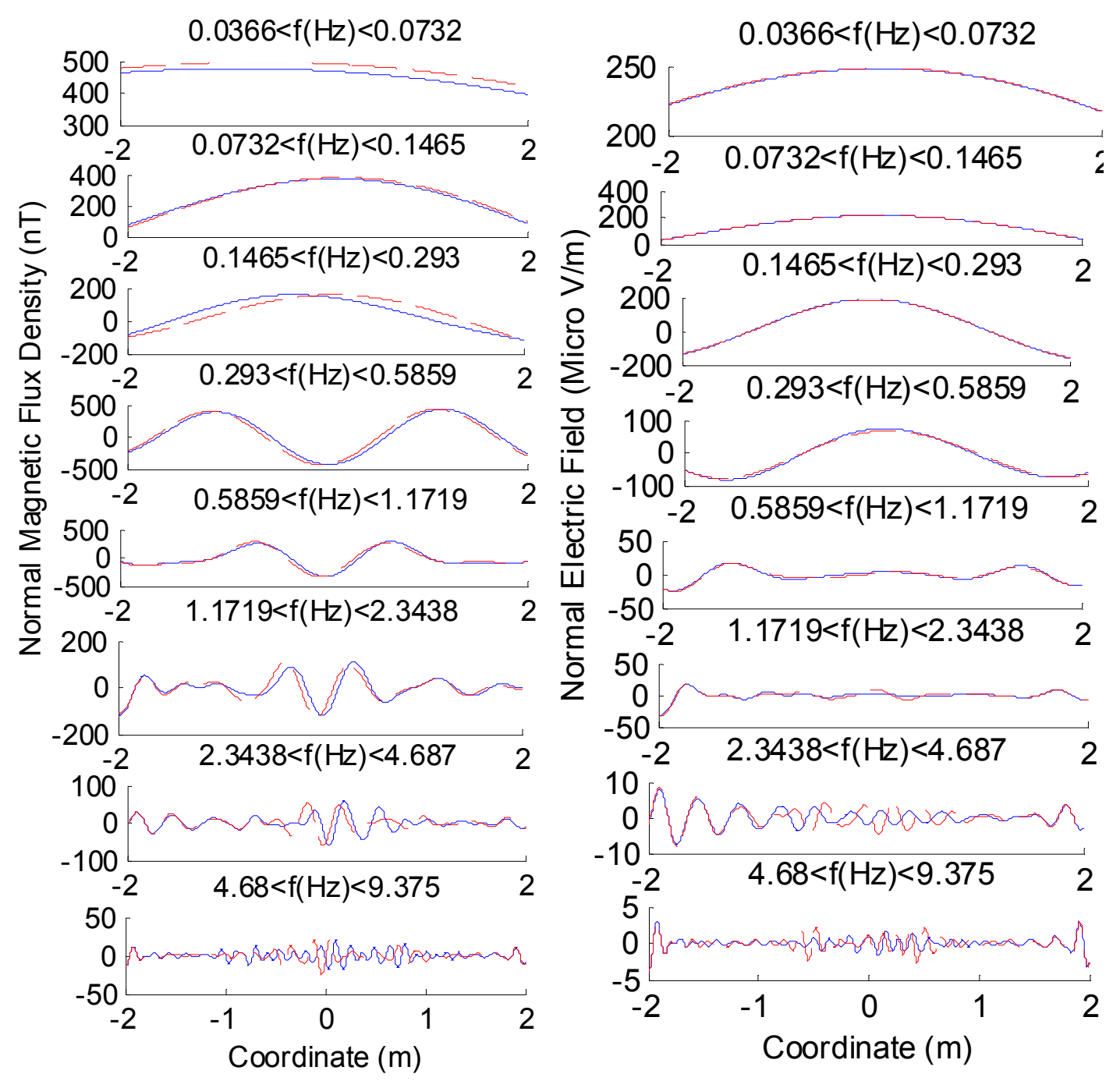

Fig. 6.15 Normal magnetic (left) and electric field (right) comparisons at different frequency bands, equivalent (solid ) real machine ( dash line), two machine case of study

Fig. 6.15 is the reproduction of fig. 6.14 for the two-machine case study. As can be observed, there is an acceptable agreement between the equivalent and real model. 
Moreover, comparison of figs. 6.14 and 6.15 proves that the linear relationship between one machine and two machine case of studies exist in all of the frequency bands. However, the found relationship between one and two machine case of studies depends on the explicit geometrical arrangement of two machine cases.

\subsubsection{Time and rotation study}

\subsection{Time-based analysis}

Since the actual induction machine carries AC current, the time-based analysis is more useful. In the previous sections, the analysis was time-based; however, the figures are just depicted in one typical moment of time. In this section, the radiated electromagnetic fields of different instances of time in one cycle are studied. For brevity, four time instances are selected $(0.0025 \mathrm{~s}, 0.005 \mathrm{~s}, 0.0075$ and $0.0125 \mathrm{~s})$. The voltage amplitude of the terminal of the model during one time cycle is shown in fig. 6.16.

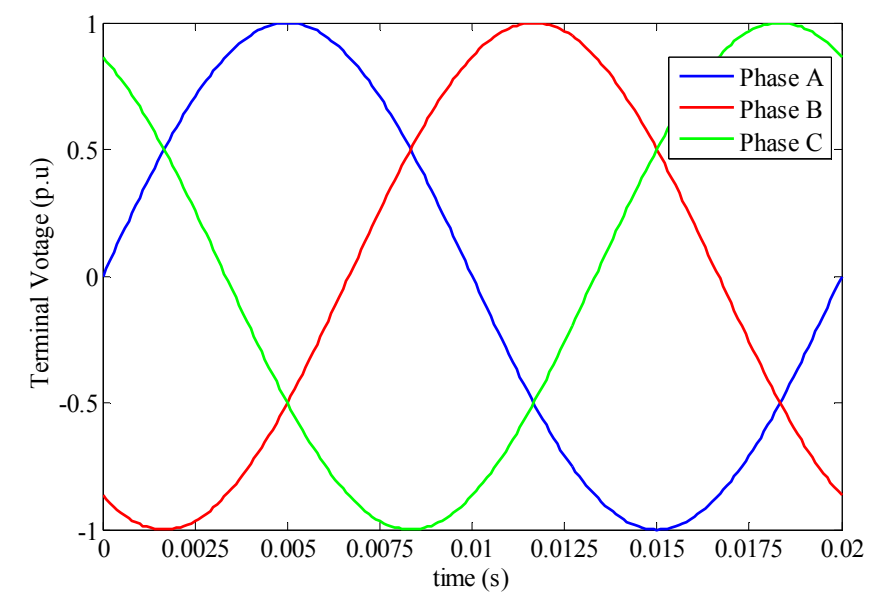

Fig. 6.16 Voltage amplitude of the terminal of the model during one time cycle

First, the radiated magnetic field in the near distance $(0.5 \mathrm{~m})$ from the machine is studied. The magnetic field density measured in four time instants is shown in fig. 6.17. The result shows that the magnetic field rotates by the variation in time, although the 
position of the maximum field point remained unchanged. It can be inferred from this result that the model resembles the machine and can be used instead of that, at all-time instants, not just one time instant, in which the model is designed. Next, the radiated magnetic field at a far distance $(\sim 10 \mathrm{~m})$ from the machine is studied. In this distance, the rule of the magnetic dipoles for these distances causes the field to become similar to a dipole, as shown in fig. 6.17 [204]. As shown in this figure, the dipoles are sensitive to time changes and they rotate when the time changes. Consequently, the equivalent source model can be used for the time-based analysis at near and far distances.

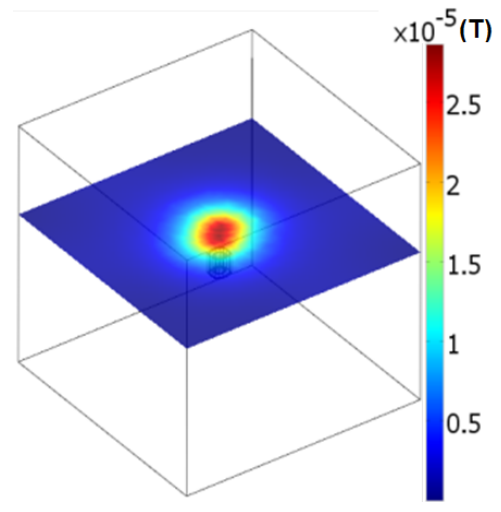

(a) $\mathrm{t}=0.0025$

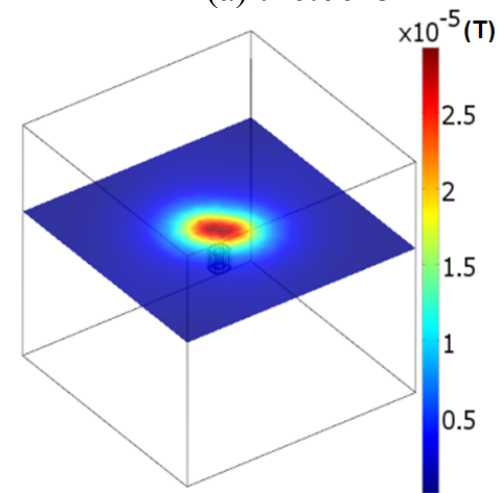

(c) $\mathrm{t}=0.0075$

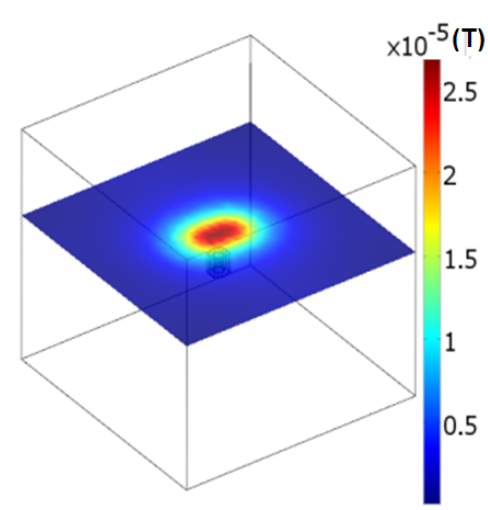

(b) $\mathrm{t}=0.005$

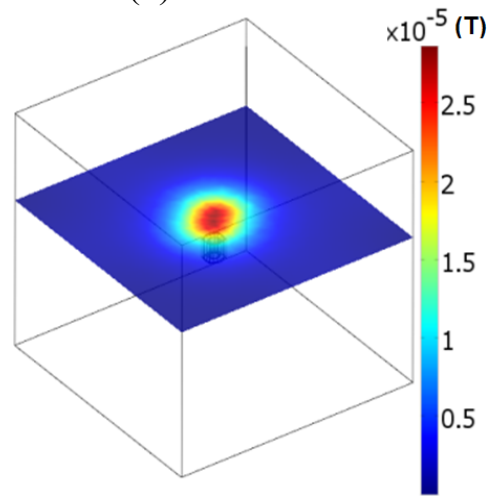

(d) $\mathrm{t}=0.0125$

Fig. 6.17 Magnetic field density (B) of equivalent source model in four different moments of time at near distance 


\subsection{The effect of rotation}

Another condition that should be studied for the induction machine is testing various positions of the machine. In many cases, the location of the motor with respect to the measured points will change. Therefore, the electromagnetic signatures are expected to be changed. Hence, a specific change of the motor is studied here. The whole machine was rotated around an axis and the results were obtained and illustrated in fig. 6.18. The magnetic field in this figure is plotted at a far distance.

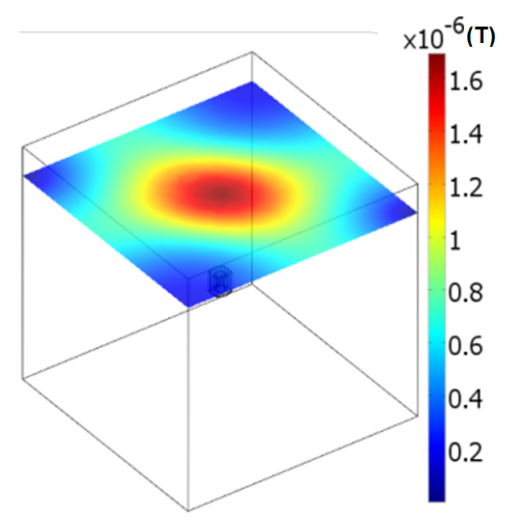

(a) $\mathrm{t}=0.0025$

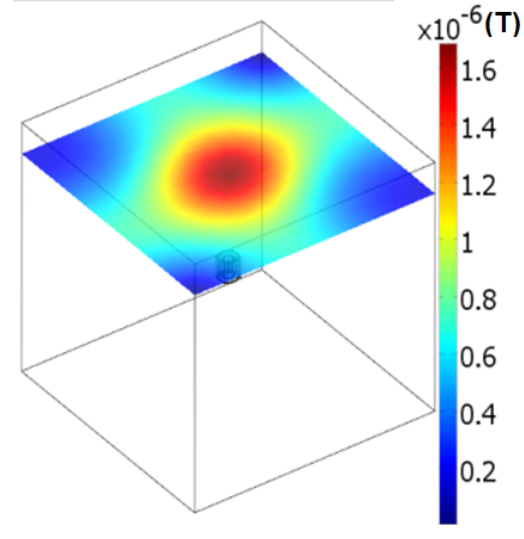

(c) $\mathrm{t}=0.0075$

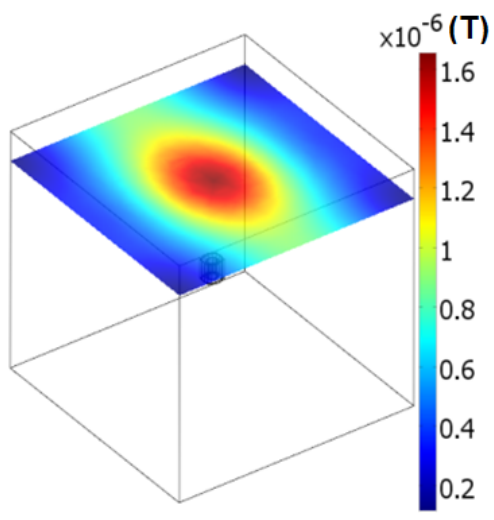

(b) $t=0.005$

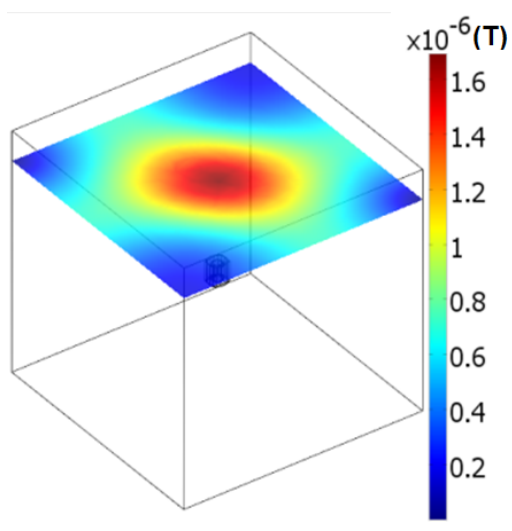

(d) $\mathrm{t}=0.0125$

Fig. 6.18 Magnetic field density (B) of equivalent source model at the four different intendances of time at far distance 


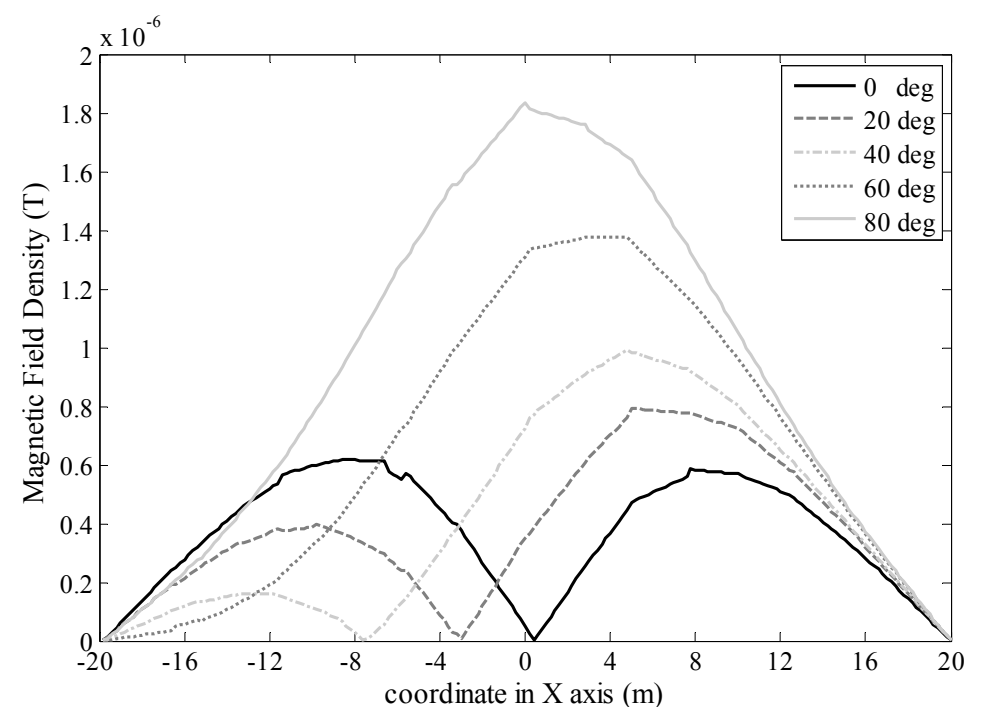

Fig. 6.19 Deviation of magnetic field density (B) of the equivalent source model due to the rotation of the whole machine around z-axis

As shown in fig. 6.19, by rotating the rotor of the induction machine around z-axis, the magnetic field moves along the perpendicular coordinates $(\mathrm{x}, \mathrm{y})$ from right to left. When plotting other angles ranging from 90 to 180 degrees, the results are exactly symmetrical with respect to the changes from 0 to 90 degrees. The magnetic field density of 180 degree change is exactly the same as the one with 0 degree change. This study is useful in identifying the situation of the source machine by looking at the electromagnetic signatures at far distances. All of these studies can be imported to an optimization program, such as genetic algorithm or neural network. Therefore, the machine in any situation can be recognized.

\subsubsection{Experimental verification}

The procedure and standard of the experimental measurement of the low frequency stray fields are explained in chapter 5. The details of the setup, which are shown in fig. 6.20, are as follows: 
The coil antenna and the real-time spectrum analyzer, which are used in the measurement, are specifically for low frequency analysis with high precision. The frequency range is between $20 \mathrm{~Hz}-500 \mathrm{kHz}$. The winding of the antenna is 36 turns of 7-41 litz wire shielded with 10-Ohms resistance and $340 \mu \mathrm{H}$ inductance. The antenna and the setup are located based on the standards (MIL-461-STD [200], MIL-462-STD). The spectrum analyzer also covers $1 \mathrm{~Hz}-3 \mathrm{GHz}$ with $\pm 0.5 \mathrm{~dB}$ absolute amplitude accuracy to $3 \mathrm{GHz}$. The details of the components are mentioned in Table 6.2.

Table 6.2 the characteristics of the components

\begin{tabular}{ll}
\hline Components & \multicolumn{1}{c}{ Description } \\
\hline $\begin{array}{l}\text { EMI } \\
\text { receiver/spectrum } \\
\text { analyzer }^{*}\end{array}$ & $\begin{array}{l}\text { Coverage between } 1 \mathrm{~Hz}-3 \mathrm{GHz}, \text { absolute amplitude accuracy: } \\
\pm 0.5 \mathrm{~dB} \text { to } 3 \mathrm{GHz}, \text { displayed average noise level: }-142 \mathrm{dBm} / \mathrm{Hz} \\
\text { at } 26.5 \mathrm{GHz},-155 \mathrm{dBm} / \mathrm{Hz} \text { at } 2 \mathrm{GHz} \text { and }-150 \mathrm{dBm} / \mathrm{Hz} \text { at } \\
10 \mathrm{kHz} .\end{array}$ \\
\hline Electric rod antenna $^{*}$ & $\begin{array}{l}\text { Active monopole antenna, Coverage between } 30 \mathrm{~Hz}-50 \mathrm{MHz}, \\
\text { impedance: } 50 \Omega .\end{array}$ \\
\hline $\begin{array}{l}\text { Magnetic coil } \\
\text { antenna }^{*}\end{array}$ & $\begin{array}{l}\text { Coverage between } 20 \mathrm{~Hz}-500 \mathrm{kHz}, 36 \text { turns of 7-41 litz wire } \\
\text { shielded with } 10-\mathrm{Ohms} \text { resistance and } 340 \mu \mathrm{H} \text { inductance. }\end{array}$ \\
\hline $\begin{array}{l}\text { Induction motor } \\
\text { (IM1) }\end{array}$ & \begin{tabular}{l} 
7.5-HP, 208-V, 1765-RPM, PF: $0.82,60 \mathrm{~Hz}$, EFF: $89.5 \%$. \\
\hline
\end{tabular}
\end{tabular}

* Measurement components are MIL-STD 461F standard compliant [200].

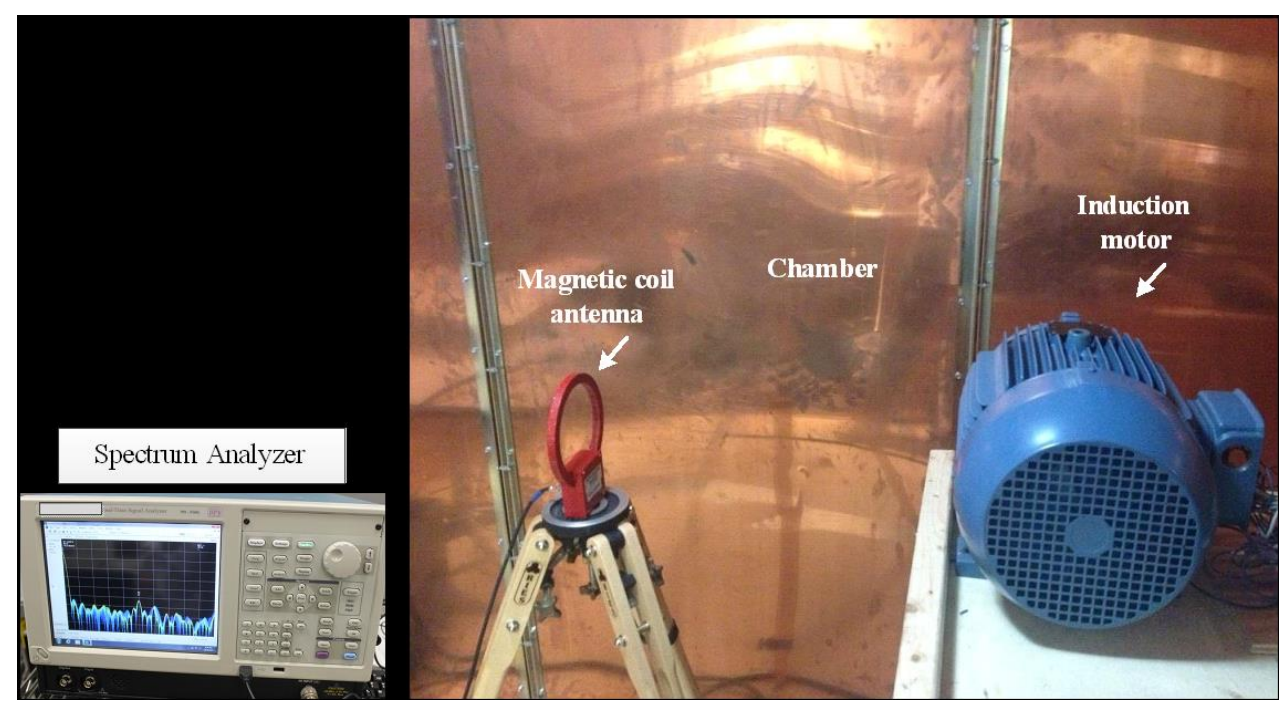

Fig. 6.20 The studied experimental setup including the machine and measurement tools 
The nominal voltage is applied to the machine and the stray magnetic field is obtained at various distances. The result of measurement, the full 3DFE model and the wire model at $60 \mathrm{~Hz}$ are obtained and shown in fig. 6.21. The magnetic field intensity, as the standard index of electromagnetic signature studies, is used with $\mathrm{dB} \mu \mathrm{A} / \mathrm{m}$ as the unit of comparative measure.

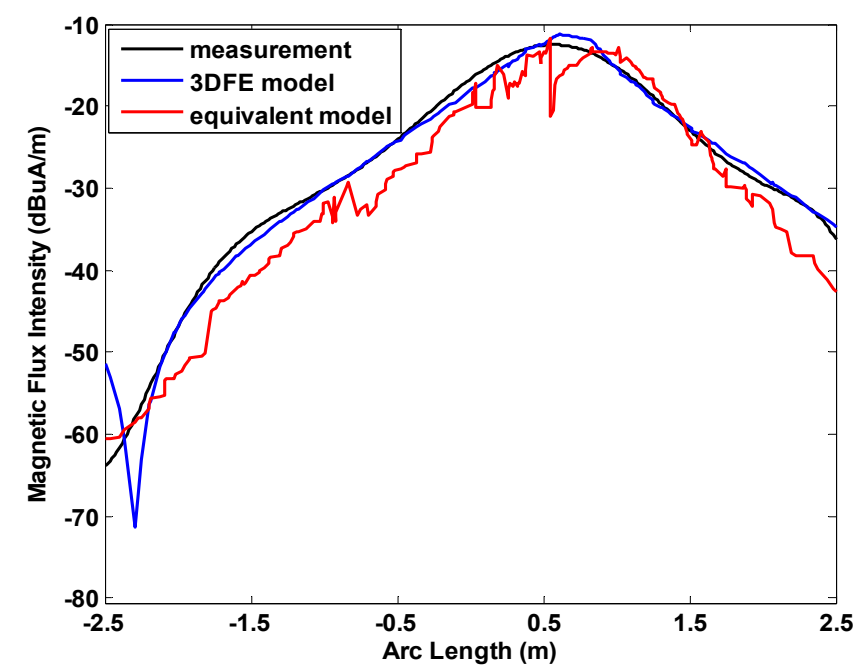

(a)

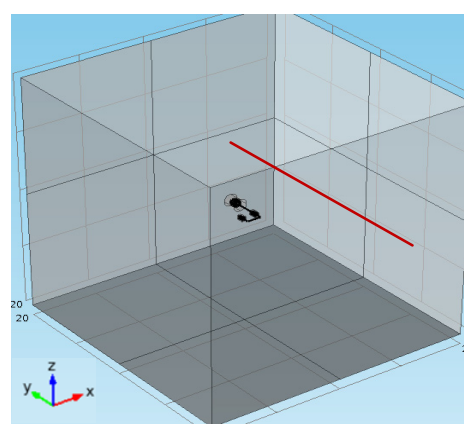

(b)

Fig. 6.21 (a) The magnetic field intensity at $55 \mathrm{~cm}$ away from the setup in $\mathrm{Y}$ axis while all components except IM were off at $60 \mathrm{~Hz}(\mathrm{~dB} \mu \mathrm{A} / \mathrm{m})$, (b) the region of the model (the model is in center, and the measured line is shown in read)

As illustrated in fig. 6.21, the electromagnetic signatures from the two simulation models match the measurement. The reason that the measurement results in the figure don't show distortions is the low number of patterns of the measured results in comparison with the simulation result, especially the wire model. The number of patterns along the line in the $\mathrm{Y}$ axis, which is used as the measured line, for the wire model are 260 points, while it is about 10 points for the measurements. The measured line is also shown in fig. 6.21. 


\subsubsection{DC motor}

\subsubsection{The modeling approach}

The induction motor which is discussed in 6.3.1 had armature and field windings and in terms of winding, it is known as the simplest machine. In contrary, the dc machine which has four types of winding, including armature, field, compensation and commutating windings. Therefore, their equivalent source modeling and merging as implemented in 6.3.1 is not easy. Each of these windings has specific design which causes specific types of electromagnetic signature. Since each winding has different shapes of the radiated field at far distances, so each of them are simulated and modeled individually and finally all of them are combined as one model.

On the other hand, the second part of the modeling is finding the appropriate size of the model, which is very important in far field and also near field computation.

Basically, dimensions of the model are based on the size of the machine, but for better and precise results, an optimization method is used. The proposed optimization process is (GA-based PSO) which was explained in chapter 4 and 6.3.1.

In this method, objective functions are dimensions of the model including the number of dimensions and their length. In addition to the length of dimensions, also the number of dimensions can be considered as objectives of the model, whereas the number of dimensions can vary from a cone and cube to polyhedral. Typical schematic of this aspect of modeling is shown in fig. 6.22. 
Finally by collecting previously mentioned methods and strategies, the equivalent source model is achieved. For better investigation and generating a more accurate model, the equivalent source model of each winding is achieved and shown in fig. 6.23 (a) - (e).

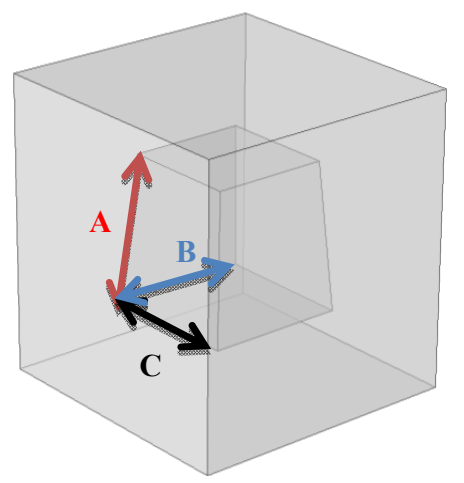

Fig. 6.22 Typical Schematic of the equivalent source model (optimization aspect)

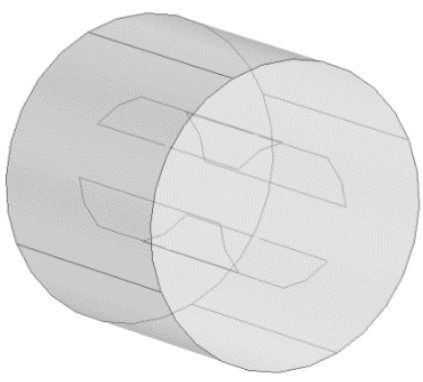

(a)

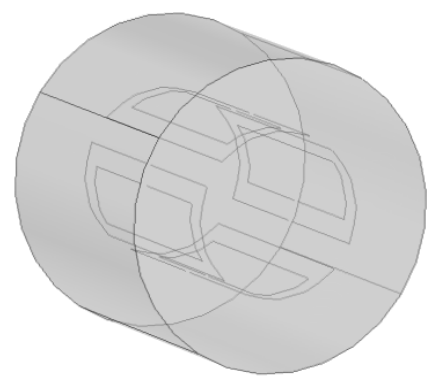

(c)

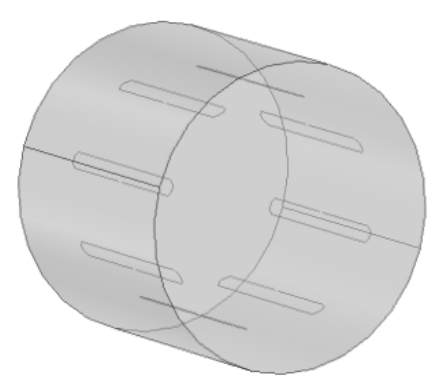

(b)

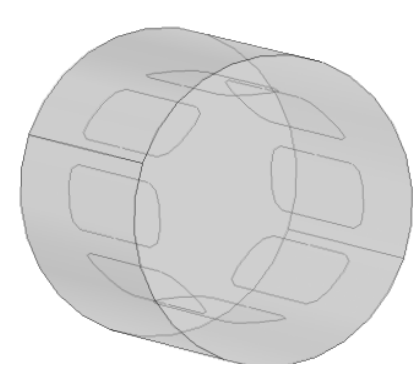

(d)

Fig. 6.23 Equivalent source models of (a) armature winding (b) commutation winding (c) compensation winding (d) field winding in equivalent dc machine 
Final Equivalent source model consists of hundreds of currents with different amplitudes and direction and tens of different voltages, which is illustrated in fig. 6.24. As discussed before, in order to have comprehensive study, appropriate switches are considered for each winding.

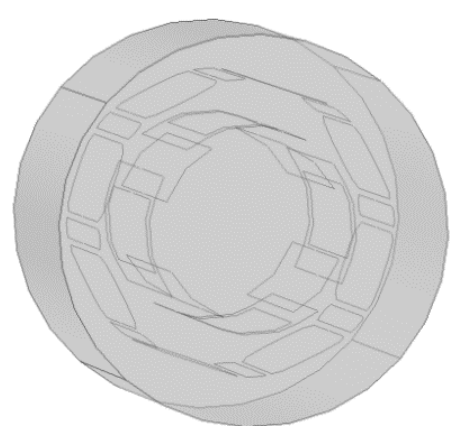

Fig. 6.24 Final Equivalent source model of the Propulsion DC Machine

\subsubsection{Results and discussion}

For simulation purposes, a $800 \mathrm{HP}, 750 \mathrm{~V}, 8$ poles and 185RPM propulsion DC machine with the length of about $3(\mathrm{~m})$ and outer diameter of $1.7(\mathrm{~m})$ is simulated in a 3D electromagnetic finite element domain for one time instance. The actual model and the mesh structure of this machine in FE domain is shown in fig. 6.25 (a) and (b).

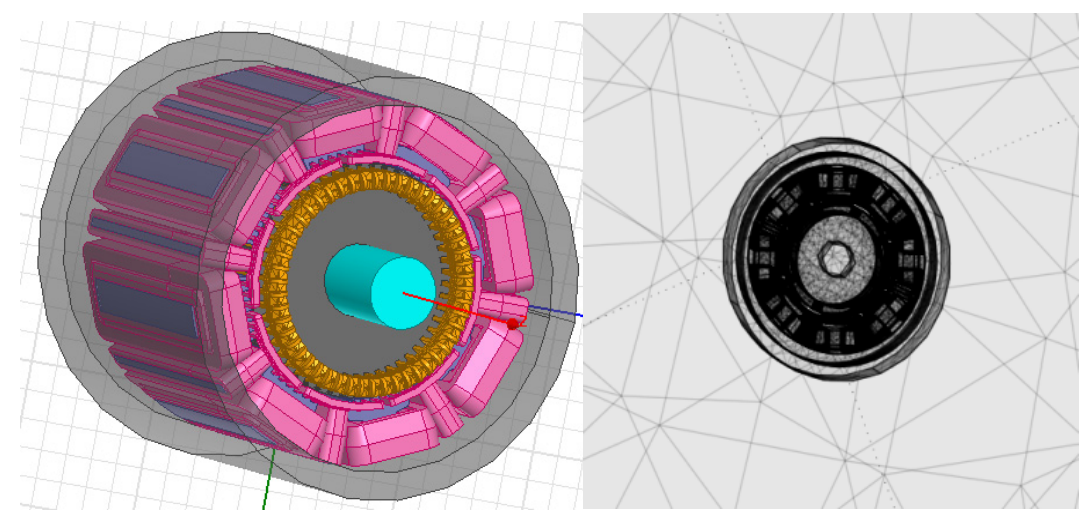

(a)

(b)

Fig. 6.25 Schematic of (a) the detailed model of the dc machine and (b) the mesh in FE domain 
The analysis for the model of the actual machine requires about 7 million degrees of freedom in FE analysis. This causes the simulation time to be about 43000 seconds ( 12hours). However, the equivalent source model with less than one million degrees of freedom takes about 300 seconds ( 6minutes).

The analysis method, which is used in this analysis, is the generalized minimal residual method (usually abbreviated GMRES) with SOR pre and post smothers, which was explained in chapter 3 and 4.

After implementing simulation of both actual and equivalent source models, the propagated electric and magnetic fields are measured in different locations at a distance from the source. fig. 6.26 shows propagated magnetic fields from both models along different lines.

Fig. 6.26 show that magnetic field propagated from the actual machines has different wave-shapes in various measured lines; so, it can be inferred that it is not possible to use a single dipole as an equivalent source model because a single dipole shows similar results in all planes. In addition to the wave-shape of fields, also their amplitudes in various measured lines are different, which can be another reason to use an embedded equivalent source model. This point can also be seen in radiated electric fields' waveshapes (fig. 6.27), although the difference of electric fields' wave-shapes measured along various lines is shallow and can hardly be recognized. For example, comparing electric fields in case (a) and (b) in fig. 6.27 and 6.28, it can be seen near the peak that both actual and equivalent source models' results are different. 


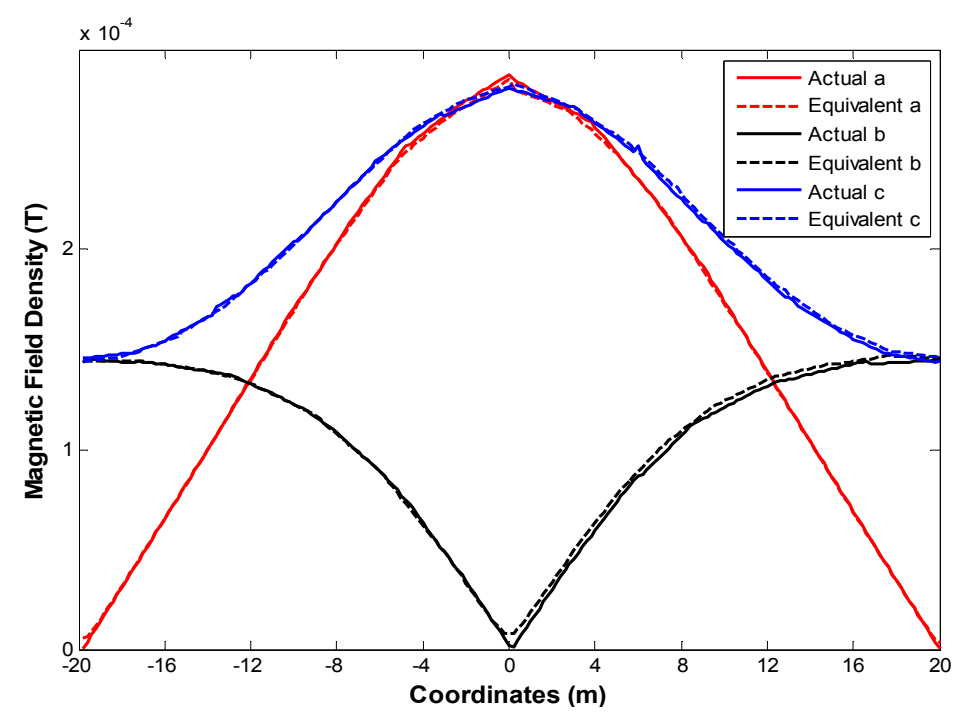

(A)

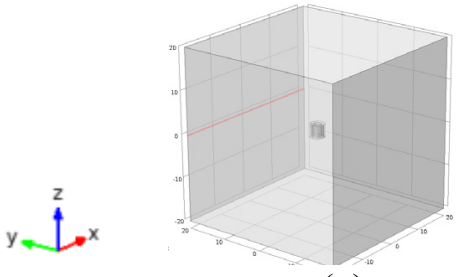

case (a)

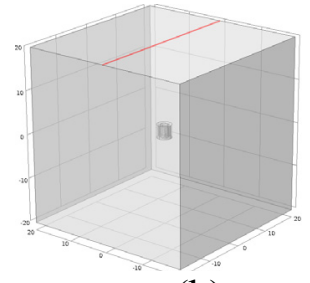

case (b)

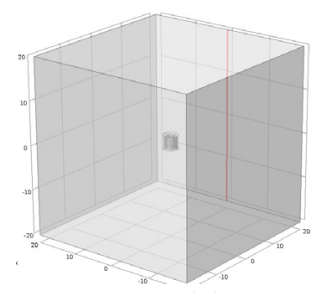

case (c)

(B)

Fig. 6.26 (A). Radiated magnetic field density in (case a): XZ plane when $\mathrm{X}$ varies between -20 to 20 (case b): XY plane when $\mathrm{X}$ varies between -20 to 20 (case c): YZ plane when $Z$ varies between -20 to 20, (B) geometry of the three cases

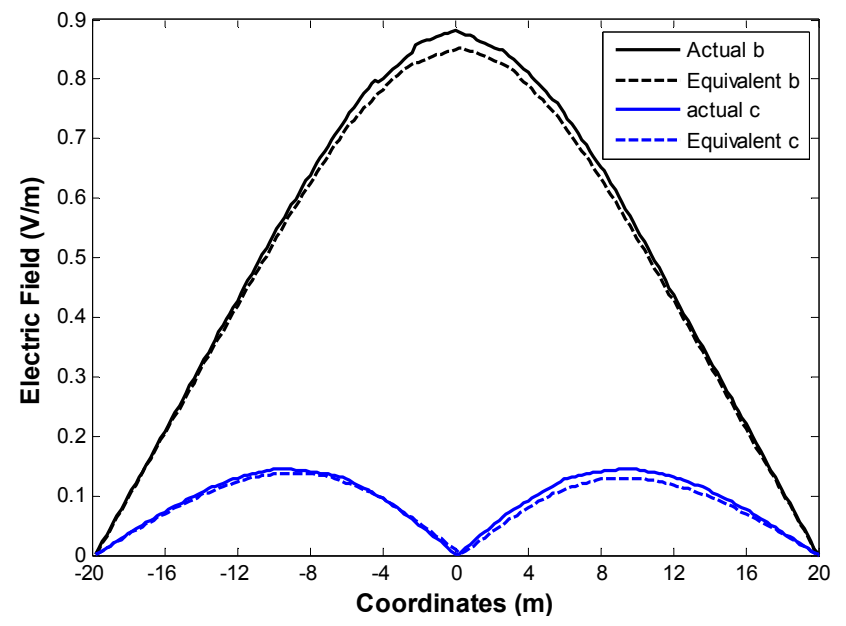

Fig. 6.27 Radiated electric field of in (case b): $\mathrm{XY}$ plane when $\mathrm{X}$ varies between -20 to 20 (case c): $Y Z$ plane when $Z$ varies between -20 to 20 


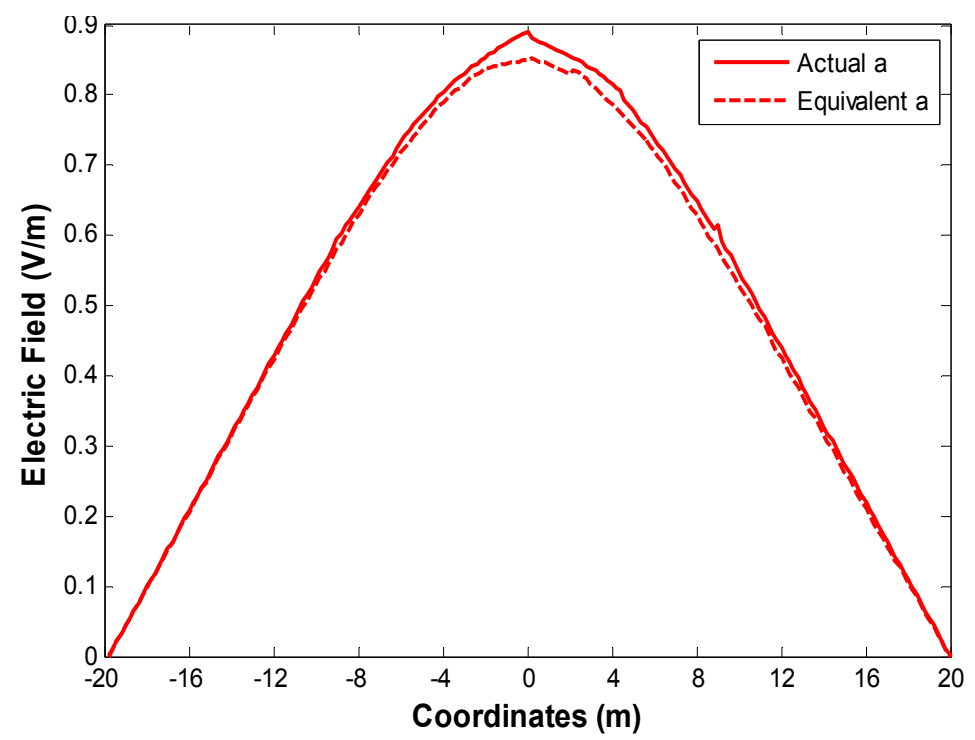

Fig. 6.28 Radiated magnetic field density in (case a): XZ plane when X varies between 20 to 20

In fig. 6.26, 6.27 and 6.28, magnetic and electric fields of two models along a single line are compared that show a reasonable similarity. However, one may say there might be dissimilarities if the fields are measured in other lines of a plane. In other words, the measured lines are in the middle of planes, so symmetric dipole of propagated field is more likely to occur in the equivalent source model, but other lines may not have this type of result. Hence, for further investigation, the measurement is implemented in planes and the results are depicted in fig. 6.29 and fig. 6.30.

Comparing fig. 6.29 (a) with fig. 6.29 (b) and also fig. 6.30 (a) with fig. 6.30 (b), it can be seen that propagated fields from the equivalent source model have very similar result to the actual model on not just one line but also on whole slice. 


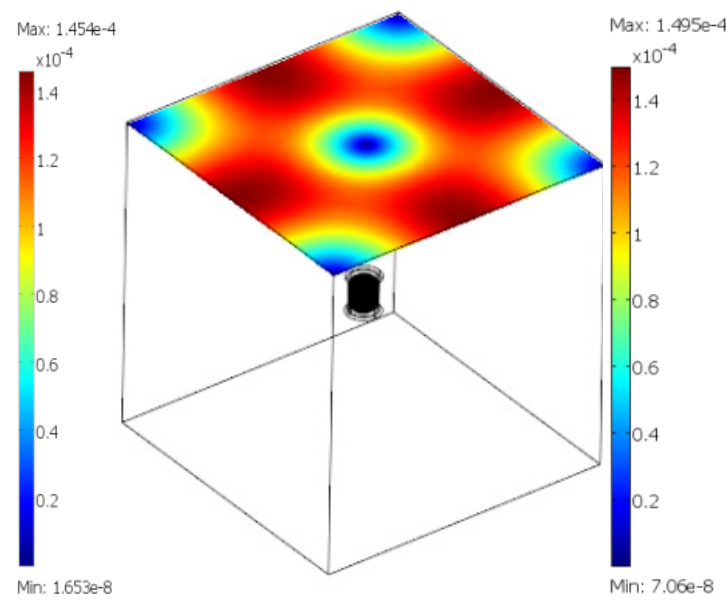

(a)

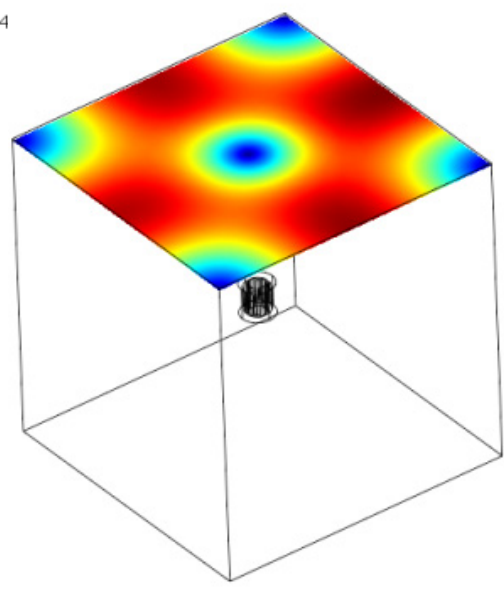

(b)

Fig. 6.29 Magnetic field density of (a) the actual machine (b) the equivalent source model (T)

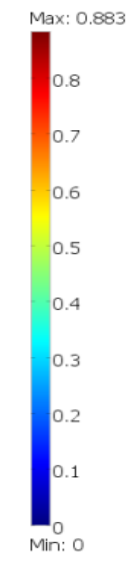

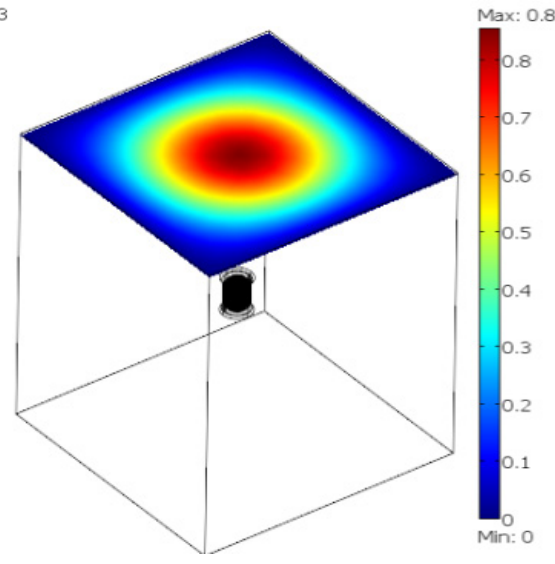

(a)

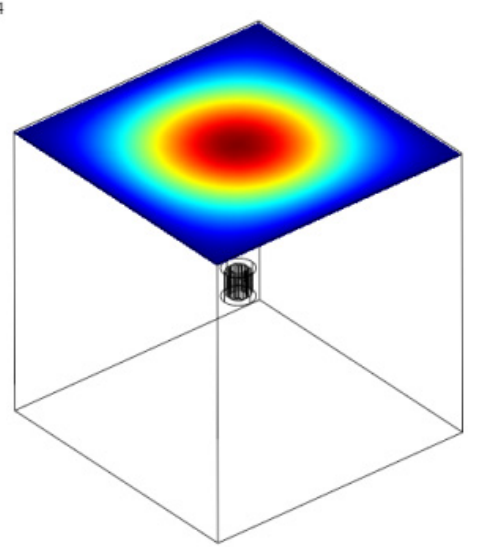

(b)

Fig. 6.30 Electric field of (a) the actual machine (b) the equivalent source model $(\mathrm{mV} / \mathrm{m})$

As mentioned in the previous case (6.3.1), the main goal of this research is to study the electromagnetic signature of multi machine system. Therefore, for more validation of the proposed equivalent source model, a two-machine system is designed. The two equivalent source models of the studied DC machine are located in a close distance to each other and then the analysis is applied. The applied current of branches and voltages of nodes of equivalents model in multi-machine study are exactly the same as the data, 
which are used for the single machine system, so there is no need for further analysis except final simulation, which takes about 18 minutes for two machine systems.

Figs. 6.31 and 6.32 show the comparison between magnetic and electric fields propagated from the actual and proposed model along several lines for two motor cases. As can be seen, the magnetic and electric fields follow the same patterns with excellent accuracy. For brevity, only some planes and lines from measured planes are considered, which are illustrated in fig. 6.31 (B). All other lines and planes show similar accuracy.

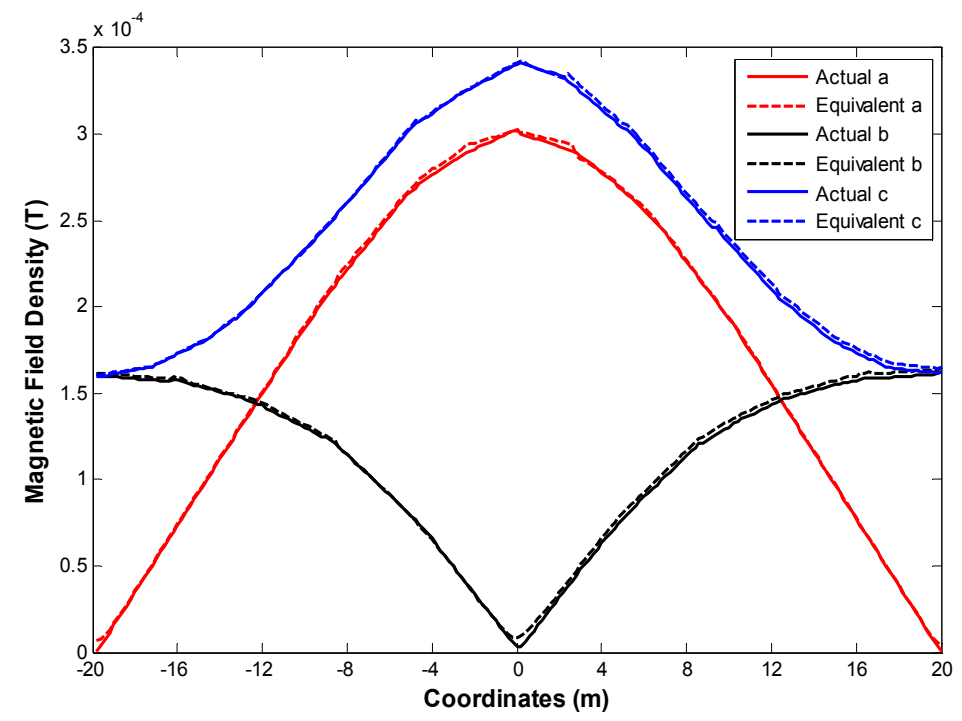

(A)

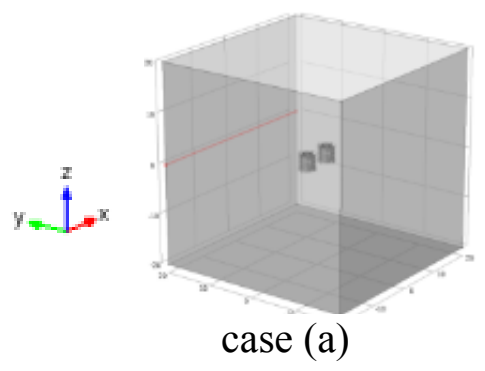

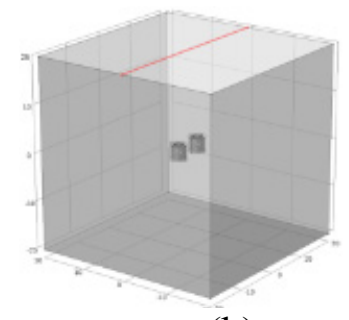

case (b)

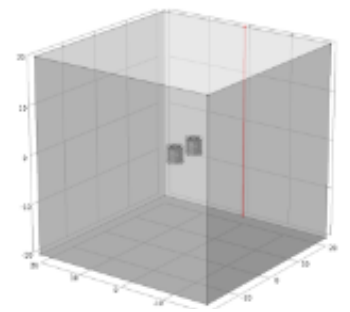

case (c)

(B)

Fig. 6.31 (A). Radiated magnetic field density of two machine case in (case a): XZ plane when $\mathrm{X}$ varies between -20 to 20 (case b): $\mathrm{XY}$ plane when $\mathrm{X}$ varies between -20 to 20 (case c): YZ plane when $\mathrm{Z}$ varies between -20 to 20, (B) geometry of the three cases 
For the situation that different rate of power is applied to the machine, some variables are considered in this model. In this case, variation coefficient of voltages and currents of each actual machine can be applied to the respective equivalent source model.

Also, for other sizes of similar types of machines, an appropriate coefficient can be applied. This coefficient can be obtained based on the size of the studied machine, whereas the coefficient for the studied machine can be considered as basic values, and for other machines any deviation can be proportional to the basic values. Similar factors, which are obtained from the study of the actual machine, can be applied to equivalent machine models.

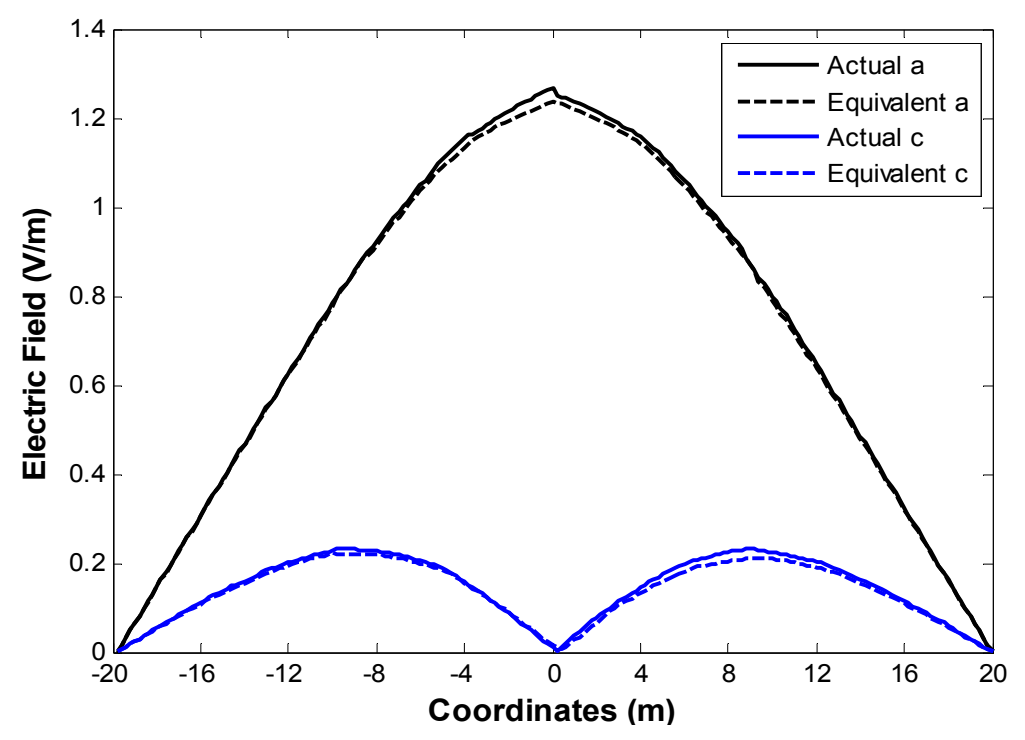

Fig. 6.32 Radiated electric field of two machine case in (case b): $X Y$ plane when $X$ varies between -20 to 20 (case c): YZ plane when $Z$ varies between -20 to 20

Similar to single machine case, electromagnetic field spectrums of two-machine cases are measured and illustrated in fig. 6.33 and 6.34 . The result shows that the similarly in two-machine (multi machine) case of the actual machine model can be replaced by the equivalent source model. 


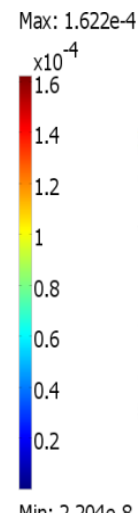

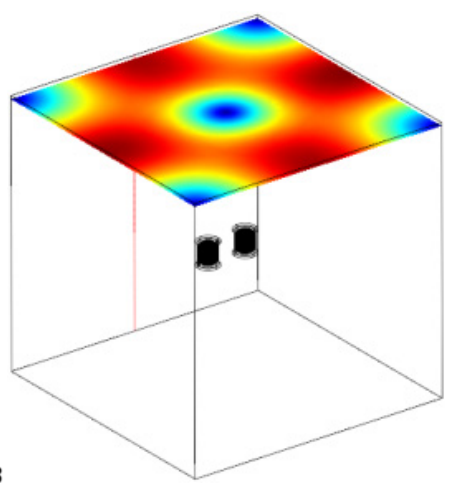

(a)

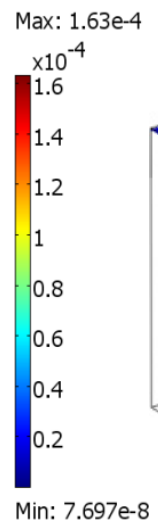

Min: 7.697e-8

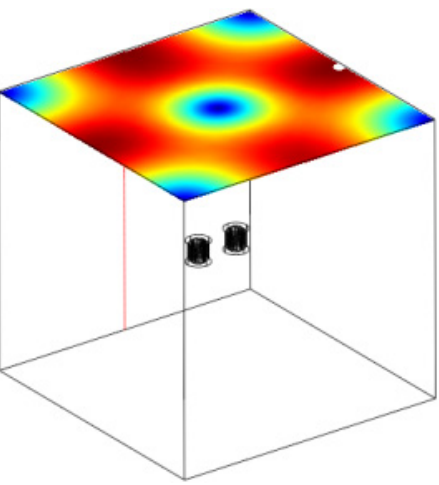

(b)

Fig. 6.33 Magnetic field density of (a) the actual machine (b) the equivalent source model

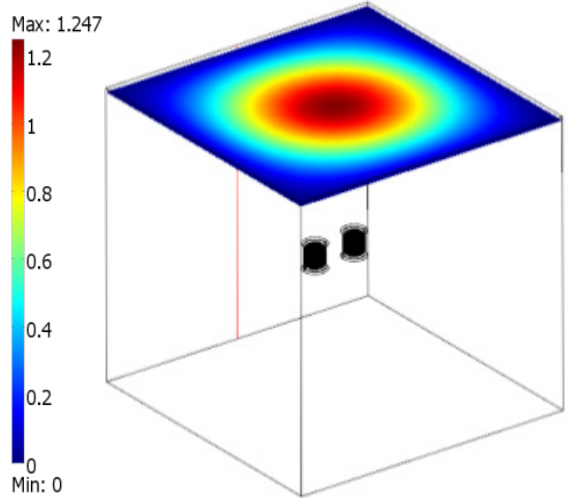

(a)

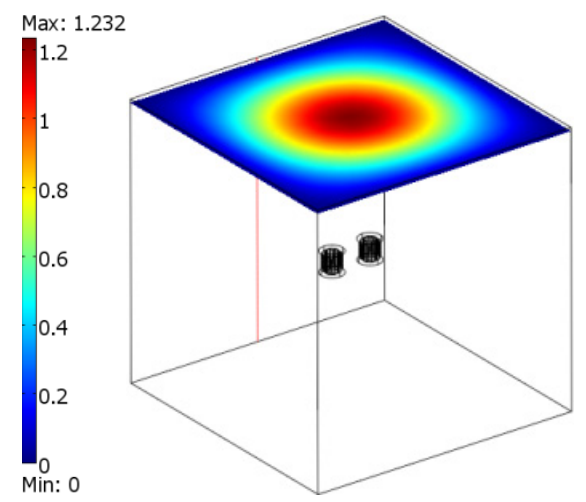

(b)

Fig. 6.34 Electric field of (a) the actual machine (b) the equivalent source model

\subsubsection{Synchronous generator}

\subsubsection{The modeling approach}

The electromagnetic signature study of the synchronous generator as the main generator in most types of power plants can be estimated at a far distance based on equations (4-14) - (4-18). However, as mentioned earlier, estimating the radiated field from electrical machines at a far distance requires significant time, especially for multicomponent studies using physics-based simulations. Therefore, a logical simplification 
used here is utilizing the edge modeling in finite element analysis. In addition, the synchronous machine has an excitation part in the rotor, which is connected to power electronic components, producing the electromagnetic interference.

The procedure of the design of wire model for resembling magnetic field stray is similar to the two previous cases. The difference is the design of the wire model for resembling the propagated electric fields. In this model, the voltages at the nodes are considered at terminal ends of the windings. The values of voltages are based on the electric field displacement of the actual machine. Consequently, the model consists of many loops with various currents and node voltages as shown in fig. 6.35 .

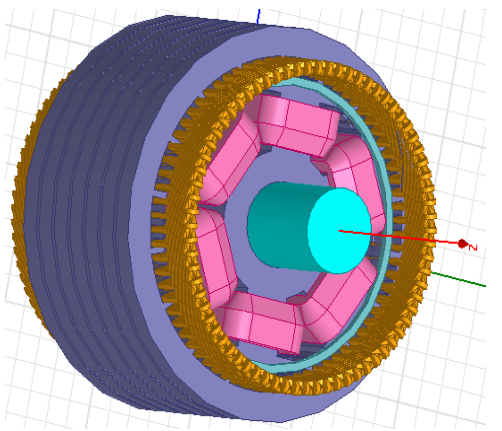

(a)

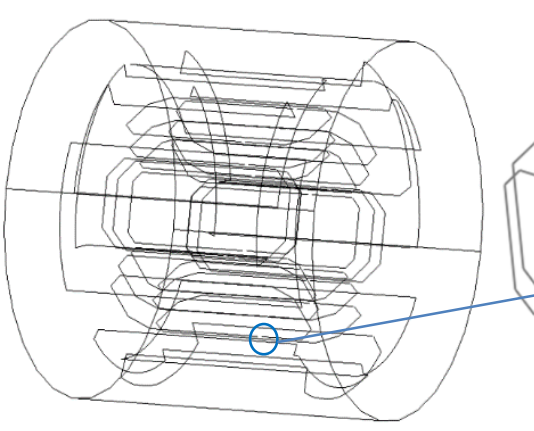

(b)

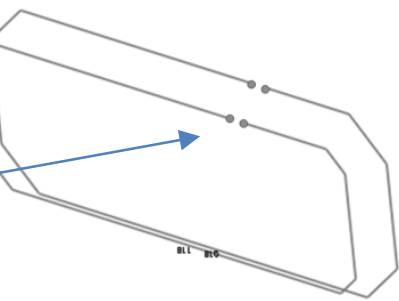

(c)

Fig. 6.35 prototype of synchronous machine (a) actual machine (b) equivalent source model (c) terminal voltage of the equivalent source model

Comparing fig. 6.35 (a) with fig. 6.35 (b), the model is replaced by a collection of lines located in the position of windings in the actual machine. Since the model doesn't have a cross section, it is not possible to apply similar current to the model. Hence, the current value of the lines will be based on the current density of the machine in each phase. Thus, the amount of current density of each phase of the winding is estimated and applied to the lines in the proposed model. Although the mentioned method would be 
helpful, the types of windings are different in synchronous machine. The field winding carrying DC current and the armature winding has AC currents. Hence, individual models should be made for each of these windings. The equivalent source models of the armature and field windings are shown in fig. 6.36 (a) and (b). The effect of each winding in the total electromagnetic signature is investigated next.

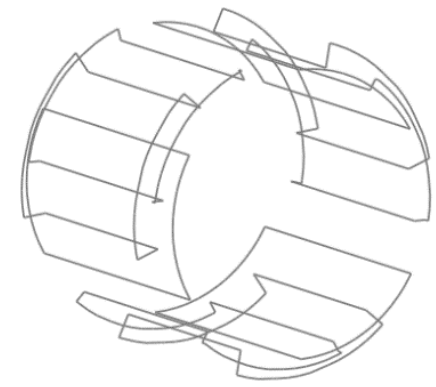

(a)

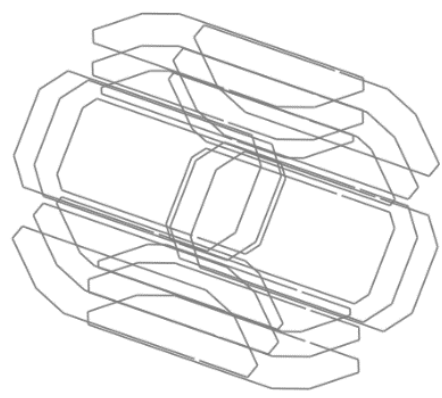

(b)

Fig. 6.36 Equivalent source model of individual windings (a) armature winding (b) field winding

\subsubsection{Simulation and discussion}

After defining the final equivalent source model, the simulation is implemented in FE domain. The 3D electromagnetic finite element method is used as an acceptable method for physics-based simulation. For implementation purposes, a 3-phase, $600-\mathrm{kW}, 600-\mathrm{V}$, 1200-rpm synchronous generator was simulated in a 3D electromagnetic finite element domain for one time instant. The analysis for the model of the actual machine required about 5.5 million degrees of freedom in the FE analysis. This caused the simulation time to be about 38000 seconds ( 10.5hours). However, the equivalent source model contained less than one million degrees of freedom and takes about 270 seconds (4.5 minutes). 
After solving the problem by the finite element model, the magnetic field density propagated from the machine with and without the armature winding in two conditions were evaluated as shown in fig. 6.37.

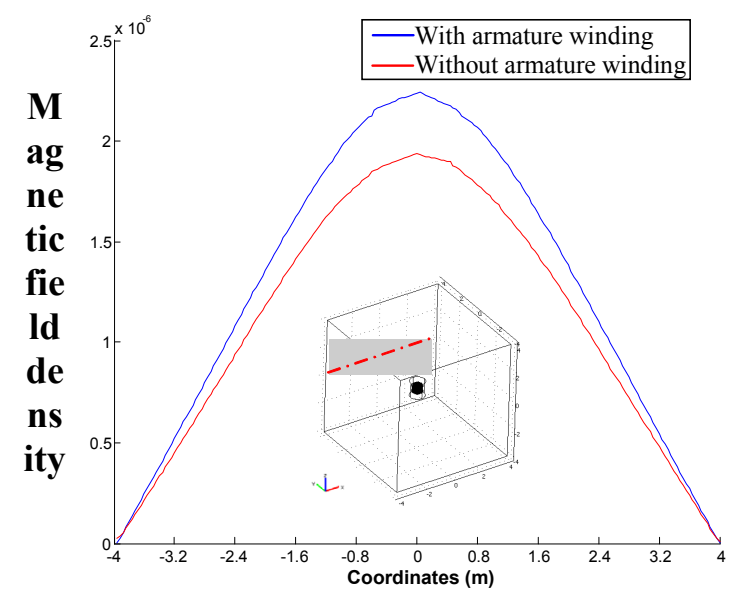

(a)

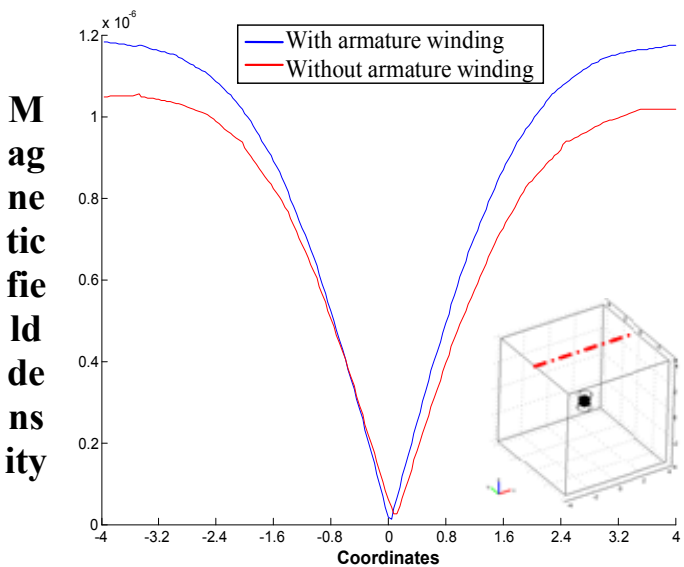

(b)

Fig. 6.37 Magnetic field density propagated with and without the armature winding along (a) $\mathrm{X}$ axis in $\mathrm{XZ}$ plane (b) $\mathrm{X}$ axis in $\mathrm{XY}$ plane

As shown in fig. 6.37, the armature winding has a direct effect in increasing the fields with the same ratio at all points. Thus, for conciseness, the armature winding can be ignored and the linear effect of it, which can be a ratio, may be considered in the current values of the field winding in the equivalent current. As a result, the simulation time will decrease dramatically, while the accuracy doesn't change. After considering this simplification, in the simulation of the equivalent source model, the propagated electric and magnetic fields are measured in different distances from the source. Fig. 6.38 shows the propagated electric and magnetic fields from both actual and equivalent source models along different lines. As can be easily seen, both radiated electric and magnetic fields from the equivalent source model (fig. 6.38 (b), (d)) accurately match the radiated 
fields from the actual model (fig. 6.38 (a), (c)) in all three shown planes. The electric field of the two other planes is negligible compared with the one of the XY plane, therefore they are not shown in fig. 6.38 (c) and (d).

Considering the accuracy and speed of simulation of the proposed model, this model can also be replaced in the multi-machine case. It should be considered that there are several machines and other power components in a typical powertrain. Consequently, simulating original model of all of them together is almost impossible, even by using very fast processor. The experimental verification of this component is explained in 6.3.5, while coupled with the induction motor.

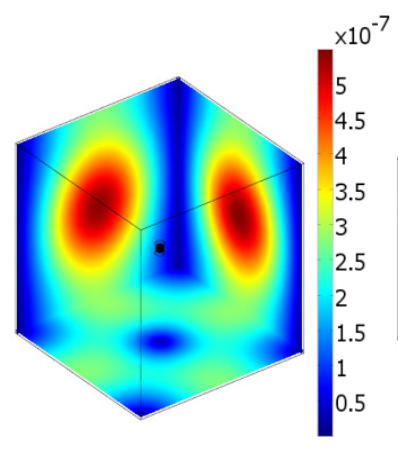

(a)

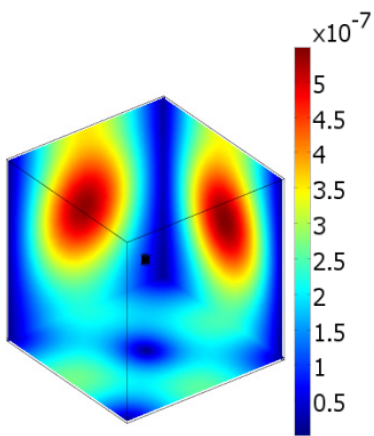

(b)

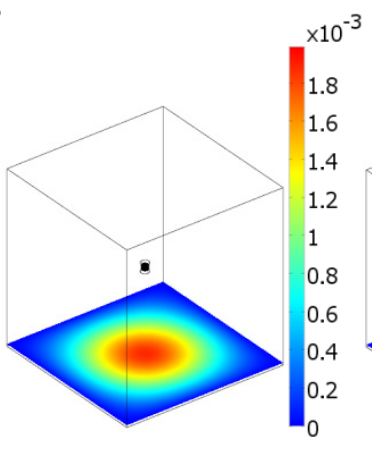

(c)

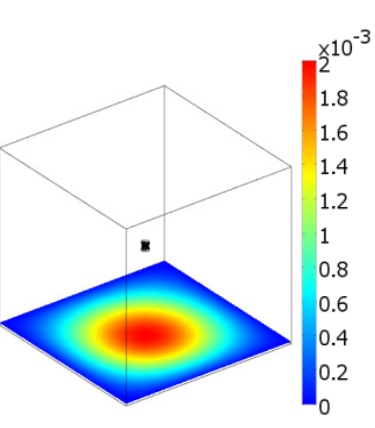

(d)

Fig. 6.38 Electromagnetic field comparisons in 3 planes: (a) Magnetic field density of actual machine (T), (b) Magnetic field density of equivalent source model (T), (c) Electric field of actual machine $(\mathrm{V} / \mathrm{m})$, (d) Electric field of equivalent source model $(\mathrm{V} / \mathrm{m})$

\subsubsection{Cable sets}

\subsubsection{Modeling approach}

The basics of the modeling of cables are similar to the previous cases. However, since this component is not electrical machine, some consideration should be employed. 
The actual physical modeling of cables for electromagnetic signature studies requires all the details to be considered, even in a large region. The XPLE cables similar to all electromagnetic sources propagate dipoles at a far distance. However, the interaction of several components such as electrical machines, and power converters modify the shape and the amplitude of dipoles. Therefore, each model should be designed and studied independently. Nevertheless, there is a problem which is the modeling of the relatively small layers of a multi-core XLPE cables. The studied region could be about 20000 times bigger. This causes the deformation of the cable's model during meshing in numerical modeling methods, such as finite element method. The present study is performed on the XLPE insulated and armored PVC sheathed cable $(0.6 / 1 \mathrm{kV})$.

Fig. 6.39 shows the typical model, as well as the original and deformed models of the studied cable in finite element analysis environment. In order to solve this issue, a specific modeling including multi-dipoles with several line currents and node voltages are designed, which resembles the actual model of the cable for electromagnetic signature studies.

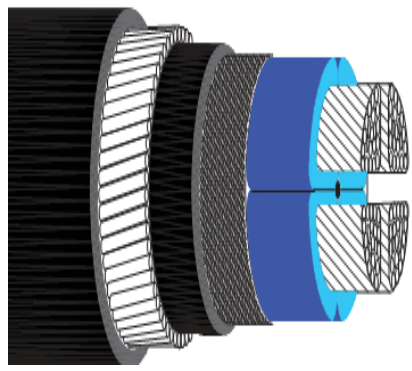

(a)

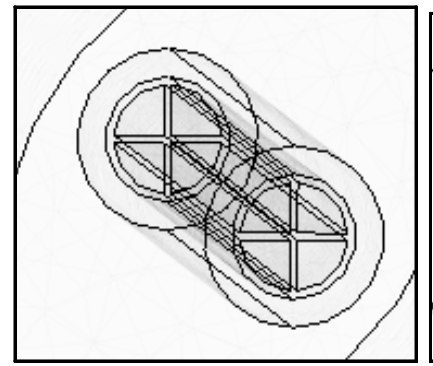

(b)

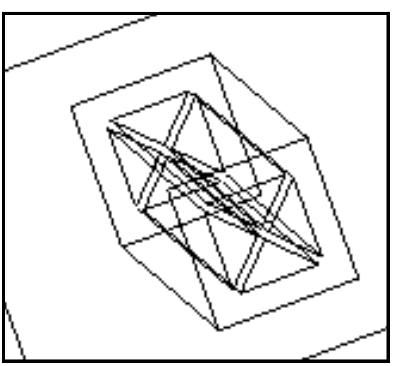

(c)

Fig. 6.39 models of the proposed cable in finite element design (a) typical model (b) original FE model (c) deformed FE model 
The multi-dipole model of the studied cable is shown in fig. 6.40. A typical node voltage and line current are displayed in the figure.

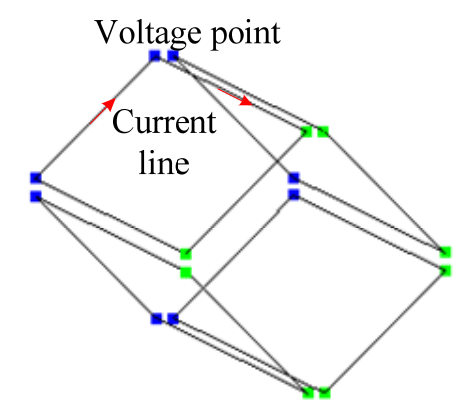

Fig. 6.40 Prototype of the multi-dipole models of the studied cable

\subsubsection{Case studies with simulation results}

For simulation purposes, first a unit length of the actual XLPE and the model cables are simulated and compared using the finite element method. Afterward, various directions of the cable are studied. The cable is then analyzed in multi-permittivity areas such as undersea. As mentioned earlier, the XLPE insulated and armored PVC sheathed cable $(0.6 / 1 \mathrm{kV})$ is the proposed cable.

\subsection{Unit length of the cable}

Initially, the radiated electromagnetic fields of the proposed model and the full model of the cable are evaluated and compared. In order to avoid deformation, the actual model is simulated by considering a large number of elements, which is only applicable in simple situations, such as a unit length of a cable. This case is studied by applying two different voltages to the ends of the cable. The field spectrums radiated from the actual and the proposed model are shown in fig. 6.41 (a) and fig. 6.41 (b), respectively. Comparing the results in fig. 6.41 (a) and fig. 6.41 (b) shows that the proposed model has a very good accuracy. 


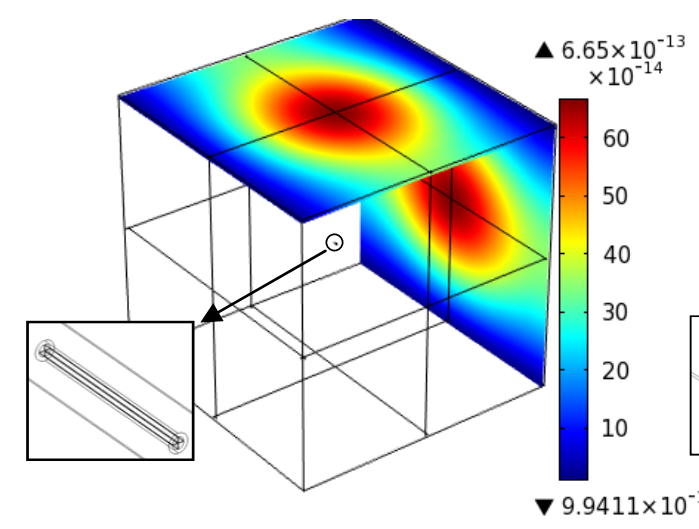

(a)

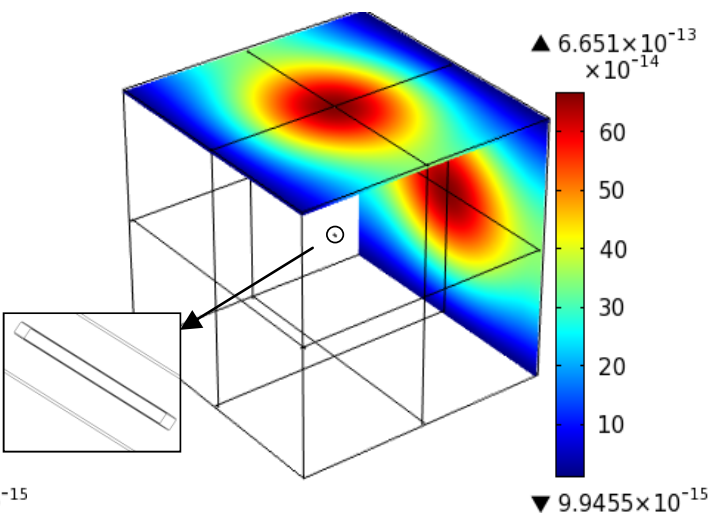

(b)

Fig. 6.41 Radiated magnetic field density of (a) actual model (b) equivalent source model in tesla $(\mathrm{T})$. Note that the cable is very small compared to the region

Since cables are symmetrical, the radiated fields are the same in all planes of the region similar to a simple dipole. Nevertheless, the radiated fields in two planes are measured, and as the result shows the proposed multi-dipole model propagates similar radiated fields as the actual model. A similar study is implemented for the radiated electric field and the result is shown in fig. 6.42. As shown in the figure, the radiated electric field of the proposed model equals the actual one. Therefore, both indices of the electromagnetic signature study of the proposed model represent accurate results, while the simulation time of this model is about 100 times less.

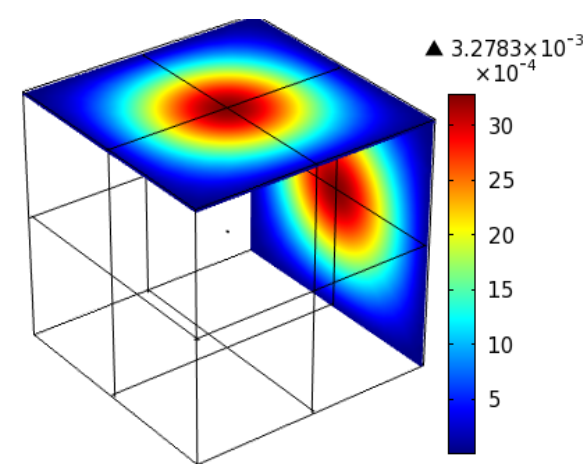

(a)

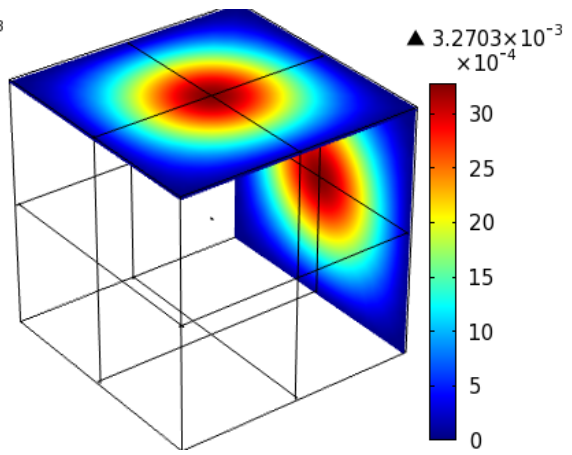

(b)

Fig. 6.42 Radiated electric field of unit length of the cable (a) actual model (b) equivalent source model $(\mathrm{mV} / \mathrm{m})$ 


\subsection{Multi-directional cables}

The XLPE cables in connection between two components may have many curves or torsions; therefore, various magnetic dipoles would be established and consequently radiated fields would become different. In multi-direction cable analysis, the simulation time increases significantly, or in the cases of coupling with other components, the simulation may become impossible due to the increase of the number of tiny spaces between fragments of each component, while the region is huge. As an initial case of multi-directional cable, perpendicular cables are located in the same region and the radiated electromagnetic fields are measured at a far distance, which is displayed in fig. 6.43 and fig. 6.44. Similar to the single cable case, the proposed model shows great accuracy. Additionally, the difference of simulation time between the actual model and the equivalent source model increases.

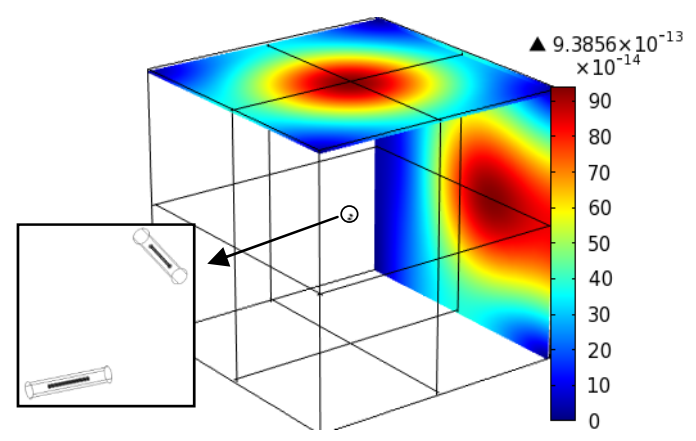

(a)

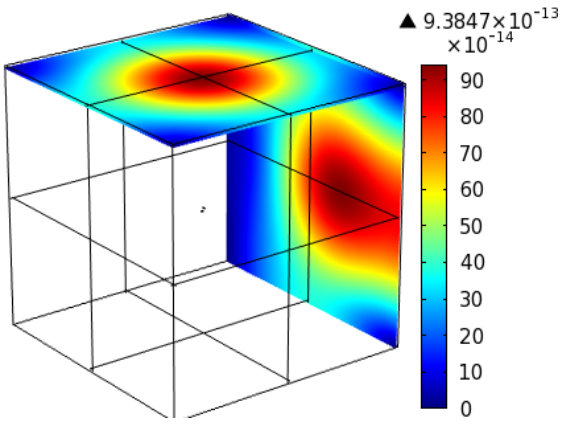

(b)

Fig. 6.43 Radiated magnetic field density of perpendicular cables case (a) actual model (b) equivalent source model in tesla $(\mathrm{T})$

Comparing fig. 6.43 and fig. 6.41, the maximum point of the radiated magnetic field density in the lateral plane is moved to the corner. This is because of the interaction of dipoles of two perpendicular cables. Since the source of electromagnetic signature is not symmetrical anymore, the radiated fields in the two shown planes are different. 


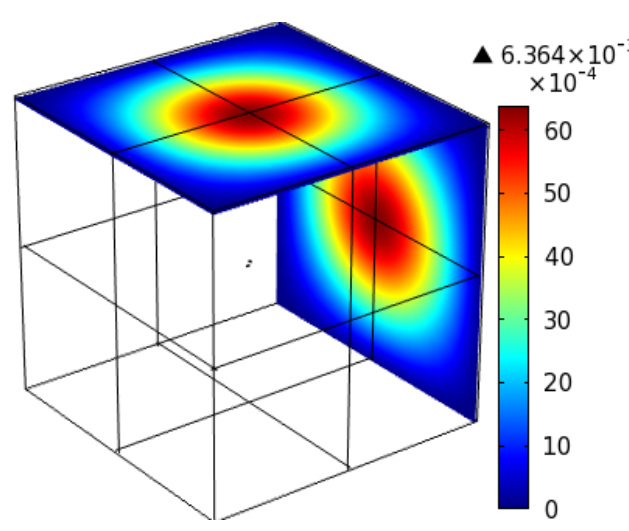

(a)

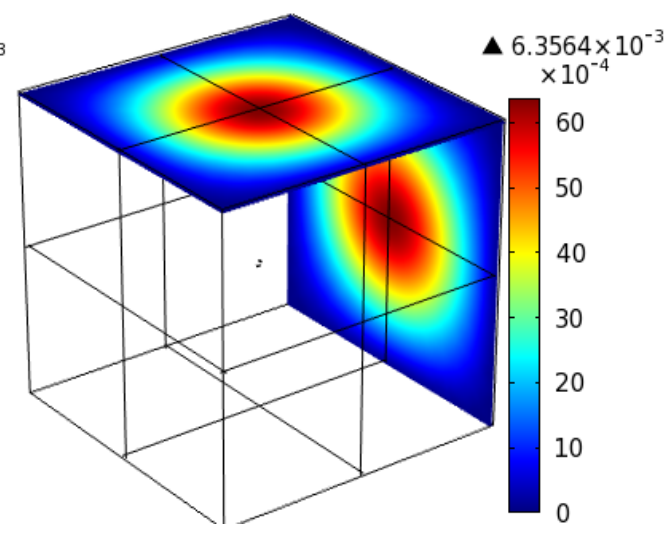

(b)

Fig. 6.44 Radiated electric field of perpendicular cables case (a) actual model (b) equivalent source model $(\mathrm{mV} / \mathrm{m})$

Moreover, in order to verify the model and have the study in all dimensions, a more complex multi-directional cable is analyzed. To do so, four discontinuous units of the cable are located arbitrarily in different angles (see fig. 6.45).
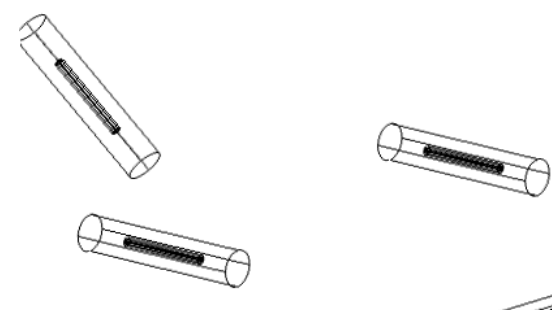

Fig. 6.45 A sample of multi-directional discontinuous cables

Similar to previous cases, magnetic and electric fields radiated from the cables are obtained and shown in fig. 6.46 and 6.47. Comparing the result of multi cable case with single case, they are not similar at all which is because of the presence of cables at various angles. The proposed model equals the actual model in this case, as well as in previous cases. Note that the lines around the region are for increasing the number of meshes in measured planes to have more accurate radiated fields. 


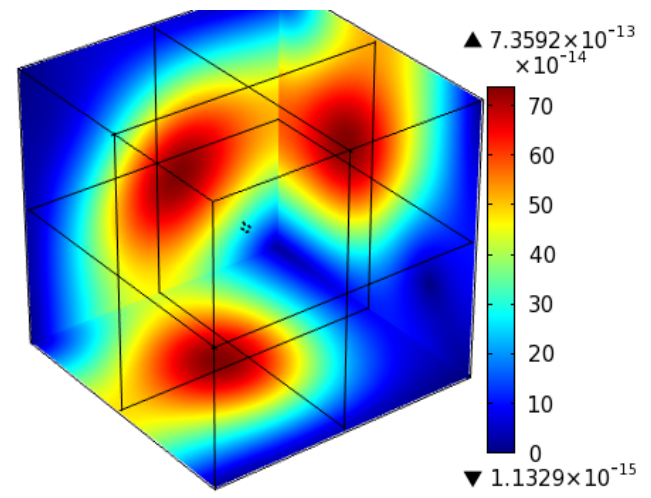

(a)

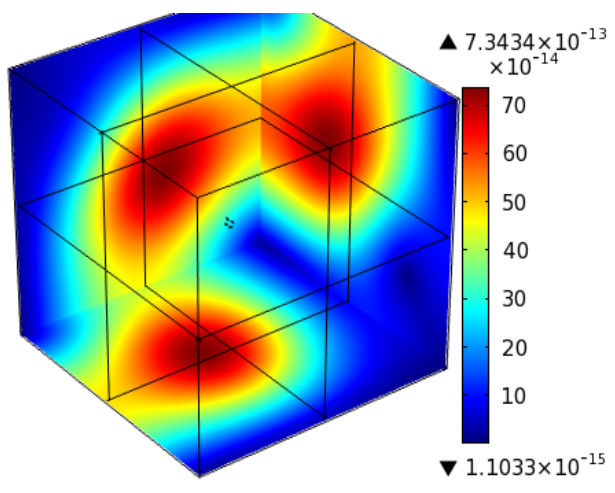

(b)

Fig. 6.46 Radiated magnetic field of multi-directional cables case (a) actual model (b) equivalent source model (T)

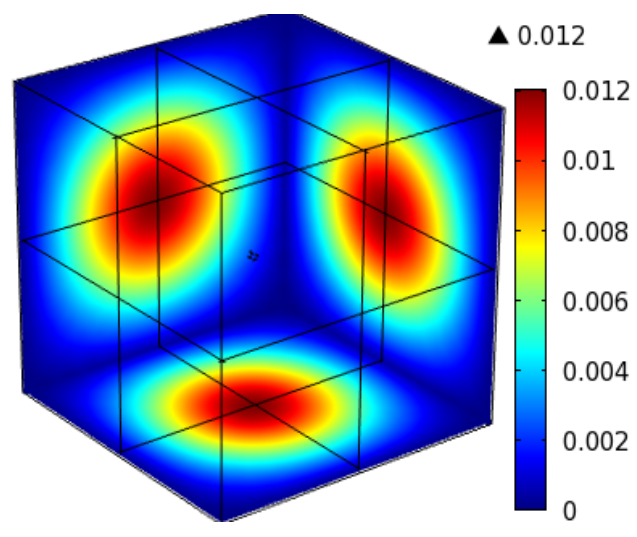

(a)

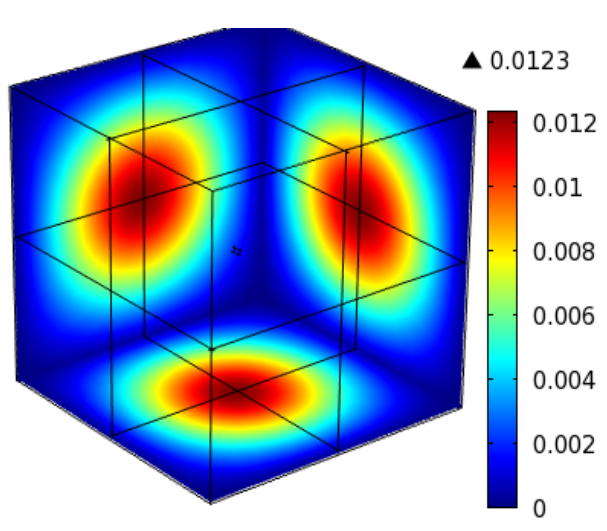

(b)

Fig. 6.47 Radiated electric field of multi-directional cables case (a) actual model (b) equivalent source model $(\mathrm{mV} / \mathrm{m})$

\subsection{The cables in multi permittivity area}

As mentioned in the introduction, one of the main applications of low-frequency EMC study is for analyzing buried, underground, and undersea cables. Since there is the area with two or more permittivity, such as soil and air or water and air, the radiated electric field at a far distance in this occasion would be different. For brevity, only the radiated electric field of the undersea cable is studied. As shown in fig. 6.48, the surface of water is illustrated, so that the region includes water, air and permittivity of the cable 
and its insulation. As expected, the radiated fields are different in the area with various permtivities. A similar area condition is applied to the equivalent source model and, as shown, the radiated field is the same as the actual model. Consequently, this model is applicable in various environmental conditions.

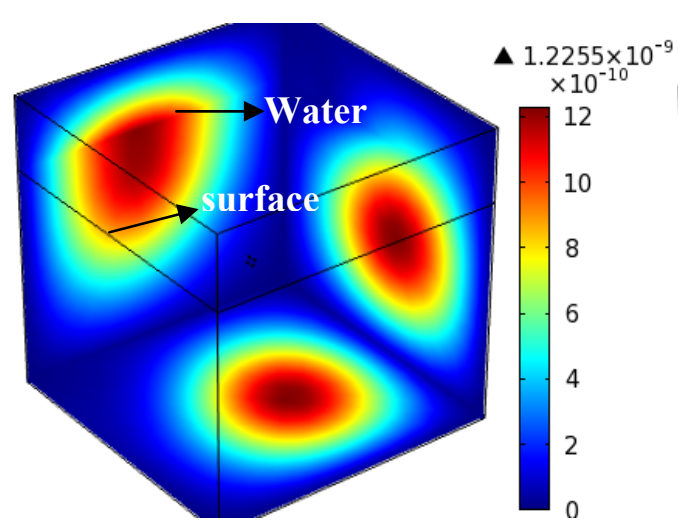

(a)

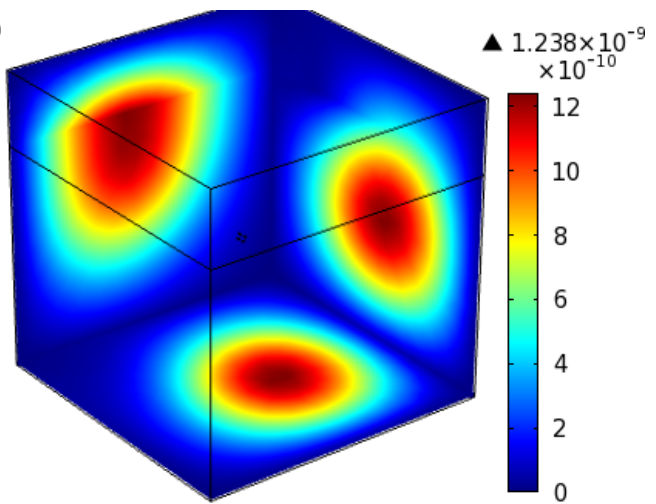

(b)

Fig. 6.48 Radiated electric field of the cables in multi permittivity area (a) actual model (b) equivalent source model (V/m)

\subsection{Coupling of the cable with synchronous machine}

For further verification and studying the application of this type of modeling, the proposed model is analyzed in connection with a power component. A synchronous generator is coupled with a multi-core XLPE cable. The modeling of synchronous generator was explained in 6.3.3. The actual and equivalent source models of the cable connected to the machine are shown in fig. 6.49 .

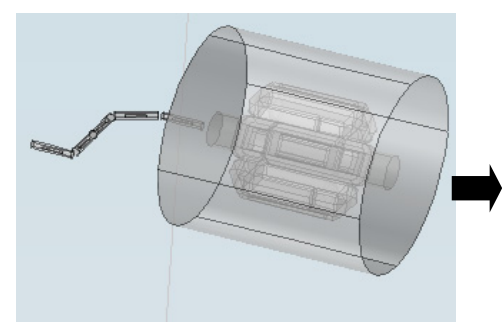

(a)

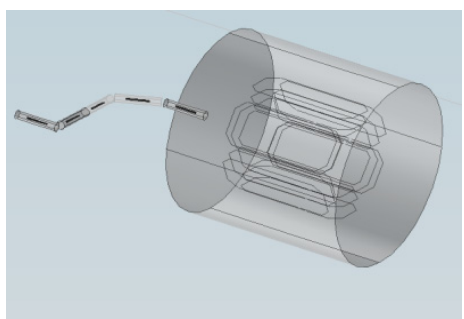

(b)

Fig. 6.49 Schematic of the synchronous machine connected to the cable (a) the detailed model (b) the equivalent source model 
The rated voltage is applied to the cable, which is connected to the machine and the radiated field, which is measured at a far distance from the sources. The current and voltage values of the equivalent source model are calculated based on the individual actual model of the machine and cable. Fig. 6.50 shows the propagated field of both models along the $\mathrm{X}$ axis in the $\mathrm{XY}$ plane. The proposed line is also shown in the figure. The difference of the amplitude between these two models is because of the superposition of materials. Since the cables and machine are so close together, there is a superposition effect in the magnetic field. The radiated magnetic field from the cable is induced into the machine and creates an induced current which radiates an additional field from the machine. This situation cannot be simulated perfectly in the proposed multi-dipole modeling, which results a difference in the curves. In order to clarify the effect fields of each component on the total radiated fields in fig. 6.50, the radiated field of each component is calculated and shown in fig. 6.51. As shown in the figure, the effect of the cable's radiated field is less than the machine's. This is because of the volume of the machine and the effect of that on the current density, which builds the magnetic field.

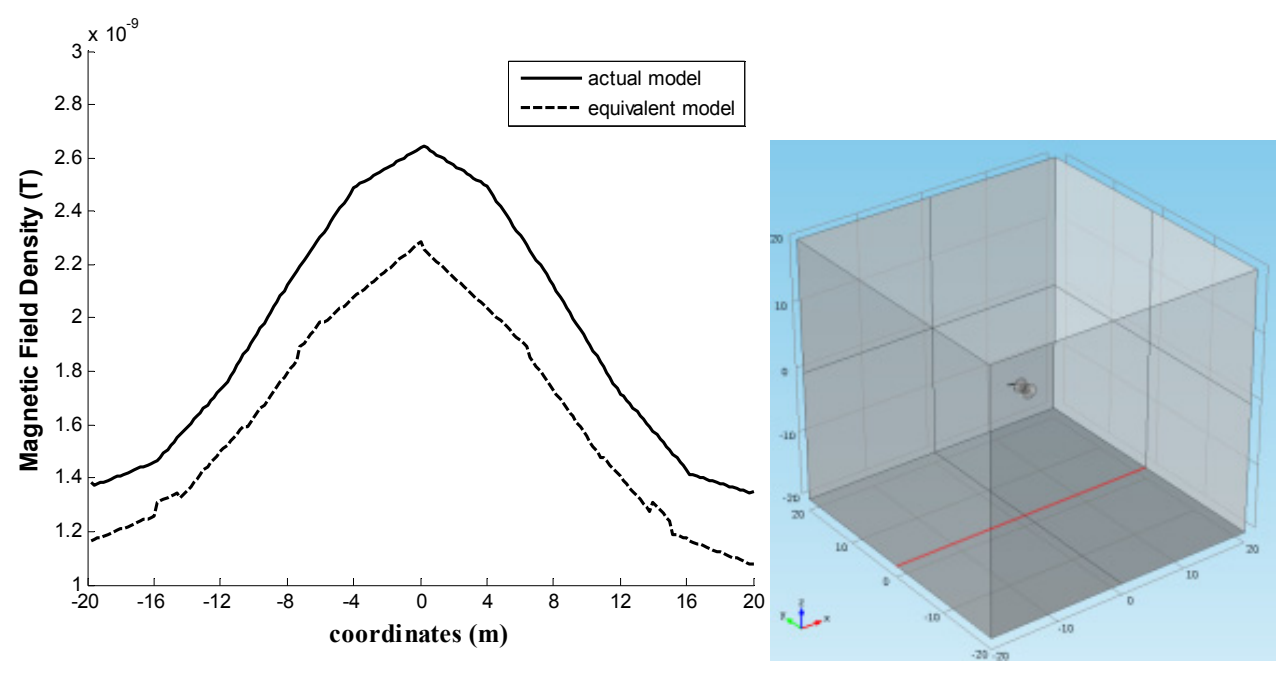

Fig. 6.50 Radiated Magnetic field density along X axis in XY plane 


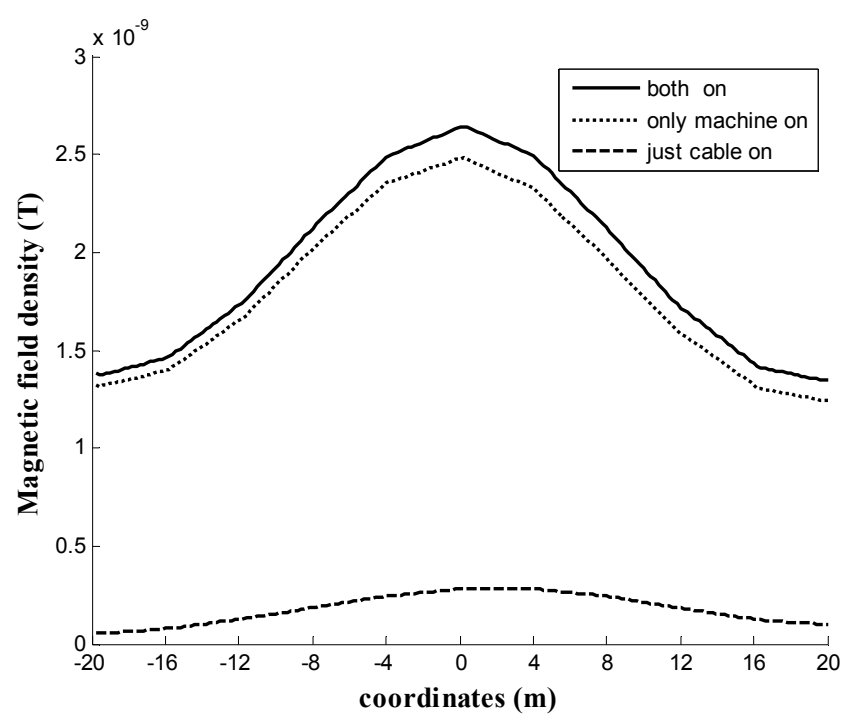

Fig. 6.51 Radiated Magnetic field density along X axis in XY plane

\subsubsection{Coupling of machines}

The studied synchronous generator in 6.3 .3 is coupled to the induction motor, which was studied in 6.3.1. The system which is implemented in finite element domain, is shown in fig. 6.52. As mentioned, the switches of other components are turned on and off for evaluating the radiated field of each component or two or several components together.

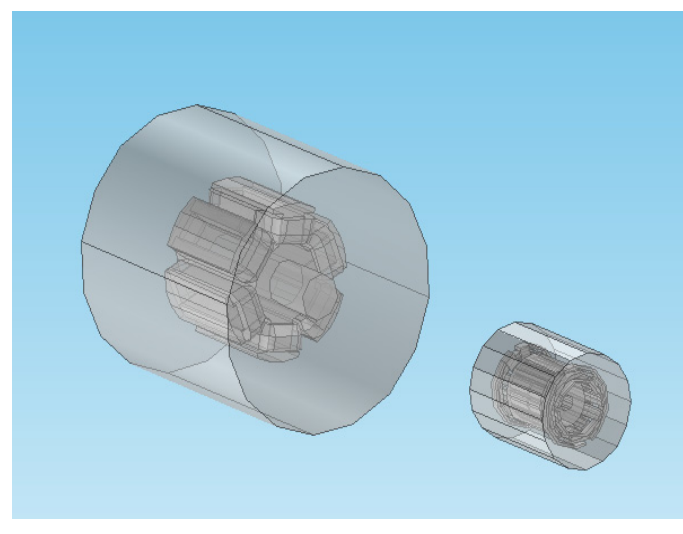

(a)

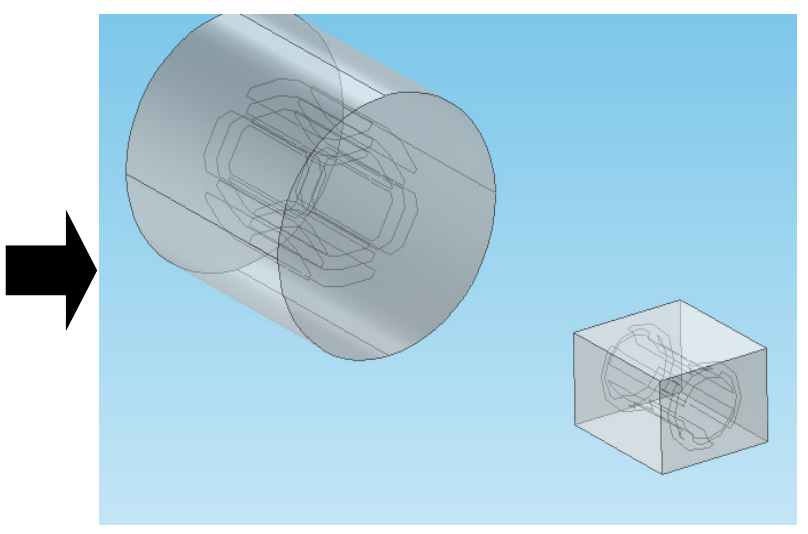

(b)

Fig. 6.52 Schematic of the power setup (a) the full FE model (b) the equivalent source model 
The synchronous generator and induction motor are switched on and off to see their effect separately and verify the wire model. In the following, the synchronous generator is turned on while other components are switched off.

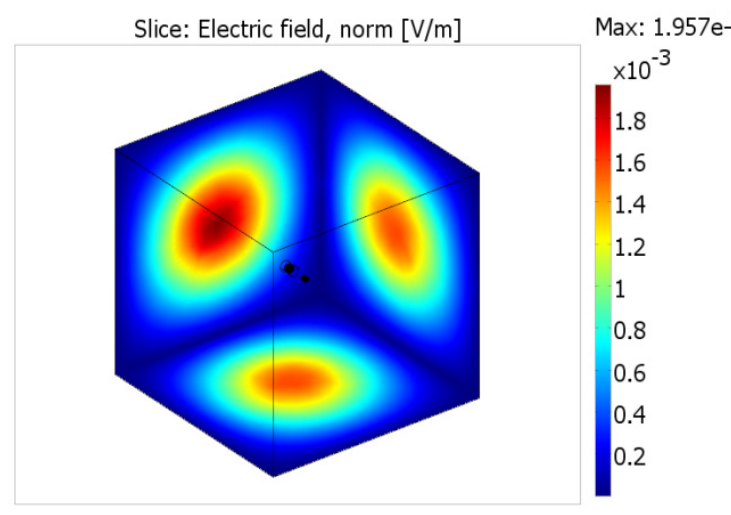

(a)

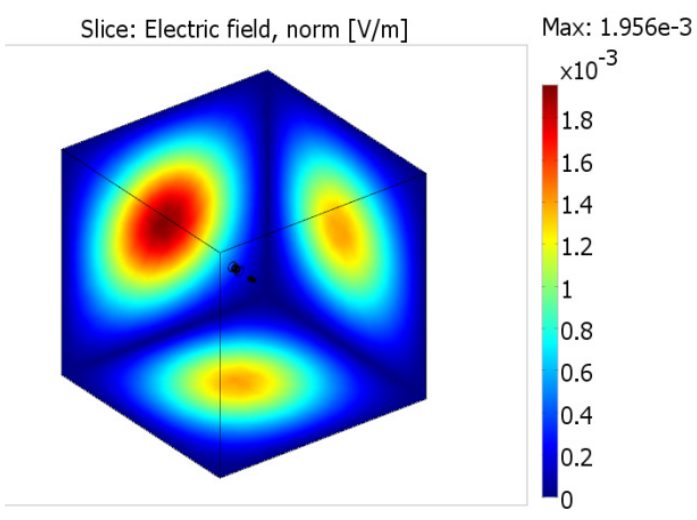

(b)

Fig. 6.53 Radiated electric field of (a) actual model (b) equivalent source model in tesla (T) while the synchronous generator is turned $\mathrm{ON}$ and other components are off

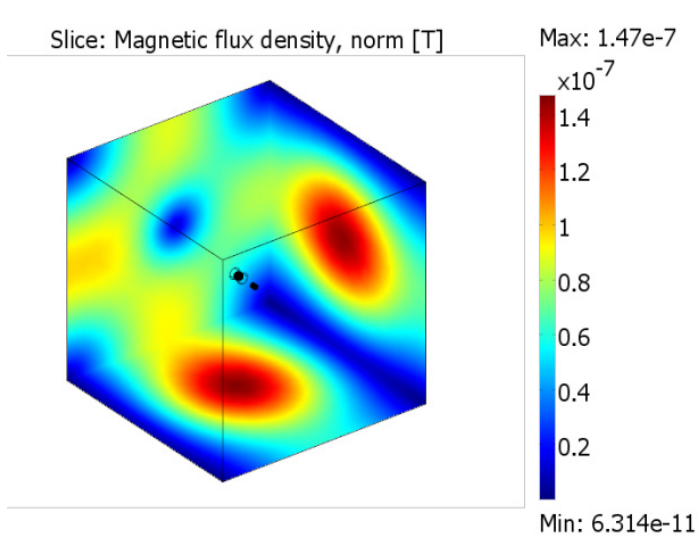

(a)

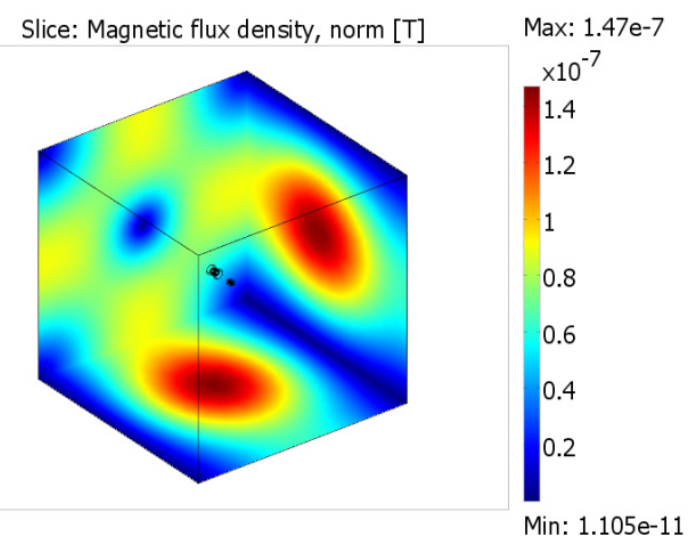

(b)

Fig. 6.54 Radiated magnetic field density of (a) actual model (b) equivalent source model in tesla $(\mathrm{T})$ while the synchronous generator is turned $\mathrm{ON}$ and other components are off

As shown in the two above figures, the electric and magnetic fields radiated from the wire-model of the synchronous generator matches the fields radiated from the actual machine. The electric fields of the fig. 6.53 match the Maxwell radiation theory, since the 
electric field is in the direction of the poles of the terminal voltage. That's why the propagated field in fig. 6.53 is more in frontal plane compared to the lateral planes. Inversely, the magnetic fields establishes in perpendicular to the direction of currents; thus, the field in lateral planes is more than frontal plane in fig. 6.54.

After testing the fields of each machine specifically, the fields of coupled motorgenerator is measured and shown in the following figures.

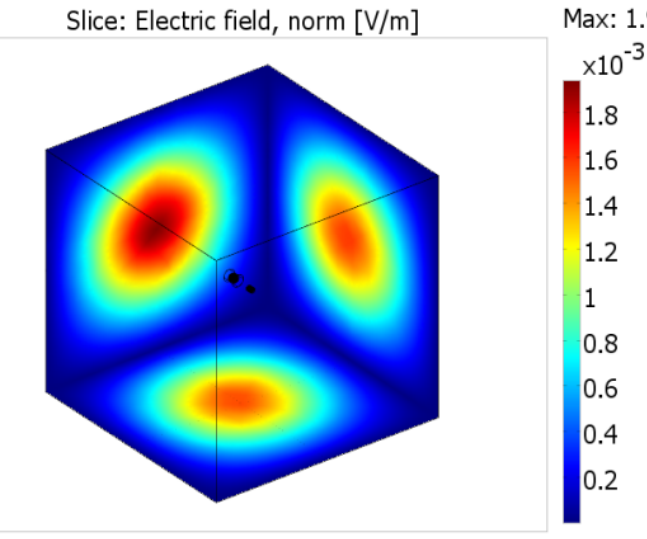

(a)

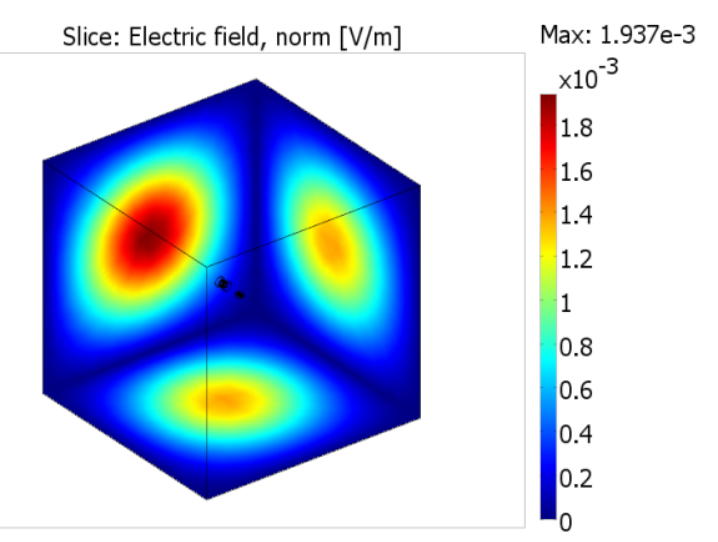

(b)

Fig. 6.55 Radiated electric field of (a) actual model (b) equivalent source model in tesla (T) while the coupling of machines (generator-motor) is turned $\mathrm{ON}$ and others are off

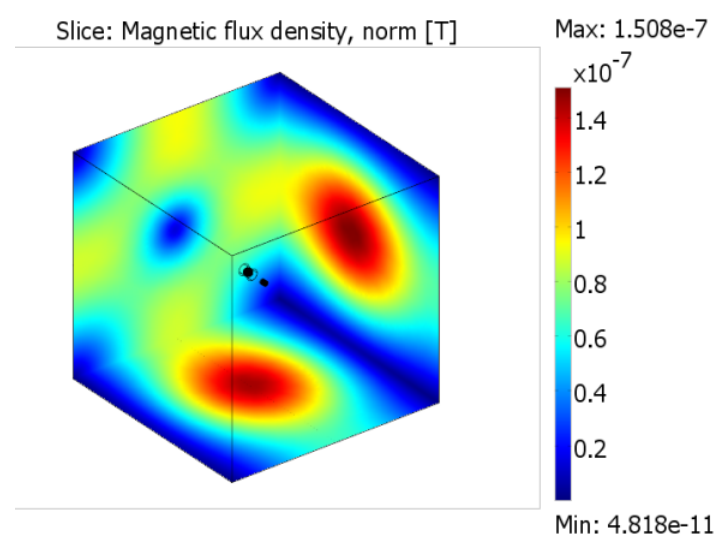

(a)

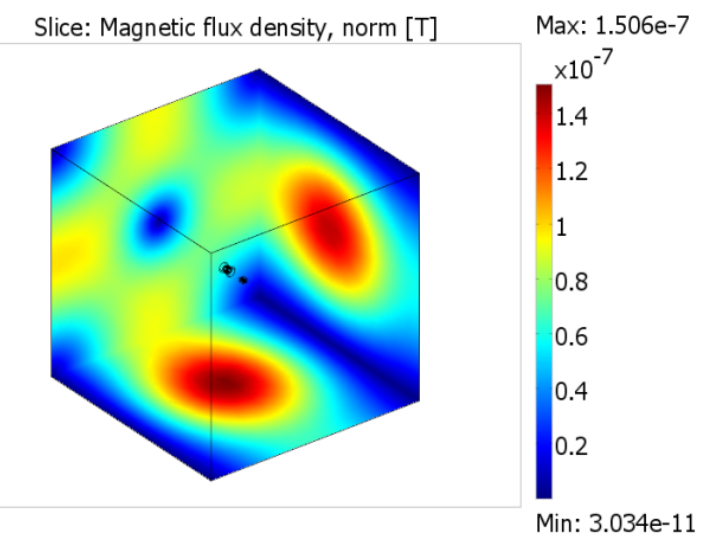

(b)

Fig. 6.56 magnetic stray field density of (a) actual model (b) equivalent source model in tesla (T) while the coupling of machines (generator-motor) is turned ON and others are off 
Comparing fig. 6.56 with fig. 6.54 , the amplitude of the electric field is decreased, while the induction motor is connected to the generator. This is because the polarity of terminal voltage in motor is against generator; so, the electric field is not cumulative. However, the direction of the current of the motor is in the direction of generator. By the way, the radiated field of the equivalent source model matches the radiated field of the actual model.

\subsubsection{Whole system setup}

\subsubsection{Model design}

Finally, all the components are gathered and the excitation is applied to the generator and motor, pulse load and the connection cable get connected to them. The details of the components are mentioned in Table 6-3. The model is analyzed in full details in finite element domain. In addition, the wire model is used to model all these components.

Table 6.3 The details of the components in the tested setup component characteristics

\begin{tabular}{|c|c|}
\hline Synchronous generator & $\begin{array}{l}13.8 \mathrm{~kW} \text {, PF: } 0.8 \text {, length: } 25 \mathrm{~cm} \text {, diameter: } 28-30 \mathrm{~cm} \text {, pole: } 4 \text {, } \\
\text { rpm: } 1800 \text {, nominal voltage: } 230 \mathrm{~V} \text {, amp: } 39.5 \mathrm{~A} \text {, exc. voltage: } \\
37 \mathrm{~V} \text {, exc. amp: } 1.9 \mathrm{~A}\end{array}$ \\
\hline Induction machine & $5.5 \mathrm{~kW}, \mathrm{PF}: 0.85$, length: $30 \mathrm{~cm}$, diameter: $25 \mathrm{~cm}$, pole: 4 \\
\hline Electric Load & $3 \mathrm{~kW}$ AC load \\
\hline Connection cable & $\begin{array}{l}\text { XLPE, Diameter: } 5 \mathrm{~cm} \text {, insulated and armored PVC sheathed } \\
\text { cable }\end{array}$ \\
\hline
\end{tabular}

The models are shown in fig. 6.57(a), (b). As shown in this figure, the wire model consists of numerous lines with specific currents flowing and voltages established at nodes of these wires. 


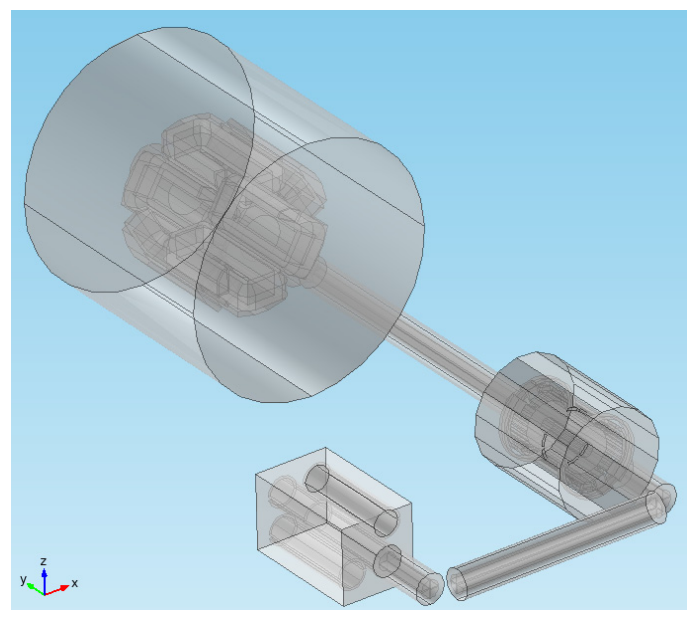

(a)

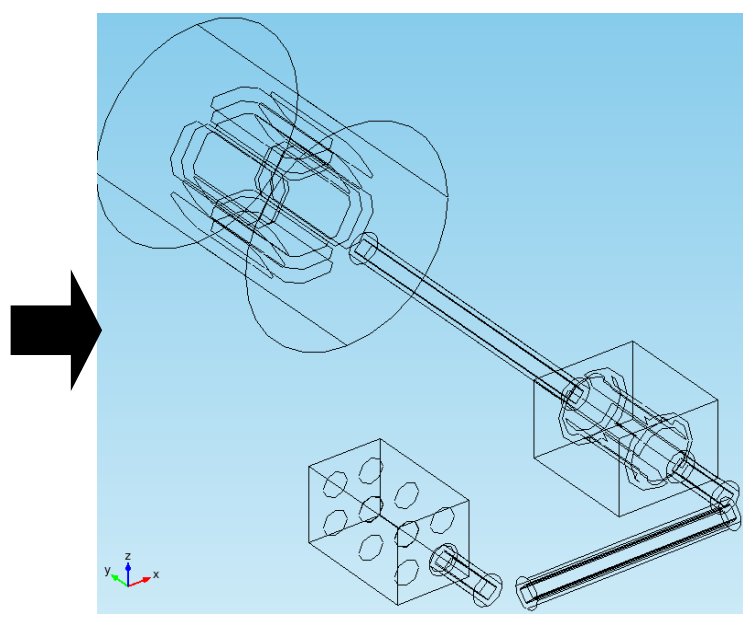

(b)

Fig. 6.57 Schematic of the power setup (a) full FE model (b) equivalent source model

The described wire models have some issues in multi-component system. The radiated electric and magnetic fields are affected not only from the voltage and current magnitudes but also from the permittivity and permeability of the materials [205]. In addition, the radiated field of one component induces fields into the other components which cause induced voltage. Consequently, additional radiated electric and magnetic fields are created. For example, the induced field of the induction motor in the vicinity of the cable is obtained as shown in fig. 6.58. This field is obtained while other components are turned off and only the induction motor is ON. On the other hand, the wire model may not have this phenomenon, since the lines in the model doesn't have the volume. Therefore, the induced voltage and consequently the additional radiated field are not produced in the wire model. In order to resolve this issue, another optimization should be applied to the wire model. The issue of superposition exists, since there are no components' cases and insulation in the wire model. Hence, each component in the embedded wire model is enclosed from the other components with a casing. The casing 
should have optimized value of permeability to avoid superposition between components. The value of permeability must be less than 1 and close to zero; however, very small value of permeability increases the simulation time dramatically. Therefore, both mentioned parameter should be considered. Less permeability of the casing reduces the amount of radiated magnetic field entering into the imposed casing; while, the magnetic field getting out of the imposed casing doesn't get affected seriously. Therefore, the superposition between the components would decrease; while, the radiated field in the area around, air, doesn't change considerably.

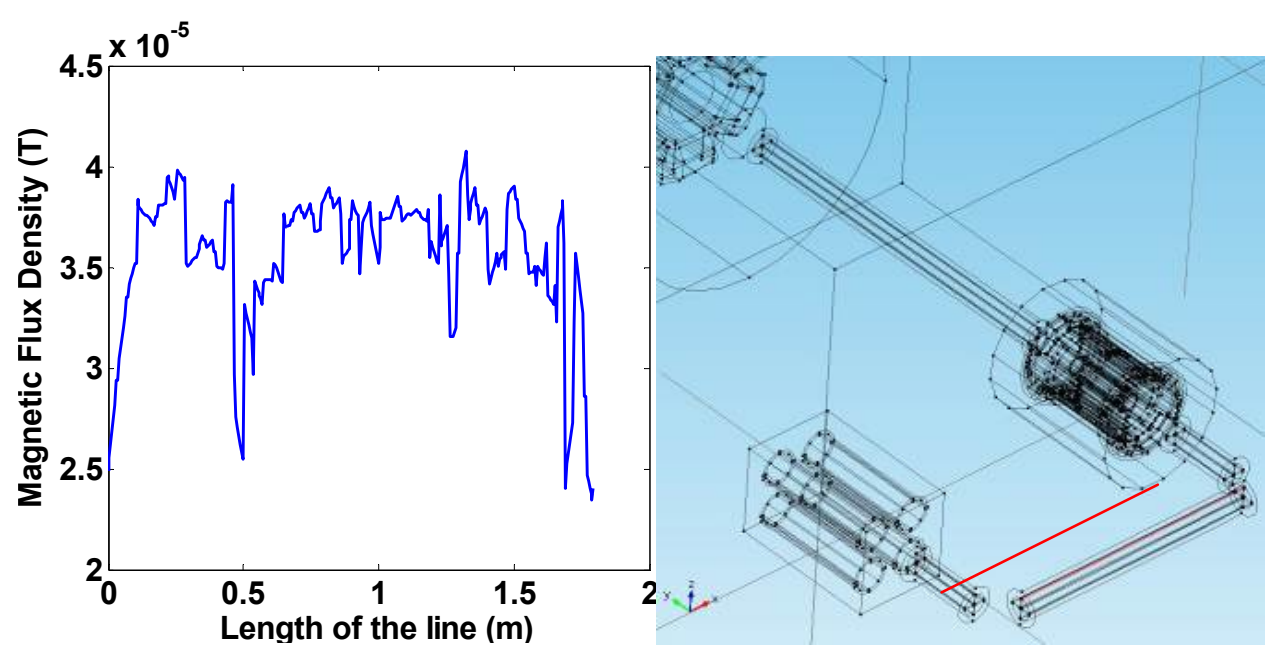

Fig. 6.58 Radiated magnetic field of the induction machine on the cable, while only the induction motor is turned on

The magnetic fluxes radiated from the actual and the wire models are derived from the simulation at $7 \mathrm{~m}$ away from the arrangement and shown in fig. 6.59 (a), (b), respectively. As illustrated in the figure, the magnetic flux radiated from the wire model is similar to the actual model. The small difference between the maximum values of 
magnetic flux densities of two models is due to the issue of the superposition of the components.

The optimized result is shown in fig. 6.60. As can be seen, the magnitude of the radiated magnetic field of the optimized wire model is almost the same as the actual model.

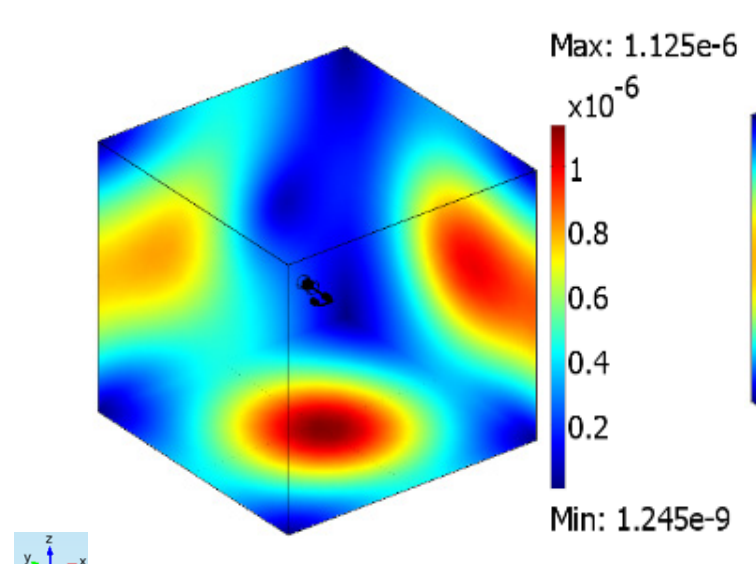

(a)

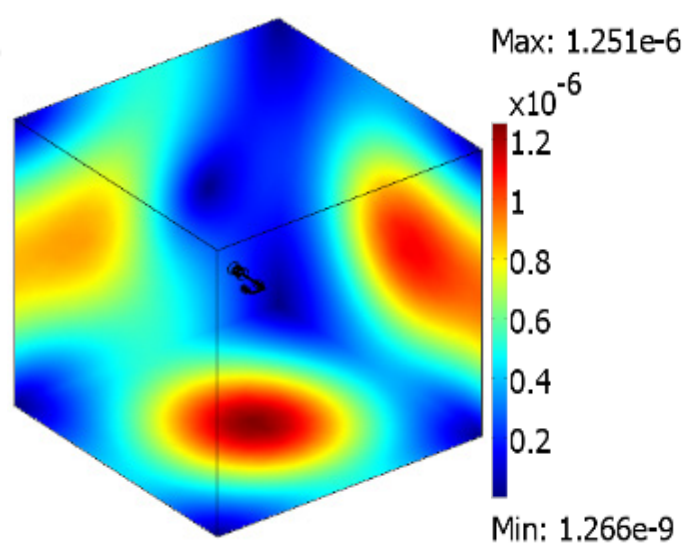

(b)

Fig. 6.59 Radiated magnetic flux density of (a) actual model (b) wire model in tesla (T)

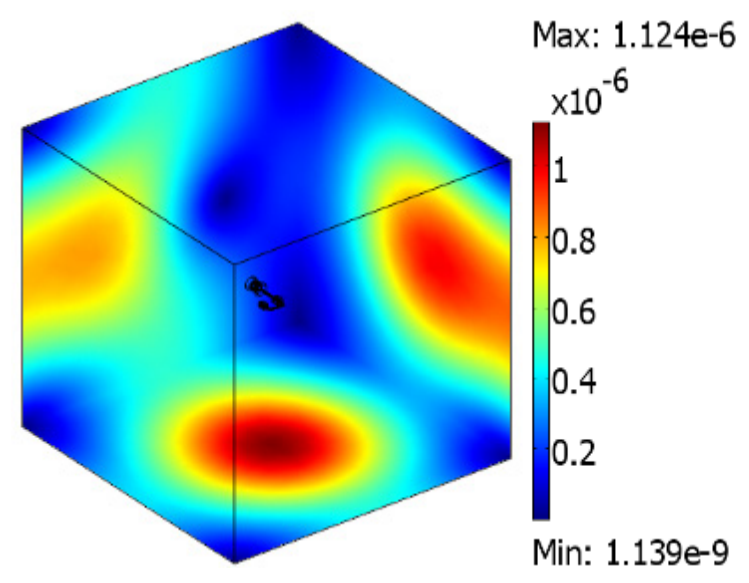

Fig. 6.60 Radiated Magnetic flux density of the optimized wire model (T) 


\subsubsection{Simulation and experimental results}

In addition to the superposition, another application of the model is using turning on and off switches for each of the components. By implementing the switches, the effect of each component could be identified and analyzed. The benefit of studying each component separately is monitoring their behavior, detecting failures and fault conditions. This can be done using both simulation and experimental methods. Various cases of the studied setup, shown in fig. 6.61, are conducted and added to the figures, obtained from the simulation and measurement results. Note that the connection cables to the loads are outside the fig. 6.61. Moreover, there is a controller connected to the drive shown in fig. 6.61 which is out of the system. The system is started up manually. Details of the measurement elements are mentioned before in 6.3.1

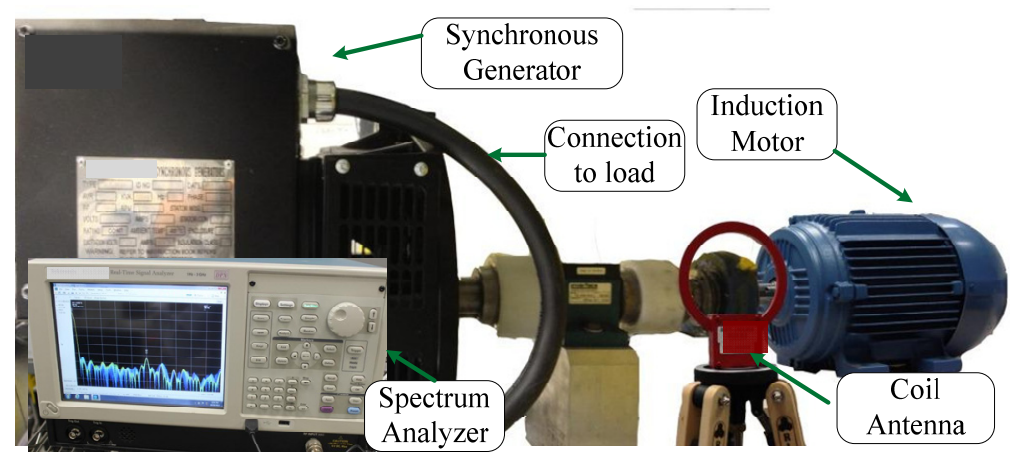

Fig. 6.61 The studied setup including machines, measurement stuff and control drive (for switching)

For the experimental test, all components including the synchronous generator, the induction motor and the electric load are turned on. The cables are passing currents, so they also can be considered ON. The same test as in the previous case is studied here. All switches are turned on and the $\mathrm{H}$-field is measured experimentally and also obtained from the simulation models. The machines are tested at their nominal voltages. The magnetic 
field intensity (H-field) of the measurement and simulation models is shown in fig. 6.62. As shown in this figure, the full finite element model and the wire model have similar radiated $\mathrm{H}$-field compared to the measurement. The small differences of the amplitudes are because of the effect of the body of the other components around the system.

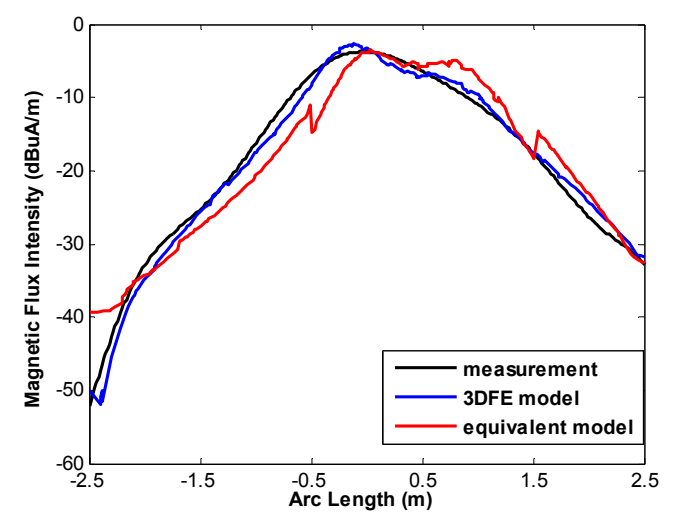

Fig. 6.62 The measured magnetic field intensity at $55 \mathrm{~cm}$ far from the setup in $\mathrm{Y}$ axis while all components were turned on at $60 \mathrm{~Hz}(\mathrm{~dB} \mu \mathrm{A} / \mathrm{m})$

The application of this study is in the system monitoring and fault diagnosis, which is studied in chapter 7.

\subsection{Generalization of the wire model}

\subsubsection{Study approach}

All designed models were suitable for only one typical situation, including terminal voltage rates and physical geometry conditions of the machines. The model proposed here is optimized in a way such that it can be utilized for various types of machine sizes and operating voltages.

The procedure involves measuring (numerically evaluating) radiated fields of an actual AC machine model with a basic size. If the model of the proposed size is not 
available, the fields can be estimated using related equations, based on the fields measured for basic size [175]. Optimization factors are then applied as follows:

$$
K_{B S}=B_{i \text { Snew }} / B_{\text {base }}, \quad K_{E S}=E_{\text {iSnew }} / E_{\text {base }}
$$

where $B_{\text {base }}$ and $E_{\text {base }}$ are the magnetic field density and electric field of the basic case and $B_{i S n e w}$ and $E_{i \text { Snew }}$ are magnetic field density and electric field of any machine size. These parameters could be measured at any random points around the component e.g. maximum B in a plane at a distance from the component. The $K_{B S}$ and $K_{E S}$ factors in eq. (6.3) are applied respectively to the currents and voltages of the wire model to optimize the model for a new machine with a different size. These factors are applied due to the fact that the magnetic field density, which is used in these equations, show strong correlation with the magnitude of the current of the lines in non-volumetric models (Biot-Savart Law). Similarly, the electric field has the same relation with the voltage at the nodes [175]. A similar procedure can be applied for variations of the terminal voltage. In this case, the factors are as follows:

$$
K_{B V}=B_{i \text { nnew }} / B_{\text {base }}, K_{E V}=E_{i \text { hnew }} / E_{\text {base }}
$$

where $B_{i \text { Vnew }}$ and $E_{i \text { Vnew }}$ are the magnetic field density and electric field of any proposed sizes. Also, $K_{B V}$ and $K_{E V}$ factors in eq. (6.4) are applied respectively to the currents and voltages of the wire model to optimize the new case with a different terminal voltage. If there is a case with both voltage and size variations, both factors will be multiplied by the current and voltage values of the basic equivalent source model.

Since some material properties of machines, such as the permeability are nonlinear, it is not possible to utilize the currents and voltages instead of B and $\mathrm{E}$ in eq. (6.3), for all 
working conditions. However, it might be possible to replace the magnetic field density (B) with the current or other parameters for specific range of currents. Hence, the most reliable parameter is magnetic field density and electric field, which are being used in (6.3) and (6.4). However, in order to avoid modeling the actual machine in each different case to obtain $\mathrm{B}_{\mathrm{iSnew}}, \mathrm{E}_{\mathrm{iSnew}}, \mathrm{B}_{\mathrm{iVnew}}$, and $\mathrm{E}_{\mathrm{iVnew}}$ in (6.3) and (6.4), the related curves of the four factors $\left(K_{B S}, K_{E S}, K_{B V}, K_{E V}\right)$ for both $\mathrm{AC}$ machines are obtained. Random examples for different cases (size and voltage variation) are measured and the related factors are obtained. These are shown in Tables 6.4 and 6.5 and based on the points in Table 6.4 and curve fitting procedure, the curves were established.

As shown in Table 6.4, the factors due to the geometrical size changes are not just based on size ratios, but many other parameters have effect on the values of these factors. A curve fitting technique was used to find an equation to obtain these factors based on size ratio as a variable. For example, the equation for $K_{B S}$ of synchronous generator is as follow:

$K B S=-0.06805 \cdot R^{3}+0.80653 \cdot R^{2}+0.28031 \cdot R+0.0028016$

where $\mathrm{R}$ is the size ratio.

The factor due to the terminal voltage changes, as illustrated in Table 6.5 , are mostly related to the terminal voltage ratios. Here, there is no need for any kind of curves for the terminal voltage variation case and the ratios that can be directly used as factors. The curves for size variation case, which is established based on Table 6.4, are shown in figs. 6.63 and 6.64 . 
Table 6.4 Some patterns of size variation of induction motor and synchronous (syn) generator

\begin{tabular}{|c|c|c|c|c|c|}
\hline & Rati & KBS & KES & KBS & KES of Induction \\
\hline 0 & & SYN Machine & SYN Machine & Induction Machine & Machine \\
\hline & 0.6 & 0.460081 & 0.558676 & 0.53685 & 0.560039 \\
\hline & 0.8 & 0.704005 & 0.773821 & 0.75928 & 0.782211 \\
\hline & 0.9 & 0.872369 & 0.88666 & 0.92806 & 0.928058 \\
\hline & 1.0 & 1.0 & 1.0 & 1.0 & 1.0 \\
\hline & 1.3 & 1.5689 & 1.34052 & 1.46037 & 1.36037 \\
\hline & 1.7 & 2.49898 & 1.81896 & 2.12283 & 1.92283 \\
\hline & 2.0 & 3.23557 & 2.16098 & 2.59692 & 2.29693 \\
\hline
\end{tabular}

Table 6.5 Some patterns of terminal voltage variation of induction motor and synchronous (syn) generator

\begin{tabular}{llllc}
\hline Ratio & $\begin{array}{l}\text { KBV of SYN } \\
\text { Machine }\end{array}$ & $\begin{array}{l}\text { KEV of SYN } \\
\text { Machine }\end{array}$ & $\begin{array}{c}\text { KBV of } \\
\text { Induction Machine }\end{array}$ & $\begin{array}{c}\text { KEV of } \\
\text { Induction Machine }\end{array}$ \\
\hline 0.6 & 0.5998 & 0.6003 & 0.60002 & 0.60001 \\
0.8 & 0.7997 & 0.8004 & 0.800066 & 0.79998 \\
0.9 & 0.8995 & 0.9002 & 0.90001 & 0.90001 \\
1.0 & 1.0 & 1.0 & 1.0 & 1.0 \\
1.3 & 1.2994 & 1.3004 & 1.30001 & 1.30002 \\
1.7 & 1.6993 & 1.7001 & 1.7000 & 1.70001 \\
2.0 & 1.9993 & 2.005 & 2.0000 & 2.00002 \\
\hline
\end{tabular}




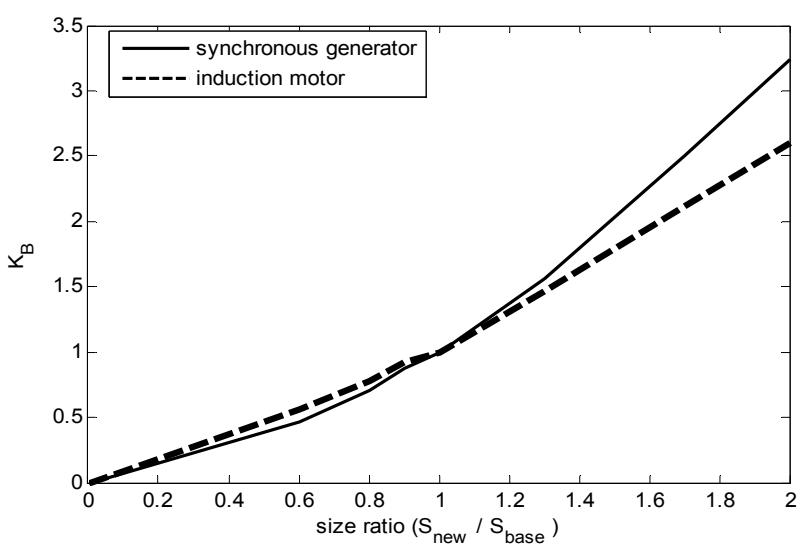

Fig. $6.63 \mathrm{~K}_{\mathrm{B}}$ due to size variation of synchronous generator and induction motor

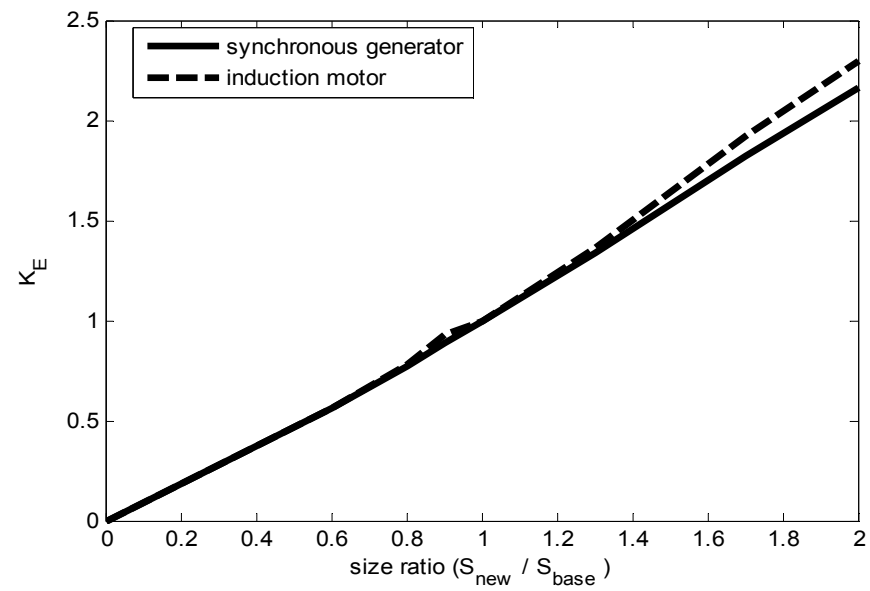

Fig. $6.64 \mathrm{~K}_{\mathrm{E}}$ due to change of size of synchronous generator and induction motor

\subsubsection{Case studies}

To verify the generalized model, both $\mathrm{AC}$ machines (induction and synchronous) were analyzed in different cases:

An induction machine case with 1.2 times the basic size ( $R: 1.2)$ is simulated in a large region. A comparison between the equivalent source and actual detailed numerical models is shown in fig. 6.65 (a)-(d). Radiated magnetic field density and electric field spectrums were used as indices. An induction machine case with 0.6 times of the original terminal voltage ( $\mathrm{R}: 0.6)$ is simulated and the comparison between the new equivalent source model and actual numerical models are shown in fig. 6.66 (a)-(d). 
This case considered both variation of geometrical size and terminal voltage. The results are shown in fig. 6.67 (a)-(d) for both the equivalent source model and the detailed numerical model.

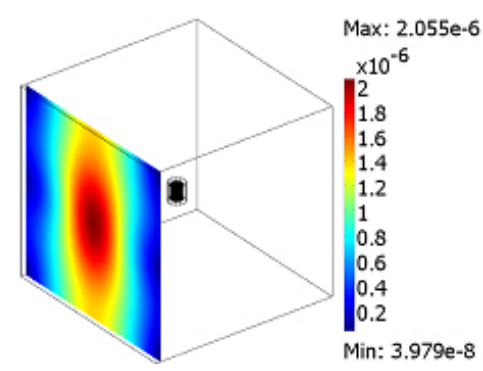

(a)

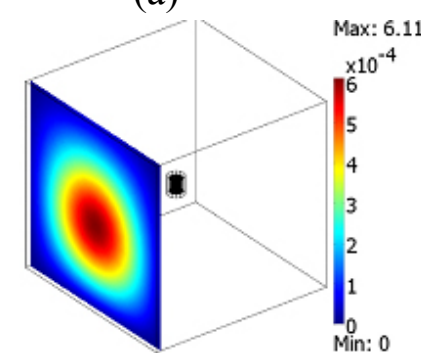

(c)

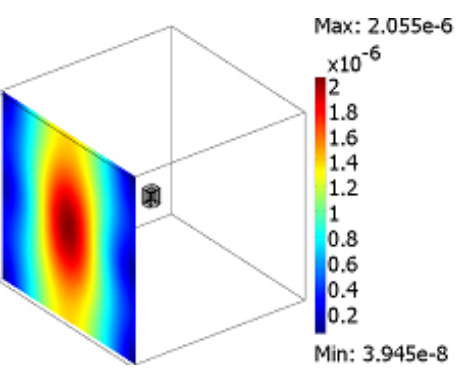

(b)

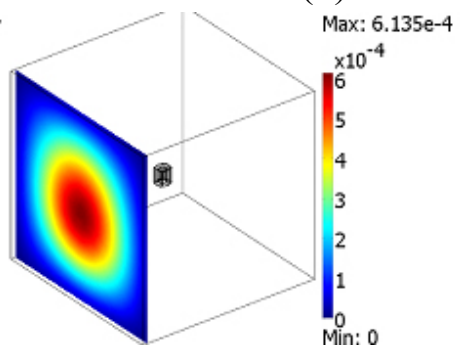

(d)

Fig. 6.65 Field spectrum of induction motor while the geometric size increased $20 \%$ (a) B of actual model (b) B of equivalent source model (c) E of actual model (d) E of equivalent source model

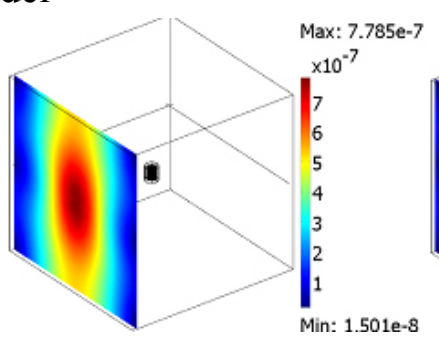

(a)

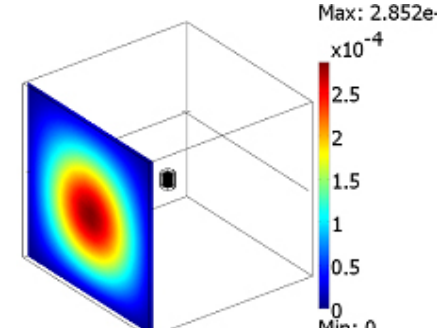

(c)

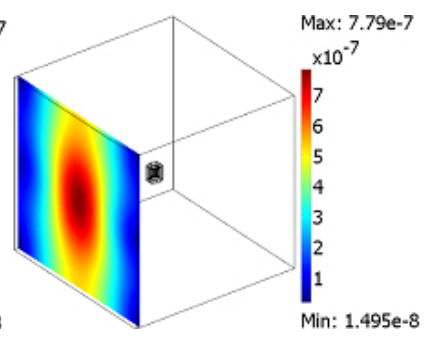

(b)

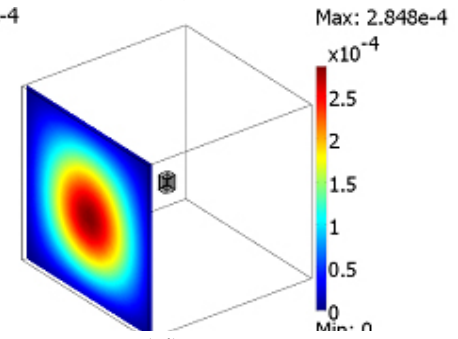

(d)

Fig. 6.66 Field spectrum of induction motor while the terminal voltage decreased $40 \%$ (a) B of actual model (b) B of equivalent source model (c) E of actual model (d) E of equivalent source model 
According to the amplitudes and spectrums in these figures, the field propagated from the equivalent source models show the significant accuracy when compared with the actual numerical models. The region in this study was $(8 \times 8 \mathrm{~m})$ region, while the diameter of the induction motor was $1.2 \mathrm{~m}$.

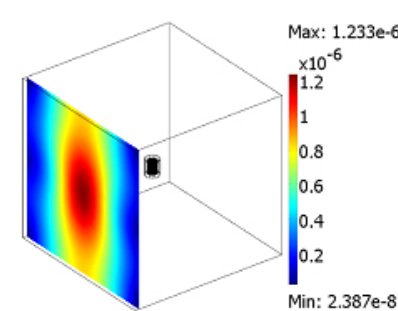

(a)

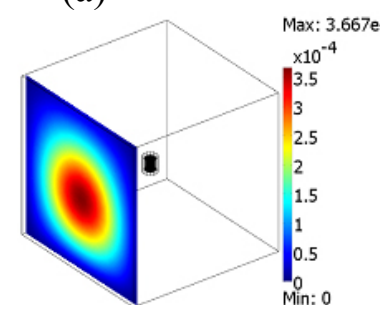

(c)

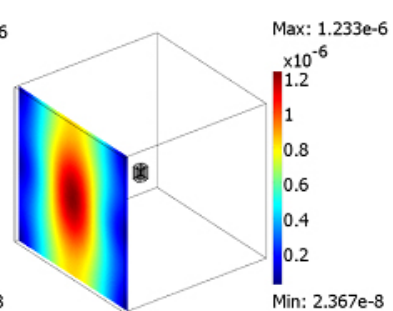

(b)

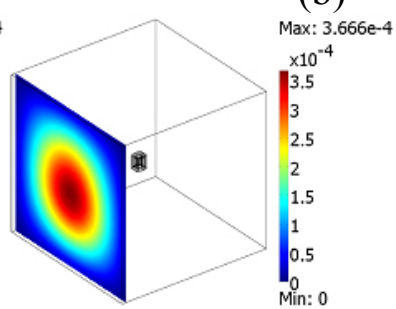

(d)

Fig. 6.67 Field spectrum of induction motor while the terminal voltage decreased $40 \%$ and geometric size increased 20\% (a) B of actual model (b) B of equivalent source model (c) E of actual model (d) E of equivalent source model

The same procedure was implemented for a synchronous generators case. In order to check the propagated fields and validate them at far distances, the machine was placed in a large region $(50 \times 50 \mathrm{~m})$. Only the similar case to the third case of induction machine is shown in this part. Hence, the variation of geometrical size with the ratio equal to 1.7 and terminal voltage with the ratio equal to 0.8 is considered. According to the Tables 6.4 and 6.5, the factors are as follow: $\mathrm{K}_{\mathrm{BS}}=2.49898, \mathrm{~K}_{\mathrm{ES}}=1.81896, \mathrm{~K}_{\mathrm{BV}}=0.8$ and $\mathrm{K}_{\mathrm{EV}}=0.8$. Therefore the two applied factors to the equivalent source model will be $K_{B}=K_{B S} \times K_{B V}$ and $\mathrm{K}_{\mathrm{E}}=\mathrm{K}_{\mathrm{ES}} \times \mathrm{K}_{\mathrm{EV}}$. These two factors were applied to the branch currents and node 
voltages of the equivalent source model, respectively. For verification, the actual and optimized equivalent source models were compared and are shown in fig 6.68 (a)-(d).

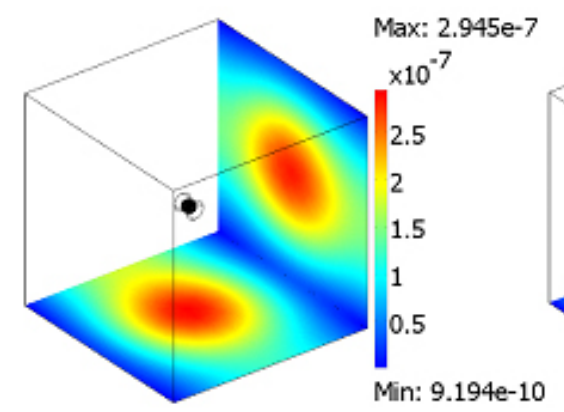

(a)

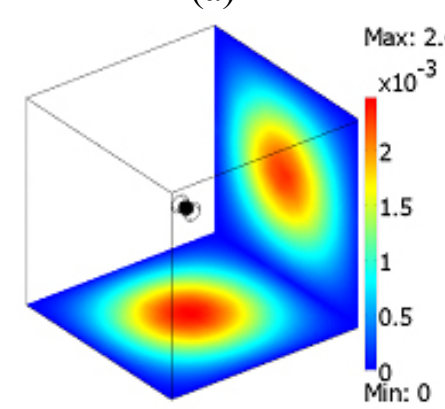

(c)

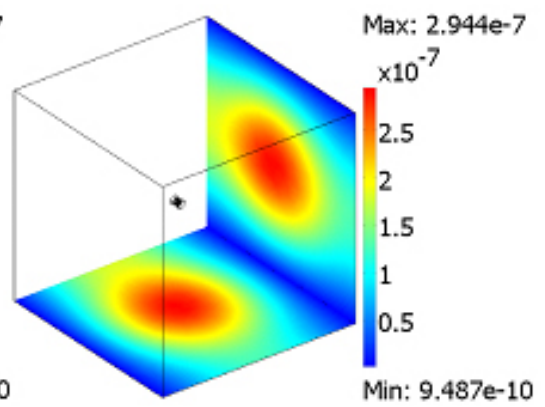

(b)

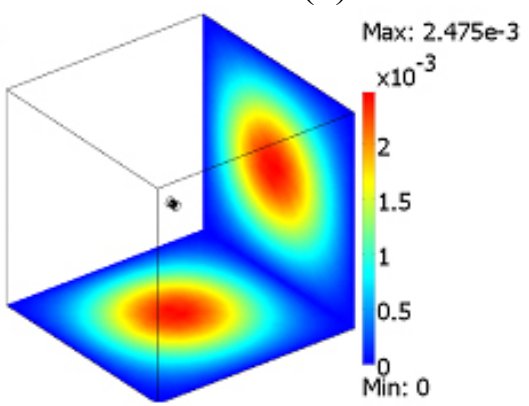

(d)

Fig. 6.68 Field spectrum of synchronous generator while the terminal voltage decreased $20 \%$ and geometric size increased $70 \%$ (a) B of the actual model (b) B of equivalent source model (c) E of actual model (d) E of equivalent source model

Comparing the amplitudes and spectrums in fig. 6.68 (a) with fig. 6.68 (b) and fig. 6.68 (c) with fig. $6.68(\mathrm{~d})$, the propagated fields from equivalent source model accurately matches in both planes around the machines at a distance of about $20-25 \mathrm{~m}$. It should be noted that unlike the induction motor cases in the previous study, the generators are located horizontally. Locating induction machine vertically is necessary in some applications [206]. As shown in fig. 6.68, the actual models are much larger than the equivalent source one, because they are tested with a new size, while the equivalent 
source model is in the same size and shape. The optimization factors were applied and the results match very accurately.

The final test case, both $\mathrm{AC}$ machine types are located in a region $(50 \times 50 \mathrm{~m})$ with a different test is implemented on both. The size ratio of the synchronous generator is chosen equal to 1.4 , while this ratio for the induction motor is chosen as 0.85 . In addition, the voltage ratios for the synchronous generator and the induction motor are selected as 1.2 and 0.7, respectively. Using Tables 6-4 and 6-5 and the curves derived earlier, the desired factors were estimated. A diagram for calculating these factors is shown in fig. 6.69. The two factors for each machine were estimated based on the four factors. The field spectrums of this test are demonstrated in fig. 6.70 (a)-(d).

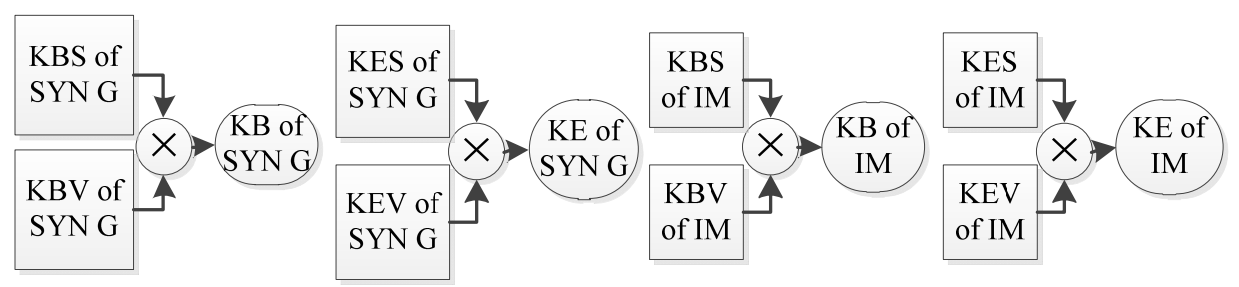

Fig. 6.69 Calculation diagram of optimization factors for the two AC machines. SYN G and IM stand for synchronous generator and induction motor

This test case shows that even with the change of all the conditions simultaneously, the equivalent source model results match the actual one. In addition to the verification of the equivalent source model, other aspects in the area of EMC evaluation can be recognized. For example, comparing fig. 6.70 with fig. 6.68 and 6.66 , it is obvious that the field spectrum of fig. 6.70 is similar to fig. 6.68. This means that coupled AC machine systems radiate similar electromagnetic signatures to the synchronous machine. This is because of the large difference between the nominal power of the synchronous 
generator and induction motor (857-kVA vs. 33- kVA). Therefore, radiated fields of induction motor only increase the amplitude of overall fields of the studied system.

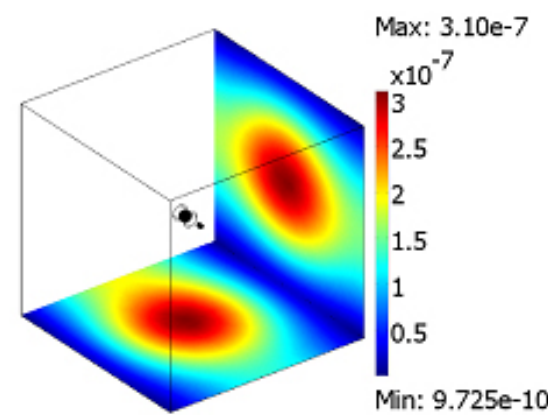

(a)

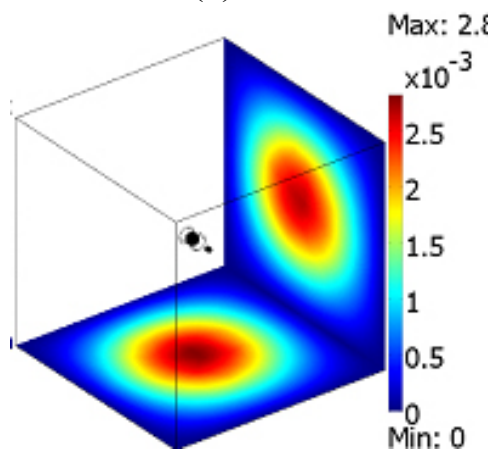

(c)

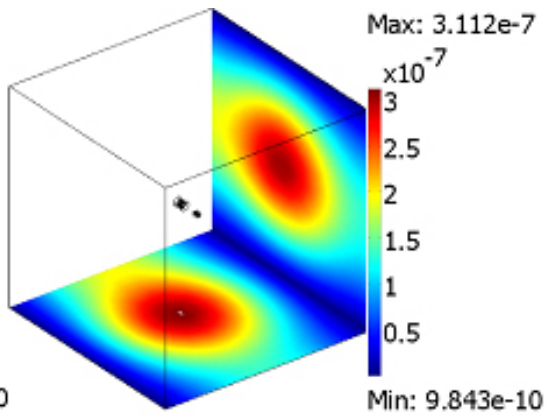

(b)

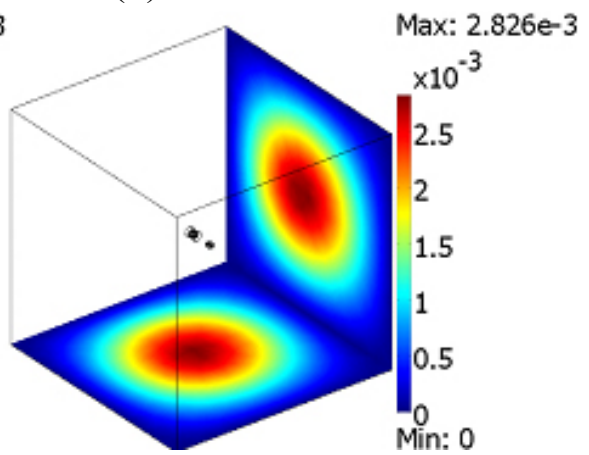

(d)

Fig. 6.70 Radiated field spectrums from both synchronous generator and induction motor (a) B of actual model (b) B of equivalent source model (c) E of actual model (d) E of equivalent source model

In order to increase the accuracy of the equivalent source model, switches were considered for each machine to turn them on and off to study the superposition concept.

Finally by comparing simulation time of the two models, the actual model of the machines implemented by full finite element model and the generalized equivalent source model, the results demonstrated in Table 6.6 are obtained.

Table 6.6 Simulation characteristics comparison 


\begin{tabular}{lcc}
\hline \hline & of freedom (million) & is time (s) \\
\hline Induction motor (actual) & 3.5 & 6200 \\
\hline Induction motor (equivalent source) & 0.9 & 140 \\
\hline synchronous generator (actual) & 4.2 & 6800 \\
\hline synchronous generator (equivalent source) & 1.1 & 170 \\
\hline Coupled machine model (actual) & 6.0 & 11000 \\
\hline Coupled machine model (equivalent source) & 1.5 & 188 \\
\hline
\end{tabular}

\subsection{Conclusion}

In order to study electromagnetic fields strayed from a typical electric power system train, the embedded equivalent source models have been designed and investigated. A new method termed wire modeling is used for designing this equivalent source model. It was created from representative cylinder loops carrying a set of currents in the cylinder branches, as well as voltages at the nodes. Since power components have several types of elements, individual equivalent source model was designed for each of them and several switches were considered for turning on and off windings. The data for the parameters in the model are based on the current density and potential difference of the actual machine. Also the GA-based PSO method was used for evaluating the dimension of the model.

For the analysis, electric and magnetic-interfaced finite element method is used. The results show that it is possible to replace actual model of electrical machines with equivalent the equivalent source model. The simulation time of the proposed equivalent source model is approximately $80-100$ times lower than using the actual model. This enables the numerical simulation of multiple sources in a reasonable time allowing the practical study of EMC issues during electric drive development stages. The 
superposition and suppression of the fields in coupling the components were also studied and verified. 


\section{Modified 3D Finite Element Modeling of Converters for System Monitoring}

\subsection{Overview}

In this chapter, detailed physics-based modeling of a power converter drive is proposed. The 3D finite element (FE) modeling of a power inverter was developed and analyzed. An approach in physical modeling of the switching activity of the inverter in FE is proposed. In addition, the solver was modified and implemented for analyzing nonlinear materials in time-harmonic study to achieve faster computation. The frequency response analysis was also implemented in the simulation and measurements at various locations from the source. To consider the modeling of the power converter-drive under different operating modes, the inverter was located in two different setups including a drive connected to an AC load and a drive connected to an induction motor. Each of these cases was studied via simulation and experimental tests and their related applications were investigated. The importance of this work is to facilitate the ability to evaluate the stray electromagnetic field levels used for evaluating EMC compliance at the design stage. In addition, the recognition of a failure condition inside each component of the system by observing the fields is another important contribution of this work. The optimum operation of the system components for lower EMI and the optimum design of related shielding for EMC evaluation studies are added benefit of this work.

The equivalent circuit modeling of power electronic drives is not applicable for studying the radiated emission in the three dimensional areas around the drive. Therefore, the physics based modeling is proposed in this chapter to evaluate the stray field in the three dimensional space around the devices. In addition, the material properties of the 
devices and shape representation were investigated in this modeling process. On the other hand, time-varying electric and magnetic fields generate MMFs and EMFs that can sustain the fields, which compromise the flux. Also, currents and voltages in electric power systems are time-varying, which causes stray electromagnetic fields. Therefore, there is a need to model the EMI generated by these systems to develop designs meeting EMC standards.

Several studies were performed in the area of radiated low-frequency electromagnetic field (EMF) analysis of power electronic devices [61], [207]-[210]. In [61], some general principles on how to allocate responsibilities between the power grid and connected equipment were investigated with the aim to achieve electromagnetic compatibility in electric power systems. It was shown, in this research, that in medium and high voltage systems, in the absence of comprehensive equipment emission and immunity standards, it is suggested provides that the grid responsible party relevant data, such as on voltage dips to the party responsible for connecting equipment to the grid. Some studies have dealt with the impact of stray EMFs on the environment including humans and the operation of devices [207]. This test is implemented from very low frequencies $5 \mathrm{~Hz}$ to medium frequencies $300 \mathrm{kHz}$. Both magnetic and electric fields are tested using gauss meters and electric field meters. On the other side, other studies were conducted to implement attenuators and shielding of the conducted and field bound EMI [208], [209]. H. Akagi et al focuses on a line EMI filter and its combination with a motor EMI filter, along with their effects on attenuation of conducted emission voltage. Furthermore, the effectiveness of an EMI filter configuration when the filter is applied to the motor was discussed [208]. In [209], EMI noises generated in the power converter and diffused on the surface of 
conductors are targeted to be controlled. Consequently, a new approach based on the distributed constant circuit theory along with multilayer power printed circuit technology was used.

For developing a model, the equivalent source representations, as well as the physics based modeling, were studied. O. Aouine et al designed high and low frequency models of the converter for magnetic near field by using modeling switching parts by circular magnetic dipoles [210]. Moreover, the physics based modeling of the power electronic drives in low-frequency EMF was studied in [201], [211]-[213]. In [201], the optimum equivalent source modeling along with the physics based modeling of an AC motor was analyzed. The idea of loops was enhanced based on optimization methods. In addition to the implementation of the physics based modeling to machines, this type of modeling was also utilized for the power converter switches for the study of the conducted EMI [213]. Since the conducted EMI mostly exist on printed circuit boards (PCB), the FE model of the PCB was studied. Also, the optimization of EMI performance was accomplished in terms of component placement on the PCB [213].

There is a need to have a physics based model, with all details, for the various system components to include the physical effects of stray fields, such as superposition and shielding. However, this model has some issues such as the problem of fast switching of the IGBTs and nonlinear commutation curves. Hence, in this chapter, the threedimensional full finite element modeling of a typical power electronic drive at low frequencies is analyzed. The identified issues with the proposed resolutions are explained. The frequency response analysis of the power converter drive was implemented in the simulation, as well as in the experimental setup in two cases of setup, 
one with an AC load and the other with an induction motor, as a dynamic load. The results of these two cases were compared and their application in EMC study was discussed.

\subsection{Modeling approach}

The stray field of a complex model with thousands of dipoles, such as the proposed drive, needs the calculation of the field of each of these dipoles using equations (4-14) (4-18), as well as a vigorous field calculated by using a numerical technique, such as the FE method. The proposed model was accurately built in FE in which the mentioned equations are calculated by modifying the default equations for obtaining electric and magnetic fields.

The proposed full finite element model is shown in fig. 7.1. This electronic drive consists of an inverter, AC load and the armored connection cable. The detail of the devices are identified in Table 7.1.

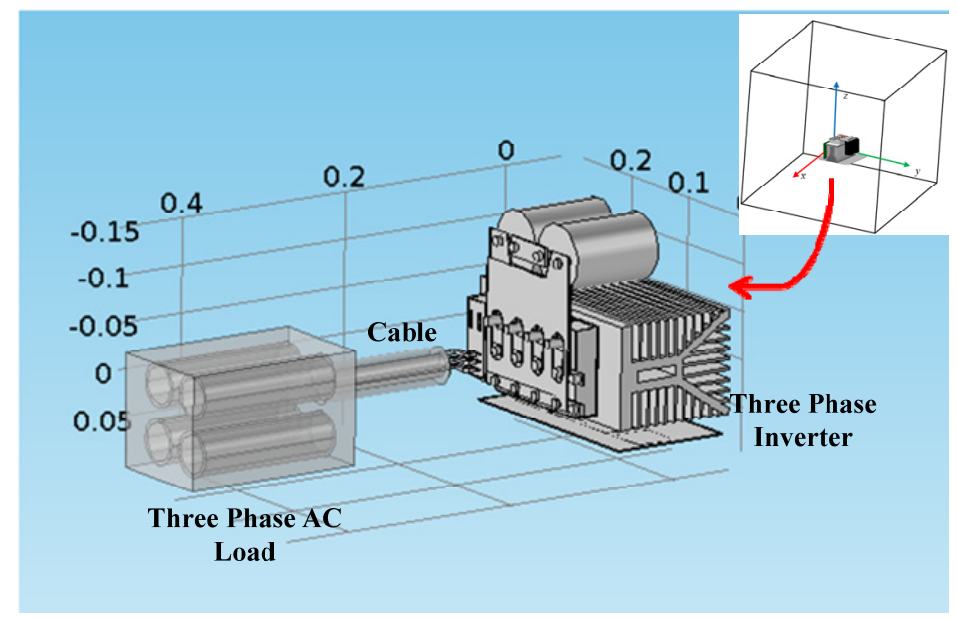

Fig. 7.1 The prototype of the inverter, load and the connection cable

The schematic of the inverter's circuit is shown in fig.7.2. In this simulation, the IGBT module was operated for a relatively low switching frequency to illustrate the 
behavior of the circuit. In the PWM inverter of fig. 7.3, the duty cycle ratio of the input signal to the IGBT gate drivers is varied using the space vector PWM technique to produce a $60-\mathrm{Hz}$ sinusoidal variation of the RL load current [214].

Table 7-1 The details of the components in the tested setup

\begin{tabular}{|c|c|}
\hline Component & Characteristics \\
\hline & $\begin{array}{l}\text { Three-phase, } 5.5 \mathrm{~kW} \text {, Switching frequency: 5-kHz, Switching } \\
\text { algorithm: SVM, Length: } 30-\mathrm{cm} \text {, Width: } 30-\mathrm{cm} \text {, Height: } 25-\mathrm{cm} \text {, } \\
\text { Nominal voltage: } 320-\mathrm{V} \text {, Amp: } 20-\mathrm{A}\end{array}$ \\
\hline Electric Load & 3-kW AC load \\
\hline $\begin{array}{l}\text { Connection } \\
\text { cable }\end{array}$ & $\begin{array}{l}\text { XLPE, Diameters: Cross-sectional area: } 1000 \mathrm{~mm}^{2} \text {, Thickness of } \\
\text { insulation: } 2.8 \mathrm{~mm} \text {, Nominal thickness of pvc sheath: } 2.4 \mathrm{~mm} \text {, Overall } \\
\text { diameter: } 51 \mathrm{~mm} \text {, insulated and armored PVC sheathed cable }\end{array}$ \\
\hline
\end{tabular}

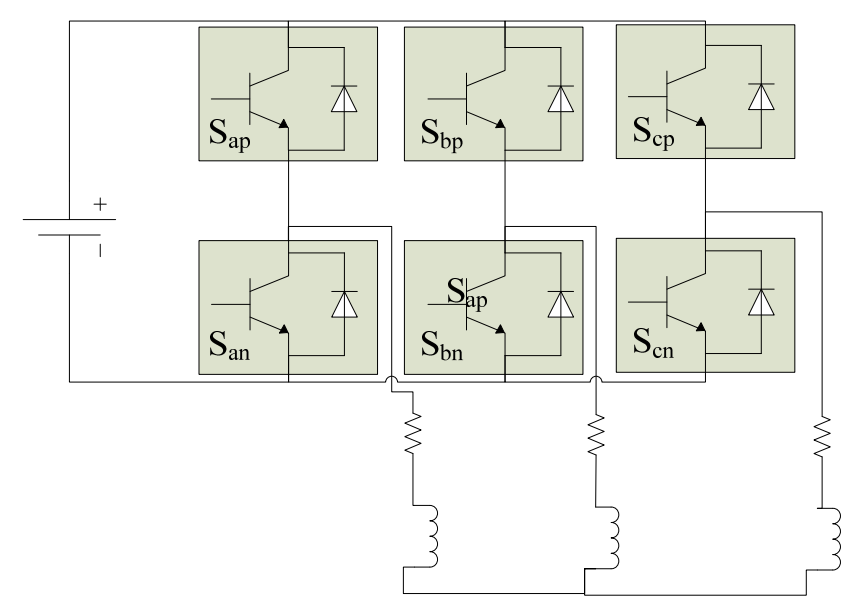

Fig. 7.2 Schematic of six switch inverter circuit, [214]

The operation of the inverter was divided into six sections. During the first $\pi / 3 \mathrm{rad}$ of 60- $\mathrm{Hz}$ inverter operation, IGBTs $\mathrm{S}_{\mathrm{ap}}, \mathrm{S}_{\mathrm{bn}}$ and $\mathrm{S}_{\mathrm{cn}}$ are switched on, while the others are in the off state. This process changes in a way to track the reference voltage as [214]: 
$V_{r e f}=\frac{3}{2}\left(V_{a}+\alpha V_{b}+\alpha^{2} V_{c}\right), \quad \alpha=e^{j \frac{2 \pi}{3}}$

The inverter operation during other sequences of the $60-\mathrm{Hz}$ reference sine wave is similar to the aforementioned sequence, except that the opposite phase of the bridge is switched on and off. The sinusoidal variations of the duty cycle ratios for each phase were specified by comparing triangular waveforms to the magnitude of the sinusoidal reference signal. When the value of the reference sine wave is larger than the value of the upper triangle wave, $\mathrm{S}_{\mathrm{ap}}$ is switched on; otherwise, it must be off. The same procedure goes within the other IGBTs as well. Fig. 7.3 shows the simulated load current for the space vector PWM (SV-PWM) operation
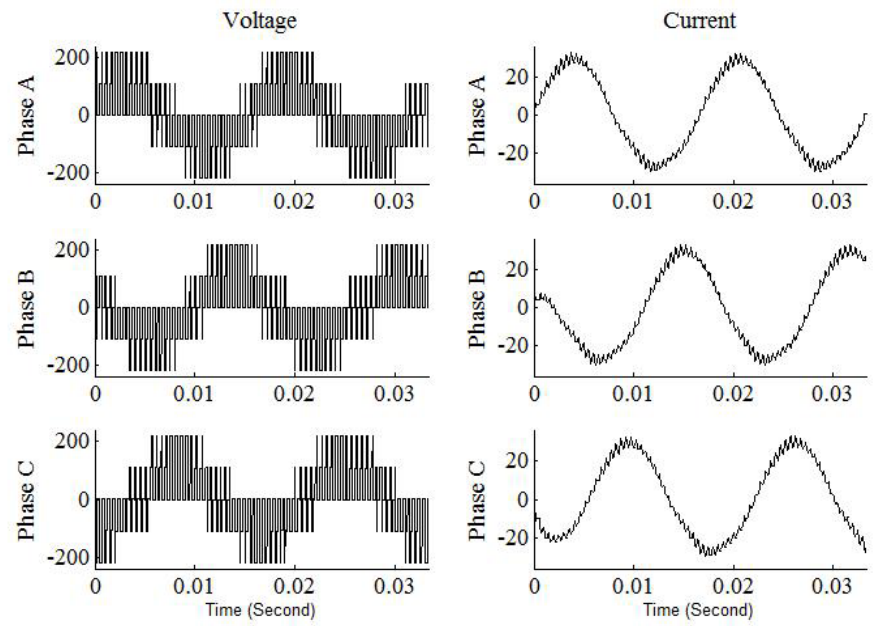

Fig. 7.3 Line Current and Voltage in the case of SVPWM

To model the IGBT switches of the inverter for electromagnetic signature studies, the switches must be considered OFF for a moment of time and then it must be considered ON for the next time instant. This shift occurs based on the switching frequency of the converter. In order to do this in the FE simulation, the plate between the load and the positive bus, shown in fig. 7.4, is considered a conductive plate for the switch-ON case. 
Subsequently, this plate is considered a non-conductive plate for the switch-OFF case. This alteration of the conductivity of the plate occurs 5000 times in a second due to the switching frequency $(5 \mathrm{kHz})$.

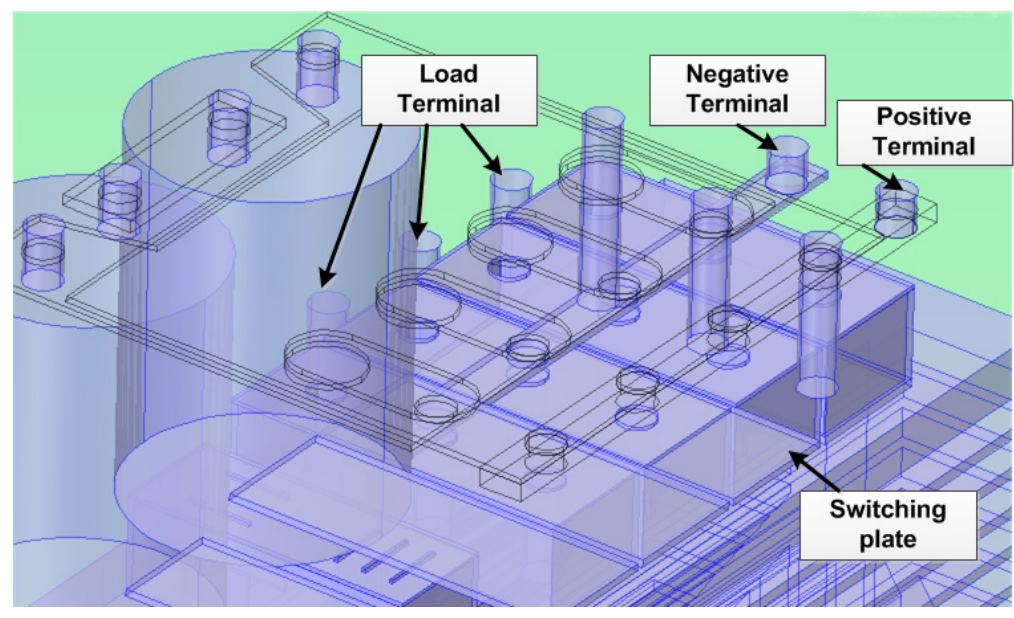

Fig. 7.4 Physical model of the inverter switches

\subsection{Simulation and experiment}

The proposed setup consists of an inverter, an induction machine, connecting cables and an AC load. The study was divided into two cases for further investigation. The converter connected to an $\mathrm{AC}$ load as the case 1 (7.3.1) and converter connected to an induction motor as case 2 (7.3.2). Each study was discussed along with its application as follows:

\subsubsection{Case 1: converter connected to the load}

The schematic of the converter shown in fig. 7.1 is implemented based on the above procedure and modification. The simulation was computed in six hours with about one million elements including face, line and node meshes in the model with six million degrees of freedom. The large number of elements is necessary because of the very small surfaces, edges and lines of the critical part of inverter and cable, as shown in fig. 7.5. 
The details of FE modeling is reflected in [215]-[217]. The simulation was implemented in a fast computer with $192 \mathrm{~GB}$ ram and 16 core Intel Xeon $3.47 \mathrm{GHz}$ CPU.

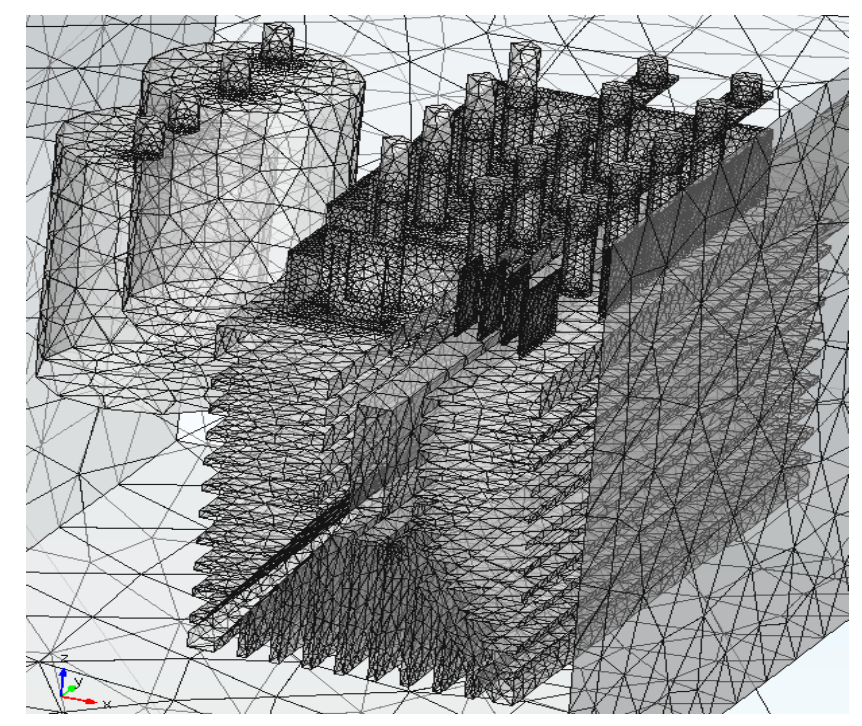

Fig. 7.5 Mesh pattern of the modeled inverter

Since there are two cases in this study, it was decided to define two types of results. In case 1, generated fields of the system on three different surfaces at a distance in space are considered as the result, and in the case 2 , the harmonics of the fields and the frequency responses are investigated. Hence, in this case, the generated stray magnetic and electric fields are obtained in three dimensions at a given distance in both switching circumstances. Fig. 7.6 (a) and (b) show that, turning on and off the switches have the effect only on the amplitude of the magnetic field density, and the spatial distribution of the stray magnetic field on the slices doesn't change significantly. This is due to the presence of the AC load, which is discussed further. On the other hand, the electric field, which is shown in fig. 7.7, illustrates that when the switches turn on, the electric field in two lateral planes, the XY-plane and YZ-plane, increases while the field in the XZ-plane decreases. The increase of the electric field, in these planes, is due to the flow of current 
in the switches. It is also due to the creation of a current loop and its reduction is because of the superposition, which is suppression in this case. The suppression occurs due to the propagation of fields into the other conductive parts of the devices in vicinity; therefore, the stray field induced from the imposed conductive parts decreases. The reason of the suppression is the inverse direction of the induced field due to Lenz's law [218]. Therefore, the induced stray field is subtracted from the main stray field and the total field decreases as in fig. 7.7 (a).

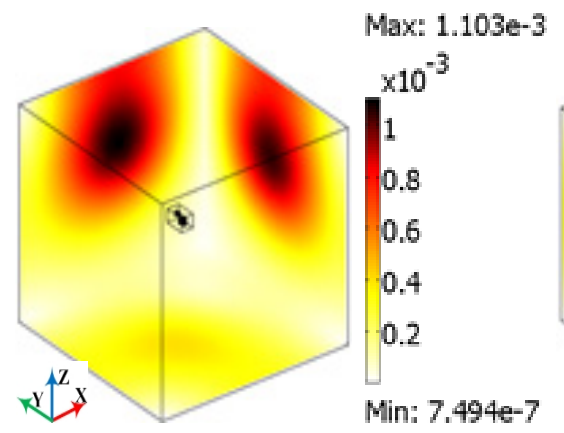

(a)

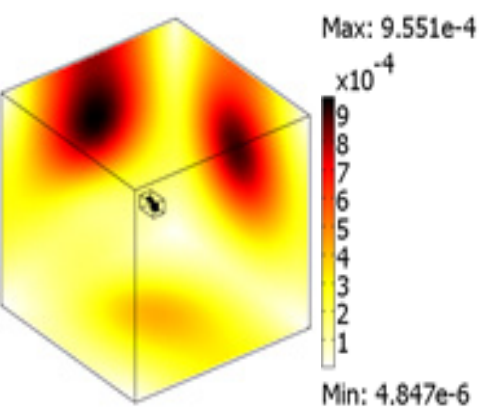

(b)

Fig. 7.6 Stray magnetic field density of the system: (a) IGBT switched on (b) IGBT switched off $(\mu \mathrm{T})$

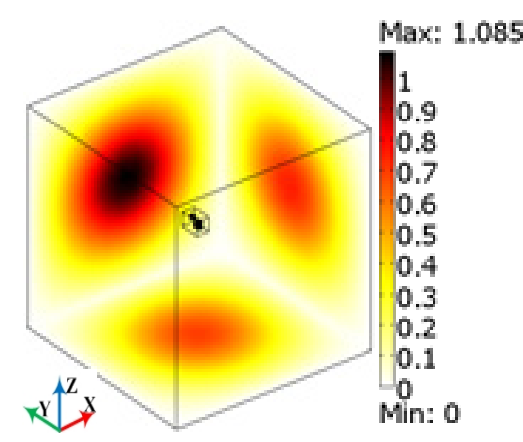

(a)

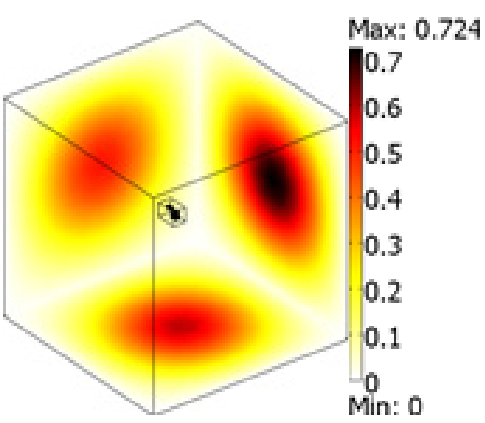

(b)

Fig. 7.7 Stray electric field of the system: (a) IGBT switched on (b) IGBT switched off $(\mu \mathrm{V} / \mathrm{m})$

To recognize which element of the setup has more effect on the total field, the stray magnetic fields of each component in this setup were analyzed individually to observe 
their spectrum and compare it with the overall fields. The results are shown in fig. 7.8 (a) - (d). Comparing fig. 7.8 (c) with fig. 7.8 (a) and fig. 7.8 (b), while only the load is switched ON, the stray magnetic fields have a higher value in comparison with fig. 7.8 (a), (b) and the total field were affected by it (compare fig. 7.8 (c) with fig. 7.8 (d)). The reason is that the $\mathrm{AC}$ load has bigger conductive elements including iron and copper materials, compared to the other elements in the setup.

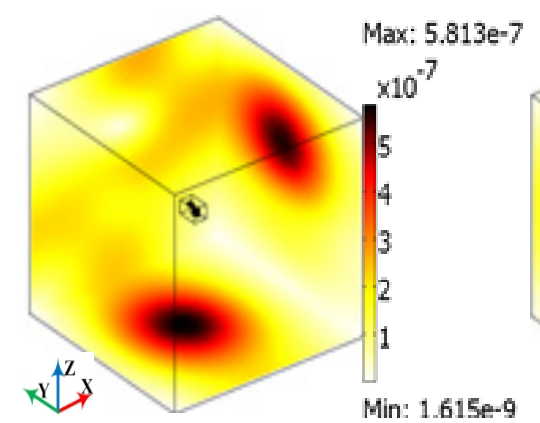

(a)

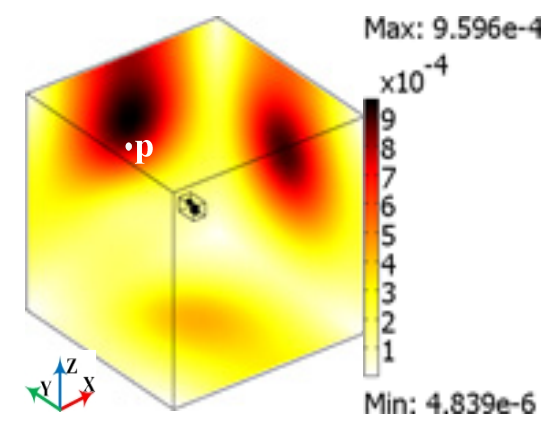

(c)

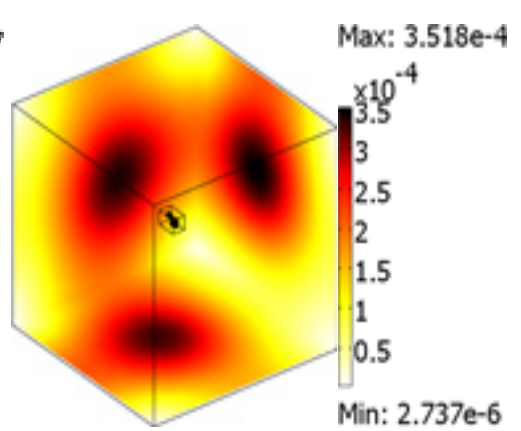

(b)

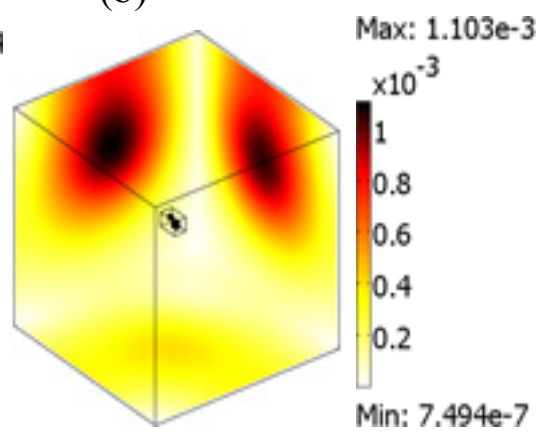

(d)

Fig. 7.8 Stray magnetic field density of the system ( $\mu \mathrm{T})$ : (a) only the cable is switched on, (b) only the inverter is switched on, (c) only the load is switched on, (d) the whole system is switched on

In order to investigate the effect of superposition, the generated field of a random point (p) of the figures, shown in fig. 7.8 (c), can be used. For instance, the values of a point of the three cases $(0,5 \mathrm{~m}, 0)$ of fig. 9 , fig. 7.8 (a) - fig. 7.8 (c), are aggregated. The 
result is $1.31 \mathrm{e}^{-3}(\mu \mathrm{T})$ while the overall maximum point is $1.10 \mathrm{e}^{-3}(\mu \mathrm{T})$ as shown in fig. 7.8 (d). This can be due to the dissimilarity of permeabilities and conductivities of the elements of the model. If the resistance of an element is less than another element in the vicinity while there is no shield between them, the EMF will be induced from the component with less conductivity into the one with higher conductivity [219]. As mentioned above, due to Lenz's law, the field radiated from the induced EMF will be the opposite of the main field. Therefore, the overall field will be less than the aggregation of the fields.

\subsubsection{Case 2: converter connected to the motor}

In this case, the inverter is connected to an induction motor. The aim of this case is investigating the radiation of harmonic fields from the inverter while the distance and the speed of the motor change. The parameters of the induction motor are: $5.5-\mathrm{kW}, 3$-phase, 208-V, PF: 0.85, length: 30-cm, diameter: $25-\mathrm{cm}$, number of poles: 4 . This case was simulated using FE shown in fig. 7.9 (a). 


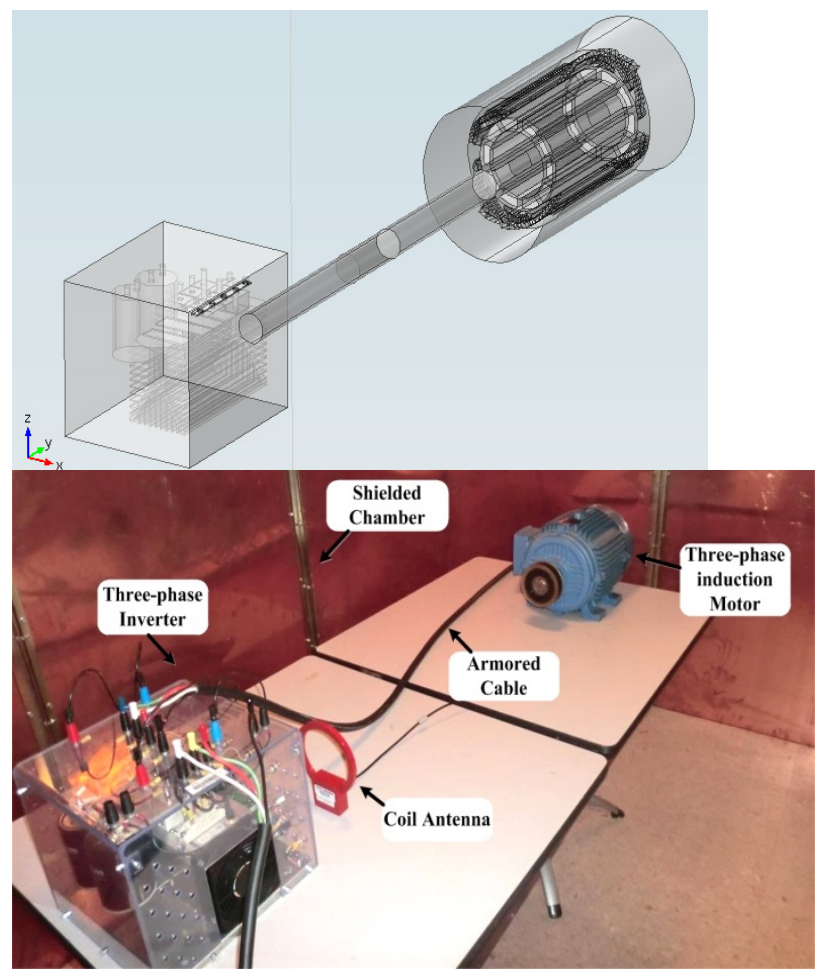

(a) (b)

Fig. 7.9 The scheme of the setup of case 2 (a) FE simulation (b) measurement

The simulation was computed in six hours with 950000 elements and 5.7 million degrees of freedom. Since the case includes very small elements and also nonlinear materials, e.g. the core of the machine, the simulation of the inverter connected to the load or motor may take 8 hours or more for only one time instant.

Generally, linear or non-linear solvers are being used the FE simulations. In this case, since there are several materials with nonlinear characteristics, the linear solver cannot be used. On the other hand, using nonlinear material rises the simulation time dramatically. Hence, a modification in choosing the solver and the associated iterative technique was employed. Instead of having linear or curved commutation curve, the ramp of the curve in several zones was calculated $\left(\mu_{\mathrm{r} 1}, \mu_{\mathrm{r} 2} \ldots\right)$ and used instead of the commutation curve in this part as shown fig. 7.10. 


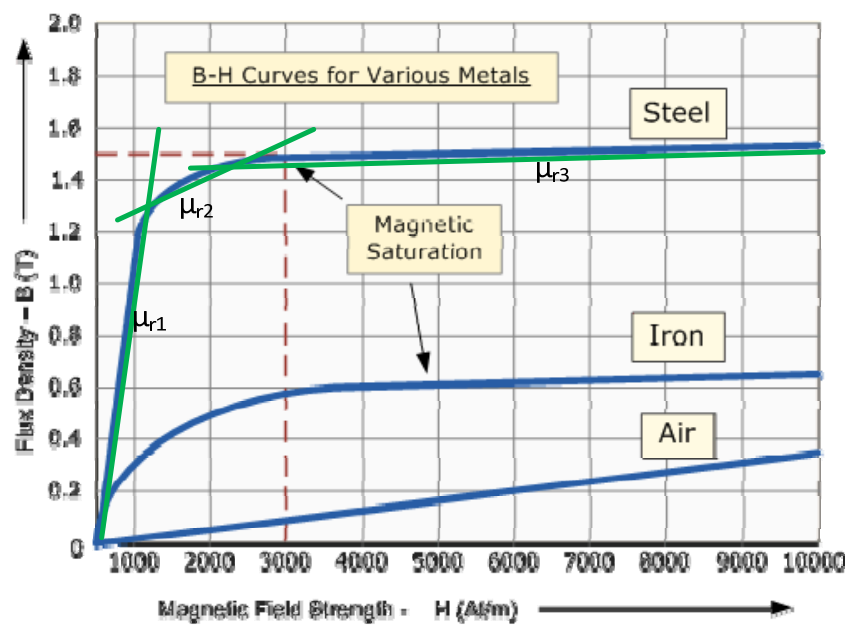

Fig. 7.10 commutation curve of some material used in the simulation

The benefit of this modification is that the magnetic flux density of a component changes in a very small period due to the steady state condition of the system. For example, the magnetic flux density of the stator core of the induction motor is about 1.5-2 $\mathrm{T}$ in power frequency analysis, 50-60 Hz. For higher frequencies, it goes down to under 1T. Therefore, in this case, a specific zone of the permeability can be chosen for this component. Similarly, the permeability of other components of the system can be chosen based on the working frequency. Therefore, having the idle parts of the commutation curves of the elements would be avoided, and the simulation time decreases. This algorithm can be defined in the material properties part of the FE simulation.

In addition to the modification in defining the material properties, some modification needs to be performed for the solver to have a flexible solution. Hence, as the iterative solver, the fast generalized minimal residual technique, GMRES, with the krylov as the pre-conditioner was used. The fast GMRES is a variant of the GMRES method with flexible preconditioning that enables the use of a different pre-conditioner at each step of the Arnoldi process. The krylov subspace is a linear subspace which enables multi- 
preconditioning [220]. In particular, a few steps of GMRES can be used as a preconditioner for fast GMRES. The flexibility of this solution method is beneficial for the problem with nonlinear material characteristics, such as the motor's core. Therefore, the simulation time decreases from about 8-9 hours to about 20 minutes. More explanation is given in [221].

In addition to the simulation, the experimental setup was implemented in a chamber, which isolates the setup from the outside environment, shown in fig. 7.9 (b). The coil antenna was located at $10 \mathrm{~cm}$ away from the inverter to obtain the stray magnetic field. The fields were transferred to an EMI receiver, real-time spectrum analyzer, with a cable of $50-\Omega$ impedance.

The magnetic field intensity ( $\mathrm{H}$-field) generated from the setup in simulation is shown in fig. 7.11. The $\mathrm{H}$-field at $5-\mathrm{kHz}$ frequency is shown on a slice at $10 \mathrm{~cm}$ away from the setup, the same as experimental setup. As illustrated in this figure, the amplitude of the stray field around the inverter box is higher than other places. The reason is that the switching frequency of the inverter is $5 \mathrm{kHz}$, the same as the frequency depicted from the simulation figure. The simulation was implemented at several other frequencies but only the switching frequency of the inverter, which is $5-\mathrm{kHz}$, is shown here. 


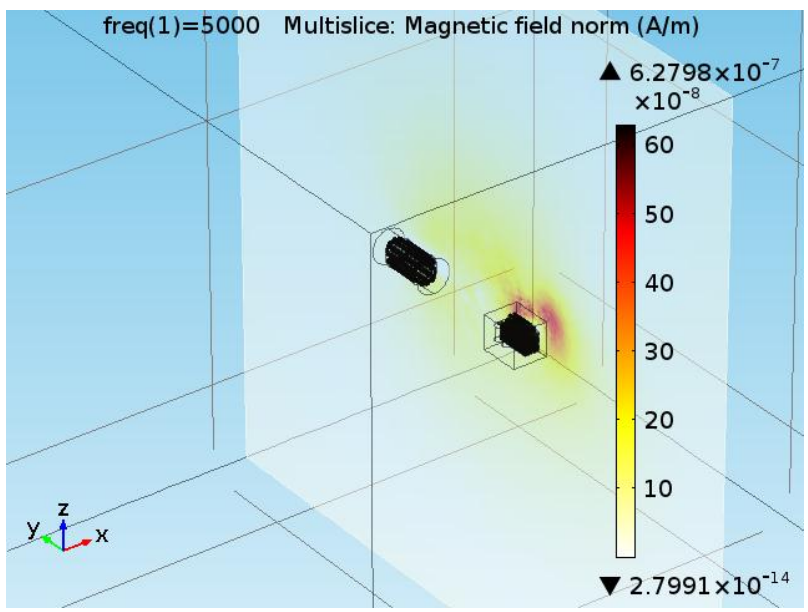

Fig. 7.11 Stray magnetic field intensity of the setup case 2 at $5 \mathrm{kHz}$ simulated in FE $(\mu \mathrm{A} / \mathrm{m})$

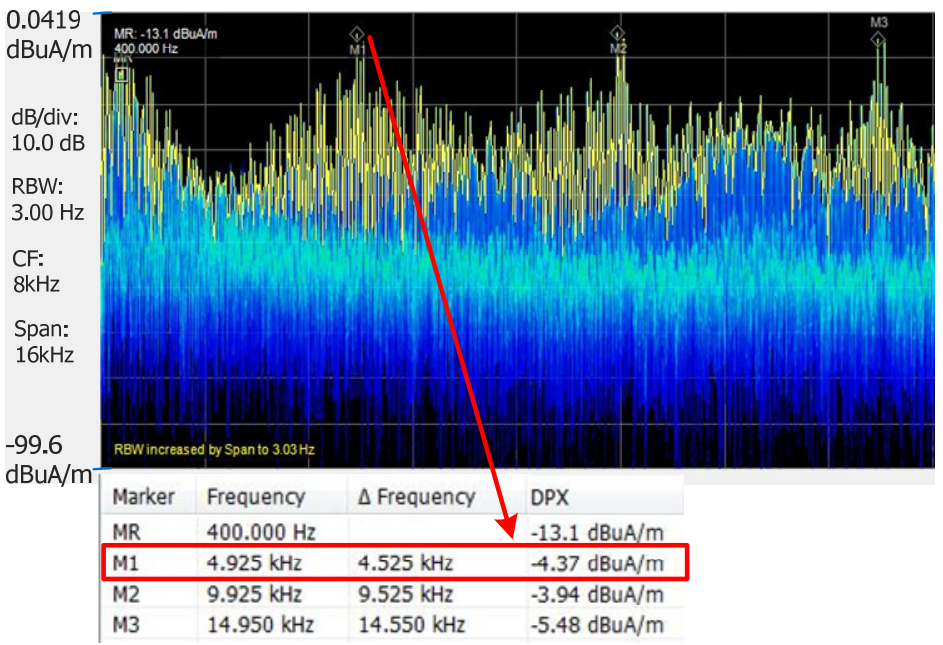

Fig. 7.12 Measured frequency response of the stray magnetic field intensity of the setup case 2 from $\mathrm{DC}$ to $20 \mathrm{kHz}(\mathrm{dB} \mu \mathrm{A} / \mathrm{m})$

The setup was also implemented experimentally. The frequency response from DC to 20-kHz was obtained and shown in fig. 7.12.

The unit of the simulation result is $\mu \mathrm{A} / \mathrm{m}$, while the unit of the experimental results is $\mathrm{dB} \mu \mathrm{A} / \mathrm{m}$. The $\mu \mathrm{A} / \mathrm{m}$ can be converted to $\mathrm{dB} \mu \mathrm{A} / \mathrm{m}$ by using eq. (7-2). Using this equation, the peak of the stray magnetic field at $5-\mathrm{kHz}$ at the given distance is $-4.37 \mathrm{~dB} \mu \mathrm{A} / \mathrm{m}$ 
experimentally, would be $0.61 \mu \mathrm{A} / \mathrm{m}$, which is very close to the value in simulation, see fig. 7.11.

$$
\mu A / m=10^{\frac{d B \mu A}{20}}
$$

\subsection{Applications of the frequency response analysis of the stray field}

Following the experimental verification of the simulation results, related applications, such as monitoring of components for the diagnosis of failures and shielding were investigated:

As shown in fig. 7.12, the first peak located at very low frequency is generated from the induction motor, since the motor is working at the power frequency, $60 \mathrm{~Hz}$. As the working frequencies of the components in the system are different, the behavior of each component can be investigated individually. This can be a very useful hint in monitoring the conditions, as well as detecting the faults of the motor and the inverter. For example in case 2 , fig. 7.12 , if a failure occurs in the motor, the peak at the power frequency and the related higher harmonic orders will shift along the frequency band or the amplitudes would change. Similarly, failures to the inverter may cause the same type of changes in switching frequency and the related higher harmonic orders. Note that, the peaks at 10$\mathrm{kHz}$ and $15-\mathrm{kHz}$ in fig. 7.12 are due to the second and the third harmonics of the inverter. The frequency responses in between the harmonics are noises and sub-harmonics.

As another application of this case, the shielding in the vicinity of the switch, $5 \mathrm{~cm}$, was tested. Fig. 7.13 shows the frequency response of the stray H-field with and without the shield between the switches and the antenna by means of simulation and measurement. Using a steel shield, Steel 1018 as an example in this test, it can be seen 
that the noises, sub-harmonics between the main harmonic orders, decrease dramatically. The experimental results show a wider band of frequency, $\mathrm{DC}-20 \mathrm{kHz}$, as shown in fig. 7.14 to illustrate the effect of shielding on the other harmonic orders.

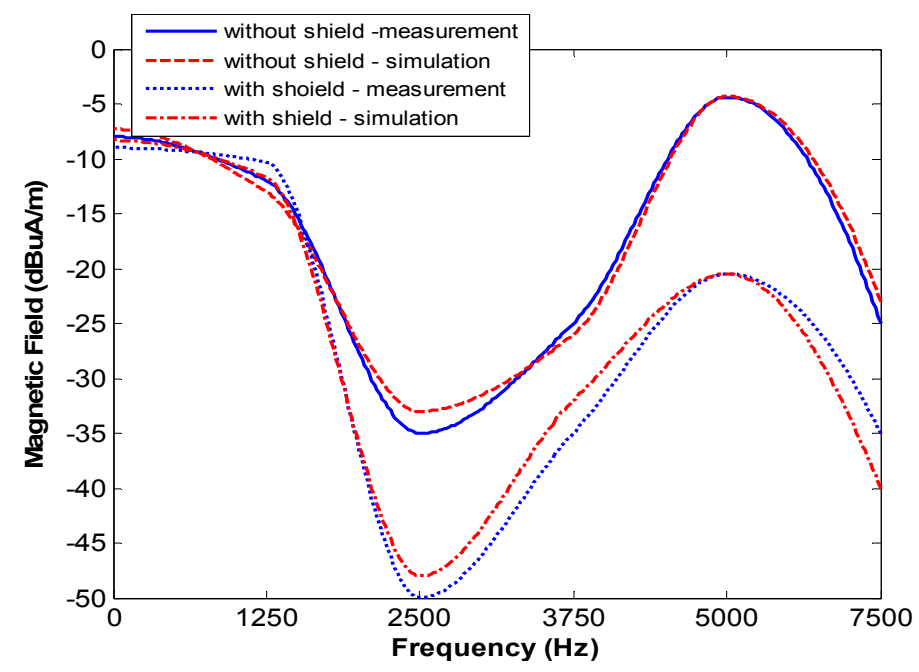

Fig. 7.13 Stray magnetic field intensity of the setup case 2 from $\mathrm{DC}$ to $7.5 \mathrm{kHz}(\mathrm{dB} \mu \mathrm{A} / \mathrm{m})$ at $5 \mathrm{~cm}$ away from the inverter with and without shield by means of simulation and measurement

Consequently, considering this test, the main harmonics and the related subharmonics can help in selecting a shield with proper characteristics including the permittivity and permeability. Comparing the curves of fig. 7.13 , the simulation result is similar to the experimental one. Hence, the proposed shield can be studied and optimized using the physics based simulation. The permittivity, permeability, conductivity and other physical characteristics of the shield can be altered and optimized for the best electromagnetic compliance or any other purposes using the simulation and experimental design. 


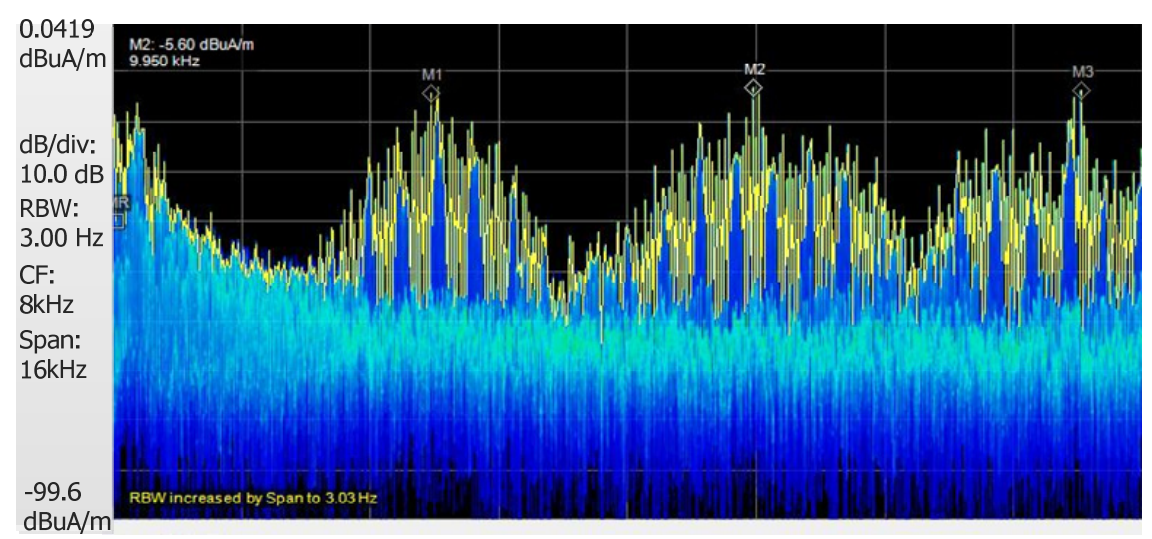

(a) Without shield

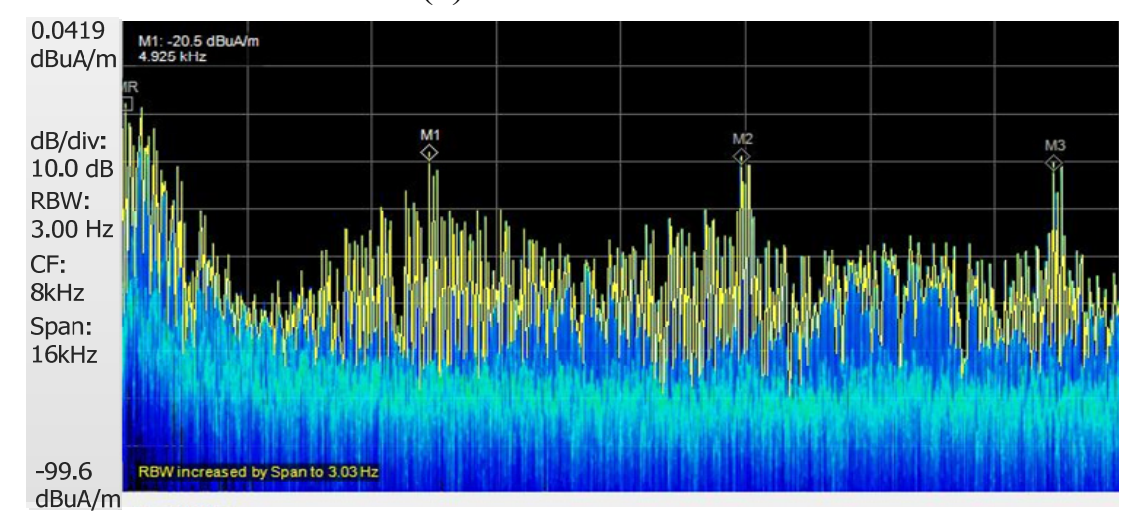

(b) With shield

Fig. 7.14 Stray magnetic field intensity of the setup at case2 from DC to $20 \mathrm{kHz}$ $(\mathrm{dB} \mu \mathrm{A} / \mathrm{m})$ at $5 \mathrm{~cm}$ away from the inverter (a) without shield and (b) with shield

\subsection{Conclusion}

In this chapter, the 3D full finite element modeling for the radiated EMI study of a typical power electronic drive was implemented. The physical approach for applying the switching activity was utilized. In order to have the detailed simulation of the model and simultaneously consider the non-linearity of commutation curve in the frequency analysis, the fast GMRES methods with proper conditioners were used as the solution method, which increases the simulation speed. The measurement was also applied for verification of the numerical results, as well as for investigating the stray fields under different operating conditions. The results show that the FE model has a good accuracy 
for evaluating the stray fields. Two cases of using the inverter in the system were studied and their applications were explained. The results show that the frequency response of the field can be used for assessing shielding arrangements, as well as for monitoring the conditions of the drive. 


\section{Fault Type Diagnosis of Induction Motor Using Magnetic Stray Field}

\subsection{Overview}

After explaining the modeling of the components for the study of electromagnetic stray field, the application will be implemented and discussed in the rest of the dissertation. This chapter presents a method for the identification of the failures in winding of the induction motors, such as unbalanced currents flowing into the motor and the winding short-circuit. The stray magnetic field of a typical induction motor was studied while various types of failures applied to the machine. Different types of unbalanced currents flow into the machine and the fields were obtained from both numerical finite element simulation, as well as experimental results. Correspondingly, the turn-to-terminal and interturn short-circuit of the motor's winding was studied. The frequency response of the three dimensional finite element (3D FE) model of the motor was implemented up to high order frequencies and they were compared with the measurement results. The fields with unbalanced currents, as well as short-circuit, were identified by studying the harmonic orders of the radiated magnetic field. This was also implemented using artificial neural networks (ANN).

Due to the vast applications of induction motors in the electromechanical energy conversion, mainly because of their low cost, roughness, low maintenance, and operation with an easily available power supply, these motors are the main workhorse of industrial prime movers.

Although induction machines are reliable, they are subjected to some undesirable stresses, causing them some faults resulting in failures. Researchers have studied a 
variety of machine- faults as winding faults, unbalanced stator and rotor, broken rotor bars, eccentricity, and bearing faults [222], [223]. The need for condition monitoring has increased recently because of the widespread use of the automation and consequent reduction in the direct man-machine interface to supervise the system operation. The condition monitoring is the graphical trend of the machine parameters for the purpose of detection, analysis, and correction of the machine problems before the failure takes place. It is used for increasing machine availability and performance, reducing consequential damage, increasing machine life, reducing spare parts inventories, and reducing breakdown maintenance.

Several studies have shown that more than $40 \%$ of induction motor failures are due to the stator winding breakdown, which can be because of short-circuits and unbalanced currents of the windings [224].

Early detection of inter turn short-circuit during the faulty operation of motor would eliminate substantial damage to the adjacent coils and the stator core reducing repair costs and motor outage time. In addition to the benefits gained from early detection of turn insulation breakdown, significant advantages would occur by locating the faulted coil within the stator winding. Fault locating would not only increase the speed of the repair, but also would allow optimal timing of the repair outage.

Based on the severity of the short-circuits, several monitoring techniques are implemented, which can be categorized into magnetic flux [225], vibration [226], instantaneous angular speed [227], noise [228], air-gap torque [229], flowing current [230], induced voltage [231], partial discharge [232], surge testing and many other methods [233]. 
In addition to the effect of short-circuit, the unbalanced current would damage the winding of the machine gradually. The proliferation of unbalanced loads on power distribution systems has resulted in increased power quality issues in a substantial manner. These unbalanced currents cause the unbalanced supply for the components such as machines [234]. Moreover, the presence of power electronic components in the system will increase the chance of having the unbalanced current. On the other hand, due to the faults inside the machine, such as grounding faults, winding malfunction and etc., the flowing currents may get unbalance.

There are several papers dealing with the reduction or elimination of the unbalanced current in the electrical network [234]-[237]. In [234], the active filter was used to reduce the effect of unbalanced currents. The symmetrical component theory was used as the proposed method. The detection of unbalanced voltages and their effect on induction motor's electromagnetic signatures as investigated in [235]. In this work [235], Mirabbasi et al also investigated the line current, power factor and efficiency, while unbalanced currents were flowing in the input lines. The Torque and speed were the index term in this study. Nabeta [236] worked on the modeling of the machines under unbalanced conditions. He used finite element as the modeling method. The analytical method was also used for validation. The modeling of the machine under unbalanced condition was also implemented using another methods involving Simulink/Matlab in [237]. The control and improvement of fault-ride capability of a doubly fed induction generator (DFIG)-based wind turbine system under both unbalanced and distorted grid voltage conditions were investigated with experimental validation [237]. 
All of the above studies explained along with several others represent valuable investigations. The methods require voltage and current of the system to detect the unbalanced current, while it's not possible to have the flowing current of all lines, such as the winding inside the machines in many applications. Hence, recently magnetic stray field is being used as another monitoring technique in fault diagnosis especially shortcircuits. In [120], medium-range frequencies are taken into consideration to determine electrical faults, when the machine is supplied through the grid. The broken bar fault and the inter-turn short-circuit is studied in this paper. Similarly, Chadebec et al [121] showed the trustworthiness of fault detection on electrical machines by analysis of the low frequency magnetic stray field. A simple model of the stray magnetic field of a synchronous machine is used to predict the generated stray magnetic field in the case of different rotor faults (short-circuit rotor windings and dynamic mechanical eccentricity). The other advantage of using stray field in fault diagnosis, such as fault in lamination, broken bars, are explained in several publications such as [122]- [126].

This chapter presents the idea of using electromagnetic signatures of the machine as the index of detecting unbalanced current and identification of the inter-turn and turnterminal short-circuits of the winding. It is proposed to obtain the radiated magnetic field through magnetic antenna and identify the effect of the mentioned faults on the amplitude of harmonics within the frequency band. This would lead to detect the specific unbalance or short-circuit types. The numerical simulations and experimental tests were implemented for this investigation. It's possible to use the experimental study to detect insignificant unbalanced currents or short-circuits; however, testing unbalanced currents or short-circuits for significant cases experimentally is dangerous for the machine. 
Therefore, the simulation is proposed for the later condition. The numerical simulation utilized here is the three-dimensional finite element method, which was explained in chapter 7. This is followed by numerical and experimental studies of a case with unbalanced current and several examples of short-circuits, and the results are discussed. Moreover, the identification of the unbalanced condition throughout the machine environment is investigated using artificial neural networks.

\subsection{Modeling details}

In order to apply the inter-turn and turn-terminal short-circuits and unbalanced current, the FE model (see fig. 8.1) requires to be coupled with the circuit-based software. To emulate the unbalanced current, the circuit is designed, which is displayed in fig. 8.2. The setup consists of a set of three phase series resistance, $2.13 \mathrm{ohm}$ each, between the power supply and the machine, as shown in fig. 8.2. The switches are opened or closed to put or take the resistors in the circuit.

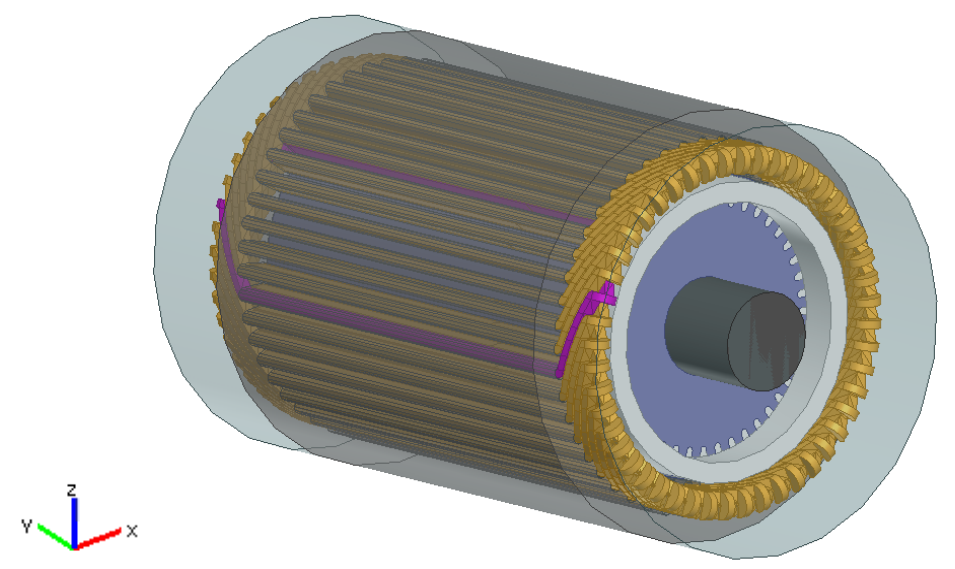

Fig. 8.1 Finite element model of the studied induction motor 


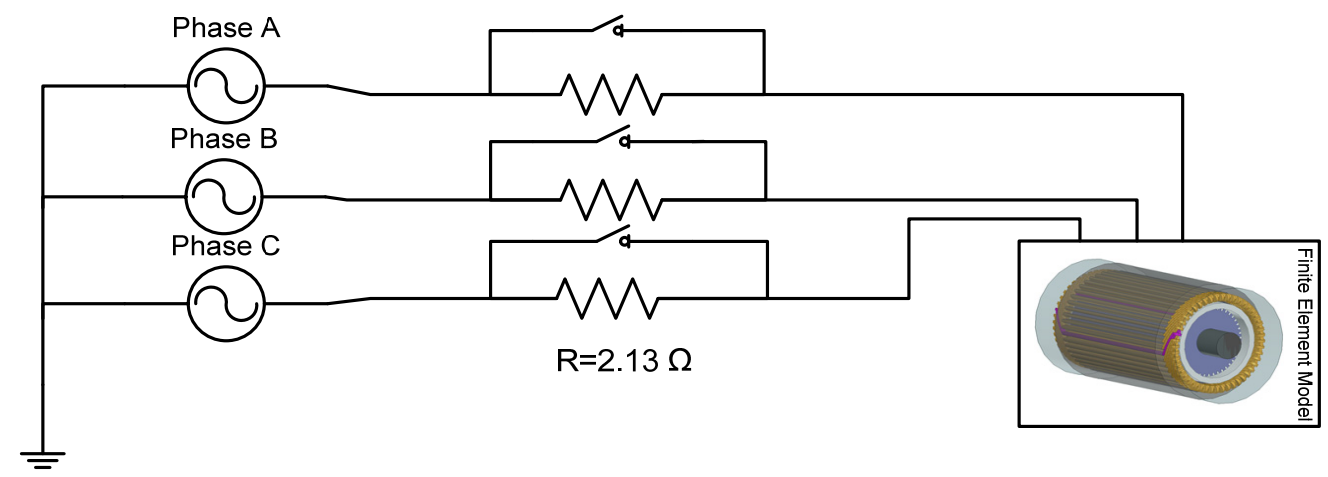

Fig. 8.2 Schematic of the circuit connected to the model for the unbalanced current study

On the other hand, to apply the inter-turn short-circuit, the proposed turns of the coil would be paralleled with a rheostat to have various levels of short-circuit, as shown in fig. 8.3. The motor and the selected terminals are illustrated in fig. 8.4. The rheostat is connected to $S_{1}$ and $S_{2}$ for inter-turn cases and between $T_{B}$ and $S_{1}$ for turn-terminal cases. Note that there are 37 turn wires in each slot and just one of them are soldered to the $\mathrm{S}_{1}$ and S2 and taken out of the casing. Similarly, the turn-terminal short-circuit is applied between terminal of the phase $B\left(T_{B}\right)$ and $S_{1}$.

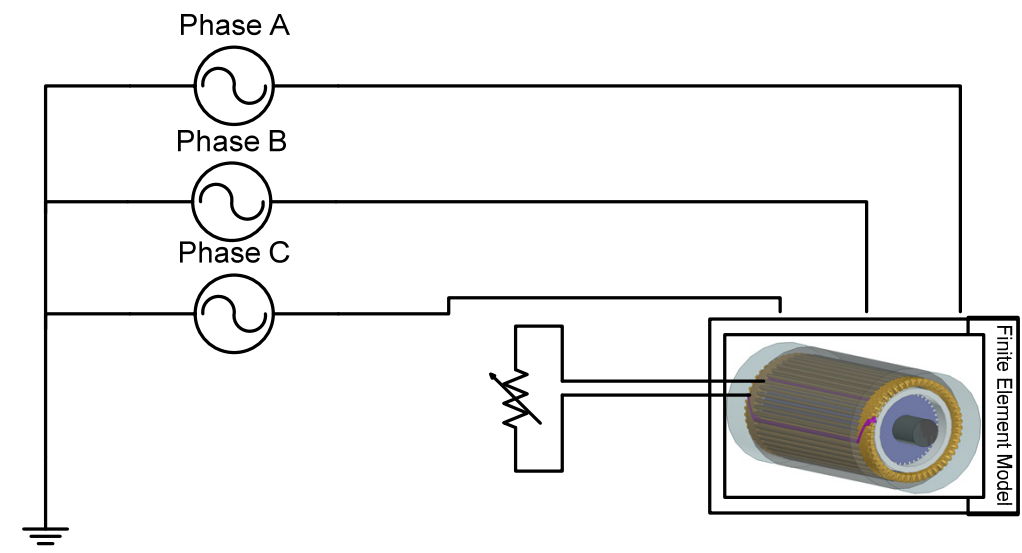

Fig. 8.3 Schematic of the circuit connected to the model for the inter-turn short-circuit study 
Since the purpose of this research is to build a model to be able to study tiny and incipient faults, details of the motor must be considered. Hence, all the details such as the slot shape, winding types and the other physical details and their magnetic characteristics, are reflected in our model. The frequency response analysis of such a detailed model connected to a circuit-based software takes a very large time to be solved for even one harmonic order, while the accurate study requires considering high orders of harmonics. Consequently, a modification in solution process is applied. We used the fast generalized minimal residual technique, GMRES, as the iterative solver with the krylov as the preconditioner. The fast GMRES is a variant of the GMRES method with flexible preconditioning that enables the use of a different pre-conditioner at each step of the Arnoldi process. In particular, a few steps of GMRES can be used as a pre-conditioner for fast GMRES. The flexibility of this solution method is beneficial for the problem with nonlinear material characteristics, such as the core of the motor. As a result, the simulation time decreases from 8-9 hours to 20 minutes. More explanation about the fast GMRES is available in [221].

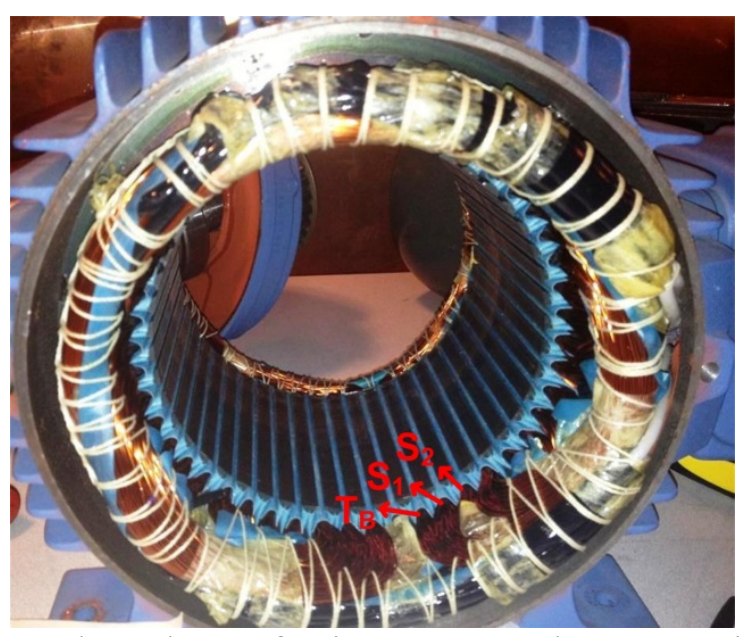

Fig. 8.4 Location of the selected turn for inter-turn and turn-terminal short-circuits (TB: terminal of phase B, S1 and S2: selected turns of phase B) 
After providing the basic circumstances of simulations and experiments, the machine is studied for the two main test cases, unbalanced current and short-circuits of the winding, which are explained in the following sections.

\subsection{Unbalanced current study, experiment and simulation}

The finite element analysis was implemented simultaneously with the circuit-based software. The radiated magnetic field density of the machine in normal condition is presented in fig. 8.5. The simulation time for solving the model in $60 \mathrm{~Hz}$ was 20 minutes. To have the reliable accuracy, the mesh is defined finely for the model with 5.7 degrees of freedom.

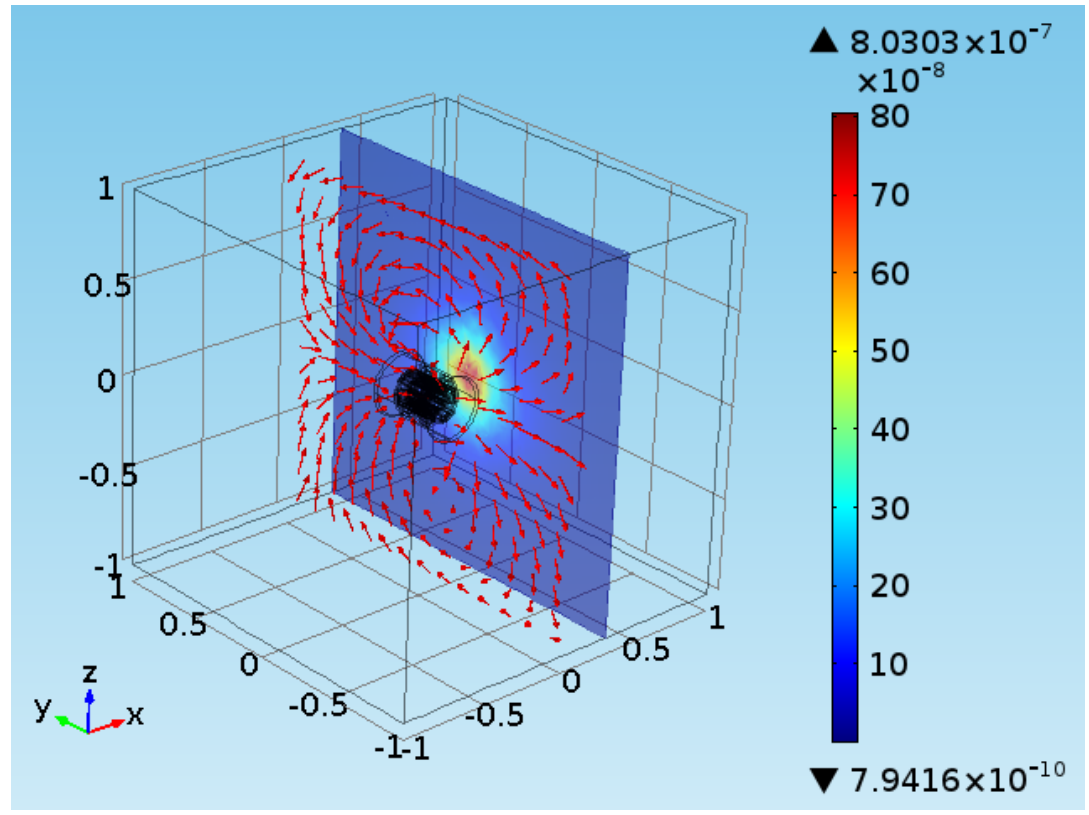

Fig. 8.5 Finite element (FE) model of the studied induction motor with the radiated magnetic field density at $30 \mathrm{~cm}(\mathrm{~T})$. The arrows show the propagated field

In order to have the unbalanced currents, various states of the resistances shown in circuit model (fig. 8.3), were tested. The states are shown in table 8.1. 
Table 8.1 The unbalanced states applied to the system

\begin{tabular}{ll}
\hline States & Description \\
\hline Normal (N) & All switches are ON \\
\hline Phase A (PHA) & The switch parallel to the phase A is open \\
\hline Phase B (PHB) & The switch parallel to the phase B is open \\
\hline Phase C (PHC) & The switch parallel to the phase C is open \\
\hline Phase AB (PHAB) & The switches parallel to the phase A and B are open \\
\hline Phase BC (PHBC) & The switches parallel to the phase B and C are open \\
\hline Phase CA (PHCA) & The switches parallel to the phase C and A are open \\
\hline
\end{tabular}

These states were tested experimentally, as well as using numerical simulation. As mentioned earlier, the radiated magnetic field was observed at a distance around the machine and the unbalanced phase(s) would be detected. In order to obtain the magnetic field radiated only from the machine, the machine was located in a chamber, which isolates the machine from any components outside in terms of fields. The other components, such as the resistance box and the power supply, were located outside the chamber. The setup is shown in fig. 8.6.

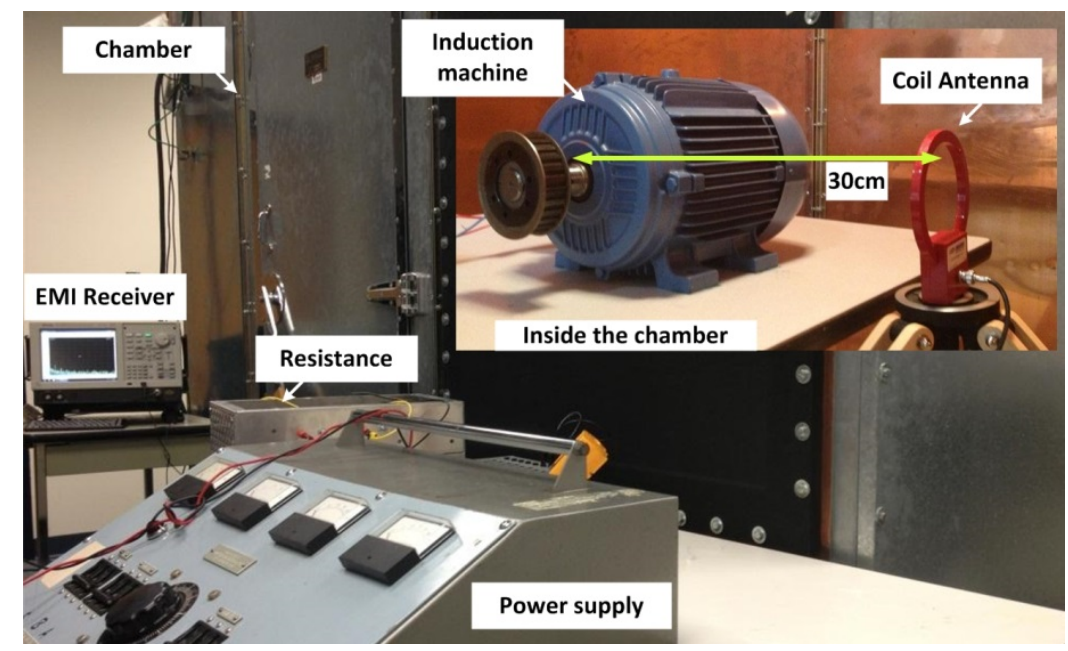

Fig. 8.6 Experimental test setup 
The radiated magnetic field intensity is measured by the low-frequency coil antenna and the results are transmitted to the EMI receiver, a real-time spectrum analyzer, which is located outside the chamber. The EMI receiver can show the field in frequency or timedomain. The coil antenna and the real-time spectrum analyzer, which is used in the measurement, are practically for low frequency analysis with high precision. The frequency range of the antenna is between $20 \mathrm{~Hz}$ to $500 \mathrm{kHz}$. The antenna and the setup are located based on the mentioned standards in chapter 5 .

At first, the setup was tested under normal conditions and the results of both simulation and experiment were obtained and shown in fig. 8.7 (a). In addition, a random unbalanced type, phase A, was selected for verification of the model under the unbalanced condition and the comparison of the simulation and experiment under this condition is depicted in fig. 8.7 (b). Note that the coil antenna, for measuring the magnetic field, was located at $30 \mathrm{~cm}$ distance from the motor, see fig. 8.6.

As it can be seen in the figures, the model has an excellent accuracy under both normal and unbalanced conditions. The small differences between the results in high order of harmonics are due to the many small bends of winding in the actual machine, which cannot be considered completely in simulation. The presence of even order harmonics and high level of their amplitudes are because of observing radiated fields rather than flowing currents in this study. Even order harmonics, as well as the third harmonic can be mitigated in the flowing current into the machine using several techniques [238]. The harmonics are still available in the radiated fields, which is a good point for the detection of abnormal conditions. However, the harmonics badly affects the efficiency of the power components. 


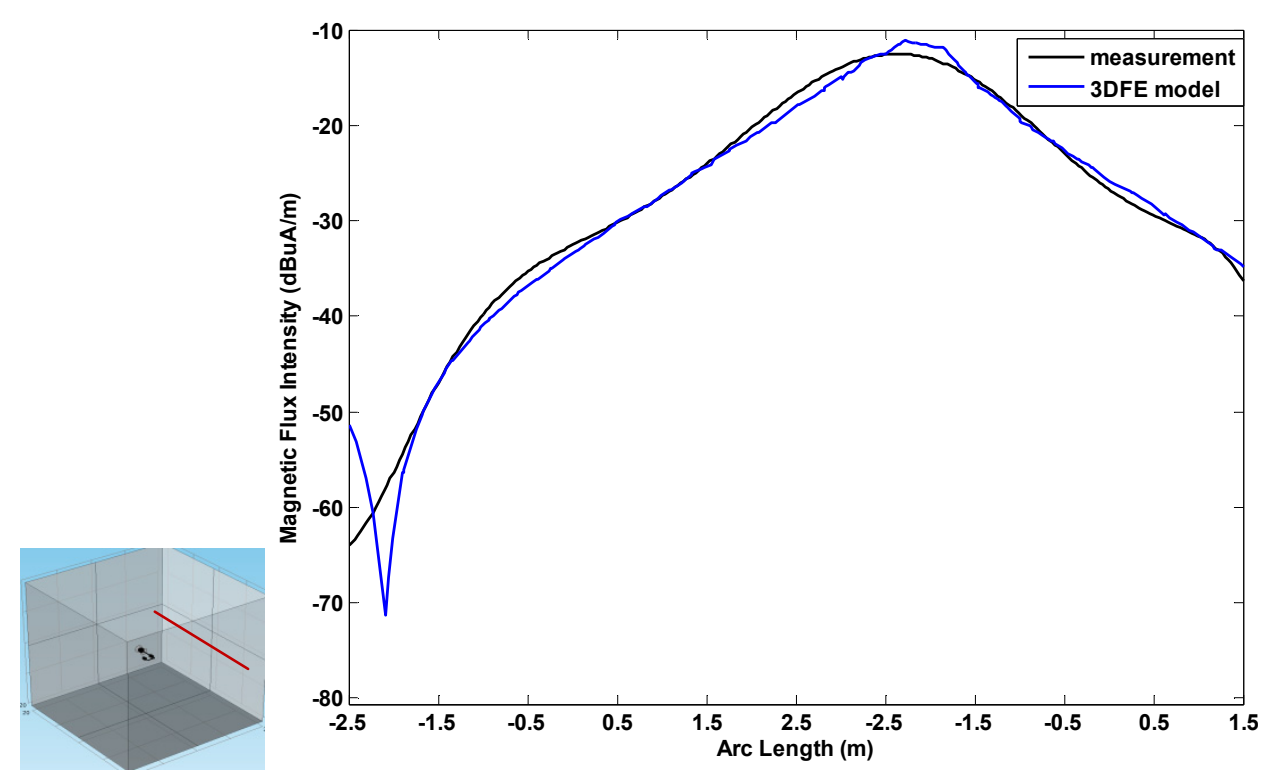

(a)

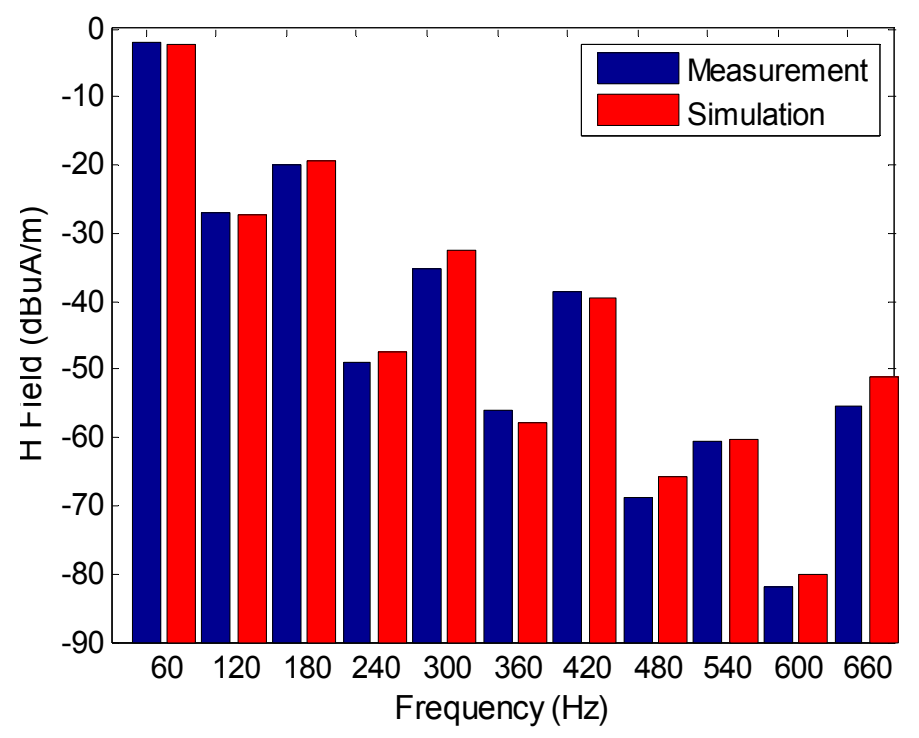

(b)

Fig. 8.7 The radiated magnetic field intensity at the given distance captured by experimental and simulation tests. (a) The normal case along a line in $\mathrm{Y}$ axis (b) frequency response of the case phase A

Subsequently, the setup was tested in various unbalanced states based on the cases of Table 8.1. The frequency responses of the seven mentioned states of Table 8.1 were 
obtained and are shown in fig. 8.8. Some harmonics were selected based on the severity of changes due to the unbalanced states. Comparing the bars in the fig. 8.8 (a)-(f), a simple algorithm can be inferred. For example, in fig. 8.8 (b), the third harmonic in case $\mathrm{PH}_{\mathrm{BC}}$ decreases, while it increases in the other cases. Hence, it can be concluded that while the third harmonic decreases, the unbalance at phases $\mathrm{B}$ and $\mathrm{C}$ may occur. Similarly, the sixth harmonic in case $\mathrm{PH}_{\mathrm{A}}, \mathrm{PH}_{\mathrm{B}}$ and $\mathrm{PH}_{\mathrm{AB}}$ decreases; therefore, there are three possibilities. Considering this with the eighth harmonic in case $\mathrm{PH}_{\mathrm{B}}$ that the amplitude decreases considerably, it can be inferred that the unbalanced current is flowing in phase B. In the same way, unbalance at phase A can be detected by looking at the ninth harmonic, and case $\mathrm{PH}_{\mathrm{C}}$ and $\mathrm{PH}_{\mathrm{BC}}$ can be identified by considering the eleventh harmonic. For more clear detection, higher harmonics should be analyzed. In addition to detecting the states manually, a well-known pattern recognition method, the artificial neural network, was used for accurate and reliable detection of abnormal conditions.

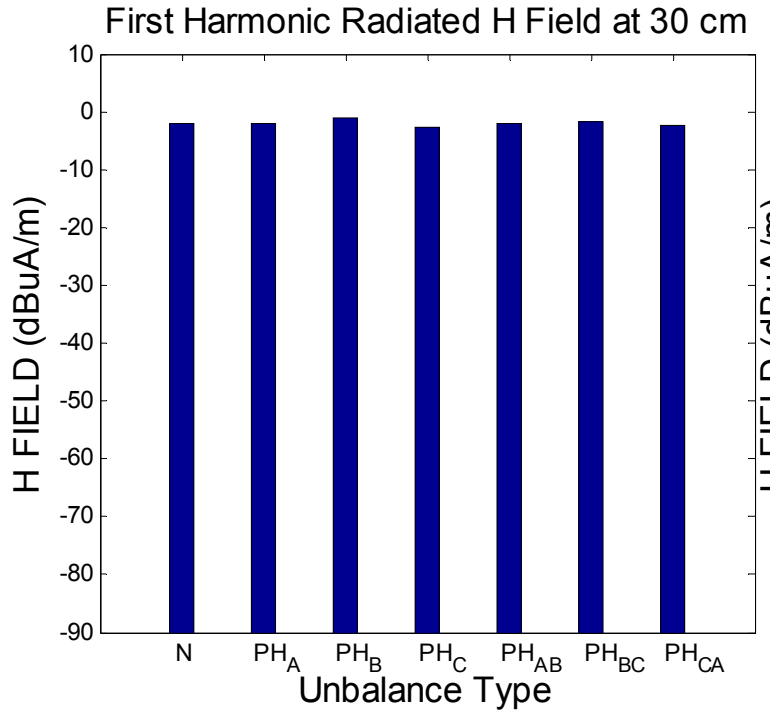

(a)

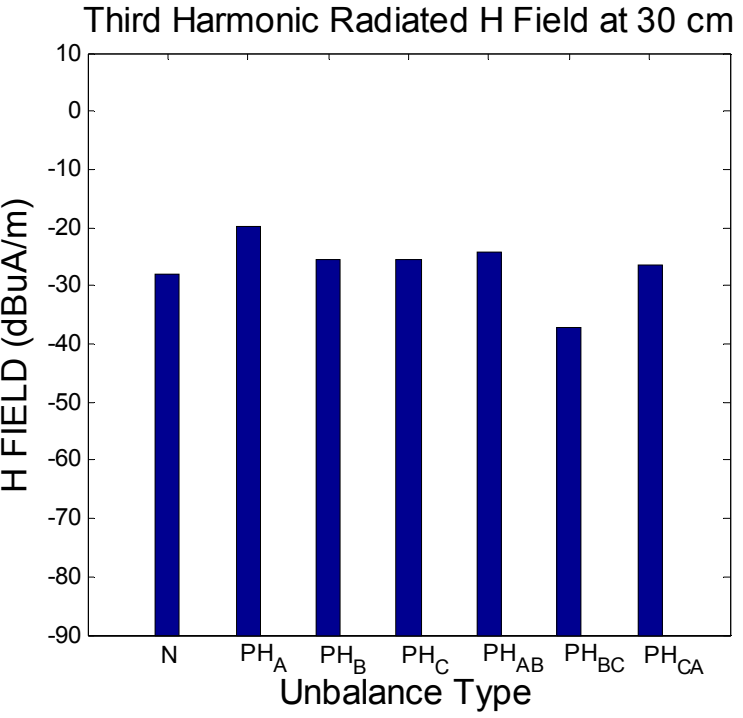

(b)

Fig. 8.8 Harmonic orders of the magnetic field radiated from the motor in the various unbalanced states. (a) first harmonic, (b) third harmonic (c) sixth harmonic, (d) eighth harmonic, (e) ninth harmonic, (f) eleventh harmonic 


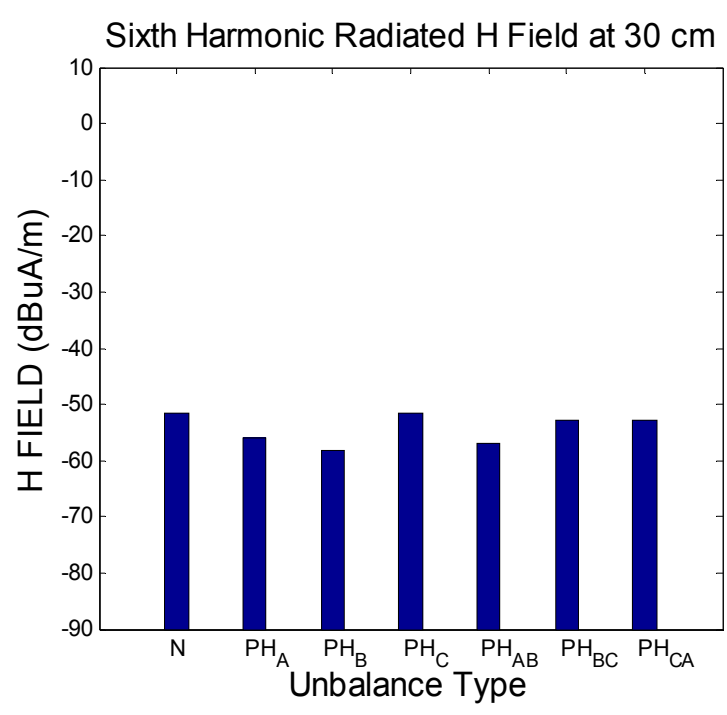

(c)

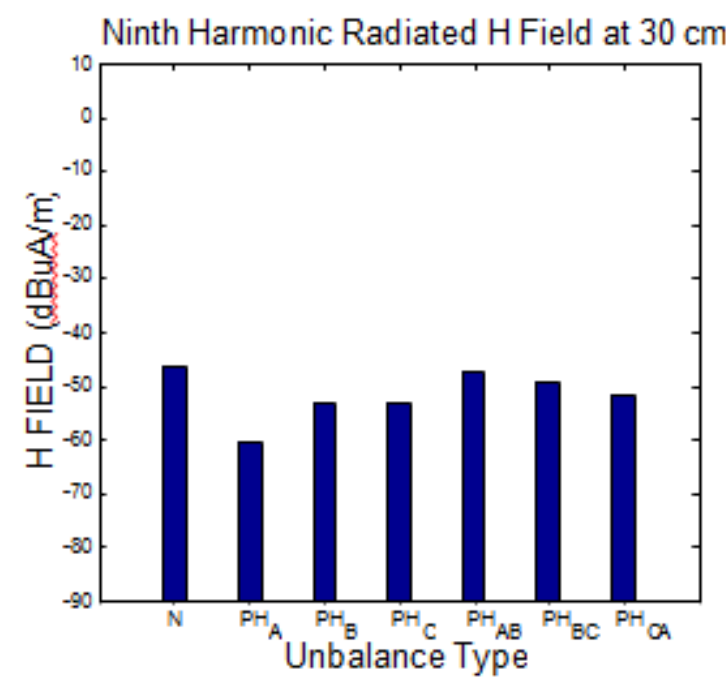

(e)

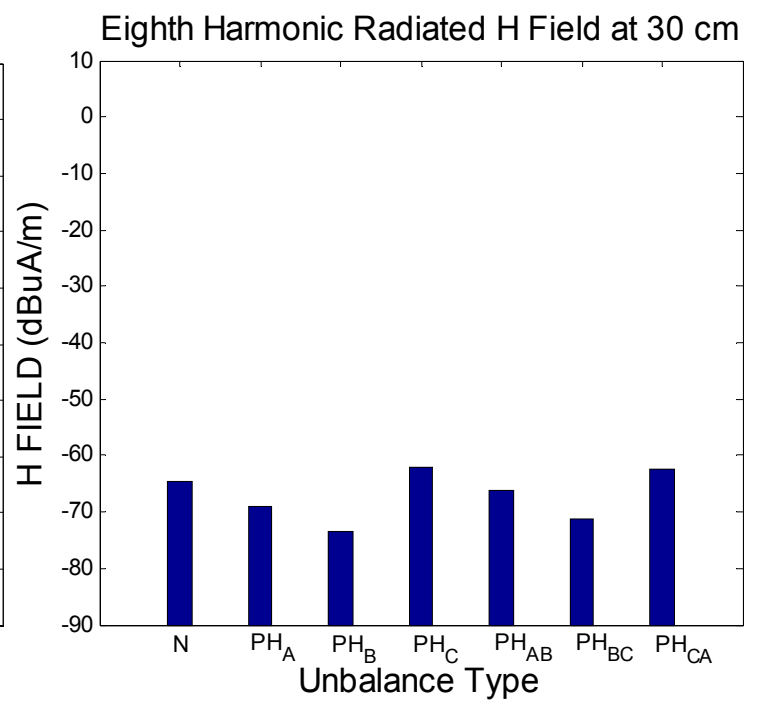

(d)

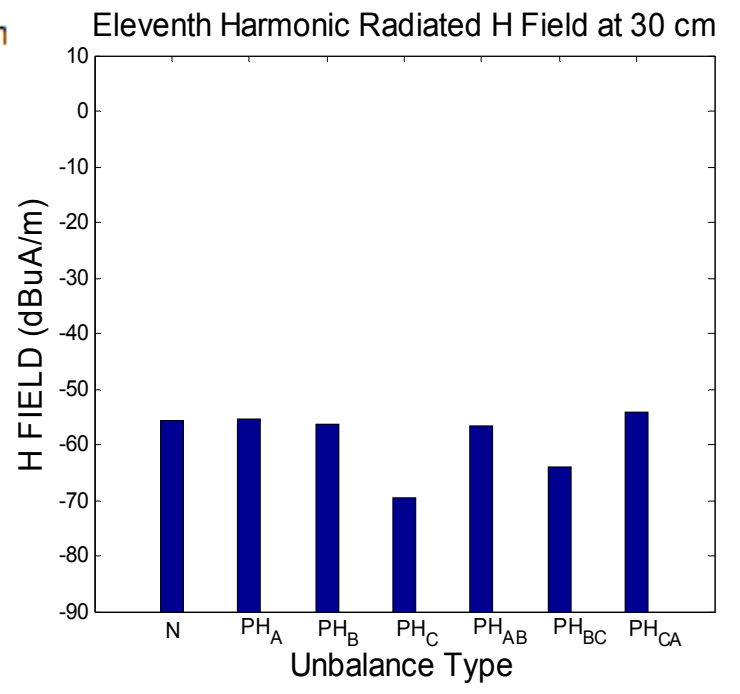

(f)

Fig. 8.9 Harmonic orders of the magnetic field radiated from the motor in the various unbalanced states. (a) first harmonic, (b) third harmonic (c) sixth harmonic, (d) eighth harmonic, (e) ninth harmonic, (f) eleventh harmonic

\subsection{Identification of unbalanced current phase through artificial neural network}

In order to have comprehensive failure identification method, the above unbalanced states were tested in various conditions, such as different levels of power and voltages in 
addition to the locations of antenna and motors. Finally, seventy cases were tested and used as the input of the artificial neural network.

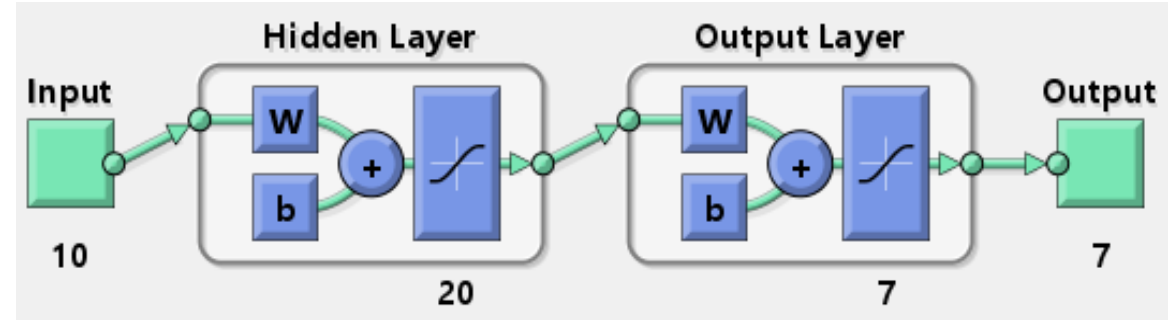

Fig. 8.10 The diagram of the neural network

The above network was implemented in MATLAB with scaled Conjugate Gradient algorithm for training the patterns. The input is a $10 \times 70$ matrix including the main 10 sensitive order of harmonics of the radiated magnetic field obtained in 70 tests. The target of the network is $7 \times 70$ matrix including the 7 mentioned states assigned for 70 cases. The diagram is shown in fig. 8.9. The 70 samples were distributed randomly for training, validating and testing, while $85 \%$ was being used for training, $10 \%$ for validating and $5 \%$ for testing. For double checking the network, the setup was tested again with different voltages, which were not tested before, while the phase B had unbalanced current. As a result, the unbalanced state identified with $0 \%$ error. Details of the training process of ANN including the performance and the training states are presented in fig. 8.10.

\subsection{Short-circuit study, experiment and simulation}

For the second case study, which is the fault in the stator winding, the short-circuit tests are implemented on one of the two coils of the phase B based on the circuit schematic in fig. 8.3. Since the short-circuit faults are more severe than the unbalanced currents, analyzing only three selected harmonic orders are enough to identify the short- 
circuit. However, for detecting very tiny short-circuits, study of higher order harmonics is suggested.

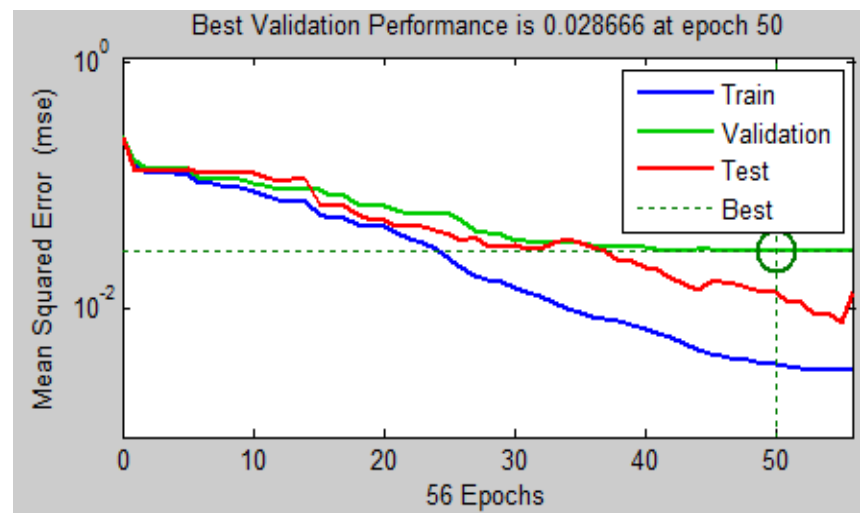

(a)

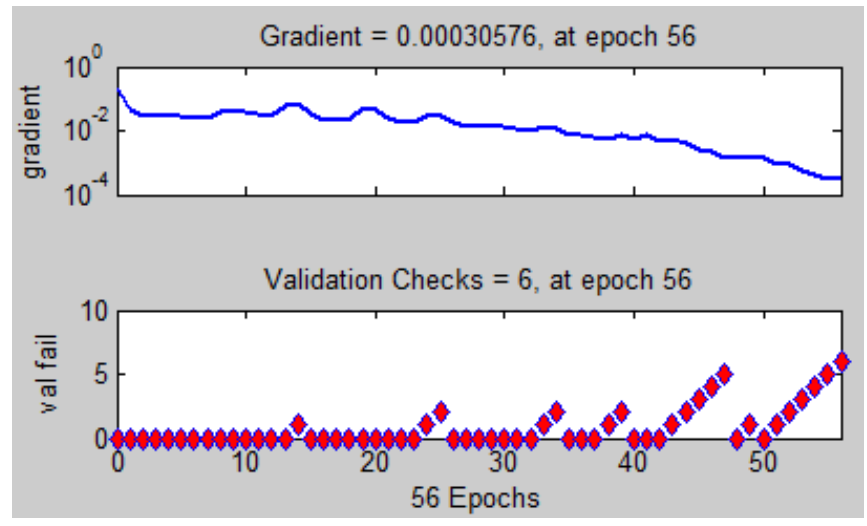

(b)

Fig. 8.11 The details of the training process of ANN (a) performance (b) training state

Similar to the unbalanced current study, some selected states of short-circuit based on the circuit of fig.8.3 and the location of short-circuited winding of fig. 8.4 are investigated and categorized in Table 8.2.

Considering Table 8.2, the short-circuit types are selected in a way to have different levels of short-circuit from the least sever, $\mathrm{SH}_{1.2}$, which had the resistance of $1.2 \Omega$, to the state $\mathrm{SH}_{140 \mathrm{v}}$, which has no resistance and the selected turns are totally short-circuited at $140 \mathrm{~V}$ of the terminal voltage. 
In order to identify short-circuit condition, the radiated field of the motor at $30 \mathrm{~cm}$ distance from the motor is obtained by the magnetic coil antenna. The field is plotted at the same distance through the simulation. The complete short-circuit between terminal $T_{B}$ and the turn $\mathrm{S}_{1}\left(\mathrm{SH}_{100 \mathrm{~V}}\right)$, which is implemented through the measurement and the simulation, is shown in fig. 8.11 (a) and (b) respectively. The 37 turns of the coil are shorted in this case. To avoid damaging the winding, the terminal voltage of the motor is reduced to $100 \mathrm{~V}$.

Table 8.2 The short-circuit states applied to the system

\begin{tabular}{|c|c|}
\hline States & Description \\
\hline Normal at $100 \mathrm{~V}(\mathrm{~N} 100 \mathrm{~V})$ & No short-circuit, Terminal Voltage $=100 \mathrm{~V}$ \\
\hline Normal at $140 \mathrm{~V}(\mathrm{~N} 140 \mathrm{~V})$ & No short-circuit, Terminal Voltage $=140 \mathrm{~V}$ \\
\hline $\begin{array}{l}\text { Short-circuit between TB } \\
\text { and } \mathrm{S} 1 \text { at } 100 \mathrm{~V}(\mathrm{SH} 100 \mathrm{~V})\end{array}$ & $\begin{array}{l}\text { The rheostat is totally shorted between terminal and } \\
\text { S1, Terminal Voltage }=100 \mathrm{~V}\end{array}$ \\
\hline $\begin{array}{l}\text { Short-circuit between TB } \\
\text { and } \mathrm{S} 1 \text { at } 140 \mathrm{~V}(\mathrm{SH} 140 \mathrm{~V})\end{array}$ & $\begin{array}{l}\text { The rheostat is totally shorted between terminal and } \\
\text { S1, Terminal Voltage }=140 \mathrm{~V}\end{array}$ \\
\hline $\begin{array}{l}\text { Short-circuit between } \mathrm{TB} \\
\text { and } \mathrm{S} 1 \text { at } 100 \mathrm{~V} \text { with } \mathrm{R}=0.4 \\
\Omega \text { (SH0.4) }\end{array}$ & $\begin{array}{l}\text { The rheostat is set to } 0.4 \Omega \text { between terminal and S1, } \\
\text { Terminal Voltage }=100 \mathrm{~V}\end{array}$ \\
\hline $\begin{array}{l}\text { Short-circuit between TB } \\
\text { and } \mathrm{S} 1 \text { at } 100 \mathrm{~V} \text { with } \mathrm{R}=0.53 \\
\Omega(\mathrm{SH} 0.53)\end{array}$ & $\begin{array}{l}\text { The rheostat is set to } 0.53 \Omega \text { between terminal and } \mathrm{S} 1 \text {, } \\
\text { Terminal Voltage }=100 \mathrm{~V}\end{array}$ \\
\hline $\begin{array}{l}\text { Short-circuit between TB } \\
\text { and } \mathrm{S} 1 \text { at } 100 \mathrm{~V} \text { with } \mathrm{R}=1.2 \\
\Omega \text { (SH1.2) }\end{array}$ & $\begin{array}{l}\text { The rheostat is set to } 1.2 \Omega \text { between terminal and } \mathrm{S} 1 \text {, } \\
\text { Terminal Voltage }=100 \mathrm{~V}\end{array}$ \\
\hline $\begin{array}{l}\text { Short-circuit between } \mathrm{S} 1 \\
\text { and } \mathrm{S} 2 \text { at } 100 \mathrm{~V} \text { with } \mathrm{R}=1.2 \\
\Omega(\mathrm{SH} 1.2 \mathrm{TT})\end{array}$ & $\begin{array}{l}\text { The rheostat is set to } 1.2 \Omega \text { between turn S1 and S2, } \\
\text { Terminal Voltage }=100 \mathrm{~V} \text {. }\end{array}$ \\
\hline
\end{tabular}


Since the FE simulation is analyzed in the frequency domain, the harmonic orders are plotted separately as shown in fig. 8.11 (b). Comparing fig. 8.11 (b) with fig. 8.11 (a) at the $\mathrm{Y}=0$ (exactly at the middle of the motor) shows that the simulation result matches the measurement with a good accuracy. To study and recognize the short-circuit types, the states based on the Table 8.2 are compared in fig. 8.12 through both simulation and experiment.

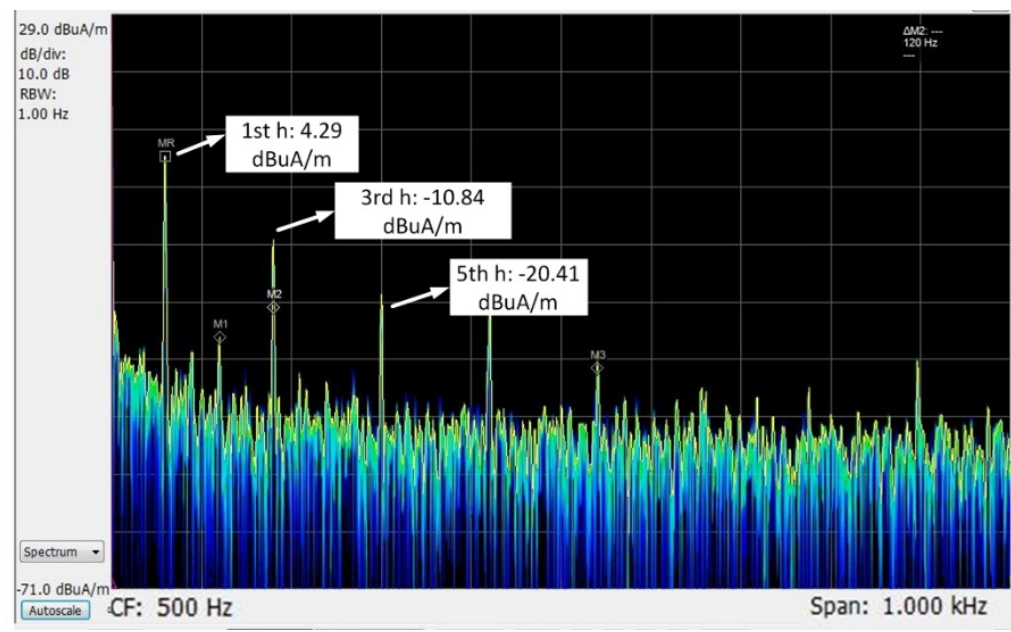

(a)
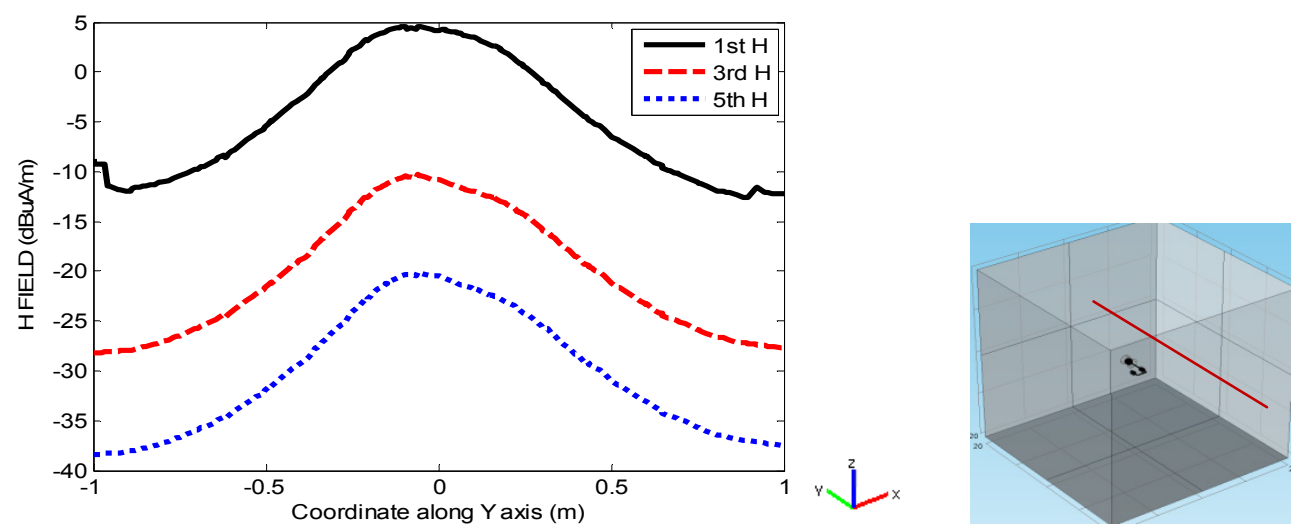

(b)

Fig. 8.12 Radiated H field of the motor, while there is a complete short-circuit between terminal $\mathrm{T}_{\mathrm{B}}$ and the turn $\mathrm{S} 1\left(\mathrm{SH}_{100 \mathrm{~V}}\right)$ (a) measurement (frequency response with the span of $1 \mathrm{kHz}$ ), (b) simulation with the plot at $\mathrm{x}=30 \mathrm{~cm}$ along the $\mathrm{Y}$ axis 
The short-circuit types can be inferred through comparing their harmonic orders. Considering the first harmonic at fig. 8.12(b), it can be easily seen that, the short-circuit states with rheostats have lower amplitude of the $\mathrm{H}$ field (magnetic field intensity). This is because of the resistance of the rheostat that allows less current to pass the rheostat in comparison with the states that there is no resistance, $\mathrm{SH}_{100 \mathrm{v}}, \mathrm{SH}_{140 \mathrm{~V}}$. Hence, the amplitude of the short-circuit current and consequently the radiated $\mathrm{H}$ field decreases. Therefore, this helps finding the intensity of the short-circuit condition. On the other hand, obviously if the terminal voltage increases, state $\mathrm{SH}_{140 \mathrm{v}}$, the higher amplitude of the current passes through the winding; therefore, the $\mathrm{H}$ field increases. Similarly comparing the first harmonic of the short-circuit states, it can be perceived that the motor at inter-turn short-circuit radiates more magnetic intensity $(\mathrm{H})$ field. For better investigation, the third and the fifth harmonics should be used. It should be noted that because of some technical reasons, the effect of some states would not be recognized through an individual harmonic order. For example, the third harmonic order of the flowing current into the rheostat doesn't change by varying the resistance of the rheostat. However, the fifth harmonic order clarifies this problem. Considering the fifth harmonic orders of the mentioned states, the radiated $\mathrm{H}$-field decreases, while the resistance of the rheostat increases. This is helpful to determine the intensity of the short-circuit condition. For analyzing the insignificant types of short-circuit, the higher order harmonics along with the artificial neural network, similar to the unbalanced current case, should be considered. Building the neural network for short-circuit cases needs testing numerous cases, similar to the unbalanced current cases. The numerous experimental testing of the 
cases would damage the machine. Therefore, using the mentioned simulation procedure is advised.

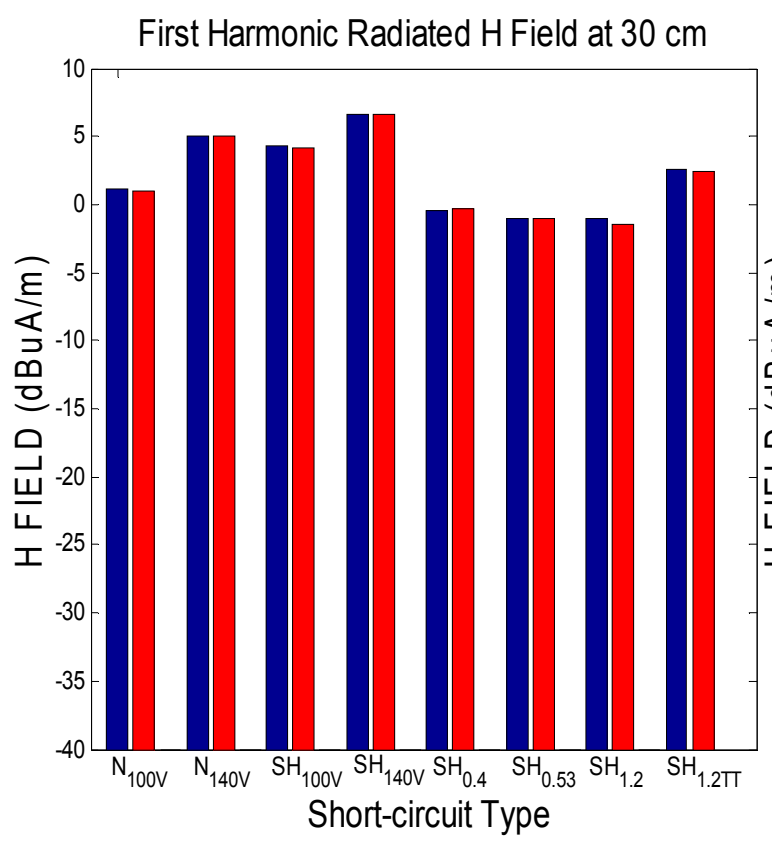

(a)

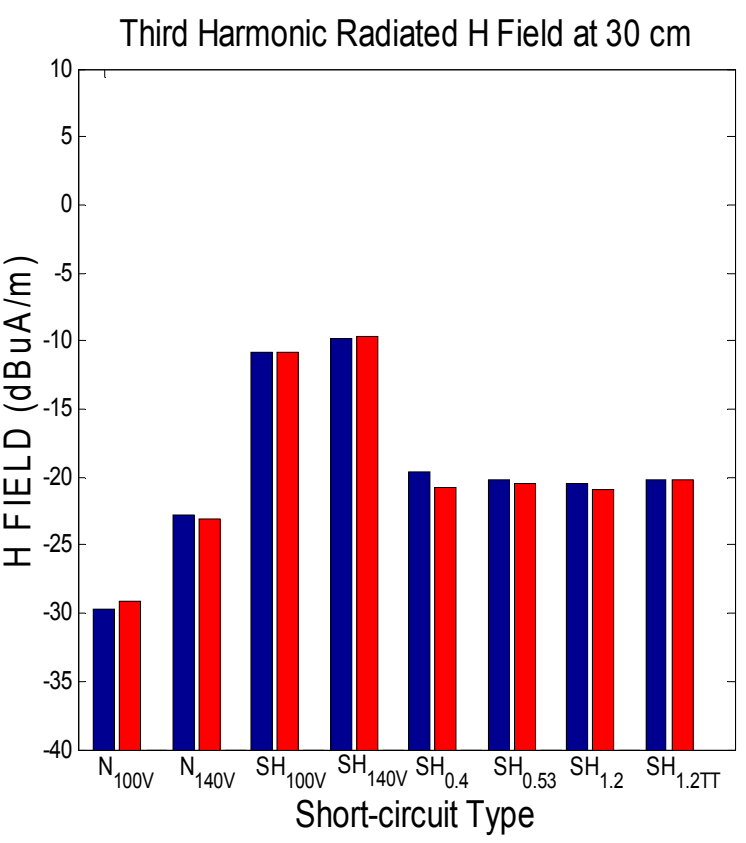

(b)

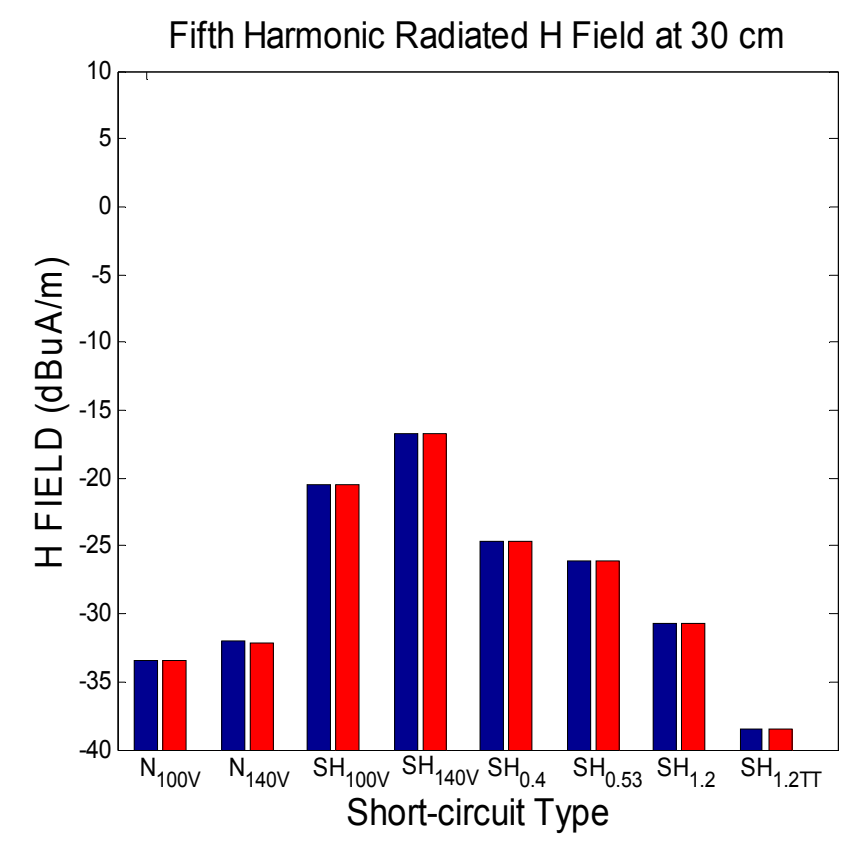


(c)

Fig. 8.13 Several harmonic orders of the magnetic field radiated from the motor in the various short-circuit states. (a) first harmonic, (b) third harmonic (c) fifth harmonic

\subsection{Conclusion}

The identification of various unbalanced input condition of flowing current, as well as the short-circuit of the stator winding of an induction motor, was developed and implemented in this chapter using 3D FE simulations and ANN. The developed process was verified through measurements. Various types of unbalanced currents and shortcircuit were analyzed. The 3D FE analysis was coupled with circuit-based software to implement the fault states. The fast generalized minimal residual technique was used for expediting the numerical analysis, while high order harmonics was proposed to be

observed in frequency domain study. Various types of unbalanced currents passed into the machine's terminal lines and the harmonic orders of the radiated magnetic field were obtained and studied. The identification was implemented manually and by using ANN for seventy various cases. Similar scenario was implemented for the various types of the short-circuit condition. The final results show that any types of unbalanced current or short-circuit states under various levels of terminal voltage and different locations of the antenna can be detected by analyzing the frequency response of the radiated magnetic field. 


\section{Identification Of Short-Circuit Location in the Stator Windings of Induction Motors Using Radiated Electromagnetic Field Electromagnetic Signatures}

\subsection{Overview}

Locating partial short-circuits in electrical machines is an important issue in industry. This enables the user to fix specific winding segment before expanding into a larger fault. This chapter investigates the identification process for locating this type of fault in the winding of an induction motor through radiated electromagnetic field signatures. It's firstly proposed to recognize the short-circuit type, such as interturn, intercoil, or terminal-turn. Then, the location of short-circuit can be identified. The frequency response of the radiated magnetic field of the motor is used for the fault location. The responses are compared based on the variations of the amplitudes of the harmonic orders. In order to be able to use this method for various industrial circumstances, two different approaches are used for the short-circuit location, the singular optimum location and the multi-optimum location. The experimental study of short-circuit conditions, especially the major and semi-major cases which could damage the winding. Instead, the full threedimensional finite element (3DFE) method is utilized in a few cases and verified experimentally. Finding the locations of the small short-circuit helps the engineers in fixing the specific wire of the winding before the major damage occurs.

Formerly, fault detection in machines involved simple techniques, such as overcurrent or overvoltage detection. After detection, it was required to bring the machine offline to clear the fault. In safety-critical applications, however, a shutdown of the motor may not be acceptable. This demands better fault-detection approaches. The first step in dealing 
with a fault in the drive is being able to detect it quickly and determine its location and severity accurately. This is particularly critical in stator short-circuit faults, which can cause catastrophic damage to the machine in a very short time making any fault compensation impossible thereafter [239]. Especially, most of the failures in the induction machines (IM) are basically due to short-circuits in the stator winding [240]. It has been reported that most short-circuit faults begin as inter-turn faults [241]. Finding the location of the partial short circuit is to prevent short-circuit conditions from spreading the fault to a serious damage in the windings. It has been shown in [240] that it takes less than 2 seconds for a single inter-turn fault to progress into a serious fault in a typical $15-\mathrm{kW}$ induction motor. However, following the recognition that the partial shortcircuit occurred, it's hard to find the location of the partial short-circuit among hundreds of wires in the machine. Therefore, advanced methods should be used to detect the interturn short-circuit.

The testing and monitoring methods can be generally divided into two different categories (online and offline). The offline techniques need the motor to be removed from the service [242]-[246], while the latter can be implemented, while the machine is working [247]-[255]. Based on the severity of the fault and also the application in which the motor is being used, these two mentioned methods can be selected.

The offline method has a great disadvantage to the online method, which is the disconnection of the machine from service. However, it has an advantage of online monitoring that meaningful tests can be performed after fabrication of the motor and that a test device can be used for several different machines, which saves costs. There are many methods in the category of offline tests such as DC connectivity test [242], AC/DC 
potential tests [243], insulation resistance (IR) [244], offline partial discharge [245], impedance [246], surge test [246], and some more methods which are discussed in [247]. They can be used based on the type of machines. For example, the PD test is not applicable to low-voltage machines.

On the other hand, online methods use different parameters of the machine, such as magnetic field, stator current and voltage, input power and temperature for the monitoring purpose. The well-known online methods include temperature monitoring [248], condition monitoring [248], online partial discharge [248], AI-based [250], current signature analysis [251], HF impedance [252], pendulous oscillation phenomenon [253], vibration signature analysis [254], axial leakage flux [255] and many other methods which are discussed in [247], [248]. Almost all of the mentioned methods are applicable for the interturn short-circuit; however, any of them have some advantages and some disadvantages based on the situation they are intended to be used. For example, temperature monitoring requires a lot of data and additional information like ambient temperature, which is a drawback. However, it can detect deterioration in phase-toground and faults in interturn insulation. Also, the leakage current technique is noninvasive, but it's weak to detect interturn short-circuits for low-voltage motors. All in all, the major advantage of these methods as mentioned before is that the machine does not have to be taken out of service. Therefore, the normal condition, while the motor is operating, can be evaluated. Since the machine is under constant monitoring, the predictive maintenance is made easier, an incipient failure can immediately be detected, and actions can be scheduled to avoid more severe process downtime. A disadvantage is that the mentioned online-monitoring techniques often require the installation of 
additional equipment, which has to be installed on every machine. Therefore, in this research, it's trying to use an online technique, which doesn't have this drawback.

The Airgap flux signature technique measures the rotating field of the machine by utilizing the sensor installed in the machine. Using this method, the interturn short-circuit can be detected. Valuable researches were presented about this technique [119]-[128], [256]. In [119], a program was developed in LabVIEW to perform the data acquisition and analysis is presented. Data is read in real-time form of the working induction motor, and the program provides three fault indicators were found: rotor bar failure, rotor end ring failure and stator winding interturn short circuit. With these three indicators failure location, the state and the cause was determined. The system was validated in a laboratory with motors with known faults. In [120], medium-range frequencies are taken into consideration to determine electrical faults, when the machine is supplied through the grid. The broken bar fault and the interturn short-circuit are studied in this paper. Similarly, Chadebec et al [121] showed the trustworthiness of the fault detection on electrical machines by analysis of the low-frequency magnetic field signature. A simple model of the stray magnetic field of a synchronous machine is used to predict the generated stray magnetic field in the case of different rotor faults (short-circuit rotor windings and dynamic mechanical eccentricity). The other advantage of using stray field in the fault diagnosis, such as the fault in lamination and broken bars, are explained in several publications such as [122]- [126]. The magnetic signature is also being used in offline method. A technique that uses motor, the electromagnetic signature analysis of the terminal voltage immediately after switch-off to diagnose turn faults, is introduced in [127]. 
The Air gap magnetic flux has good results in detecting interturn short-circuit; however, installing and maintaining the sensor are the drawback of this method. Hence, in this research, it's trying to measure the radiated magnetic field outside the motor using the magnetic antenna and use the frequency response analysis for interturn fault detection. Since it's possible to locate the field antenna close to the machine, the magnetic behavior of only the faulty machine would be evaluated. This is useful while there are some other machines around the faulty one.

In this chapter, the radiated field of the machine is observed while short-circuit occurred in different locations of a coil and winding. The changes of the harmonic orders of the radiated field help in the identification of short-circuit conditions. The test setup details along with the procedure of monitoring the system, using magnetic field is explained in 9.2. As the first case study, the intercoil short-circuit, as well as terminal-toturn short-circuit are investigated in 9.3. Since these fault may be hazardous for the winding, the full 3D finite element modeling connecting with the circuit-based software is used for the modeling of short-circuit. The simulation results were verified by the experimental tests. Furthermore, short-circuit between two adjacent wires, the interturn short-circuit, changes the magnitude of the higher harmonic orders of the radiated field. Identifying the location of short-circuit requires finding the optimum location of the magnetic coil antenna for the machine. Two different types of the optimization of the antenna's position along with the interturn fault location are investigated and discussed in section 9.4. Finally, analyzing the harmonic orders of the frequency response for the purpose of identifying the interturn short-circuits is employed. 


\subsection{Magnetic stray field monitoring of the short-circuit in the winding}

\subsubsection{Motor setup}

Fig. 9.1 shows the block diagram of the test setup implemented to characterize the fault detection under controlled conditions in the laboratory experiment. The characteristics of the equipment used in the test are:

Induction motor: 7.5HP, 208V, 1800RPM, PF 0.82, 60Hz

Adjustable power resistor: $3.0 \Omega$, Max. Power 225W, with 58 adjustable points.

The Magnetic coil antenna and EMI receiver were the same as chapter 8 .

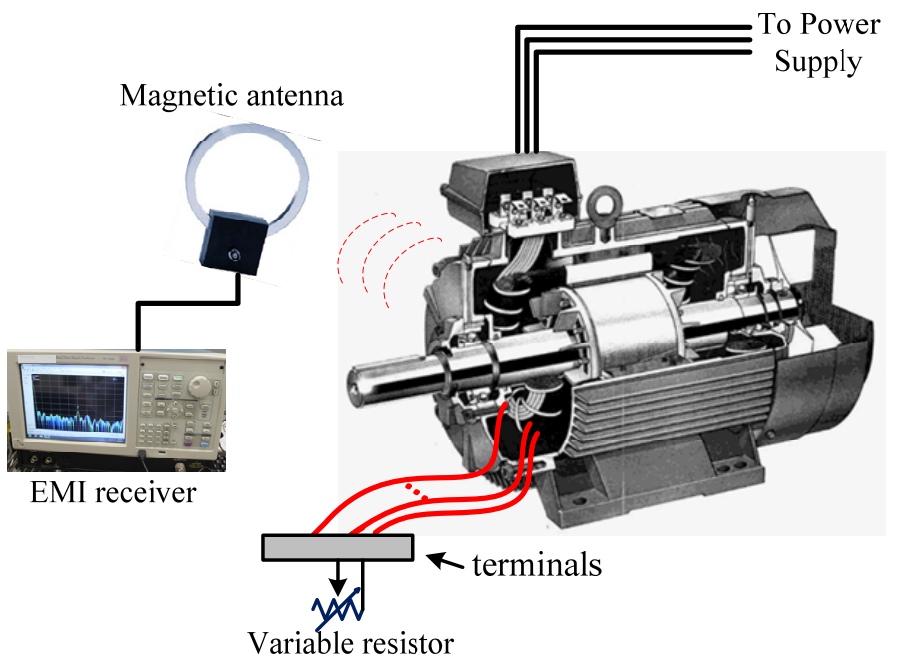

Fig. 9.1 The schematic of the test setup

The radiated magnetic field intensity can be observed at given distances close to the machine by using the magnetic coil antenna. The data is sent through the BNC cable to the EMI receiver. Then, the frequency response or time domain response of the radiated field can be seen and investigated. The radiated fields show the behavior of the machine and the changes in the windings or other components of the motor. Hence, it's a valuable parameter for monitoring the condition of the machine. Since the load may have a 
significant effect on the fields and may be variable in different application, the machine in this research is investigated at no-load to have a general and valuable result.

The laboratory setup is shown in the fig. 9.2. In order to avoid possible noises around the setup, the motor is located inside a chamber which isolates the setup from the other electric nearby components. Therefore, the components of this setup including power supply and EMI receiver are located outside the chamber and connected through an isolated duct into the chamber. In order to have the maximum invulnerability of the motor from undesirable fields, the table, on which the machine in located, is made up of wood. The antenna and the setup are located based on the standards explained in chapter 5.

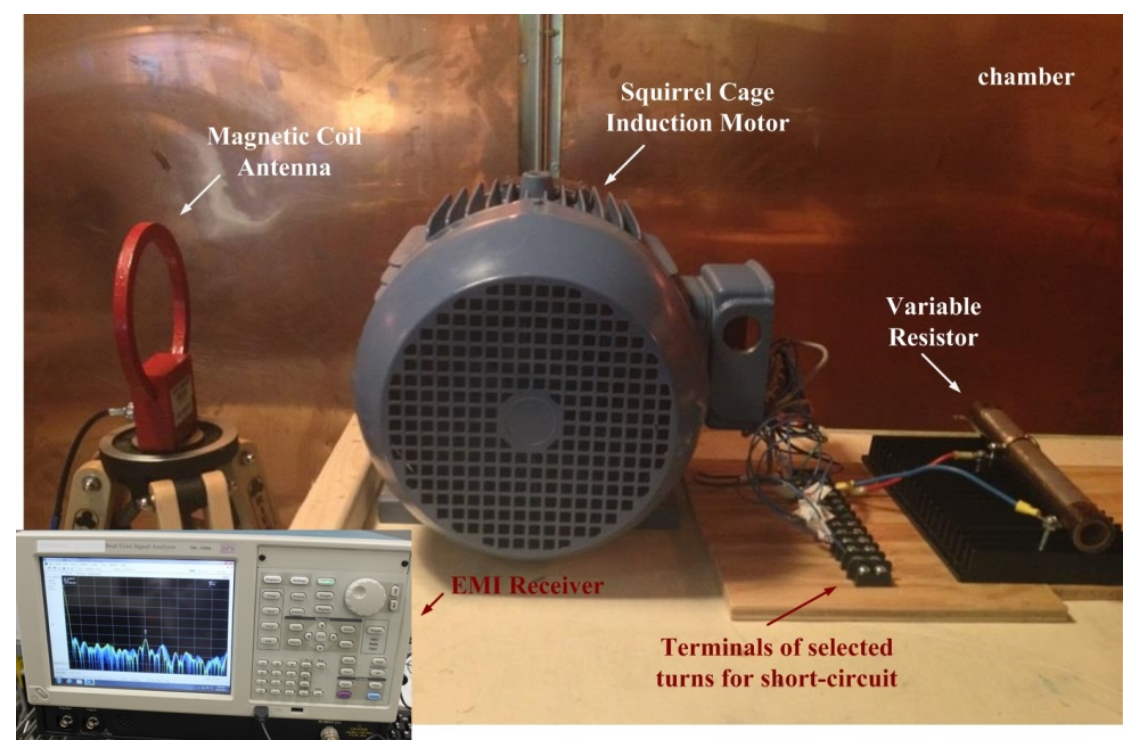

Fig. 9.2 The studied setup including machines, measurement stuff

\subsubsection{Monitoring the radiated magnetic field}

After preparing the components, the machine is started and the magnetic antenna captured the field and sent to the EMI receiver. The EMI receiver is set in the frequency domain mode. The frequency-domain analysis is based on the transformed signal in the 
frequency domain. The advantage of the frequency-domain analysis over the timedomain analysis is its ability to easily identify and isolate certain frequency components of interest. In other words, the main idea of the spectrum analysis is to either look at the whole spectrum or look closely at certain frequency components of interest and thus extract features from the signal. Since the proposed motor is working at $60 \mathrm{~Hz}$, the frequency components of interest which help in the detection process are between power frequency $(60 \mathrm{~Hz})$ and $3 \mathrm{kHz}$. The bandwidth based on the severity of the fault can be shortened or expanded. This is explained in the next section.

In order to have comprehensive analysis of the frequency response of the system, the harmonics of the machine should be explained.

The harmonic order is directly related to the slot number per 2 times the polar step, and its magnitude depends on the distribution winding factor [128]. Every stator harmonic creates synchronous torques and requires special attention on the teeth wave. These kinds of harmonics are in the order

$$
f_{\text {slot }}=f_{s}\left[k \frac{m}{p} \pm 1\right]
$$

where $k$ is an integer, $\mathrm{m}$ is the stator slots number, and $p$ is the number of pairs of poles.

The stator $\mathrm{h}$ harmonic turns at $\mathrm{fh}= \pm \mathrm{fs} / \mathrm{h}$ from main harmonic fs and the harmonic slip could be expressed as:

$$
S_{h}=\frac{f_{h}-f_{r}}{f_{h}}=1 \pm(1-s) h
$$

where $S_{h}$ is the harmonic's h slip and $f_{r}$ is the rotor frequency. The induced rotor current frequency of the $h$ harmonic magnetic field is determined by: 
$f_{2 h}=f_{s}[1 \pm(1-s) h]$

The harmonic orders of the stator and rotor, as wells as synchronous harmonics, which appears for mutual action between stator and rotor are affected by the occurrence of faults including broken bar, bearing damage, air-gap eccentricity [256], as well as short-circuit in the machine. In an induction machine, the frequencies produced by interturn shorts in the stator current around the base frequency are given by:

$f_{s t h}=f_{s}\left[1 \pm k m\left(\frac{1-s}{p}\right)\right]$

The harmonic orders can be analyzed using much frequency-domain signal-processing technique in order to extract the fault information. Due to the presence of voltage unbalances and other asymmetries, only frequencies corresponding to specific combinations of $\mathrm{k}$ and $\mathrm{m}$ in (4) can be used for the short-circuit fault diagnosis. Based on the severity of short-circuit, the higher or lower order of harmonics get affected. That is to say, when short-circuit occurs between only two turns, the amplitude of higher order of harmonics (9th-31th) changes. Inversely, short-circuit between more number of turns can easily be recognized by analyzing lower order of harmonics (3th-7th). This is shown and discussed in the following two sections.

\subsection{Intercoil short-circuit study, experimental and simulation}

After explaining the basics and procedure of the study, the explained setup is implemented for different short-circuit types. The major goal of this study as mentioned before is to detect the location of the short-circuit. Hence, the minor goal of each part of this section is set to obtain a way to find out the proper harmonic orders for studying the 
different levels of short-circuits. This needs to attain the proper distance of the antenna from the machine and the proper location of the antenna around the machine, while faulted with the interturn short-circuits

First, short-circuit between the coils are studied. The short-circuit tests are implemented on one of the two coils of the phase B, by setting the terminals shown in fig. 9.2 and fig. 9.3. The part of the coil selected to be shorted has 37 turns. Therefore, the terminals are connected to the variable resistance to avoid damages.

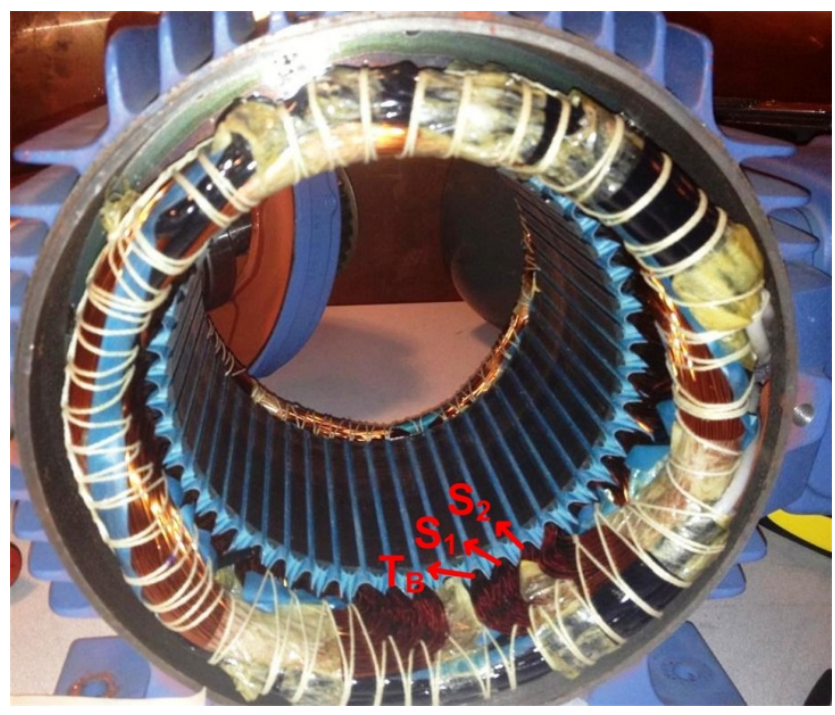

Fig. 9.3 Location of the selected turn for inter-turn and turn-terminal short-circuits (TB: terminal of phase B, S1 and S2: selected turns of phase B)

The short-circuit is implemented for the terminal-to-turn case (TB-S1) and the interturn $\left(\mathrm{S}_{1}-\mathrm{S}_{2}\right)$ case with and without variable resistance in different voltages. Fig. 9.4 and fig. 9.5 show the radiated magnetic field intensity (H-field), while short-circuit was between $T_{B}-S_{1}$. The fig. 9.4 is without variable resistance (rheostat) and the fig. 9.5 is with variable resistance set to $0.4 \Omega$. Both tests are implemented at $100 \mathrm{~V}$. As illustrated in the figures, the short-circuit affects the radiated field measured at $30 \mathrm{~cm}$ away from the 
shaft of the motor. Since the short-circuit is significant, the changes at the important harmonic orders such as $2 \mathrm{nd}, 3 \mathrm{rd}, 5$ th, and 7 th are easily noticeable, especially in the case without rheostat.

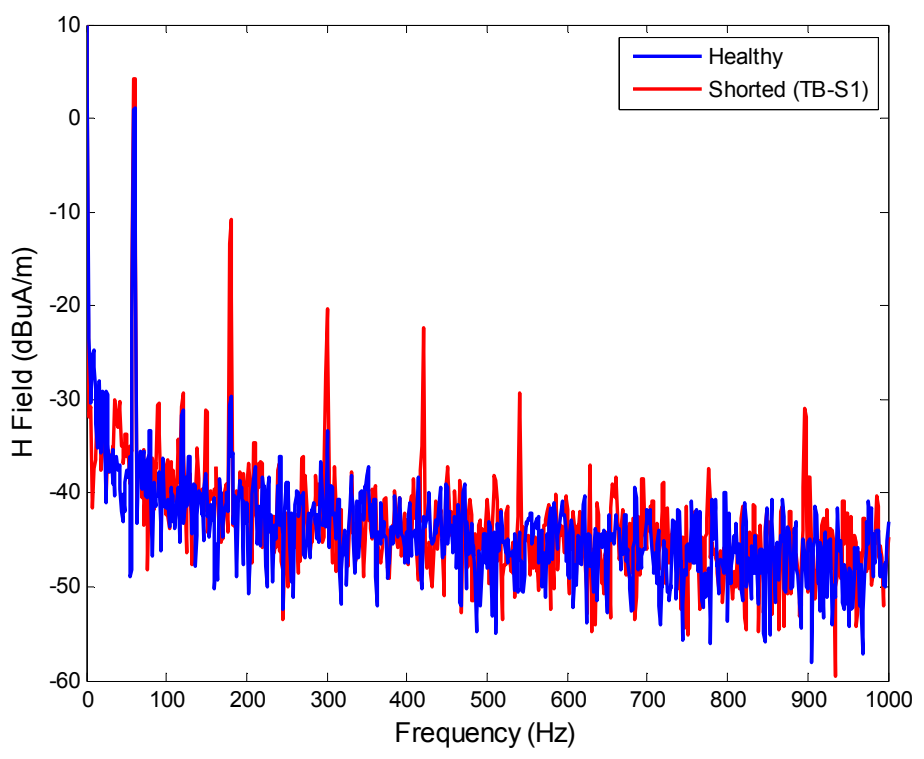

Fig. 9.4 Radiated $\mathrm{H}$-field of the motor, while there is a complete short-circuit between terminal $\mathrm{T}_{\mathrm{B}}$ and the turn $\mathrm{S}_{1}$

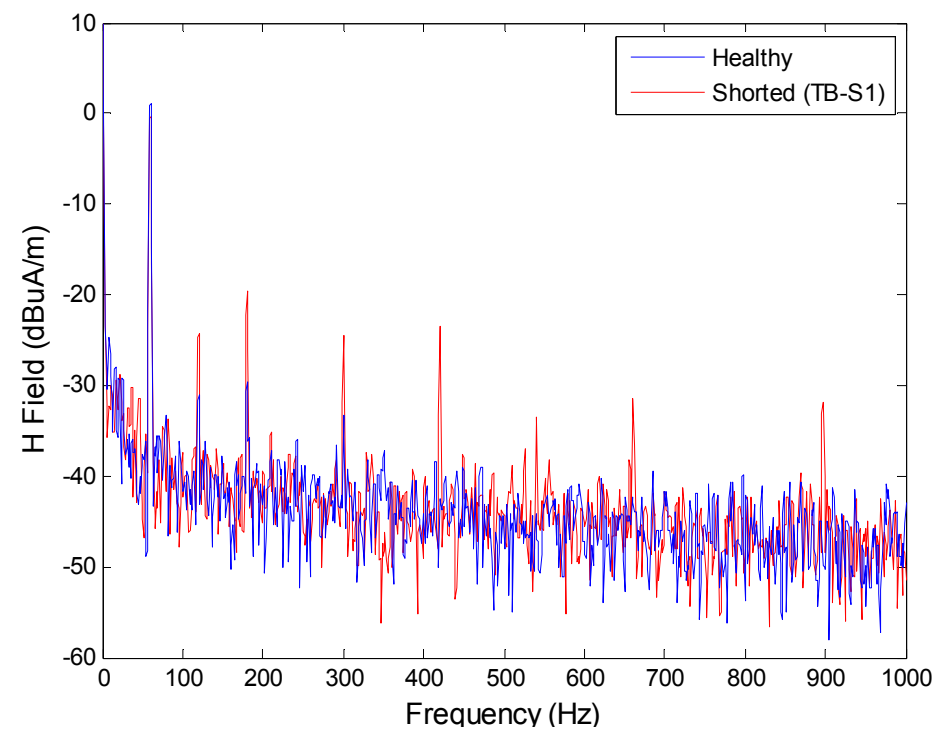

Fig. 9.5 Radiated H-field of the motor, while there is a short-circuit between terminal TB and the turn S1 while the rheostat is set to $0.4 \Omega$ 
The experimental testing of the coils to find out the difference of terminal-to-turn short-circuit and intercoil short-circuit is damageable for the winding. Therefore, for these cases, which have significant short-circuit, the setup is simulated by means of the full three-dimensional finite element (3DFE) method and verified experimentally.

All the details of the machine are considered in this model, which is shown in fig. 9.6. In order to apply the interturn short-circuit, the proposed turns of the coil would be paralleled with a rheostat to have various levels of short-circuit as shown in fig. 9.7. The motor and the selected terminals were illustrated in fig. 9.3. The rheostat is connected to $\mathrm{S} 1$ and S2 for interturn cases and between TB and S1 for turn-terminal cases. Note that there are 37 turn wires in each slot and just one of them is soldered to the S1 and S2 and taken out of the casing. Similarly, the turn-terminal short-circuit is applied between terminals of the phase $B\left(T_{B}\right)$ and $S_{1}$.

The simulation time for solving the model in $60 \mathrm{~Hz}$ was 20 minutes with the modified solution settings. To have the reliable accuracy, the model is meshed finely with 5.7 degrees of freedom.

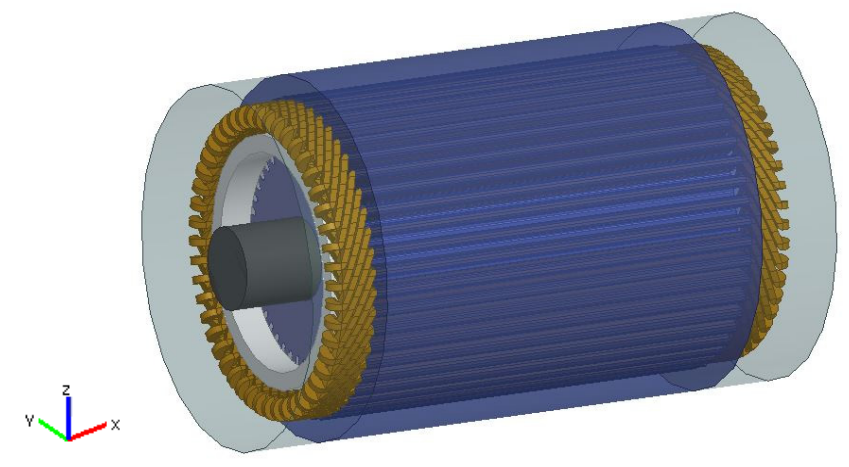

Fig. 9.6 Finite element model of the studied induction motor 


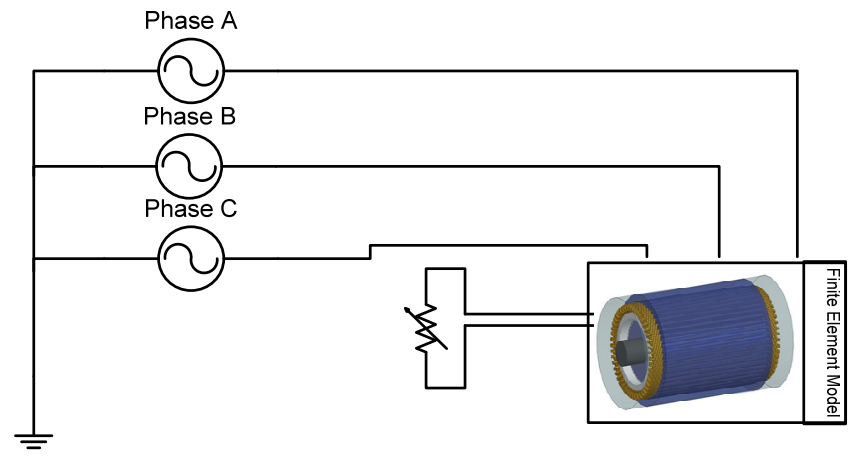

Fig. 9.7 Schematic of the circuit connected to the model for the short-circuit study

Since the important harmonics can be enough to recognize this type of short-circuit, the other harmonics and noises are cleared out for easier evaluation. The comparison of the intercoil and terminal-turn short-circuit is shown in fig. 9.8.

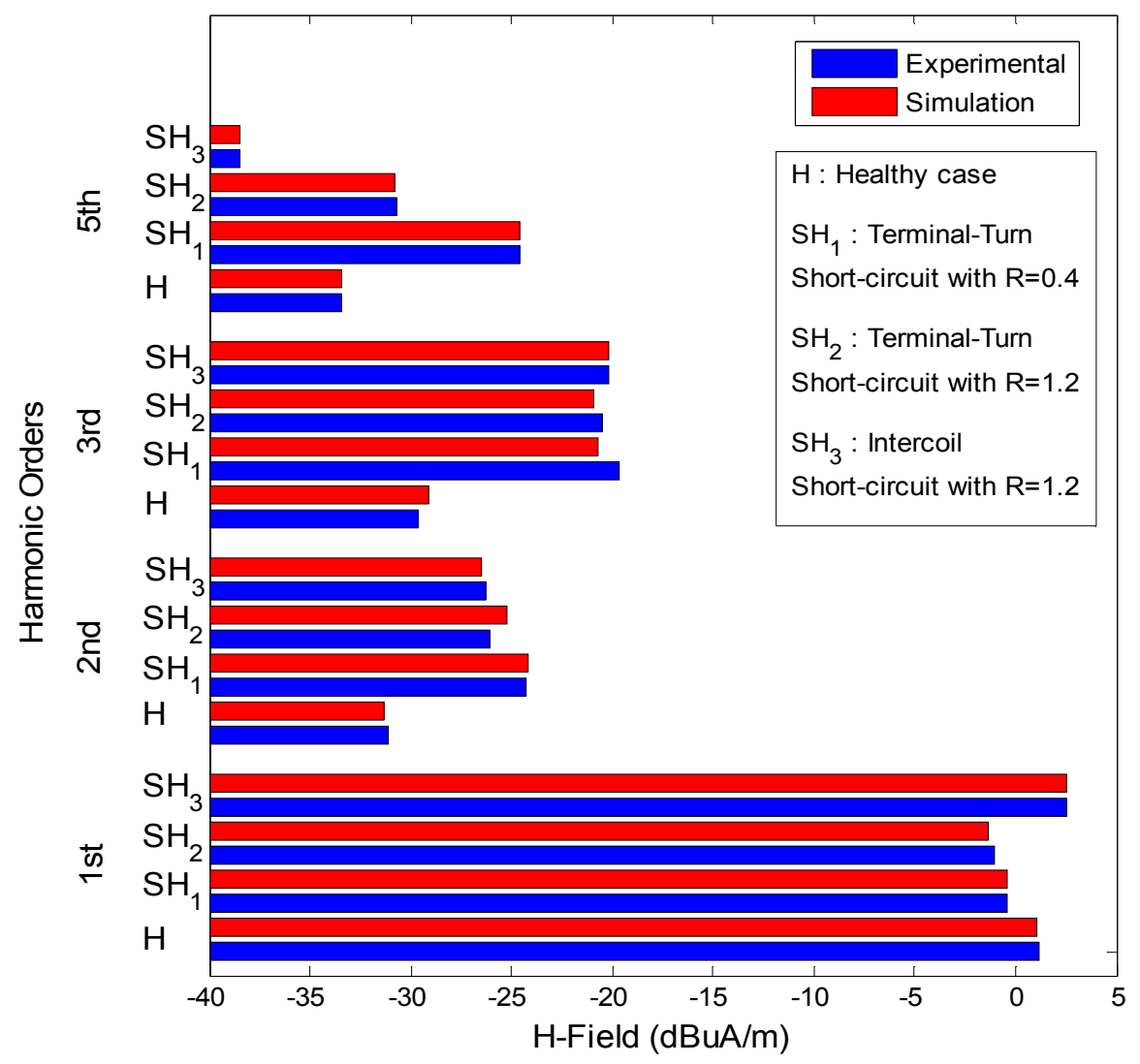

Fig. 9.8 Radiated H-field in various cases of the significant short-circuits in the induction motor's stator winding $(\mathrm{dB} \mu \mathrm{A} / \mathrm{m})$. Note that the terminal-turn short-circuit is between 
$\mathrm{T}_{\mathrm{B}}-\mathrm{S}_{1}$ of fig. 9.3 and the intercoil short-circuit is between $\mathrm{S}_{1}-\mathrm{S}_{2}$. The $\mathrm{R}$ is the re resistance of the rheostat

Fig. 9.8 verifies the accuracy of the 3DFE model, since the simulation results substantially equal the experimental ones. As illustrated in the figure, the first harmonics of the cases don't get affected significantly from the proposed short-circuit. However, the 2nd, 3rd, and 5th orders of the harmonics change. Based on the types of the fault, specific harmonic orders should be used to identify the type of short-circuit. For example, for identifying the intercoil short-circuit from the terminal-turn short-circuit, the 5 th harmonic is applicable. Comparing the 5th harmonic order of the $\mathrm{SH}_{2}$ case with $\mathrm{SH}_{3}$ case in fig. 9.8, it can be perceived that the current loop produced due to short-circuit in between $S_{1}$ and $S_{2}$ is inverse the main current loop. Therefore, it decreases the aggregate amount of the radiated $\mathrm{H}$-field at 5th harmonic. In reverse, the loop due to short-circuit between $T_{B}$ and $S_{1}$ increases the aggregate amount of the radiated $\mathrm{H}$-field. By decreasing the resistance of the rheostat, the field increases more. Therefore, this harmonic order can be used for identifying the severity of short-circuit between $\mathrm{SH}_{1}$ and $\mathrm{SH}_{2}$ as well. Besides, higher harmonic orders may be useful for this purpose. The 2nd and 3rd harmonics can be used for identifying the short-circuit cases from the healthy case but they are not useful for identifying the type of short-circuits.

\subsection{Interturn short-circuit}

The proposed machine has 48 coils and in each coil it has 37 turns, which totally becomes 1776 turns. Detecting short-circuit between two turns between 1776 turns is not possible by observing the low harmonic orders as explained above. 
Firstly, it needs to be mentioned that the location of the antenna is very important, since the radiated magnetic fields of the coils are closed loop, up to a distance based on the passing current through the wires as shown in fig. 9.9. After this distance, the radiated fields will not establish the loops. Therefore, the appropriate location of the antenna to capture the major part of the radiated field is concerned.

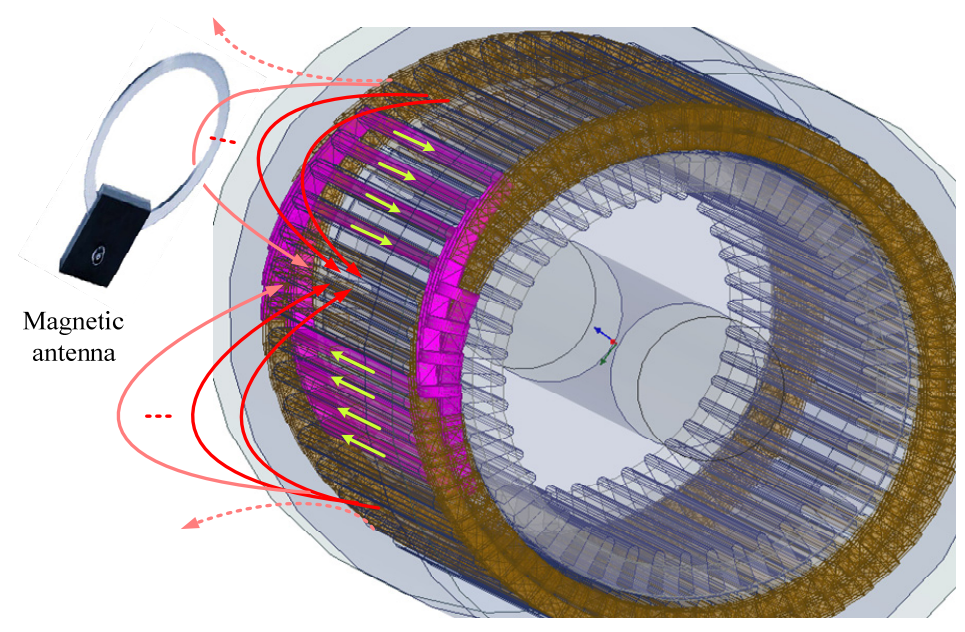

Fig. 9.9 The coil of the winding and the necessity of the appropriate location of antenna

As illustrated in the figure, one parameter of this optimized location is the angle vs. machine's shaft in which the antenna should be located to capture the specific field of the shorted turns. This is termed the angle index in the rest of the chapter. The other parameter is the distance of the antenna from the motor. This is termed the distance index in the rest of the chapter. In order to have the proper location of the antenna, the optimization with these two parameters as the indices should be implemented. However, the optimum angle for each turn is unique. In other words, if it's intended to find out the optimized location of antenna for whole winding, the angle index could not be obtained; 
because, each turn of the 1776 turn has the appropriate location of the radiated field in the area around to be captured. Therefore, the optimum angle index could be for a particular group of the winding such as each phase. Namely, it can be expressed that the optimum location of antenna in terms of angle for the interturn short-circuits in phase A can be obtained. On the other hand, the distance index can be optimized based on the level of the passing current through the winding. For example in the cases in section 9.3, the distance of the antenna from the shaft of the motor was $30 \mathrm{~cm}$, which is $16 \mathrm{~cm}$ from the casing. Locating the antenna at a closer distance from the machine causes the presence of many sub-harmonics in the frequency response, which makes the investigation complex. Also, locating farther than $30 \mathrm{~cm}$ would not show the radiated field properly. This is more critical in the interturn case due to the weaker effect of this fault in the frequency response.

Six turns in three coils of the winding of phase A are selected to be tested for interturn short-circuit. The selected turns are depicted in fig. 9.10. The turns $S_{25-1}$ and $S_{25-2}$ are located next to each other, as well as $S_{27-1}$ and $S_{27-2}$ and in the same manner $S_{28-1}$ and $S_{28-2 .}$. To verify the equality of these three interturn short-circuits, the resistance in between two selected adjacent turns of this three short-circuits are tested by multi-meter and confirmed. 


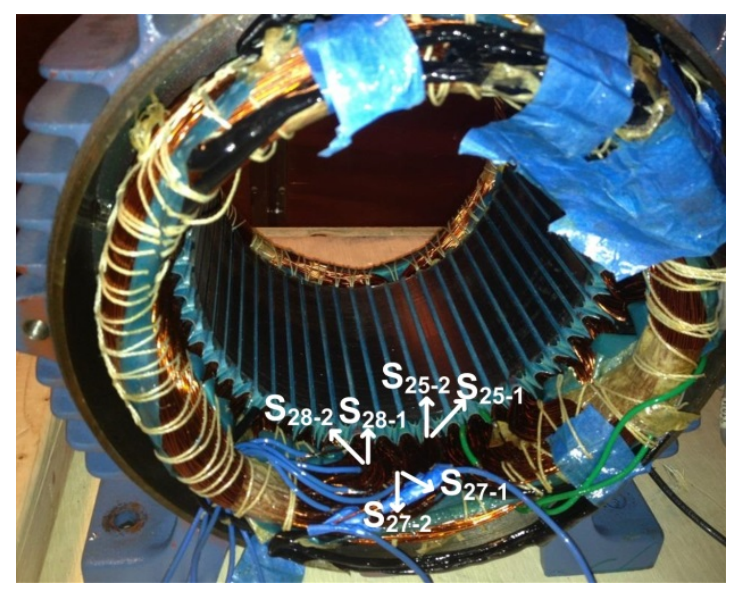

Fig. 9.10 The selected six points in the winding of phase A for the interturn short-circuit. Note that the displayed terms such as S25-1 means the turn number 1 in the 25 th slot

Based on the optimization types of the antenna, the interturn short-circuit study is classified into two types. This classification is due to the constraints of the particular applications in which the detection may be used. This is explained in 9.4.1 and 9.4.2.

\subsubsection{Singular-optimum location of antenna}

In this section, the mentioned three interturn short-circuits in phase A are compared with each other in different possible situations of antenna to find the optimum location with the hints to detect the location of the short-circuit.

It's not possible in some of the industrial applications to get near to the motor to place the antenna in variable angles versus the motor shaft. In other words, it's just possible to have the antenna(s) at a fixed location. Hence, a particular optimum location of the antenna can be obtained, which at least should be attained for each phase.

The radiated $\mathrm{H}$-field is perpendicular to the passing current. Therefore, the antenna shows the maximum sensitivity of the position where is perpendicular to the short-circuit coil. This is a good hint to find out the short-circuited turn; however the turns of each coil 
are so tightened together, so it's not possible to detect the short-circuited turns just by using this hint. Therefore, the optimum location of antenna is used here for detecting to the level of faulty coil. For detecting more precisely, the frequency response at the higher harmonic orders around $0.5-2 \mathrm{kHz}$ should be investigated.

The index in finding the most sensitive location of the antenna is the number of harmonic orders, which varies compared with the normal case. The location of antenna with more changes in the frequency response is considered as the most sensitive or optimum location. When the optimum location of the antenna is identified, the frequency response would be investigated.

In order to find the main hint in identifying the location of the fault, the physical theory of short-circuit is studied. By implementing interturn short-circuit, a new loop of current is created, which radiates a new field. This leads to the appearance of a new peak at a specific frequency and/or change of the amplitude of an existed peak. This change can be a decrease or an increase based on the direction of the passing current in the shortcircuited turns. If it is in the same direction as the main coil, the change would be an increase. Otherwise, it would be a decrease. This is a very helpful hint in finding the location of the fault. More details about the theory of the interturn short-circuit is explained in [256].

The short-circuit at the three mentioned locations of the fig. 9.10 is implemented from zero to 180 degrees of $\alpha$ around the machine as shown in the fig. 9.11. 


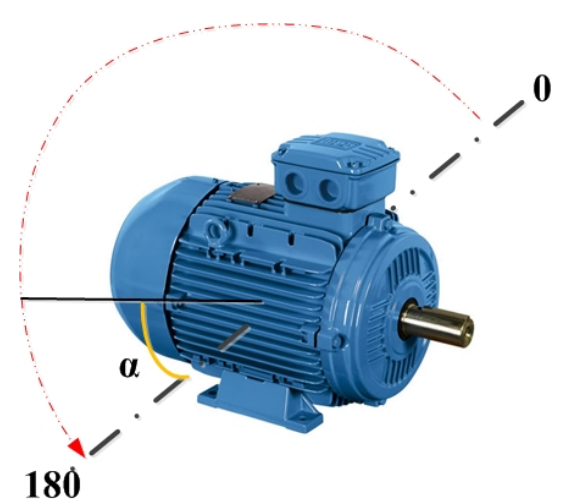

Fig. 9.11 The rotation angle of the magnetic antenna around the machine

Fig. 9.12 shows the radiated $\mathrm{H}$-field of the motor while interturn short-circuit implemented between $S_{28-1}$ and $S_{28-2}$ and compared with the healthy condition at $\alpha=90^{\circ}$. The distance of the antenna from the motor is $7 \mathrm{~cm}$ from the casing. Since the differences in the figure is not so recognizable for comparing the faulty cases, the $\Delta \mathrm{H}=\mathrm{H}_{\text {Short-circuit }}{ }^{-}$ $\mathrm{H}_{\text {Healthy }}$ is obtained. For brevity, only the results of two selected $\alpha$ are shown in fig. 9.13 $\left(\alpha=90^{\circ}\right)$ and fig. $9.14\left(\alpha=60^{\circ}\right)$.

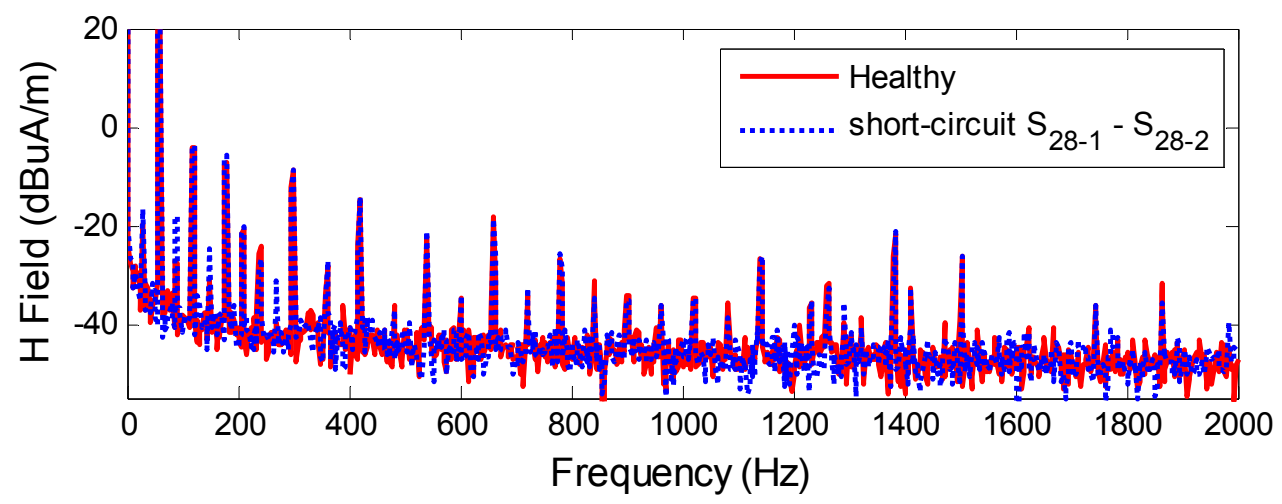

Fig. 9.12 Radiated H-field of the interturn short-circuit between S28-1 - S28-2 and normal case at $\alpha=90^{\circ}(\mathrm{dB} \mu \mathrm{A} / \mathrm{m})$ 

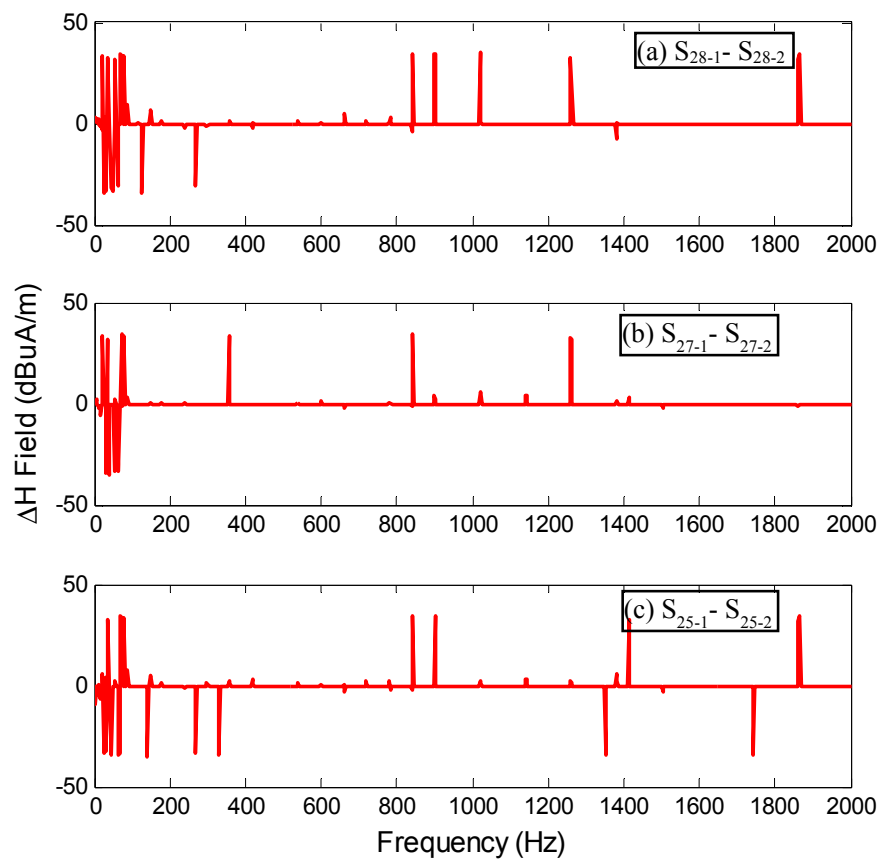

Fig. 9.13 Difference of the Radiated H-field of the 3 interturn short-circuit cases and normal case at $\alpha=90^{\circ}(\mathrm{dB} \mu \mathrm{A} / \mathrm{m})$, (a): interturn short-circuit between S28-1-S28-2, (b): interturn short-circuit between S27-1-S27-2, (c): interturn short-circuit between S25-1-S
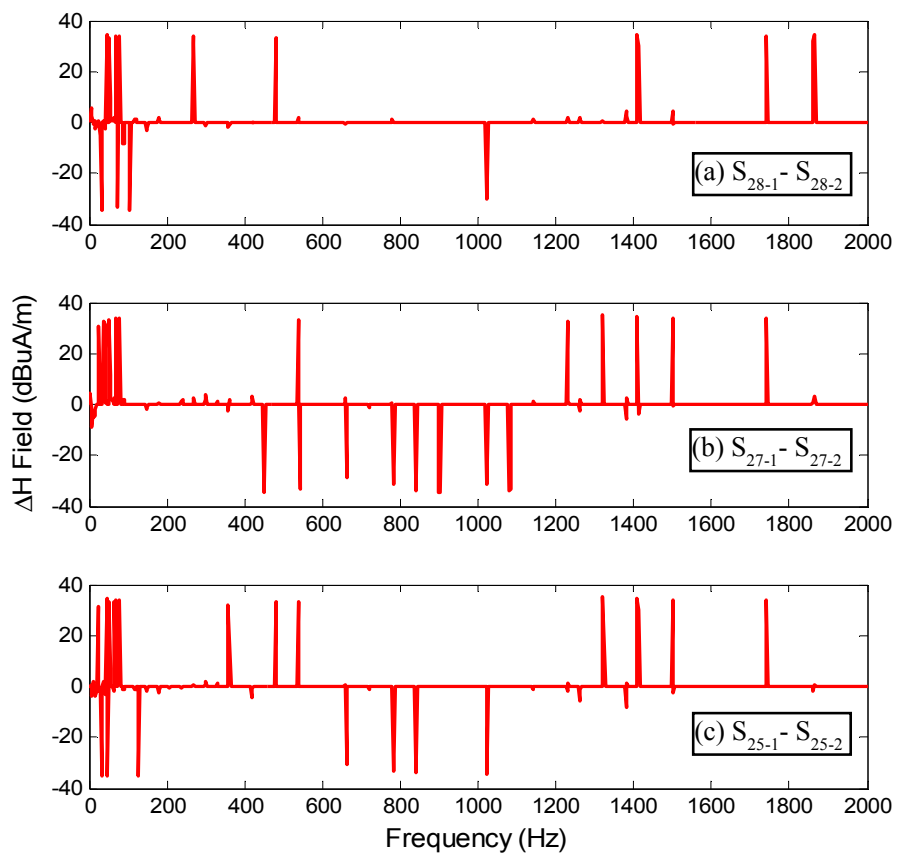

Fig. 9.14 Comparison of the Radiated H-field of the 3 interturn short-circuit cases at $\alpha=60^{\circ}(\mathrm{dB} \mu \mathrm{A} / \mathrm{m}),(\mathrm{a})$ : interturn short-circuit between S28-1-S28-2, (b): interturn shortcircuit between S27-1-S27-2, (c): interturn short-circuit between S25-1-S25-2 
Comparing these two figures, the number of peaks in fig. $9.14(\alpha=60)$ is more than the other one for all cases; however, the results at $\alpha=90^{\circ}$ is also helpful in fault location. By measuring all $\alpha$ angles, the most sensitive $\alpha$ for all three mentioned faults is $60^{\circ}$. The most sensitive $\alpha$ for each particular case could be slightly different, but the singular angle for the applications in which it's not possible to have the several antennas around the machine is more applicable.

The next step is finding the faulty coil in which the short-circuit occurred, based on the $\alpha$ angle. Considering the schematic of the stator winding in fig. 9.15, the perpendicular passing current to the $\mathrm{H}$ - field at $\alpha=60^{\circ}$ is through winding $\mathrm{A}_{2}$ of phase $\mathrm{A}$, which is correct.

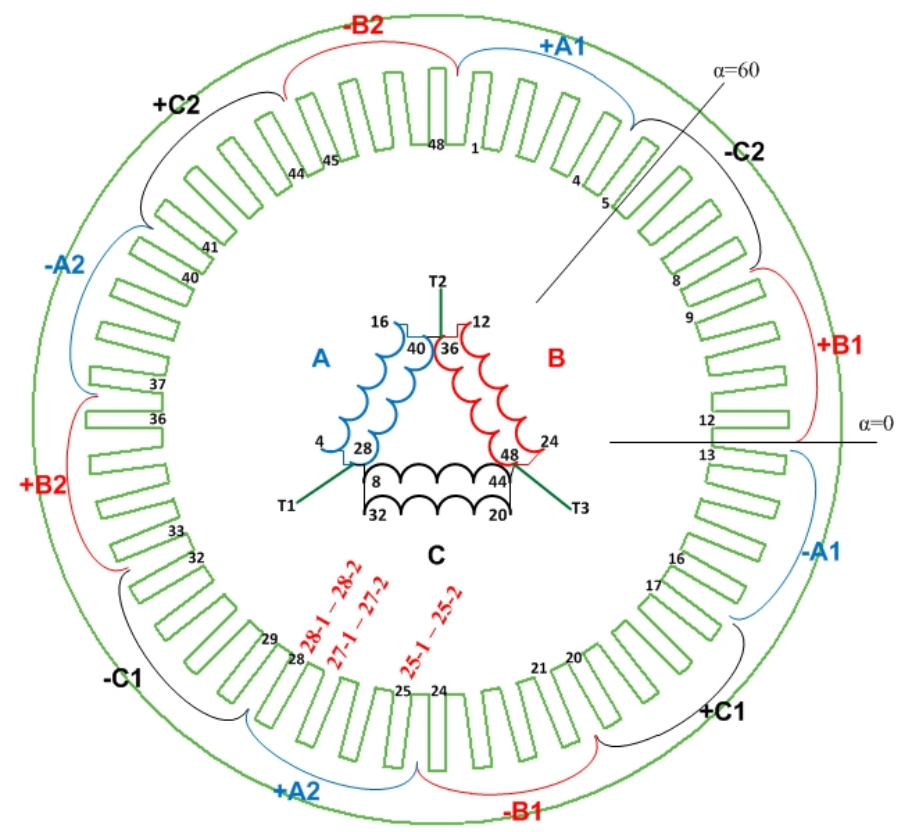

Fig. 9.15 The schematic of the stator winding

After identifying the faulty coil, the location of short-circuit can be detected by investigating the peaks of fig. 9.14 (a) -(c). Comparing fig. 9.14 (b) with (a) and (c), the 
number of peaks is more when short-circuit is between $S_{27-1}$ and $S_{27-2}$. This means that the impact of short-circuit, occurred in the middle of the winding, is more than the two ends. Furthermore, the number of peaks when short-circuit is between $S_{25-1}$ and $S_{25-2}$ is more than the one at the terminal end $\left(\mathrm{S}_{28-1}\right.$ and $\left.\mathrm{S}_{28-2}\right)$. Therefore, the effect of the interturn short-circuit in the other end of the winding is more than the terminal end. In all three cases, some peaks are created in about $1400 \mathrm{~Hz}$ and some peaks are diminished in about $1000 \mathrm{~Hz}$. However, the number of peaks created or diminished in this area is different in these cases. Considering all of these hints, the location of short-circuits can be identified.

As the conclusion for the related application of this section, it's suggested to locate three antennas with $120^{\circ}$ difference to be able to monitor the three phases of the winding continuously. Certainly, the fixed location of the antenna is more sensitive for some faults and less for some others, based on the angle of antenna perpendicular to the fault location. For these cases, it's possible to find the location of fault by focusing on the location of the peaks along the frequency.

\subsubsection{Multi-optimum locations of antenna}

In opposite to the previous section, there is no limit in many applications for changing the location of antennas around the machine. Therefore, it's possible to have the optimum location of the antenna for each individual interturn short-circuit. Consequently, the location of the fault can be identified more precisely.

The same faults as part 9.4.1 are tested in this section. However, each of the three interturn short-circuits are tested from $\alpha=0^{\circ}$ to $\alpha=180^{\circ}$. As the result, optimum location of the antenna is obtained for each case demonstrated in fig. 9.16 (a) -(c). The optimum 
distance of the antenna from the casing is $7 \mathrm{~cm}$, similar to the previous part. The optimum $\alpha$ for the fault at $S_{28-1}-S_{28-2}$ obtained $45^{\circ}$, while the ideal $\alpha$ for the faults at $S_{28-1}-S_{28-2}$ and $\mathrm{S}_{28-2-}-\mathrm{S}_{28-3}$ are achieved $40^{\circ}$ and $30^{\circ}$, respectively. Note that, this situation of antenna is more sensitive than the others, therefore the numerous numbers of peaks shows up which makes the study hard. Accordingly, the small peaks are cancelled.
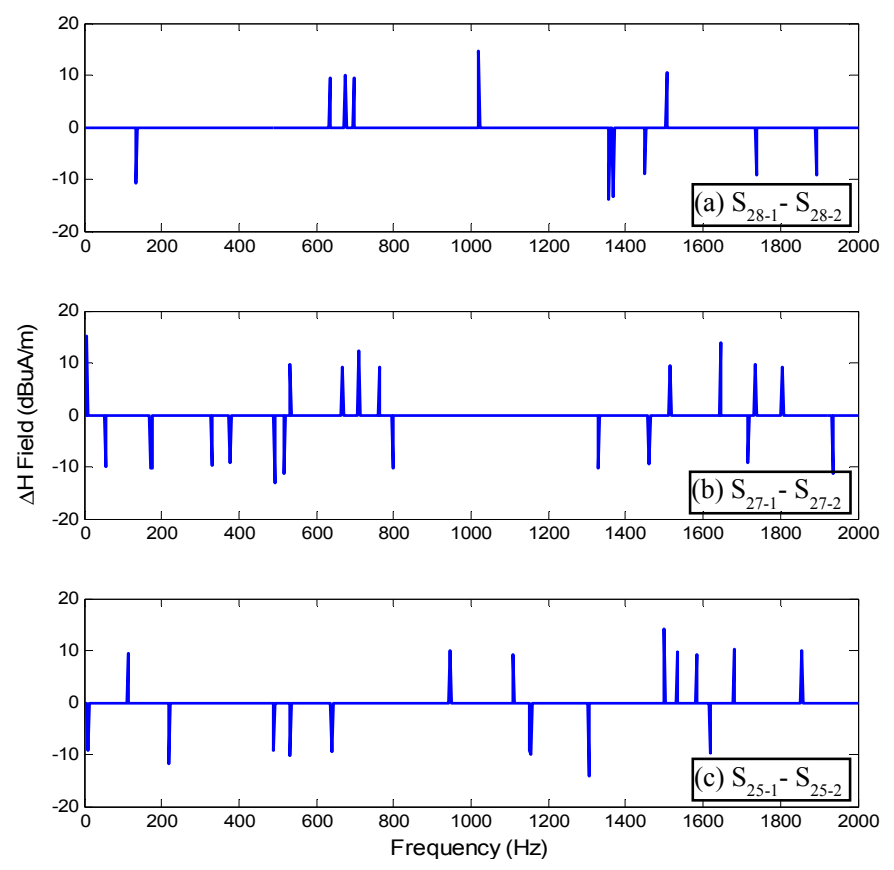

Fig. 9.16 Comparison of the Radiated $\mathrm{H}$-field of the 3 interturn short-circuit cases $(\mathrm{dB} \mu \mathrm{A} / \mathrm{m})$, (a): interturn short-circuit between S28-1-S28-2 at $\alpha=45^{\circ}$ (b): interturn shortcircuit between S27-1-S27-2 at $\alpha=40^{\circ}$ (c): interturn short-circuit between S25-1-S25-2 at $\alpha$

Comparing fig. 9.16 (b) with (a) and (c), short-circuit between $\mathrm{S}_{27-1}$ and $\mathrm{S}_{27-2}$, which is in the middle of the coil, has more effect on the frequency response due to the number of creation and dissipation of the peaks. Also, short-circuit near to the other end $\left(\mathrm{S}_{25-1}-\mathrm{S}_{25-2}\right)$ has more peaks in the frequency response comparing with the one near to the terminal 
end $\left(\mathrm{S}_{28-1}-\mathrm{S}_{28-2}\right)$. Consequently, the inferences of the section 9.4.1 are proven. To detect the location of the interturn short-circuit, unique changes of each response should be considered. As mentioned in section 9.4.1, by occurring the interturn short-circuit, a new current pass and subsequently a new radiated $\mathrm{H}$-field are generated. Therefore, new peaks in the frequency response appear. The location of the peak along the frequency response helps in finding the location of the fault. For example, for the fault at $S_{28-1}-S_{28-2}$, the peaks at 11 th harmonic order $(660 \mathrm{~Hz})$ and 17 th harmonic order $(1020 \mathrm{~Hz})$ arise. These peaks didn't appear in the two other faults. On the other hand, for the fault at $\mathrm{S}_{27-1}-\mathrm{S}_{27-2}$, some sub-harmonics between 11th and 13th harmonic orders $(660 \mathrm{~Hz}-780 \mathrm{~Hz})$ come out. Also some others showed up between 23 th and 31 th harmonic orders $(1380 \mathrm{~Hz}-1860 \mathrm{~Hz})$. For the fault at $\mathrm{S}_{25-1}-\mathrm{S}_{25-2}$, dissimilar to the other two faults, the peaks appeared between 15 th and 21th harmonic orders $(900 \mathrm{~Hz}-1260 \mathrm{~Hz})$. These hints are helpful in finding out the location of the fault. Note that appearing these peaks has also the effect on the low harmonic orders (3th-7th), but theses impacts are small; therefore, they cannot be helpful in finding out the location of faults and cancelled out.

\subsection{Conclusion}

Identification of the type and location of short-circuits are implemented in this chapter. Hence, the analysis of radiated magnetic field intensity was achieved to differentiate the major faults, such as intercoil short-circuit, from the minor ones, such as interturn shortcircuit. An experimental setup was devised for testing and verifying the developed condition monitoring process using the magnetic field signature. The intercoil shortcircuit was implemented and studied experimentally. Since the intercoil short-circuit 
might damage the winding, a F3DFE model was developed and connected to the circuitbased software. The simulation results were verified experimentally. For the interturn short-circuit, two different optimizations for finding the most sensitive location of the antenna were considered, based on industrial constraint. The optimum rotation angle of the antenna $(\alpha)$, as well as the distance to the machine's casing, were obtained and used for identifying fault location. The number of peaks in the field signature was used as part of the information for distinguishing the faults. The location of the created or diminished harmonic or sub-harmonic orders along the frequency were considered as the final information in locating the interturn short-circuits. This type of fault diagnosis is nondestructive and has the ability to be applied, while the motor is running. Moreover, it has excellent accuracy without a need to add any sensor inside the motor. 


\section{Power Source Condition Monitoring Based On the Electromagnetic Signatures}

\subsection{Overview}

The monitoring and fault detection of typical power components through the stray fields are proposed in this chapter. The frequency response of stray electromagnetic field from the components is measured through low-frequency antennas and signal analyzer. The proper algorithms are developed for the identification of the components, as well as the detection of some faults and failures. These algorithms are designed based on the active performance of the components including induction, DC and synchronous machines and converter. More than 170 tests of various combinations of the components and their conditions are implemented to fulfill this identification. The results show that the propose technique performs a reliable monitoring the components. Since there is no need to dismantle the components to detect the faults in this method, it can be considered nondestructive for the industry. Moreover, this method can be used for the applications in which it's impossible to access the parameters of the components for online monitoring.

Modern technology promotes using power electronics in the power system, which has become more and more popular in recent years. Their electrical systems are becoming increasingly complex, as they are composed of microprocessor circuits, power delivery networks, safety control circuits, communication circuits, snubber circuits, sensors, drives, motors, electronic ignitions, and more. These components, which many of them are nonlinear, could create numerous harmonic currents to downgrade the power quality. Harmonic distortions cause problems, such as equipment overheating, motor failures, mis-operation of protective equipment, inaccurate metering, and sometimes interference 
with communication circuits. To ensure the power quality now and in a future deregulated industry, the harmonic source detection is important [117]. In addition to harmful harmonics, the inter-harmonics sources are more troublesome and harmful than harmonics. Moreover, the typical problems caused by harmonics, such as overheating and useful life reduction, inter-harmonics create some new problems, such as subsynchronous oscillations, voltage fluctuations, and light flicker, even for low-amplitude levels [111]. Inter-harmonics can be observed in an increasing number of components, such as static frequency converters, cycloconverters, sub-synchronous converter cascades, adjustable speed drives for induction or synchronous machines, arc furnaces, and all loads not pulsating synchronously with the fundamental power system frequency. In addition to all these components, the partial failures, such as inter-turn short-circuits, cause the presence of inter-harmonics. The detection of the inter-harmonic polluters has also become a concern, as a utility company has the responsibility to provide customers with a power supply of a fixed quality. Identifying the inter-harmonic sources leads to the illustration of the power quality problem, and further mitigation process can be applied. To accelerate the mitigation process, practical and reliable methods for identifying the harmonic and inter-harmonic polluters are required. Many researches have been implemented in characterization and detection of the source of harmonics and interharmonics [111]-[115]. In [111], the principle of the harmonic and inter-harmonic is discussed and the proposed model is suggested. Then, the inter-harmonic assessment is considered with particular attention to the problem of the frequency resolution and of the computational burden associated with the analysis of periodic steady-state waveforms. Finally, the modeling of different kinds of inter-harmonic sources and the extension of 
the classical models developed for power system harmonic analysis to include interharmonics are discussed. The modeling for assessment of the sources is discussed more in [112]. A new algorithm termed hybrid blind source separation is used for the localization. Although, the application of this localization is for high frequency components, identifying and locating the sources on the basis of predicted measures, which are used in this research, can be inferred and used for the low-frequency components. Some literature, such as [113], [114], focused on the improving the measurement and extraction techniques to enhance the investigation of harmonics and inter-harmonics. The enhancements are in about higher degree of accuracy, structural/performance robustness, and frequency adaptivity. In [115], simulation and experimental study of the identification of the inter-harmonic source location in power systems are implemented. The method is based on the inter-harmonic impedances measuring at the metering points and comparing them with the harmonics of the utility system. The main idea behind this method is that the inter-harmonic impedance of the system is much smaller than that of an inter-harmonic-generating load.

In addition to the inter-harmonic, some other methods are used for source identification. In [116], an approximate technique is proposed for the reconstruction of magnetic-field distribution in the proximity of unknown sources based on two nested optimization algorithm. Moreover, in [117], the cascade correlation network is used for the harmonic source detection. The current-injection-based harmonic power flow was used to calculate bus voltages and total harmonic distortion. D. Srinivasan et al [118] proposes a neural-network (NN)-based approach to the nonintrusive harmonic source identification. In this approach, NNs are trained to extract important features from the 
input current waveform to uniquely identify various types of devices using their distinct harmonic "signatures." The identification is particularly implemented, based on the measurement of current at the incoming supply point and comparing the magnitude of different components from home appliances to computer apparatuses and power components.

In this chapter, the identification of the power components, such as induction motor, DC motor, synchronous generator and power converter is proposed. The magnetic and electric signatures are observed in several distances in different conditions. The components are monitored in healthy and faulty conditions. Several cases of short-circuit in the machine's winding as well as the unbalanced currents to the system, are applied to the machines and converter to differentiate the healthy and faulty components. Moreover, many other conditions are studied. The procedure of the identification method is discussed in section 10.2. The test procedure and different case studies are mentioned in section 10.3. Finally, the fault diagnosis and detection of converter requires specific strategies which are investigated in 10.4 .

\subsection{Power component monitoring procedure}

The recognition is based on nonintrusive electromagnetic signature identification, without human intervention. The harmonic components of the stray electric and magnetic fields are measured as the sources of valuable information for electromagnetic signature identification. Then, a processing network was trained to identify the devices from which the harmonics strayed. The overall typical scheme of the studied system with the procedure of the recognition is shown in fig. 10.1. As shown, the proposed components 
consist of the power converter and typical rotating machines such as DC motor, induction motor and synchronous generator.

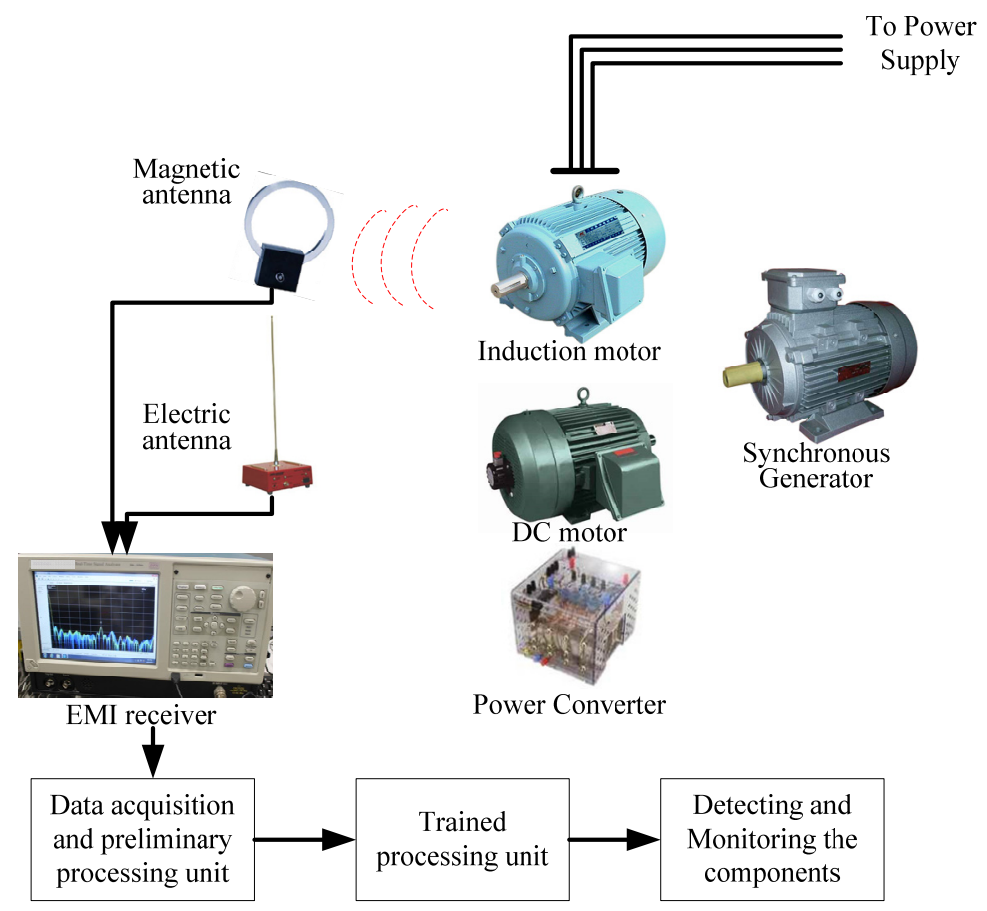

Fig. 10.1 Scheme of the system and identification procedure

The monitoring process is implemented in the following steps. The procedure of these processes is explained in details in the following sections.

\subsubsection{Data acquisition and preliminary processing unit}

In order to analyze the electromagnetic signatures, which show the characteristics of the components, the strayed magnetic or electric fields are measured using the magnetic coil and the electric rod antennas. These specific antennas can capture low-frequencies down to $30 \mathrm{~Hz}$, so the power frequencies are covered. The electric rod antenna is used for the most component identifications and healthy and faulty components detection, because it can capture the electric stray fields at far distances. On the other hand, the magnetic antenna doesn't capture the magnetic stray further than $30-40 \mathrm{~cm}$, which is because of the 
weakness of the stray fields in far distances. Therefore, the magnetic coil antenna is used for more specific investigation, such as inter-turn short-circuits.

After capturing the fields with the antennas, the data are sent to the EMI receiver. The technical details of the measurement devices are mentioned in the next section. The frequency responses of the stray fields are monitored through the EMI receiver. Then, these data are sent to the data acquisition unit for further processing.

The acquired frequency response consists of the main harmonic orders of the stray fields and the inter-harmonics in between the main harmonics. The main harmonics and critical sub-harmonic orders are picked up for the comparison between cases. The attained data are used in the trained processing unit.

\subsubsection{Trained processing unit}

In order to investigate the stray harmonic and sub-harmonic orders, the physics of them in the machines and converters should be investigated. The comprehensive study about the origin and details of the harmonic sources including machines, transformer, converters and other components are explained in [257], [258]. The related discussion of the harmonics produced by the components is expressed in the following.

The speed of the synchronous rotating field of the stator of an induction motor is the fundamental frequency times the wavelength, i.e. $f_{1} \lambda$. For a slip s, the rotor speed is thus $f_{1} \lambda(1-s)$ and the frequency of the rotor currents $s f_{1}$.

Time harmonics are produced by induction motors as a result of the harmonic content of the field and m.m.f. distribution and are speed dependent. 
A harmonic of order $\mathrm{n}$ in the rotor m.m.f. (i) has a wavelength $\lambda / \mathrm{n}$; (ii) travels at a speed $\pm(s f) \lambda / n$ with respect to the rotor; and (iii) travels at a speed $f \lambda(1-s) \pm(s f) \lambda / n$ with respect to the stator.

The harmonic respect to the stator induces an e.m.f. in the stator at a frequency equal to the ratio speed/wavelength, i.e.

$$
f_{i i i}^{n}=\frac{f \lambda(1-s) \pm s f(\lambda / n)}{\lambda / n}=f(n-s(n \pm 1))
$$

The positive sign of the harmonic is due to the opposite direction of the rotor m.m.f to the fundamental. If the electrical asymmetry due to the fault or unbalanced current or any other abnormal reasons occur, the positive and negative phase sequence currents will flow, giving field forward and reverse directions. The induced field due to these asymmetry travels at speed $\pm s f \lambda$ with respect to the asymmetric part, which can be stator or rotor. The induced frequencies by these fields could be $f$ and $(1-2 s) f$. These are the indices in the investigation of the frequency response to detect the faults or abnormal conditions of the induction machines.

The harmonic behavior of the power converter as the purpose of speed drive, pulse width modulation-adjustable speed drives (PWM-ASD), is different in the DC - link, supply side, and output side [259]. Therefore, each of them should be considered for the diagnosis purpose. For the DC link, the harmonic is:

$$
f_{h d c}^{i}\left(m_{f}, j, r\right)=h_{d c}\left(m_{f}, j, r\right) \cdot f_{o}=\left|m_{f} j \pm r\right| \cdot f_{o}
$$

where $j$ is corresponds to the harmonic orders of the inverter; while, $\mathrm{j}$ and $\mathrm{r}$ integers depend on the modulation ratio [259]. The $f_{o}$ is the output frequency. The dependency 
from modulation ratio $(\mathrm{mf})$ is related to the switching strategy. For the supply side, the harmonic is:

$f_{h s}^{i}(v)=h_{s}(v) \cdot f_{s}=\left|(v-1) q_{s} \pm 1\right| \cdot f_{s} \quad v=1,2,3, \ldots$

where $h_{s}$ is the order of the supply side harmonic and $v$ corresponds to the harmonic order of the rectifier. The $f_{o}$ is the output frequency. The $q_{s}$ is the rectifier number of pulses. Finally, the harmonic for the output side is:

$f_{h o}^{i}\left(m_{f}, j, k\right)=h_{o}\left(m_{f}, j, k\right) \cdot f_{o}=\left|m_{f} j \pm k\right| \cdot f_{o}$

where $h_{s}$ is the order of the supply side harmonic and $k$ corresponds to the harmonic order of the output side [260].

The harmonics in DC machines, as well as synchronous generators are characteristics. The harmonics radiated from the DC motors are mainly from the winding of the commutation side, which creates high frequency harmonics due to the presence of the brushes. As the current to the rotor coils is frequently connected and disconnected to the DC source through the commutator segments, arcing at the brushes is produced as a result of the periodic interruption of the current in the rotor coils (inductors). This arcing has a spectral content [219]. Similarly, the excitation part of the synchronous generator produces the harmonic orders in addition to the harmonic orders of the power frequency. The harmonic contents of the synchronous generator are studied in [261].

All of the aforementioned hints help in the differentiation of the components, as well as the recognition of their normal or faulty condition. This process is considered as the detection unit, which is discussed in the next part. 


\subsubsection{Detecting and monitoring unit}

After importing the radiated field and extracting the harmonic orders, the harmonics are investigated based on the physics of the components as discussed in section 10.2.2 and the identification of the type of component is implemented. The flowchart of this unit is shown in fig. 10.2. 


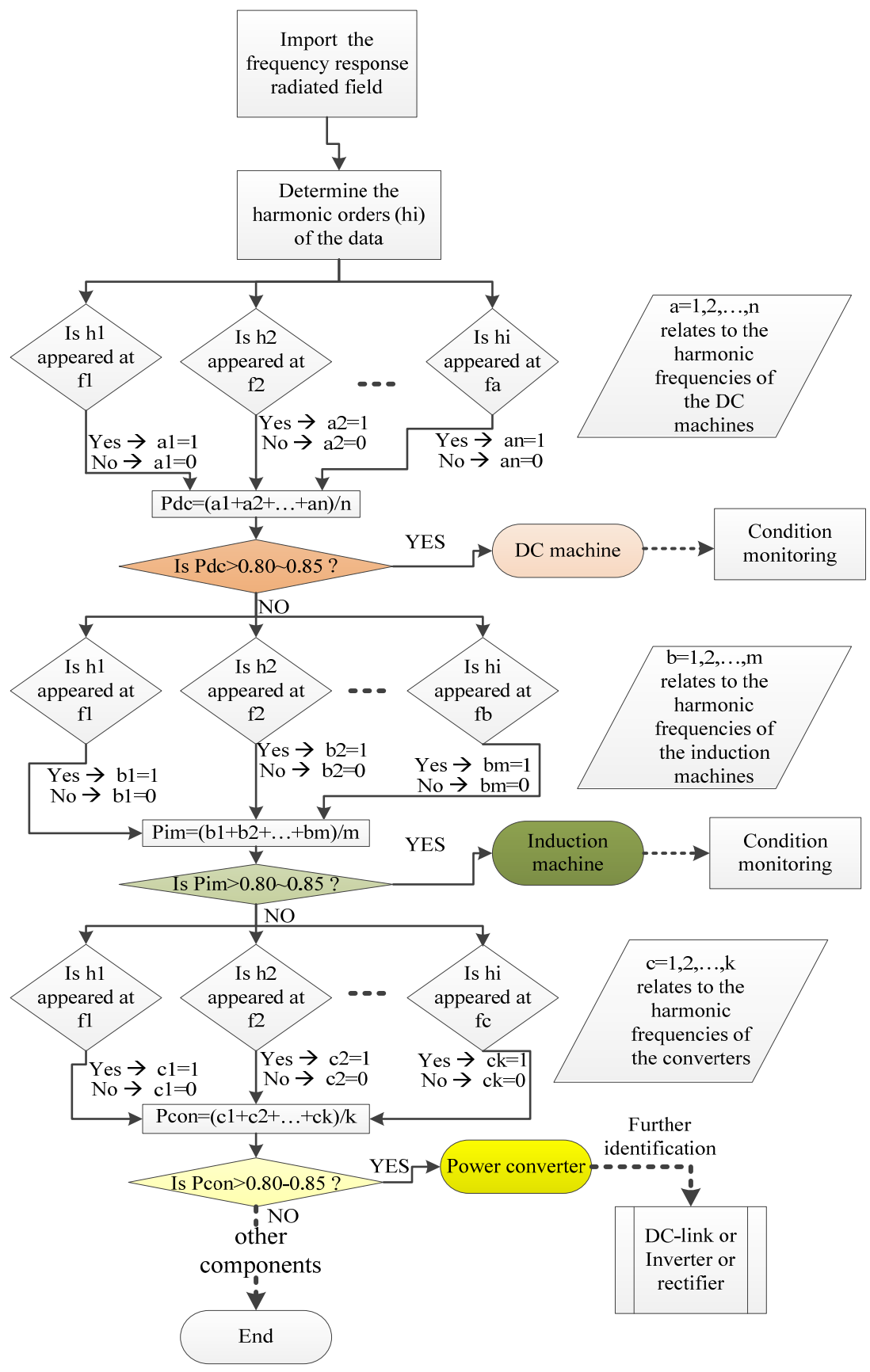

Fig. 10.2 Flowchart of the component identification

The processing unit of each decision block in fig. 10.2 was trained based on the characteristics of the mentioned component. The $f a$ frequencies, in which the harmonic orders of the DC motor (hi) appear, can be considered from the first to the higher orders (31th). Similarly $f b$ for the induction motor, $f_{c}$ for the converter and the other frequencies 
for synchronous generator and other components can be considered. It is mentioned in the main decision blocks, shown in colors, that if more than $80 \sim 85 \%$ of the harmonic orders were located in the right (expected) frequencies; then, the component would be identified. The mentioned percentage $(\mathrm{P})$ is because of the possible failures or abnormal condition of the proposed components. That is to say, the occurrence of short-circuits or unbalanced current may lead to the appearance of a new harmonic order or disappearance of an usual harmonic order. Therefore, some harmonic order may be missed and $100 \%$ of the harmonic orders may not match the expectance. The $80-85 \%$ number is obtained based on the experience. The percentage $(\mathrm{P})$ varies between 80 and 85 due to the power level of the machine and the distance of the antenna to the machine.

Since the converter can consist of the DC-link, inverter, and rectifier, further identification of the specific part can be employed by considering the related equations (10-2)-(10-4). Identifying the other components is also possible by locating their charts in the continuation of the main flowchart. So the extension of main flowchart is based on the number of available components in the system.

After detecting the type of the components, the condition monitoring in order to find out any failures or faults was also considered. The related flowchart is shown in fig. 10.3. As shown in the figure, two symptoms are considered for diagnosing the faults. The first one is the percentage of "the harmonic orders located in the expected frequencies" (P) and the second one is the difference of the amplitude of harmonic orders in comparison with the healthy one $\left(A_{h i}\right)$. Both indices are defined based on the characteristics of the components. If any component meets both indices, the component is healthy; otherwise, it's faulty. The type and severity of the fault can be identified by further analysis by 
looking at the level of deviation from the healthy condition. This can be achieved by analyzing the data using signal processing techniques.

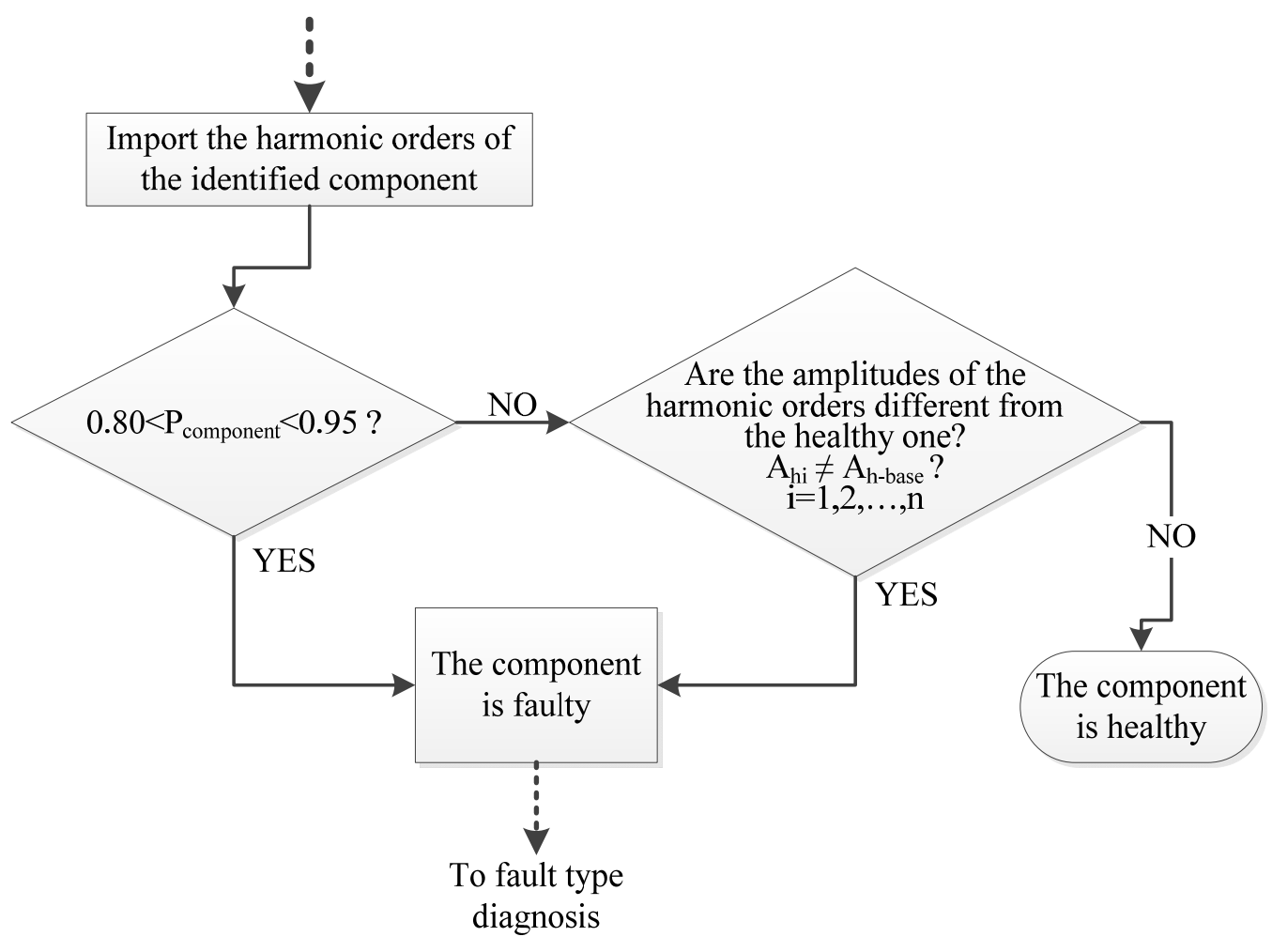

Fig. 10.3 Flowchart of the component identification

\subsection{Test implementation and case studies}

Following the explanation of the basics and procedure of the study, the setups were implemented through various case studies.

The characteristics of the equipment used in the test through the different case studies are mentioned in Table 10.1.

Table 10.1 The characteristic of the components

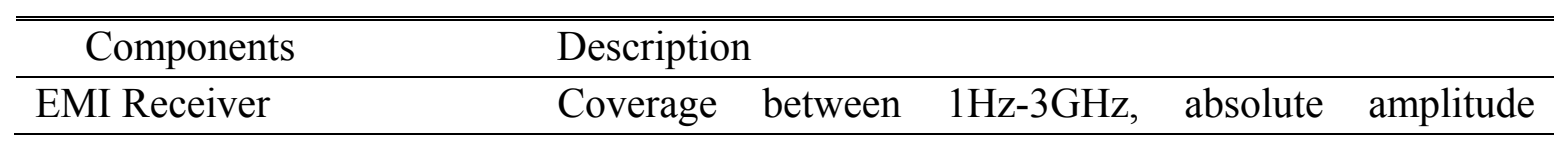




\begin{tabular}{|c|c|}
\hline /spectrum analyzer* & $\begin{array}{l}\text { accuracy: } \pm 0.5 \mathrm{~dB} \text { to } 3 \mathrm{GHz} \text {, displayed average noise level: - } \\
142 \mathrm{dBm} / \mathrm{Hz} \text { at } 26.5 \mathrm{GHz},-155 \mathrm{dBm} / \mathrm{Hz} \text { at } 2 \mathrm{GHz} \text { and }- \\
150 \mathrm{dBm} / \mathrm{Hz} \text { at } 10 \mathrm{kHz} \text {. }\end{array}$ \\
\hline Electric rod antenna* & $\begin{array}{l}\text { Active monopole antenna, Coverage between } 30 \mathrm{~Hz}-50 \\
\mathrm{MHz} \text {, impedance: } 50 \Omega \text {. }\end{array}$ \\
\hline Magnetic coil antenna* & $\begin{array}{l}\text { Coverage between } 20 \mathrm{~Hz}-500 \mathrm{kHz}, 36 \text { turns of } 7-41 \text { litz } \\
\text { wire shielded with } 10-\mathrm{Ohms} \text { resistance and } 340 \mu \mathrm{H} \\
\text { inductance. }\end{array}$ \\
\hline $\begin{array}{l}\text { Synchronous } \\
\text { Generator (GEN) }\end{array}$ & $\begin{array}{l}13.8 \mathrm{kVA}, 3 \text { phase, } 60 \mathrm{~Hz}, 208-\mathrm{V}, 1750-\mathrm{RPM} \text {, Vexc: } 37- \\
\text { V. Iexc: } 1.9-\mathrm{A} \text {. }\end{array}$ \\
\hline Induction motor (IM1) & 7.5-HP, 208-V, 1765-RPM, PF: 0.82, 60Hz, EFF: 89.5\%. \\
\hline Induction motor (IM2) & 10-HP, 208-V, 1760-RPM, PF: 0.83, 60Hz, EFF: $91.7 \%$. \\
\hline Induction motor (IM3) & 3-HP, 208-V, 1740-RPM, PF 0.76, 60Hz, EFF: 87.5\%. \\
\hline DC motor (DC) & 2-HP, 208-V, 1750-RPM, Varm: 180-V, Vf: 200/100-V. \\
\hline $\begin{array}{l}\text { Multifunctional } \\
\text { Converter }\end{array}$ & $\begin{array}{l}\text { B6CI, with IGBT switches, Switching algorithm: SVM, I: } \\
20 \sim 1500 \text {-A, VCES: } 600,1200,1700-\mathrm{V}\end{array}$ \\
\hline
\end{tabular}

* Measurement components are MIL-STD 461F standard compliant.

The case studies are designed in a way to monitor various examples of power system setups and they are set from the simplest setup to the setup with the most components. The case studies are implemented in the following six steps:

Step1: IM2 (10-HP induction motor) with and without mechanical load

Step2: IM1 (7.5-HP induction motor) and IM2 (10-HP induction motor) at different locations of antenna

Step3: IM1 (faulty: intercoil short-circuit), IM2 (healthy) at different locations of antenna

Step4: IM1 (unbalanced current, healthy), IM2 (unbalanced current, healthy) at different locations of antenna

Step5: Converter connected to the IM1 at different switching frequencies at different locations of antenna with and without fault 
Step6: Synchronous Generator connected to the DC motor and IM3 (3-HP induction motor) at different locations of antenna

\subsubsection{Step 1: IM2 with and without mechanical load}

As the first case, the 10-HP induction motor is tested with and without load to figure out the effect of mechanical load on the electromagnetic signatures. The purpose of this step is to investigate the effect of the mechanical load on the harmonic orders.

The test is employed at $180 \mathrm{~V}$ with three different loads (0-NM, 3-NM, 10-NM).

As mentioned in the procedure, the harmonic and inter-harmonic orders of these three cases are extracted and shown in fig. 10.4 and fig. 10.5, respectively.

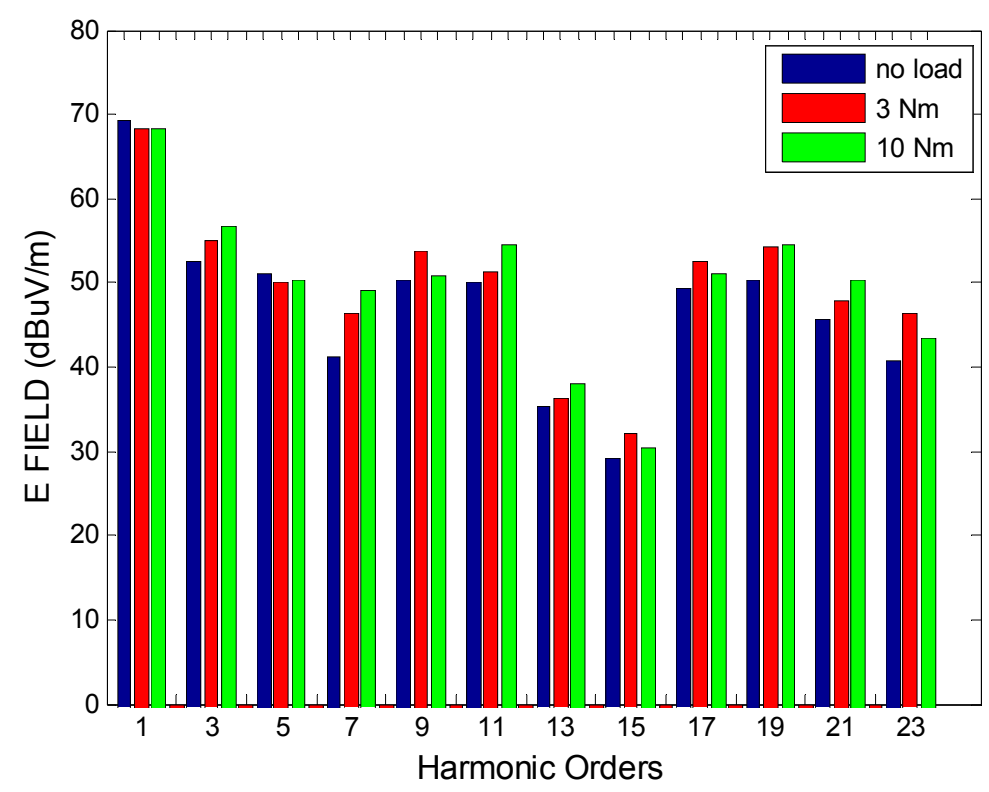

Fig. 10.4 Electric stray field of the step 1 in $\mathrm{dB} \mu \mathrm{V} / \mathrm{m}$ (main harmonic orders)

As shown in the fig. 10.4, the harmonic orders of the electric stray field (E-field) slightly increase, while the load is connected. However, the harmonic orders don't change orderly by increasing the amount of load. Therefore, for identifying the motor with load from the motor without load, the study of inter-harmonics is essential. 
The inter-harmonics of the setup of step1 are shown in fig. 10.5. The main frequency of the setup is $60 \mathrm{~Hz}$ and the machine $\left(\mathrm{IM}_{2}\right)$ is 4 pole. Based on the fact that the mechanical frequency is (2/number of Pole) times the electrical frequency, the mechanical frequency is $30 \mathrm{~Hz}$. Hence, the convolution of the mechanical frequency and electrical frequency yields the inter-harmonics at $90 \mathrm{~Hz}, 210 \mathrm{~Hz}$ and so forth, which are the 1.5th, 3.5th harmonic orders of the main frequency, respectively. As shown in fig. 10.5, the inter-harmonic orders show more changes by applying the mechanical load. Therefore, the algorithm for monitoring the machine with load can be designed in a way that the inter-harmonics compare with and without load. The amplitude of inter-harmonic orders of the machine with load is more than the one without load. Identifying the machine with load helps in the monitoring of the system with several machines to diagnose the faults.

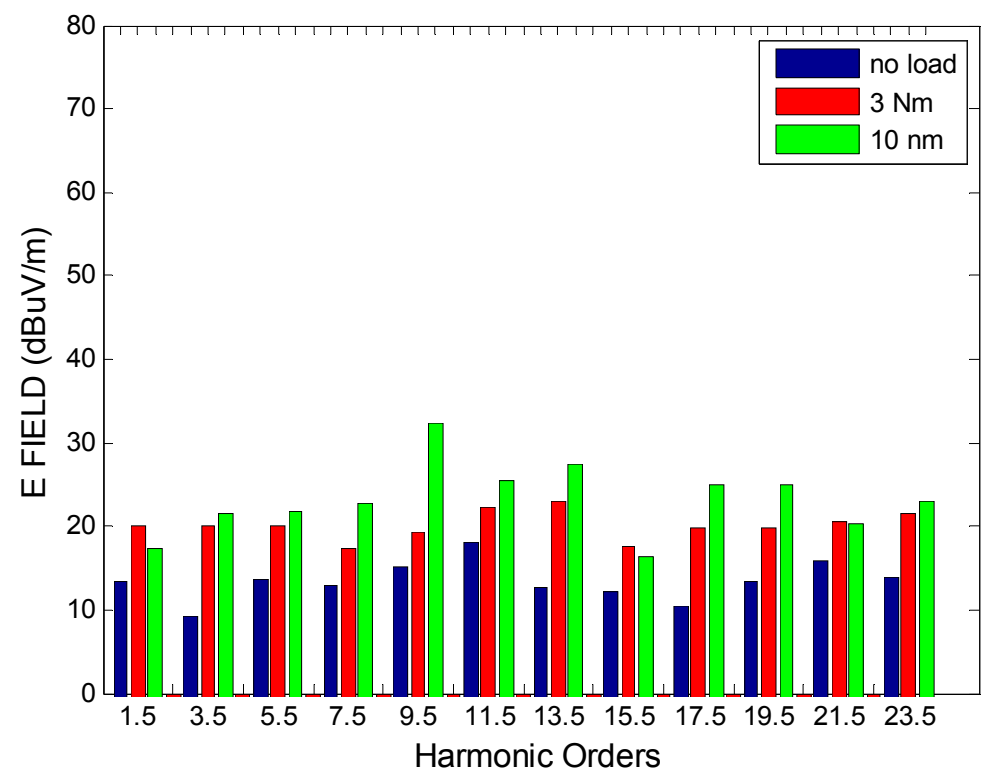

Fig. 10.5 Electric stray field of the step 1 in $\mathrm{dB} \mu \mathrm{V} / \mathrm{m}$ (inter-harmonic orders)

Note that amplitudes of harmonic or inter-harmonic orders don't decrease based on the theory of harmonic orders of current/voltage, which is because of the effect of the 
previous harmonics, see fig. 10.4, 10.5. In other words, the previous harmonic orders of a specific harmonic order produce sub-harmonic orders themselves. Therefore, these subharmonic orders are being convolved with harmonic orders of the main frequency and build a higher magnitude than it was expected.

\subsubsection{Step 2: IM1 and IM2 on and off}

After recognizing the effect of the mechanical load on the electric signature, the load is removed from the setup and another motor is added. Adding another induction motor with the same number of poles but different power level was for the reason of the identification of the components with similar operational mechanism but different power level. This is the required step before studying the detection of the faulty machine, which is explained the next step.

This test is implemented while the two IM motors were fed with $180 \mathrm{~V}$. The location of the antenna is changed from A to F, as shown in fig. 10.6.

The machines are tested in all six positions to recognize the effect of each of them at different locations. In addition, the optimum location of antenna for better investigation is the other reason for testing at different locations and height of the antenna. In addition, the machines are switched on and off and located at different locations, as shown on the bottom side of the fig. 10.6. Consequently, the setup was tested in 38 circumstances. The results are applied to the algorithm and the identification is fulfilled. As an example, the comparison of some of the results, in which the antenna was located at point A, is shown in fig. 10.7 . 

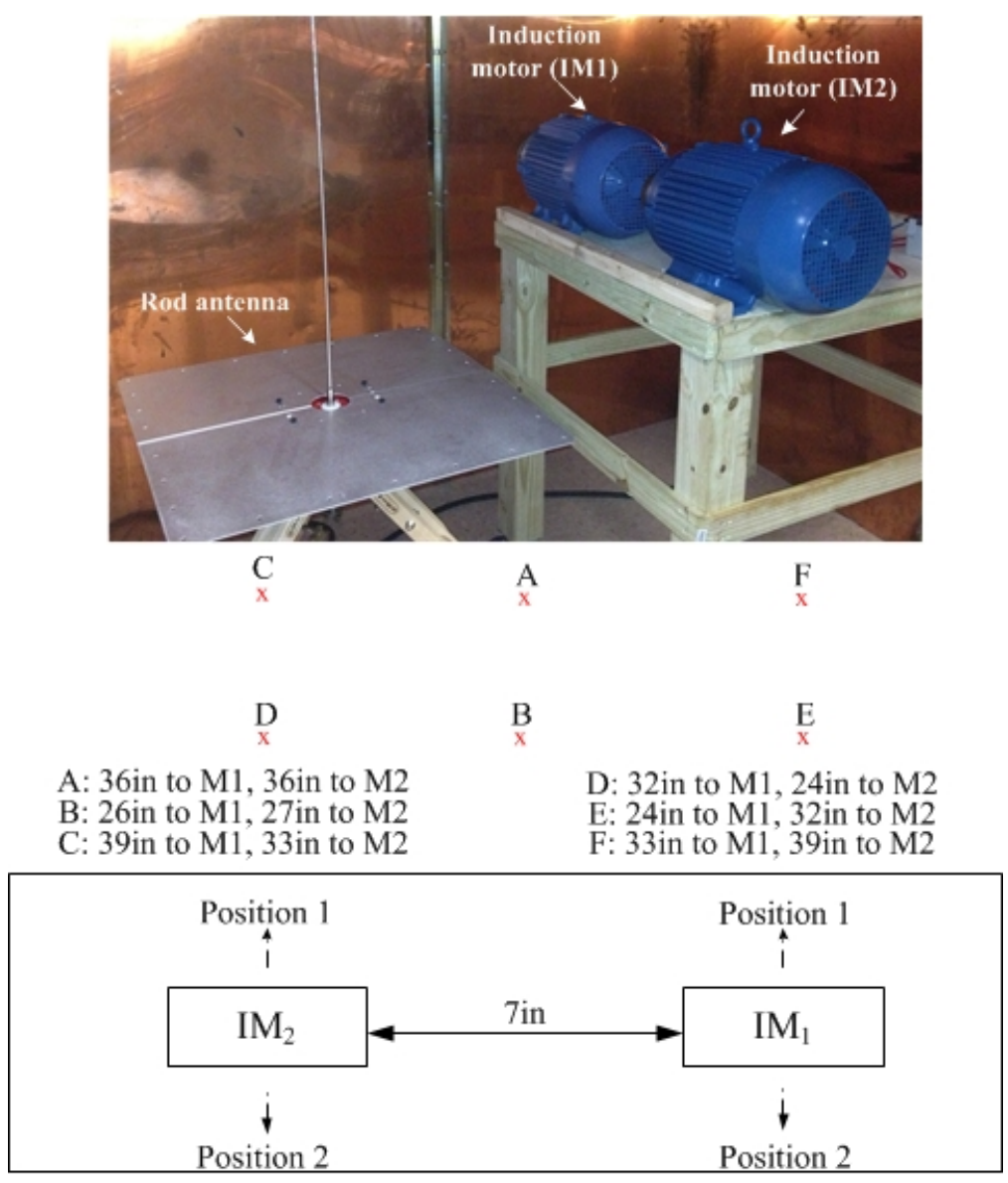

Fig. 10.6 The locations of the antennas and motor in the tests of step2, 3, 4

As illustrated in fig. 10.7, the amplitudes of more than 90 percent of the harmonic orders of the case, in which $\mathrm{IM}_{2}$ is switched on and $\mathrm{IM}_{1}$ is switched off, is more than the case, in which $\mathrm{IM}_{1}$ is switched on and $\mathrm{IM}_{2}$ is switched off. The reason is that the horsepower of $\mathrm{IM}_{2}$ is more than $\mathrm{IM}_{1}$. Therefore, higher current passes through the winding of $\mathrm{IM}_{2}$ and consequently the induced voltage is higher. This fact leads to the generation of more electric stray field. Accordingly, the amplitudes of the harmonic orders of the case in which both motors are switched on is higher than the two other cases. 
This case along with the other 36 tested cases built an algorithm to identify the larger machine in different locations.

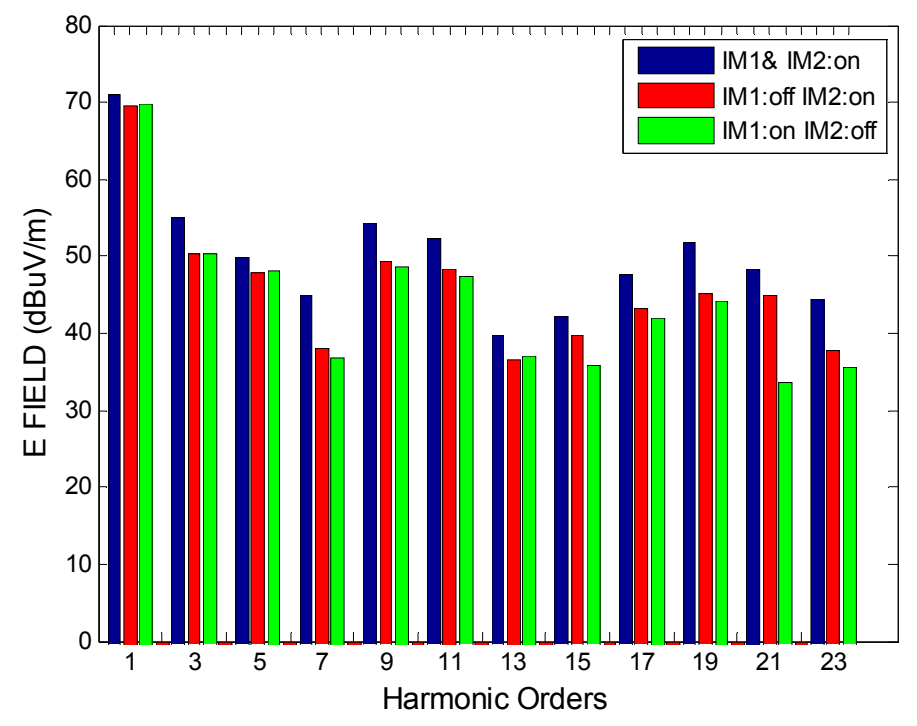

Fig. 10.7 Electric stray field of the step 2 in $\mathrm{dB} \mu \mathrm{V} / \mathrm{m}$ (the antenna is located at point $\mathrm{A}$ )

\subsubsection{Step 3: IM1 faulty: short-circuited) and IM2 (healthy)}

Following the identification of the machine in terms of the power level, the recognition of the faulty machine is investigated, while a healthy machine is located very close to the faulty one, see fig. 10.6.

The inter-coil and the turn-terminal short-circuits are implemented into $\mathrm{IM}_{1}$. The locations of the short-circuited turns are shown in fig. 10.8. The $T_{B}$ is connected to $S_{1}$ for the turn-terminal and $S_{1}$ to $S_{2}$ for the inter-coil short-circuit. The leads of these points are connected through a rheostat to avoid damages. This test is also done, while the two IM motors were fed with $180 \mathrm{~V}$. The location of the antenna is changed from A to F. 


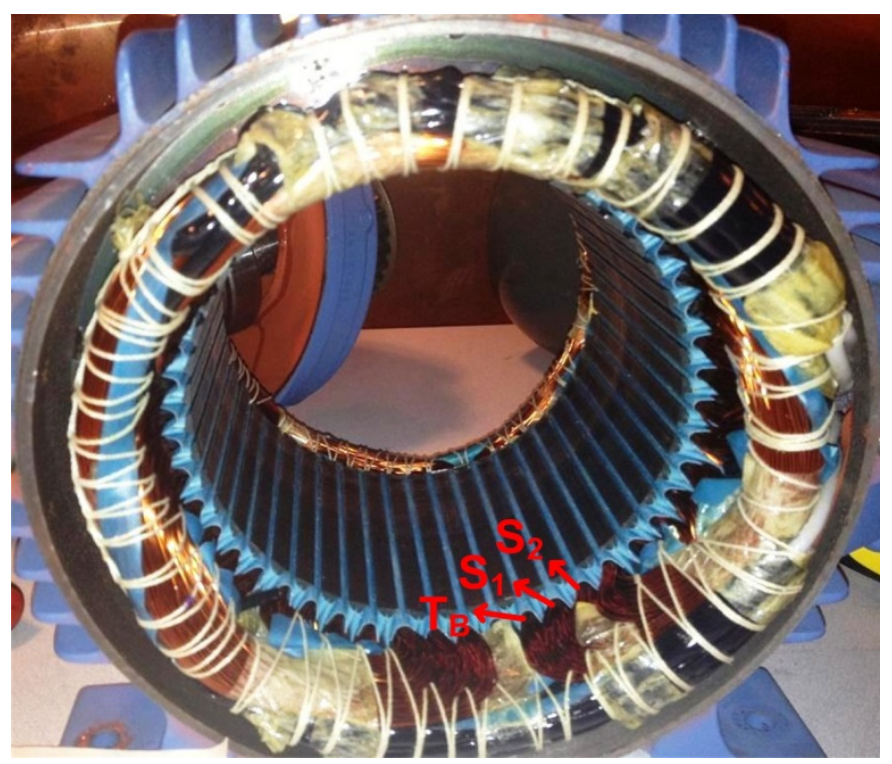

Fig. 10.8 Location of the selected turn for inter-coil and turn-terminal short-circuits $\left(\mathrm{T}_{\mathrm{B}}\right.$ : terminal of phase $\mathrm{B}, \mathrm{S}_{1}$ and $\mathrm{S}_{2}$ : selected turns of phase $\mathrm{B}$ )

The test cases in this step are implemented similar to the cases in step 2 and the algorithm, which is shown in fig. 10.3 is used for fault detection. For instance, the antenna is located at point $\mathrm{B}$ and the two mentioned types of short-circuits are implemented. The harmonic orders of the stray E-field are shown in fig. 10.9.

As demonstrated in fig. 10.9, the harmonic orders of the faulty cases have higher amplitude compared to the healthy one. As the result, $\mathrm{A}_{\mathrm{hi}}$ of fig. 10.3 is not equal to $A_{h \text { - }}$ base. Accordingly, the faulty system can be identified. In addition to the identification of faulty component, the type of fault can be detected. Comparing the harmonic orders of the two faulty cases, the harmonic orders of the turn-terminal short-circuit have higher amplitude.

To identify which of the placed machines are faulty, the level of harmonic order variations $\left(\Delta \mathrm{A}_{\mathrm{h}}=\mathrm{A}_{\mathrm{hi}}-\mathrm{A}_{\mathrm{h}-\text { base }}\right)$ can be compared. Perceiving from the step2 and 3 , the $\Delta \mathrm{A}_{\mathrm{h}}$ 
is larger while the fault is placed in the larger machine. This is discussed more in the next step.

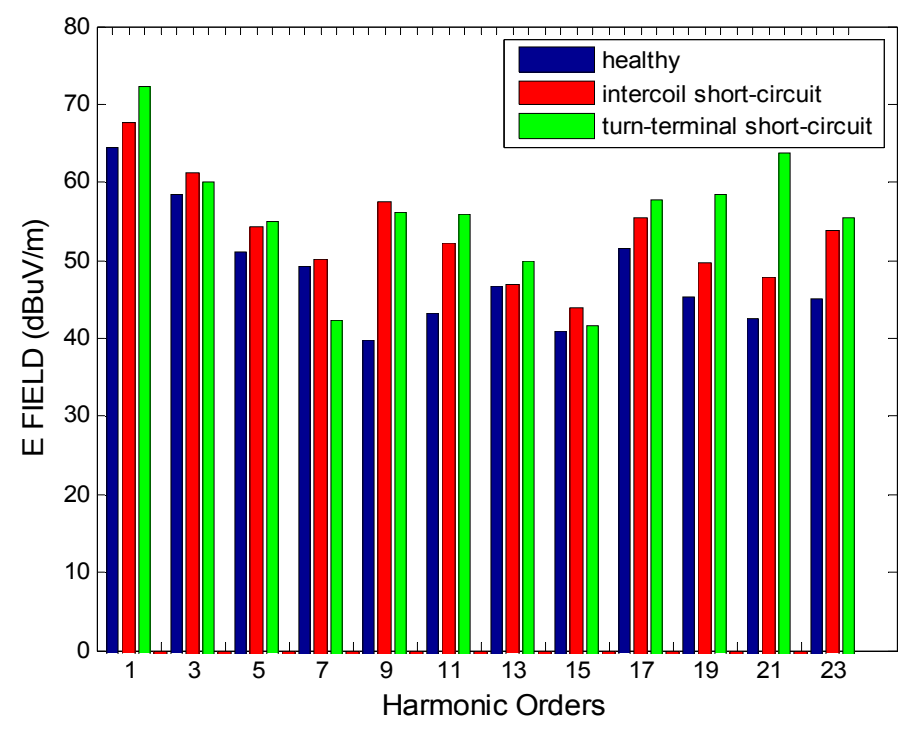

Fig. 10.9 Electric stray field of the step3 in $\mathrm{dB} \mu \mathrm{V} / \mathrm{m}$ (the antenna is located at point $\mathrm{B}$ and both machines are switched on)

\subsubsection{Step 4: $I M_{1}$ (unbalanced current, healthy) and $\mathrm{IM}_{2}$ (unbalanced current, healthy)}

The other abnormal condition of the electrical machine, which is tested here, is passing unbalanced currents into the phases of the windings. A resistor box is located in the way of the cables to the machines and each three phases of both motors are unbalanced individually. The results are measured similar to the two previous cases, as shown in fig. 10.6.

Since this step is alike faulty step, the procedure of detecting the faulty component is similar to the previous one. The $\mathrm{P}_{\text {component }}$ and $\mathrm{A}_{\mathrm{hi}}$ of each case are compared with the healthy case, and the faulty component would be identified. To identify which machine 
of the system is faulty, the unbalanced current at the phase $\mathrm{A}$ of the $\mathrm{IM}_{1}$ and $\mathrm{IM}_{2}$ are compared with each other, and the healthy case and displayed in fig. 10.10. The result shows that the harmonic orders of both machines cases increase, which is due to the increase of current in the machines. Note that the unbalanced current in $\mathrm{IM}_{2}$ has more effect in comparison with $\mathrm{IM}_{1}$. As mentioned before, the reason is the level of the $\mathrm{IM}_{2}$ 's power that is larger than $\mathrm{IM}_{1}$. Accordingly by comparing the level of changes $\Delta \mathrm{A}_{\mathrm{h}}$, the machine, which carries the unbalanced current, can be detected.

The figure shows that, some harmonic orders may decrease or do not change. For these kinds of cases and especially for the cases with insignificant faults, the other locations of antenna, such as points B and E, should be tested.

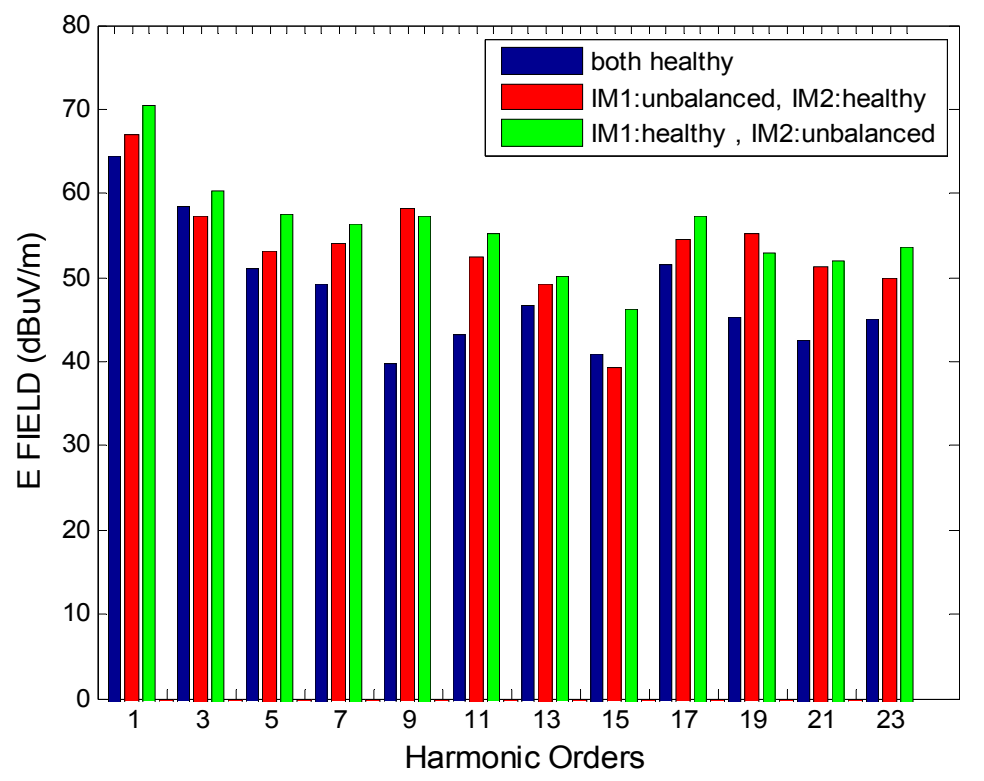

Fig. 10.10 Electric stray field of the step4 in $\mathrm{dB} \mu \mathrm{V} / \mathrm{m}$ (the antenna is located at point $\mathrm{B}$, both machines are switched on while there is an unbalance current in phase A) 


\subsubsection{Step 5: Converter connected to the $I M_{1}$ at different switching frequencies with and without fault}

Following the fault and abnormal conditions monitoring, the healthy and faulty conditions of the $\mathrm{IM}_{1}$ motor connected to a multifunctional converter is studied in this case. The converter is controlled through a dSpace, connected to the MATLAB/SIMULINK control blocks. Through this, the switching frequency of the converter, as well as the frequency of the machine, is adjusted. The locations and distances of the components in this step are shown in fig. 10.11.

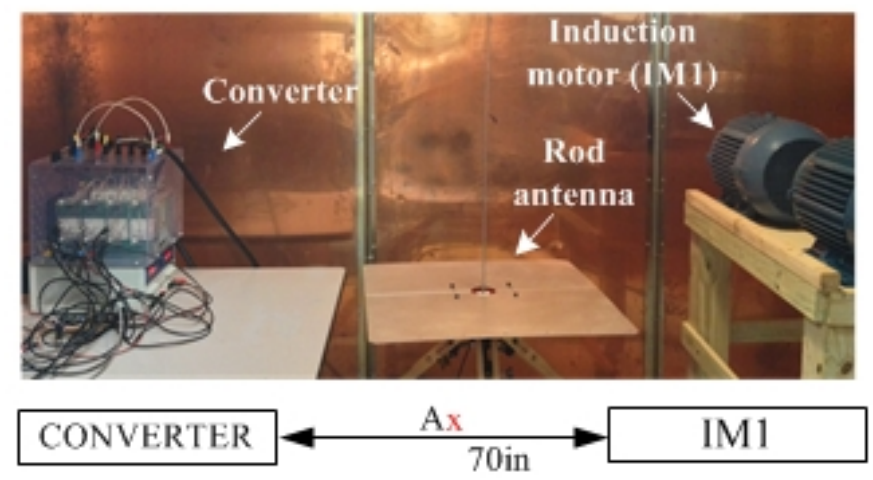
A: 35 in to IMl, 35 in to CONV C: 75 in to IM1, 36in to CONV
B: 45 in to IM1, 45 in to CONV D: 36 in to IM1, 75 in to CONV

$\mathrm{Cx} \quad \mathrm{Bx} \quad \mathrm{Dx}$

Fig. 10.11 The locations of the antennas and components in the tests of step5

The converter is tested in three switching frequencies $(2,2.5$, and $3 \mathrm{kHz})$ to see the effect of changing the switches in the stray E-field. Moreover, the frequency of motor is altered from $60 \mathrm{~Hz}$ to $59 \mathrm{~Hz}$ and $58 \mathrm{~Hz}$.

Fig. 10.12 displays the stray E-field from the setup, while the three switching frequencies $(2,2.5$, and $3 \mathrm{kHz})$ are applied. Since the frequency band is expanded due to the presence of the converter, it's impossible to demonstrate the comparison of all 
harmonic orders, as shown in previous sections. Therefore, the continuous frequency response is used. As data tips of the figure show, the main frequencies are the power frequency of the machine $(60 \mathrm{~Hz})$ and the switching frequency and its harmonics. Therefore, the type of components can be identified. Through this, not only the type of components can be identified, but also the failures or fault can be monitored. For example, if there is any problem in the switches, the peaks at the switching frequency may shift along the frequency band. Moreover, if the slip of the induction motor changes, the harmonic orders of the machine may not be located at the expected frequencies.

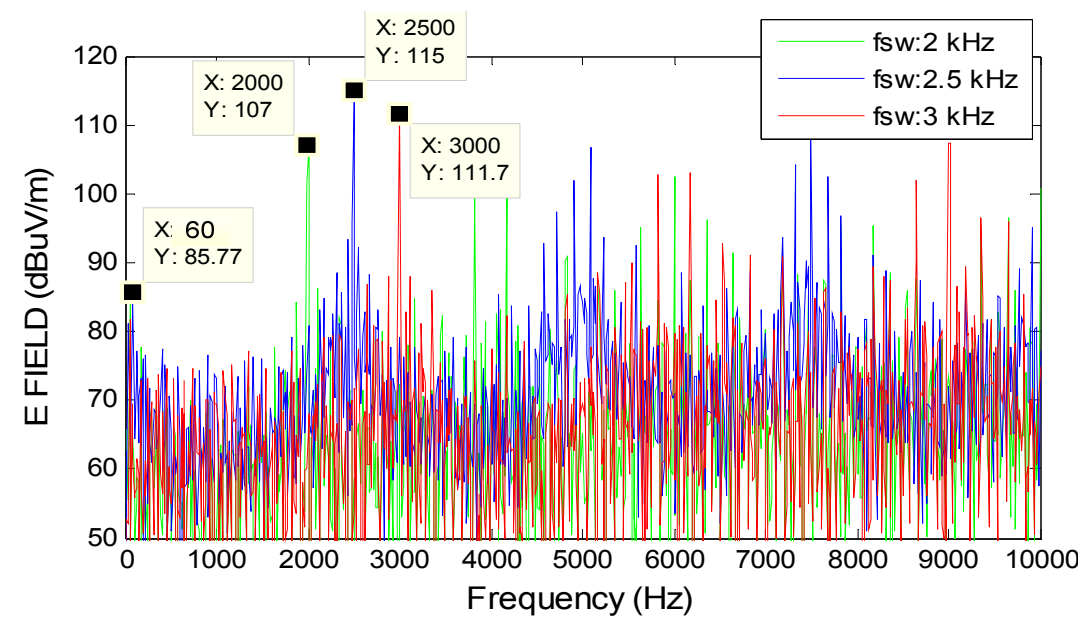

Fig. 10.12 Electric stray field of the step $5 \mathrm{in} \mathrm{dB} \mu \mathrm{V} / \mathrm{m}$ (the antenna is located at point $\mathrm{B}$ )

The short-circuit, which is employed in step 3, is applied again to the $\mathrm{IM}_{1}$ and the effect of the faults on the stray E-fields at the switching frequencies are shown in fig. 10.13. It is shown that the fault in one component, $\mathrm{IM}_{1}$ in this case, may not affect the other components significantly; however, the peaks at the switching frequency and its harmonics are changed marginally. Consequently, the faults of the inverter can be investigated without significant interference of the other components. 


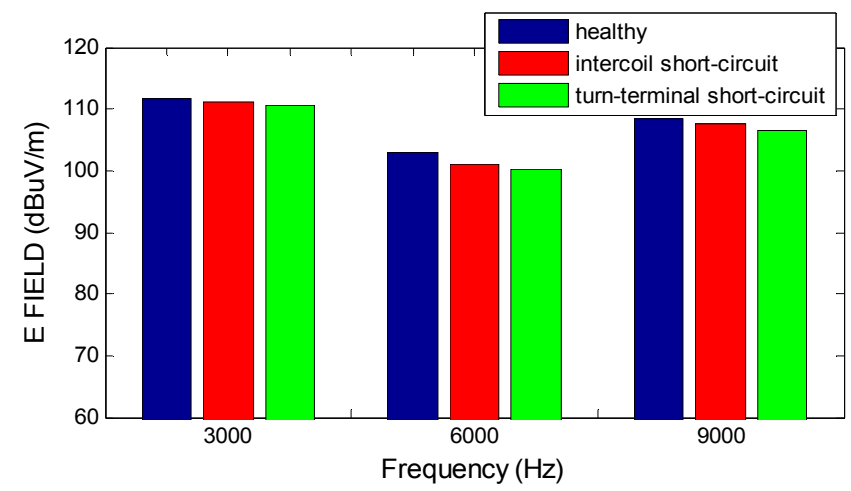

Fig. 10.13 Electric stray field of the step5 in $\mathrm{dB} \mu \mathrm{V} / \mathrm{m}$ while the $\mathrm{IM}_{1}$ is faulty (the antenna is located at point B)

\subsubsection{Step 6: Synchronous Generator connected to the DC motor and $\mathrm{IM}_{3}(3-\mathrm{HP}$ induction motor)}

As the last case study, the synchronous generator is connected to a DC and induction motors to identify the different type of machines. The purpose of this step is to identify the different type of machines. The situation of the machines and antenna is shown in fig. 10.14

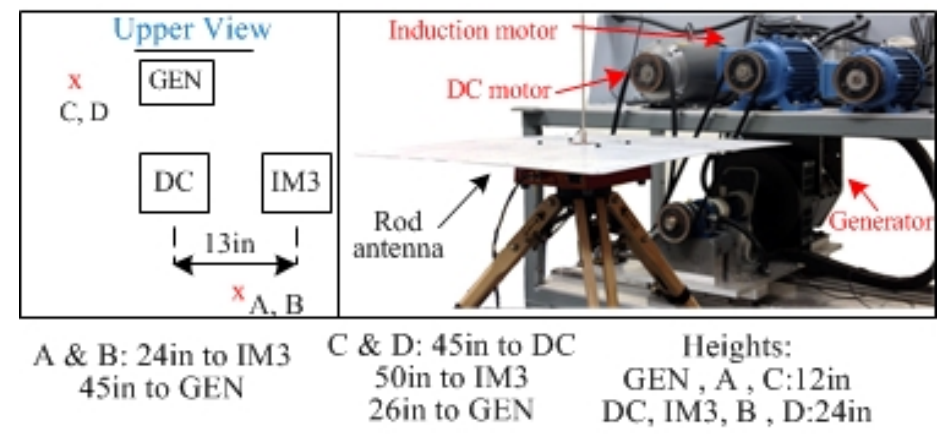

Fig. 10.14 The locations of the antennas and components in the tests of step6

As demonstrated in the fig. 10.14, the generator and motors are not at the same height. Similar to the previous cases, the stray fields are measured with several combinations of these components. Three cases are selected among numerous tests to be shown in fig. 
10.15. As displayed, the harmonic orders of the case, in which the generator is connected to the DC machine (GEN+DC), are considerably different from the other two cases in terms of amplitude. This is due to the difference between the structures of the DC motor from the induction motor. The presence of four types of winding in DC motor, such as field, armature, compensation and commutation winding, creates different amplitude of the stray E-field. It is also shown that the presence of all machines in one of the test cases would lead to the highest amplitudes of the harmonic orders. However, the difference between the case GEN+IM3 and GEN+IM3+DC is less, which is due to the existence of two similar machines, generator and induction motor, in terms of working frequency.

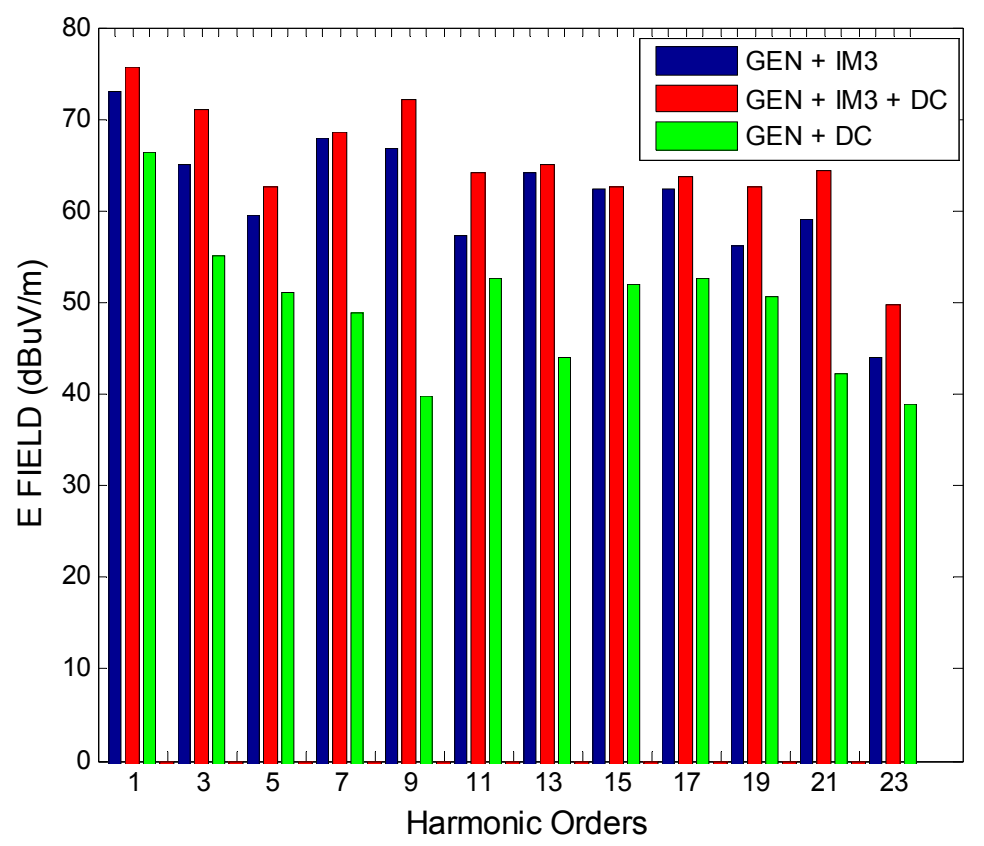

Fig. 10.15 Electric stray field of the step6 in $\mathrm{dB} \mu \mathrm{V} / \mathrm{m}$ (the antenna is located at point $\mathrm{A}$ )

In addition to the difference of amplitudes between the above cases, the location of the harmonic orders along the frequency band should be compared, as mentioned in the main flowchart. Fig. 10.16 shows the frequency response of the above cases measured at point 
B. The amplitudes of the 12th harmonic order in the three cases are shown in the data tips. As revealed, the harmonics of the first two cases appeared at $727.5 \mathrm{~Hz}$ instead of $720 \mathrm{~Hz}$, which is because of the presence of the induction motor in these two cases that have slip frequency. The slip frequency shifts the rotors frequency, which yields to the shift of the harmonic orders along the frequencies. This is one of the main hints in identifying the type of machines.
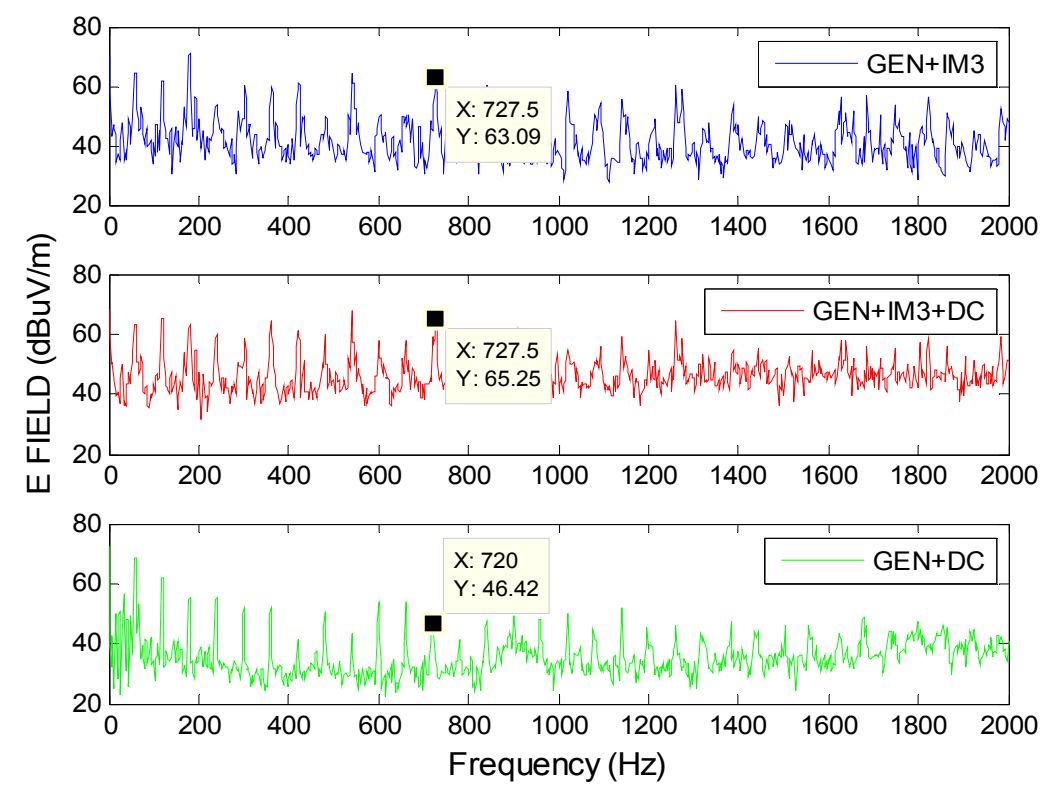

Fig. 10.16 Electric stray field of the step6 in $\mathrm{dB} \mu \mathrm{V} / \mathrm{m}$ (the antenna is located at point $\mathrm{B}$ )

\subsection{Fault diagnosis and monitoring of power converter}

The stray magnetic and electric fields of a multifunctional power converter operation, while connected to a system involving an induction motor is investigated in this part. The impacts of the switching techniques on the radiated fields of the converter were studied for the purpose of evaluating EMI and for fault detection. This was achieved through the inspection of the magnetic and electric fields. Two of the most commonly used switching techniques were implemented through a hardware-in-the-loop system (HIL), studied in 
terms of the stray. Various fault and failure conditions were applied to the converter to monitor the converter condition through the stray field observations. A coil and a rod antenna were connected to an EMI receiver to capture the fields. The fields were employed in further analyses. The harmonics and inter-harmonic of the stray fields were studied in details for system monitoring purposes. The importance of this study is enabling the evaluation of the optimum switching pattern for lower EMI field levels from the converter and for condition monitoring and fault diagnosis purposes

\subsubsection{Harmonic and Inter-Harmonic Study of the Radiated Fields}

By increasing the speed of switches, the switching loss will be lower and the controllability of the system increases. However, the increase of $d v / d t$ is accompanied by an increase in the EMI level. Therefore, more inter-harmonics appear in the frequency response of the stray fields. Consequently, for a comprehensive electromagnetic stray field study of the drive, the harmonics and inter-harmonics of the drive are studied.

The schematic of the proposed converter connected to the machine is shown in Fig. 10.17. The common feature of such a double energy conversion system is that it contains an ac-dc rectifier and a dc-ac inverter. The rectifier and inverter are coupled through a dc link filter, which is either capacitor or a reactor. If the reactor or the capacitor has infinite value, there will be no ripples on the dc side, and consequently the ideal rectifier will only generate the characteristic harmonics $\left(f_{h-R}\right)$ :

$f_{h-R}=(k n \pm 1) f$

where $\mathrm{k}$ is the pulse number of the rectifier, $\mathrm{n}$ is an integer, and $f$ is the power frequency. However, the reactor or the capacitor values are finite in practice and the ripples at the dc 
side are inevitable. As a consequence of not having a flat dc link current, its ac side will be modulated by the dc ripple and the inter-harmonics could be produced.

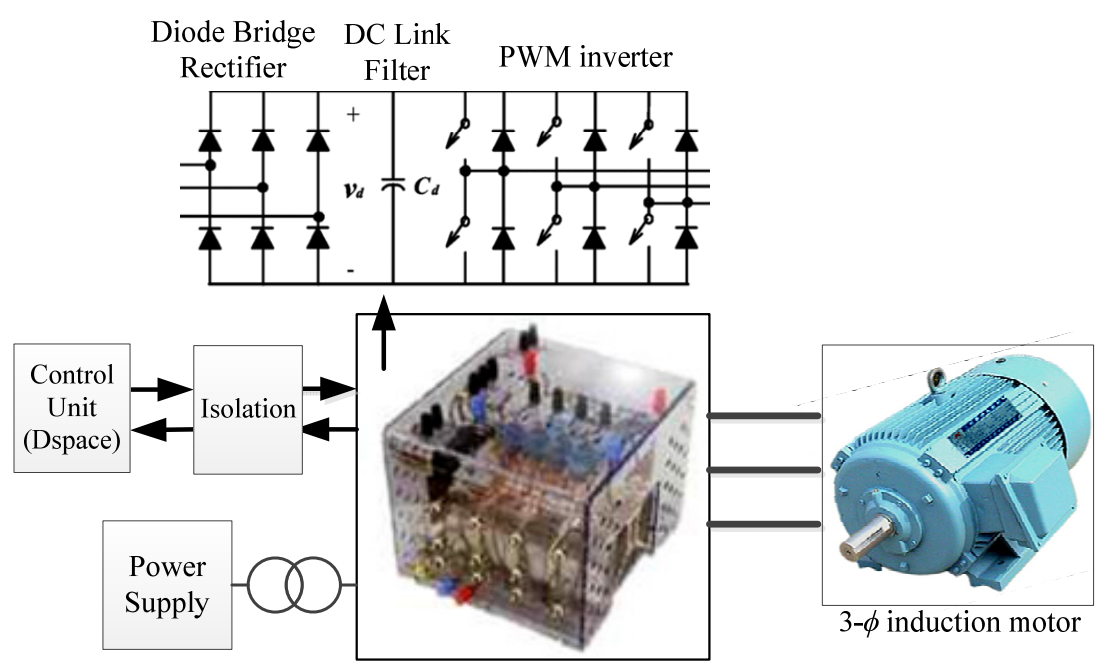

Fig. 10.17 Schematic of the test setup (PWM VSI drive)

For example, for a 6-pulse rectifier, based on (10-5), its characteristic frequencies are the 1st, 5th, 7th, 11th, 13th, and so on harmonic orders. But if the dc side has a ripple of for example $165 \mathrm{~Hz}$, the ac side current will be modulated as (1st, 5 th, 7 th, 11 th, 13 th ...) $\pm 175 \mathrm{~Hz}$. These are inter-harmonic components.

In voltage source converters (VSC), in comparison with current source converters (CSC), more complex formulas are required to determine the dc ripple generated by the inverter [111]. In the case of synchronous PWM modulation technique (SPWM), the harmonic frequencies generated by the inverter are evaluated as:

$$
f_{\text {ripple }}\left(m_{f}, i, j\right)=\left|m_{f} i \pm j\right| \cdot f_{\text {output }}
$$

where $\mathrm{mf}$ is the modulation ratio with $i$ and $j$ as the integers depending on the modulation ratio. The $\mathrm{f}_{\text {output }}$ is the output frequency. The dependency of $\mathrm{mf}$ is related to the switching strategy adopted. The value of $i$ and $j$ for the various $\mathrm{mf}$ is shown in table 10.2 [111]. 
Table 10.2 value of $i$ and $j$ for the various $\mathrm{mf}[111]$

\begin{tabular}{lll}
\hline $\mathrm{mf}$ & $\mathrm{i}$ & $\mathrm{j}$ \\
\hline Non triple \& odd & Even $\rightarrow$ & Even \\
& Odd $\rightarrow$ & Odd \\
\hline Non triple \& even & Even $\rightarrow$ & Even or Odd \\
& Odd $\rightarrow$ & Even triple \\
Triple \& odd & Even $\rightarrow$ & Odd triple \\
\hline Triple \& even & Odd $\rightarrow$ & Triple even or odd \\
\hline
\end{tabular}

Note that the frequencies generated by inverters as shown in (10-6), will modulate with the rectifier's characteristic harmonic of (10-5) and subsequently the supply-side frequencies will be generated:

$$
f_{s s}=f_{h-R} \pm f_{\text {ripple }}
$$

The supply-side frequencies $\left(f_{s s}\right)$ are actually the inter-harmonics of the power frequency as long as the $f_{\text {ripple }}$ is not synchronous with $f$.

The discussed, the harmonics and inter-harmonics appear in the frequency response of the radiated electric and magnetic fields. Since the location of the peaks along the frequency band is related to the setting parameters and characteristic of the converter, the behavior of the converter or the malfunction of the switches would be recognized. In order to do so, the two common switching patterns are briefly explained. 


\subsubsection{Switching Patterns (SPWM, SVPWM)}

Amongst the most commonly used, PWM techniques are the sinusoidal PWM (SPWM) and the Space Vector PWM (SVPWM). A schematic diagram of the inverter circuit is shown in fig. 10.18 (a).

In the SPWM technique, the control signal is simply compared to a high-frequency triangular waveform. The high-frequency signal represents a carrier to the control signal, which results in shifting the harmonic content of the output voltage to higher frequencies. The later can be suppressed by the high-frequency impedance of the machine. Using a higher switching frequency theoretically leads to a cleaner output voltage. However, the switching losses in the converter impose a limitation on the switching frequency that can be used. The aforementioned limitation is actually imposed on all PWM techniques not only SPWM. In terms of harmonic study, the modulation index (ma) plays an essential role in the harmonic content of the output voltage. This ratio does not exceed 1 in the SPWM technique and therefore this method does not provide full utilization of the DC supply voltage. The SPWM is commonly used, and further details about its implementation can be found in the reference [262].

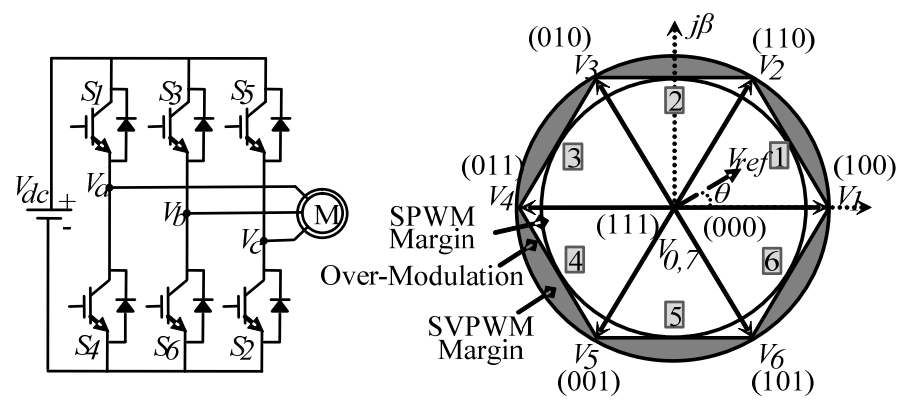

Fig. 10.18 The drive system schematic diagram: (a) the inverter circuit; (b) A vector representation of the SVPWM technique and the margins of SPWM versus SVPWM 
The output in SVPWM is represented by eight switching vectors $\left[\mathrm{V}_{0}-\mathrm{V}_{7}\right]$ as shown in fig. 10.18 (b). Each of these vectors represents a switching state for the six switches. The control signal can be looked as a vector that is rotating in the $\alpha-\beta$ plane at the fundamental frequency with a length that corresponds to the desired fundamental amplitude. As the control vector rotates within any of the sectors, the two switching vectors bounding this sector along with the zero vectors are used to develop the desired voltage. The time is shared among the switching vectors according to the projection of the control vector over the switching ones (time averaging). The equations, used to calculate the duty cycles, are well-developed in the literature [262].The SVPWM generates less harmonic distortion as compared to the SPWM technique [263]. Moreover, it provides a more efficient utilization of the supply voltage, since the locus of the reference voltage in SVPWM is greater than that of the SPWM. The latter is depicted in fig. 10.18 (b). The locus of the reference for the SPWM technique is the inner circle with a radius of $\mathrm{Vdc} / 2$, whereas the locus of the reference for the SVPWM is the outer circle with a radius of $\mathrm{Vdc} / \sqrt{3}$. The drawback of the SVPWM technique is that it requires more computational power than the SPWM.

\subsubsection{Experimental Result and Discussion}

The electromagnetic signature study of the proposed setup was performed experimentally while the converter was controlled by simulation (MATLAB/SIMULINK). The proposed test setup consists of a multifunctional converter connected to an induction motor controlled by a dSpace 1104 controller development environment. For measurement, the proper electric and magnetic field antennas for low- 
frequencies were used. The data were transferred from the antennas to the EMI receiver based on the MIL-STD 461F standard. To eliminate any extra noises, the converter and the motor were located inside an isolated chamber. The controller, isolation circuit and transducers were all located outside the chamber to avoid any unwanted signatures. The details of the devices are identified in Table 10.1.

The study was divided into three case studies. The first one was proposed to find the optimum switching algorithm for lower stray field production and the optimum algorithm for fault diagnoses. An open circuit fault was applied to one of the switches and the converter was tested with both SPWM and SVPWM switching algorithms with two different switching frequencies. Finally, an unbalanced voltage was generated by decreasing the voltage of one of the inverter legs.

\subsubsection{Optimum switching algorithm}

As mentioned in the previous paragraph, there are two reasons for optimum switching algorithm; lower EMI and fault detection. Fig. 10.17 shows the stray electric field (Efield) for the SPWM and SVPWM switching algorithms with switching frequencies of $1380 \mathrm{~Hz}$ and $1740 \mathrm{~Hz}$. Comparing figures 10.19 and 10.20, the first inference is that the amplitudes of the harmonic orders of the switching frequencies have significant amplitude in fig. 10.19, which corresponds to the SPWM switching technique. The significant harmonic orders help considerably in diagnosing the faults of the converter. Applying the faults, the amplitudes or frequencies of the harmonic orders change. Considering fig. 10.20, the amplitude of the harmonic orders is not obvious. Therefore, 
the space vector technique may not be helpful and consequently, the SPWM technique is optimum for fault diagnosis.

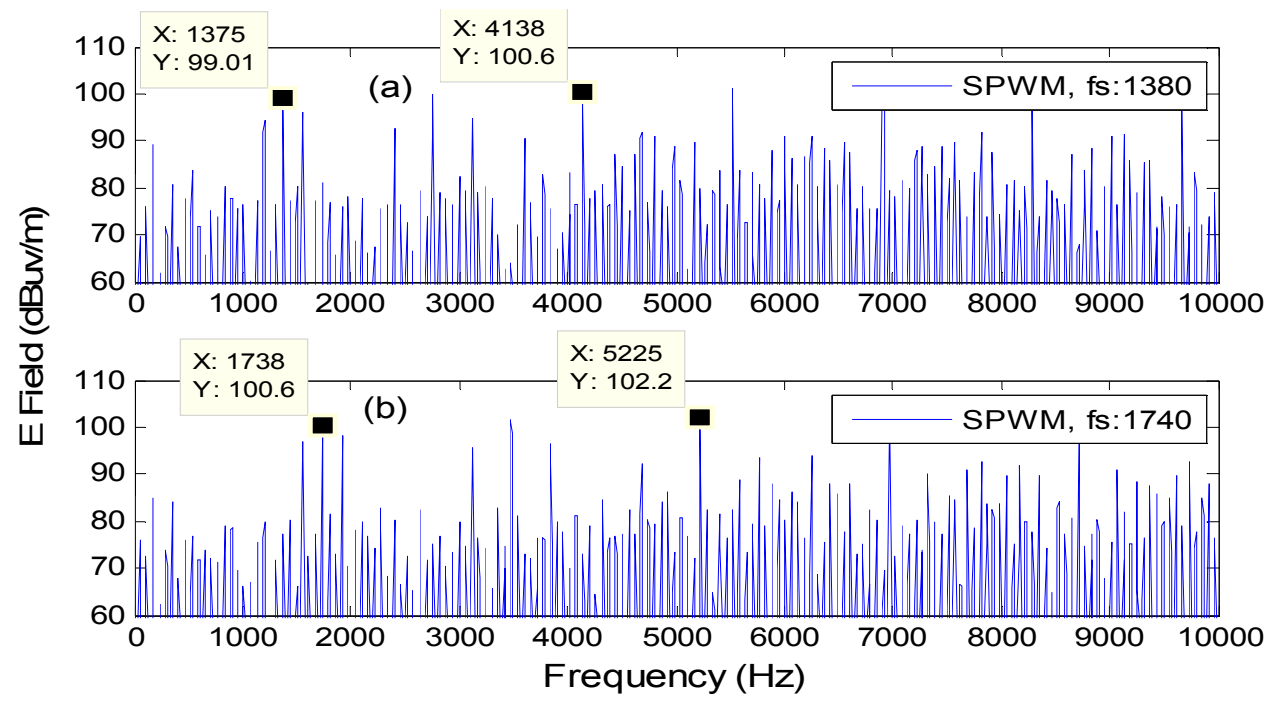

Fig. 10.19 The radiated electric field from the setup with SPWM pattern in two different switching frequencies (fs), (a) fs: $1380 \mathrm{~Hz}$, (b) fs: $1740 \mathrm{~Hz}$

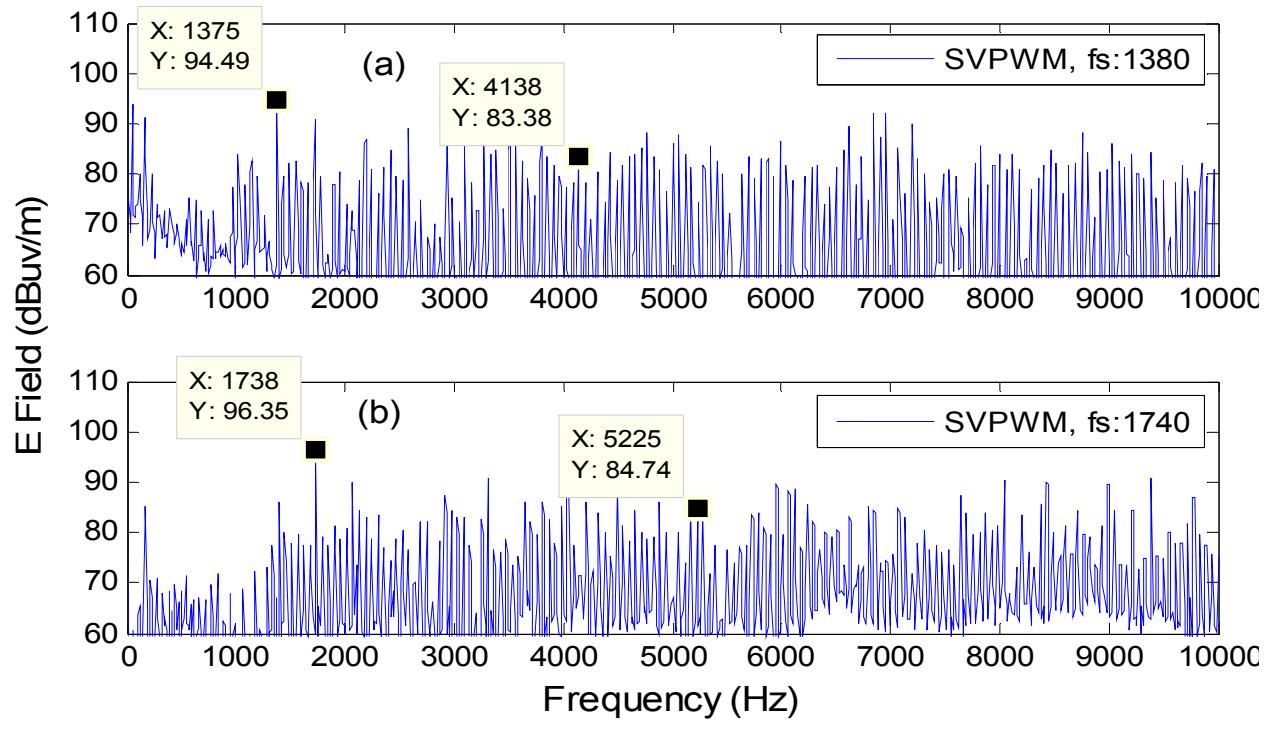

Fig. 10.20 The radiated electric field from the setup with SVPWM pattern in two different switching frequencies (fs), (a) fs: $1380 \mathrm{~Hz}$, (b) fs: $1740 \mathrm{~Hz}$

On the other hand, the same point is a hint for the other purpose but in an inverse fashion. Comparing fig. 10.20(a) with fig. 10.19(a) and also fig. 10.20(b) with fig. 
10.19(b), the amplitude of the harmonic orders and inter harmonics are lower when the SVPWM is used. This is due to the lower distortion produced by this method, which was explained in section 10.4.2. The data tips are located at the amplitudes of harmonic orders to facilitate the comparison. As can be seen, the difference between the amplitudes is increasing by an increase of the harmonic orders. Note that the deviation of the harmonic orders from the switching frequency (1380 to 1375) is because of the $f_{\text {ripple }}$, as mentioned in section 10.4.1. Consequently, the SVPWM produces lower stray fields. This makes this strategy an optimum choice for the application in which lower EMI is essential. The stray magnetic field (H-field) shows the same notes. For brevity, they are not mentioned in this part.

\subsubsection{Open-circuit fault}

An open-circuit fault was applied to the bottom switch at phase $\mathrm{C}$ and the effects of this fault on the stray fields were analyzed. The results with the SPWM technique are studied here, since this method was found to be optimum.

The stray E-field and H-field of the faulty state was compared with the healthy state, as shown in fig. 10.21.

Inspecting fig. 10.21(a), it can be noticed that the variation in the radiated fields at different switching frequencies has an observable trend. For instance, there is a repeated decrease in the range from $7-20 \mathrm{~dB} \mu \mathrm{V} / \mathrm{m}$ in at several harmonic orders with the switching frequency $(1380 \mathrm{~Hz})$. The reduction is because the applied open-circuit condition decreases the overall stray electric field. The analysis of the $\mathrm{H}$-field spectrum is slightly more complex. The ripples due to the DC link, mentioned in section 10.4.1, which is 
about triple the fundamental frequency $(\sim 175)$ convolves with the switching frequency. Therefore, the harmonic orders would be observed around $1380 \pm 175 \mathrm{~Hz}$. This was verified in fig. 10.21 (b) by having the peaks at about 1200 and $1550 \mathrm{~Hz}$ and their harmonic orders. Due to the occurrence of the fault, the $\mathrm{H}$-field increases in an inverse fashion to the E-field. This is due to the DC current component when the switch is opencircuited that flows through the faulty phase and creates magnetic field in the rotor.
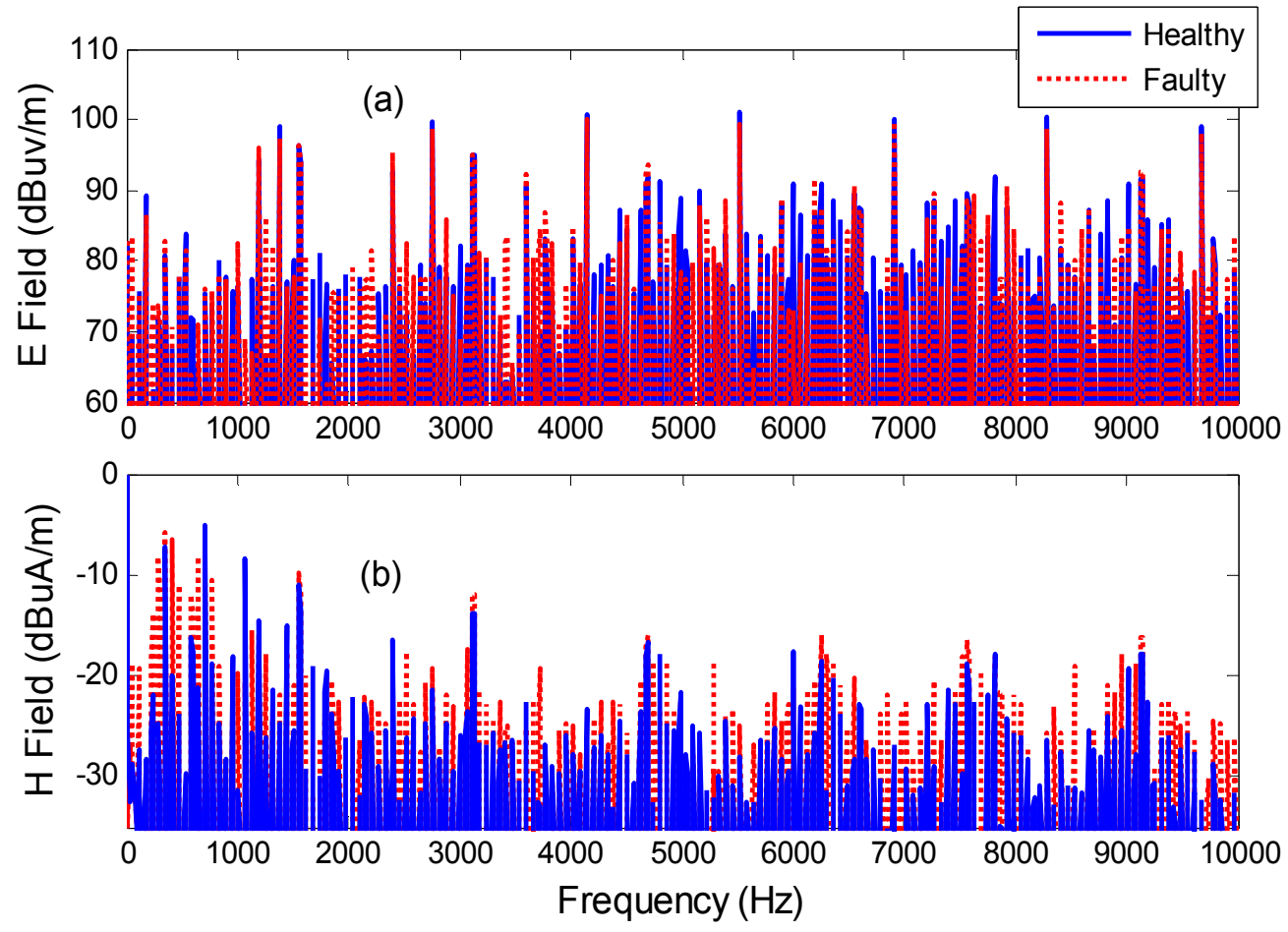

Fig. 10.21 The radiated (a) E-field and (b) H-field from the setup with SPWM strategy with $\mathrm{fs}=1380 \mathrm{~Hz}$ in healthy and faulty states

\subsubsection{Unbalanced voltage fault}

The second type of fault that was applied is the loss of one of the phases (the upper and lower switches of one of the legs). This fault creates an unbalanced voltage, which has an impact on the stray filed spectrum that was measured. As shown in fig. 10.22(a), it 
can be noticed that significant changes occur at the fifth harmonic order around $7 \mathrm{kHz}$. The shift of the inter-harmonics is the other symptom in this figure. Decreasing specific harmonic order, 5th in this case, helps in distinguishing this fault from the other faults. Observing the $\mathrm{H}$-field in fig. 10.22(b), the main harmonic orders do not change dramatically, while the significant amounts of inter-harmonics appear. The reason is that the unbalanced voltage generates unbalanced current in the inverter and motor, which makes non sinusoidal m.m.f. Therefore, the unwanted harmonics and accordingly interharmonics appear.

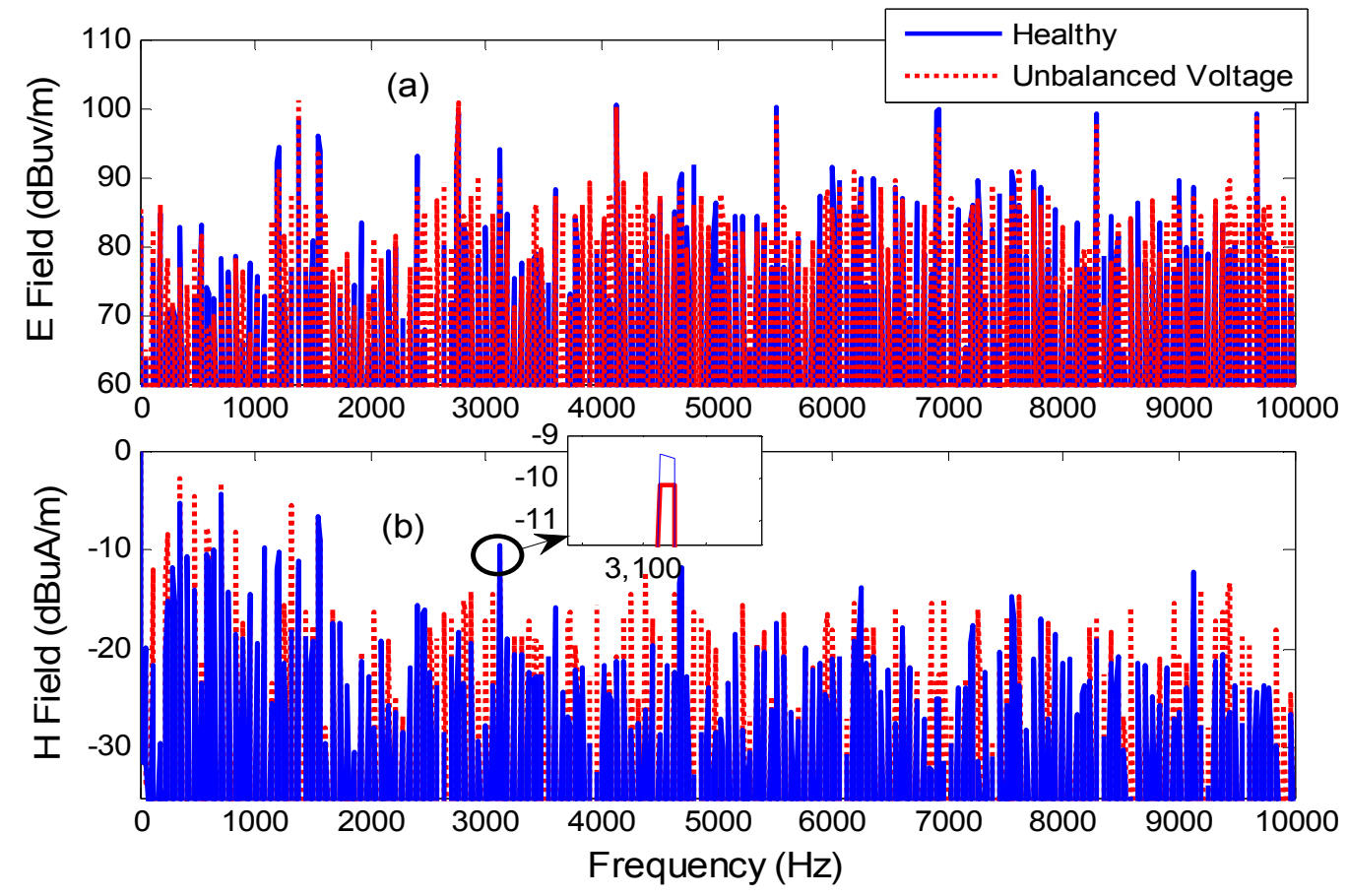

Fig. 10.22 The radiated E-field from the setup with SPWM strategy with fs $=1380 \mathrm{~Hz}$ in healthy and unbalanced voltage faulty states

\subsection{Conclusion}

The identification of the types of power system components was implemented in this chapter. The electromagnetic stray field of the component was used for the study. An 
algorithm was designed for the identification and monitoring. More than 170 circumstances of the combinations of the typical power components were tested experimentally and the identification was explained. The application of the identification, the fault and failure detections were also experimented and studied.

The results show that, it's possible to identify the type of components, as well as the faulty components by comparing the amplitudes of their harmonic orders, as well as the location of the location of harmonic orders along the frequency band. This comparison can be processed through the explained flowcharts. The identification using the stray fields is nondestructive and can be used for the setups that cannot go offline and dismantled. 


\section{Conclusions and Future Work}

\subsection{Conclusions}

This dissertation presented research and results on the study of low-frequency electromagnetic signature of power components. The physics based modeling of the power components through the computational techniques were implemented. Numerical modelling applications, including fault diagnosis and system condition monitoring, were investigated.

Electromagnetic compatibility of power components was studied with the review of electromagnetic computational models and the applications, including system monitoring and fault, failure diagnosis. In detail, the advances in modeling electromagnetic compatibility were studied by classifying the various EMC models. Analytical and numerical modeling were compared. Details are presented for numerical modeling and the reason for selecting the finite element for physics based modeling of low-frequency EMC and electromagnetic signature studies.

A method for frequency response model of the converter's components was presented using coupled FE-transmission line method computations. FE analysis was performed to obtain the frequency behavioral model of the converter. The natural frequencies computed from the proposed s-domain model were used to analyze the circuit's electrical behavior and operation. The results showed that the s-domain model of the converter has the ability to reveal the behavior of parasitic elements, as well as higher resonances, which has critical impact in studying the EMI problems. This model can also be implemented for other types of converters, making it practical for the evaluation on 
EMI/EMC issues at the design and development stages. The power converter is not a representative component of all power converter designs, but it can show the value of considering the effects of parasitic components on operation of the power converters, especially when their switching frequencies are increased. By considering the effects of parasitic components, one can modify the converter's circuit design in a way to reduce the effects of parasitic components and set the operating condition so it satisfies the converter's behavioral characteristics.

Since there is considerable necessity for analyzing the radiated electromagnetic field around electrical machines and also considering that simulating multi-machine environment with actual models require significant time and computing resources, designing an equivalent source model was studied in chapter 4. Several models were investigated in this study and the voltage-current cube model was shown to have the best result among these proposed models. The GA-based PSO method was used for evaluating currents of the cube branches as well as voltages at the nodes. The finite element analysis was used for simulation. The results show that it is possible to replace actual model of electrical machines with equivalent rectangular cube model. The simulation time of the cube model was approximately 100 times less than the actual model. This enables numerical simulation of multiple cases. The model was validated based upon the simulation of two similar induction machine with two cube models with the same center of coordinates.

Although the final model has great accuracy in resembling the stray fields, it can only be used in one particular occasion. In other words, by changing the voltage, power or construction of the machine, such as number of poles and size, the model should be re- 
optimized. Therefore, a new type of modeling was designed, which was discussed with experimental verification as was given in chapter 6 .

The requirements and procedure of electromagnetic compatibility testing was explained before the use of experimental studies. The procedure for the measurement of radiated emission, as well as conducted emissions, was described. All the details were related to the low-frequency analysis. Note that the requirements for high frequency analysis are different for both emission and susceptibility studies.

Considering the pros and cons of the voltage-current cube models, in order to study electromagnetic fields radiated from a typical connected electric power system, the embedded equivalent source models were designed and investigated. A new method termed wire modeling was used for designing this equivalent source model. It was created from representative cylindrical loops carrying a set of currents in the branches, as well as voltages at the nodes. Since power components have several types of elements, individual equivalent source model was designed for each of them. Several switches were considered for turning on and off windings. The data for the model parameters are based on the current density and potential difference of the actual machine. Also, the GA-based PSO method was used for evaluating the dimension of the model. For the analysis, electric and magnetic interfaced finite element method is used. The results show that it is possible to replace actual model of electrical machines with the equivalent source model. The simulation time of the proposed equivalent source model was approximately 80-100 times lower than using the actual model. This enables the numerical simulation of multiple sources in a reasonable time, allowing the practical study of EMC issues during 
electric drive development stages. The superposition and suppression of the fields in coupling the components were also studied and verified.

The full 3D finite element modeling for the radiated EMI study of a typical power electronic drive was implemented. The physical approach for applying the switching activity was utilized. In order to have the detailed simulation of the model and simultaneously consider the non-linearity of commutation curve in the frequency analysis, the fast GMRES methods with proper conditioners were used as the solution method, which increases the simulation speed. The laboratory measurements was also utilized for verification of the numerical results, as well as for investigating the stray fields under different operating conditions. The results show that the FE model has a good accuracy for evaluating the stray fields. Two cases of using the inverter in the system were studied and their applications were explained. The results show that the frequency response of the field can be used for assessing shielding arrangements, as well as for monitoring the conditions of the drive.

The application of EMC and electromagnetic signature studies were investigated. The identification of various unbalanced input condition of flowing current, as well as shortcircuit condition in the stator winding of an induction motor was developed and implemented in chapter 8 using the 3D FE simulations and ANN. The developed process was verified through measurements. Various types of unbalanced currents and shortcircuits were analyzed. The 3D FE analysis was coupled with circuit-based software to implement the fault states. The fast generalized minimal residual technique was used for expediting the numerical analysis, while high order harmonics were observed in frequency domain study. Various types of unbalanced currents passed into the machine's 
terminals, and the harmonic orders of the radiated magnetic field were obtained and studied. The identification was implemented manually and by using ANN for seventy various cases. The similar scenario was implemented for the various types of shortcircuit. The final results show that any types of unbalanced current or short-circuit states under various levels of terminal voltage and different locations of antenna can be detected by analyzing the frequency response of the radiated magnetic field.

The identification of the type and location of short-circuits were implemented in chapter 9. The analysis of radiated magnetic field intensity was achieved to differentiate the major faults, such as intercoil short-circuit from the minor cases which could be an interturn short-circuit. An experimental setup was devised for testing and verifying the developed condition monitoring process using the magnetic field signature. The intercoil short-circuit was implemented and studied experimentally. Since the intercoil shortcircuit might damage the winding, a F3DFE model was developed and connected to the circuit-based software. The simulation results were verified experimentally. For the interturn short-circuit, two different optimizations for finding the most sensitive location of the antenna were considered, based on industrial constraint. The optimum rotation angle of the antenna $(\alpha)$, as well as the distance to the machine's casing were obtained and used for identifying the fault location. The number of peaks in the field signature was used as part of the information for distinguishing the faults. The location of the created or diminished harmonic or sub-harmonic orders along the frequency were considered as the final information in locating the interturn short-circuits. This type of fault diagnosis is nondestructive and has the ability to be applied, while the motor is running. Moreover, it has excellent accuracy without the need to add any sensor inside the motor. 
Finally, the identification of the types of power system components was implemented in chapter 10. The electromagnetic stray field of the component was used for the study. An algorithm was designed for the identification and monitoring. More than 170 circumstances of the combinations of the typical power components were tested experimentally and the identification was explained. The application of fault identification for fault and failure detections were also experimented and studied.

The results show that, it's possible to identify the type of components as well as the faulty components, by comparing the amplitudes of their harmonic orders, and the location of harmonic orders along the frequency band. This comparison can be processed through the explained flowcharts. The identification using the stray fields is nondestructive and can be used for practical cases that cannot go offline or get dismantled.

\subsection{Future works}

The equivalent source modeling of the power components enables the investigation of electromagnetic signature studies of the power system, such as fault diagnosis, system condition monitoring and improvement to the performance of the system. The typical power setup, including synchronous generator, induction motors, DC motors and power converter, were modeled and the above applications were studied. It is suggested to expand this modeling for various power setups, such as electric vehicles, sustainable grid and dc grids. The fault diagnosis and condition monitoring of the mentioned setups with such a method would be nondestructive and reliable. 
The fault type detection could be implemented using 3-D full finite modeling; however, the fault location could not be implemented because of the need to model all turns of winding. That is to say, the stator of one of the studied machine has 1776 turns, which is impossible to be simulated using full finite element method. On the other hand, since the detection is based on the harmonic and inter-harmonic study, the turns of windings must be simulated. It's suggested to extend the wire-model for the machines to simulate the full structure of the winding to enable fault location using the simulation. Moreover, modified 3-D FE model was designed for the electromagnetic signature study of the converter. However, the model was successful; the combination of this model with many other components can be implemented in full finite element. Therefore, the wire model of the converter is advised to be designed, even though the wire modeling of switching activities through the wire modeling method would be complex. 


\section{LIST OF REFERENCES}

[1] Barnes M., Practical variable speed drives and power electronics, Oxford: Newnes, 2003.

[2] "Eueopean Energy roadmap 2050," EUROPEAN COMMISSION, Brussels, 15.12.2011.

[3] Wacket M., "Germany Eyes Cutting Solar Incentives Faster”," Reuters UPDATE 1, Berlin, 2012.

[4] Std, I. E. C. "60050-321,"International Electrotechnical Vocabulary (IEV)-Chapter 327: Instrument transformers"." International Electrotechnical Commission, Geneva.

[5] I. G. Math H.Bollen, Signal Processing of Power Quality Disturbances, Wiley-IEEE Press, 2006.

[6] Martinez-Velasco, Juan A., and J. Martin-Arneclo. "Distributed Generation Impact on Voltage Sags in Distribution Networks," in 9th International Conference Electric Power and Distribution, Barcelona, 9 - 11 October 2007.

[7] Olofsson, U. G. M. "Voltage Quality in the Context of EMC, Paper 21R4-4," in 2009 International Symposium on Electromagnetic Compatibility, Kyoto, Japan, 20 - 24 July 2009.

[8] IEC 600 50-161 Std, "International Electrotechnical Vocabulary (IEV)".

[9] Olofsson, M. "Low Frequency EMC and Power Quality," Incompliance Magazine, pp. 34-41, April 2012.

[10] Amoruso F. L. V., "Thundercloud pre-stroke electrostatic modeling," Journal of electrostatics, vol. 56, no. 2, pp. 255-276, 2002.

[11] Ianoz M., "Lightning and EMC," in in The lightning flash, V. Cooray Ed., IEE Power \& Energy Series, London, 2003.

[12] Amoruso A. F. L. V., "Lightning precursory electrostatics and a critique to some protection systems," in IEEE Power Tech Conference Proceedings 2003, Bologna, 2003.

[13] Berta I., "Static control," Journal of electrostatics , vol. 63, no. 6, pp. 679-685, 2005.

[14] Chowdhuri P., Electromagnetic transients in power systems, New York: John Wiley \& 
Sons, 1996.

[15] Carpenter Jr, Roy B., and Mark M. Drabkin., "Protection against direct lightning strokes by charge transfer system," in IEEE International Symposium on Electromagnetic Compatibility Vol. 2, Seatle, 1998.

[16] Ette, A. I. I., J. I. Aina, and E. U. Utah. "Point discharge characteristics and charge configuration in thunderclouds." Electrical Processes in Atmospheres. Steinkopff, 1977. 389-393.

[17] Horvath T., "Standardization of lightning protection based on the physics or on the tradition?," Journal of electrostatics, vol. 60, no. 2, pp. 265-275, 2004.

[18] Mazzetti C., "Principles of protection of structures against lightning," V. Cooray Ed., IEE Power \& Energy Series 34, London, 2003.

[19] D'Alessandro, F., and J. R. Gumley., "A Collection Volume Method" for the placement of air terminals for the protection of structures against lightning," Journal of electrostatics, vol. 50, no. 4, p. 2001, 279-302.

[20] Y. Takahashi, "Thickness and luminosity distribution of positive corona sheath in air," Journal of Physics D: Applied Physics, vol. 15, no. 4, p. 639, 1982.

[21] Rust, W. David, and C. B. Moore., "Electrical Conditions Near the Bases of Thunderclouds in the Southwestern United States," Steinkopff, 1977, pp. 354-361.

[22] Amoruso, Vitantonio, and F. Lattarulo. "The electromagnetic field of an improved lightning return-stroke representation." Electromagnetic Compatibility, IEEE Transactions on 35.3 (1993): 317-328.

[23] Bleistein, Norman, and Jack K. Cohen. "Nonuniqueness in the inverse source problem in acoustics and electromagnetics." Journal of Mathematical Physics18 (1977): 194.

[24] Amoruso, V., and F. Lattarulo. "Remarks on lightning electromagnetics."Engineering analysis with boundary elements 27.4 (2003): 375-387.

[25] Cooray, Vernon. "Predicting the spatial and temporal variation of the electromagnetic fields, currents, and speeds of subsequent return strokes," Electromagnetic Compatibility, IEEE Transactions on 40.4 (1998): 427-435.

[26] Cveti' c J.M., Stani' c B.V., Heidler F., "Properties of the channel discharge function in the generalized lightning travelling current source return stroke model", Proc. 13th Zurich Symposium of Electromagnetic Compatibility, Zurich, Feb 16-18, 1999. 
[27] Gardet, Robert L. Lightning electromagnetics. CRC PressI Llc, 1990.

[28] Cvetic, Jovan, Fridolin Heidler, and Adolf Schwab. "Light intensity emitted from the lightning channel: comparison of different return stroke models." Journal of Physics D: Applied Physics 32.3 (1999): 273.

[29] De la Rosa, F., et al. "Discussion about the inducing effects of lightning in an experimental power distribution line in Mexico." Power Delivery, IEEE Transactions on 3.3 (1988): 1080-1089.

[30] Heidler, F., J. M. Cvetic, and B. V. Stanic. "Calculation of lightning current parameters." Power Delivery, IEEE Transactions on 14.2 (1999): 399-404.

[31] Le Vine, D. M., and R. Meneghini. "Electromagnetic fields radiated from a lightning return stroke: Application of an exact solution to Maxwell's equations."Journal of Geophysical Research: Oceans (1978-2012) 83.C5 (1978): 2377-2384.

[32] Nucci, Carlo Alberto, et al. "On lightning return stroke models for LEMP calculations." 19th International Conference on Lightning Protection, Graz. 1988.

[33] Nucci, C. A., Rachidi, F., Ianoz, M. V., \& Mazzetti, C. (1993). Lightning-induced voltages on overhead lines. Electromagnetic Compatibility, IEEE Transactions on, 35(1), $75-86$.

[34] Paul C.R., "Crosstalk", inHandbook of electromagnetic compatibility, R. Perez Ed., Academic Press, New York, 1995.

[35] Plumer J.A., "Lightning", inHandbook of electromagnetic compatibility, R. Perez Ed., Academic Press, New York, 1995.

[36] Tesche F.M., Ianoz M.V., Karlsson T.,EMC analysis methods and computational models, John Wiley, New York, 1997.

[37] Thottappillil R., Uman M.A., Theethayi N., "Electric and magnetic fields from a semiinfinite antenna above a conducting plane", J. Electrostat.,62(2004) 209

[38] Rakov, V. Vladimir A., and Martin A. Uman. Lightning: physics and effects. Cambridge University Press, 2003.

[39] Albertson, V. D., et al. "Solar-induced-currents in power systems: cause and effects." Power Apparatus and Systems, IEEE Transactions on 2 (1973): 471-477.

[40] Boteler, D. H., et al. "Characteristics of geomagnetically induced currents in the BC Hydro $500 \mathrm{kV}$ system." Power Apparatus and Systems, IEEE Transactions on 6 (1982): 1447-1456. 
[41] Pirjola, Risto. "On currents induced in power transmission systems during geomagnetic variations." Power Apparatus and Systems, IEEE Transactions on10 (1985): 2825-2831.

[42] Bolduc L., Aubin J., "Effects of direct currents in power transformers - Part I - A general theoretical approach", Electr. Pow. Sys. Res., (1), (1977a).

[43] Albertson V.D., Kappenman J.G., Mohan N., Skarbakka G.A., "Load-flow studies in the presence of geomagnetically-induced currents", IEEE Trans. on Power Apparatus and Systems, PAS-100(2)Feb. (1981).

[44] Aspnes J.D., Akasofu S.I., "Effect of solar induced current on autotransformer tertiary windings", IEEE Trans. on Power Apparatus and Systems,PAS-101(3)Mar. (1981).

[45] Boteler D.H., Shier R.M., Watanabe T., Horita R.E., "Effects of geomagnetically induced currents in the B.C. Hydro $500 \mathrm{kV}$ system", IEEE Trans. on Power Delivery, 4(1)Jan. (1989)

[46] Ringlee R.J., Stewart J.R., "Geomagnetic effects on power systems", IEEE Power Eng. Rev., July (1989)

[47] Albertson V.D., Miske S.A., Thorson Jr. J.M., "The effects of geomagnetic storms on electrical power systems", IEEE Trans. on Power Apparatus and Systems, PAS-93(4) July/August(1974).

[48] CIGRÉ Working Group 36.02, Report on "Electromagnetic Compatibility with Telecommunication Circuits, Low Voltage Networks and Metallic Structures", Guide on the Influence of High Voltage AC Power Systems on Metallic Pipelines, 1995.

[49] DIN EN 12954-2001 European Standard, Cathodic Protection of Buried or Immersed Metallic Structures - General Principles and Application for Pipelines, 2001.

[50] Ying, Zhang, and Gao Ben-Qing. "Propagation of cylindrical waves in media of timedependent permittivity." Chinese Physics Letters 22.2 (2005): 446.

[51] Williams, Tim. EMC for product designers. Newnes, 2001.

[52] Buccella, C., M. Feliziani, and V. Fuina. "ELF magnetic field mitigation by active shielding." Industrial Electronics, 2002. ISIE 2002. Proceedings of the 2002 IEEE International Symposium on. Vol. 3. IEEE, 2002.

[53] Buccella, Concettina, and Mauro Feliziani. "A hybrid model to compute the effects of a direct lightning stroke on three-dimensional structures." Magnetics, IEEE Transactions on 39.3 (2003): 1586-1589.

[54] Payne, L. E. "Isoperimetric inequalities for eigenvalues and their applications."Autovalori e autosoluzioni. Springer Berlin Heidelberg, 2011. 107-167. 
[55] Sten, JC-E. "Magnetic moment and surface dipole distributions of circular holes in a conducting screen." Electromagnetic Compatibility, IEEE Transactions on41.4 (1999): 290-297.

[56] Cerri, G., and R. Russo. "Hybrid MFIE/FDTD method for analysis of metallic enclosures with slots." Electronics Letters 39.6 (2003): 502-504.

[57] Bollen, Math HJ. Understanding power quality problems. Vol. 3. New York: IEEE press, 2000.

[58] Olofsson, Magnus. "Power quality and EMC in smart grid." Electrical Power Quality and Utilization, 2009. EPQU 2009. 10th International Conference on. IEEE, 2009.

[59] Haixue, Lin. "Main problems of modern power quality." POWER SYSTEM TECHNOLOGY-BEIJING- 25.10 (2001): 5-12.

[60] Moreno-Munoz, Antonio. Power quality: mitigation technologies in a distributed environment. Springer, 2007.

[61] Olofsson, M., and U. Grape. "Framework for Electromagnetic Compatibility in Electric Power Systems." VIII International Symposium and Exhibition on Electromagnetic Compatibility and Electromagnetic Ecology, St. Petersburg, Russia. 2009.

[62] Tokuda, M. "History and Recent Topics of IEC TC 77 (EMC Standards)."Workshop at 2009 International Symposium on Electromagnetic Compatibility, Kyoto, Japan. 2009.

[63] Norberg, P., et al. "Introducing Network Strength to Handle Power Quality in System Planning." Cigré General Session, Paris (2008).

[64] Erinmez, I. A., et al. "Application of modeling techniques to assess geomagnetically induced current risks on the NGC transmission system,"CIGRE Paper (2002): 39-304.

[65] Smoleński, R. "Selected conducted electromagnetic interference issues in distributed power systems." (2009): 383-393.

[66] Smolenski, Robert, et al. "EMI generated by Power Electronic Interfaces in Smart Grids." Electromagnetic Compatibility (EMC EUROPE), 2012 International Symposium on. IEEE, 2012.

[67] Chung, H., S. Y. R. Hui, and K. K. Tse. "Reduction of power converter EMI emission using soft-switching technique." Electromagnetic Compatibility, IEEE Transactions on 40.3 (1998): 282-287.

[68] Kuisma, M. "Variable frequency switching in power supply EMI-control: an overview." Aerospace and Electronic Systems Magazine, IEEE 18.12 (2003): 18-22. 
[69] Mainali, K.; Oruganti, R., "Conducted EMI Mitigation Techniques for Switch-Mode Power Converters: A Survey," Power Electronics, IEEE Transactions on, vol.25, no.9, pp.2344,2356, Sept. 2010

[70] M. Nave, Power Line Filter Design for Switched-Mode Power Supplies. New York: Springer-Verlag, 1991.

[71] D. M. Mitchell, "Power line filter design considerations for DC-DC converters," IEEE Ind. Appl. Mag., vol. 5, no. 6, pp. 16-26, Nov./Dec. 1999.

[72] W. Zhang, M. T. Zhang, F. C. Lee, J. Roudet, and E. Clavel, "Con-ducted EMI analysis of a boost PFC circuit," inProc. IEEE Appl. Power Electron. Conf., 1997, pp. 223-229.

[73] S. Wang, F. C. Lee, and W. G. Odendaal, "Improving the performance of boost PFC EMI filters," inProc. IEEE Appl. Power Electron. Conf.,2003, pp. 368-374.

[74] J. C. Crebier, M. Brunello, and J. P. Ferrieux, "A new method for EMI study in boost derived PFC rectifiers,” inProc. IEEE Power Electron.Spec. Conf., 1999, pp. 855-860.

[75] T. Ninomiya, M. Nakahara, H. Tajima, and K. Harada, "Backward-noise generation in forward DC-to-DC converters, ’IEEE Trans. Power Electron., vol. PE-2 no. 3, pp. 208217, Jul. 1987.

[76] F. Lin, "Reduction of power supply EMI emission by switching frequency modulation," M.S. thesis, Virginia Polytechnic Institute and State University Blacksburg, VA, 1992.

[77] J. Paramesh and A. von Jouanne, "Use of sigma-delta modulation to con-trol EMI from switch-mode power supplies," IEEE Trans. Ind. Electron., vol. 48, no. 1, pp. 111-117, Feb. 2001.

[78] T. Ninomiya and K. Harada, "Common-mode noise generation in a DC-to-DC converter," IEEE Trans. Aerosp. Electron. Syst., vol. AES-16, no. 2, pp. 130-137, Mar. 1980.

[79] M. J. Schutten, "EMI causes, measurement, and reduction techniques for switch-mode power converters,” inProc. IEEE Appl. Power Electron.Conf. Prof. Educ. Semin., 2006.

[80] R. L. Ozenbaugh, EMI Filter Design. Boca Raton, FL: CRC Press, 2000.

[81] R. A. Southwick, "Impedance characteristics of single-phase power-lines," inProc. IEEE EMC Symp., 1973, pp. 241-252.

[82] F. Y. Shih, D. Y. Chen, Y. P. Wu, and Y. T. Chen, "A procedure for designing EMI filters for AC line applications," IEEE Trans. Power Electron., vol. 11, no. 1, pp. 170181, Jan. 1996. 
[83] B. Mammano and B. Carsten, "Understanding and optimizing electro-magnetic compatibility in switch mode power supplies," in Proc. Uni-trode (TI) Power Supply Design Semin. Topics, 2002, SEM1500.

[84] L. M. Schneider, "Noise source equivalent circuit model for off-line converters and its use in input filter design," inProc. Powercon 10, 1983, pp. 1-11, Paper C-1.

[85] L. E. LaWhite and M. F. Schlecht, "Active filters for $1 \mathrm{MHz}$ power cir-cuits with strict input/output ripple requirements," in Proc. IEEE Power Electron. Spec. Conf., 1986, pp. 255-263.

[86] N. K. Poon, J. C. P. Liu, C. K. Tse, and M. H. Pong, "Techniques for input ripple current cancellation: Classification and implementation," IEEE Trans. Power Electron., vol. 15 , no. 6, pp. 1144-1152, Nov. 2000.

[87] L. Rossetto, S. Buso, and G. Spiazzi, "Conducted EMI issues in a 600-W single-phase boost PFC design,” IEEE Trans. Ind. Appl., vol. 36, no. 2, pp. 578-585, Mar./Apr. 2000.

[88] L. Rossetto, G. Spiazzi, and P. Tenti, "Boost PFC with 100-Hz switching frequency providing output voltage stabilization and compliance with EMC standards," IEEE Trans. Ind. Appl., vol. 36, no. 1, pp. 188-193, Jan./Feb. 2000.

[89] F. Lin and D. Y. Chen, "Reduction of power supply EMI emission by switching frequency modulation," IEEE Trans. Power Electron., vol.9, no. 1, pp. 132-137, Jan. 1994.

[90] F. Mihalic and D. Kos, "Reduced conductive EMI in switched-mode DC-DC power converters without EMI filters: PWM versus randomized PWM," IEEE Trans. Power Electron., vol. 21, no. 6, pp. 1783-1794, Nov. 2006.

[91] J. Mahdavi, M. Tabandeh, and A. K. Shahriari, "Comparison of conducted RFI emission from different unity power factor AC/AC converters," inProc. IEEE Power Electron. Spec. Conf., 1996, pp. 1979-1985.

[92] Revol, B., et al. "EMI study of a three phase inverter-fed motor drives." Industry Applications Conference, 2004. 39th IAS Annual Meeting. Conference Record of the 2004 IEEE. Vol. 4. IEEE, 2004.

[93] Crebier, J. C., M. Brunello, and J. P. Ferrieux. "A new method for EMI study in boost derived PFC rectifiers." Power Electronics Specialists Conference, 1999. PESC 99. 30th Annual IEEE. Vol. 2. IEEE, 1999.

[94] Kodali, V. Prasad, and V. Prasad. Engineering electromagnetic compatibility: Principles, measurements, technologies, and computer models. IEEE, 2001.

[95] Montrose, Mark I. EMC and the printed circuit board: design, theory, and layout made simple. Vol. 6. John Wiley \& Sons, 2004. 
[96] Zhong, Yu-Lin, et al. "HF circuit model of conducted EMI of ground net based on PEEC." Zhongguo Dianji Gongcheng Xuebao(Proceedings of the Chinese Society of Electrical Engineering). Vol. 25. No. 17. 2005.

[97] Zhu, Huibin, et al. "Analysis of conducted EMI emissions from PWM inverter based on empirical models and comparative experiments." Power Electronics Specialists Conference, 1999. PESC 99. 30th Annual IEEE. Vol. 2. IEEE, 1999.

[98] Mainali, Krishna, and Ramesh Oruganti. "Simple analytical models to predict conducted EMI noise in a power electronic converter." Industrial Electronics Society, 2007. IECON 2007. 33rd Annual Conference of the IEEE. IEEE, 2007.

[99] Liu, D. H., and J. G. Jiang. "High frequency characteristic analysis of EMI filter in switch mode power supply (SMPS)." Power Electronics Specialists Conference, 2002. pesc 02. 2002 IEEE 33rd Annual. Vol. 4. IEEE, 2002.

[100] Pei, Xuejun, et al. "Analytical estimation of common mode conducted EMI in PWM inverter." Industry Applications Conference, 2004. 39th IAS Annual Meeting. Conference Record of the 2004 IEEE. Vol. 4. IEEE, 2004.

[101] Chen, Chingchi, and Xingyi Xu. "Modeling the conducted EMI emission of an electric vehicle (EV) traction drive." Electromagnetic Compatibility, 1998. 1998 IEEE International Symposium on. Vol. 2. IEEE, 1998.

[102] Poljak, Dragan. Advanced modeling in computational electromagnetic compatibility. John Wiley \& Sons, 2007.

[103] Zhu, Huibin, et al. "Modeling-based examination of conducted EMI emissions from hard and soft-switching PWM inverters." Industry Applications, IEEE Transactions on 37.5 (2001): 1383-1393.

[104] Sevgi, Levent. "EMC and BEM engineering education: physics-based modeling, hands-on training, and challenges." Antennas and Propagation Magazine, IEEE45.2 (2003): 114-119.

[105] Carpes Jr, Walter P., et al. "TLM and FEM methods applied in the analysis of electromagnetic coupling." Magnetics, IEEE Transactions on 36.4 (2000): 982-985.

[106] Dixon, David S., Michael Obara, and Nicholas Schade. "Finite-element analysis (FEA) as an EMC prediction tool." Electromagnetic Compatibility, IEEE Transactions on 35.2 (1993): 241-248.

[107] Laroussi, R., and G. I. Costache. "Finite-element method applied to EMC problems [PCB environment]." Electromagnetic Compatibility, IEEE Transactions on 35.2 (1993): 178-184. 
[108] Rosales, Andrew, Ali Sarikhani, and Osama A. Mohammed. "Evaluation of radiated electromagnetic field interference due to frequency swithcing in PWM motor drives by 3D finite elements." Magnetics, IEEE Transactions on 47.5 (2011): 1474-1477.

[109] Nejadpak, A., A. Sarikhani, and O. A. Mohammed. "Analysis of Radiated EMI and Noise Propagation in Three-Phase Inverter System Operating Under Different Switching Patterns." IEEE Transactions on Magnetics 49.5 (2013): 2213.

[110] Arash Nejadpak, and Osama Mohammed. "Physics-Based Modeling of Power Converters from Finite Element Electromagnetic Field Computations." (2013): 1-1.

[111] A. Testa, et al, "Interharmonics: theory and modeling," IEEE Trans. on Power Delivery, V22, No.4, pp. 2335-2348, 2007.

[112] S. Fiori, A. Faba, L. Albini, E. Cardelli, and P. Burrascano,"Numerical modeling for the localization and the assessment of electromagnetic field sources," IEEE Trans. Magn., Vol. 39, No. 3, pp. 1638-1641, May2003.

[113] T. Zhu, "Exact harmonics/interharmonics calculation using adaptive windows width," IEEE Trans. on Power Delivery, Vol. 22, No. 4, pp.2279-2288, Oct. 2007.

[114] M. K. Karimi and M. R. Iravani, "Measurement of harmonics/inter-harmonics of time-varying frequencies," IEEE Trans. on Power Delivery, Vol. 20, No. 1, pp. 23-31, Jan. 2005.

[115] A. B. Nassif, Jing Yong, H. Mazin, X. Wang, and Wilsun Xu, "An impedance-based approach for identifying interharmonic sources," IEEE Trans. on Power Delivery, Vol. 26, No. 1, pp. 333-340, 2011.

[116] A. Canova, F. Freschi, M. Repetto, and M. Tartaglia. "Identification of an equivalentsource system for magnetic stray field evaluation," IEEE Trans. on Power Delivery, Vol. 24, No. 3, pp. 1352-1358, 2009.

[117] Whei-Min Lin et al, "Multiple harmonic source detection and equipment identification with cascade correlation network," IEEE Trans. on Power Delivery, Vol. 20, No.3, pp. 2166-2173, 2005

[118] D. Srinivasan, W. S. Ng, and A. C. Liew, "Neural-network-based signature recognition for harmonic source identification," IEEE Trans. on Power Delivery, Vol. 21, No. 1, pp. 398-405, 2006.

[119] I. Rodriguez, R. Alves, and V. Guzman, "Analysis of air gap flux to detect induction motor faults," in Proc. 41st International UPEC, Vol. 2, pp. 690-694, Sep. 6-8, 2006.

[120] R. Romary, R. Corton, D. Thailly, and J. F. Brudny, "Induction machine fault diagnosis using an external radial flux sensor," The European Physical Journal Applied Physics, Vol. 32, No. 2, pp. 125-132, 2005. 
[121] Chadebec, Olivier, Viet Phuong Bui, Pierre Granjon, L-L. Rouve, Nicolas Le Bihan, and J-L. Coulomb, "Rotor fault detection of electrical machines by low frequency magnetic stray field analysis," IEEE 5th International Symposium on Diagnostics for Electric Machines, Power Electronics and Drives (SDEMPED), pp. 1-6. 2005.

[122] R. Romary, S. Jelassi, J.F. Brudny, "Stator-Interlaminar-Fault Detection Using an External-Flux-Density Sensor," IEEE Transactions on Industrial Electronics, Vol.57, No.1, pp.237-243, Jan. 2010.

[123] Didier, Gaëtan, et al. "A new approach to detect broken rotor bars in induction machines by current spectrum analysis." Mechanical systems and signal processing, Vol. 21, No.2, pp. 1127-1142, 2007.

[124] A. Ceban, R. Pusca, R. Romary, "Eccentricity and broken rotor bars faults - Effects on the external axial field," 19th International Conference on Electrical Machines (ICEM), pp.1-6, 6-8 Sept. 2010.

[125] R. Romary, S. Jelassi, J.F. Brudny, M. Mimouni, "Detection of short-circuits between stator laminations of electrical machine by analysis of external magnetic field," 18th International Conference on Electrical Machines (ICEM), pp.1-5, 6-9 Sept. 2008.

[126] A. Sarikhani, O.A. Mohammed, "Inter-turn fault detection in PM synchronous machines by physics-based EMF estimation," IEEE Energy Conversion Congress and Exposition (ECCE), pp.1254-1261, 15-20 Sept. 2012.

[127] J. Cusido, J. A. Rosero, J. A. Ortega, A. Garcia, and L. Romeral. "Induction motor fault detection by using wavelet decomposition on dq0 components." IEEE International Symposium on Industrial Electronics 2006, Vol. 3, pp. 2406-2411. 2006.

[128] Jordi Cusido, Luis Romeral, Juan A. Ortega, Javier A. Rosero, and A. Garcia Espinosa, "Fault detection in induction machines using power spectral density in wavelet decomposition," IEEE Transactions on Industrial Electronics, Vol. 55, No. 2, pp. 633643, Feb. 2008.

[129] Reduction of a ship's Magnetic Field Signature, John J. Claypool, published by Morgan \& Claypool 2008

[130] Degano, M.; Zanchetta, P.; Clare, J.; Empringham, L.; , "HF induction motor modeling using genetic algorithms and experimental impedance measurement," Industrial Electronics (ISIE), 2010 IEEE International Symposium on , vol., no., pp.1296-1301, 4-7 July 2010

[131] Boglietti, A.; Carpaneto, E., "An accurate high frequency model of AC PWM drive systems for EMC analysis," Industry Applications Conference, 2001. Thirty-Sixth IAS Annual Meeting. Conference Record of the 2001 IEEE, vol.2, no., pp.1111-1117 vol.2, 30 Sep-4 Oct 2001 
[132] Roger D., Henneton A. "II-16 AC Motor Magnetic Signature: Contribution of Stator Lamination," international symposium on Electromagnetic Fields in Electrical Engineering, Maribor, Slovenia, September 18-20, 2003

[133] J. C. Maxwell: A Treatise on Electricity and Magnetism, Oxford University Press, 1865, 1904 (also,Dover, New York, 1954, republication of the 3rd ed.,Clarendon Press, 1891).

[134] J. A. Aharoni: An Introduction to Their Theory,Oxford University Press, 1946.

[135] R. C. Hansen: Early computational electromagnetics, IEEE Antennas Propagat. Mag., Vol. 38, No. 3, June 1996, pp 60-61.

[136] M. G. Andreasen: Scattering from bodies of revolution, IEEE Trans. Antennas Propagat.,Vol. 13, March 1965, p 303.

[137] K. K. Mei: On the integral equation of thin wire antennas, IEEE Trans. Antennas Propagat., Vol. 13, 1965, pp 59-62.

[138] J. H. Richmond: Digital computer solutions of the rigorous equations for scattering problems, Proc. IEEE, Vol. 53, August 1965, pp 796-804.

[139] K. S. Yee: Numerical solution of initial boundary value problems involving Maxwell's equations in isotropic media, IEEE Trans. Antennas Propagat., Vol. 14, May 1966, pp 302-307.

[140] E. P. Sayre, R. F. Harrington: Transient response of straight wire scatterers and antennas, Int. Antennas and Propogat. Symp., Boston, MA, September 1968.

[141] E. K. Miller, A. J. Poggio, G. J. Burke: An integro-differential equation technique for the time-domain analysis of thin wire structures-I. The numerical method, J. Comput. Phys., Vol. 12, No. 1, May 1973, pp 24-48.

[142] E. K. Miller, A. J. Poggio, G. J. Burke: An integro-differential equation technique for the time-domain analysis of thin wire structures - II. Numerical results, J. Comput. Phys., Vol. 12, No. 2, June 1973, pp 210-233.

[143] Gérard Meunier, ed. The finite element method for electromagnetic modeling. Vol. 33. Wiley. com, 2010.

[144] William Hall, S. Boundary Element Method. Springer Netherlands, 1994.

[145] D. Poljak, C. A. Brebbia: Boundary Element Methods for Electrical Engineers, WIT Press, Southampton-Boston, 2005.

[146] P. R. Foster: CAD for antenna systems, IEE Electronics \& Commun. Eng. J., Vol. 12, No. 1, February 2000, pp 3-14. 
[147] J. L. Volakis, L. C. Kempel: Electromagnetics: computational methods and considerations, IEEE Computational Sci. \& Eng., 1995, pp 42-57.

[148] Andrew Peterson, Scott L. Ray, and Raj Mittra. Computational methods for electromagnetics. Vol. 24. New York: IEEE press, 1998.

[149] M. N. O. Sadiku: Numerical Techniques in Electromagnetics, 2nd ed., CRC Press, Boca Raton, 2001.

[150] F. Tesche, M. Ianoz, T. Karlsson: EMC Analysis Methods and Computational Models, John Wiley \& Sons, New York, 1997.

[151] D. Poljak: Electromagnetic Modeling of Wire Antenna Structures, WIT Press, Southampton-Boston, 2002.

[152] D. Poljak, C. Y. Tham: Integral Equation Techniques in Transient Electromagnetics, WIT Press, Southampton-Boston, 2003.

[153] D. Poljak (Ed.): Time Domain Techniques in Computational Electromagnetics, WIT Press, Southampton-Boston, 2004.

[154] F. J. K. Buesink: Engineering Electromagnetic Compatibility, EMV 99, Workshop Notes, Dusseldorf, Germany, March 1999.

[155] Richard Courant, and David Hilbert. Methods of mathematical physics. Vol. 1. Wiley, 2008.

[156] M. Kchikach, R. Lee, H.F. Weinner, Y. Zidani, Y.S. Yuan, and Z.M. Qian, "Study of the resonance phenomenon in switching mode power supply (SMPS)," Power Electronics Specialists Conference, 2004. PESC 04. 2004 IEEE 35th Annual, vol.4, no., pp. 30163020 Vol.4, 2004.

[157] S.V.Kulkarni, "Transformer Engineering: Design and Practice," Marcel Dekker (Taylor \& Francis Group), New York, May 2004.

[158] S. Hashino and T. Shimizu, "Characterization of parasitic impedance in a power electronics circuit board using TDR," Power Electronics Conference (IPEC), 2010 International, vol., no., pp.900-905, 21-24 June 2010.

[159] Tin-ho Li, Jianjing Wang, and H.S.-H. Chung, "Effect of parasitic elements in a power converter on the switching performance of a MOSFET-snubber-diode configuration," Applied Power Electronics Conference and Exposition (APEC), 2011 Twenty-Sixth Annual IEEE , vol., no., pp.364-371, 6-11 March 2011

[160] M.R. Abdul-Gaffoor, H.K. Smith, A.A. Kishk, and A.W. Glisson, "Simple and efficient full-wave modeling of electromagnetic coupling in realistic RF multilayer PCB 
layouts," Microwave Theory and Techniques, IEEE Transactions on , vol.50, no.6, pp.1445-1457, Jun 2002.

[161] Jong-Gwan Yook, L.P.B. Katehi, K.A. Sakallah, R.S. Martin, L. Huang, and T.A. Schreyer, "Application of system-level EM modeling to high-speed digital IC packages and PCBs," Microwave Theory and Techniques, IEEE Transactions on, vol.45, no.10, pp.1847-1856, Oct 1997.

[162] W. Chen, X. Yang, and Z. Wang, "Application of Wavelets and Auto-CorrelationFunction For Cancellation of High-Frequency EMI Noise," ACES Journal, vol. 24, no.3, pp. 332 - 336, June 2009.

[163] A. Bhargava, D. Pommerenke, K.W. Kam, F. Centola, and C. Lam, "DC-DC Buck Converter EMI Reduction Using PCB Layout Modification," Electromagnetic Compatibility, IEEE Transactions on, vol.53, no.3, pp.806-813, Aug. 2011

[164] R. W. Erickson, D. Maksimovic, Fundamentals of Power Electronics, Springer, 912 p., 2nd ed., 2001.

[165] J. Yunas, N.A. Rahman, Lem Tien Chai, and B.Y. Majlis, "Study of coreless planar inductor at high operating frequency," Semiconductor Electronics, 2004. ICSE 2004. IEEE International Conference on , vol., no., pp. 5 pp., 7-9 Dec. 2004.

[166] J. Vanek, I. Szendiuch, and J. Hladik, "Optimization of Properties of Planar Spiral Inductors," Electronics Technology, 30th International Spring Seminar on , vol., no., pp.235-238, 9-13 May 2007.

[167] Jae-Jin Lee, Yang-Ki Hong, Seok Bae, Ji-Hoon Park, J. Jalli, G.S. Abo, R. Syslo, Byoung-Chul Choi, and G.W. Donohoe, "High-Quality Factor Ni-Zn Ferrite Planar Inductor," Magnetics, IEEE Transactions on , vol.46, no.6, pp.2417-2420, June 2010.

[168] A. Eroglu, "Microstrip Inductor Design and Implementation," ACES Journal, vol.25, no. 9, pp. 794 - 800, September 2010.

[169] Dong Hui, Zhu Yisheng, and Zhao Baishan, "Research on the electromagnetic radiation of a PCB planar inductor," Microwave Conference Proceedings, 2005. APMC 2005. Asia-Pacific Conference Proceedings, vol.1, no., pp. 3 pp., 4-7 Dec. 2005.

[170] Z. Jonsener, "A new calculation for designing multilayer planar spiral inductors," Electronics Design Strategy, News. EDN, July 29, 2010.

[171] N. Y. Abed and O. A. Mohammed, "Frequency dependent coupled field-circuit modeling of armored power cables using finite elements," Electromagnetic Field Computation (CEFC), 2010 14th Biennial IEEE Conference on, vol., no., pp.1-1, 9-12 May 2010. 
[172] M. R. Barzegaran, and M. Mirzaie. "Detecting the position of winding short circuit faults in transformer using high frequency analysis." European Journal of Scientific Research 23 (2008): 644-658.

[173] L. Satish and S. K. Sahoo, "An effort to understand what factors affect the transfer function of a two-winding transformer," Power Delivery, IEEE Transactions on, vol.20, no.2, pp. 1430- 1440, April 2005.

[174] N.N. Rao, Elements of Engineering Electromagnetics, Prentice Hall, 6th Edition, pp. 321-324, 2004.

[175] Fawwaz T. Ulaby, Fundamental of Applied Eectromagnetics, Prentice Hall, 5th Edition, pp. 321-324, 2006.

[176] M. R. Barzegaran, A. Nejadpak, and O. A. Mohammed, "High Frequency Electromagnetic Field Model for the Evaluation of Parasitic Elements in Power Converters," 27th Annual Review of Progress in Applied Computational Electromagnetics, ACES March 27-31, pp 623-629 2011.

[177] C. R. Paul, "Inductance Loop and Partial," published by John Wiley and Sons, 2010.

[178] McLyman, Colonel William T. Transformer and inductor design handbook. Vol. 121. CRC press, 2004.

[179] Y. Saad, Iterative Methods for Sparse Linear Systems, 2nd edition, Society for Industrial and Applied Mathematics, 2003.

[180] A. Nejadpak, M.R. Barzegaran, A. Sarikhani, and O.A. Mohammed, "Design of planar inductor based Z-source inverter for residential alternate energy sources," Applied Power Electronics Conference and Exposition (APEC), 2011 Twenty-Sixth Annual IEEE , vol., no., pp.1698-1703, 6-11 March 2011.

[181] D. E. Goldberg, Genetic Algorithms in Search, Optimization and Machine Learning, Addison-Wesley Longman Publishing Co., Inc. Boston, MA, USA, 1989.

[182] O.A. Mohammed, S. Ganu, "FE-Circuit Coupled Model of Electric Machines for Simulation and Evaluation of EMI Issues in Motor Drives," IEEE Transactions on Magnetics, vol. 46, no. 8, pp. 3389-3392, Aug. 2010.

[183] O. A. Mohammed, S. Ganu, N. Abed, S. Liu, Z. Liu, "High frequency phase variable model of electric machines from electromagnetic field computation," Applied Computational Electromagnetics Society (ACES) Journal, vol. 22, no. 1, pp. 164-171, Mar. 2007.

[184] G. Coat, A. Foggia, JP. Bongiraud, P. Thiec, "Electromagnetic Signature of Induction Machines," IEEE Transaction on Energy Conversion, vol. 14, no. 3, pp. 628-632, Sep. 1999. 
[185] H. Hasper, "Reduction of Magnetic Stray field from Squirrel-cage Induction Motors," COMBIMAC document, Feb 1991.

[186] X. Brunotte, G. Meunier and J.P. Bongiraud, "Ship Magnetizations Modeling by the Finite Element Method," IEEE Transactions on Magnetics, vol. 29, no. 2, pp. 1970-1975, Mar. 1993.

[187] G.G. Karady, Sh. FI. Berisha, J.A. Demcko and M. Samotyj, "Variable Speed Motor Drive Generated Magnetic Fields," IEEE Transactions on Power Delivery, vol. 9, no. 3, pp. 1639-1646, July 1994.

[188] A. Sarikhani, O. A. Mohammed, "Coupled electromagnetic field computation with external circuit for the evaluation the performance of electric motor designs," Applied Computational Electromagnetics Society (ACES) Journal, vol. 26, no. 12, pp. 997-1006, Dec. 2011.

[189] J. H. Alwash, L. J. Qaseer, "Three-dimension finite element analysis of a helical motion induction motor," Applied Computational Electromagnetics Society, (ACES) Journal, vol. 25, no. 8, pp. 703-712, Aug. 2010.

[190] A. kashefi kaviani, G.H. Riahy, SH.M. Kouhsari, "Optimal design of a reliable hydrogen-based stand-alone wind/PV generating system, considering component outages," Elsevier, Renewable Energy Vol.34, pp.2380-2390, April 2009

[191] R.R. Tan, "Hybrid evolutionary computation for the development of pollution prevention and control strategies," J Clean Prod 2007;15:pp. 902-906

[192] American National Standard for Testing Unlicensed Wireless Devices," ANSI C63.10-2009, pp.C1,119, Sept. 102009

[193] American National Standard Dictionary for Technologies of Electromagnetic Compatibility (EMC), Electromagnetic Pulse (EMP), and Electrostatic Discharge (ESD)," ANSI C63.14-1998, 1998

[194] American National Standard Dictionary for Technologies of Electromagnetic Compatibility (EMC), Electromagnetic Pulse (EMP), and Electrostatic Discharge (ESD) (Dictionary of EMC/EMP/ESD Terms and Definitions)," ANSI C63.14-1992, 1992

[195] American National Standard Dictionary of Electromagnetic Compatibility (EMC) including Electromagnetic Environmental Effects (E3)," ANSI Std C63.14-2009, pp.146, Oct. 232009

[196] IEEE Standard for Power Line Communication Equipment--Electromagnetic Compatibility (EMC) Requirements--Testing and Measurement Methods," IEEE Std 1775-2010, pp.1-66, Jan. 72011 
[197] IEEE Recommended Practice--Adoption of IEC 61000-4-15:2010, Electromagnetic compatibility (EMC)--Testing and measurement techniques--Flickermeter--Functional and design specifications," IEEE Std 1453-2011 , pp.1-58, Oct. 212011

[198] American National Standard for Electromagnetic Noise and Field Strength Instrumentation, $10 \mathrm{~Hz}$ to $40 \mathrm{GHz}$ - Specifications," ANSI Std C63.2-1996, pp.0_1, 1996

[199] IEEE Recommended Practice for Validation of Computational Electromagnetics Computer Modeling and Simulations," IEEE Std 1597.2-2010 , , pp.1-124, Feb. 252011

[200] Department of defence interface standard, Requirements for The Control of Electromagnetic Interference Characteristics of Subsystems And Equipment, DEC 2007

[201] A. Sarikhani, M. Barzegaran, and O. A. Mohammed, "Optimum equivalent models of multi-source systems for the study of electromagnetic signatures and radiated emissions from electric drives," IEEE Trans. on Magnetic, vol. 48, no. 2, pp. 1011-1014, Feb. 2012.

[202] M Vetterli and C. Herley "Wavelets and Filter Banks: Theory and Design," IEEE Trans. on Signal Processing, Vol.40, no.9, pp.2207-2232, Sep 1992.

[203] RR Coifman, Y Meyer and MV. Wickerhauser "Wavelet analysis and signal processing. In Wavelets and their Applications," Jones and Bartlett: Boston, MA, pp.153-178, 1992.

[204] Chow, Tai L, Introduction to electromagnetic theory: a modern perspective, Jones \& Bartlett Learning, Massachusetts, 2006

[205] M F. Ubeid, M. M. Shabat, M. O. Sid-Ahmed, "Effect of negative permittivity and permeability on the transmission of electromagnetic waves through a structure containing left-handed material," Natural Science Magazine, Vol.3, No.4, pp. 328-333, 2011

[206] F. Lattarelo, Electromagnetic Compatibility in Power Systems, Elsevier 1st edition, 2006

[207] Electromagnetic Health Organization, "EMF test of 2007 Toyota Prius hybrid", Electromagn. Health, 2008.

[208] H. Akagi, T. Shimizu, "Attenuation of Conducted EMI Emissions from an InverterDriven Motor", Power Electronics, IEEE Transactions on, vol. 23, pp. 282 - 290, 2008.

[209] Mutoh, N.; Nakashima, J.; Kanesaki, M.; , "Multilayer power printed structures suitable for controlling EMI noises generated in power converters," Industrial Electronics, IEEE Transactions on, vol.50, no.6, pp. 1085- 1094, Dec. 2003. 
[210] Aouine, O.; Labarre, C.; Costa, F.; , "Measurement and Modeling of the Magnetic Near Field Radiated by a Buck Chopper," Electromagnetic Compatibility, IEEE Transactions on, vol.50, no.2, pp.445-449, May 2008

[211] G. Ala, M.C. Di Piazza, G. Tine, F. Viola, G. Vitale, "Numerical Simulation of Radiated EMI in 42V Electrical Automotive Architectures," Magnetics, IEEE Transactions on, vol.42, no.4, pp.879-882, April 2006.

[212] Z. Cheng, H. Takahashi, B. Forghani, L. Liu, Y. Fan, T. Liu, J. Zhang and X. Wang, "3-D Finite Element Modeling and Validation of Power Frequency Multi-shielding Effect", Magnetics, IEEE Transactions on, vol. 48, pp. 243-246, Feb. 2012.

[213] A. Nejadpak, O.A. Mohammed, "Physics-Based Modeling of Power Converters From Finite Element Electromagnetic Field Computations," Magnetics, IEEE Transactions on , vol.49, no.1, pp.567-576, Jan. 2013.

[214] A. Nejadpak, B. Mirafzal, O. Mohammed and Lixiang Wei, "Effects of different switching algorithms on the thermal behavior of IGBT modules under pulse-load conditions," IECON 2010 - 36th Annual Conference on IEEE Industrial Electronics Society, vol., no., pp.451-456, 7-10 Nov. 2010

[215] M.R. Barzegaran, A. Nejadpak, O.A. Mohammed, "Evaluation- of High Frequency Electromagnetic Behavior of Planar Inductor Designs for Resonant Circuits in Switching Power Converters," applied computational electromagnetic society (ACES) journal, vol.26, no.9, pp.737-748, Sep 2011

[216] G. L. Skibinski, R. J. Kerkman and D. Schlegel "EMI emissions of modern PWM AC drives", IEEE Ind. Appl. Mag., vol. 5, no. 6, pp.47 -80 1999

[217] O. Martins , S. Guedon and Y. Marechal "A new methodology for early stage magnetic modeling and simulation of complex electronic systems", IEEE Trans. Magn., vol. 48, no. 2, pp.319-322 2012

[218] Giancoli, Douglas C. Physics: principles with applications. Pearson Education, 2005, pp. 624.

[219] Paul, Clayton R. Inductance: loop and partial. Wiley-IEEE Press, 2011, pp. 195.

[220] W. E. Arnoldi, "The principle of minimized iterations in the solution of the matrix eigenvalue problem," Quarterly of Applied Mathematics, volume 9, pages 17-29, 1951

[221] J. Stoer and R. Bulirsch, Introduction to numerical analysis, 3rd edition, springer, New York, 2002

[222] S. Nandi and H. A. Toliyat, "Condition monitoring and fault diagnosis of electrical machines-A review,” Proc. Ind. Applicat. Soc., vol. 1, 1999, pp. 197-204. 
[223] P. Vas, Parameter Estimation, Condition Monitoring, and Diagnosis of Electrical Machines. Oxford, U.K. Clarendon Press, 1993.

[224] Motor Reliability Working Group, "Report of large motor reliability survey of industrial and commercial installations Part I, and II," IEEE Trans. Ind. Appl., vol. IA-21, no. 4, pp. 853-872, Jul./Aug. 1985.

[225] J. R. Cameron, W. T. Thomson, and A. B. Dow,"Vibration and current monitoring for detecting air gap eccentricity in large induction motors," Proc. Inst. Elect. Eng. B, vol. 133, no. 3, pp. 155-163, May 1986.

[226] R. K. Singal, K. Williams, and S. P. Verma, "Vibration behavior of stators of electrical machines, Part II: Experimental study,” J. Sound Vib., vol.115, pp. 13-23, 1987.

[227] A. B. Sasi, B. Payne, A. York, F. Gu, and A. Ball. "Condition Monitoring of Electric Motors Using Instantaneous Angular Speed," [Online]. Available: www. Maintenance org.uk/RESEARCH/Marcon\%2001\%20publications/Ahmed.pdf

[228] Y.-S. Lee, J. K. Nelson, H. A. Scarton, D. Teng, and S. A. -Ghannad, "An acoustic diagnostic techniques for use with electric machine insulation," IEEE Trans. Dielectr. Electr. Insul., vol. 1, no. 6, pp. 1186-1193, Dec.1994

[229] J. S. Hsu, "Monitoring of defects in induction motors through air-gap torque observation,” IEEE Trans. Ind. Appl., vol. 31, no. 5, pp. 1016-1021, Sep./Oct. 1995.

[230] W. T. Thomson and M. Fenger, "Current signature analysis to detect induction motor faults," IEEE Ind. Appl. Mag., vol. 7, no. 4, pp. 26-34, July /Aug. 2001.

[231] M. A. Cash, T. G. Habetler, and G. B. Kliman, "Insulation failure prediction in induction machines using line-neutral voltages," Proc. IEEE Ind. Applicat. Soc. Annu. Meeting, vol. 1, Oct. 1997, pp. 208-212.

[232] G. C. Stone, H. G. Sedding, and M. J. Costello, "Application of partial discharge testing to motor and generator stator winding maintenance," IEEE Trans. Ind. Appl., vol. 32, no. 2, pp. 459-464, Mar./Apr. 1996.

[233] Siddique, Arfat, G. S. Yadava, and Bhim Singh, "A review of stator fault monitoring techniques of induction motors,", IEEE Trans. on Energy conversion, Vol. 20, no. 106$1141,2005$.

[234] Ch. Siva Kumar, A.V.R.S. Sarma, R. Somanatham, "Application of active power filter for compensating unbalanced load currents using symmetrical component theory," Information and Communication Technology in Electrical Sciences (ICTES 2007), ICTES. International Conference on IET-UK, pp.496-502, 20-22 Dec. 2007. 
[235] D. Mirabbasi, G. Seifossadat, M. Heidari, "Effect of unbalanced voltage on operation of induction motors and its detection," Electrical and Electronics Engineering, 2009, International Conference on ELECO 2009, pp.I-189-I-192, 5-8 Nov. 2009.Letter Symbols for Quantities, ANSI Standard Y10.5-1968.

[236] S.I. Nabeta, A. Foggia, J.-L. Coulomb, G. Reyne, "Finite element simulations of unbalanced faults in a synchronous machine," IEEE Transactions on Magnetics, vol.32, no.3, pp.1561-1564, May 1996.Letter Symbols for Quantities, ANSI Standard Y10.51968.

[237] Xu Hailiang, Hu Jiabing, He Yikang, "Integrated Modeling and Enhanced Control of DFIG Under Unbalanced and Distorted Grid Voltage Conditions," IEEE Transactions on Energy Conversion, vol.27, no.3, pp.725-736, Sept. 2012.Letter Symbols for Quantities, ANSI Standard Y10.5-1968.

[238] T. Tanaka, N. Koshio, H. Akagi, A. Nabae, "Reducing supply current harmonics," , IEEE Industry Applications Magazine, vol.4, no.5, pp.31-37, Sep/Oct 1998.

[239] A. Gandhi, T. Corrigan, L. Parsa, "Recent Advances in Modeling and Online Detection of Stator Inter-turn Faults in Electrical Motors," IEEE Transactions on Industrial Electronics, vol. 58, no. 5, pp. 1564-1575, May 2011.

[240] C. Gerada, K. Bradley, M. Sumner, P. Wheeler, S. Pickering, J. Clare, C. Whitley, and G. Towers, "The results do mesh," IEEE Industrial Application Magezine, vol. 13, no. 2, pp. 62-72, Mar./Apr. 2007.

[241] A. Bonnett and G. Soukup, "Cause and analysis of stator and rotor failures in threephase squirrel-cage induction motors," IEEE Transaction on Industry Application, vol. 28, no. 4, pp. 921-937, Jul./Aug. 1992.

[242] D. E. Schump, "Testing to assure reliable operation of electric motors," in Proc. IEEE Industry Application Society 37th Annu. Petrol. Chem. Ind. Conf., pp. 179-184, Sep. 1012, 1990.

[243] H. D. Kim, J. Yang, J. Cho, S. B. Lee, and J.-Y. Yoo, "An advanced stator winding insulation quality assessment technique for inverter-fed machines," IEEE Transaction on Industry Application, vol. 44, no. 2, pp. 555-564, Mar./Apr. 2008.

[244] C. Lanham, "Understanding the Tests That Are Recommended for Electric Motor Predictive Maintenance," Fort Collins, CO: Baker Instrument Co, 2002.

[245] H. D. Kim and Y. H. Ju, "Comparison of off-line and on-line partial discharge for large motors," in Conf. Rec. IEEE International Symposium Electrical Insulation, pp. 2730, Apr. 7-10, 2002. 
[246] G. C. Stone, E. A. Boulter, I. Culbert, and H. Dhirani, "Electrical Insulation for Rotating Machines: Design, Evaluation, Aging, Testing, and Repair," Vol. 21, Piscataway, NJ:Wiley-IEEE Press, 2004.

[247] S. Grubic, Jose M. Aller, Bin Lu, Thomas G. Habetler, "A Survey on Testing and Monitoring Methods for Stator Insulation Systems of Low-Voltage Induction Machines Focusing on Turn Insulation Problems," IEEE Transaction on Industrial Electronics, Vol. 55, No. 12, pp. 4127-4136, Dec 2008.

[248] G. C. Stone, "Advancements during the past quarter century in on-line monitoring of motor and generator winding insulation," IEEE Transaction on Dielectrics and Industrial Insulation, Vol. 9, No. 5, pp. 746-751, Oct. 2002.

[249] M. S. Ballal, Z. J. Khan, H. M. Suryawanshi, and R. L. Sonolikar, "Adaptive neural fuzzy inference system for the detection of inter-turn insulation and bearing wear faults in induction motor," IEEE Transaction on Industrial Electronics, Vol. 54, No. 1, pp. 250258, Feb. 2007.

[250] J. F. Martins, V. F. Pires, and A. J. Pires, "Unsupervised neural-network based algorithm for an on-line diagnosis of three-phase induction motor stator fault," Transaction on Industrial Electronics, Vol. 54, No. 1, pp. 259-264, Feb. 2007.

[251] S. H. Chetwani, M. K. Shah, and M. Ramamoorty, "Online condition monitoring of induction motors through signal processing," in Proc. 8th ICEMS, vol. 3, pp. 2175-2179, Sep. 27-29, 2005.

[252] P. Werynski, D. Roger, R. Corton, and J. F. Brudny, "Proposition of a new method for in-service monitoring of the aging of stator winding insulation in AC motors," IEEE Transaction on Energy Conversion, Vol. 21, No. 3, pp. 673-681, Sep. 2006.

[253] B. Mirafzal, R. J. Povinelli, and N. A. O. Demerdash, "Interturn fault diagnosis in induction motors using the pendulous oscillation phenomenon," IEEE Transaction on Energy Conversion, Vol. 21, No. 4, pp. 871-882, Dec. 2006.

[254] A. Stavrou, H. G. Sedding, and J. Penman, "Current monitoring for detecting interturn short circuits in induction motors," IEEE Transaction on Energy Conversion, Vol. 16, No. 1, pp. 32-37, Mar. 2001.

[255] T. Assaf, H. Henao, and G.-A. Capolino, "Simplified axial flux spectrum method to detect incipient stator inter-turn short-circuits in induction machine," in Proc. IEEE International Symposium on Industrial Electronics, Vol. 2, pp. 815-819, May 4-7, 2004.

[256] Juha Pyrhönen, Tapani Jokinen, and Valéria Hrabovcová, "Design of Rotating Electrical Machine”, Chichester, UK:John Wiley \& Sons, Ltd, 2008.

[257] J. Arrillaga, and N. Watson, Power system harmonics. Wiley, UK, 2004. 
[258] R. Yacamini, "Power system harmonics. Part 1. Harmonic sources." Power Engineering Journal, Vol. 8, No.4, pp. 193-198, 1994.

[259] F. D. Rosa, R. Langella, A. Sollazzo, and A. Testa, "On the inter harmonic components generated by adjustable speed drives," IEEE Trans. Power Del., vol. 20, no. 4, pp. 2535-2543, Oct. 2005.

[260] J. M. D. Murphy and F. G. Turnbull, Power Electronic Control of AC Motors. New York: Pergamon, 2005.

[261] Wang Jin-quan; Song Peng-chao; Cui Chen-hua; Li Jian-ke; Yang Tao, "Analysis of Operation of Synchronous Generator under the Distortion of Harmonic Current," Power and Energy Eng. Conf. (APPEEC), 2012.

[262] K. Zhou and D. Wang, " Relationship between space-vector modulation and threephase carrier-based PWM: A comprehensive analysis," IEEE Trans. Ind. Elec., vol. 49, no. 1, pp. 186-196, Feb. 2002.

[263] H. Van Der Broeck, H. Skudenly, and G. Stanke, "Analysis and realization of a pulsewidth modulator based on voltage space vectors," IEEE Trans. Ind. Appl., vol. 24, no. 1, pp. 142-150, Jan./Feb. 1988. 
VITA

\section{MOHAMMADREZA BARZEGARANBABOLI}

DEC 20, 1984 Born, Babol, Iran

2003-2007 B.Sc. University of Mazandaran

2007-2010 M.Sc. University of Mazandaran

2010 Lecturer, Hadaf University, Iran

2010-2012 Research Assistant, Florida International University

2012-2013 Doctoral Evidence Acquisition Fellowship, Florida

International University

2013-2014 Research Assistant, Florida International University

\section{SELECTED PUBLICATION AND PRESENTATIONS}

J1- M.R Barzegaran, Ali Mazloomzadeh, O.A. Mohammed "Fault Diagnosis of the Asynchronous Machines through Magnetic Signature Analysis using Finite Element Method and Neural Networks," IEEE Transaction on Energy Conversion, Vol. 28, No. 4, pp.1064-1071, DEC 2013.

J2- M.R Barzegaran, O.A. Mohammed, "Multi-Dipole Modeling of XLPE Cable for Electromagnetic Field Studies in Large Power Systems," The International Journal for Computation and Mathematics in Electrical and Electronic Engineering (COMPEL), vol. 33, No. 1, 2014.

J3- M.R Barzegaran, Ahmed Mohamed, Tarek Yussef, O.A. Mohammed "Electromagnetic signature study of the power converter connected to an electric motor drives," IEEE Transaction on Magnetics [In Press].

J4- M.R Barzegaran, Ali Sarikhani, Osama A. Mohammed, "An Optimized Equivalent Source Modeling for the Evaluation of Time Harmonic Radiated Fields from Electrical Machines and Drives" Applied Computational Electromagnetics Society Journal, Vol. 28, No. 4, pp. 273-282, Apr. 2013.

J5- M.R Barzegaran, O.A. Mohammed, "A Generalized Equivalent Source Model of AC Electric Machines for Numerical Electromagnetic Field Signature Studies," IEEE Transaction on Magnetics, Vol. 48, No. 11, Nov 2012. 
J6- Ali Sarikhani, M. Barzegaran, O. A. Mohammed, "Optimum Equivalent Models of Multi-source Systems for the Study of Electromagnetic Signatures and radiated Emissions from Electric Drives," IEEE Transaction on Magnetics, Vol. 48, Issue 2, pp. 1011-1014, 2012.

J7- A. Nejadpak, M.R Barzegaran, O.A. Mohammed, "Evaluation of High Frequency Electromagnetic Behavior of Planar Inductor Designs for Resonant Circuits in Switching Power Converters," Applied Computational Electromagnetic Society Journal (ACES), Vol.26, No 9, pp. 737-748, Sep 2011.

J8- M.R Barzegaran, O.A. Mohammed, "3-D FE Equivalent Source Modeling and Analysis of Electromagnetic Signatures from Electric Power Drive Components and Systems," IEEE Transaction on Magnetics, vol.49, no.5, pp.1937, 1940, May 2013.

J9- M.R Barzegaran, Arash Nejadpak, O.A. Mohammed, "Numerical Simulation of Electromagnetic Field Correlation between Components of Power Converter-Pulse Load System," Submitted to Applied Computational Electromagnetics Society Journal.

\section{INTERNATIONAL CONFERENCE PAPERS}

C1- $\quad$ M.R Barzegaran, A. Sarikhani, O.A. Mohammed, "Equivalent Current Loop Model for the Study of Radiated Electromagnetic Field Interference in Multi-Machine Electric Drives" ACES 2011, VA, USA 27-31 March 2011.

C2- M.R Barzegaran, A. Nejadpak, O.A. Mohammed, "High Frequency Electromagnetic Field Model for the Evaluation of Parasitic Elements in Power Converters" ACES 2011, VA, USA 27-31 March 2011.

C3- A. Nejadpak, M.R Barzegaran, A Sarikhani, O.A. Mohammed, "Design of Planar Inductor Based Z-Source Inverters for Residential Alternate Energy Sources," IEEE APEC 2011, TX USA, pp.1-8, 6-10 March 2011.

C4- A. Nejadpak, M.R Barzegaran, O.A. Mohammed, "Study of the high frequency electromagnetic behavior of planar inductor design for resonant circuits in switching power converters" IEEE CEM 2011, Poland, 6-10 April 2011.

C5- A. Sarikhani, M.R Barzegaran, O.A. Mohammed, "Optimum Equivalent source models of Multi-Conductor Systems for the Study of Electromagnetic Signatures and Radiated Emissions from Electric" IEEE COMPUMAG 2011, Australia 12-15 July 2011.

C6- $\quad$ M.R Barzegaran, A. Sarikhani, O.A. Mohammed, "An Equivalent Source Model for the Study of Radiated Electromagnetic Fields in Multi-Machine Electric Drive Systems" IEEE EMC 2011, CA, USA, 25-29 Aug 2011. 
C7- $\quad$ M.R Barzegaran, O.A. Mohammed, "A Generalized Equivalent Source Model of AC Electric Machines for Numerical Electromagnetic Field Signature Studies" IEEE INTERMAG 2012, Vancouver, Canada 7-11 MAY 2012.

C8- $\quad$ M.R Barzegaran, O.A. Mohammed, "Near Field Evaluation of Electromagnetic Signatures from Wound Rotor Synchronous Generators using Wire Modeling in Finite Element domain" 28th Annual Review of Progress in Applied Computational Electromagnetics, Columbus, OH, USA Apr. 2012.

C9- M.R Barzegaran, O.A. Mohammed, "Multi-Dipole Modeling of XLPE Cable for Electromagnetic Field Studies in Large Power Systems," The Sixth International Conference on Electromagnetic Field Problems and Applications (ICEF), June 1921 2012, Dalian, China.

C10- M.R Barzegaran, O.A. Mohammed, "3DFE Wire Modeling and Analysis of Electromagnetic Signatures from Electric Power Drive Components and Systems," Fifteenth Biennial IEEE Conference on Electromagnetic Field Computation (CEFC2012).

C11- A. Nejadpak, M.R Barzegaran, O.A. Mohammed, "Numerical Simulation of Low Frequency Far Fields form an Operating Multi-Component System Including Power Converter Load Switching," Fifteenth Biennial IEEE Conference on Electromagnetic Field Computation (CEFC2012).

C12- M.R Barzegaran, O.A. Mohammed, "Investigation of the Magnetic Signatures of Unbalanced Currents of Electric Motor Operation Using Finite Element Method and Neural Networks" 29th Annual Review of Progress in Applied Computational Electromagnetics, Monterey, CA, USA Mar. 2013.

C13- M.R Barzegaran, A. Nejadpak, O.A. Mohammed, "Condition Monitoring of PM Synchronous Motor Using High-Frequency Signal Injection in Low Speed Senseless Control Algorithm," IEEE International Electric Machines and Drives Conference IEMDC 2013, pp. 716-721, 12-15 May 2013.

C14- M.R Barzegaran, A. Nejadpak, O.A. Mohammed, "Electromagnetic signature study of the power converter connected to an electric motor drives," Presented at Conference on the Computation of Electromagnetic Fields (COMPUMAG 2013).

C15- M.R Barzegaran, A. Mazloomzadeh, O.A. Mohammed, "Identification of Shortcircuit Location in Induction Motors using Radiated Electromagnetic Field Signatures," 2013 International Conference on Electrical Machines and Systems (ICEMS), pp.484,489, 26-29 Oct. 2013.

C16- M.R Barzegaran, O.A. Mohammed, "Combined Edge Modelling Technique for Electromagnetic Signature Study of the Large Direct Current Machines", 30th Annual Review of Progress in Applied Computational Electromagnetics, Jacksonville, FL, USA Mar. 2014. 